NASA/CR-2010-216188

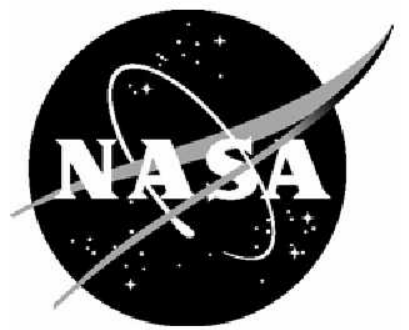

\title{
Integrated Analysis of Airport Capacity and Environmental Constraints
}

Shahab Hasan, Dou Long, George Hart, Jeremy Eckhause, Robert Hemm, and Andrew Busick LMI Government Consulting, McLean, Virginia

Michael Graham, Terry Thompson, and Charles Murphy

Metron Aviation, Dulles, Virginia

James Poage

JPL Performance Consulting, Lexington, Massachusetts 


\section{NASA STI Program ... in Profile}

Since its founding, NASA has been dedicated to the advancement of aeronautics and space science. The NASA scientific and technical information (STI) program plays a key part in helping NASA maintain this important role.

The NASA STI program operates under the auspices of the Agency Chief Information Officer. It collects, organizes, provides for archiving, and disseminates NASA's STI. The NASA STI program provides access to the NASA Aeronautics and Space Database and its public interface, the NASA Technical Report Server, thus providing one of the largest collections of aeronautical and space science STI in the world. Results are published in both non-NASA channels and by NASA in the NASA STI Report Series, which includes the following report types:

- TECHNICAL PUBLICATION. Reports of completed research or a major significant phase of research that present the results of NASA programs and include extensive data or theoretical analysis. Includes compilations of significant scientific and technical data and information deemed to be of continuing reference value. NASA counterpart of peerreviewed formal professional papers, but having less stringent limitations on manuscript length and extent of graphic presentations.

- TECHNICAL MEMORANDUM. Scientific and technical findings that are preliminary or of specialized interest, e.g., quick release reports, working papers, and bibliographies that contain minimal annotation. Does not contain extensive analysis.

- CONTRACTOR REPORT. Scientific and technical findings by NASA-sponsored contractors and grantees.
- CONFERENCE PUBLICATION. Collected papers from scientific and technical conferences, symposia, seminars, or other meetings sponsored or co-sponsored by NASA.

- SPECIAL PUBLICATION. Scientific, technical, or historical information from NASA programs, projects, and missions, often concerned with subjects having substantial public interest.

- TECHNICAL TRANSLATION. Englishlanguage translations of foreign scientific and technical material pertinent to NASA's mission.

Specialized services also include creating custom thesauri, building customized databases, and organizing and publishing research results.

For more information about the NASA STI program, see the following:

- Access the NASA STI program home page at http://www.sti.nasa.gov

- E-mail your question via the Internet to help@sti.nasa.gov

- Fax your question to the NASA STI Help Desk at $443-757-5803$

- Phone the NASA STI Help Desk at 443-757-5802

- Write to:

NASA STI Help Desk NASA Center for AeroSpace Information 7115 Standard Drive Hanover, MD 21076-1320 
NASA/CR-2010-216188

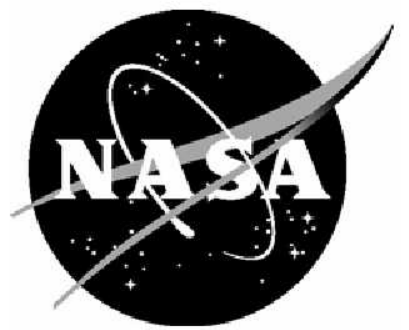

\section{Integrated Analysis of Airport Capacity and Environmental Constraints}

Shahab Hasan, Dou Long, George Hart, Jeremy Eckhause, Robert Hemm, and Andrew Busick LMI Government Consulting, McLean, Virginia

Michael Graham, Terry Thompson, and Charles Murphy

Metron Aviation, Dulles, Virginia

James Poage

JPL Performance Consulting, Lexington, Massachusetts

National Aeronautics and

Space Administration 
Available from:

NASA Center for AeroSpace Information 7115 Standard Drive

Hanover, MD 21076-1320 443-757-5802 


\section{Integrated Analysis of Airport Capacity and Environmental Constraints}

DECEMBER 2009

\section{Executive Summary}

NASA's goal for the Next Generation Air Transportation System (NextGen) Air Traffic Management (ATM) Airportal Project is to develop concepts, capabilities, and technologies that mitigate airport system constraints to NextGen capacity goals. Through a NASA Research Announcement award, LMI conducted an integrated analysis of airport capacity and environmental constraints, and the identification and ranking of the key factors limiting the achievement of the capacity goals for NextGen.

The primary metric used for the airport constraints analysis was projected throughput, which is an estimate of the number of daily or annual operations that an airport can support in nominal operational conditions given the capacity and environmental constraints faced by the airport. This metric can also be expressed as a percentage of unconstrained demand such that the ultimate desired projected throughput is $100 \%$ of unconstrained demand. It is a robust capacity metric developed by LMI that has been applied to prior tasks sponsored by both NASA and the Joint Planning and Development Office (JPDO).

The projected throughput was estimated for 2015 and 2025, the two years which the JPDO has used for its studies, based on the unconstrained demand forecast from the Federal Aviation Administration (FAA), planned runway improvements and ATM programs, and other operational improvements proposed in the NextGen research plan. The study identified a set of 310 critical airports, which collectively account for more than 99 percent of domestic air traffic volume, and used a one-off analytical approach to isolate the constraint being assessed and to identify each airport's primary and secondary constraints affecting airport throughput. The study considered three airport capacity constraints (runway, taxiway, and gate) and three environmental constraints (fuel, NOx emissions, and noise).

In addition to identifying the constraints at individual airports, LMI aggregated the results by the following airport groups, where each is a superset of the previous one by including the next largest airports:

- Busiest 10 airports. For this group of airports, runway and noise are the primary and secondary constraints in both 2015 and 2025. From 2015 to 
2025 , their projected throughput percentages drop by about 4 percent, from the low 90 s to high $80 \mathrm{~s}$. The drop in throughput percentage under the runway constraint is due to increasing traffic compared to relatively stable airport capacities between the 2 years. The feasible throughput for airports under the other four constraints (taxiway, gate, emissions, and $\mathrm{NOx}$ ) are comparable (in the 90s for both years), but is generally lower in 2025 .

- OEP 35 airports. The most binding constraint for OEP 35 airports is noise. The projected throughput percentages for both 2015 and 2025 are in the mid 80 s. In contrast, the throughput percentages for the other five constraints are in the mid to upper $90 \mathrm{~s}$. For the three capacity constraints, the projected throughput percentages drop by a few points from 2015 to 2025 . For airports under the NOx constraint, the throughput percentage drops about one point, but under the emissions constraint, the throughput percentage increases slightly from 2015 to 2025 .

- LMI 110 airports and LMI 310 airports. For the two LMI airport groups, the results are virtually identical. The primary constraint is noise, under which the projected throughput percentages for the two years are in the mid 80 s, but the percentage increases slightly from 2015 to 2025 . The throughput percentages for the remaining five constraints are in the mid 90s.

Of the 310 airports, 32 airports, including 6 of the 10 busiest, will face runway constraints in 2025, and 95 will face gate constraints; only 12 airports will face taxiway constraints by 2025, but all 12 are large airports (OEP 35). In addition, nearly every airport will be subject to constraints due to emissions and NOx, and 237 airports will be subject to noise constraints.

To policymakers, the results of this study mean that there are still significant capacity shortfalls even under NextGen. The runway and taxi constraints are more concentrated in the large airports and the environmental constraints are present at almost every airport regardless of its size. Other strategies ought to be explored to meet the traffic growth. The widespread environment constraints suggest new accelerated engine and airframe programs are needed to mitigate the environmental constraints. 


\section{Contents}

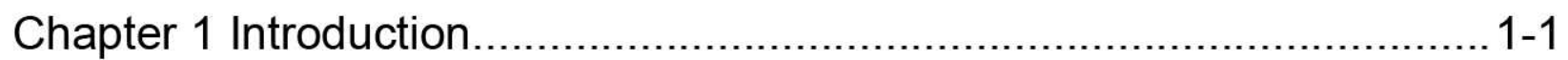

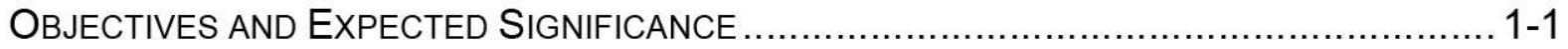

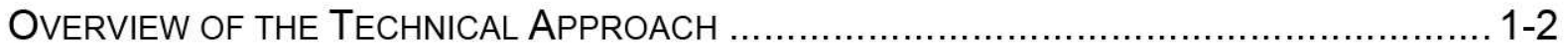

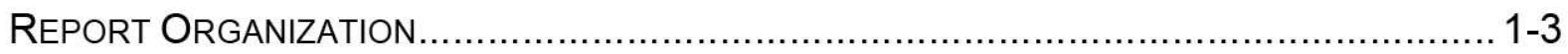

Chapter 2 Develop a Set of Scenarios ................................................. 2-1

SCENARIO DIMENSIONS AND PARAMETERS ....................................................... 2-1

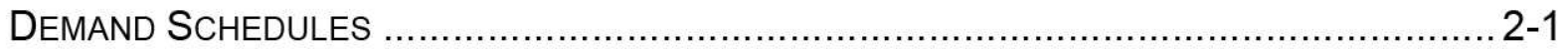

Chapter 3 Develop a Set of Metrics …........................................... 3-1

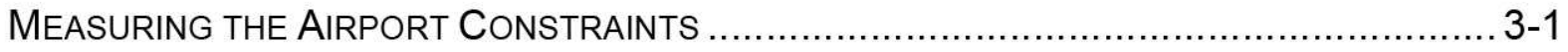

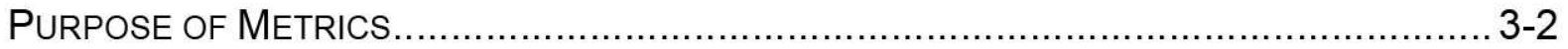

Approach to Developing Metrics for the Airportal Project ........................... 3-3

SUMMARY OF RECOMMENDED METRICS AND FRAMEWORKS ..................................... 3-6

Top-Level Airport Metrics …................................................................... 3-6

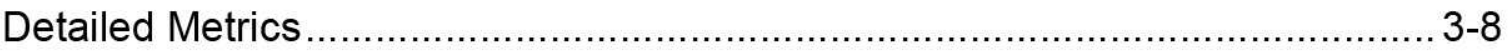

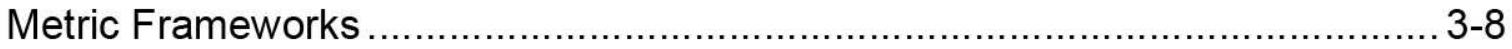

Chapter 4 Develop a Set of Critical Airports ......................................... 4-1

DEFINE THE SUPERSET OF CANDIDATE AIRPORTS ............................................... 4-1

DeVElop and Apply Assumptions to IDENTIFy a Subset of CRitical AirPorts ...... 4-2

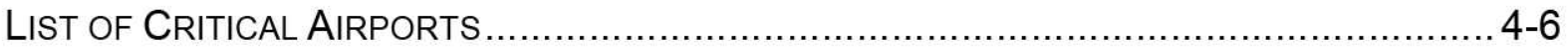

Chapter 5 Analyze Airport Capacity Constraints ................................... 5-1

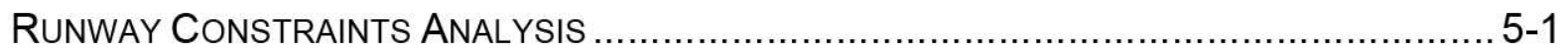

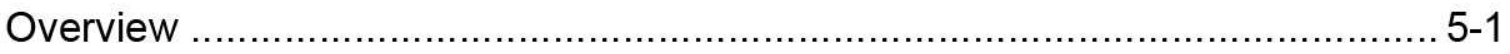

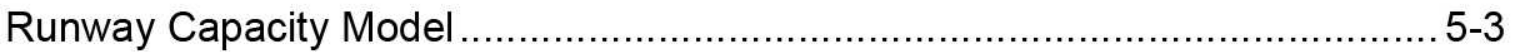

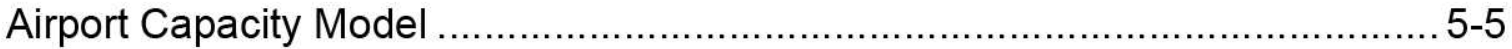

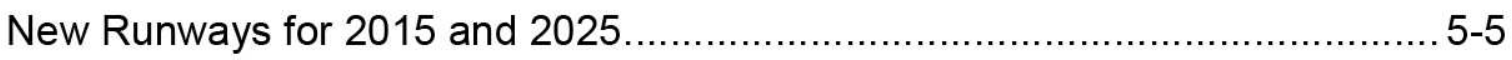

NextGen Technology Improvements ........................................................

MIT-Limited and ROT-Limited Capacities ................................................... 5-9 
Projected Throughput Results for NextGen

Projected Throughput Results for ROT-Limited and MIT-Limited Operations

Delay Results for NextGen Case

Delay Results for ROT-Limited and MIT-Limited Cases

TAXIWAY CONSTRAINTS ANALYSIS

Overview

Taxiway Delay Model

Taxiway Demand

Taxiway Delay and Its Relationship to Demand …................................... 5-30

Surface Interaction and Taxi Delays ........................................................... 5-31

Simulation-Based Taxiway Model..................................................................

Demand-Based Airport Elimination......................................................... 5-40

Configuration-Based Airport Elimination ................................................... 5-41

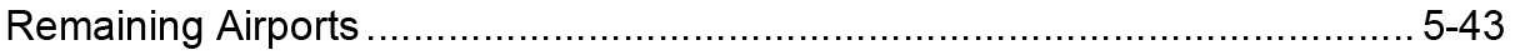

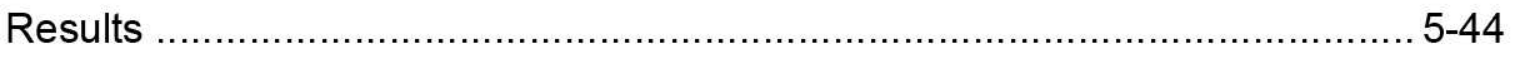

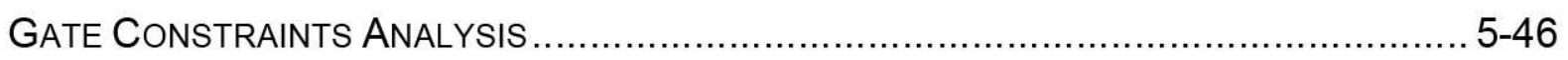

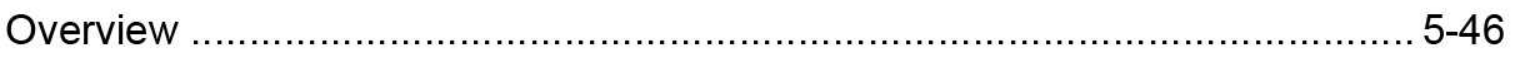

Factors Affecting Gate Capacity .......................................................... 5-47

Approaches to Modeling Gate Usage ................................................... 5-48

Modeling the Current and Future States.................................................. 5-49

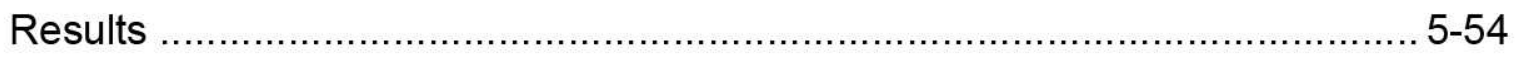

Chapter 6 Analyze Airport Environmental Constraints ..........................6-1

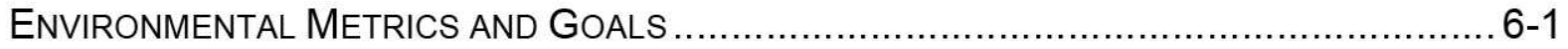

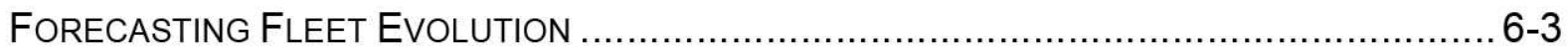

Modeling OF FUeL EfFICIENCY, EmISSIONS, AND NOISE ...................................... 6-7

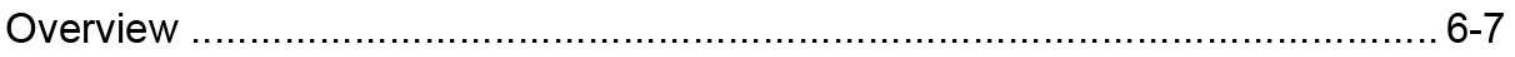

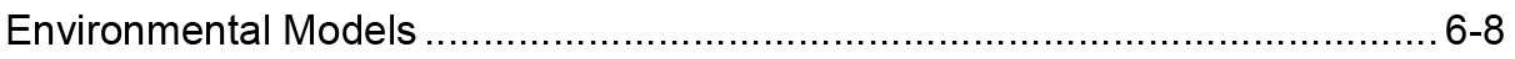

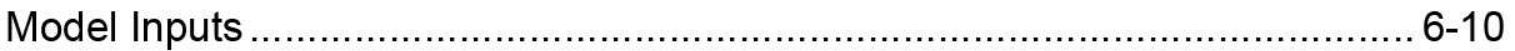

Fuel Efficiency and Emissions Computations ........................................... 6-18

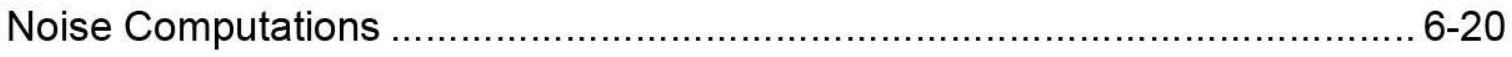


ANALYSIS of ENVIRONMENTAL CONSTRAINTS AT CRITICAL AIRPORTS 6-23

Fuel Efficiency 6-24

Emissions 6-25

Noise 6-27

RESULTS 6-30

Chapter 7 Catalog the Primary and Secondary Airport Constraints $7-1$

UNCERTAINTIES AND LIMITATIONS 7-1

BUSIEST 10 AIRPORTS. 7-2

OEP 35 AIRPORTS. $7-4$

LMI 110 AIRPORTS 7-5

LMI 310 AIRPORTS $7-6$

Chapter 8 Analyze Shift to Metroplex Operations 8-1

METROPLEX CAPACITY CONSTRAINTS...................................................................... 8-2

Method 8-2

Results 8-3

Summary of Metroplex Capacity Constraints 8-9 METROPLEX ENVIRONMENTAL CONSTRAINTS $8-10$

Overview $8-10$

Method $8-12$

Results $8-14$

Summary of Metroplex Environmental Constraints 8-20

Appendix A LMI Runway Capacity Model

Appendix B Airport Capacities

Appendix C IFR Delays at 310 LMINET Airports

Appendix D Abbreviations 


\section{Figures}

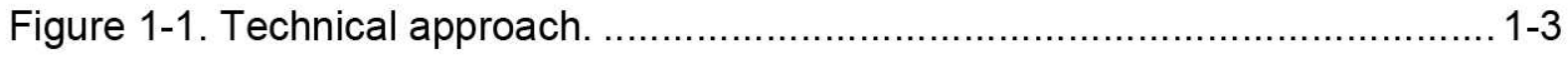

Figure 3-1. Metrics for phases of flight for aircraft using an airport: example from terminal airspace arrival through terminal airspace departure................ 3-11

Figure 4-1. NPIAS airport types................................................................. 4-1

Figure 4-2. Airport selection process. ................................................................ 4-4

Figure 4-3. Air carrier operations by airport sets.......................................... 4-5

Figure 4-4. Air taxi operations by airport sets. .............................................. 4-5

Figure 4-5. Locations of 310 critical airports. .................................................... $4-6$

Figure 5-1. Single runway capacity Pareto. .................................................... 5-3

Figure 5-2. 2015 NextGen VMC1 single runway capacities............................. 5-12

Figure 5-3. 2015 NextGen VMC2 single runway capacities.............................. 5-12

Figure 5-4. 2015 NextGen IMC1 single runway capacities. ............................... 5-13

Figure 5-5. 2025 NextGen VMC1 single runway capacities.............................. 5-13

Figure 5-6. 2025 NextGen VMC2 single runway capacities.............................. 5-14

Figure 5-7. 2025 NextGen IMC1 single runway capacities. ................................ 5-14

Figure 5-8. Comparison of operations at 10 busiest airports. .......................... 5-18

Figure 5-9. Comparison of aggregate airport operations. ................................ 5-19

Figure 5-10. Throughput under NextGen and ROT-limited cases for the 10

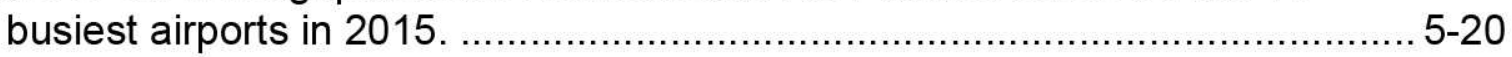

Figure 5-11. Throughput comparison for NextGen and ROT-limited cases by

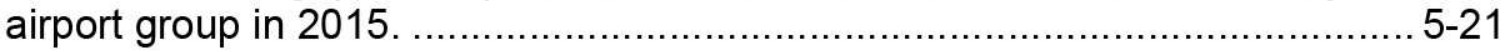

Figure 5-12. Throughput under NextGen, ROT-limited, and MIT-limited cases for the 10 busiest airports in 2025 .

Figure 5-13. Throughput comparison for NextGen, ROT-limited, and MITlimited cases by airport group in 2025 .

Figure 5-14. Average 2007 IFR per flight-delay minutes at 10 busiest airports. ... 5-24

Figure 5-15. Average 2015 IFR per flight-delay minutes at 10 busiest airports. ... 5-24

Figure 5-16. Average 2025 IFR per flight-delay minutes at 10 busiest airports. ... 5-25

Figure 5-17. Average 2015 IFR arrival delay minutes at 10 busiest airports........ 5-26

Figure 5-18. Average 2015 IFR departure delay minutes at 10 busiest airports.

Figure 5-19. Average 2025 IFR arrival delay minutes at 10 busiest airports. 
Figure 5-20. Average 2025 IFR departure delay minutes at 10 busiest airports.

Figure 5-21. LGA taxi-in times as a function of demand.

Figure 5-22. Average LGA taxi-in times as a function of demand. 5-31

Figure 5-23. ORD runway layout.. $5-32$

Figure 5-24. LGA runway layout. $5-33$

Figure 5-25. Average ORD taxi-in time as a function of demand. $5-33$

Figure 5-26. LGA airport layout. 5-35

Figure 5-27. Arena model screen shot for ORD. $5-36$

Figure 5-28. Hypothetical worst-case airport design. $5-40$

Figure 5-29. JFK under the most common runway configuration. $5-42$

Figure 5-30. Breakdown of taxiway analysis. $5-43$

Figure 5-31. Taxiway throughput by airport group and year.

Figure 5-32. 2015 and 2025 unconstrained and taxiway-constrained throughput for 10 busiest airports.

Figure 5-33. Aircraft on the ground at IAD in 2025 .........................................

Figure 5-34. Gate throughput by airport group and year.

Figure 5-35. 2015 and 2025 unconstrained and gate-constrained throughput out of eligible operations for 10 busiest airports.

Figure 5-36. 2015 and 2025 unconstrained and gate-constrained throughput out of all operations for 10 busiest airports.

Figure 6-1. Fleet evolution process.......................................................... 6-4

Figure 6-2. Environmental constraints modeling.......................................... 6-8

Figure 6-3. Wind rose sample for Sterling, Virginia.................................... 6-13

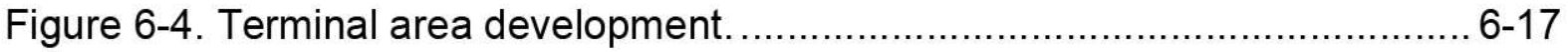

Figure 6-5. Approach to airport environmental constraints analysis................... 6-24

Figure 6-6. Cincinnati/Northern Kentucky International Airport (CVG) with a census population point on the airport grounds....................................... 6-28

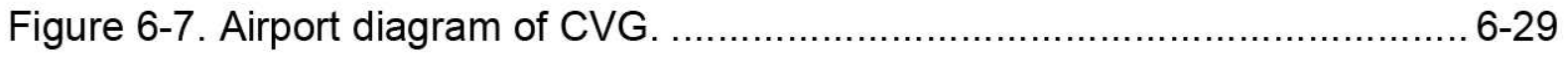

Figure 6-8. Hayward Executive Airport (HWD) with a census population point near the runway.

Figure 6-9. Unconstrained and fuel-constrained throughput by airport groups. .... 6-31

Figure 6-10. 2015 and 2025 unconstrained and NOx-constrained throughput by airport groups. 
Figure 6-11. 2015 and 2025 unconstrained and noise-constrained throughput by airport groups.

Figure 6-12. 2015 and 2025 unconstrained and fuel-constrained throughput for the 10 busiest airports.

Figure 6-13. 2015 and 2025 unconstrained and NOx-constrained throughput for the 10 busiest airports.

Figure 6-14. 2015 and 2025 unconstrained and noise-constrained throughput for the 10 busiest airports.

Figure 7-1. Comparison of throughput in 2015 under constraints at busiest 10 airports.

Figure 7-2. Comparison of throughput in 2025 under constraints at busiest 10 airports.

Figure 7-3. Comparison of throughput in 2015 under constraints at OEP 35 airports.

Figure 7-4. Comparison of throughput in 2025 under constraints at OEP 35 airports.

Figure 7-5. Comparison of throughput in 2015 under constraints at LMI 110 airports.

Figure 7-6. Comparison of throughput in 2025 under constraints at LMI 110 airports.

Figure 7-7. Comparison of throughput in 2015 under constraints at LMI 310 airports.

Figure 7-8. Comparison of throughput in 2025 under constraints at LMI 310 airports.

Figure 8-1. Locations of the 77 airports in ASPM.

Figure 8-2. Potomac TRACON metroplex.

Figure 8-3. Southern Florida metroplex.

Figure 8-4. San Francisco Bay Area metroplex. 8-6

Figure 8-5. Chicago area metroplex 8-6

Figure 8-6. Los Angeles metroplex. 8-7

Figure 8-7. Houston metroplex. $8-8$

Figure 8-8. Dallas metroplex. 8-8

Figure 8-9. New York City metroplex.

Figure 8-10. Counties designated "Nonattainment" or "Maintenance" for NAAQS pollutants

Figure 8-11. Characteristic interaction for noise: area. 
Figure 8-12. Characteristic interaction for noise: elliptical major axis.................. 8-13

Figure 8-13. Characteristic interaction for air quality and noise ....................... 8-14

Figure 8-14. New York metroplex: 2015 noise contours. .............................. 8-15

Figure 8-15. New York Region: counties designated "Nonattainment" or

"Maintenance" for NAAQS pollutants........................................................ 8-16

Figure 8-16. New York metroplex: aggregate constraints................................ 8-16

Figure 8-17. Southern California metroplex: 2015 noise contours. .................... 8-17

Figure 8-18. Southern California region: counties designated "Nonattainment" or "Maintenance" for NAAQS pollutants. ................................................. 8-17

Figure 8-19. Southern California metroplex: aggregate constraints.................. 8-18

Figure 8-20. Chicago metroplex: 2015 noise contours.................................. 8-19

Figure 8-21. Chicago region: counties designated "Nonattainment" or

"Maintenance" for NAAQS pollutants.

Figure 8-22. Chicago metroplex: aggregate constraints.

\section{Tables}

Table 3-1. Suggested Metrics for Supporting Goals ..................................... 3-7

Table 3-2. Metrics for Airport Runway Management ......................................... 3-8

Table 3-3. Metrics for Airport Taxi Route Planning ........................................ 3-9

Table 3-4. Sample Metrics for Airportal Project Topics of Interest ....................... 3-10

Table 3-5. Metrics for Benefit-Creating Mechanism: Example of Improving Aircraft Sequencing and Scheduling for Arrivals at a Runway ........................ 3-12

Table 4-1. Description of NPIAS Airport Types ............................................... 4-2

Table 4-2. LMINET-110 Airports .............................................................. 4-7

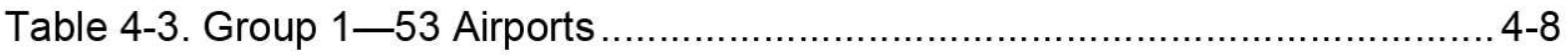

Table 4-4. Group 2-40 Airports ................................................................. 4-9

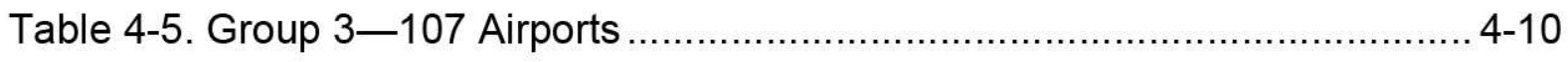

Table 5-1. LMI Capacity Model Parameters................................................... 5-4

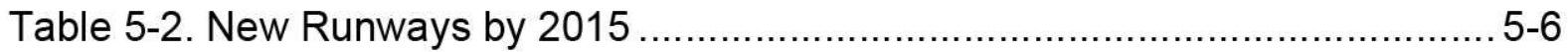

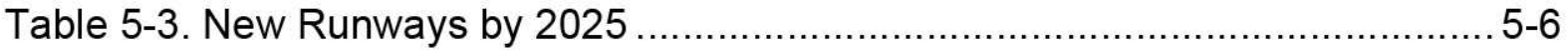

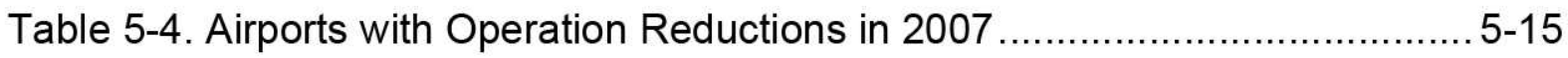

Table 5-5. Airports with Operation Reductions in 2015 ................................. 5-16

Table 5-6. Airports with Operation Reductions in 2025 ................................. 5-17

Table 5-7. Details of Flight Trimming and Delay at ORD and STL in 2025 .......... 5-39 
Table 5-8. Taxiway Throughput at Constrained Airports. $5-46$

Table 5-9. Gate Throughput for 310 Airports, 2015 and 2025 $5-57$

Table 6-1. Environmental Targets for 2015 and 2025 6-3

Table 6-2. Environmental Substitutes for New Aircraft. 6-5

Table 6-3. Environmental Projections from the National Aeronautics Research and Development Plan

Table 6-4. Environmental Projections from the Environmental Working Group's Technology Standing Committee 6-6

Table 6-5. Surrogate Aircraft for Future Fleet Performance 6-7

Table 6-6. ICAO Default Times in Mode 6-26

Table 7-1. 2015 Throughput under Constraints at Busiest 10 Airports 7-3

Table 7-2. 2025 Throughput under Constraints at Busiest 10 Airports 7-3

Table 7-3. Number of Constrained Airports by Category in 2015 7-7

Table 7-4. Number of Constrained Airports by Category in 2025 7-7

Table 7-5. 2015 Throughput under Constraints at 310 Critical Airports . 7-8

Table 7-6. 2025 Throughput under Constraints at 310 Critical Airports 7-15 


\section{Chapter 1 \\ Introduction}

This report is the third and final deliverable of our task under NASA Research Announcement NNH06ZEA001N, "Research Opportunities in Aeronautics-2006," addressing the Airspace Systems Program, Next Generation Air Transportation System (NextGen) Air Traffic Management (ATM) Airportal Project, Subtopic 3, "Constraints to Achieving Airportal Capacity Requirements." The first deliverable laid the foundation and outlined the research method, the second report built upon the first one by describing our progress in developing the constituent modeling tools and some initial results, and this report adds the final results and preliminary analysis of metroplex constraints.

The goal of the NASA NextGen ATM-Airportal Project is to develop concepts, capabilities, and technologies that mitigate airport system constraints to NextGen capacity goals. The airport domain includes the airport surface (taxiways and runways) and the immediate terminal airspace used for metering and spacing to final approach and for the initial climb during departure. The airport domain also includes groups of closely situated airports (collectively referred to as a "metroplex") that have the potential to be operated as a system to improve capacity or that may impose constraints on each other. NASA research on achieving NextGen capacity goals requires analysis of the constraints to achieving these goals and development of a modeling capability to periodically assess technology and concept options as research efforts and NextGen definitions evolve. Maximizing the value of the NASA research requires prioritizing potential research areas, establishing realistic performance metrics, identifying "breakthrough" research areas, and understanding the dependencies among technologies and concepts. Research is also needed to determine the point at which improvements in particular technology domains are no longer costeffective and entirely new approaches ought to be pursued.

\section{ObJectives ANd EXPECTEd SignificAnce}

The NASA NextGen ATM-Airportal Project asked LMI to conduct an integrated analysis of airport capacity and environmental constraints. Specifically, NASA asked us to identify and rank the key factors limiting the achievement of the capacity goals for NextGen and to identify capabilities required, and gaps in available tools, for conducting system-level trade and benefit studies. This report presents the results of our research. Our research results will assist with the identification, focusing, and prioritization of fundamental research to enable NextGen. 
This research also supports the following NASA strategic subgoal and outcome:

- Strategic Subgoal 3E-Advance knowledge in the fundamental disciplines of aeronautics, and develop technologies for safer aircraft and higher capacity airspace systems.

- Outcome 3E.2-By 2016, develop and demonstrate future concepts, capabilities, and technologies that will enable major increases in air traffic management effectiveness, flexibility, and efficiency, while maintaining safety, to meet capacity and mobility requirements for the Next Generation Air Transportation System.

\section{OVERVIEW OF THE TECHNICAL APPROACH}

We organized our research into seven subtasks. Figure 1-1 identifies those subtasks, shows how the subtasks relate to each other, and shows the nominal timeline for our research. We considered two major categories of airport constraints: capacity and environmental. Capacity constraints - which we subdivide into runway, taxiway, and gate constraints - are the limits to safely moving aircraft within airports. Environmental constraints - which we further subdivide into fuel, emission, and noise constraints in the vicinity of airportscan limit the number of aircraft allowed to use the airports due to environmental mandates.

Before conducting the constraint analyses, we first undertook three subtasks to help scope the work:

- Develop the scenarios of interest, which specify the traffic and technology parameters to be used in the analyses

- Develop the metrics to be used to measure the constraints

- Develop the set of airports that constitute a critical set covering the significant airport operations in the National Airspace System (NAS).

As shown in the figure, we did the first three subtasks in parallel, and the results fed subtasks 4 and 5. Subtasks 4 and 5, in turn, fed subtask 7, but there is also iteration between these three subtasks. Subtask 6 was largely done independently of the other subtasks. 


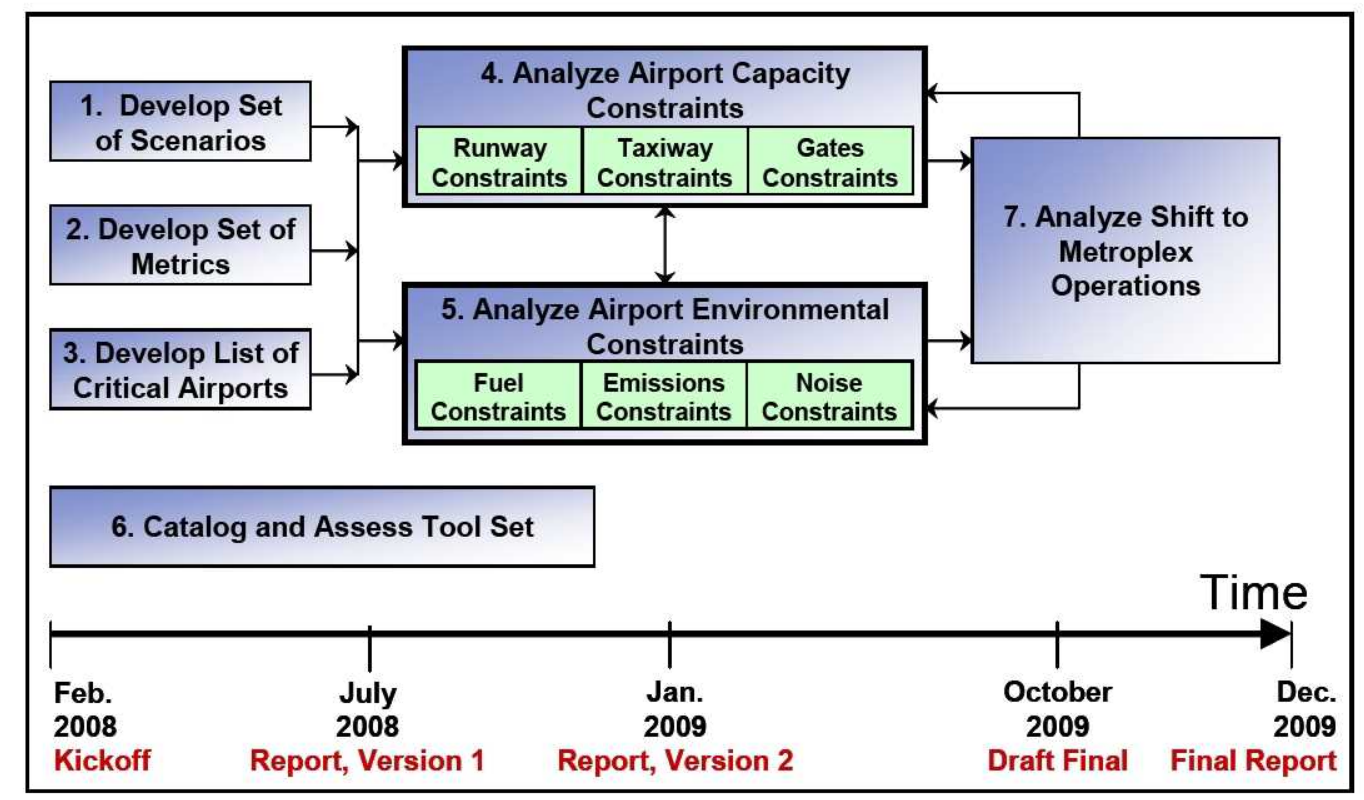

Figure 1-1. Technical approach.

\section{REPORT ORGANIZATION}

Each subtask is covered in a subsequent chapter of this report:

- Chapter 2, Develop a Set of Scenarios (subtask 1), explains the dimensions of the scenarios we intend to analyze, such as the level of air traffic demand and the projected capacity of the system. It then describes the particular scenarios that we chose.

- Chapter 3, Develop a Set of Metrics (subtask 2), introduces the primary metric we use to quantify airport constraints, contains research on prior relevant efforts to define metrics, and recommends a common set of metrics to be used by the NextGen ATM-Airportal Project to support its ongoing research.

- Chapter 4, Develop a Set of Critical Airports (subtask 3), looks at a master set of U.S. airports and then identifies a subset to serve as a critical set of airports suitable for our research effort and for other Airportal Project studies.

- Chapter 5, Analyze Airport Capacity Constraints (subtask 4), details our modeling approach and assessment of three categories of airport capacity constraints: runways, taxiways, and gates.

- Chapter 6, Analyze Airport Environmental Constraints (subtask 5), details our modeling approach and assessment of three categories of airport environmental constraints: fuel, emission, and noise. 
- Chapter 7, Catalog the Primary and Secondary Airport Constraints, ranks the constraints based on the results in Chapters 5 and 6 .

- Chapter 8, Analyze Shift to Metroplex Operations (subtask 7), examines the definitions associated with metroplex operations and the effect of these operations on airport capacity and environmental constraints.

The appendixes contain additional detail on the LMI runway capacity model, the capacities at the 310 critical airports identified as part of this research effort, detailed delay results, and abbreviations used in the report.

The results of subtask 6 are in a companion report: Catalog of Models for Assessing the Next-Generation Air Transportation System. That report surveys and classifies the modeling and simulation tools available to assess airport capacity and environmental constraints. 


\section{Chapter 2 \\ Develop a Set of Scenarios}

For our analysis, we needed to develop a set of scenarios such as the level of air traffic demand and the projected capacity of the system. This chapter explains our approach; it included determining scenario dimensions and parameters, obtaining demand schedules, and forecasting the evolution of the fleet. Each scenario will need to specify the anticipated level of demand, which, when compared to the anticipated capacity of the system, will indicate the airport capacity and environmental constraints.

\section{SCENARIO DIMENSIONS AND PARAMETERS}

The LMI team had several discussions with members of the NASA NextGen Airportal Project staff to collaborate on developing a set of scenarios under which airport constraints will be analyzed. The basic parameters that define such scenarios are the level of future demand and the state of future NAS capacity.

After consultation with the NASA task sponsor, we selected the unconstrained demand at 2015 and 2025 as the demand levels for use in this analysis. Unconstrained demand is forecast purely based on socioeconomic factors regardless of the feasibility of whether the NAS can sustain such traffic levels. In addition, we selected 2007 as the baseline year, which we use for delay comparisons and especially for the environmental constraints analysis because future constraints are specified as the relative increase or decrease from the baseline values.

For the NAS capacities, we selected the NextGen airport capacities at 2015 and 2025 that are used by the Joint Planning and Development Office (JPDO). The NextGen capacities include the planned runway improvements, the existing Federal Aviation Administration (FAA) ATM programs, and other operational improvements proposed in the NextGen research plan. The reason to select the NextGen capacities is to see whether the proposed NextGen can support the traffic growth to 2025 and to determine the effect on the constraints we are assessing. The capacities for the baseline year are also the ones used by the JPDO. Chapter 4 and Appendix A provide further details regarding the airport capacities.

\section{DEMAND SCHEDULES}

Generating or obtaining future air traffic demand schedules is a fundamental component of the required analysis. Although "demand" often refers to the overall traffic volume - which usually takes the forms of total number of operations, total 
number of passengers, or total number of revenue passenger miles in a year in the United States - the flight schedule in a day is more specific; it includes the origin, destination, equipment, and time of operations for throughput and environmental constraints analyses. Aggregating all flights will give us the typical measures of traffic demand.

Although LMI has demand-generation techniques and algorithms that we have applied to numerous NASA analyses, we decided, in consultation with NASA, to obtain and use future demand schedules directly from the FAA Air Traffic Organization-Planning (ATO-P). This group has expressed its desire to NASA and the JPDO to be the source of record for future demand schedules. The motivation for this is that just as the FAA's projections for overall and airportspecific traffic forecasts are regarded as "official" (specifically, the FAA Aerospace Forecasts and the FAA Terminal Area Forecast, or TAF), the FAA should also generate and provide air traffic schedules to the aviation research and analysis community. The TAF has the forecast of airport annual operations, among other annual measures. When forecasting the traffic flight schedule for a day in the future, ATO-P first selects a day in the baseline year and applies the Fratar algorithm to grow the flight schedule according to the different operational growth rates from the baseline year to the target year as specified in TAF. In this study, the seed date in the baseline year is August 2, 2007, which ATO-P has selected as one of the representative days; that date has also been used in JPDO studies. 


\section{Chapter 3 \\ Develop a Set of Metrics}

The LMI team developed a set of performance metrics for use in future system benefit and trade studies related to the NextGen ATM-Airportal Project. We designed the performance metrics to provide information needed by Airportal Project managers and staff members to make decisions regarding the research portfolio; to reflect realistically the benefits, costs, and other attributes of potential concepts or technology; to reflect the NextGen metrics developed by JPDO; and to help the Airportal Project communicate with its stakeholders. We delivered three documents to NASA in April 2008:

- Metrics for NextGen ATM-Airportal Project. This document contains details about the metrics developed.

- Examples of Metrics Narrative Frameworks for the NextGen ATMAirportal Project. This document presents possible metrics frameworks that are designed to aid decision making and communications about the Airportal Project.

- Airportal-Related Metrics Proposed in NextGen, NASA, and FAA Documents. This document lists metrics that have been used in other NextGen, NASA, and FAA programs. We reviewed them to assess their relevance for the Airportal Project.

In this chapter, we first discuss the specific metric we used to measure airport constraints. We then examine the general purpose of metrics and describe our approach to developing a set of metrics for the Airportal Project. The chapter concludes with a summary of recommended metrics and frameworks to be used in the Airportal Project's ongoing research.

\section{MEASURING THE AIRPORT CONSTRAINTS}

The primary metric we use for the airport constraints analyses is projected throughput. ${ }^{1}$ Projected throughput is an estimate of the number of daily or annual operations that an airport can support in nominal - visual flight rules (VFR) operational conditions. This is a consolidated capacity metric developed by LMI that has been applied to prior tasks sponsored by both NASA and JPDO. Unlike other methods that try to predict the impacts (delays, noise, or emissions) under different scenarios, we take the view that some flights would not be flown that

${ }^{1}$ D. Long, J. Eckhause, and S. Hasan, "Using Enabled Throughput Instead of Reduced Delay to Quantify Capacity Improvement Benefits," AIAA 2003-6809, November 2003. 
had been in forecasts, which are developed purely on the basis of socioeconomic projections. Our premise is that airlines are in the business of providing punctual transportation service, so known excessive flight delays will result in demand reduction either by the ATM authorities or by the airlines themselves. Similarly, some number of flights may not be allowed to fly because of environmental mandates set by the public or authorities. In our analysis, we do not speculate on which flights would not be flown, only how many. Chapters 4 and 5 have the details of the method and analyses on how the projected throughput is estimated.

To estimate the magnitude of the constraints, in terms of projected throughput, we take a "one-off" analytical approach. In other words, we isolate the constraint being assessed while assuming there is no other constraint in effect. For example, when estimating the constraint of gate capacity, we assume there are no constraints from runways, taxiways, noise, or emissions. Obviously, this is a hypothetical situation because, in reality, multiple constraints are typically present. However, this approach directly serves our purpose to identify and estimate each constraint in isolation so that we can assess which is the most binding and which is secondary.

Projected throughput is an applicable metric only for nominal operations because that is how airlines construct their flight schedules. However, airport operations under inclement weather are also important. To estimate its resiliency, we compare the airport delays under the baseline, and varying capacity parameters, which are presented in Chapter 4. To have a fair comparison, we based all the delays on the same airport operations when they are under instrument flight rules (IFR) for the entire day.

\section{PURPOSE OF METRICS}

For the Airportal Project, metrics will likely have two main functions:

- Support decision making on airport research activities

- Communicate airport research activities to stakeholders.

Such decision-making and communication needs will likely be at several levels of detail:

- Overall impacts of the Airportal Project

- Impacts of technology and operational procedures in research areas addressed by airport research

- Impacts of individual research initiatives such as taxi times, runway capacity, and metroplex operations

- Views of stakeholders. 
Due to recognition of their value in supporting decision making, metrics have received considerable attention in government and in the private sector in the last 20 years. Some government initiatives concerning metrics are the Government Performance and Results Act of 1993, which encourages federal agencies to use metrics for both strategic planning and annual performance planning; President's Management Agenda, which requires the use of metrics as evidence in the Program Assessment Rating Tool developed by the Office of Management and Budget (OMB) to assess government agency program efficiency; and OMB Exhibit 300 , which requires reporting of benefits and costs for all major federal investments before the investment can be approved.

Over the years, the approach used to develop performance metrics has evolved:

- Data-driven metrics. Early performance metric development focused on looking for data that can be used for metrics and then constructing metrics from those data. The problem with this approach is that the metrics are not likely to provide information needed for decisions on a research portfolio because there is little connection to project or program needs.

- Model-driven metrics. This approach moves metrics closer to providing needed information by relying on an established framework to link metrics to project or program goals. The Balanced Scorecard used by industry and government agencies is an example of such a framework. For aviation research, this approach often takes the form of using common general metrics categories, such as benefits, costs, and environment. This approach moves toward providing information to support decisions, but the metrics developed for each desired category do not always provide the exact information that is needed.

- Decision-driven metrics. This approach features working with the managers who will use the metrics to identify what decisions they must make and what information is needed to make those decisions. This approach might be viewed as developing requirements for metrics before developing the metrics themselves.

\section{APPROACH TO DEVELOPING METRICS FOR THE AIRPORTAL PROJECT}

To develop metrics for the Airportal Project, the LMI team used the decisiondriven approach because it provides the most useful metrics. We built on experience working with other NASA and FAA programs on metrics. For example, the LMI team has worked with the JPDO's Interagency Portfolio and Systems Analysis Division (IPSA) on evaluations of NextGen issues and capabilities; has developed metrics for NASA's Virtual Airspace Modeling and Simulation (VAMS) and Advanced Air Transportation Technologies (AATT) projects; and has developed metrics for FAA's overall research and development 
(R\&D) program, FAA's weather R\&D program, and FAA's Safe Flight 21 program.

Our specific approach was as follows:

- Review NASA, JPDO, FAA, and other documents related to possible metrics for the Airportal Project.

> We reviewed the NASA Airportal Project proposal-Next Generation Air Transportation System (NGATS) Air Traffic Management (ATM): Airportal Project, May 23, 2007, Reference Material - to ensure that the metrics we developed would address the goals and objectives of the Airportal Project and would include the various conceptual elements likely to be part of the Airportal Project.

> We reviewed JPDO metrics documents to ensure that the airport metrics we developed would reflect JPDO metrics to the extent appropriate and feasible, as well as address possible impacts of the JPDO concept elements. The JPDO metric documents also delineated 10 desired attributes for JPDO metrics, such as relevant, amenable to projection, and convertible to dollars. We reviewed these attributes for their applicability to the Airportal Project metrics. The documents we reviewed are as follows:

- JPDO metrics: NextGen Metrics JPDO, Metrics Team, March 27, 2008, and Shareholder Benefits: Test Case Benefit Metrics, January 10, 2008

- JPDO concept: Concept of Operations for the Next Generation Air Transportation System, Version 2.0, June 13, 2007.

$>$ We reviewed FAA metrics documents to ensure that the metrics developed are consistent with FAA metrics and to gain ideas for possible metrics. The documents are as follows:

- Operational evolution plan (OEP): OEP Airport Metrics, Industry Coordination Draft, September 2002, Section 5

- FAA performance plan: FAA Performance Plan, 2004, http://www.dot.gov/PerfPlan2004/mobility_delay.html, and Airport Capacity Benchmark Report, September 2004.

- FAA R\&D plan: FAA R\&D Strategic Plan, Review Draft, September 30, 2001. 
We reviewed metrics used by the NASA VAMS and AATT projects to gain ideas for possible metrics:

- VAMS metrics: common set of metrics as maintained by the VAMS Project, September 2004

- AATT metrics: Technical Performance Metrics Description Document for the NASA Advanced Air Transportation Technologies Project (AATT), Draft Report, Benefits and Safety Assessment Sub-Element, Advanced Air Transportation Technologies Project Office, NASA Ames Research Center, November 1, 1999.

$>$ We reviewed metrics used by the Communication Navigation Surveillance/Air Traffic Management Focused Team (C/AFT) to gain ideas for possible metrics. C/AFT is a non-governmental organization whose membership comprises airline and manufacturing companies. $\mathrm{C} / \mathrm{AFT}$ compiled metrics for evaluating the performance of air traffic services and published them in Airline Metric Concepts for Evaluating Air Traffic Service Performance, Report of the Air Traffic Services Performance Focus Group, February 1, 1999.

Metrics relating to the Airportal Project from this review are documented in Airportal-Related Metrics Proposed in NextGen, NASA, and FAA Documents, delivered in April 2008.

- Study possible criteria for making decisions and potential Airportal Project research activities.

Criteria are needed to ensure that the metrics will reflect both decisionmaking needs and research activities. Because increasing capacity is the primary goal of the Airportal Project, we looked for possible top-level metrics for capacity. At the same time, the project may have supporting goals such as increasing the efficiency and predictability of air traffic operations and flights and reducing environmental impacts; therefore, we also looked for metrics reflecting decisions related to the supporting goals.

Airport research initiatives will focus on technology and operational procedures to address the capacity goal and other supporting goals. We thought that Airportal Project decision makers would be interested in the contribution of individual research initiatives to the goals, as well as in metrics that relate to the impacts of individual research initiatives on particular aspects of surface operations, such as taxi times, runway capacity, and metroplex operations. Thus, we developed metrics at a higher level for capacity and supporting goals and for impacts on specific aspects of airport operations. 
- Develop possible frameworks for presenting metrics to support decision making.

The usefulness of metrics for making decisions about research activities in the Airportal Project can be increased by presenting the metrics in different narrative frameworks that are designed to aid understanding of the benefits created by individual research efforts, by collections of research efforts, and by the project as a whole. The term "narrative" indicates that the metric frameworks help convey the story of the project and its benefits from different perspectives. For example, one narrative framework might focus on the top-level benefits of the project portfolio and the benefits of the individual research efforts that support the top-level benefits. Another framework might focus on the project portfolio's effects on the various phases of flight constituting the Airportal Project. Yet another might focus on showing how a particular research effort creates a benefit. (We documented possible frameworks in Examples of Metrics Narrative Frameworks for the NextGen ATM-Airportal Project, delivered in April 2008). We developed four sample metric narrative frameworks for the Airportal Project.

- Develop metrics.

We developed metrics to express the overall impact of airport research on air transportation capacity. In addition, we developed metrics to express the overall impact of airport research on supporting goals, such as increasing the efficiency and predictability of air traffic operations and flights and reducing environmental impacts. We also developed metrics relating to the impacts of individual research initiatives on particular aspects of surface operations, such as taxi times, runway capacity, and metroplex operations. (The metrics are documented in Metrics for NextGen ATM-Airportal Project, delivered in April 2008.)

\section{SUMMARY OF RECOMMENDED METRICS AND FRAMEWORKS}

\section{Top-Level Airport Metrics}

For the primary goal of the Airportal Project-increase capacity-LMI suggests the following metric: "number of aircraft arriving and departing per unit of time." This measure could be summed for all airports in the NAS, summed for a set of airports, or applied to one airport.

Table 3-1 contains suggested metrics for supporting goals. 
Table 3-1. Suggested Metrics for Supporting Goals

\begin{tabular}{|c|c|c|}
\hline Goal & Description & Possible measures \\
\hline Efficiency & $\begin{array}{l}\text { Resources required by users to } \\
\text { provide a given level of service }\end{array}$ & $\begin{array}{l}\text { Change in aircraft travel time } \\
\text { (for constant demand) } \\
\text { Change in aircraft miles flown } \\
\text { (for constant demand) } \\
\text { Change in passenger flight time } \\
\text { (for constant demand) }\end{array}$ \\
\hline Predictability & $\begin{array}{l}\text { Variation in the air traffic control } \\
\text { system, particularly in arrival times } \\
\text { (i.e., delays) }\end{array}$ & $\begin{array}{l}\text { Number of flights more than } 15 \text { minutes } \\
\text { late from scheduled arrival time } \\
\text { Number of passengers more than } \\
15 \text { minutes late from scheduled arrival } \\
\text { time } \\
\text { Average of difference between actual } \\
\text { and planned flight time } \\
\text { Standard deviation of difference between } \\
\text { actual and planned flight time }\end{array}$ \\
\hline Flexibility & $\begin{array}{l}\text { Ability of users to optimize } \\
\text { operations, based on their } \\
\text { objectives and constraints (user } \\
\text { request for routes, runways, etc.) }\end{array}$ & - Total number of user requests honored \\
\hline Environment & $\begin{array}{l}\text { Impacts on noise level, fuel } \\
\text { consumption, and air quality }\end{array}$ & $\begin{array}{l}\text { Projected percentage reduction in } \\
\text { number of people exposed to }>65 \mathrm{~dB} \\
\text { day-night level (DNL) } \\
\text { - Tons of pollutants emitted ( } \mathrm{CO}, \mathrm{HC} \text {, } \\
\text { SOx, and NOx) } \\
\text { Fuel consumption in gallons or pounds }\end{array}$ \\
\hline Economic & Dollar value of benefits & $\begin{array}{l}\text { Direct aircraft operator costs, including } \\
\text { fuel, flight crew, ground crew, and other } \\
\text { Value of passenger time saved }\end{array}$ \\
\hline Access & $\begin{array}{l}\text { Ability of users to obtain access to } \\
\text { airport, airspace, and air traffic } \\
\text { control (ATC) services on demand }\end{array}$ & $\begin{array}{l}\text { Number of airports newly accessible with } \\
\text { virtual towers } \\
\text { Number of airports requiring advanced } \\
\text { equipage by aircraft }\end{array}$ \\
\hline Safety & $\begin{array}{l}\text { Hazards contributing to aviation } \\
\text { accidents/incidents that may } \\
\text { cause fatalities, injuries, or } \\
\text { property damage (the Airportal } \\
\text { Project is not aimed directly at } \\
\text { reducing accidents, but will likely } \\
\text { want to maintain safety) }\end{array}$ & $\begin{array}{l}\text { Number of runway incursions } \\
\text { incursion accidents } \\
\text { Number of fatalities from runway } \\
\text { accidents } \\
\text { Number of fatalities in terminal airspace } \\
\text { accidents } \\
\text { Number of fatal terminal airspace } \\
\text { accidents }\end{array}$ \\
\hline
\end{tabular}




\section{Detailed Metrics}

In addition to the top-level airport metrics, we believe detailed metrics are needed to understand the effects of various airport enhancements on capacity and supporting goals. We did not, however, identify them, because the specific research initiatives were still being defined by the Airportal Project at the time the metrics work was completed. Numerous additional metrics can be developed for almost any airport improvement action that may arise.

In addition to developing detailed metrics for terminal airspace, surface, and metroplex operations, we developed benefit-creating mechanisms for concepts in these areas, as well as metrics to understand these mechanisms.

\section{Metric Frameworks}

Four metric narrative frameworks are likely to be useful to the Airportal Project:

- Metrics for airport runway management

- Metrics for airport taxi route planning

- Metrics for topics of interest to the Airportal Project

- Metrics for a benefit-creating mechanism.

Table 3-2 and Table 3-3 list metrics for two airport concepts: runway management and taxi route planning. The tables are organized by concept subelement.

Table 3-2. Metrics for Airport Runway Management

\begin{tabular}{|c|c|}
\hline Concept subelement & Metric \\
\hline $\begin{array}{l}\text { Optimize runway and taxiway } \\
\text { configuration change }\end{array}$ & $\begin{array}{l}\text { - Number of aircraft arriving and departing on a runway per } \\
\text { unit time } \\
\text { - Time to change from runway configurations } \\
\text { - Taxi distance/time/fuel use for runway configuration } \\
\text { change } \\
\text { - Airborne (for arriving aircraft) distance/time/fuel use for } \\
\text { runway configuration change }\end{array}$ \\
\hline $\begin{array}{l}\text { Enable dynamic allocation of } \\
\text { runways for a given } \\
\text { configuration (i.e., runway } \\
\text { balancing) }\end{array}$ & $\begin{array}{l}\text { - Number of aircraft arriving and departing on a runway per } \\
\text { unit time }\end{array}$ \\
\hline $\begin{array}{l}\text { Ensure more efficient } \\
\text { departure sequences and } \\
\text { schedules }\end{array}$ & $\begin{array}{l}\text { - Number of aircraft arriving and departing on a runway per } \\
\text { unit time } \\
\text { - Reduction in spacing between aircraft using a runway } \\
\text { - Reduction in runway occupancy time }\end{array}$ \\
\hline
\end{tabular}


Table 3-3. Metrics for Airport Taxi Route Planning

\begin{tabular}{|c|c|}
\hline Concept subelement & Metric \\
\hline $\begin{array}{l}\text { Plan efficient taxi routes under } \\
\text { specific airport constraints } \\
\text { Increase density of surface } \\
\text { operations }\end{array}$ & $\begin{array}{l}\text { Number of aircraft taxi operations per unit time } \\
\text { - Average taxi-out time during peak period } \\
\text { - Average taxi-in time during peak period } \\
\text { - Standard deviation taxi-out time during peak period } \\
\text { - Total taxi-out time per year } \\
\text { Total taxi-in time per year }\end{array}$ \\
\hline $\begin{array}{l}\text { Minimize queuing and provide } \\
\text { clearances for longer taxiing } \\
\text { segments }\end{array}$ & $\begin{array}{l}\text { Average length of queue at departure runway } \\
\text { - Average length of queue to cross active runway }\end{array}$ \\
\hline $\begin{array}{l}\text { Provide for equipage differences, } \\
\text { user preferences, and fairness }\end{array}$ & - Number of user-selected taxi routes \\
\hline $\begin{array}{l}\text { Integrate ground vehicle movement } \\
\text { into surface traffic flow }\end{array}$ & $\begin{array}{l}\text { Average taxi-out time during peak period } \\
\text { - Average taxi-in time during peak period } \\
\text { - Standard deviation taxi-out time during peak period } \\
\text { Standard deviation taxi-in time during peak period }\end{array}$ \\
\hline $\begin{array}{l}\text { Execute and adhere to precision taxi } \\
\text { routes }\end{array}$ & $\begin{array}{l}\text { Reduction in minimum spacing between taxiing } \\
\text { aircraft } \\
\text { - Average spacing between taxiing aircraft } \\
\text { - Average taxi-out time during peak period } \\
\text { - Average taxi-in time during peak period } \\
\text { Standard deviation taxi-out time during peak period } \\
\text { Standard deviation taxi-in time during peak period }\end{array}$ \\
\hline
\end{tabular}

Table 3-4 lists metrics that apply to particular topics of interest in the Airportal Project. $^{2}$

${ }^{2}$ The topics of interest are from NextGen Air Traffic Management (ATM): Airportal Project, May 23, 2007, Reference Material. Other topics of interest could be used. 
Table 3-4. Sample Metrics for Airportal Project Topics of Interest

\begin{tabular}{|c|c|}
\hline Topic of interest & Metric \\
\hline $\begin{array}{l}\text { Equivalent } \\
\text { visual operations }\end{array}$ & $\begin{array}{l}\text { Number of aircraft arriving and departing from airport per unit time } \\
\text { compared in visual meteorological conditions (VMC) and poor visibility } \\
\text { conditions }\end{array}$ \\
\hline Metroplex & $\begin{array}{l}\text { Number of aircraft arriving and departing through metroplex per unit } \\
\text { time }\end{array}$ \\
\hline $\begin{array}{l}\text { Converging } \\
\text { and intersecting } \\
\text { runways }\end{array}$ & $\begin{array}{l}\text { Number of aircraft arriving and departing on converging/intersecting } \\
\text { runways per unit time }\end{array}$ \\
\hline Virtual towers & $\begin{array}{l}\text { Number of aircraft arriving and departing from airport per unit time, in } \\
\text { both VMC and poor visibility conditions }\end{array}$ \\
\hline $\begin{array}{l}\text { Real-time wake } \\
\text { vortex models }\end{array}$ & $\begin{array}{l}\text { Number of aircraft arriving and departing on a runway per unit time } \\
\text { - Change in spacing of arriving and departing aircraft }\end{array}$ \\
\hline Weather & $\begin{array}{l}\text { Number of aircraft arriving and departing on a runway and in airport } \\
\text { per unit time compared in good weather and in different poor weather } \\
\text { conditions }\end{array}$ \\
\hline $\begin{array}{l}\text { Merging and } \\
\text { spacing }\end{array}$ & $\begin{array}{l}\text { Average flight time from } 40 \text { miles out (or distance in which airport } \\
\text { domain begins) to touchdown per flight during peak period } \\
\text { Standard deviation of flight time from } 40 \text { miles out (or distance in } \\
\text { which airport domain begins) to touchdown per flight during peak } \\
\text { period } \\
\text { Average flight time from wheels-up to } 40 \text { miles out (or distance in } \\
\text { which airport domain begins) per flight during peak period } \\
\text { Standard deviation of flight time from wheels-up to } 40 \text { miles out (or } \\
\text { distance in which airport domain begins) per flight during peak } \\
\text { period }\end{array}$ \\
\hline
\end{tabular}

Figure 3-1 illustrates how metrics could be presented by phases of flight for an aircraft using an airport and for different levels of detail on airport operations. In the figure, six phases of flight for airport operations are shown with a drill-down example with metrics for "taxi to runway." The figure could be filled with details for the other phases and with metrics for the other phases. Of course, other phases of airport operations and other detailed drill-downs can be displayed. Such a figure could also display metroplex operations. 


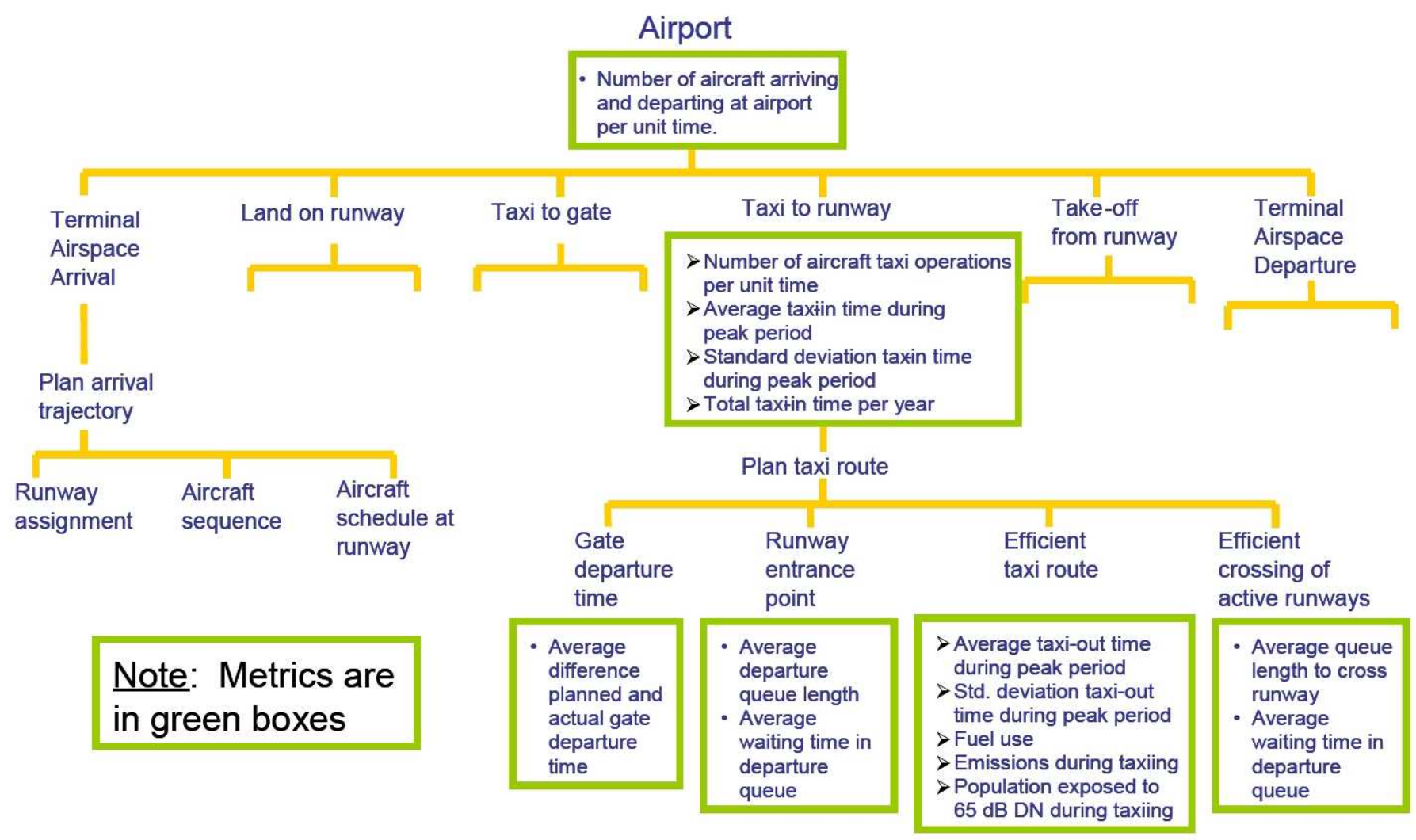

Figure 3-1. Metrics for phases of flight for aircraft using an airport: example from terminal airspace arrival through terminal airspace departure.

Table 3-5 illustrates how metrics could be displayed to indicate the benefitcreating mechanism of an element of the Airportal Project, specifically, improve aircraft sequencing and scheduling for arrival at runway. The benefit flow framework shown in the table identifies the capability, then describes the direct impacts of this capability, presents metrics for the direct impacts, describes the top-level benefits provided by the capability, and presents metrics for the toplevel benefits provided by the capability. (Other columns can be used to show and understand the benefit-creating mechanism of any topic or element in the Airportal Project.) 
Table 3-5. Metrics for Benefit-Creating Mechanism:

Example of Improving Aircraft Sequencing and Scheduling for Arrivals at a Runway

\begin{tabular}{|c|c|c|c|c|}
\hline Capabilities & $\begin{array}{l}\text { Direct } \\
\text { Impacts }\end{array}$ & Direct Impact Metrics & Benefit Impacts & $\begin{array}{l}\text { Benefit Impact } \\
\text { Metrics }\end{array}$ \\
\hline $\begin{array}{l}\text { Improve aircraft } \\
\text { sequencing and } \\
\text { scheduling for arrival } \\
\text { at runway }\end{array}$ & $\begin{array}{l}\text { - Aircraft } \\
\text { sequenced in } \\
\text { terms of } \\
\text { small/large aircraft } \\
\text { to minimize } \\
\text { effects of wake on } \\
\text { number of aircraft } \\
\text { that can land per } \\
\text { unit time } \\
\text { - Increased } \\
\text { accuracy in } \\
\text { aircraft arriving at } \\
\text { runway at time } \\
\text { planned }\end{array}$ & $\begin{array}{l}\text { - Spacing of aircraft compared } \\
\text { to wake separation } \\
\text { standards } \\
\text { - Number of aircraft arriving } \\
\text { and departing on a runway } \\
\text { per unit time analyzed for } \\
\text { different sets of aircraft size } \\
\text { mixes } \\
\text { - Average difference between } \\
\text { Estimated Time of Arrival } \\
\text { and Actual Time of Arrival } \\
\text { Standard deviation of } \\
\text { difference between } \\
\text { Estimated Time of Arrival } \\
\text { and Actual Time of Arrival }\end{array}$ & $\begin{array}{l}\text { Increased number of } \\
\text { aircraft landing on } \\
\text { runway per unit time } \\
\text { Reduced arrival } \\
\text { delays }\end{array}$ & $\begin{array}{l}\text { - Number of aircraft arriving } \\
\text { and departing on a } \\
\text { runway per unit time } \\
\text { Average delay between } \\
\text { Estimated Time of Arrival } \\
\text { and Actual Time of } \\
\text { Arrival }\end{array}$ \\
\hline
\end{tabular}




\section{Chapter 4 \\ Develop a Set of Critical Airports}

For our research effort, and for other Airportal Project studies, we needed a set of critical airports. This chapter describes how we developed that set and then presents the results.

\section{DEFINE THE SUPERSET OF CANDIDATE AIRPORTS}

To define a superset of candidate airports, we started with the master list of U.S. airports as compiled by the National Plan for an Integrated Airport System (NPIAS). ${ }^{1}$ Figure 4-1 identifies airport types, and Table 4-1 describes them.

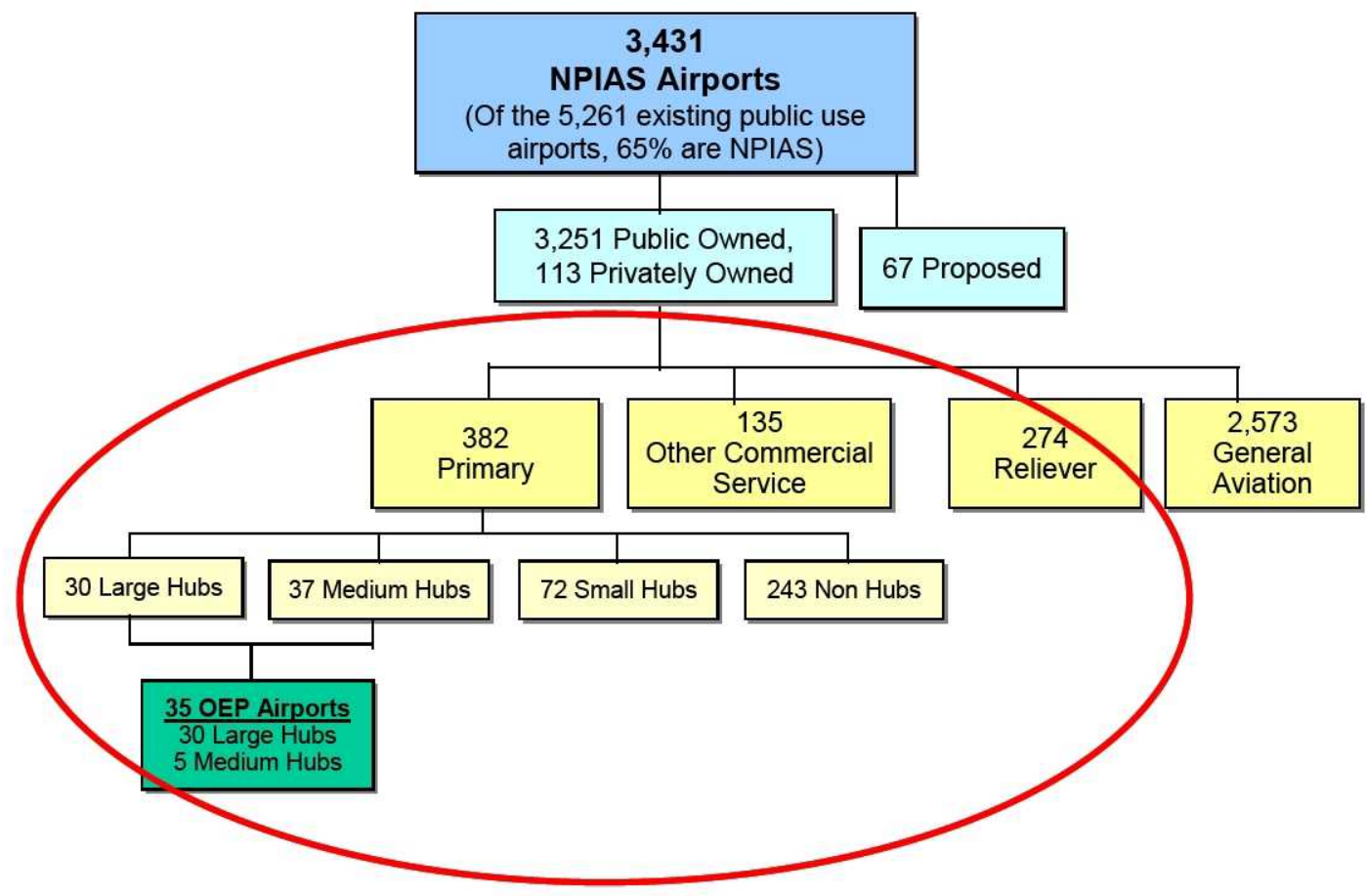

Figure 4-1. NPIAS airport types.

\footnotetext{
${ }^{1}$ See http://www.faa.gov/airports_airtraffic/airports/planning_capacity/npias/reports/.
} 
Table 4-1. Description of NPIAS Airport Types

\begin{tabular}{|c|c|c|}
\hline Type of airport & Description & Enumeration \\
\hline \multicolumn{3}{|l|}{ Primary airports } \\
\hline Large hub & $\begin{array}{l}\text { A large-hub airport has at least } 1 \% \text { of } \\
\text { the total U.S. passenger enplanements. }\end{array}$ & $\begin{array}{l}30 \text {, accounting for } 69 \% \text { of all } \\
\text { passenger enplanements }\end{array}$ \\
\hline Medium hub & $\begin{array}{l}\text { A medium-hub airport has } 0.25-1 \% \text { of } \\
\text { the total U.S. passenger enplanements. }\end{array}$ & $\begin{array}{l}37 \text {, accounting for } 20 \% \text { of all } \\
\text { passenger enplanements }\end{array}$ \\
\hline Small hub & $\begin{array}{l}\text { A small-hub airport has } 0.05-0.25 \% \text { of } \\
\text { the total U.S. passenger enplanements. }\end{array}$ & $\begin{array}{l}72 \text {, accounting for } 8 \% \text { of all } \\
\text { passenger enplanements }\end{array}$ \\
\hline Non-hub & $\begin{array}{l}\text { A non-hub primary airport has less than } \\
0.05 \% \text { and more than } 10,000 \text { annually } \\
\text { of the total U.S. passenger } \\
\text { enplanements. }\end{array}$ & $\begin{array}{l}243, \text { accounting for } 3 \% \text { of all } \\
\text { passenger enplanements }\end{array}$ \\
\hline $\begin{array}{l}\text { Other commercial } \\
\text { service airports }\end{array}$ & $\begin{array}{l}\text { Other commercial service airports have } \\
2,500 \text { to } 10,000 \text { annually of the total } \\
\text { U.S. passenger enplanements. }\end{array}$ & $\begin{array}{l}135 \text {, accounting for } 0.1 \% \text { of all } \\
\text { passenger enplanements }\end{array}$ \\
\hline Reliever airports & $\begin{array}{l}\text { Reliever airports are high-capacity } \\
\text { general aviation airports in major } \\
\text { metropolitan areas. }\end{array}$ & 274 \\
\hline
\end{tabular}

This categorization of airports and the accompanying statistics indicate that 517 airports - large-hub, medium-hub, small-hub, non-hub, and other commercial service airports - account for virtually all U.S. passenger enplanements. Adding the reliever airports brings the total number of airports to 791 .

\section{DeVELOP ANd APPLy AsSUMPTIONS TO IDENTIFY A SUBSET OF CRITICAL AIRPORTS}

A set of 791 airports is too large for modeling, leading us to identify a subset of airports. At the outset, we knew that our subset of critical airports needed to include the 110 LMINET airports. (The 110 LMINET airports are the set of the most significant airports in the NAS used for airport queuing delay calculations in LMINET. This set of 110 airports includes all the 35 OEP airports, almost all of the medium-hub airports, and about half of the small-hub airports.) This left us with 681 smaller airports to be considered for inclusion in our subset of airports. We wanted to include smaller airports, including non-hub and reliever airports, because we believe that some of the scenarios of how the air transportation system may evolve - such as metroplex operations - could drive a significant portion of the traffic to these airports. In those scenarios, the operations (and the delays) will be important.

Data on the 681 airports were merged with 2007 TAF data to determine the number of air carrier (AC), air taxi (AT), and general aviation (GA) operations that occurred at each airport. These data provided information on the relative importance of each airport in terms of current flight activity. We then considered 
demographic data provided by GRA, Inc. These data include population and income data for areas within 10,20, and 50 miles of an airport. No demographic data were available for 69 of the airports, all of which are small, leaving 612 airports.

Next, we looked at the remaining 612 airports, eliminating any that did not have at least one paved runway that is 5,000 feet or longer. This cutoff serves as a measure for determining whether the airport can support jet operations. Of the 612 airports, 453 have at least one paved runway that is at least 5,000 feet long.

We examined the 453 airports in some detail to determine which of them should be included in the list of critical airports for our research. The following are the main criteria we considered:

- Location of the airport, with its associated demographics, primarily, population and income (GRA data)

- Extent of AC, AT, and GA operations (TAF data)

- Identification as a BizShift (reliever) airport ${ }^{2}$

- Availability of a tower

- Presence of lights and Instrument Landing System (ILS).

The first three criteria were the biggest factors in our selection process. We considered the last two criteria to be less important; we did not automatically exclude an airport if it lacks a tower, lights, or an ILS.

We categorized the 453 airports into four groups in decreasing order of criticality:

- Group 1-53 airports. Airports in Group 1 tend to be commercial and other significant airports not in the LMINET 110 list.

- Group 2-40 airports. Airports in Group 2 are generally commercial airports in smaller population regions with fewer operations or are obvious candidate reliever airports.

- Group 3-107 airports. Group 3 generally consists of candidate reliever airports in significant metropolitan areas.

- Group 4-253 airports. This group consists of airports that we believe are not critical; therefore, we excluded them from further consideration.

After eliminating the 253 airports assigned to Group 4, we were left with 200 critical airports that are not LMINET airports.

\footnotetext{
${ }^{2}$ BizShift is a JPDO scenario in which excess traffic is distributed to smaller airports within the vicinity of the 34 CONUS OEP airports.
} 
Figure 4-2 depicts the airport selection process.

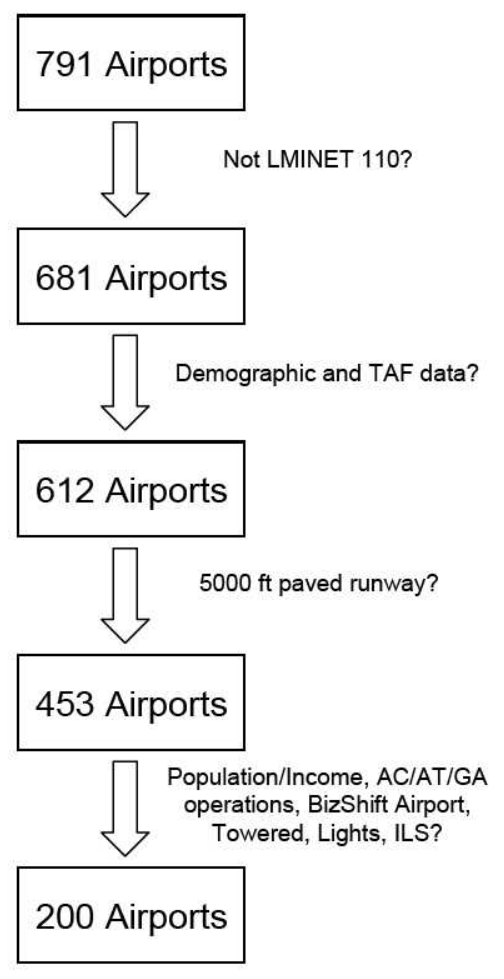

Figure 4-2. Airport selection process.

Combining the 200 airports with the 110 LMINET airports results in a list of 310 critical airports:

- 30 large-hub airports

- 37 medium-hub airports

- 71 (of the 72) small-hub airports

- 94 (of the 243) non-hub airports

- 12 (of the 135) other commercial service airports

- 66 (of the 274) reliever airports.

The one small-hub airport excluded is Saipan International (GSN). Though the set of 310 airports includes several airports in Hawaii and Puerto Rico, GSN's remoteness from the continental United States made it less critical in terms of its impact on the NAS.

Figures 4-3 and 4-4 show the share of total NAS air carrier and air taxi operations for various airport sets, respectively; the solid bars represent the total operation of 
the selected airport set For example, a total of about 13.5 million AC operations occur each year; of those, OEP 35 represents about 9.5 million of them.

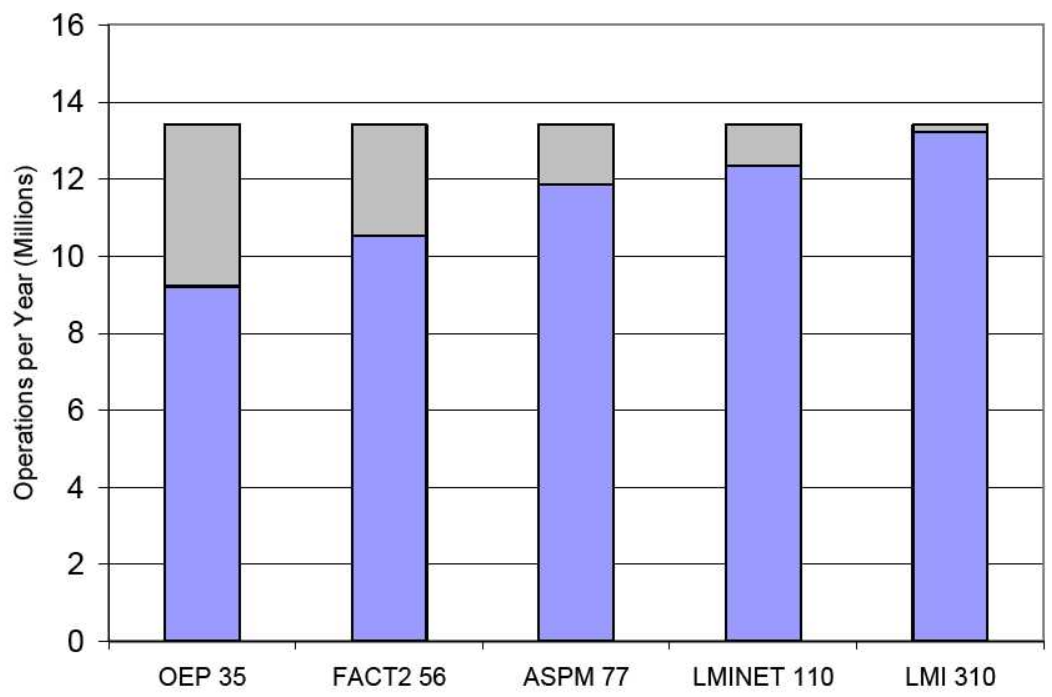

Note: FACT2 = Future Airport Capacity Task 2, and ASPM = Aviation System Performance Metrics.

Figure 4-3. Air carrier operations by airport sets.

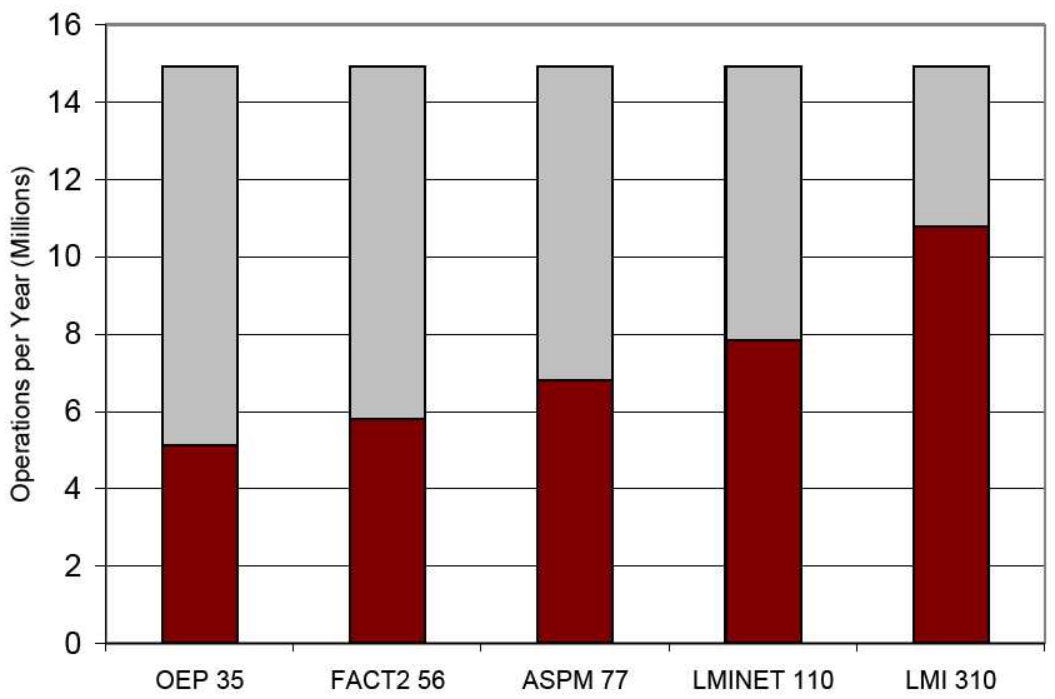

Figure 4-4. Air taxi operations by airport sets.

The 310 airports selected for modeling represent 98.6 percent of all air carrier activity as measured by operations and 99.8 percent of air carrier enplanements. The set of 310 airports includes only 72 percent of all air taxi operations, but achieving 95 percent coverage of air taxi operations would require modeling an additional 475 airports. Figure 4-5 shows the locations of the 310 airports. 


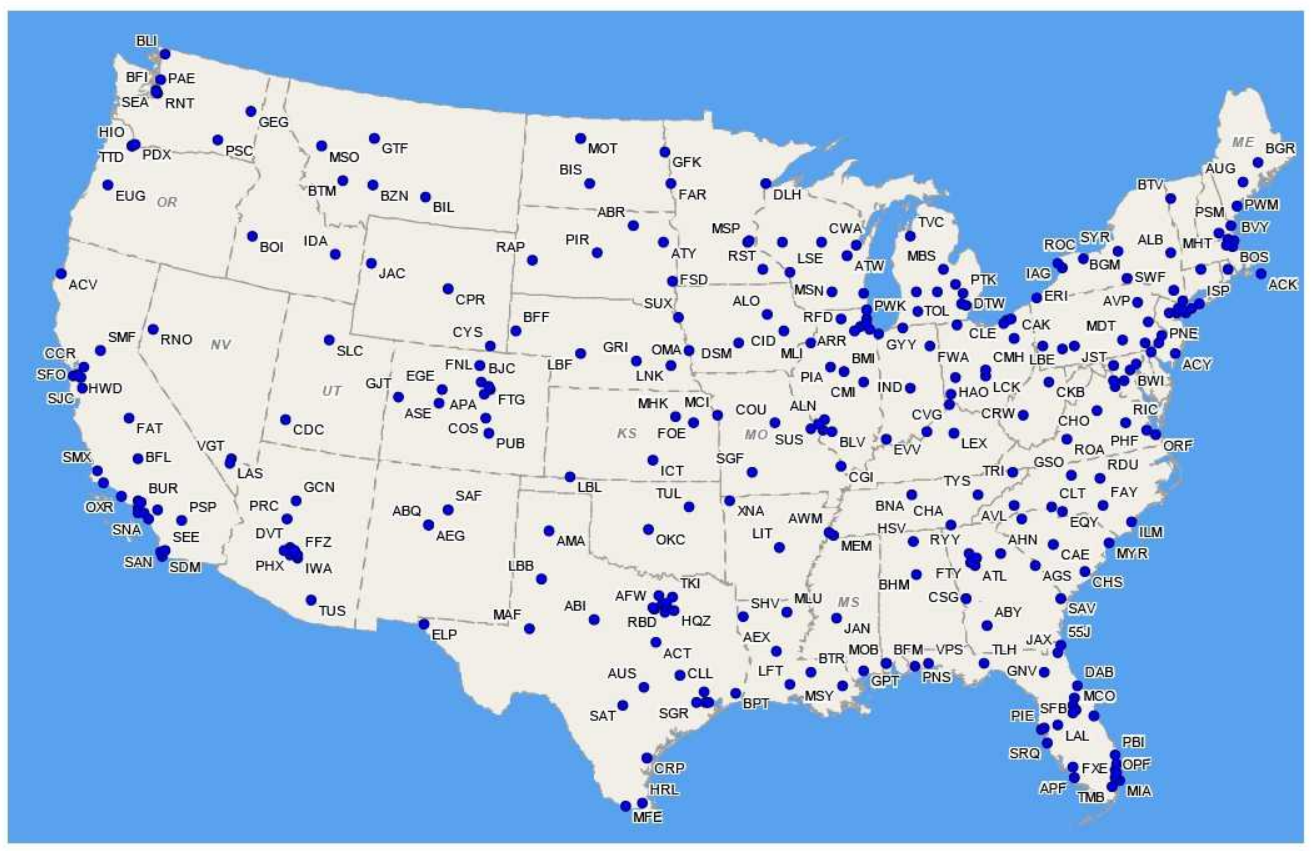

Figure 4-5. Locations of 310 critical airports.

\section{LIST OF CRITICAL AIRPORTS}

Tables 4-2 through 4-5 list the 310 critical airports - from LMINET, Group 1, Group 2, and Group 3-that we included in our subset for modeling. 
Table 4-2. LMINET-110 Airports

\begin{tabular}{|c|c|c|c|c|c|c|c|c|c|}
\hline Code & Airport Name & State & Lat. & Long. & Code & Airport Name & State & Lat. & Long. \\
\hline$\overline{\mathrm{ABQ}}$ & Albuquerque Intl Sunport & NM & 35.040 & -106.609 & LGA & La Guardia & $\overline{\mathrm{NY}}$ & 40.777 & -73.873 \\
\hline ALB & Albany County & NY & 42.748 & -73.803 & LGB & Long Beach /Daugherty Field/ & CA & 33.818 & -118.152 \\
\hline ANC & Anchorage Intl & AK & 61.174 & -149.996 & LIT & Adams Field & AR & 34.729 & -92.225 \\
\hline ATL & The William B Hartsfield Atlanta Intl & $\mathrm{GA}$ & 33.640 & -84.427 & $\mathrm{MCl}$ & Kansas City Intl & MO & 39.298 & -94.714 \\
\hline AUS & Robert Mueller Muni & TX & 30.299 & -97.702 & $\mathrm{MCO}$ & Orlando Intl & $\mathrm{FL}$ & 28.429 & -81.316 \\
\hline $\mathrm{BDL}$ & Bradley Intl & CT & 41.939 & -72.683 & MDW & Chicago Midway & $\mathrm{IL}$ & 41.786 & -87.752 \\
\hline $\mathrm{BFL}$ & Meadows Field & $\mathrm{CA}$ & 35.434 & -119.057 & MEM & Memphis Intl & TN & 35.044 & -89.977 \\
\hline $\mathrm{BHM}$ & Birmingham Intl & $\mathrm{AL}$ & 33.563 & -86.754 & MHT & Manchester & $\mathrm{NH}$ & 42.934 & -71.438 \\
\hline BNA & Nashville International & TN & 36.125 & -86.678 & MIA & Miami Intl & $\mathrm{FL}$ & 25.793 & -80.290 \\
\hline $\mathrm{BOI}$ & Boise Air Terminal/Gowen Fld & ID & 43.564 & -116.223 & MKE & General Mitchell International & WI & 42.947 & -87.897 \\
\hline BOS & General Edward Lawrence Logan Intl & MA & 42.364 & -71.005 & MLB & Melbourne International & $\mathrm{FL}$ & 28.103 & -80.646 \\
\hline BTR & Baton Rouge Metropolitan & LA & 30.533 & -91.150 & MSN & Dane County Regional-Truax Field & WI & 43.139 & -89.338 \\
\hline BUF & Buffalo Niagara Intl & $\overline{\mathrm{NY}}$ & 42.941 & -78.732 & MSP & Minneapolis-St Paul Intl & $\mathrm{MN}$ & 44.881 & -93.217 \\
\hline BUR & Burbank-Glendale-Pasadena & $\mathrm{CA}$ & 34.201 & -118.359 & MSY & New Orleans Int//Moisant Fld/ & $\mathrm{LA}$ & 29.994 & -90.258 \\
\hline BWI & Baltimore-Washington Intl & $\mathrm{MD}$ & 39.175 & -76.668 & OAK & Metropolitan Oakland Intl & CA & 37.721 & -122.221 \\
\hline $\mathrm{CHS}$ & Charleston Afb/Intl & SC & 32.899 & -80.041 & OKC & Will Rogers World & OK & 35.393 & -97.601 \\
\hline CLE & Cleveland-Hopkins Intl & $\mathrm{OH}$ & 41.411 & -81.849 & OMA & Eppley Airfield & $\mathrm{NE}$ & 41.303 & -95.894 \\
\hline $\mathrm{CLT}$ & Charlotte/Douglas Int| & NC & 35.214 & -80.943 & ONT & Ontario Intl & $\overline{C A}$ & 34.056 & -117.601 \\
\hline $\mathrm{CMH}$ & Port Columbus Intl & $\mathrm{OH}$ & 39.996 & -82.889 & ORD & Chicago O'Hare Intl & $\frac{\mathrm{IL}}{\mathrm{L}}$ & 41.980 & -87.905 \\
\hline $\cos$ & City Of Colorado Springs Muni & $\mathrm{CO}$ & 38.806 & -104.700 & ORF & Norfolk Intl & VA & 36.895 & -76.201 \\
\hline CRP & Corpus Christi Intl & $\overline{T X}$ & 27.770 & -97.501 & OXR & Oxnard & $\mathrm{CA}$ & 34.201 & -119.207 \\
\hline CVG & Cincinnati/Northern Kentucky Intl & $\overline{\mathrm{KY}}$ & 39.046 & -84.662 & $\mathrm{PBI}$ & Palm Beach Intl & $\mathrm{FL}$ & 26.683 & -80.096 \\
\hline$\overline{D A B}$ & Daytona Beach Intl & $\mathrm{FL}$ & 29.180 & -81.058 & PDX & Portland Intl & OR & 45.589 & -122.598 \\
\hline DAL & Dallas Love Field & $\mathrm{TX}$ & 32.847 & -96.852 & PHF & Newport News/Williamsburg Int| & $\overline{V A}$ & 37.132 & -76.493 \\
\hline DAY & James M Cox Dayton Intl & $\mathrm{OH}$ & 39.902 & -84.219 & PHL & Philadelphia Intl & $\mathrm{PA}$ & 39.870 & -75.245 \\
\hline$\overline{D C A}$ & Washington National & $\overline{D C}$ & 38.852 & -77.038 & $\mathrm{PHX}$ & Phoenix Sky Harbor Intl & $\overline{A Z}$ & 33.436 & -112.010 \\
\hline DEN & Denver Intl & $\mathrm{CO}$ & 39.858 & -104.667 & PIE & St Petersburg/Clearwater Intl & $\mathrm{FL}$ & 27.911 & -82.687 \\
\hline DFW & Dallas/Fort Worth International & $\overline{T X}$ & 32.896 & -97.037 & PIT & Pittsburgh International & PA & 40.492 & -80.233 \\
\hline DSM & Des Moines Intl & IA & 41.535 & -93.661 & PVD & Theodore Francis Green State & RI & 41.724 & -71.427 \\
\hline DTW & Detroit Metropolitan Wayne County & MI & 42.212 & -83.349 & RDU & Raleigh-Durham International & $\mathrm{NC}$ & 35.878 & -78.787 \\
\hline ELP & El Paso Intl & TX & 31.807 & -106.378 & RFD & Greater Rockford & $\mathrm{IL}$ & 42.195 & -89.097 \\
\hline EUG & Mahlon Sweet Field & $\mathrm{OR}$ & 44.123 & -123.219 & RIC & Richmond International & $\overline{V A}$ & 37.505 & -77.320 \\
\hline EWR & Newark Intl & NJ & 40.693 & -74.169 & RNO & Reno/Tahoe International & NV & 39.499 & -119.768 \\
\hline FAT & Fresno Yosemite International & $\mathrm{CA}$ & 36.776 & -119.718 & ROC & Greater Rochester International & $\mathrm{NY}$ & 43.119 & -77.672 \\
\hline FLL & Fort Lauderdale/Hollywood Intl & $\mathrm{FL}$ & 26.073 & -80.153 & RSW & Southwest Florida Intl & $\mathrm{FL}$ & 26.536 & -81.755 \\
\hline FNT & Bishop International & $\mathrm{Ml}$ & 42.966 & -83.744 & SAN & San Diego Intl-Lindbergh Fld & $\mathrm{CA}$ & 32.734 & -117.190 \\
\hline FXE & Fort Lauderdale Executive & $\mathrm{FL}$ & 26.197 & -80.171 & SAT & San Antonio Intl & TX & 29.534 & -98.470 \\
\hline GFK & Grand Forks Intl & ND & 47.949 & -97.176 & SBA & Santa Barbara Muni & $\mathrm{CA}$ & 34.426 & -119.840 \\
\hline GRR & Kent County Intl & MI & 42.881 & -85.523 & SDF & Louisville Intl-Standiford Field & KY & 38.174 & -85.736 \\
\hline GSO & Piedmont Triad International & $\mathrm{NC}$ & 36.098 & -79.937 & SEA & Seattle-Tacoma Intl & $\overline{\text { WA }}$ & 47.449 & -122.309 \\
\hline GYY & Gary/Chicago Regional & IN & 41.616 & -87.413 & SFO & San Francisco Intl & $\mathrm{CA}$ & 37.619 & -122.375 \\
\hline $\mathrm{HNL}$ & Honolulu Int| & HI & 21.319 & -157.922 & $\mathrm{SJC}$ & San Jose International & $\mathrm{CA}$ & 37.362 & -121.929 \\
\hline $\mathrm{HOU}$ & William P Hobby & TX & 29.645 & -95.279 & SLC & Salt Lake City Intl & UT & 40.788 & -111.978 \\
\hline HPN & Westchester County & NY & 41.067 & -73.708 & SMF & Sacramento International & $\mathrm{CA}$ & 38.695 & -121.591 \\
\hline IAD & Washington Dulles International & $\overline{D C}$ & 38.945 & -77.456 & SNA & John Wayne-Orange County & $\mathrm{CA}$ & 33.676 & -117.868 \\
\hline $\mathrm{IAH}$ & George Bush Intercontinental & $\overline{T X}$ & 29.981 & -95.340 & STL & Lambert-St Louis Intl & $\mathrm{MO}$ & 38.748 & -90.360 \\
\hline ICT & Wichita Mid-Continent & KS & 37.650 & -97.433 & SWF & Stewart Int'L & NY & 41.504 & -74.105 \\
\hline IND & Indianapolis Intl & IN & 39.717 & -86.294 & SYR & Syracuse Hancock Intl & NY & 43.111 & -76.106 \\
\hline ISP & Long Island Mac Arthur & $\overline{\mathrm{NY}}$ & 40.795 & -73.100 & TEB & Teterboro & $\mathrm{NJ}$ & 40.850 & -74.061 \\
\hline JAX & Jacksonville Intl & $\mathrm{FL}$ & 30.494 & -81.688 & TPA & Tampa Intl & $\mathrm{FL}$ & 27.976 & -82.533 \\
\hline JFK & John F Kennedy Intl & NY & 40.640 & -73.779 & TUL & Tulsa Intl & OK & 36.198 & -95.888 \\
\hline JNU & Juneau Intl & $\mathrm{AK}$ & 58.355 & -134.576 & TUS & Tucson Intl & $\overline{A Z}$ & 32.116 & -110.941 \\
\hline LAN & Capital City & $\mathrm{Ml}$ & 42.779 & -84.587 & TVC & Cherry Capital & $\mathrm{MI}$ & 44.741 & -85.583 \\
\hline LAS & Mc Carran Intl & NV & 36.081 & -115.152 & TYS & Mc Ghee Tyson & TN & 35.813 & -83.993 \\
\hline LAX & Los Angeles Intl & $\mathrm{CA}$ & 33.943 & -118.408 & VNY & Van Nuys & $\mathrm{CA}$ & 34.210 & -118.490 \\
\hline
\end{tabular}


Table 4-3. Group 1-53 Airports

\begin{tabular}{|c|c|c|c|c|}
\hline Code & Airport Name & State & Lat. & Long. \\
\hline $\mathrm{ABE}$ & Lehigh Valley International & PA & 40.652 & -75.440 \\
\hline $\mathrm{ACY}$ & Atlantic City International & NJ & 39.458 & -74.577 \\
\hline AMA & Amarillo Intl & $\overline{T X}$ & 35.219 & -101.706 \\
\hline$\overline{A V L}$ & Asheville Regional & $\mathrm{NC}$ & 35.436 & -82.542 \\
\hline AVP & Wilkes-Barre/Scranton Intl & PA & 41.338 & -75.724 \\
\hline AZO & Kalamazoo/Battle Creek International & $\mathrm{MI}$ & 42.235 & -85.552 \\
\hline $\mathrm{BFI}$ & Boeing Field/King County Intl & WA & 47.530 & -122.302 \\
\hline BIL & Billings Logan Intl & MT & 45.808 & -108.544 \\
\hline$\overline{B I S}$ & Bismarck Muni & ND & 46.774 & -100.748 \\
\hline $\mathrm{BLI}$ & Bellingham Intl & WA & 48.792 & -122.538 \\
\hline BLV & Scott Afb/Midamerica & $\mathrm{IL}$ & 38.545 & -89.834 \\
\hline BMI & Central II Regl Arpt At Bloomington-Normal & $\mathrm{IL}$ & 40.479 & -88.919 \\
\hline BTV & Burlington Int| & $\mathrm{VT}$ & 44.473 & -73.150 \\
\hline$\overline{\mathrm{CAE}}$ & Columbia Metropolitan & $\mathrm{SC}$ & 33.939 & -81.120 \\
\hline CAK & Akron-Canton Regional & $\mathrm{OH}$ & 40.916 & -81.443 \\
\hline CID & The Eastern lowa & $\mathrm{IA}$ & 41.885 & -91.711 \\
\hline CRW & Yeager & WV & 38.373 & -81.593 \\
\hline CSG & Columbus Metropolitan & $\overline{\mathrm{GA}}$ & 32.516 & -84.939 \\
\hline CYS & Cheyenne & WY & 41.156 & -104.812 \\
\hline$\overline{\mathrm{DLH}}$ & Duluth Intl & $\overline{\mathrm{MN}}$ & 46.842 & -92.194 \\
\hline FAl & Fairbanks Intl & AK & 64.815 & -147.856 \\
\hline FAR & Hector International & ND & 46.919 & -96.815 \\
\hline FSD & Joe Foss Field & SD & 43.581 & -96.742 \\
\hline GEG & Spokane Intl & WA & 47.620 & -117.534 \\
\hline GPT & Gulfport-Biloxi Rgnl & MS & 30.407 & -89.070 \\
\hline GRB & Austin Straubel International & WI & 44.486 & -88.132 \\
\hline GSP & Greenville-Spartanburg Intl & SC & 34.898 & -82.217 \\
\hline HRL & Valley Intl & TX & 26.229 & -97.654 \\
\hline HSV & Huntsville Intl-Carl T Jones Field & $\mathrm{AL}$ & 34.640 & -86.773 \\
\hline ILM & New Hanover International & $\mathrm{NC}$ & 34.271 & -77.903 \\
\hline ITO & Hilo International & $\mathrm{HI}$ & 19.720 & -155.048 \\
\hline JAN & Jackson International & MS & 32.311 & -90.076 \\
\hline KOA & Kona International At Keahole & $\mathrm{HI}$ & 19.739 & -156.046 \\
\hline LBB & Lubbock Intl & $\overline{T X}$ & 33.664 & -101.823 \\
\hline LEX & Blue Grass & $\mathrm{KY}$ & 38.037 & -84.605 \\
\hline $\mathrm{LIH}$ & Lihue & $\mathrm{HI}$ & 21.976 & -159.339 \\
\hline MDT & Harrisburg International & PA & 40.194 & -76.763 \\
\hline MFE & Mc Allen Miller Intl & TX & 26.176 & -98.239 \\
\hline $\mathrm{MLI}$ & Quad City Intl & $\mathrm{IL}$ & 41.449 & -90.507 \\
\hline$\overline{\mathrm{MOB}}$ & Mobile Regional & $\mathrm{AL}$ & 30.691 & -88.243 \\
\hline MYR & Myrtle Beach Intl & SC & 33.680 & -78.928 \\
\hline OGG & Kahului & $\mathrm{HI}$ & 20.899 & -156.430 \\
\hline PNS & Pensacola Regional & $\mathrm{FL}$ & 30.473 & -87.188 \\
\hline PSP & Palm Springs Regional & $\mathrm{CA}$ & 33.829 & -116.506 \\
\hline PWM & Portland Intl Jetport & $\overline{\mathrm{ME}}$ & 43.646 & -70.309 \\
\hline SAV & Savannah International & GA & 32.128 & -81.202 \\
\hline SFB & Orlando Sanford & $\mathrm{FL}$ & 28.779 & -81.239 \\
\hline SJU & Luis Munoz Marin Intl & PR & 18.439 & -66.002 \\
\hline SRQ & Sarasota/Bradenton Intl & $\mathrm{FL}$ & 27.395 & -82.554 \\
\hline STT & Cyril E King & $\mathrm{VI}$ & 18.337 & -64.973 \\
\hline TLH & Tallahassee Regional & $\mathrm{FL}$ & 30.397 & -84.350 \\
\hline TOL & Toledo Express & $\mathrm{OH}$ & 41.587 & -83.808 \\
\hline VPS & Eglin Afb & $\mathrm{FL}$ & 30.487 & -86.526 \\
\hline
\end{tabular}


Table 4-4. Group 2-40 Airports

\begin{tabular}{|c|c|c|c|c|}
\hline Code & Airport Name & State & Lat. & Long. \\
\hline$\overline{A B I}$ & Abilene Regional & $\overline{T X}$ & 32.411 & -99.682 \\
\hline$A B Y$ & Southwest Georgia Regional & $\mathrm{GA}$ & 31.536 & -84.195 \\
\hline ACK & Nantucket Memorial & $\mathrm{MA}$ & 41.253 & -70.060 \\
\hline ADS & Addison & $\mathrm{TX}$ & 32.969 & -96.836 \\
\hline AEX & Alexandria Intl & $\mathrm{LA}$ & 31.327 & -92.549 \\
\hline AGS & Bush Field & $\overline{\mathrm{GA}}$ & 33.370 & -81.965 \\
\hline$\overline{\mathrm{APA}}$ & Centennial & $\mathrm{CO}$ & 39.570 & -104.849 \\
\hline$\overline{\text { ATW }}$ & Outagamie County & WI & 44.257 & -88.520 \\
\hline BED & Laurence G Hanscom Fld & $\overline{\mathrm{MA}}$ & 42.470 & -71.289 \\
\hline BFF & William B. Heilig Field & $\mathrm{NE}$ & 41.874 & -103.596 \\
\hline$\overline{\mathrm{BGR}}$ & Bangor Intl & $\overline{\mathrm{ME}}$ & 44.807 & -68.828 \\
\hline $\mathrm{BKL}$ & Burke Lakefront & $\mathrm{OH}$ & 41.518 & -81.684 \\
\hline$\overline{\mathrm{BPT}}$ & Jefferson County & $T \mathrm{TX}$ & 29.951 & -94.021 \\
\hline $\mathrm{BVY}$ & Beverly Muni & $\mathrm{MA}$ & 42.584 & -70.917 \\
\hline$\overline{B Z N}$ & Gallatin Field & $\mathrm{MT}$ & 45.777 & -111.153 \\
\hline CGF & Cuyahoga County & $\mathrm{OH}$ & 41.565 & -81.487 \\
\hline $\mathrm{CHA}$ & Lovell Field & $\mathrm{TN}$ & 35.035 & -85.204 \\
\hline $\mathrm{CHO}$ & Charlottesville-Albemarle & VA & 38.139 & -78.453 \\
\hline $\mathrm{CMI}$ & University Of Illinois-Willard & $\mathrm{IL}$ & 40.039 & -88.278 \\
\hline CPS & St Louis Downtown - Parks & $\mathrm{IL}$ & 38.571 & -90.156 \\
\hline$\overline{\mathrm{DPA}}$ & Dupage & IL & 41.907 & -88.248 \\
\hline ERI & Erie Intl & $\overline{\mathrm{PA}}$ & 42.082 & -80.176 \\
\hline FAY & Fayetteville Regional/Grannis Field & $\mathrm{NC}$ & 34.992 & -78.880 \\
\hline$\overline{\mathrm{FNL}}$ & Fort Collins-Loveland Muni & $\mathrm{CO}$ & 40.452 & -105.011 \\
\hline FOE & Forbes Field & KS & 38.950 & -95.664 \\
\hline$\overline{\text { FRG }}$ & Republic & $\mathrm{NY}$ & 40.729 & -73.413 \\
\hline FTW & Fort Worth Meacham Intl & $\mathrm{TX}$ & 32.819 & -97.362 \\
\hline GJT & Walker Field & $\mathrm{CO}$ & 39.122 & -108.527 \\
\hline GNV & Gainesville Regional & $\mathrm{FL}$ & 29.690 & -82.272 \\
\hline GTF & Great Falls Intl & MT & 47.482 & -111.371 \\
\hline GUM & Guam International & $\mathrm{GU}$ & 13.484 & 144.797 \\
\hline $\mathrm{IAG}$ & Niagara Falls Intl & NY & 43.107 & -78.945 \\
\hline ILG & New Castle County & $\mathrm{DE}$ & 39.679 & -75.607 \\
\hline KTN & Ketchikan Intl & $\mathrm{AK}$ & 55.354 & -131.711 \\
\hline LNK & Lincoln Muni & $\mathrm{NE}$ & 40.851 & -96.759 \\
\hline MAF & Midland International & $\mathrm{TX}$ & 31.943 & -102.202 \\
\hline$\overline{\mathrm{MSO}}$ & Missoula International & $\overline{\mathrm{MT}}$ & 46.916 & -114.091 \\
\hline PTK & Oakland County International & $\mathrm{MI}$ & 42.665 & -83.419 \\
\hline RAP & Rapid City Regional & SD & 44.045 & -103.057 \\
\hline SHV & Shreveport Regional & LA & 32.447 & -93.826 \\
\hline
\end{tabular}


Table 4-5. Group 3-107 Airports

\begin{tabular}{|c|c|c|c|c|}
\hline Code & Airport Name & State & Lat. & Long. \\
\hline $40 \mathrm{~N}$ & Chester County G O Carlson & $\overline{\mathrm{PA}}$ & 39.979 & -75.866 \\
\hline $55 \mathrm{~J}$ & Fernandina Beach Muni & $\mathrm{FL}$ & 30.610 & -81.461 \\
\hline ABR & Aberdeen Regional & SD & 45.449 & -98.422 \\
\hline ACT & Waco Regional & TX & 31.611 & -97.231 \\
\hline $\mathrm{ACV}$ & Arcata & $\mathrm{CA}$ & 40.978 & -124.109 \\
\hline $\mathrm{ADQ}$ & Kodiak & AK & 57.750 & -152.494 \\
\hline $\mathrm{AEG}$ & Double Eagle li & $\overline{N M}$ & 35.145 & -106.795 \\
\hline AFW & Fort Worth Alliance & $\overline{T X}$ & 32.988 & -97.319 \\
\hline $\mathrm{AHN}$ & Athens/Ben Epps & $\overline{\mathrm{GA}}$ & 33.949 & -83.326 \\
\hline$\overline{A L N}$ & St Louis Regional & $\mathrm{IL}$ & 38.890 & -90.046 \\
\hline$\overline{A L O}$ & Waterloo Muni & $\overline{\mathrm{IA}}$ & 42.557 & -92.400 \\
\hline APF & Naples Muni & $\mathrm{FL}$ & 26.153 & -81.775 \\
\hline$\overline{A R R}$ & Aurora Muni & $\overline{\mathrm{IL}}$ & 41.771 & -88.473 \\
\hline ASE & Aspen-Pitkin Co/Sardy Field & $\mathrm{CO}$ & 39.223 & -106.869 \\
\hline ATY & Watertown Muni & SD & 44.914 & -97.155 \\
\hline AUG & Auqusta State & $\overline{\mathrm{ME}}$ & 44.321 & -69.797 \\
\hline AWM & West Memphis Muni & AR & 35.135 & -90.234 \\
\hline $\mathrm{BCT}$ & Boca Raton & $\mathrm{FL}$ & 26.379 & -80.108 \\
\hline BET & Bethel & $\overline{\mathrm{AK}}$ & 60.780 & -161.838 \\
\hline$\overline{B F M}$ & Mobile Downtown & $\overline{\mathrm{AL}}$ & 30.627 & -88.068 \\
\hline BGM & Binghamton Regional & NY & 42.209 & -75.980 \\
\hline $\mathrm{BJC}$ & Jeffco & $\mathrm{CO}$ & 39.909 & -105.117 \\
\hline BTM & Bert Mooney & MT & 45.955 & -112.498 \\
\hline CCR & Buchanan Field & $\overline{\mathrm{CA}}$ & 37.990 & -122.057 \\
\hline $\mathrm{CDC}$ & Cedar City Regional & UT & 37.701 & -113.099 \\
\hline CGI & Cape Girardeau Regional & $\mathrm{MO}$ & 37.225 & -89.571 \\
\hline CKB & Benedum & $\overline{W V}$ & 39.294 & -80.229 \\
\hline $\mathrm{CLL}$ & Easterwood Field & $\mathrm{TX}$ & 30.589 & -96.364 \\
\hline $\mathrm{COU}$ & Columbia Regional & $\mathrm{MO}$ & 38.818 & -92.220 \\
\hline CPR & Natrona County Intl & $\overline{W Y}$ & 42.908 & -106.464 \\
\hline CWA & Central Wisconsin & WI & 44.778 & -89.667 \\
\hline DTO & Denton Muni & TX & 33.201 & -97.198 \\
\hline DVT & Phoenix-Deer Valley Muni & $\mathrm{AZ}$ & 33.688 & -112.083 \\
\hline EAU & Chippewa Valley Regional & WI & 44.865 & -91.485 \\
\hline EFD & Ellington Field & $\mathrm{TX}$ & 29.607 & -95.159 \\
\hline EGE & Eagle County Regional & $\mathrm{CO}$ & 39.643 & -106.918 \\
\hline$\overline{E Q Y}$ & Monroe & $\mathrm{NC}$ & 35.019 & -80.620 \\
\hline EVV & Evansville Regional & IN & 38.038 & -87.531 \\
\hline FDK & Frederick Muni & $\mathrm{MD}$ & 39.418 & -77.374 \\
\hline FFZ & Falcon Fld & $\mathrm{AZ}$ & 33.461 & -111.728 \\
\hline$\overline{\text { FTG }}$ & Front Range & $\mathrm{CO}$ & 39.785 & -104.544 \\
\hline FTY & Fulton County Airport & $\mathrm{GA}$ & 33.779 & -84.521 \\
\hline FWA & Fort Wayne International & IN & 40.979 & -85.195 \\
\hline GCN & Grand Canyon National Park & $\overline{A Z}$ & 35.952 & -112.147 \\
\hline GEU & Glendale Municipal & $\mathrm{AZ}$ & 33.527 & -112.295 \\
\hline GRI & Central Nebraska Regional & $\mathrm{NE}$ & 40.968 & -98.309 \\
\hline $\mathrm{HAO}$ & Hamilton-Fairfield & $\mathrm{OH}$ & 39.365 & -84.525 \\
\hline HEF & Manassas Regional & $\overline{V A}$ & 38.721 & -77.516 \\
\hline $\mathrm{HIO}$ & Portland-Hillsboro & OR & 45.540 & -122.950 \\
\hline HQZ & Mesquite Metro & $\mathrm{TX}$ & 32.747 & -96.530 \\
\hline HWD & Hayward Air Terminal & $\mathrm{CA}$ & 37.659 & -122.122 \\
\hline IDA & Fanning Field & ID & 43.515 & -112.070 \\
\hline ISM & Kissimmee Muni & $\overline{F L}$ & 28.290 & -81.437 \\
\hline IWA & Williams Gateway & $\overline{A Z}$ & 33.308 & -111.656 \\
\hline
\end{tabular}

\begin{tabular}{|c|c|c|c|c|}
\hline Code & Airport Name & State & Lat. & Long. \\
\hline JAC & Jackson Hole & WY & 43.607 & -110.738 \\
\hline JST & Johnstown-Cambria County & PA & 40.316 & -78.834 \\
\hline $\mathrm{LAL}$ & Lakeland Linder Regional & $\mathrm{FL}$ & 27.989 & -82.019 \\
\hline LBE & Westmoreland County & $\mathrm{PA}$ & 40.276 & -79.405 \\
\hline LBF & North Platte Rgnl Airport & $\mathrm{NE}$ & 41.126 & -100.687 \\
\hline LBL & Liberal Muni & $\mathrm{KS}$ & 37.044 & -100.960 \\
\hline$\overline{\mathrm{LCK}}$ & Rickenbacker International & $\mathrm{OH}$ & 39.814 & -82.928 \\
\hline$\overline{\mathrm{LFT}}$ & Lafayette Regional & $\mathrm{LA}$ & 30.205 & -91.988 \\
\hline LSE & La Crosse Muni & $\overline{\mathrm{WI}}$ & 43.879 & -91.256 \\
\hline LWM & Lawrence Muni & MA & 42.717 & -71.123 \\
\hline MBS & Mbs International & $\mathrm{Ml}$ & 43.533 & -84.080 \\
\hline$\overline{\mathrm{MHK}}$ & Manhattan Rgnl & $\overline{\mathrm{KS}}$ & 39.141 & -96.671 \\
\hline$\overline{M L U}$ & Monroe Regional & $\overline{\mathrm{LA}}$ & 32.511 & -92.038 \\
\hline MMU & Morristown Muni & $\mathrm{NJ}$ & 40.799 & -74.415 \\
\hline MOT & Minot Intl & ND & 48.260 & -101.281 \\
\hline MTN & Martin State & $\mathrm{MD}$ & 39.326 & -76.414 \\
\hline OPF & Opa Locka & $\mathrm{FL}$ & 25.907 & -80.278 \\
\hline ORL & Executive & $\mathrm{FL}$ & 28.546 & -81.333 \\
\hline$\overline{\mathrm{PAE}}$ & Snohomish County (Paine Fld) & $\overline{W A}$ & 47.908 & -122.282 \\
\hline$\overline{P D K}$ & Dekalb-Peachtree & $\overline{\mathrm{GA}}$ & 33.876 & -84.302 \\
\hline $\mathrm{PIA}$ & Greater Peoria Regional & $\mathrm{IL}$ & 40.664 & -89.693 \\
\hline PIR & Pierre Regional & SD & 44.383 & -100.286 \\
\hline PNE & Northeast Philadelphia & PA & 40.082 & -75.011 \\
\hline PRC & Ernest A. Love Field & $\overline{A Z}$ & 34.652 & -112.421 \\
\hline PSC & Tri-Cities & $\overline{\text { WA }}$ & 46.265 & -119.119 \\
\hline PSM & Pease Intern & $\mathrm{NH}$ & 43.078 & -70.823 \\
\hline PUB & Pueblo Memorial & $\mathrm{CO}$ & 38.289 & -104.497 \\
\hline PWK & Palwaukee Muni & $\mathrm{IL}$ & 42.114 & -87.902 \\
\hline RBD & Redbird & $\mathrm{TX}$ & 32.681 & -96.868 \\
\hline$\overline{\mathrm{RNT}}$ & Renton Muni & $\overline{\text { WA }}$ & 47.493 & -122.215 \\
\hline ROA & Roanoke Regio & VA & 37.326 & -79.975 \\
\hline RST & enational & $\mathrm{MN}$ & 43.909 & -92.498 \\
\hline RYY & Cobb County-Mc Collum Field & GA & 34.013 & -84.599 \\
\hline SAF & Santa Fe Muni & $\mathrm{NM}$ & 35.617 & -106.088 \\
\hline$\overline{\text { SBN }}$ & Michiana Rgnl & $\mathrm{IN}$ & 41.709 & -86.319 \\
\hline$\overline{S D L}$ & $\widehat{S c o t t}$ & $\overline{A Z}$ & 33.623 & -111.911 \\
\hline$\overline{\text { SDM }}$ & $\overline{B r o w}$ & $\overline{\mathrm{CA}}$ & 32.572 & -116.980 \\
\hline SEE & Gillespie Field & $\mathrm{CA}$ & 32.826 & -116.972 \\
\hline SGF & Springfield-Branson Regional & $\mathrm{MO}$ & 37.244 & -93.387 \\
\hline SGR & Sugar Land Muni/Hull Field & TX & 29.622 & -95.657 \\
\hline$\overline{\text { SMX }}$ & Santa Maria Public & $\mathrm{CA}$ & 34.899 & -120.458 \\
\hline STP & St Paul Downtown Holman Fld & $\mathrm{MN}$ & 44.935 & -93.060 \\
\hline SUS & Spirit Of St Louis & $\mathrm{MO}$ & 38.662 & -90.651 \\
\hline SUX & Sioux Gateway & $\mathrm{IA}$ & 42.403 & -96.384 \\
\hline TKI & Mc Kinney Muni & TX & 33.178 & -96.591 \\
\hline TMB & Kendall-Tamiami Executive & $\mathrm{FL}$ & 25.648 & -80.433 \\
\hline TOA & ini Field & $\mathrm{CA}$ & 33.803 & -118.340 \\
\hline TRI & Tri-Cities Regional Tn/Va & $\mathrm{TN}$ & 36.475 & -82.407 \\
\hline TTD & Portland-Troutdale & OR & 45.549 & -122.401 \\
\hline UGN & Waukegan Regional & $\mathrm{IL}$ & 42.422 & -87.868 \\
\hline VGT & North Las Vegas & NV & 36.212 & -115.196 \\
\hline$\overline{\mathrm{XNA}}$ & Northwest Arkansas Rgnl & $\overline{A R}$ & 36.282 & -94.307 \\
\hline YIP & Willow Run & $\mathrm{Ml}$ & 42.238 & -83.530 \\
\hline
\end{tabular}




\section{Chapter 5 \\ Analyze Airport Capacity Constraints}

This chapter details our modeling approach for assessing three categories of airport capacity constraints: runways, taxiways, and gates. Our approach for each of these categories follows our general approach of using the flight throughput as the primary metric. Because many of the NextGen technologies are envisioned to help operations in bad weather, when most of the flight delays occur, we also include the delay results for the runway capacity analysis.

\section{RUNWAY CONSTRAINTS ANALYSIS}

\section{Overview}

Our basic approach to airport capacity modeling is to calculate runway capacities for three types of runway operations and then to combine the results to generate airport capacities. We have done this at various levels of detail to model the effects of specific technologies and airport issues. Very detailed models, including all airport configurations, have been built for several airports to support analysis of several NASA technologies. A semi-automated, primary configuration model for 110 airports has been built to support LMINET analysis of NASA and JPDO technologies. For this effort, we expanded the semi-automated capacity models used with LMINET from 110 airports to 310 airports. The outputs of the models are airport Pareto capacity curves for the baseline and two technologies with separate curves for two visual meteorological conditions (VMC) and two instrument meteorological conditions (IMC). ${ }^{1}$

The main tool for the projected throughput under the runway capacity constraint analysis is LMINET, which is a queuing network model of the NAS, including the major airports and all air traffic control sectors. ${ }^{2}$ For the analyses conducted in this report, only the airport part of the model is used. Simply put, LMINET can be

\footnotetext{
${ }^{1} \mathrm{VMCl}$ : Operations under VFR in which the aircrew is responsible for safe separation.

VMC2: Marginal VMC (MVFR) in which the ceiling is $\geq 1,000$ feet and the visibility is $\geq 3$ statute miles, but approaches are still under radar control with the air traffic controller responsible for safe separation.

IMC1: IFR Category 1 (Cat 1$)$ operations with ceilings and visibilities $<(1,000-3)$ but $\geq$ Cat 1 minimums, typically $\geq(200-1 / 2)$.

IMC2: IFR Cat 2 and Cat 3 operations extending down to airport minimums. The lowest minimums found at U.S. airports are Cat $3 \mathrm{~b}$ with 0 -foot ceiling and 300 -foot runway visual range.

${ }^{2}$ NASA, Modeling Air Traffic Management Technologies with a Queuing Network Model of the National Airspace System, NASA Contractor Report 208988, Dou Long, David A. Lee, Jesse P. Johnson, Eric M. Gaier, and Peter F. Kostiuk, March 1998.
} 
used to estimate the flight delays for a given demand, or flight schedule, or to estimate the feasible throughput at airports based on given flight delay tolerances or demand-to-capacity ratios. ${ }^{3}$

In this study, we applied the same rules as the ones adopted by JPDO in its studies. For each airport, the demand is allowed to be as large as 1.2 times the airport's VMC capacity for any 15-minute epoch. The arrival and departure demands are considered in quarter-hour bins (e.g., 9:00-9:14, 9:15-9:29), and the capacity is the point on the Pareto curve that corresponds with that demand. The exact point on the Pareto curve is specific to each airport's capacity curve and the arrival and departure demands, but that point corresponds to the point that minimizes the steady-state delay of combined arrival and departure delay to the greatest extent allowable by the curve. In addition to the 1.2 demand-to-capacity $(\mathrm{D} / \mathrm{C})$ ratio quarter-hour restriction, we also assume that any rolling-hour (9:009:59, 9:15-10:14, etc.) demand for either arrivals or departures cannot exceed 0.9 times their respective VMC capacities. For demand balancing, we trim an even number of departures and arrivals.

The 0.9 rolling-hour D/C and the 1.2 quarter-hour rules essentially allow for significant short-term demand spikes while restricting the overall demand to less than capacity over longer periods. Due to the exponential nature of delay growth as demand increases, combined with the infinite steady-state delay for values of $\mathrm{D} / \mathrm{C}$ greater than or equal to $1.0, \mathrm{a} \mathrm{D} / \mathrm{C}$ ratio of 0.9 generally allows for a reasonable throughput while ensuring schedule integrity and flexibility to accept delays if the demand spikes or if weather conditions become degraded for moderate periods. Therefore, for some of the busiest airports, our model would trim a modest number of flights under their 2007 demand, which is for a summer peak day. This result is consistent with late afternoon delays experienced in summer 2007 at these airports, even under good weather conditions.

In addition to the baselines, which are the NextGen capacities in 2015 and 2025, respectively, we have also conducted the runway capacity constraint analysis under two conditions: runway occupancy time (ROT) limits and miles-in-trail (MIT) separation limits. ROT and MIT separation are the two functional constraints on runway capacities. In the 2015 and 2025 scenarios, we have made one of the constraints restricting and the other irrelevant to the NextGen capacities. The results should help NASA better understand the source of runway constraints beyond NextGen capacities.

Because many of the NextGen technologies are envisioned to help NAS have robust operations in bad weather, we have estimated flight delays at the selected 310 airports under the hypothetical assumption that the airports are under IMC for the entire day. The results of this analysis should not change the results of the capacity throughput analysis if the delays are not extensive, because the

\footnotetext{
${ }^{3}$ D. Long, J. Eckhause, and S. Hasan, "Using Enabled Throughput Instead of Reduced Delay to Quantify Capacity Improvement Benefits," AIAA 2003-6809, November 2003.
} 
throughput analysis assumes that the effect of weather will not be more than that in the current NAS. Also, the current delay information is useful to estimate delays in the future.

\section{Runway Capacity Model}

Our runway capacity model uses closed-form analytic algorithms to model the task of the controller (or pilot) to maintain adequate spacing to avoid multiple runway occupancy (controller and pilot) and minimum assigned MIT separation (controller) in the face of uncertainties in leader and follower speeds, positions, and winds. Specifically, we calculate, for each aircraft pair in a given aircraft class matrix, the minimum required spacing necessary to maintain an assigned MIT separation and a single ROT requirement. Additional algorithms and logic are applied to model-specific FAA rules, such as no departures when an arrival is within 2 miles of the runway, minimum departure separations of 1 minute, and 2minute versus MIT separation spacing for departures behind B757s and heavyclass aircraft.

Our runway capacity model includes algorithms for a single runway, parallel runway dependent (staggered) arrivals and departures, and very closely spaced parallel runway arrivals and departures. The model also has features that address crossing runway configurations. The basic capacity model output is an arrival/departure Pareto frontier, one form of which is shown in Figure 5-1 and described here.

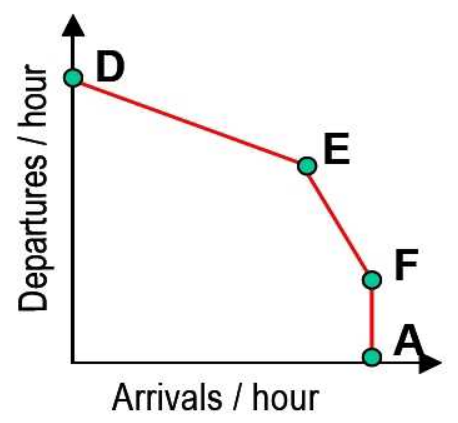

Figure 5-1. Single runway capacity Pareto.

Separate airport capacity Pareto frontiers are generated for each technology and each weather condition. Point $\mathrm{D}$ represents the runway being used for maximum departures; point $\mathrm{A}$ is maximum arrivals; point $\mathrm{E}$ is balanced arrivals and departures; and point F is maximum arrivals, with some "free" departure slots available. Free departures are those that can be accommodated even when the airport is configured for maximum arrivals. Table 5-1 lists the parameters for the basic capacity model. Appendix A describes the derivation of the mathematical algorithms used in the runway capacity model. 
Table 5-1. LMI Capacity Model Parameters

\begin{tabular}{|c|l|c|}
\hline Parameter & \multicolumn{1}{|c|}{ Description } & $\begin{array}{c}\text { Airport } \\
\text { unique }\end{array}$ \\
\hline$P_{\mathrm{i}}$ & Fraction of aircraft that are type i (small, large, B757, heavy, A380) & $\mathrm{X}$ \\
\hline $\mathrm{D}$ & Length of common approach path & \\
\hline $\mathrm{S}_{\mathrm{ij}}$ & Miles-in-trail minimums, type i behind type $\mathrm{j}$ & \\
\hline $\mathrm{V}_{\mathrm{i}}$ & Mean approach speed of aircraft type $\mathrm{i}$ & \\
\hline$\sigma_{\mathrm{Vi}}$ & Standard deviation speed (speed uncertainty) of aircraft type i & \\
\hline$\sigma_{\mathrm{Xi}}$ & Standard deviation of position (position uncertainty) of aircraft type i & \\
\hline$\sigma_{\mathrm{Wi}}$ & Standard deviation of wind (wind uncertainty) of aircraft type i & \\
\hline $\mathrm{R}_{\mathrm{ai}}$ & Mean arrival runway occupancy time (ROT) of aircraft type i & $\mathrm{X}$ \\
\hline$\sigma_{\mathrm{Rai}}$ & Standard deviation of arrival ROT of aircraft type $\mathrm{i}$ & \\
\hline $1 / \lambda$ & TRACON delivery inefficiency parameter & $\mathrm{X}$ \\
\hline $\mathrm{R}_{\mathrm{di}}$ & Mean departure runway occupancy time of aircraft type i & \\
\hline$\sigma_{\mathrm{Rdi}}$ & Standard deviation of departure ROT of aircraft type $\mathrm{i}$ & $\mathrm{X}$ \\
\hline $\mathrm{DD}$ & Distance to turn on departure & \\
\hline $\mathrm{C}$ & Mean communication time delay for departure & \\
\hline$\sigma_{\mathrm{C}}$ & Standard deviation of communication time delay & \\
\hline
\end{tabular}

Note: TRACON = Terminal Radar Approach Control.

As the table shows, the model includes both common and airport-unique input variables. When necessary, common variables can be transformed into airportunique variables, and airport idiosyncrasies can be implemented in the model.

The airport-unique mixes of small, large, B757, and heavy aircraft are determined in three ways. For the 56 FACT2 airports, we used the mixes developed by MITRE. For the rest of the 110 basic airports, we used mixes developed by LMI for JPDO. For the additional 200 airports, we developed mix information from Official Airline Guide data for commercial operations, supplemented by FAA TAF data for general aviation and military operations. The model is coded for five weight classes to accommodate the A380 aircraft and includes the provisional separation requirements for the $\mathrm{A} 380$.

The runway model is coded in Pascal with a Microsoft Excel workbook handling input/output and run control. The workbook includes a spreadsheet with nominal input values for all the variables, plus two spreadsheets containing airport-specific inputs for the 310 airports. Inputs are included for a baseline and two technology cases, with four meteorological conditions for each (12 sets in all). When run, the results for the nominal value case are presented numerically and graphically on spreadsheets in the workbook, and the results for the 310 airports are written directly to text files for subsequent input into the airport model. The model can be set to run only the nominal value case for quick what-if analyses, or for the nominal value case and some or all of the 310 airports. 


\section{Airport Capacity Model}

The airport capacity model is an extension of the LMINET model used for JPDO analyses. It is limited to one configuration per meteorological condition per airport; it does not include separate capacities for IMC2 (ILS Cat 2 and Cat 3) configurations. These are the same conditions modeled by FAA ATO-F for the basic 110 airports and by Boeing and MITRE for the 56 FACT2 airports. If necessary, our model can be readily expanded to include VMC2 and additional configurations. The model consists of a single Microsoft Excel workbook containing both spreadsheet and Visual Basic coding for input/output, analysis support, and model control.

Airport capacity curves differ from runway capacity curves in that they do not necessarily contain the Dmax, Amax, Equal, and Free points. An airport with only arrival-only and departure-only runways will have a single-point, (Amax, Dmax), curve. Airports with both arrival-only (Amin) and mixed-operations runways will add an (Amin, Dmax) point and shift the Equal, Free, and Amax points by Amin. Similarly, an airport with a departure-only (Dmin) and mixed runway will shift the Dmax, Equal, and Free points by adding the Dmin to the mixed-runway values. Airports that alternate between all-arrival and all-departure operations will have a two-point (0, Dmax), (Amax, 0) curve. Airports with crossing, converging, diverging, and auxiliary runways are modeled with fractional increases or decreases in the basic capacity curve parameters.

The airport capacity model loads the runway model output text files. These data are linked to the airport capacity models for $\mathrm{VMC1}, \mathrm{VMC} 2$, and $\mathrm{IMC} 1$ for the baseline and the two technology cases. The airport models are built using the appropriate combinations of single, dependent pair, and closely spaced parallel runway capacities for each airport.

For the large 110 airports, the results are similar to the FAA ATO-F and FACT2 curves. These capacities are derived using the methods described above. For the remaining 200 airports, we reviewed the airport diagrams and the FAA terminal area procedures to make informed estimates of the operating configurations. We sometimes reviewed aerial photographs to observe arrival skid marks. The model is able to plot, for each meteorological condition, an airport's baseline and technology curves and the ATO-F curve, when available, to help check and calibrate the model.

The airport capacity model also generates airport capacity text files for use by airport delay models. Appendix B includes the VMC1 and IMC1 airport capacities for the 310 airports in 2007, which is our baseline year.

\section{New Runways for 2015 and 2025}

For the 56 FACT2 airports, we have limited the new runways to those included in FACT2. This is consistent with assumptions used in JPDO analyses. Also, because we are using the 2006 FACT2 configurations as the baseline for the 
FACT2 airports, we do not include in the baseline the new runways at IAD, ORD, and SEA that opened in 2008. These new runways are included in 2015 and 2025. A second new runway is forecast for IAD by 2025 .

For the airports beyond the 56 contained in the FACT2 study, we considered a variety of sources: FAA/ATO-P, FAA/ATO-F, and the FAA 2003 Aviation Capacity Enhancement (ACE) Plan, supplemented by interviews and individual airport master plans.

Tables 5-2 and 5-3 show the new runways or runway improvements that are assumed to be in place by 2015 and 2025 , respectively. In the tables, " $X$ " indicates a new runway. The tables also indicate which airports were included in the FACT studies.

Table 5-2. New Runways by 2015

\begin{tabular}{|c|c|c|c|c|c|c|}
\hline Airport & $\begin{array}{c}\text { FACT } \\
\text { airport? }\end{array}$ & FACT 2 & ATO-F & ACE 2003 & ATO-P & LMI \\
\hline BOS & Yes & $\mathrm{X}$ & $\mathrm{X}$ & $\mathrm{X}$ & & $\mathrm{X}$ \\
\hline FLL & Yes & $\mathrm{X}$ & $\mathrm{X}$ & $\mathrm{X}$ & $\mathrm{X}$ & $\mathrm{X}$ \\
\hline IAD & Yes & $\mathrm{X}$ & $\mathrm{X}$ & $\mathrm{X}$ & $\mathrm{X}$ & $\mathrm{X}$ \\
\hline ORD & Yes & $\mathrm{X}$ & $\mathrm{X}$ & $\mathrm{X}$ & $\mathrm{X}$ & $\mathrm{X}$ \\
\hline PHL & Yes & Extension & $\mathrm{X}$ & $\mathrm{X}$ & $\mathrm{X}$ & \\
\hline SEA & Yes & $\mathrm{X}$ & $\mathrm{X}$ & $\mathrm{X}$ & $\mathrm{X}$ & $\mathrm{X}$ \\
\hline CLE & Yes & $\mathrm{X}$ & $\mathrm{X}$ & $\mathrm{X}$ & & $\mathrm{X}$ \\
\hline CLT & Yes & $\mathrm{In} 2025$ & $\mathrm{X}$ & $\mathrm{X}$ & $\mathrm{X}$ & $\mathrm{In} 2025$ \\
\hline MKE & Yes & $\mathrm{X}$ & & $\mathrm{X}$ & $\mathrm{X}$ & $\mathrm{X}$ \\
\hline PBI & Yes & Extension & & $\mathrm{X}$ & $\mathrm{X}$ & \\
\hline TUS & Yes & Modifications & & & Modifications & \\
\hline DAY & No & & & $\mathrm{X}$ & & \\
\hline GSO & No & & & $\mathrm{X}$ & & \\
\hline
\end{tabular}

Table 5-3. New Runways by 2025

\begin{tabular}{|c|c|c|c|c|c|c|}
\hline Airport & $\begin{array}{c}\text { FACT } \\
\text { airport? }\end{array}$ & FACT 2 & ATO-F & ACE 2003 & ATO-P & LMI \\
\hline BNA & No & & & $\mathrm{X}$ & & \\
\hline BWI & Yes & $\mathrm{X}$ & & $\mathrm{X}$ & $\mathrm{X}$ & $\mathrm{X}$ \\
\hline CMH & No & & & $\mathrm{X}$ & & $\mathrm{X}$ \\
\hline DEN & Yes & & & $\mathrm{X}$ & & \\
\hline DFW & Yes & $\mathrm{X}$ & $\mathrm{X}$ & $\mathrm{X}$ & & $\mathrm{X}$ \\
\hline HOU & Yes & & & & $\mathrm{X}$ & \\
\hline GEG & No & & & $\mathrm{X}$ & & $\mathrm{X}$ \\
\hline
\end{tabular}


Table 5-3. New Runways by 2025

\begin{tabular}{|c|c|c|c|c|c|c|}
\hline Airport & $\begin{array}{c}\text { FACT } \\
\text { airport? }\end{array}$ & FACT 2 & ATO-F & ACE 2003 & ATO-P & LMI \\
\hline GSP & No & & & $\mathrm{X}$ & & $\mathrm{X}$ \\
\hline IAD & Yes & $\mathrm{X}$ & $\mathrm{X}$ & $\mathrm{X}$ & $\mathrm{X}$ & $\mathrm{X}$ \\
\hline IAH & Yes & $\mathrm{X}$ & $\mathrm{X}$ & $\mathrm{X}$ & & $\mathrm{X}$ \\
\hline IND & No & & $\mathrm{X}$ & $\mathrm{X}$ & & $\mathrm{X}$ \\
\hline JAX & No & & $\mathrm{X}$ & & & $\mathrm{X}$ \\
\hline MSY & No & & & $\mathrm{X}$ & & $\mathrm{X}$ \\
\hline OKC & No & & & $\mathrm{X}$ & & $\mathrm{X}$ \\
\hline ORF & No & & & $\mathrm{X}$ & & $\mathrm{X}$ \\
\hline PDX & Yes & & $\mathrm{X}$ & $\mathrm{X}$ & & \\
\hline PIT & Yes & & & $\mathrm{X}$ & & \\
\hline RDU & No & & & $\mathrm{X}$ & & $\mathrm{X}$ \\
\hline RSW & No & & $\mathrm{X}$ & $\mathrm{X}$ & & $\mathrm{X}$ \\
\hline SAT & Yes & $\mathrm{X}$ & $\mathrm{X}$ & $\mathrm{X}$ & & $\mathrm{X}$ \\
\hline SAV & No & & & $\mathrm{X}$ & & $\mathrm{X}$ \\
\hline SYR & No & & & $\mathrm{X}$ & & \\
\hline TPA & Yes & $\mathrm{X}$ & $\mathrm{X}$ & $\mathrm{X}$ & & $\mathrm{X}$ \\
\hline TUL & No & & $\mathrm{X}$ & $\mathrm{X}$ & & $\mathrm{X}$ \\
\hline TUS & Yes & & & $\mathrm{X}$ & & \\
\hline
\end{tabular}

\section{NextGen Technology Improvements}

For 2015 and 2025 capacities, we consider the added benefits of technology increases due to planned NextGen improvements. These benefits are consistent with the modeling assumptions made by JPDO. We assume these technological benefits will be implemented only at the large 110 airports. Thus, the only benefits for the smaller 200 airports in 2015 and 2025 are due to runway improvements (in other words, only GEG, GSP, and SAV receive capacity increases in that set of 200 airports).

The primary 2015 technology benefit is the reduction in TRACON delivery inefficiency at the $35 \mathrm{OEP}$ airports due to deployment of airport and multicenter Traffic Management Advisor (TMA) technology. This technology is also assumed to allow sequencing that prevents small class aircraft from following Boeing 757 and heavy class aircraft at those 35 airports. The TRACON inefficiency parameter is reduced from 0.25 nautical mile $(\mathrm{nm})$ to $0.10 \mathrm{~nm}$ at all the 35 airports except SFO. The baseline TRACON inefficiency for SFO in VMC1 and VMC2 is set to $0.50 \mathrm{~nm}$ to accommodate the challenges of setting up paired approaches and is reduced to $0.25 \mathrm{~nm}$ in 2015 . In IMC, SFO operates a single arrival runway, and the TRACON inefficiency is the same as for the other airports $(0.25$ baseline, 0.10 technology). 
NextGen technology assumptions for 2025 include the following:

- Terminal area airborne merging and spacing

- 4D time-based trajectories

$\bullet$ TMA

- En route descent advisor

- Required navigation performance trajectories and timing

- Wake vortex MIT reductions

- Expedited departure-path technology

- Surface management system

- Airborne information for lateral spacing, allowing independent approaches on runways with 2,500 -foot centerline separation.

To model these technologies for 2025, we made several changes to the parameter values. The changes are based on educated estimates and are consistent with those made by JPDO for benefit analysis of the 2025 technology portfolio. The changes are as follows:

- Common path of $3.0 \mathrm{~nm}$ for all meteorological conditions because speed corrections occur until approach is stabilized

- Standard deviation of arrival speed reduced from $5 \mathrm{kt}$ to $2 \mathrm{kt}$

- Standard deviation of position reduced from $0.25 \mathrm{~nm}$ to $100 \mathrm{ft}=0.016 \mathrm{~nm}$ based on GPS accuracy

- Standard deviation of wind reduced from $7.5 \mathrm{kt}$ to $5 \mathrm{kt}$

- Standard deviation of departure ROT reduced from 5 seconds to 4 seconds

- Standard deviation of arrival ROT reduced from 8 seconds to 4 seconds due to required navigation performance (RNP), airborne merging and spacing, dynamic runway occupancy management, etc.

- Hold distance for release of departure between arrivals reduced from $2 \mathrm{~nm}$ to $1 \mathrm{~nm}$ due to RNP

- TMA prevention of small aircraft following B757s and heavy aircraft

- TRACON inefficiency reduced to $0.05 \mathrm{~nm}$ due to TMA, RNP, and airborne merging and spacing 
- Mean departure communication time delay reduced from 1.5 seconds in VMC1 and VMC2 and 6.0 seconds in IMC to 1.0 second in all conditions

- Standard deviation of departure communication time delay reduced from 0.15 second in VMC1 and VMC2 and 0.6 second in IMC to 0.1 second in VMC and IMC

- Minimum arrival MIT separation reduced from $2.5 \mathrm{~nm}$ to $2.0 \mathrm{~nm}$

- Average arrival wake vortex separation reduction of $1 \mathrm{~nm}$, i.e., $4,5,6 \mathrm{~nm} \rightarrow 3,4,5 \mathrm{~nm}^{4}$

Appendix B contains the values for the 2015 and 2025 capacities.

\section{MIT-Limited and ROT-Limited Capacities}

In this section, we discuss the modeling of purely MIT-limited and purely ROTlimited runway capacities. We model both the 2015 and 2025 NextGen technology sets. Basic cases for 2015 and 2025 represent the expected NextGen operating conditions, and the MIT-limited and ROT-limited cases are excursions to determine the capacity limits of those constraints. We first extend the discussions above about how NextGen capacities are modeled under the joint MIT and ROT constraints, and then examine how they can be modeled under only one or the other. Finally, we present and discuss single runway capacity model results.

We model runway arrival capacity for the 2015 and 2025 NextGen cases using the joint requirements of maintaining regulatory MIT separations with 95 percent confidence and single runway occupancy with 97.5 percent confidence, subject to uncertainties in aircraft speeds, positions, wind environments, and ROT. For each technology, we calculate the pairwise time and distance spacing among five weight classes of aircraft - small, large, B757, heavy, and A380 - resulting in separation times and distances for 25 different pairs for each meteorological condition.

We model capacities for the following four meteorological conditions.

- VMC1, which refers to full visual conditions when visual approaches are offered by the controller to arrivals on instrument flight plans

- VMC2, which refers to conditions with ceilings greater than 1,000 feet and visibilities greater than 3 statute miles $(1,000-3)$ where terrain or other features require use of radar approaches

\footnotetext{
${ }^{4}$ The 1-mile reduction used for all weather conditions is consistent with JPDO and FACT2 modeling assumptions. The 1-mile average reduction allows estimates of delay benefits without tracking of wind data. In actual practice, when weather conditions permit, the separations can be reduced to safe separation minimums of 2.5 or 3 nautical miles.
} 
- IMC1, which refers to FAA Category 1 conditions of less than 1,000-3 but typically at least $200-1 / 2$ (ceiling of 200 feet and visibility of $1 / 2$ statute mile)

- IMC2, which refers to FAA Category 2 and 3 conditions of less than $200-1 / 2$ and runway visual range (RVR) of at least 300 or 600 feet.

It is common for IMC1 and IMC2 to be combined for throughput and delay analyses. For this task, we report capacities only for VMC1, VMC2, and IMC1.

Modeling VMC1 operations provides a unique challenge, because there are no regulatory MIT requirements for visual approaches. For such approaches, the aircrew is responsible for safe separation. The MIT separations we and others use for modeling visual approaches are inputs that, when used in the models, reflect observed reality and not regulatory requirements. The MIT separations we use in our model for VMC1 are those recommended in FAA-EM-78-8A (June 1978) for use in the FAA Airfield Capacity Model and "are not operational minima as consciously maintained by the controller, but rather reflect what field data shows under saturated conditions." ${ }^{5}$ When used in our model, the FAA-EM-78-8A visual separations result in ROT-limited approaches for large, B757, and heavy class aircraft following large class aircraft, and spacing less than that required by MIT regulatory requirements for aircraft following heavier aircraft. This behavior is supported by the VMC1 interarrival time data, ROT data, and anecdotal information we have reviewed over the past several years.

We assume that the current use of visual approaches in VMC1 conditions will continue for 2015 NextGen. Because there are no minimum regulatory MIT separation limits for visual approaches, an MIT-limited case could logically imply the unreasonable result of infinite MIT-limited arrival capacity. In lieu of this, we maintain that calculation of MIT-limited operation is inappropriate for 2015 NextGen VMC1 operations. It is appropriate for all other meteorological conditions where minimums apply.

For 2025 NextGen VMC1, on the other hand, it is logical to consider that regulatory MIT limits will apply, because we assume that airborne merging and spacing or some other tool will support continuous ATC separation control in visual conditions. We model 2025 NextGen VMC1 capacity using reduced FAA 7110.65 (Controller Handbook) separations rather than the FAA-EM-78-8A visual separations.

\section{MODELING MIT-LIMITED CAPACITY}

As mentioned above, MIT-limited capacity is applicable only when MIT separations represent actual requirements, i.e., in VMC1 for NextGen 2025 and in VMC2 and IMC for both 2015 and 2025 NextGen. Even in these cases, we cannot

\footnotetext{
${ }^{5}$ FAA-EM-78-8A, June 1978, p. 3-3.
} 
simply reduce ROTs to low levels when calculating mixed operations, because the arrival ROTs largely determine the number of departures that can be fit between arrivals. Arbitrary arrival ROT reductions result in pathological model results. The approach we adopt is to modify the capacity model to force selection of the MIT constraint for the all-arrival and all-departure capacities. We retain the existing ROTs for calculation of the mixed capacity points. The mixed capacities are the "Equal" arrivals and departures point and the "Free" departures that can be inserted when operating with maximum arrivals.

\section{MODELING ROT-LIMITED CAPACITY}

ROT-limited operations are applicable to all technologies because they logically model the elimination of wake vortex and ATC separation requirements. ROTlimited capacity can be modeled by simply reducing the MIT requirements to arbitrarily low numbers, such that all spacing is controlled by ROT.

\section{SINGLE RUNWAY MODEL RESULTS}

The figures below show the single runway capacity Pareto curves for basic NextGen, MIT-limited, and ROT-limited 2015 and 2025 NextGen under VMC1, VMC2, and IMC. The capacity points on the Pareto curves are calculated for maximum arrivals, maximum departures, equally mixed arrivals and departures, and free departures that can be inserted when operating at the maximum arrival capacity. As maximum arrival capacity increases, the number of free departures diminishes or disappears.

Figures 5-2 through 5-4 show the single runway Pareto curves for 2015 NextGen. The basic and MIT-limited curves are identical for two reasons. For VMC1 operations, they are identical because MIT-limited operation is not applicable to VMC1 operations. For VMC2 and IMC operations, the curves are identical because all pairwise separations are already MIT-limited in the basic case.

ROT-limited curves show improvements in maximum arrivals and departures. This is because, for basic NextGen operations, many pairs in VMC1 and all pairs in VMC2 and IMC are MIT limited. The percentage improvement for maximum arrivals increases as we progress from VMC1 to IMC. The departure capacity increases to the point where all departures are constrained by the 1-minute minimum departure interval. 


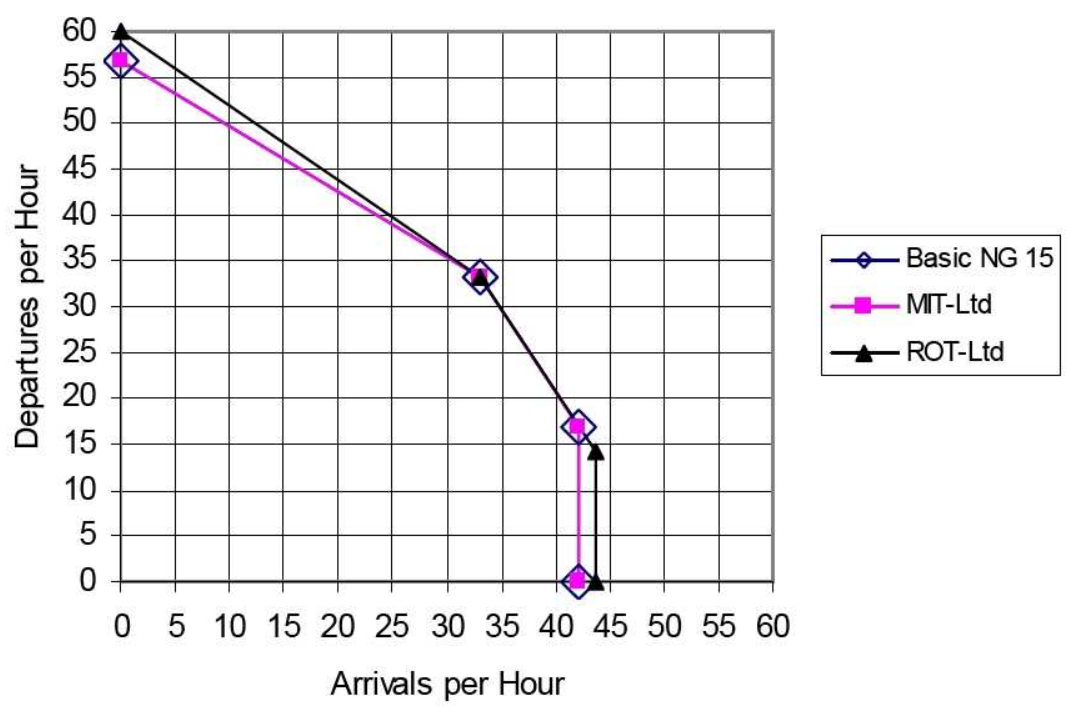

Figure 5-2. 2015 NextGen VMC1 single runway capacities.

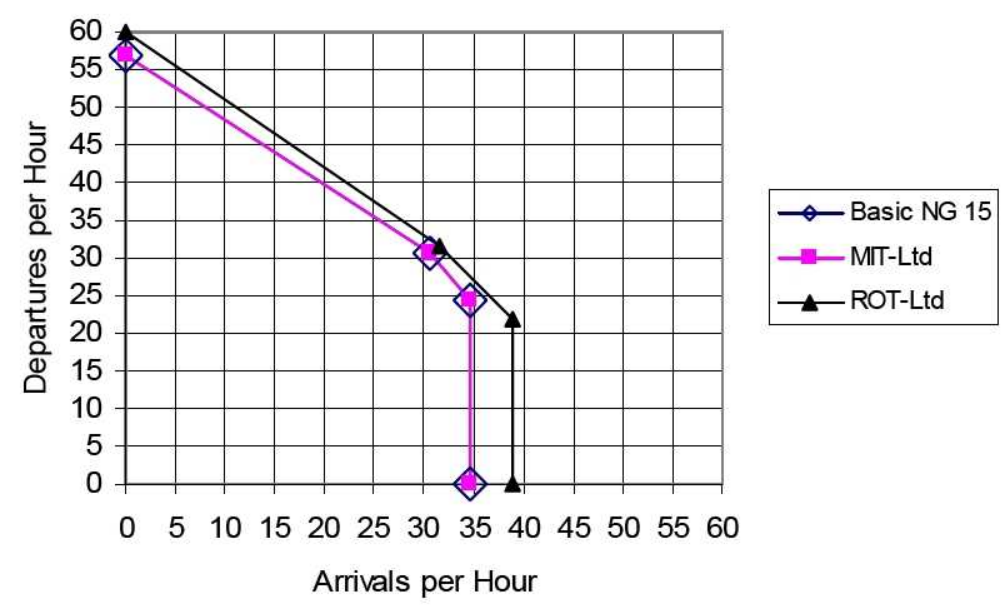

Figure 5-3. 2015 NextGen VMC2 single runway capacities. 


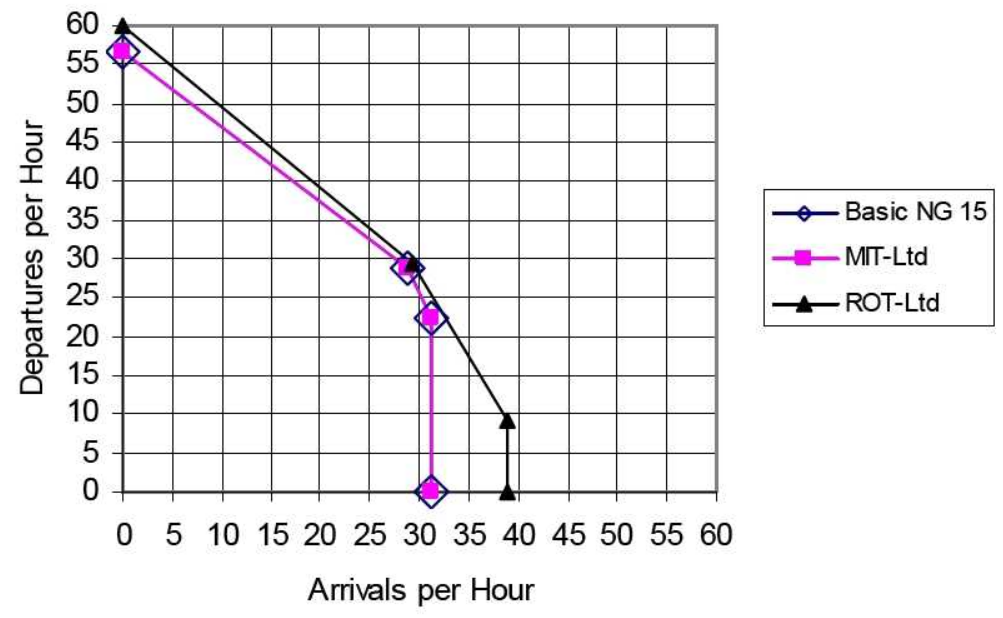

Figure 5-4. 2015 NextGen IMC1 single runway capacities.

Figures 5-5 through 5-7 show the single runway Pareto curves for 2025 NextGen. In the basic 2025 NextGen, arrivals of large, B757, and heavy class aircraft behind large aircraft in VMC1 and VMC2 are ROT limited. This is because 2025 NextGen technology improves uncertainties in speed, position, and wind to the extent that the spacing required for ATC to guarantee MIT separation is less than that needed to guarantee single runway occupancy. The other aircraft pairs are still MIT limited. In this situation, both MIT-limited and ROT-limited operations improve arrival capacity.

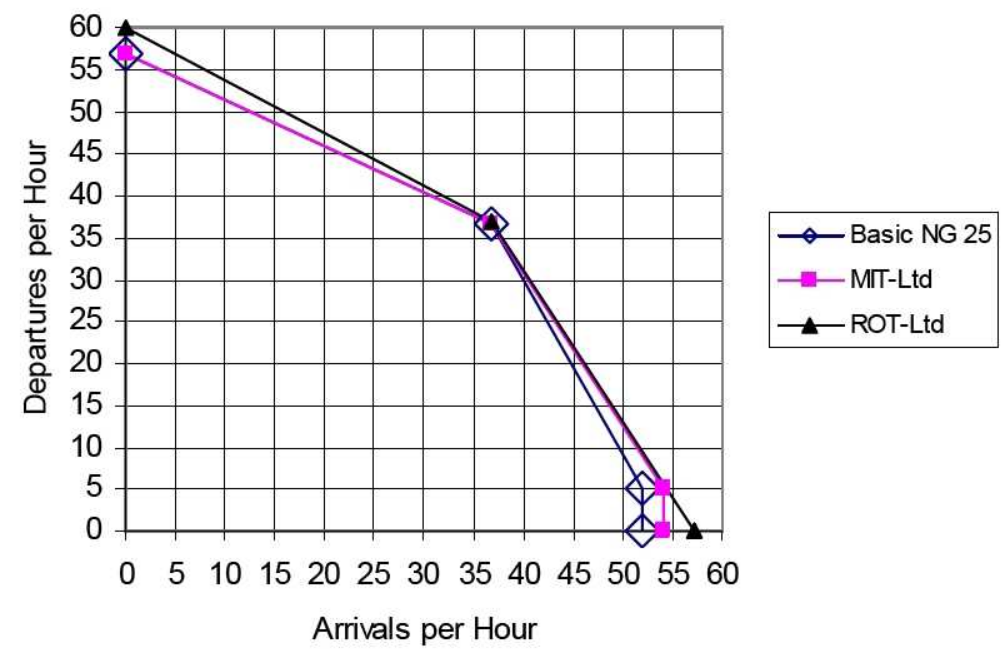

Figure 5-5. 2025 NextGen VMC1 single runway capacities. 


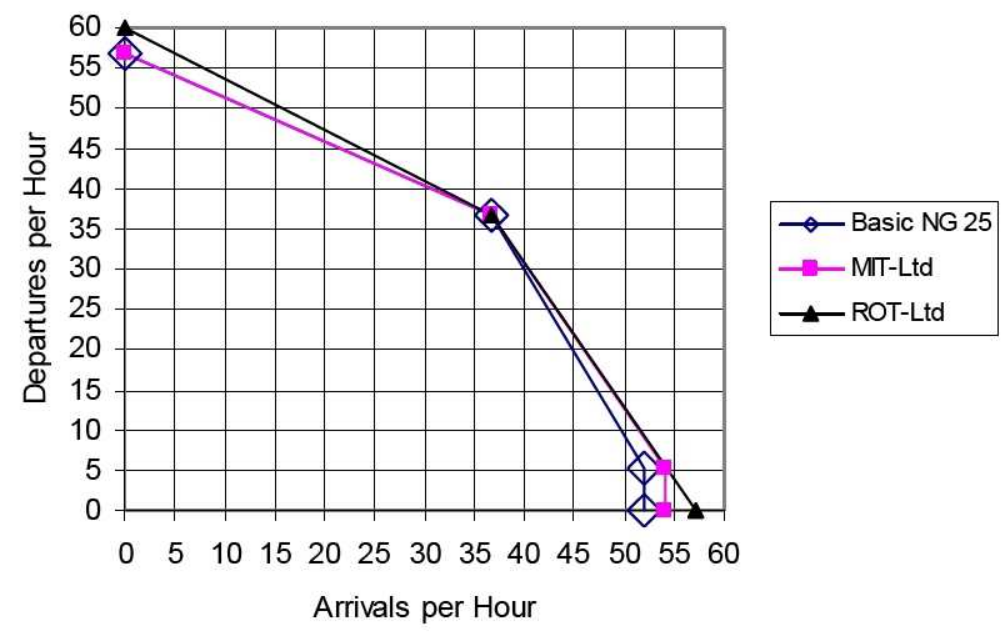

Figure 5-6. 2025 NextGen VMC2 single runway capacities.

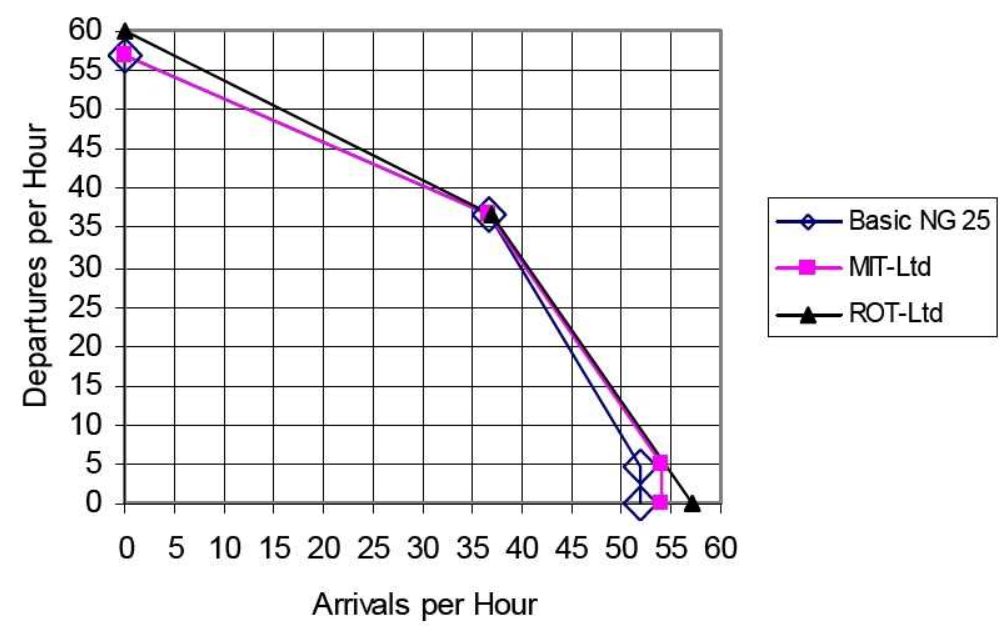

Figure 5-7. 2025 NextGen IMC1 single runway capacities.

In general, the curves above show the following:

- ROT-limited operation, i.e., eliminating MIT restrictions, provides improved maximum arrival and departure capacity, but does not substantially change the mixed arrival/departure capacity.

- MIT-limited operation, i.e., eliminating ROT restrictions, provides no benefits for 2015 NextGen and modest benefits for 2025 NextGen.

Results for closely spaced parallel arrival and departure runways and closely spaced parallel runways with dependent operations are qualitatively similar to results for a single runway. 
We used the capacity results discussed above to develop the MIT-limited and ROT-limited airport capacities contained in Appendix B.

\section{Projected Throughput Results for NextGen}

Tables 5-4 through 5-6 list the reductions in operations (arrivals plus departures) for airports under the 2007, 2015, and 2025 demand scenarios. Airports with no reductions are omitted. For the 2007 demand reductions, the significant reductions occur at today's busiest hub airports. Although the reductions for some of these airports are significant (e.g., LGA), we chose a relatively high level of service in our throughput calculations. This restriction ensures that calculated future throughput benefits due to NextGen or other technologies will correspond to a higher level of schedule integrity than is currently experienced at these airports. Reductions in later years typically grow, because demand increases at a rate generally faster than some of the NextGen improvements. However, for many airports, reductions are decreased or eliminated in future years, as NextGen improvements outpace demand growth (e.g., ORD in 2025).

Table 5-4. Airports with Operation Reductions in 2007

\begin{tabular}{|c|c|c|c|}
\hline Airport & $\begin{array}{c}\text { Actual } \\
\text { operations }\end{array}$ & $\begin{array}{c}\text { Projected } \\
\text { operations }\end{array}$ & $\begin{array}{c}\text { Percentage } \\
\text { reduction }\end{array}$ \\
\hline ANC & 1,022 & 1,002 & $2.0 \%$ \\
\hline ATL & 3,019 & 2,869 & $5.0 \%$ \\
\hline BOS & 1,339 & 1,335 & $0.3 \%$ \\
\hline CLT & 1,549 & 1,375 & $11.2 \%$ \\
\hline DAL & 708 & 706 & $0.3 \%$ \\
\hline DCA & 854 & 852 & $0.2 \%$ \\
\hline EWR & 1,390 & 1,298 & $6.6 \%$ \\
\hline HOU & 737 & 735 & $0.3 \%$ \\
\hline HPN & 668 & 664 & $0.6 \%$ \\
\hline IAD & 1,179 & 1,165 & $1.2 \%$ \\
\hline JFK & 1,412 & 1,392 & $1.4 \%$ \\
\hline LAS & 1,525 & 1,391 & $8.8 \%$ \\
\hline LAX & 2,030 & 1,972 & $2.9 \%$ \\
\hline LGA & 1,243 & 1,091 & $12.2 \%$ \\
\hline PHL & 1,576 & 1,434 & $9.0 \%$ \\
\hline PHX & 1,477 & 1,449 & $1.9 \%$ \\
\hline SAN & 710 & 708 & $0.3 \%$ \\
\hline VNY & 767 & 765 & $0.3 \%$ \\
\hline BFI & 790 & 774 & $2.0 \%$ \\
\hline
\end{tabular}


Table 5-5. Airports with Operation Reductions in 2015

\begin{tabular}{|c|c|c|c|}
\hline Airport & $\begin{array}{c}\text { Unconstrained } \\
\text { operations }\end{array}$ & $\begin{array}{c}\text { Projected } \\
\text { operations }\end{array}$ & $\begin{array}{c}\text { Percentage } \\
\text { reduction }\end{array}$ \\
\hline ANC & 1,234 & 1,104 & $10.5 \%$ \\
\hline ATL & 3,561 & 3,063 & $14.0 \%$ \\
\hline BOS & 1,518 & 1,516 & $0.1 \%$ \\
\hline DAL & 913 & 883 & $3.3 \%$ \\
\hline DCA & 874 & 864 & $1.1 \%$ \\
\hline EWR & 1671 & 1,421 & $15.0 \%$ \\
\hline HNL & 1,067 & 1,061 & $0.6 \%$ \\
\hline HOU & 894 & 870 & $2.7 \%$ \\
\hline HPN & 757 & 741 & $2.1 \%$ \\
\hline IAD & 1,490 & 1,484 & $0.4 \%$ \\
\hline IAH & 2,256 & 2,244 & $0.5 \%$ \\
\hline JFK & 1,775 & 1,591 & $10.4 \%$ \\
\hline JNU & 650 & 628 & $3.4 \%$ \\
\hline LAS & 1,992 & 1,494 & $25.0 \%$ \\
\hline LAX & 2,805 & 2,407 & $14.2 \%$ \\
\hline LGA & 1,277 & 1,129 & $11.6 \%$ \\
\hline MDW & 1,058 & 1,028 & $2.8 \%$ \\
\hline MEM & 1,345 & 1,281 & $4.8 \%$ \\
\hline MSP & 1,662 & 1,646 & $1.0 \%$ \\
\hline ORD & 3,217 & 3,121 & $3.0 \%$ \\
\hline PHL & 1,923 & 1,763 & $8.3 \%$ \\
\hline PHX & 1,840 & 1,718 & $6.6 \%$ \\
\hline SAN & 890 & 838 & $5.8 \%$ \\
\hline SFO & 1,443 & 1,439 & $0.3 \%$ \\
\hline SLC & 1,443 & 1,399 & $3.0 \%$ \\
\hline TEB & 812 & 806 & $0.7 \%$ \\
\hline VNY & 827 & 815 & $1.5 \%$ \\
\hline ACK & 865 & 819 & $5.3 \%$ \\
\hline BED & 459 & 449 & $2.2 \%$ \\
\hline BFI & 907 & 837 & $7.7 \%$ \\
\hline PWK & 401 & 397 & $1.0 \%$ \\
\hline & & & \\
\hline & & & 1,07 \\
\hline
\end{tabular}


Table 5-6. Airports with Operation Reductions in 2025

\begin{tabular}{|c|c|c|c|}
\hline Airport & $\begin{array}{l}\text { Unconstrained } \\
\text { operations }\end{array}$ & $\begin{array}{l}\text { Projected } \\
\text { operations }\end{array}$ & $\begin{array}{l}\text { Percentage } \\
\text { reduction }\end{array}$ \\
\hline ANC & 1,563 & 1,333 & $14.7 \%$ \\
\hline ATL & 4,383 & 3,605 & $17.8 \%$ \\
\hline BOS & 1,750 & 1,722 & $1.6 \%$ \\
\hline CVG & 1,542 & 1,526 & $1.0 \%$ \\
\hline DAL & 1,186 & 1,172 & $1.2 \%$ \\
\hline DCA & 893 & 889 & $0.4 \%$ \\
\hline DTW & 1,979 & 1,909 & $3.5 \%$ \\
\hline EWR & 2,111 & 1,545 & $26.8 \%$ \\
\hline HNL & 1,270 & 1,260 & $0.8 \%$ \\
\hline $\mathrm{HOU}$ & 1,055 & 1,023 & $3.0 \%$ \\
\hline HPN & 893 & 871 & $2.5 \%$ \\
\hline IAD & 2,083 & 1,999 & $4.0 \%$ \\
\hline IAH & 2,848 & 2,810 & $1.3 \%$ \\
\hline JFK & 2,327 & 1,899 & $18.4 \%$ \\
\hline JNU & 784 & 760 & $3.1 \%$ \\
\hline LAS & 2,760 & 1,684 & $39.0 \%$ \\
\hline LAX & 3,678 & 2,834 & $22.9 \%$ \\
\hline LGA & 1,287 & 1,279 & $0.6 \%$ \\
\hline MDW & 1,333 & 1,229 & $7.8 \%$ \\
\hline MEM & 1,611 & 1,457 & $9.6 \%$ \\
\hline MSP & 2,026 & 1,964 & $3.1 \%$ \\
\hline PHF & 718 & 692 & $3.6 \%$ \\
\hline PHL & 2,518 & 2,002 & $20.5 \%$ \\
\hline PHX & 2,516 & 2,230 & $11.4 \%$ \\
\hline SAN & 1,146 & 978 & $14.7 \%$ \\
\hline SFO & 1,791 & 1,721 & $3.9 \%$ \\
\hline SLC & 1,698 & 1,598 & $5.9 \%$ \\
\hline VNY & 890 & 884 & $0.7 \%$ \\
\hline ACK & 1,030 & 942 & $8.5 \%$ \\
\hline APA & 726 & 724 & $0.3 \%$ \\
\hline BFI & 1,051 & 949 & $9.7 \%$ \\
\hline OGG & 597 & 587 & $1.7 \%$ \\
\hline
\end{tabular}

Figure 5-8 shows the unconstrained and projected operations for the 10 busiest U.S. airports in 2015 and $2025 .^{6}$ The percentages on the top of the bars are the

\footnotetext{
${ }^{6}$ These are the top 10 airports by operations count in 2007 . We do not anticipate substantial changes in the makeup of this group by 2015 or 2025 .
} 
ratios of the projected operations versus the unconstrained operations. One can see that ATL, LAX, LAS, PHX, and PHL are significantly constrained, even with NextGen. Some airports, like DFW and DEN, have no runway constraints. Each airport should be viewed individually. Some, like CLT, are constrained currently and then will be helped by NextGen; others, like IAH, are fine currently but will be constrained in the future due to the unbalanced demand growth.

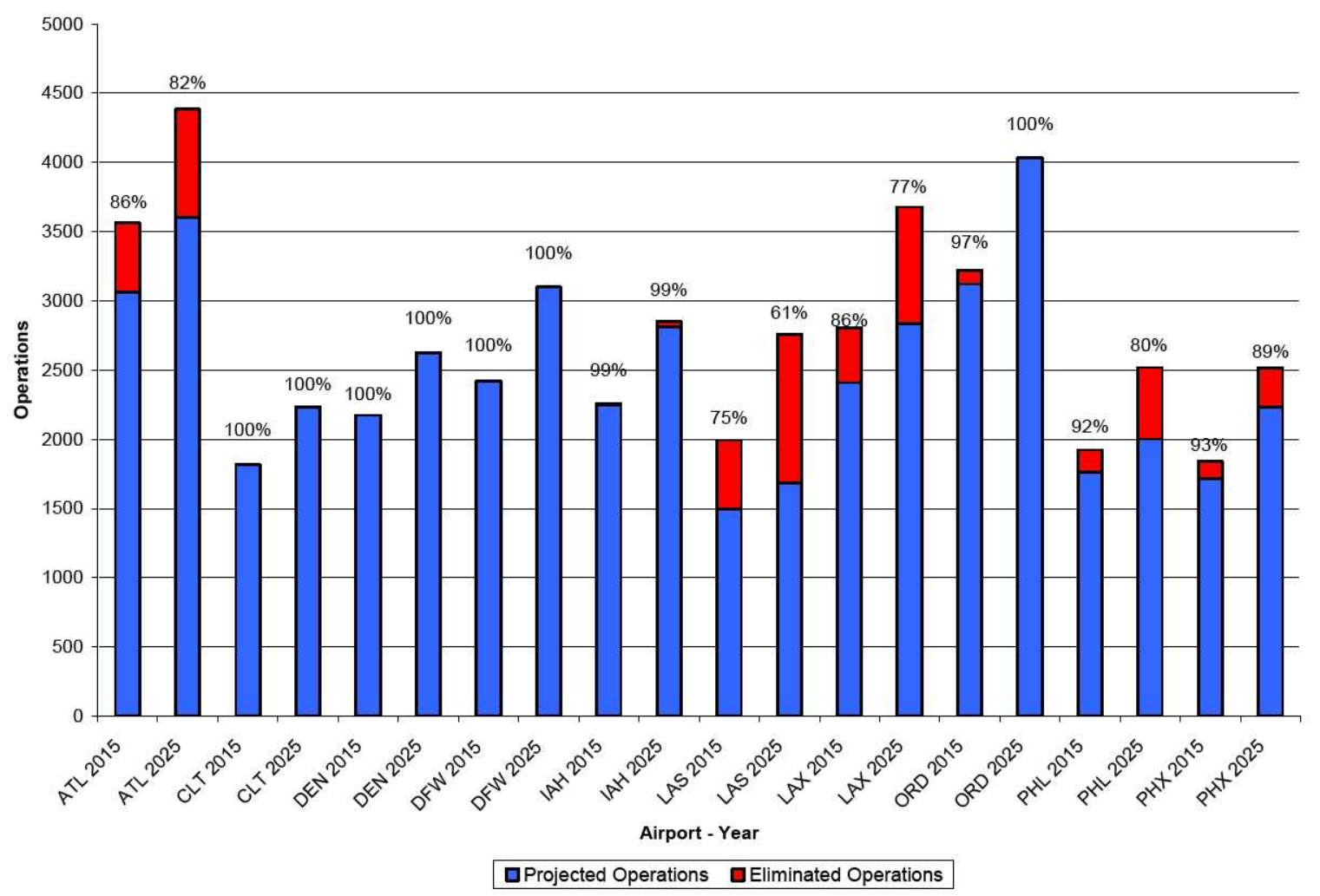

Figure 5-8. Comparison of operations at 10 busiest airports.

Figure 5-9 shows the same type of information aggregated for OEP 35 airports, LMINET 110 airports, and LMINET 310 airports. In general, the OEP 35 airports are more constrained than the LMINET 110 airports, which are more constrained than the LMINET 310 airports. Also, airports are more constrained in 2015 than in 2007, and they are more constrained in 2025 than in 2015. The constraint in 2025 is significant, regardless of the set of airports. 


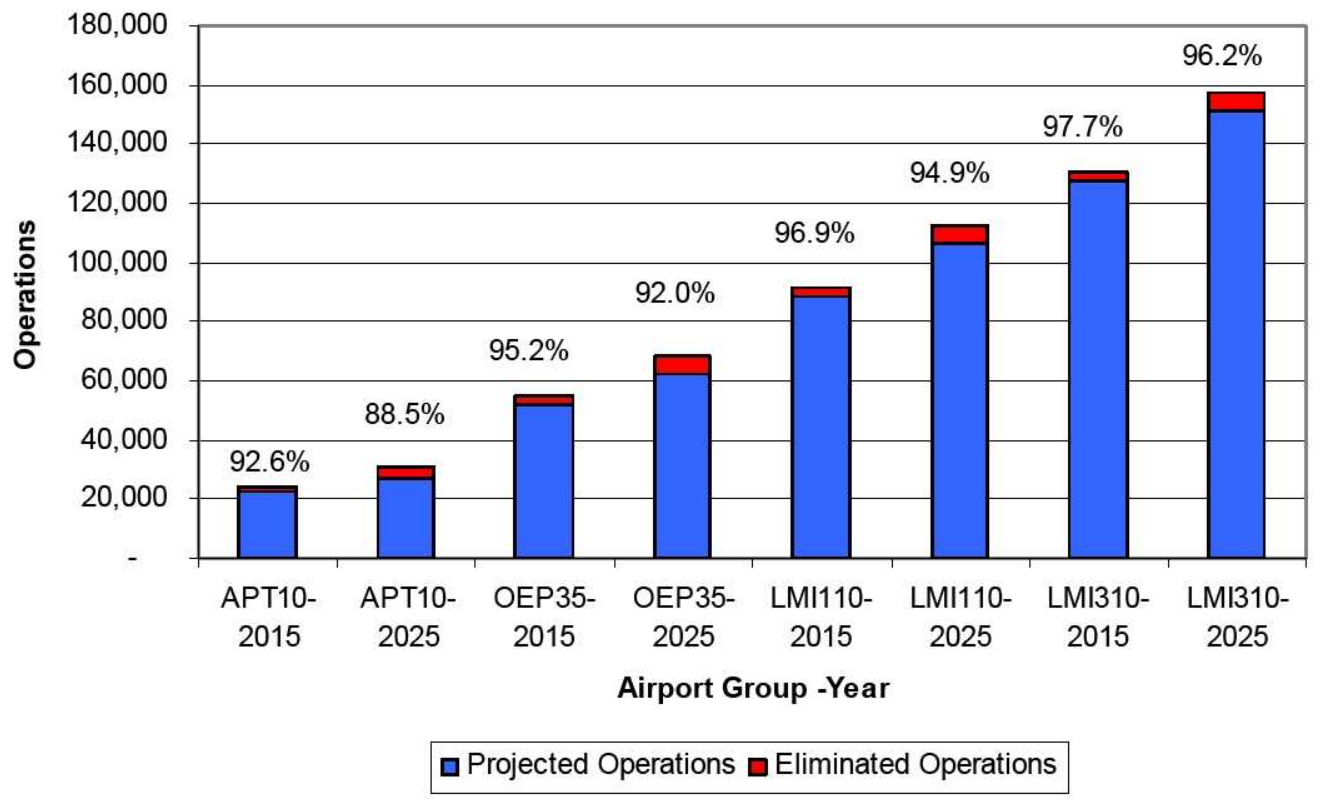

Figure 5-9. Comparison of aggregate airport operations.

\section{Projected Throughput Results for ROT-Limited and MIT-Limited Operations}

To calculate the throughput values for ROT-limited and MIT-limited operations, we used the same method that we used to calculate values for NextGen. In other words, we calculated the reduction in operations using the same demand-tocapacity $(\mathrm{D} / \mathrm{C})$ ratios of 1.2 quarter-hour and 0.9 rolling-hour values. Because both the ROT-limited and MIT-limited cases reduce restrictions on the runway capacities outlined previously in this chapter, the throughput for these cases will be greater than or equal to the NextGen throughput values.

As noted in the capacity section of this chapter, there is no benefit in the MITlimited case in 2015. Therefore, the throughput benefits in 2015 when no ROT restrictions exist (but MIT restrictions do) are identical to the standard 2015 NextGen case. There are, however, modest capacity increases in the ROT-limited case (provided in Appendix B). This benefit will increase throughput at the congested airports in 2015. Figure 5-10 shows the throughput for the 10 busiest airports, under both the NextGen and the ROT-limited case. Additional throughput benefits for airports that experience demand trimming in 2015 range from 1 percent to 4 percent of the unconstrained demand among these 10 airports. 


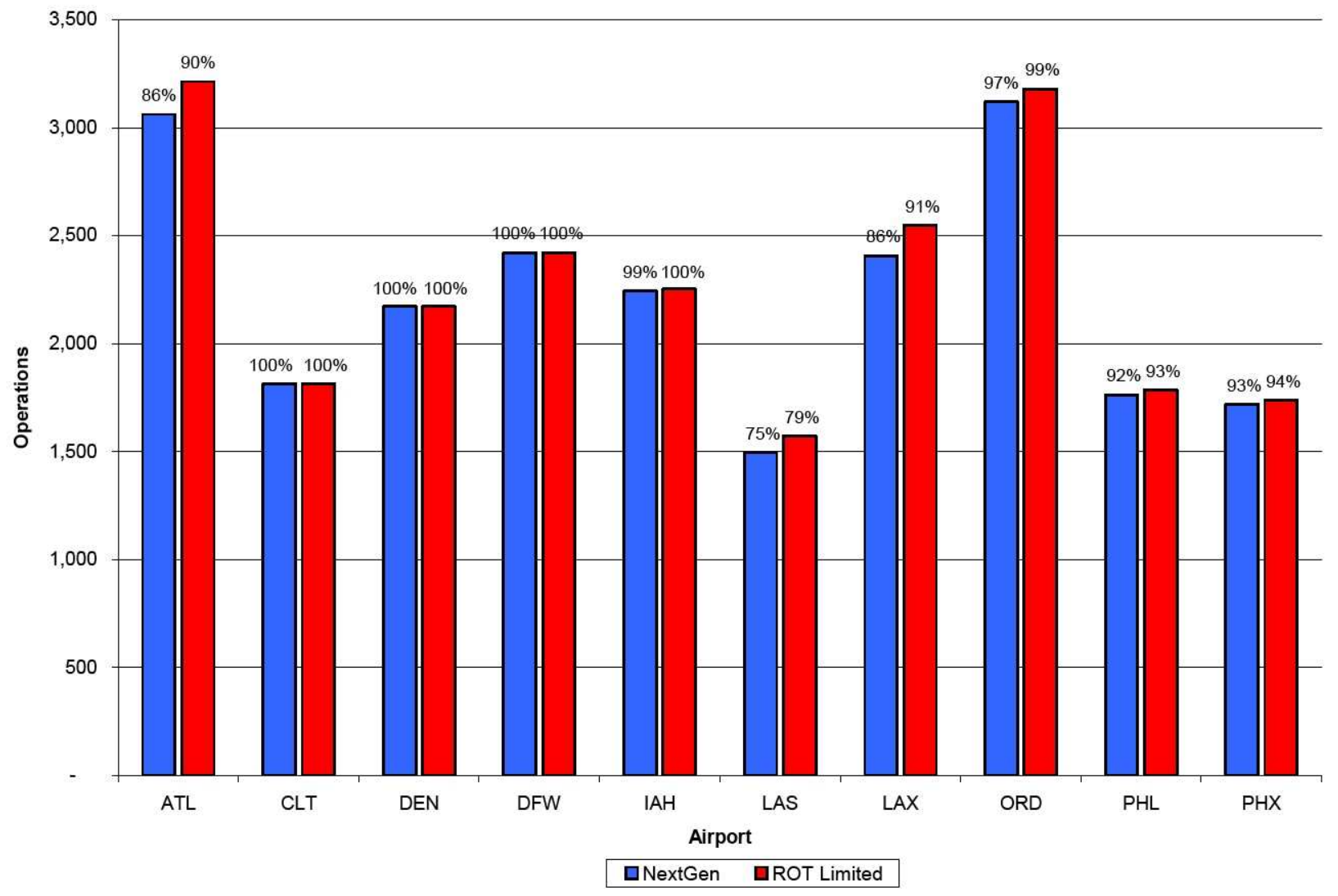

Figure 5-10. Throughput under NextGen and ROT-limited cases for the 10 busiest airports in 2015.

Figure 5-11 shows the aggregate throughput for 2015 under the NextGen and ROT-limited cases for the 10 busiest, OEP 35, LMINET 110, and LMINET 310 airports. Because the OEP 35 airports are the largest airports in the NAS, they typically are the most constrained. Although the increase in the throughput in the ROT-limited case over the NextGen case is modest, the amount of trimming at most of these airports is relatively small in 2015 under the NextGen case. 


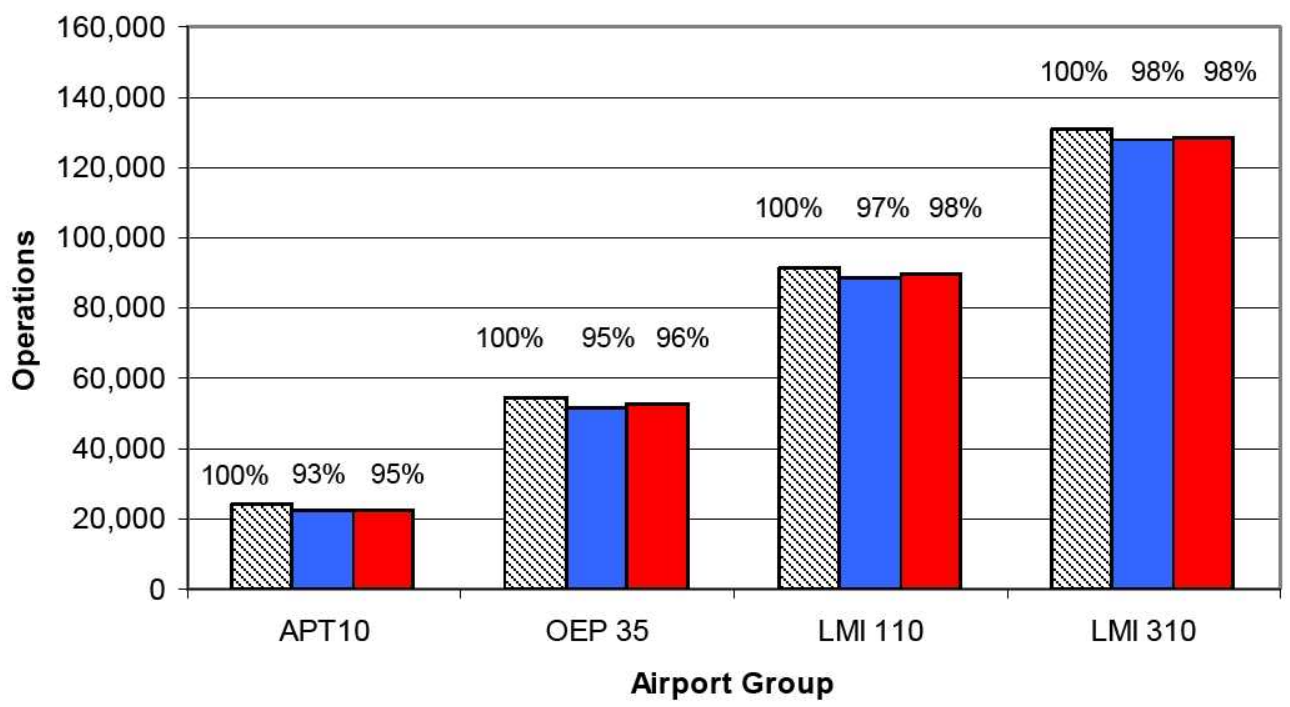

\$ Unconstrained $\square$ NextGen $\square$ ROT Limited

Figure 5-11. Throughput comparison for NextGen and ROT-limited cases by airport group in 2015.

Due to the assumptions of maturing NextGen technologies and the addition of some new runways by 2025 , the ROT-limited case experiences a greater capacity increase than in 2015. In addition, the MIT-limited case experiences a modest benefit beyond NextGen in 2025, as opposed to no additional benefit in 2015 . Although the amount of the capacity benefit under the ROT-limited case depends greatly on the configuration of the airport, many airports receive a significant increase in feasible throughput. Figure 5-12 provides the throughput values for the 2025 NextGen, ROT-limited, and MIT-limited cases for the 10 busiest airports. At LAX, the ROT-limited case provides 13 percent of the total unconstrained demand above what is achievable under NextGen only. This increase represents more than half of the unmet demand at LAX. PHL, on the other hand, has a relatively minor increase under the ROT-limited case. It is worth noting that the throughput increases in the MIT-limited case are relatively modest. Figure 5-13 provides these data by the same airport categories as Figure 5-11. 


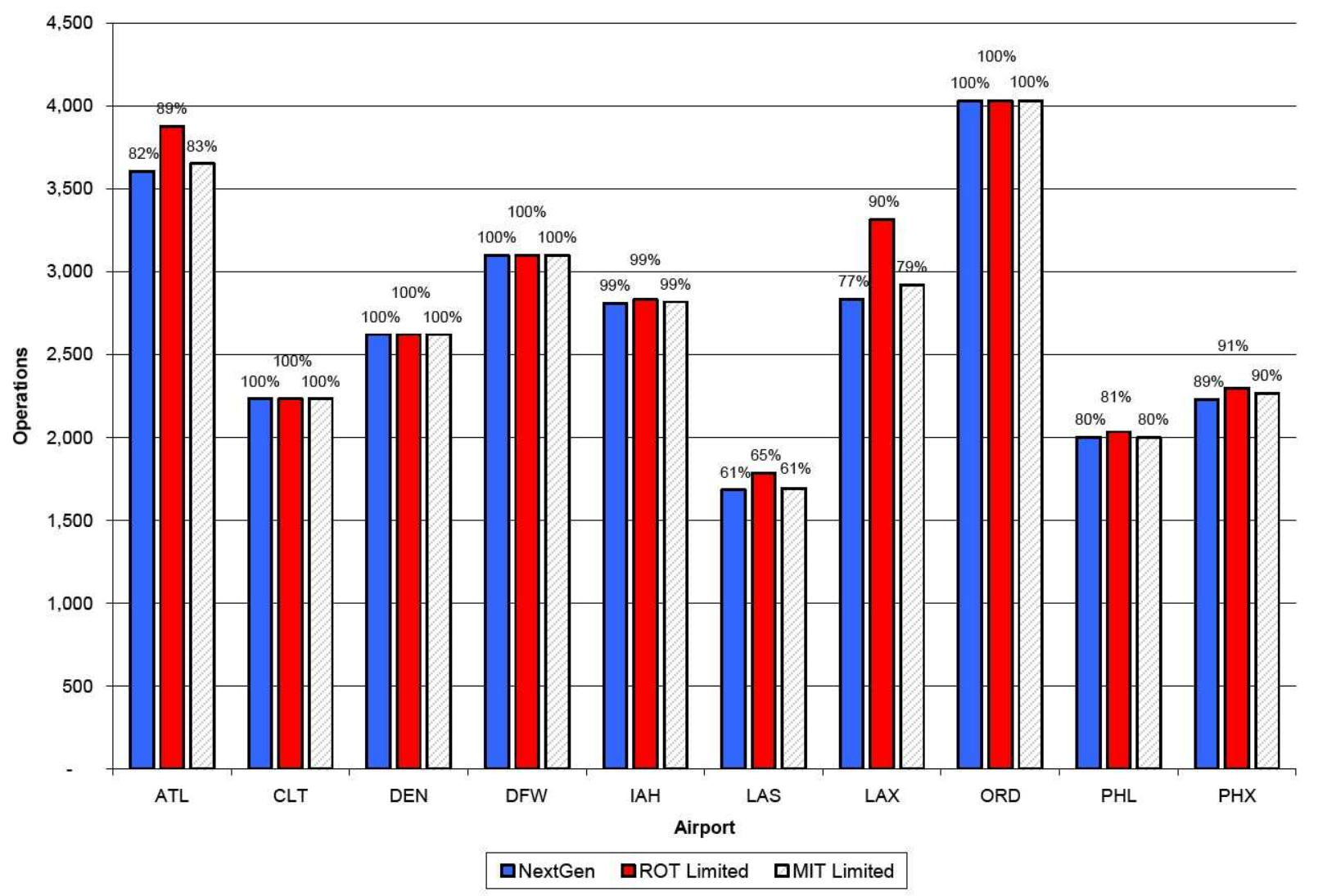

Figure 5-12. Throughput under NextGen, ROT-limited, and MIT-limited cases for the 10 busiest airports in 2025.

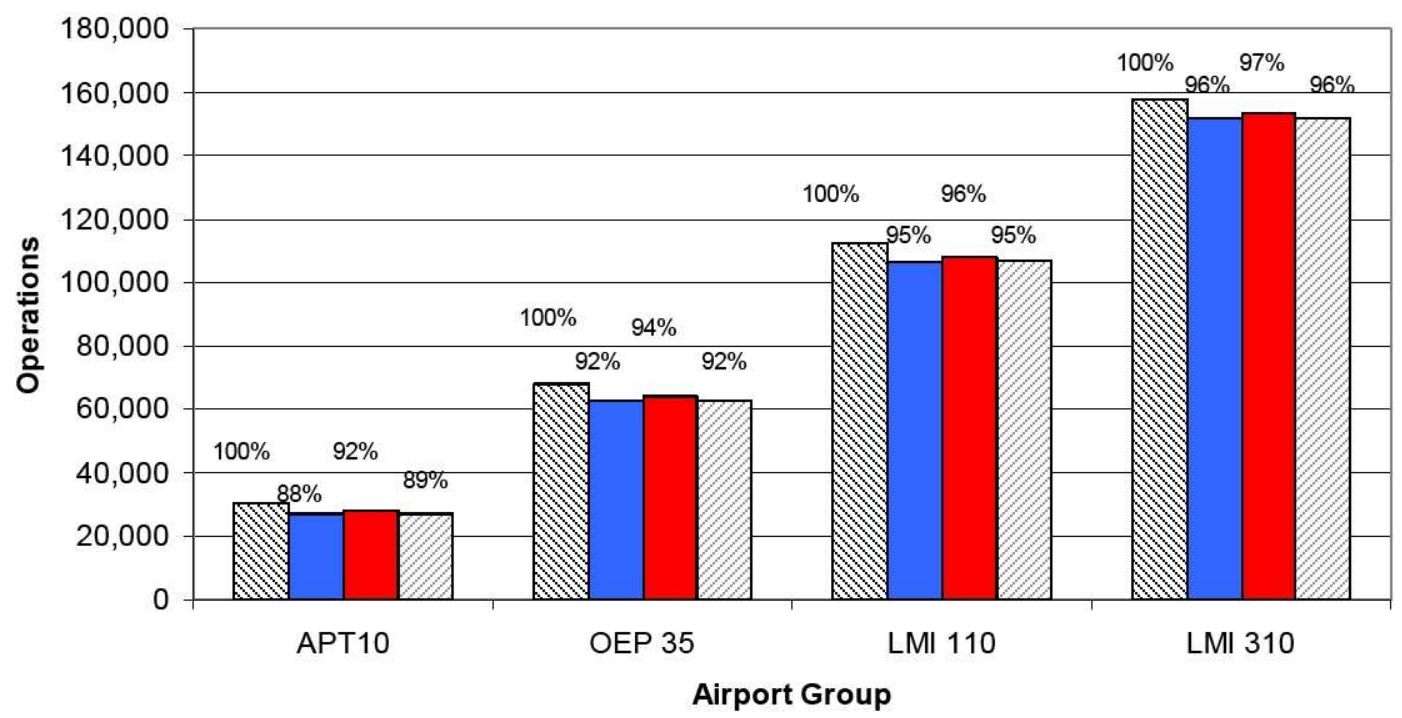

⿴囗U一 Unconstrained $\square$ NextGen $\square$ ROT Limited $\square$ MIT Limited

Figure 5-13. Throughput comparison for NextGen, ROT-limited, and MIT-limited cases by airport group in 2025. 


\section{Delay Results for NextGen Case}

The previous section outlined the analysis and results of projected airport throughput based on the NextGen runway capacity modeling. In particular, the projected throughput depends on the runway capacities in good weather and on the selected D/C ratio parameters. Although it is true that the airlines base their schedules mostly on good weather conditions, they also consider the impact of bad weather. This consideration is reflected in the selected $\mathrm{D} / \mathrm{C}$ ratios, which make the overall delay statistics tolerable. To be sure, the estimated projected throughput results in the previous section reliably reflect this fact, but we also needed to check whether the delays in the future are tolerable. To do that, we calculated the most conservative possible estimate of delays due to bad weather; specifically, we ran LMINET for each airport under IFR conditions throughout the day (conditions that rarely occur). In fact, many NextGen operational initiatives are envisioned to improve operations in times of bad weather, thus our delay estimates are another way of checking whether NextGen can provide the needed capacity to keep up with the growth in traffic demand. The following paragraphs summarize how we generated the delay results. (The detailed delay results are in Appendix C.)

Figures 5-14 through 5-16 show, for the 10 busiest airports, the arrival and departure delays, respectively, for 2007, 2015, and 2025. (Note that the scale in Figure 5-16 is radically different than those in the other two figures.) The huge imbalances of the arrival and departure delays at some airports are caused by two factors: (1) the assumption of IFR conditions throughout the day, which leaves no recovery time, and (2) the airport configuration under IFR, which can require the airport to close some runways. The drastic changes of delays across the years are caused by the increases in demand and in capacity due to NextGen, which are also magnified by the IFR conditions throughout the day. These figures show that by 2025, NextGen should be able to reduce the flight delays significantly for the bad weather days and for the overall flight delays. The reconfiguration at ORD and the subsequently large capacity increase contribute to the massive reduction in IFR delays from 2007 to 2015. 


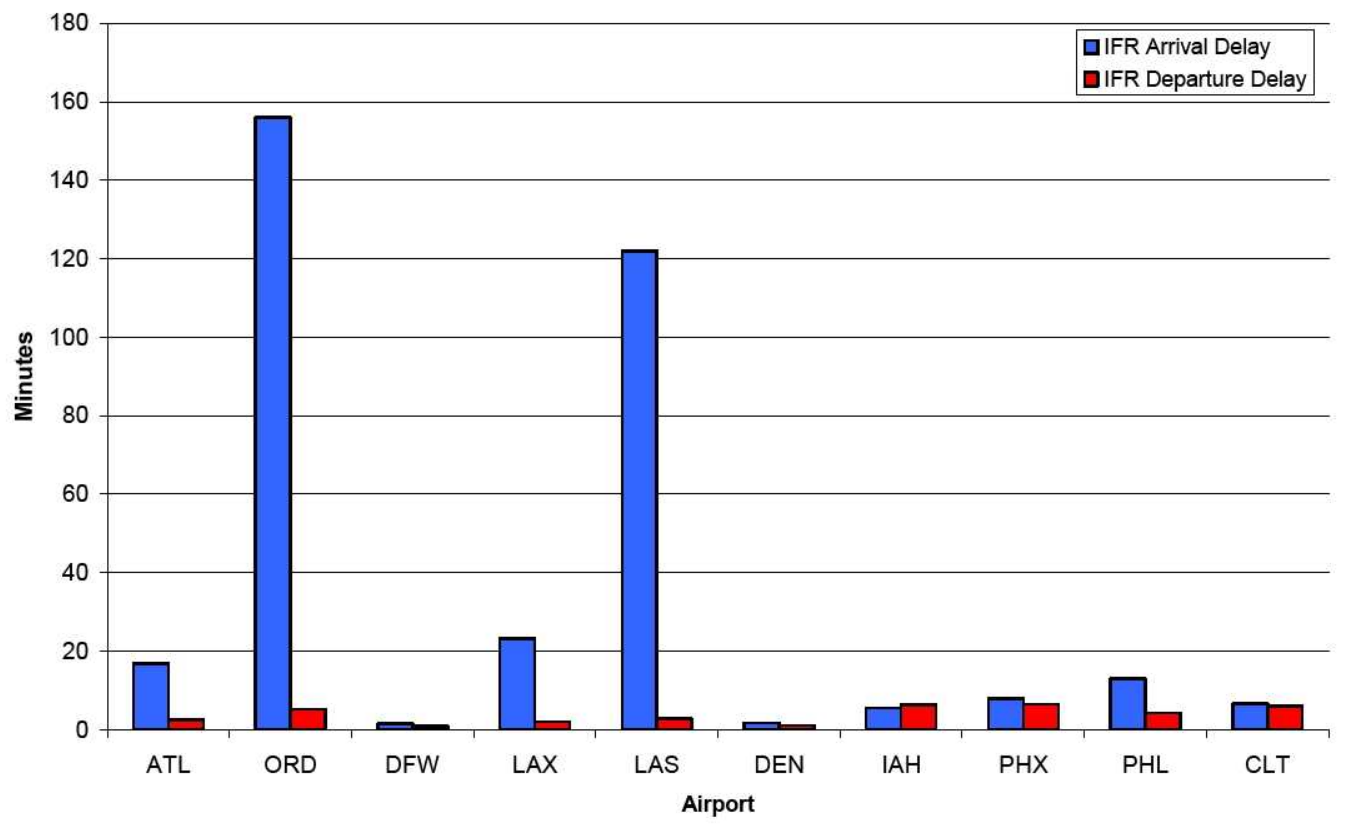

Figure 5-14. Average 2007 IFR per flight-delay minutes at 10 busiest airports.

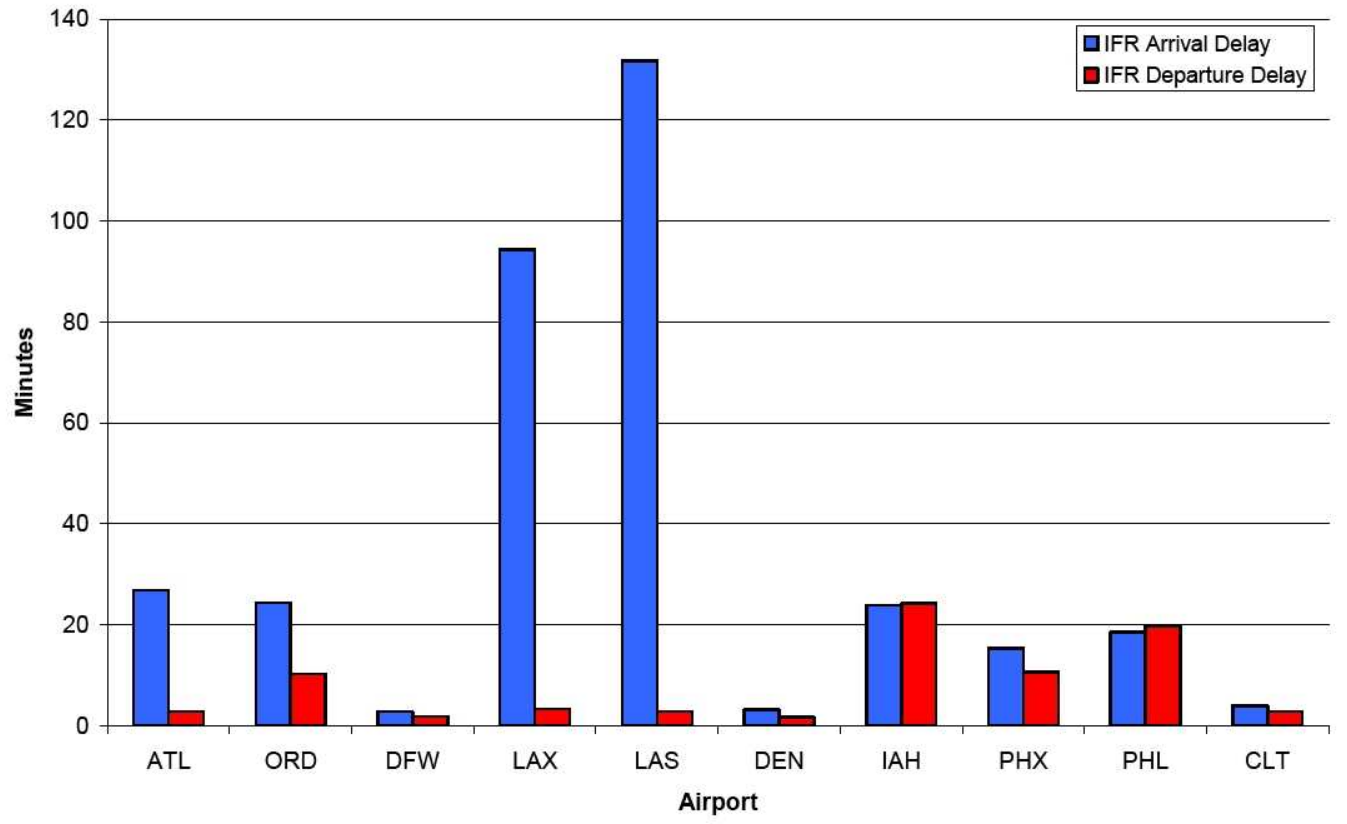

Figure 5-15. Average 2015 IFR per flight-delay minutes at 10 busiest airports. 


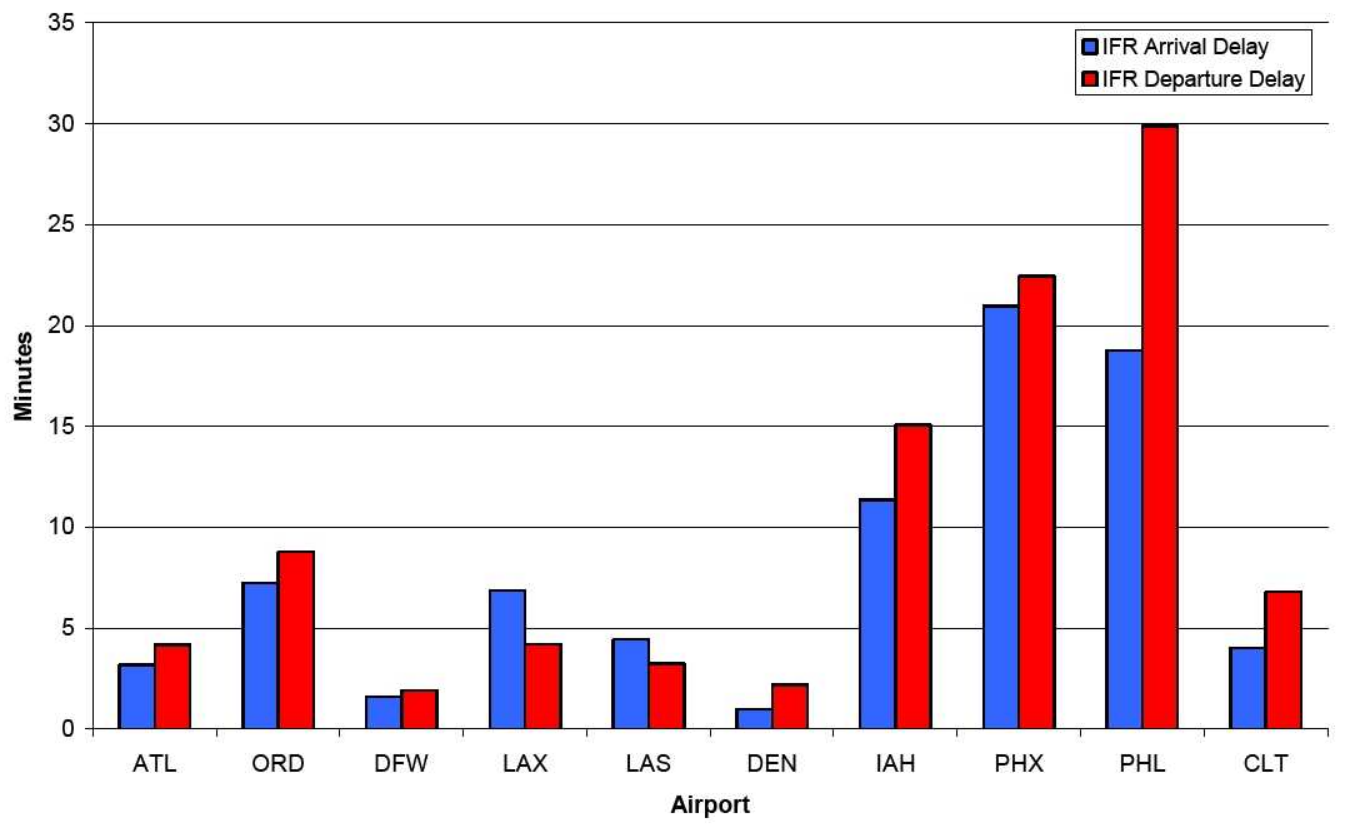

Figure 5-16. Average 2025 IFR per flight-delay minutes at 10 busiest airports.

\section{Delay Results for ROT-Limited and MIT-Limited Cases}

The delay values for the ROT-limited and MIT-limited cases are calculated under identical assumptions as those used in the NextGen case. Again, the projected throughput depends on the runway capacities in good weather and the selected $\mathrm{D} / \mathrm{C}$ ratio parameters. Again, we calculated the delays for a pure IFR day. A pure bad weather day across the NAS is extreme, but it provides a bound on the types of delays experienced. It also captures the extent to which the ROT-limited and MIT-limited cases help in off-nominal weather conditions. It is important to note that although the throughput is higher under the ROT-limited and MIT-limited cases compared to NextGen in 2025, the IFR delays are actually slightly lower on average than in NextGen. This illustrates the robustness of the capacities for the ROT-limited and MIT-limited cases in all weather conditions. Figures 5-17 and 5-18 provide the 2015 arrival and departure delay statistics for the 10 busiest airports for the ROT-limited and NextGen cases. Figures 5-19 and 5-20 provide the 2025 delay statistics for those same airports for ROT-limited, MIT-limited, and NextGen cases. Appendix $\mathrm{C}$ has the airport-specific delays for the 310 LMINET airports for both years and all cases. 


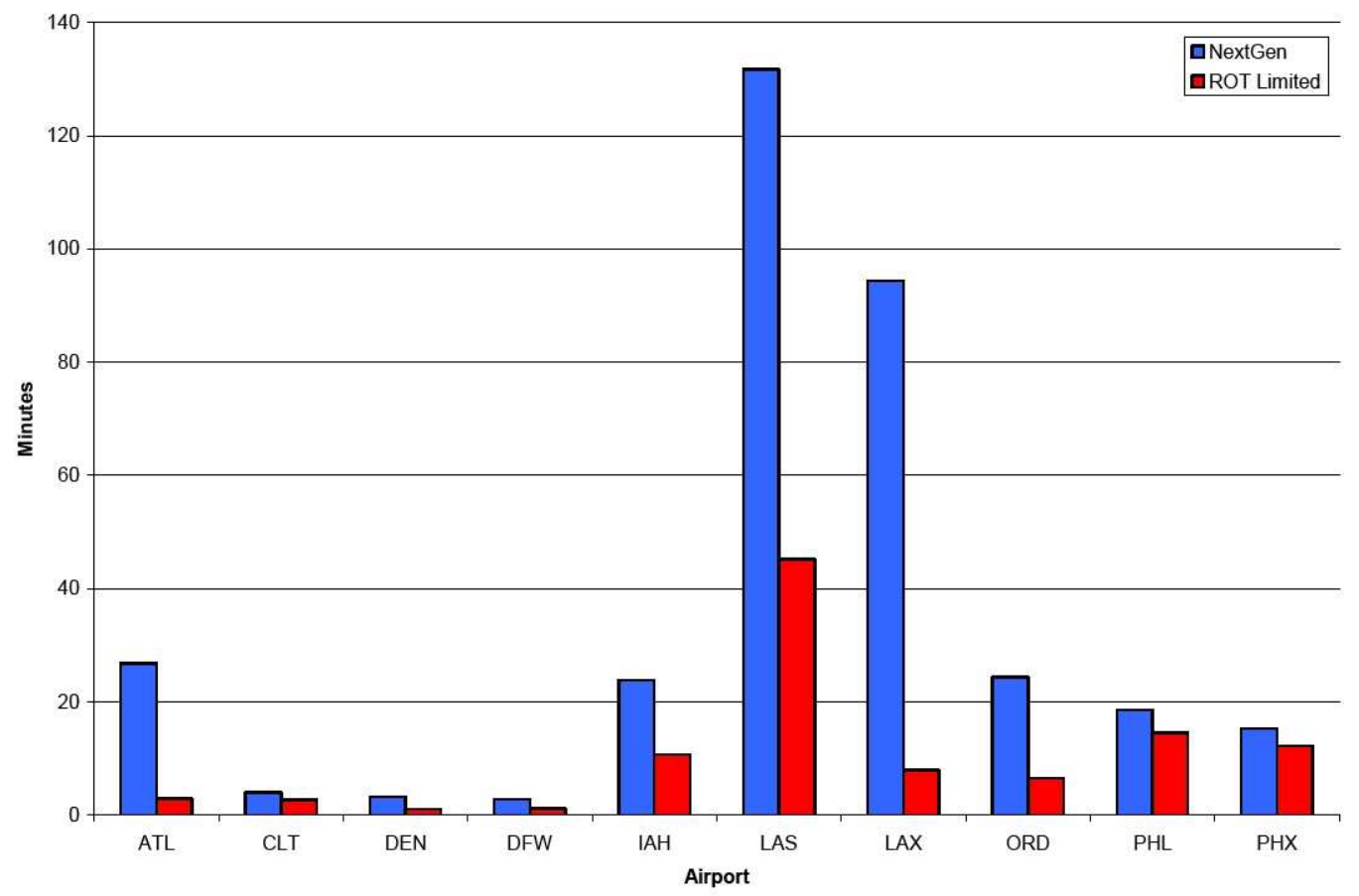

Figure 5-17. Average 2015 IFR arrival delay minutes at 10 busiest airports.

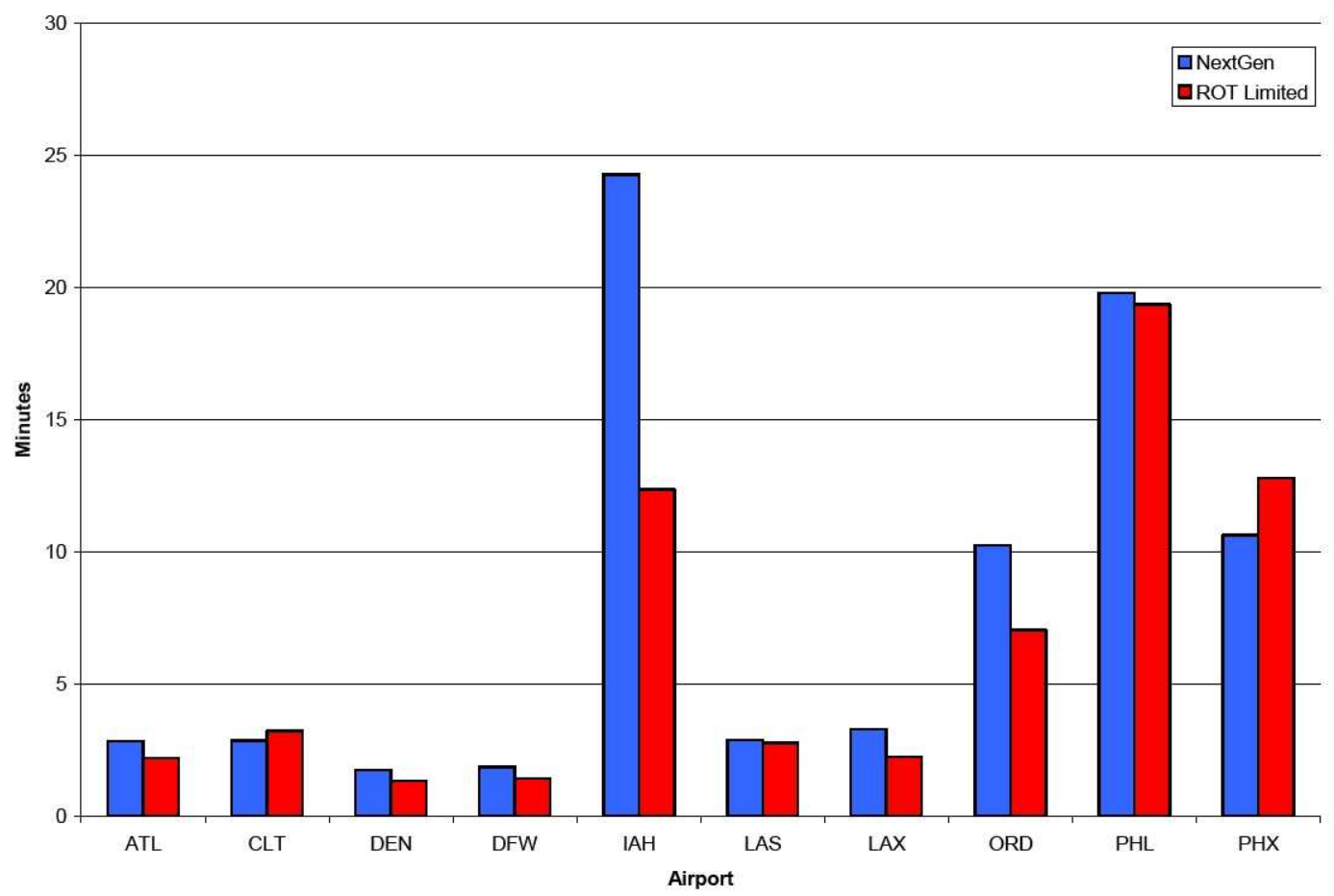

Figure 5-18. Average 2015 IFR departure delay minutes at 10 busiest airports. 


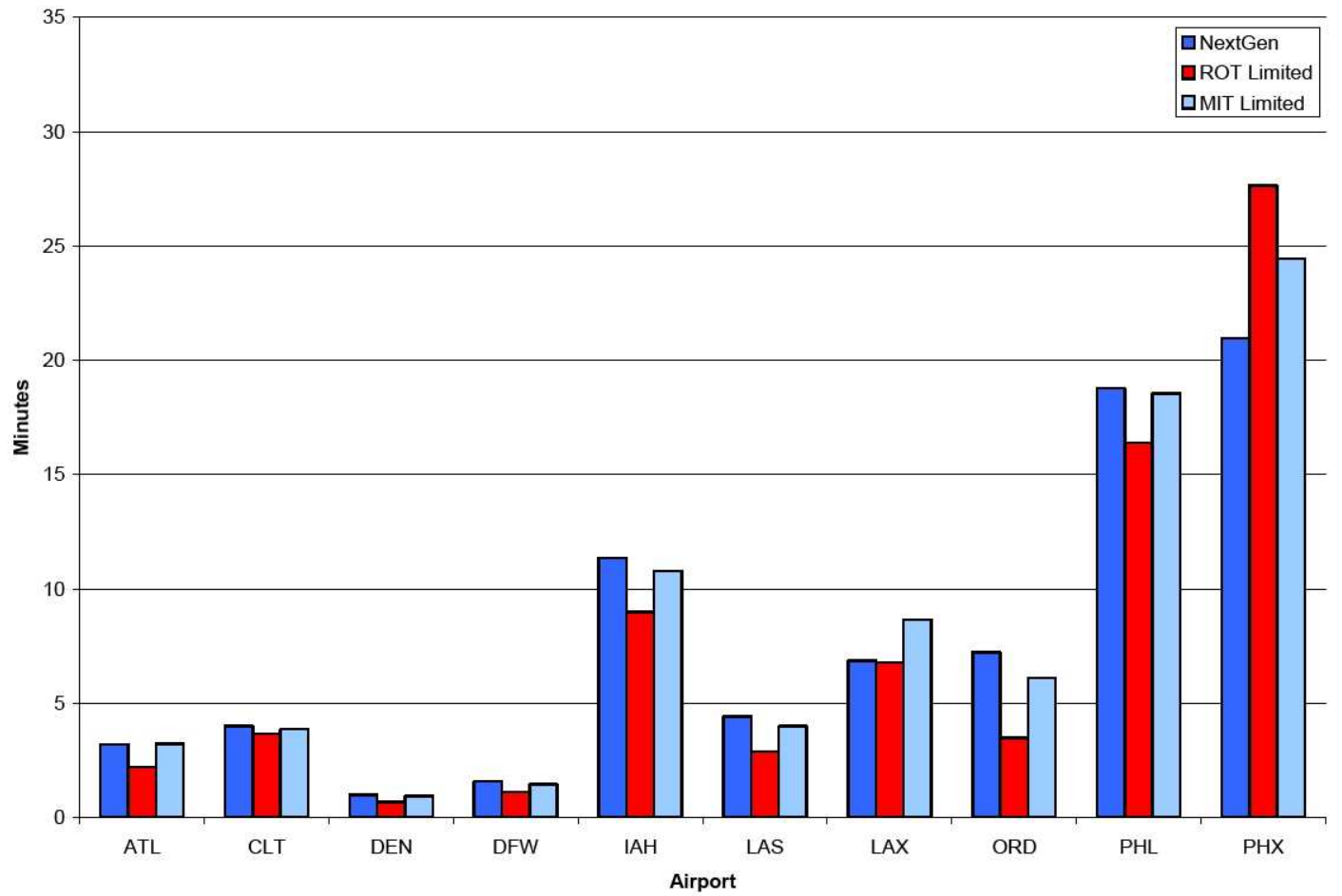

Figure 5-19. Average 2025 IFR arrival delay minutes at 10 busiest airports.

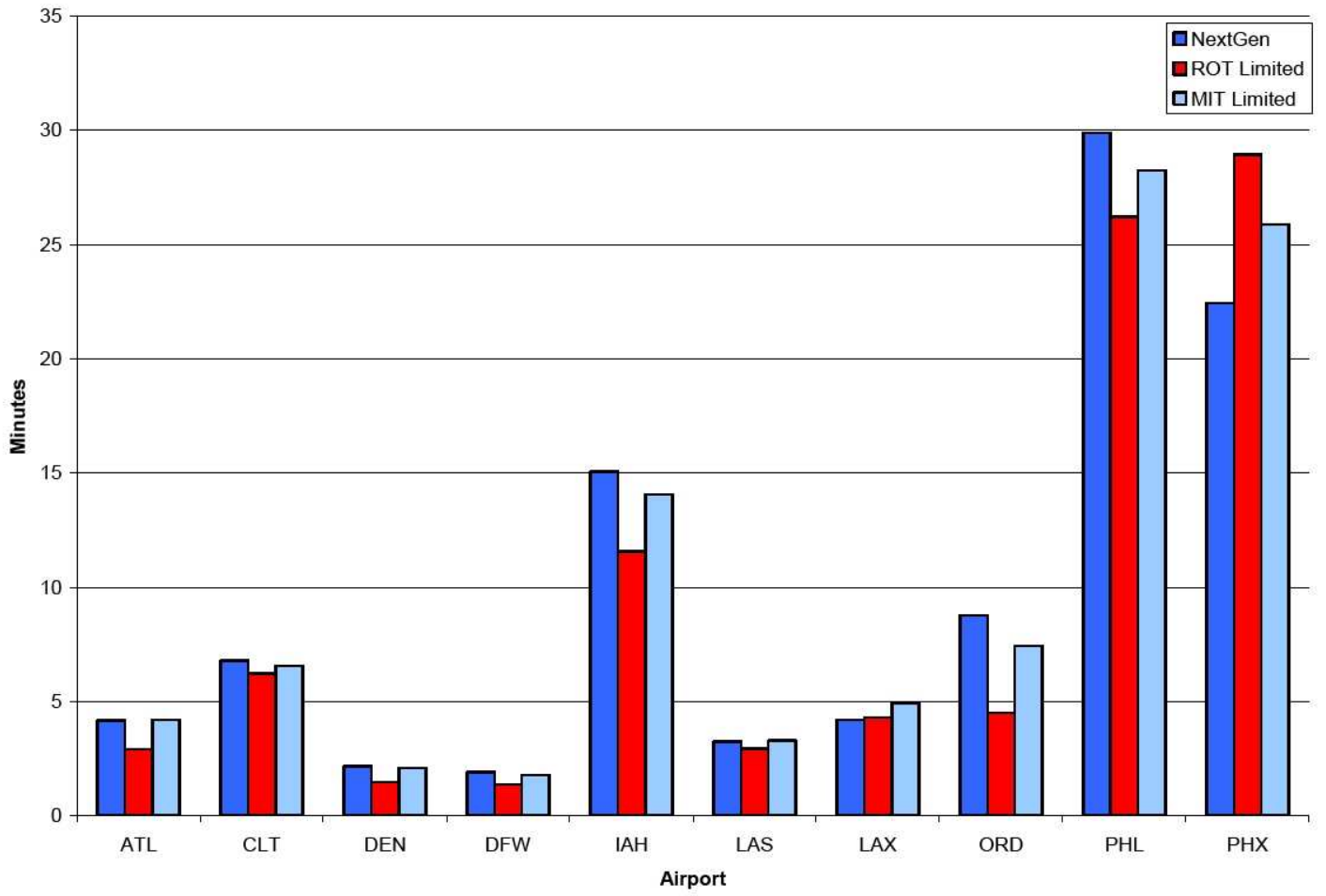

Figure 5-20. Average 2025 IFR departure delay minutes at 10 busiest airports. 


\section{TAXIWAY CONSTRAINTS ANALYSIS}

\section{Overview}

As part of our task of understanding the effect of future growth in air traffic demand on airport operations as a whole, we must study the potential impact of this growth on taxiways. LMI has a well-developed airport operations model, LMINET, which includes consideration of taxi delays. LMINET explicitly models these delays as part of its analysis of total airport delay. These delay calculations are based on taxiway capacities. However, taxiway capacity is not derived empirically from physical characteristics of the airport. Rather, the capacities are developed by calibrating the LMINET model until the model's reported taxi delays match the most recent ASPM taxi delay data. The result is a standard queuing model representation of taxi capacity, with exponential service time. Under this representation, greater arrival and departure demand leads to greater taxi delays. Although this approach is sufficient for modeling the general operations of the NAS, LMI decided to develop a more realistic, empirically based approach for this airport constraints analysis.

Our task was complicated by the existence of a number of interpretations of taxiway delay. The Department of Transportation's ASPM reports taxiway delay as the extra time beyond the "nominal time," which is defined as the 10th percentile of all taxi times at the airport. Therefore, by ASPM definition, reportable taxiway delay is subject to change if there is a change in experienced taxi times. This could lead to a misstatement of taxi delays. In addition, the ASPM approach does not take into consideration the physical distance the aircraft has to travel from the runway to the gate. A short taxi route should take very little time; however, if an aircraft traveling this route is delayed, but not beyond the 10th percentile of all taxi times, the delay is not reported under the ASPM system.

\section{Taxiway Delay Model}

The LMI team recognized that we needed to develop a new model to properly assess taxiway capacity constraints and that we needed a new way of determining whether a taxiing aircraft was delayed. Our first approach was to build a model using actual taxi time data. To do so, the team turned to Airline Service Quality Performance (ASQP) out-off-on-in (OOOI) data. These data correspond to the time when aircraft leave the gate (out), take off (off), land (on), and arrive at the gate (in). Taxi-out time can be calculated by subtracting the off time from the out time, and taxi-in time can be calculated by subtracting the on time from the in time.

However, taxi-out time is confounded by a number of other factors, including weather and downstream delays stemming from congested runways and airspace. By contrast, taxi-in time is less exposed to outside factors; the only factor external to the taxiway system that has the potential to skew reported taxi time is gate 
unavailability. Because delays due to gate unavailability are relatively rare, their effect can be minimized by averaging across large numbers of flights.

Baseline taxi-in times can be gathered by collecting data on flights occurring under nominal conditions and at off-peak times. To estimate best-case taxi-in times, the LMI team collected data for nighttime operations taking place between March and October 2007. This eliminated most congestion- and weather-related delays (which occur in November through February).

Most airports have taxiways that are constructed in parallel - that is, taxi-in capacity is equivalent to taxi-out capacity. For this model, we assumed that each airport's inbound and outbound taxiway capacities are equal. As a result, the baseline non-delay taxi-in times can be applied to taxi-out times as well.

Under our new taxiway model, taxi-in time, $X$, is treated as a random variable composed of two parts: the nominal time, $X n$, from the runway exit to the gate, and the delay, $X c$, due to demand congestion. Both values are non-negative:

$$
X=X n+X c .
$$

Nominal time is determined by route or by the assignment of the gate, and it is independent of congestion delay. For the model, the expectation of the nominal time, $E(X n)$, is assumed to be independent of demand and, therefore, is considered a constant. The expectation of the delay due to congestion, $E(X c)$, is assumed to be a non-decreasing function of the demand level.

\section{Taxiway Demand}

Under these assumptions, only the aircraft on the taxiways during the time the aircraft in question is moving from the runway exit to the gate can cause its delay. $X c$ is therefore driven entirely by these aircraft, which in our model, represent taxiway demand.

The LMI team spent a considerable amount of effort to develop a generic definition for taxiway demand to apply to the model system-wide. We considered a number of definitions. Because the national average taxi-in time is under 15 minutes, the definitions employed generally restrict the taxiway demand period to 15 minutes. Using one definition, the taxiway demand experienced by an aircraft was defined as the number of aircraft arriving in the 15 minutes prior to the aircraft in question. Under another definition, taxiway demand was defined as those aircraft arriving within 7.5 minutes before and within 7.5 minutes after the arrival of the aircraft in question. A third definition considered aircraft that arrived within 15 minutes prior to and aircraft that departed within 15 minutes after the arrival of the relevant aircraft. Finally, the team considered a triangular function centered on the arrival of the aircraft. 
The team found that the model was not very sensitive to the definition of the time period used. That is, considering taxiway demand in the 15 minutes prior to the arrival of the aircraft, versus in the 7.5 minutes before and 7.5 minutes after, produced very little difference in the quality of the model. Therefore, because it is computationally much simpler, the team decided to define the relevant taxiway demand epoch as a simple 15-minute block. We also discovered that the total number of arrival and departure operations, not simply arrivals, is the best predictor of congestion delay. This is likely because both arriving and departing aircraft share the same taxiways, ramps, and aprons.

\section{Taxiway Delay and Its Relationship to Demand}

After developing this model, the team turned to real-world data to check our assumptions. Figure 5-21 shows the reported taxi-in times at LGA during October 2007. As can be seen in the graph, there is large variation in taxi-in time for any given demand level. This is particularly true when demand levels are high.

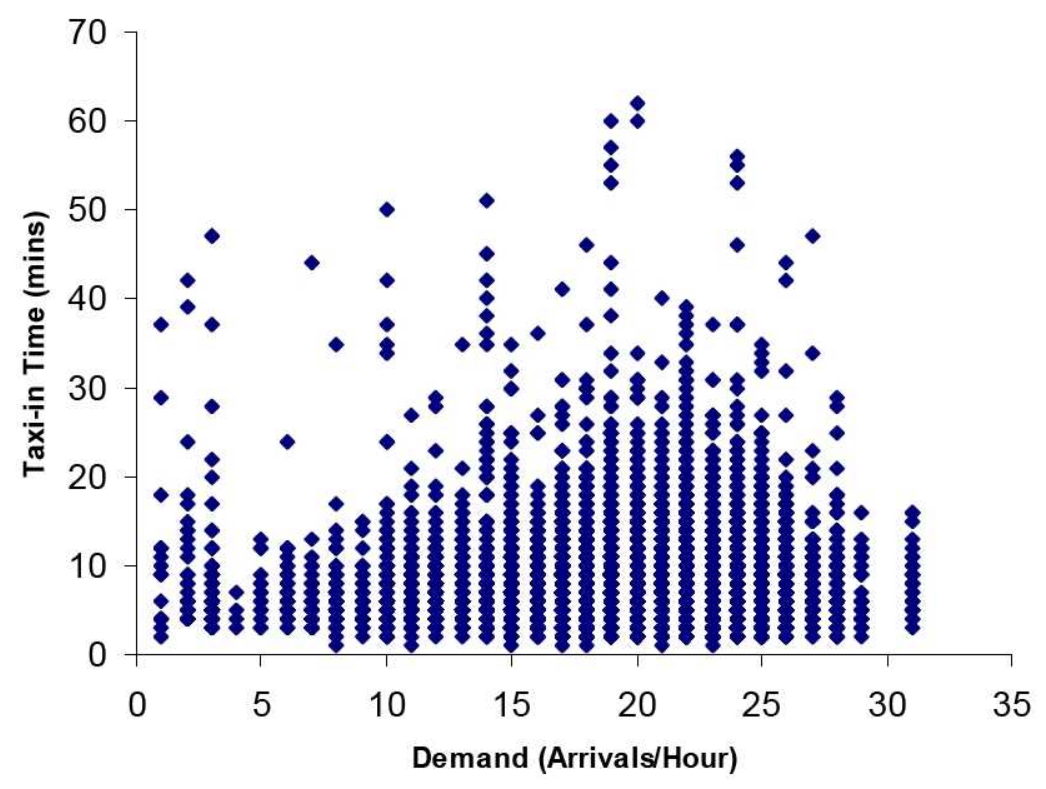

Figure 5-21. LGA taxi-in times as a function of demand.

Figure 5-22 shows the average taxi-in times at LGA and emphasizes taxi-in time's relative independence from arrival and departure demands. Like the majority of airports analyzed, LGA's average taxi-in time curve remains relatively flat, increasing only slightly with demand. The spikes at the end of the curve are due to the fact that demand at this level is infrequent and the average is based on only a few points. We can therefore conclude that for LGA and many other airports, demand is not necessarily the primary driver of excess taxi-in time. 


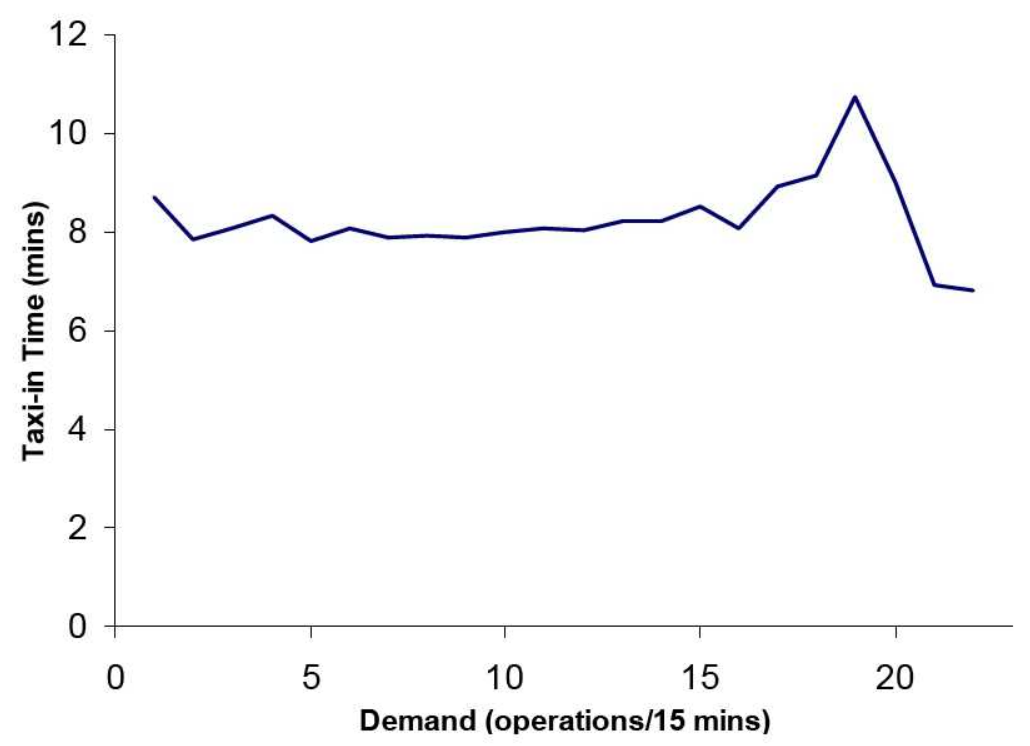

Figure 5-22. Average LGA taxi-in times as a function of demand.

\section{Surface Interaction and Taxi Delays}

One possible explanation for these findings is that taxi delay is not driven by high demand for taxiways alone, but by the number of interactions between aircraft on these taxiways as well. To help explain this hypothesis further, the team developed a conceptual model of aircraft arrival operations.

Generally, a single runway has several exit points to the taxiways. From the runway, there are usually multiple potential pathways for the aircraft to follow to reach the gate. If we assume that all aircraft exiting the runway maintain a consistent taxi speed, there will be no congestion on the taxiway provided the taxiway does not intersect other taxiways or runways. Because aircraft arrive sequentially on a particular runway and then exit using one of several taxiways, taxiway capacity will always be greater than runway capacity. Under these assumptions, as demand increases, the runway will always become a constraint before the taxiways do.

However, we know from common sense and experience that taxiways do occasionally become constraints on airport operations. This is because, unlike the idealized world described above, taxiways often intersect one another or cross active runways.

Rather than being driven by demand alone, taxi delays are generally driven by a combination of demand and the degree to which the assigned taxiways, ramps, and runways intersect one another. This phenomenon is represented well by the real-world examples of ORD and LGA. ORD has a complex airport layout that requires aircraft to interact with one another on numerous occasions as they make 
their way from the runways to the gates and from the gates to the runways. LGA's layout is relatively straightforward, lending itself well to streamlined, simple ground operations. These layouts are shown in Figures 5-23 and 5-24.

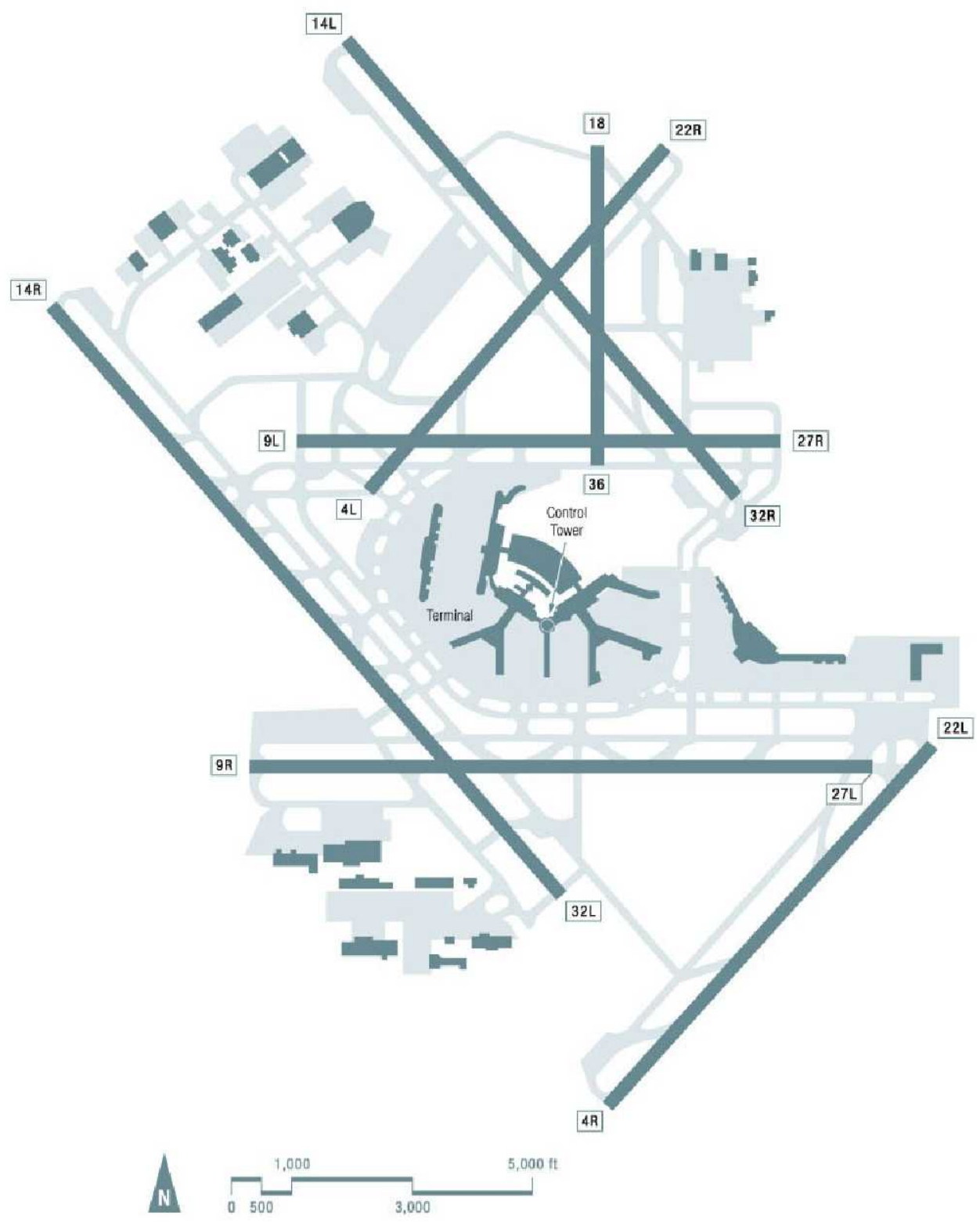

Figure 5-23. ORD runway layout. 


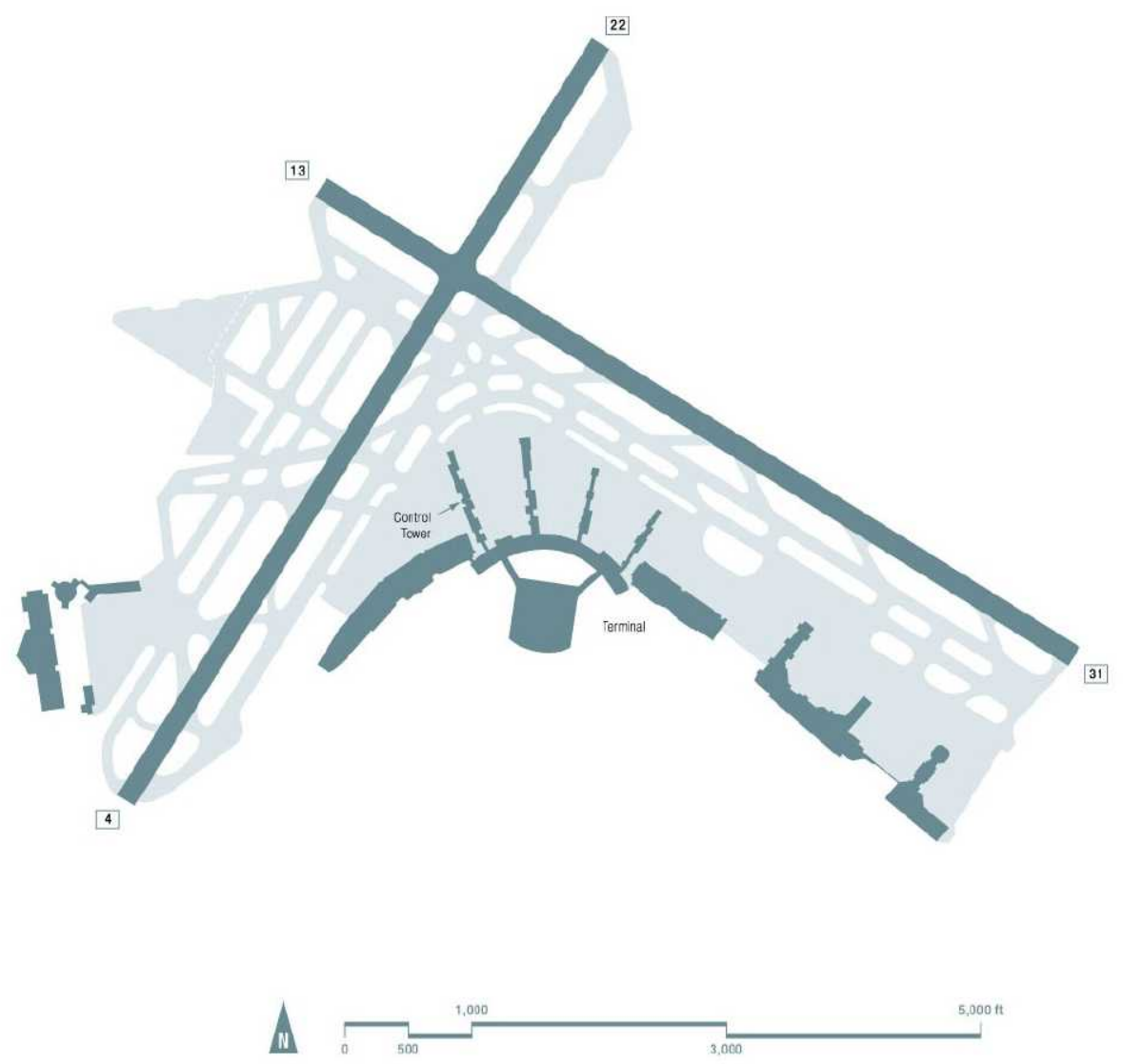

Figure 5-24. LGA runway layout.

The impact of these layout differences can be seen by comparing Figures 5-22 and 5-25. As arrivals and departures increase at LGA, little difference is observed in the taxi-in times. However, under similar increases at ORD, average taxi-in times experience a significant increase.

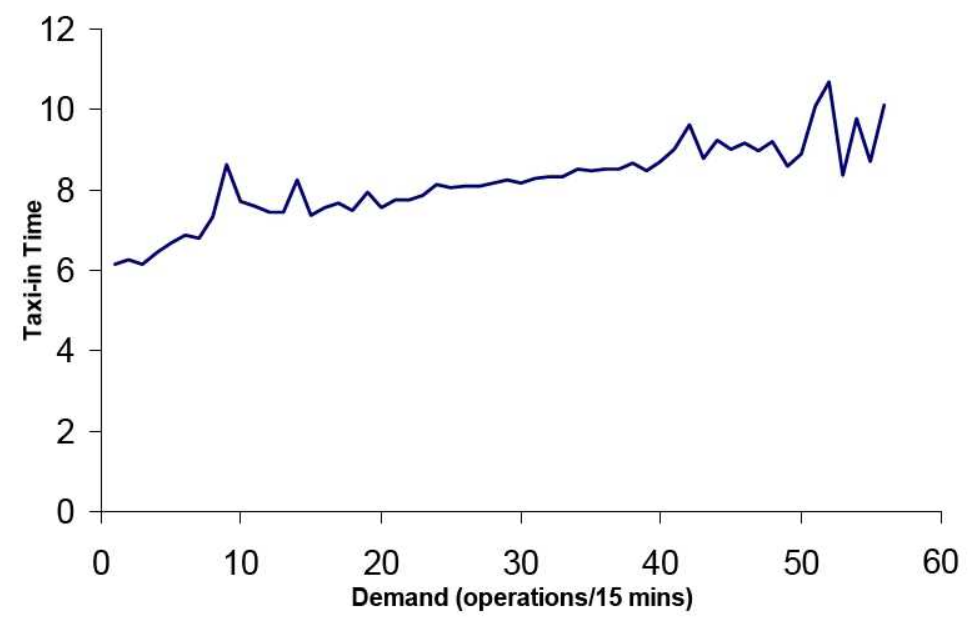

Figure 5-25. Average ORD taxi-in time as a function of demand. 


\section{Simulation-Based Taxiway Model}

Despite the progress described above, LMI discovered that the taxiway problem does not lend itself well to queuing theory or statistical analysis. This is primarily because observations made at today's demand levels do not provide highconfidence estimates for what taxi delay will look like at future demand levels, particularly if future demand is significantly higher than present levels.

It is not valid to extend the trends described above beyond demand levels seen here; we needed to find an alternative method for estimating future taxi delays. One alternative for doing so is to explicitly simulate taxiway traffic flows on the airport surface. This requires the development of individualized models for each airport that accurately represent taxiways and typical traffic flow patterns - an enormously labor-intensive process. Nevertheless, it is the only way to develop estimates of taxi delays as a function of forecasted demand levels.

This approach has a number of challenges beyond the time and effort required to create accurate models for each airport. First, pathway data - data on which particular taxiways each aircraft took on its way from the terminal to the runway or from the runway to the terminal - are limited. This means that we would need to refer to airport handbooks or infer from airport diagrams the routes that aircraft typically take. Second, each airport generally operates under one of several possible runway configurations depending on weather and other factors. This means that taxi procedures could vary substantially under different conditions, even within the same airport. Finally, the taxiway and runway systems of many airports are extremely complex; the number of possible pathways and potential interaction points along these pathways can be overwhelming.

In light of these challenges, rather than creating surface models for all 310 airports, many of which are trivially small, we began by identifying 4 of the 35 OEP airports to serve as a test bed for our approach. Ultimately, as we became more comfortable with the modeling process, we created models for an additional 16 of the 35 OEP airports.

To identify the most common runway configuration for each airport, we turned to the FAA's 2004 Airport Capacity Benchmark Report. ${ }^{7}$ For simplicity, we chose to model only each airport's most frequently used configuration. In addition, using airport layout diagrams, we divided each airport's surface into two separate regions: the terminal or apron area, and the outer taxiway system (taxiways used to go from the terminal area to the runway). Figure 5-26 shows the LGA layout, which is divided in this manner.

\footnotetext{
${ }^{7}$ Federal Aviation Administration, Airport Capacity Benchmark Report 2004, October 2004.
} 


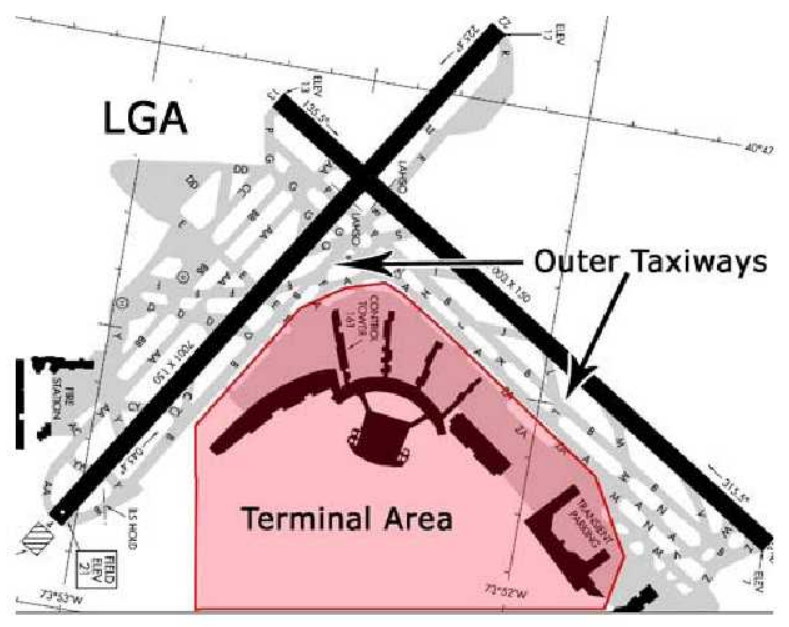

Figure 5-26. LGA airport layout.

This division breaks the problem down into two subproblems. The first focuses on congestion and delay in the terminal area. The second focuses on delays caused by aircraft interaction on the outer taxiways, usually at active-runway crossings.

Terminal area delays are the product of a complex system of interacting components: gate capacity, placement of terminal buildings, movement of service vehicles, and other aircraft all contribute to terminal area congestion. Airports plagued by these delays can be identified by a number of characteristics, such as narrow alleyways or gate areas directly adjacent to major taxiways (where aircraft pushback can interfere with taxiway operation). The vast majority of the airports considered in this study are at very low risk for major delays from surface operations in this terminal area. In fact, only a subset of the top 110 airports have enough traffic or are laid out in such a way that terminal area congestion is likely to have an impact on taxi delay. For this study, we focused our efforts on gauging the effects of congestion on the outer taxiways on overall airport capacity.

\section{OVERVIEW OF THE MODEL}

LMI modeled the outer taxiway system using Rockwell's Arena simulation software. Arena represents aircraft as entities passing through a number of processes. Each process represents a component of landing and taxi-in or taxi-out and takeoff. During each of these processes, aircraft seize resources, which model the various elements of the airport surface, including individual runways, taxiways, and intersection points. In keeping with the findings from our earlier statistical analysis, the model focuses on bottlenecks caused by intersections between taxiways and active runways. These bottlenecks occur whenever aircraft must cross an active runway to reach either the terminal area or another runway.

We made a number of assumptions, based on reviews of FAA guidebooks and consultations with former FAA controllers, about how aircraft move through the 
system. These include acceleration and deceleration rates for aircraft on the runways, taxiing speed, the time required to safely cross an active runway (we take into account whether an aircraft starts from a standstill or is already underway), and routing to and from the terminal area. Our assumptions reflect standard operating procedures.

Figure 5-27 shows the model built in Arena for ORD configured such that arrivals land on runways 9L, 9R, and $4 \mathrm{R}$ and departures take off on runways $32 \mathrm{~L}, 32 \mathrm{R}$, $4 \mathrm{~L}$, and 9L. Blocks enclosed in blue represent the arrival process at each of the arrival runways. Blocks enclosed in green represent the departure process at each of the departure runways. Blocks enclosed in yellow address taxiway bottlenecks caused by taxiway-runway interaction. Those blocks not enclosed in colored rectangles control the simulation itself. A brief summary of each of the steps and the various processes modeled in the simulation follows.

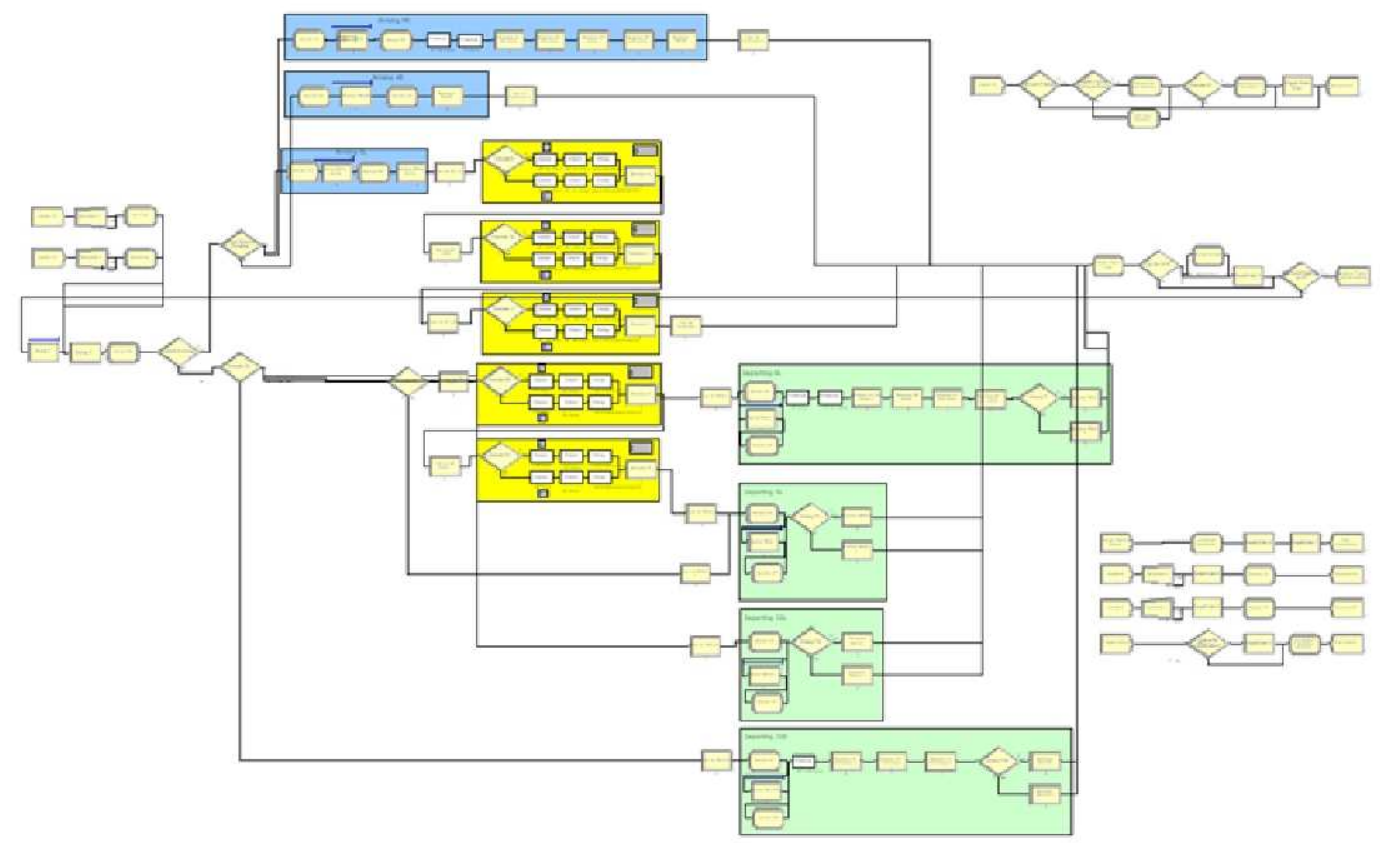

Figure 5-27. Arena model screen shot for ORD.

Initialization

During initialization, the model populates a number of parameters that control the model's execution. In addition, the schedule differentiates between aircraft size (small, large, 757, heavy), which is used to determine the timing of the creation and destruction of the entities representing aircraft in the model. 
During execution, the model runs through the arrival and departure schedules and creates entities to represent the aircraft in the schedule. Arrivals are created when aircraft enter onto the taxiway after landing on the runway, and then travel to the terminal where they exit the model. Departures are created at the terminal, travel along the taxiways to the departure runway, and exit the model when they are ready to take off. Decisions about which runway configurations and taxiways to use were made based on the FAA's 2004 Airport Capacity Benchmark Report. Only the most commonly used configuration was modeled; therefore, there are no configuration changes during simulation.

Routing decisions (which runway to use, which runway crossing to use, which taxiway path to use, etc.) are made so as to balance demand across parallel resources. That is, when there are multiple alternatives, aircraft are routed so that each alternative receives roughly the same amount of traffic. For example, if aircraft are taxiing into the terminal and must cross an active runway, the aircraft are routed to a variety of crossing points along this runway so that no single crossing point is overwhelmed, resulting in unnecessary delay. However, aircraft are routed only to resources that can accommodate them. In the case of runways, for example, a heavy aircraft would not be routed to a short runway simply because that runway is underutilized, but rather would be routed to the first available runway that is long enough for it to land safely.

Once an arrival is created, it is routed to a landing runway. The arriving aircraft seizes the resources it needs for landing, including the right to land on the runway, the right to block any runway crossing points, and the right to block any active crossing runways. Landing and departing aircraft are given a higher priority than aircraft on the taxiways. Therefore, if a taxiing aircraft wants to cross the runway, the landing aircraft can block it from doing so. However, fellow landing and departing aircraft have equivalent priority levels, so one landing or departing aircraft cannot seize a resource in use by another landing or departing aircraft.

The aircraft lands and decelerates on the runway. After the aircraft passes runway crossing points, these crossing points reopen to taxiing aircraft. Similarly, as the aircraft passes runway intersections, the intersecting runway is released so that other aircraft may use it. Once the aircraft leaves the runway, it releases any remaining resources still in its control.

The aircraft then enters the taxiway system and moves toward the terminal. As described above, the aircraft is routed among alternatives based on demand levels at each of the alternatives. When the aircraft must cross an active runway, it is sent to the crossing point with the shortest queue, with preference given to those crossing points that are most convenient for the aircraft. Aircraft can cross an active runway whenever there is sufficient time between arrivals or departures. The model differentiates between the amount of time required for a stopped aircraft to accelerate to taxi speed and cross the runway, and the time required for 
an already taxiing aircraft to cross the runway. Aircraft are allowed to cross runways after the arriving or departing aircraft has moved past the crossing point, provided another aircraft is not on final approach or about to take off.

These crossing points represent the core driver of taxi delay, and the time spent by each aircraft waiting for sufficient taxiway resources is tracked throughout the model. Time spent by arriving or departing aircraft waiting for runway availability is separated from this figure, so as to capture taxiway delay only. The arrival entities exit the model when the aircraft arrives at the terminal.

The departure process works in much the same way as the arrival process. An entity representing each departing aircraft is created according to the schedule. The aircraft is assigned a feasible departure runway according to demand levels, and proceeds to taxi out. As before, the aircraft is routed along the taxiways and across active runways according to the queue lengths at each resource, while attempting to keep the aircraft on the shortest path. Again, departing aircraft on the taxiways are assigned a lower priority level than those aircraft using the runways to take off or land and, as a result, must wait at runway crossing points until there is sufficient time to cross the runway between runway operations.

Once the departing aircraft arrives at its assigned runway, it attempts to seize the necessary resources: the right to take off the runway, the right to block taxiing aircraft from crossing the runway, and the right to block other aircraft from using intersecting runways. If the runway is in use, the aircraft must wait for that aircraft to depart (and allow for separation standards) before it can take off. The aircraft then accelerates down the runway. After it passes runway crossing points and runway intersection points, those resources once again become available for use. The aircraft exits the model upon becoming airborne.

Termination

Termination occurs once the last flight in the 24-hour schedule exits the model. The model then analyzes total taxi delay for each flight. If the model encounters a flight whose total taxi delay exceeds 15 minutes, this flight is trimmed from the schedule, and the model is run again with the trimmed schedule. This process repeats until no flights exceed the maximum taxi delay of 15 minutes.

\section{SUMMARY OF RESULTS}

The team built models for 20 of the OEP 35 airports: ATL, BOS, CLE, CLT, CVG, DCA, DFW, EWR, HNL, LAS, LAX, LGA, MCO, MDW, ORD, PHX, SAN, SEA, SLC, and STL.

Overall, trimming rates at these airports tended to be relatively low, with a number of airports experiencing no trimming whatsoever. BOS, CVG, DFW, HNL, LAS, LGA, MCO, MDW, ORD, SAN, SEA, SLC, and STL all had zero or close to zero percent of their flights trimmed in 2015, with only modest increases 
in 2025. DCA's percentage of flights trimmed actually declined, reflecting a subtle distribution of delay across a broader set of flights at that airport. ATL experienced the greatest trimming of flights, with up to 21 percent of all flights trimmed in 2025. Other notably high trimming rates occurred at CLE with 12 percent in 2025, CLT with 11 percent in 2025, and PHX with 9 percent in 2025. It should be noted that both CLE and CLT have relatively low total operations counts.

The substantial gains in worst-case delay reduction that can be made by trimming the worst offender flights can be seen here. By removing those flights in excess of the delay tolerance of 15 minutes, the schedule can be strategically trimmed to reduce demand at peak times. Removing these flights eliminates their contribution to overall delay, but also reduces congestion for the aircraft that remain, further reducing system delay.

For both target years, each airport's average delay was less than 2 minutes when averaged across all aircraft and less than 6 minutes when averaged across those aircraft experiencing delay, with most airports' average delay below 2 minutes per delayed aircraft.

For many airports, large portions of operations experience at least some taxi delay (defined as anything above zero). As many as two-thirds of operations at EWR experience taxi delay. Other airports with high percentages of delayed operations are SEA (51 percent in 2025), LGA (41 percent in 2025), ATL (38 percent in 2015), and LAS (31 percent in 2025).

Table 5-7 is a more detailed look at two airports: ORD and STL. ORD has a large amount of traffic and experiences a small amount of trimming (3.4 percent of scheduled flights). STL has a much smaller amount of traffic and experiences no trimming. Aircraft at ORD experience delays both in arrivals and departures, while only arrivals at STL experience delay. This is a result of the airports' layouts. At STL, departures leave the terminal area and can taxi unimpeded directly to the departure runway, but this is not the case for all ORD departures under the configuration modeled. For both airports, the number of remaining flights, when trimmed flights are subtracted from the total scheduled operations, represents the number of flights that the airport's taxiways can support under typical scheduling.

Table 5-7. Details of Flight Trimming and Delay at ORD and STL in 2025

\begin{tabular}{|l|c|c|c|c|c|c|}
\hline \multirow{2}{*}{ Airport } & \multicolumn{2}{|c|}{ Operations } & \multicolumn{2}{c|}{ Average taxi-in delay (min.) } & \multicolumn{2}{c|}{ Average taxi-out delay (min.) } \\
\cline { 2 - 7 } & Total & Trimmed & $\begin{array}{c}\text { Before } \\
\text { trimming }\end{array}$ & $\begin{array}{c}\text { After } \\
\text { trimming }\end{array}$ & $\begin{array}{c}\text { Before } \\
\text { trimming }\end{array}$ & $\begin{array}{c}\text { After } \\
\text { trimming }\end{array}$ \\
\hline ORD & 4,031 & 139 & 3.13 & 2.98 & 3.46 & 2.76 \\
\hline STL & 1,052 & 0 & 3.32 & 3.32 & 0 & 0 \\
\hline
\end{tabular}




\section{Demand-Based Airport Elimination}

A large number of airports considered in this study simply do not have sufficient demand to cause significant taxi delay problems. Aviation industry experts and lay travelers alike understand this; however, given the resource-intensive nature of modeling a single airport, we needed a simple way to prove that taxiway capacity is not an issue at a large number of airports. To do so, we developed a technique for quickly determining whether an airport requires further study or can safely be eliminated from consideration based on its demand levels. We created a hypothetical worst-case scenario airport designed to maximize the opportunity for taxi delay. This airport consists of a single runway and a single taxiway that crosses this runway at only one point. The taxiway is assumed to be wide enough for bi-directional traffic except at the runway crossing point, where it can accommodate only a single aircraft at a time. Both arrivals and departures must cross the runway on their way to and from the airport. Figure 5-28 depicts this theoretical airport.

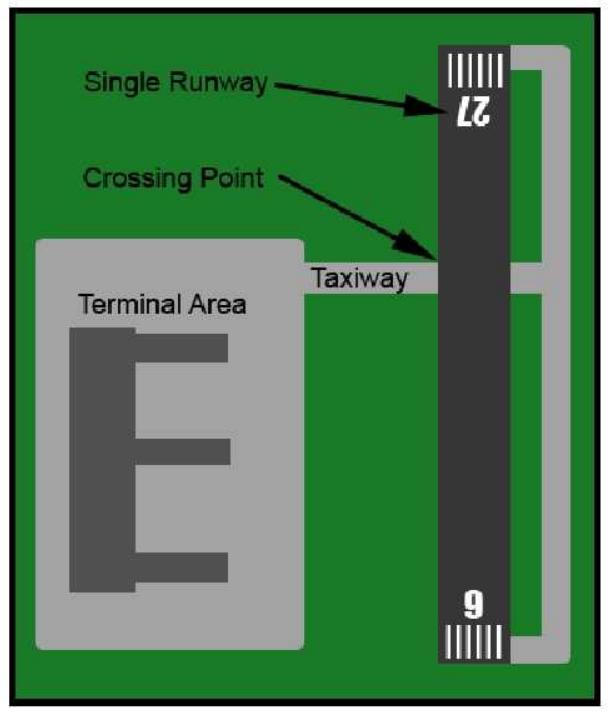

Figure 5-28. Hypothetical worst-case airport design.

We then analyzed the demand at each of the 310 airports considered in the study and grouped the arrivals and departures by 15-minute epoch. Regardless of each airport's actual layout, we projected its epoch-by-epoch demand onto the hypothetical airport, with all traffic using the single runway and taxiway system.

Assuming an average ROT of 45 seconds and an average runway crossing time of 30 seconds, and assuming that the runway crossing remains closed when an aircraft is taking off or landing on the runway, each operation would require the exclusive use of the runway and runway crossing for a total of $45+30=75$ seconds, or 1.25 minutes. 
Based on these assumptions, in a given 15-minute epoch, there is time for a maximum of 15/1.25 = 12 operations before some minor delay occurs. Because most airports' demand schedules exhibit a peak-and-trough pattern of demand, it is likely that at most airports this delay would be resolved in subsequent lowdemand epochs. Taken together with the poor design of the hypothetical airport, these assumptions provide a safe estimate of an airport's proclivity for delay.

The team flagged those airports with more than five epochs in 24-hour period (96 epochs total) with operations exceeding the limit of 12 based on any year's schedule. These flagged airports require further examination. Those that remained were determined to have demand levels too low to exceed taxiway capacity. Using this method, the team determined that 213 of the 310 airports did not merit further study.

\section{Configuration-Based Airport Elimination}

During our simulation analysis, we occasionally considered an OEP 35 airport whose most common configuration was such that there were no taxiway bottlenecks. Because taxi delay is driven by these bottlenecks, building a complex simulation of such an airport would simply confirm that taxi delay was not a major problem facing the airport. We decided to skip these airports and spend our time simulating airports that clearly faced serious taxi delay problems.

An example best illustrates this concept. JFK is the 10th busiest airport in the United States and has a complex layout, as can be seen in Figure 5-29. According to the 2004 Benchmark Capacity Report, the most common configuration at JFK is mixed arrivals and departures on both runway $31 \mathrm{~L}$ and runway $31 \mathrm{R}$. At JFK, the terminal is directly between these runways. Therefore, traffic using these runways will never need to cross an active runway on its way to or from the terminal.

Further analysis using 2007 ASPM configuration data revealed that, 64 percent of the time, JFK uses configurations that do not create bottlenecks in the taxiway system. It may seem difficult to believe that such a busy airport would not have serious taxiway capacity problems, especially given anecdotal evidence to the contrary. However, it is important to keep in mind that while an aircraft may wait on the taxiway, the cause of the delay is often capacity problems at downstream elements like runways or gates. No amount of taxiway enhancements can resolve a runway or gate capacity issue. 


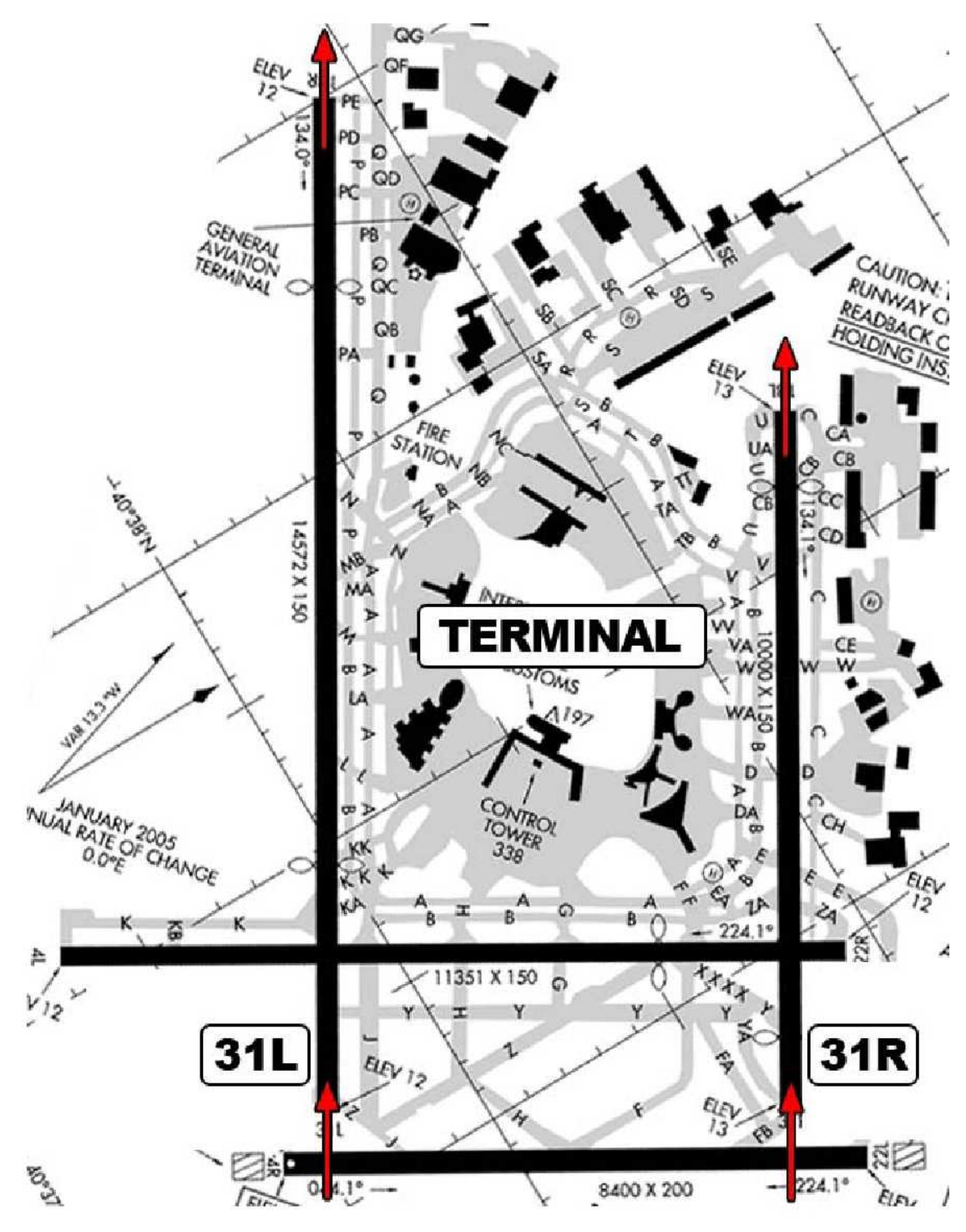

Figure 5-29. JFK under the most common runway configuration.

Using a combination of the ASPM configuration data and the FAA's 2004 Airport Capacity Benchmark Report, we conducted a broad configuration analysis on those airports not modeled in the simulation effort and not eliminated using the demand-based elimination technique described earlier.

The team decided that overall airport capacity was unlikely to be hindered by taxiway capacity if the airport operated in configurations not prone to congestion at least 60 percent of the time. We believe this proportion is sufficiently conservative because a configuration is considered congestion-prone even if demand levels are very low. Airports tend to operate so that congestion is minimized when demand is high and taxiway convenience is maximized when demand is low. Therefore, during periods of low demand, an airport may be configured in a way that would cause taxiway congestion under high demand, but 
allows aircraft to land closer to the terminal or to follow a more direct path to the gate. Because, during high demand periods, airports will choose configurations that do not lead to taxiway bottlenecks whenever possible, we can assume that a significant proportion of the time in which an airport is not configured to reduce congestion takes place during periods of low demand.

We were able to perform this analysis on only the 77 airports for which ASPM collects data. Nevertheless, we were able to determine that taxiway capacity was unlikely to be a binding constraint for an additional 43 airports, including all of the OEP 35 airports not modeled with simulation. Most of these airports were rarely in congestion-prone configurations: 25 airports were in bottleneck-free configurations at least 90 percent of the time and another 13 were in such configurations 70 percent to 90 percent of the time; only 6 airports were configured in this manner between 60 percent and 70 percent of the time.

\section{Remaining Airports}

LMI built simulation models for 20 of the OEP 35 airports. The team eliminated an additional 247 airports, including the remainder of the OEP 35, based on an analysis of their demand levels or their most commonly used configurations. Figure 5-30 shows this breakdown.

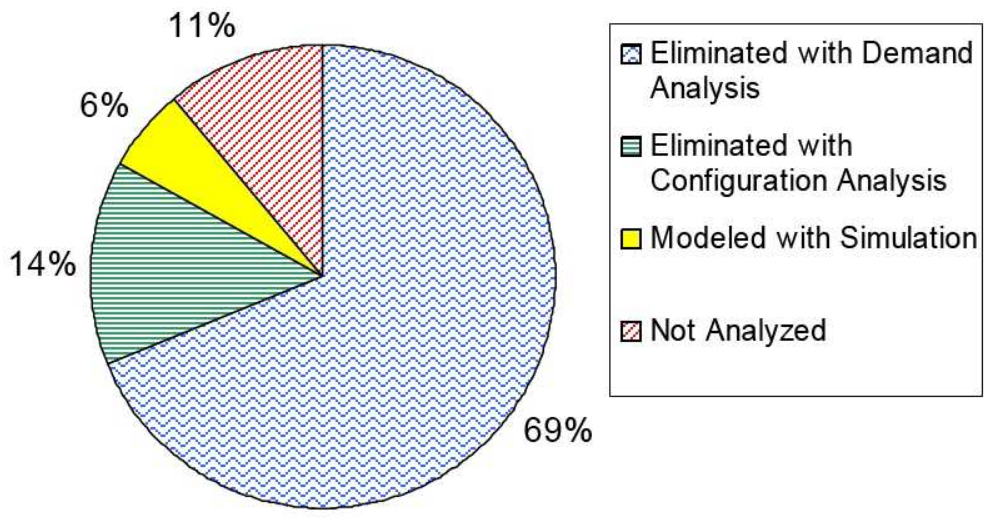

Figure 5-30. Breakdown of taxiway analysis.

Of the original 310 airports, 34 were not modeled using simulation (because resource-intensive simulation was impractical for airports of relatively low importance) and could not be eliminated using either the demand- or configuration-based approaches. ${ }^{8}$ This gap represents 11 percent of the total. These airports tend to be small, with modest levels of commercial traffic. The

\footnotetext{
${ }^{8}$ Those airports are ACK, ALB, ANC, APA, BDL, BED, BHM, BOI, BUR, DAB, DVT, FAI, FFZ, FRG, FTY, FXE, GSO, HIO, MMU, OAK, OGG, OMA, PAE, PBI, PDK, PHF, PWK, RIC, RNO, SEE, STP, TEB, TMB, and VGT.
} 
majority are ranked between 55th and 110th out of the 310 airports, in terms of traffic volume. Only four are ranked under 50: ACK, ANC, OAK, and TEB. For the purposes of summary results, LMI assumed that these airports will not be constrained by their taxiways.

\section{Results}

Figure 5-31 shows the total operations throughput for the 10 busiest (Apt10), OEP 35, LMI 110, and LMI 310 airports.

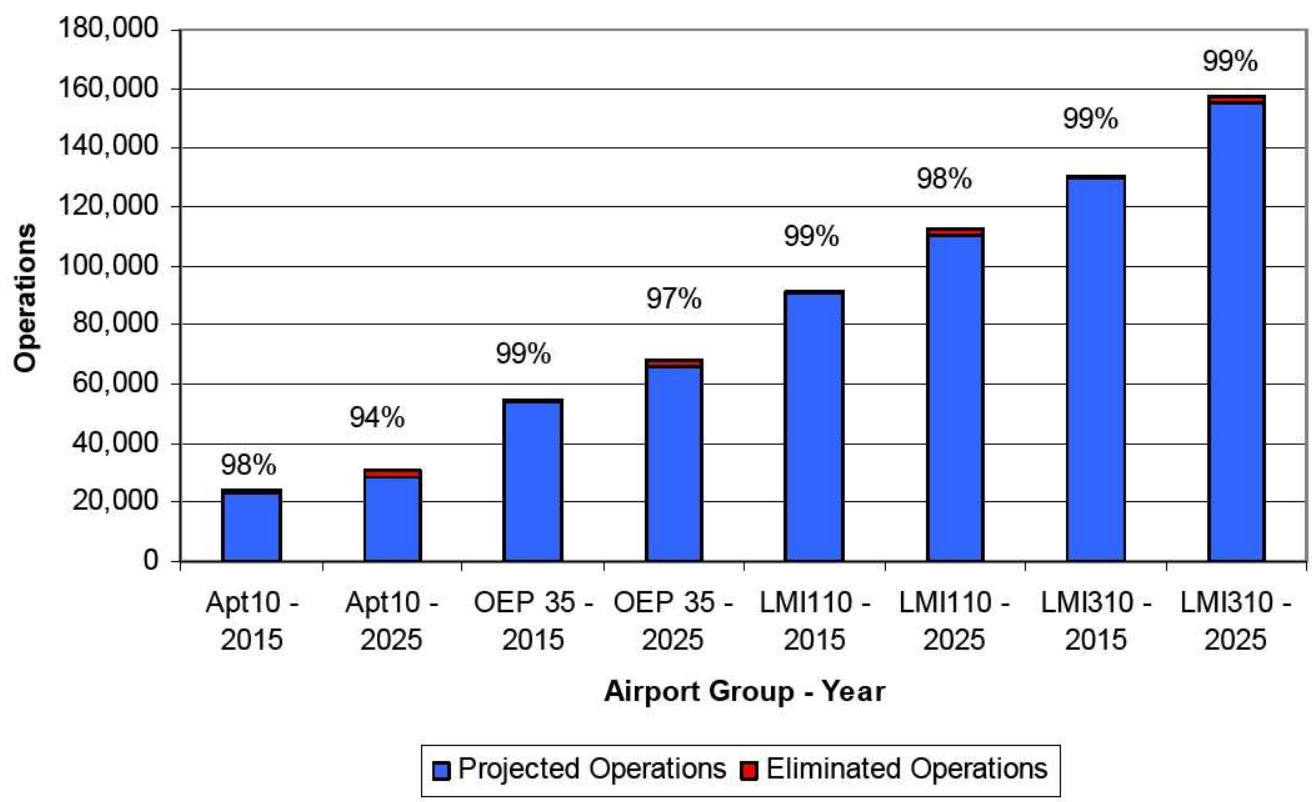

Figure 5-31. Taxiway throughput by airport group and year.

Based on our analysis, the only airports with taxiway capacity constraints are in the OEP 35 airport set. As a result, the absolute size of the shortfall in capacity stays constant across the groups, while the relative portion of flights that this shortfall represents naturally decreases. In 2015, a total of 731 operations were trimmed. In 2025, a total of 2,353 operations were trimmed. As the figure shows, trimmed operations make up only a small portion of total operations.

Figure 5-32 shows 2015 and 2025 throughput for the top 10 busiest airports based on this analysis. In 2015, the top 10 airports are only slightly affected by taxiway limitations. By 2025, taxiway constraints play a more pronounced role, particularly at ATL; however, most airports still maintain good throughput rates. 


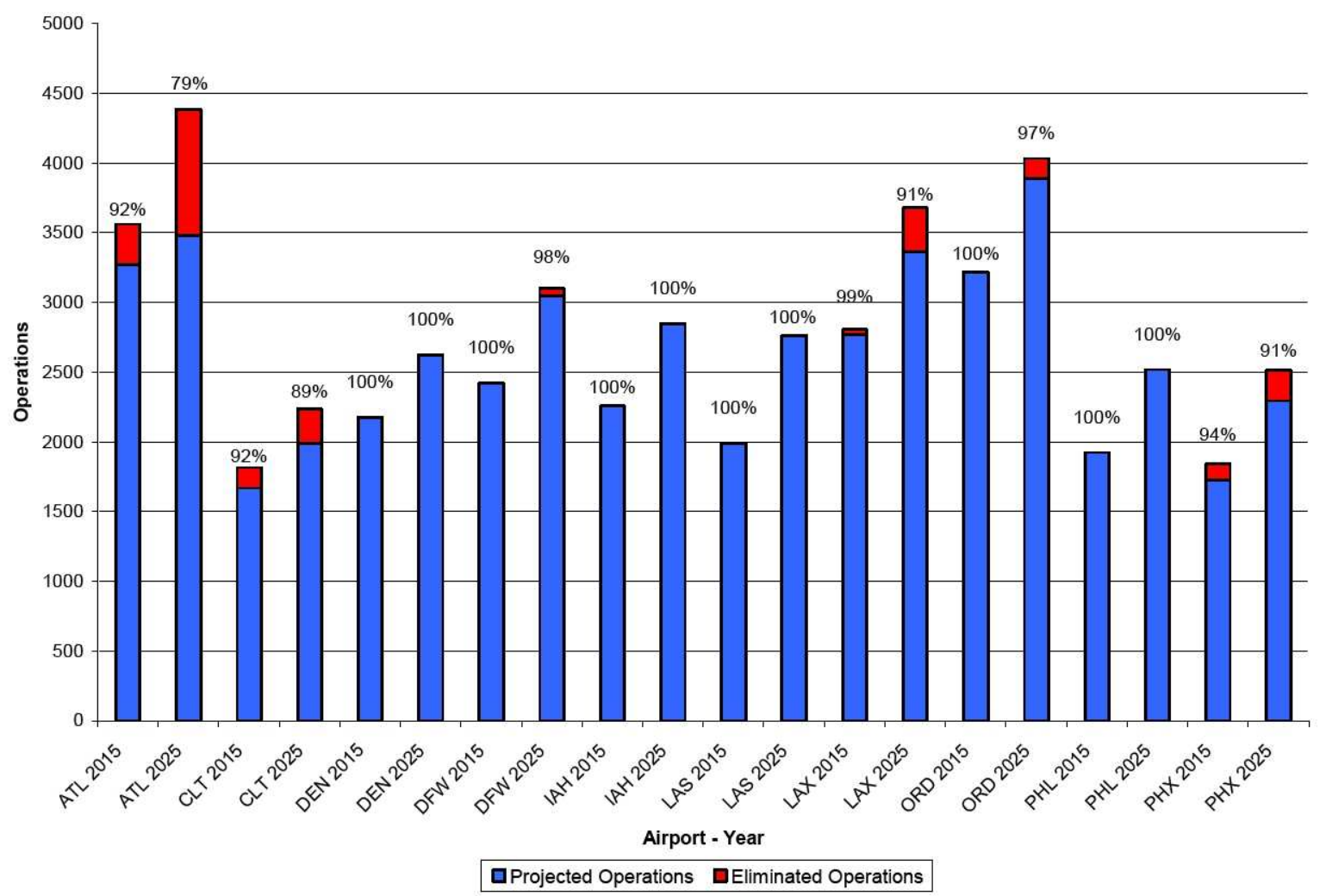

Figure 5-32. 2015 and 2025 unconstrained and taxiway-constrained throughput for 10 busiest airports.

In Figures 5-31 and 5-32, the projected throughputs for the groups of LMI 110 and LMI 310 airports are overly optimistic, or higher than what would be predicted by using more precise models, because we assumed that there are no eliminated operations at the 34 airports for which we have not done a complete analysis. But given the facts that many OEP 35 airports are not taxiway constrained based on our detailed model and vast numbers of airports are not constrained beyond OEP 35 based on most stringent assumptions, we believe that the error is minimal when neglecting the taxi constraints at the identified 34 airports. Table 5-8 shows taxiway throughput, for 2015 and 2025, at 12 constrained airports. 
Table 5-8. Taxiway Throughput at Constrained Airports

\begin{tabular}{|c|c|c|c|c|c|c|}
\hline \multirow{2}{*}{ Airport } & \multicolumn{3}{|c|}{2015 operations } & \multicolumn{3}{c|}{2025 operations } \\
\cline { 2 - 7 } & Unconstrained & Constrained & Reduction & Unconstrained & Constrained & Reduction \\
\hline ATL & 3,561 & 3,269 & $8 \%$ & 4,383 & 3,481 & $21 \%$ \\
\hline BOS & 1,518 & 1,518 & $0 \%$ & 1,750 & 1,743 & $0 \%$ \\
\hline CLE & 952 & 891 & $6 \%$ & 1,194 & 1,047 & $12 \%$ \\
\hline CLT & 1,815 & 1,668 & $8 \%$ & 2,234 & 1,987 & $11 \%$ \\
\hline DCA & 874 & 860 & $2 \%$ & 893 & 886 & $1 \%$ \\
\hline DFW & 2,421 & 2,420 & $0 \%$ & 3,099 & 3,050 & $2 \%$ \\
\hline EWR & 1,671 & 1,643 & $2 \%$ & 2,111 & 1,949 & $8 \%$ \\
\hline LAX & 2,805 & 2,768 & $1 \%$ & 3,678 & 3,362 & $9 \%$ \\
\hline ORD & 3,217 & 3,216 & $0 \%$ & 4,031 & 3,892 & $3 \%$ \\
\hline PHX & 1,840 & 1,727 & $6 \%$ & 2,516 & 2,293 & $9 \%$ \\
\hline SEA & 1,297 & 1,297 & $0 \%$ & 1,594 & 1,578 & $1 \%$ \\
\hline SLC & 1,443 & 1,441 & $0 \%$ & 1,708 & 1,690 & $1 \%$ \\
\hline
\end{tabular}

\section{GATE ConstrainTS ANALYSIS}

\section{Overview}

As the demand for air travel grows, the number of gates available for commercial aviation at each of the critical airports could have a major impact on the capacity of NextGen. To better understand that effect, LMI developed an add-on model that focuses on gate usage. This model identifies which of the critical airports are likely to experience gate shortages under the current 2015 and 2025 growth projections. It also quantifies the degree to which these airports exceed their gate capacities.

Developing such a model requires a thorough understanding both of gate operations and of the available techniques and approaches for modeling these operations. Our approach to the development of this model followed these steps:

- Determine which factors affect airport gate capacity

- Consider alternative approaches to modeling gate usage

- Choose an approach

- Model the current state

> Get the inventory of the number of gates at critical airports 
> Model the current state to calibrate the model and determine baseline

- Model the future state

> Model gate demand for the future

> Determine which airports are likely to experience gate congestion in 2015 and 2025

> Quantify the degree to which problem airports violate gate constraints.

\section{Factors Affecting Gate Capacity}

Determining an airport's gate capacity - its ability to accommodate commercial aircraft at the terminal - is difficult, because an airport's gate capacity is a function of a broad array of factors. Below, we discuss three major elements affecting the calculation of gate capacity.

\section{Number of Gates AVAILABLE}

One crucial element in determining an airport's gate capacity is the total number of gates available at the airport. Unfortunately, determining this number is not as straightforward as it may seem. First, there is the challenge of inconsistent definitions: what is the definition of a gate? Most gates serve just one aircraft at a time. However, sometimes a single gate serves many aircraft by routing passengers to aircraft with buses or by having passengers walk across tarmac to reach their aircraft. Should these multi-aircraft gates be counted as a single gate or as multiple gates? Because LMI got most of its gate information from reports or airport factsheets, how do these sources differ in their definitions of gates?

Second, some gates can service only certain types of aircraft. Some passenger bridges accommodate only very small or very large aircraft, and depending on the airport's layout, the spacing between gates can be prohibitively close for certain aircraft types. This means that while some gates may be available, certain incoming aircraft may not be able to use them.

Finally, most airports lease gate-access rights to airlines. Often these rights are either exclusively for one airline or can be shared through an agreement between airlines. As a result, some gates may be unoccupied, but inaccessible to aircraft belonging to those airlines not party to the leasing agreement. Because these agreements are subject to change over time, it is difficult to accurately include these restrictions in the model.

\section{REQUIRED TURNAROUND TIME}

A second major element of airport gate capacity is the required turnaround time: once a gate is occupied, what minimum time is required before the gate becomes available to another aircraft? Turnaround time is itself affected by a number of 
factors, including aircraft type; the number of passengers; enplaning/deplaning procedures; availability of crewmembers; maintenance, cleaning, and restocking requirements; and the need for the flight to accommodate connecting passengers, as is often the case at hub airports. Many of these factors are highly dependent on airline policies, making them difficult to model, especially in the future state.

\section{ARRIVAL AND DEPARTURE DEMAND}

The third major element in calculating airport gate capacity is the arrival and departure demand for the airport. An airport's ability to accommodate new arrivals at its gates obviously depends on whether the aircraft that arrived previously have since departed. Of particular interest is variation in the arrival and departure demands throughout the day; an airport may have ample excess gate capacity at off-peak times, but may exceed capacity during peak times. These arrival and departure demands are dependent on airline flight schedules, but are likely to reflect passenger demand.

For our model, we have relied exclusively on the traffic schedules provided by FAA for 2007, 2015, and 2025, outlined in Chapter 2.

\section{Approaches to Modeling Gate Usage}

The LMI team considered two approaches to modeling airport gate capacity. The first approach focuses on calculating throughput capacity, while the second approach focuses on the feasibility of arrival and departure demands.

In the first approach, throughput capacity is calculated based on the total number of gates, the aircraft size and fleet mix, turnaround times for these aircraft, and whether the airport is a hub. This calculated throughput capacity is then compared with projected demands. If projected net arrival rates exceed gate throughput capacity, aircraft will experience delays. This approach necessarily relies on a number of assumptions, like projected fleet mix values, turnaround times, and airline policies, in addition to the projected demand values.

The second approach focuses only on arrivals and departures. In this approach, a gate is reserved for each aircraft in the terminal area. A running total of change in this gate demand is calculated, based on current or forecasted schedules, where each arrival is represented by $(+1)$ and each departure is represented by $(-1)$ :

\section{Change in Gate Demand $(G D)=\Sigma$ (Arrivals) $-\Sigma$ (Departures).}

When an airport is operating in equilibrium over a period of time, gate demand will remain steady and around zero. During imbalances in arrivals and departures, gate demand will become strongly positive or negative. Of particular concern for gate capacity is when gate demand becomes strongly positive. 
This approach provides the net change in the number of aircraft at the gates at any time relative to any other point in time. Using this method, it is possible to determine whether an airport's gate capacity can satisfy demand over time given that the following information is available:

- A schedule of arrivals and departures of aircraft requiring gate access (to determine the net change in gate demand)

- The airport's total number of gates (gate capacity)

- A known number of aircraft at the gates at some point in time (a reference point).

An airport's gate capacity is insufficient at time $t$ if the number of aircraft at the reference point plus the net change in gate demand from that reference point to time $t$ exceeds the airport's gate capacity.

If one assumes that arriving aircraft that cannot be provided a gate are turned away, the extent to which the gate capacity constraint is violated can be measured by tallying these denied arrivals.

Both approaches rely on assumptions that could prove invalid by 2025 . This is unavoidable to some extent; however, LMI believes that the first approach depends too heavily on assumptions and airline policies that are subject to change. The second approach's assumptions are limited to the projected demand levels and consistent gate-use policies.

\section{Modeling the Current and Future States}

As stated above, the necessary inputs to the model are a schedule of arrivals and departures, the gate capacity for each airport, and a known number of aircraft at the gates at a point in time. The following subsections describe how each of these components was obtained. We also discuss the details of the software tools designed and used for this analysis.

\section{ARRIVAL AND DEPARTURE INFORMATION}

The model requires the arrival and departure information for a 24-hour period. As mentioned earlier, we used the flight schedules provide by the FAA.

For both the baseline (2007) and the forecasted schedules, the data include only those flights to and/or from the 310 airports considered in this study. We excluded those flights that do not require gate access (general aviation, cargo, military, fractional jets, and prop and turboprop aircraft).

For this analysis, the 24-hour period used corresponds to August 2 in 2007, 2015, and 2025 , as in the schedule files. 


\title{
GATE INVENTORY
}

\author{
Definitions
}

To accurately model airport gate capacity, we first need to know the total number of gates at each airport. As was mentioned earlier, the definition of a gate can vary somewhat from airport to airport and from source to source. Before we could begin tallying the number of gates at each airport, we needed to settle on a working definition.

Airports often ground-load certain aircraft (serve aircraft remotely through a single gate, asking passengers to walk or use buses to reach their aircraft). Relative to the infrastructure costs of adding new passenger-bridge-equipped gate space, adding additional remote parking is relatively easy to do. In fact, many airports already have extra tarmac space that, if necessary, could accommodate additional ground-loaded aircraft. Because constraints on ground-loading aircraft are relatively flexible at most airports, it is more appropriate to focus instead on the constraints imposed by the number of passenger-bridge-equipped gates. Discussions with airport operators and passenger-bridge manufacturers have reinforced this logic. Passenger bridges increase the safety and comfort of passengers, protecting them from the dangers of the tarmac and shielding them from weather. They also speed loading and unloading of aircraft and simplify aircraft service operations performed at the gate. As a result, airlines are using passenger-bridge-equipped gates to serve an increasing proportion of their aircraft, including regional jets, when possible. A number of discussions with airport operators have confirmed that the current trend is toward greater use of passenger bridges.

Given that passenger-bridge-equipped gates pose a more unyielding constraint to gate capacity and that current trends indicate greater future dependence on such gates, we calculated airport gate capacities using only those gates that can accommodate passenger bridges. Using this definition, aircraft that are groundloaded do not consume gate capacity.

Once we had more narrowly defined an airport's gate capacity, we needed to also create some rules regarding which types of aircraft would be ground-loaded and which would be served by passenger-bridge-equipped gates. To reflect the airlines' preference for serving all aircraft at gates with passenger bridges, we assumed that all jet aircraft, including regional jets, would be served by gates with passenger bridges at the LMI 110 airports. To reflect the continued use of groundloading of regional aircraft common at smaller airports, all regional jets would be ground-loaded at airports not included in the LMI 110. All other jet aircraft at these airports would be served by gates with passenger bridges. All non-jet aircraft (props and turbo-props) are automatically assumed to be ground-loaded at all airports. Finally, all noncommercial aircraft (general aviation, cargo, military, etc.) are not considered in the gate analysis. 
For this analysis, the aircraft that qualify as regional jets are Bombardier CRJ200, Bombardier CRJ700, Bombardier CRJ900, Embraer 145, Embraer 170, Embraer 190 , their closely related derivatives, and any other passenger jet aircraft with fewer than 100 seats.

\section{Additional Assumptions}

We made two additional simplifying assumptions:

- All gates can service all aircraft types. This assumption acknowledges that airports tend to build gates to accommodate a range of aircraft. In addition, although we know that gate spacing can affect aircraft-gate compatibility, obtaining airport-specific information about gate spacing and gates is problematic.

- All airlines have equal access to all gates, regardless of current gateaccess leasing agreements. This assumption implies that airports can maximize the collective capacity of their gates, which aligns well with our overall objective of determining each airport's maximum gate capacity.

\section{Gathering the Data}

The team gathered gate information from a variety of sources. The typical information search process started with an Internet search for the airport's website. Many airports list the number of gates on a factsheet or similar information page. When this was available, we used the numbers provided without modification or interpretation. If a factsheet was unavailable, we turned to terminal maps to manually count the gates listed. If maps were unavailable, or if a website for the airport did not exist, we relied on publicly available satellite imagery to count the physical number of gates. If this too was unavailable, LMI tried to contact the airport directly.

As a result of these efforts, the team was able to establish the number of gates with passenger bridges for all 310 airports. For LMI 110 airports, gate information was significantly easier to come by, and the data are very reliable. Less-reliable data sources such as satellite imagery were used at times, particularly for the smallest airports. However, the impact of this on the quality of the data is slight. These airports tend to be very small and are unlikely to have experienced rapid expansion; therefore, possibly outdated satellite photos still fairly accurately depict the airports' passenger-bridge inventories. Furthermore, the overall impact on the model of slight inaccuracies in these airports' gate counts (especially since the majority of their aircraft tend to be regional jets or props not served by passenger bridges) is minimal.

\section{Calculating the Baseline Reference Point}

At least one reference point - a known absolute number of occupied gates at a particular point in time - is required to determine the number of occupied gates at 
any other time and thus to determine whether and to what extent projected demand exceeds the airport's gate capacity. Because no such data exist, providing this reference point is a major challenge.

The team needed to develop a means for estimating the number of aircraft on the ground at a given time. Our first step was to contact a sample of airports' ground operations directors. The team surveyed about 15 airports, contacting large hubs as well as medium and small origin/destination airports. We found that most airports do not track the number of occupied or available gates throughout the day; nevertheless, most airport operations departments were willing to investigate and provide us with the requested data. It would have been too time consuming and costly to continue this process for all 310 airports considered in this study. But the information gathered provided a means for verifying that our estimation method was acceptably accurate.

To estimate the number of aircraft at the gates at a point in time, we turned to the baseline schedule. For each airport, we selected a start time (9:00 a.m. GMT) and then identified all subsequent departures not preceded by a corresponding arrival at least 25 minutes (to account for turnaround time) prior to the departure. Those departures for which there is no corresponding preceding arrival must be aircraft that were on the ground at the start time. If departures took place less than 25 minutes after the start time of 9:00 a.m. GMT, we did not search for a corresponding preceding arrival. Instead, it was automatically assumed that these aircraft were on the ground at the start time.

Because the baseline schedule does not include tail numbers, exactly identifying corresponding arrivals and departures was impossible. The team considered using ASQP data, which do include tail number information, but we found that because of limitations on the reporting airports and airlines, the ASQP data captured only 63 percent of the flights in the baseline schedule. Instead, we used a combination of airline and aircraft type as a proxy for the tail number. Although this is not as precise, the team found it to be accurate enough to provide a reasonable estimate.

This estimate of the number of aircraft on the ground contextualizes the tracking of arrivals and departures. The net arrivals at any point in time can then be added to this baseline reference point, giving the total number of aircraft on the ground at that time.

For 2015 and 2025, the baseline reference point is calculated following the same process described above but using the 2015 and 2025 flight schedules. Again, only those operations that require passenger bridges, as defined previously, are considered.

MODEL EXECUTION

LMI developed the necessary software utilities to support the computerized execution of the model. The model relies on two Java-based utilities. The first 
analyzes the arrival and departure times for all 310 airports to provide the baseline reference point for the number of aircraft on the ground. The second utility uses the baseline or FAA forecasted schedules to build an airport-by-airport, epoch-byepoch schedule of arrivals and departures. It then cycles through each 15-minute epoch, creating a running count of the change in the number of aircraft at the gate for each airport. These net change values are added to the baseline value to provide the total aircraft at the gates throughout the day. These values are compared with the airport's gate capacity.

If the number of gates is exceeded by the number of aircraft present at the gate, the airport is marked as gate constrained. When gate capacity is exceeded, the utility creates an alternative arrival schedule. Under this arrival schedule, any arrival that would bring the total number of aircraft on the ground over the airport's limit is trimmed from the schedule. A future departure of the same aircraft type that is at least 45 minutes after the arrival is also removed from the departure schedule. The first such departure encountered by the utility is trimmed from the schedule.

If an airport is gate constrained, the number of aircraft on the ground at the beginning of the day, the baseline reference point, is decremented by one, and the original schedule is rerun with this new baseline reference point. This process is repeated until the reduction in the baseline reference point is proportionate to the reduction in flights resulting from trimming. This keeps the calculation of the number of flights on the ground at the beginning of the day realistic relative to the trimmed schedule. The total number of arrivals trimmed and the resulting arrival acceptance rate are recorded.

Flight trimming takes place only between 5:30 a.m. and 11:00 p.m. local time. Flights arriving after 11:00 p.m. or before 5:30 a.m. are not subject to gate constraints. This no-trim policy is designed to account for airports' practice of shuffling aircraft off the gates and into overnight parking areas when gate space is limited.

Finally, the utility cycles through each airport's arrival and departure schedules to calculate its total aircraft spacing requirements. The terminal frontage requirements for 2015 and 2025 can then be compared to those of 2007 to provide an idea of the rate of anticipated growth in this requirement.

A third Visual Basic- and Microsoft Excel-based utility facilitates data analysis and allows for epoch-by-epoch charting of the aircraft on the ground under both alternative arrival schedules.

Model runtime is very short, generally 5 minutes or less, which allows for rapid adjustment, calibration, and easy exploration of alternative scenarios.

Figure 5-33 depicts the number of aircraft on the ground at IAD throughout a 24hour period in 2025. Time $t=0$ corresponds to 9:00 a.m. GMT, with the x-axis 
being divided into 96 15-minute epochs. The red dashed line indicates the airport's gate capacity. The blue curve shows the natural ebb and flow of aircraft on the ground. The green curve shows the same ebb and flow modified to remain within the constraints imposed by gate capacity. The difference between the blue and green curves represents trimmed arrivals. The green curve starts out below the blue curve at the beginning of the day because of the process through which the baseline reference number of aircraft is reduced in response to iterative trimming of the schedule.

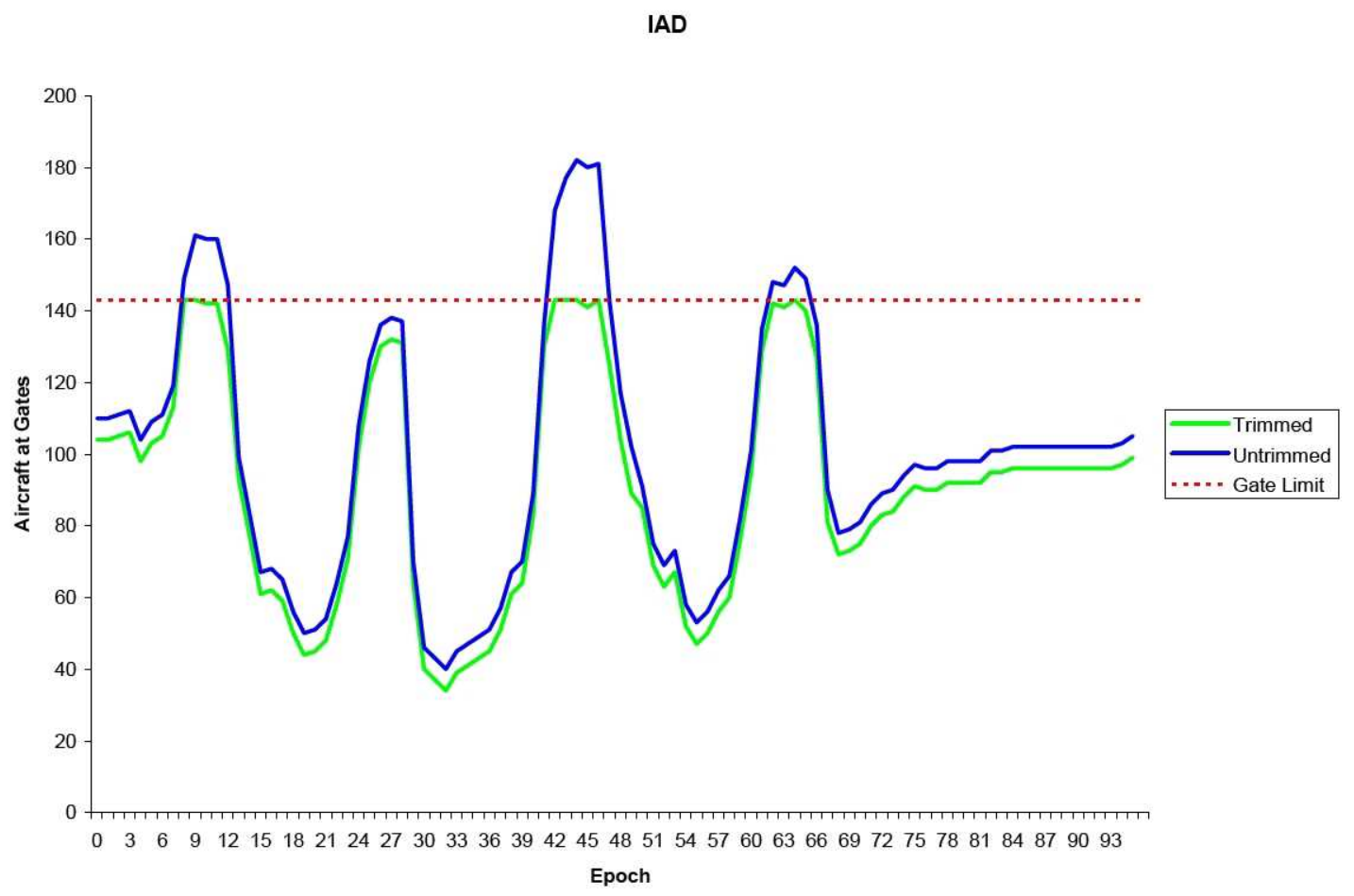

Figure 5-33. Aircraft on the ground at IAD in 2025.

\section{Results}

In general, the largest airports tend to be least capable of accommodating significant growth in gate demand. According to this analysis, in 2015, 55 percent of the LMI 110 airports will be gate constrained, and by 2025 , that number will grow to 72 percent. These gate-constrained airports will need to trim an average of 7 percent of their flights in 2015 and 10 percent in 2025. In other words, not only will demand surpass capacity at a greater number of large airports, but it will do so by a greater amount. In contrast, the percentage of gate-constrained small airports is not expected to grow beyond current, already low levels. Figure 5-34 shows gate throughput by airport group and year. 


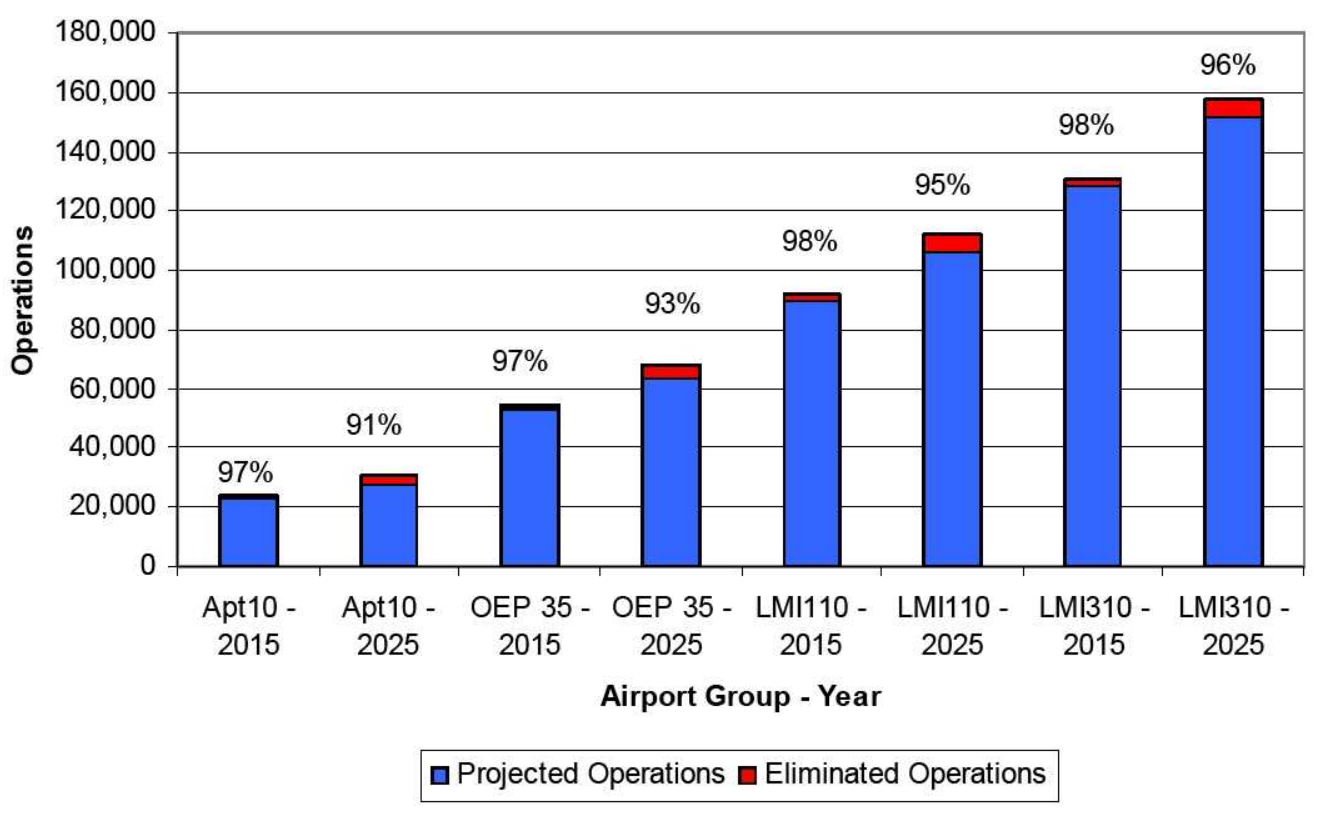

Figure 5-34. Gate throughput by airport group and year.

Most of the LMI 110 airports have trimming rates of less than 10 percent. This corresponds to some small amount of gate-related delays at peak operating periods. Trimming rates well above this range are cause for concern. As expected, this is more common in 2025. (Exceptionally poor acceptance rates at Hawaiian airports such as HNL and HPN are thought to be due to the tendency of these airports to service large aircraft without passenger-bridge-equipped gates. Due to their unique climate, these airports are able to operate well without them. We believe that these artificially low acceptance rates can safely be ignored.)

For smaller airports, the results are slightly more nuanced. Many small airports do not have any passenger bridges, making their capacity to serve large, nonregional jet aircraft, as defined in this model, zero. Because most of these airports' commercial traffic is exclusively regional aircraft, this is not typically a problem. Exceptions to this general rule show up here as trimmed flights. Also, because these airports receive low volumes of traffic, small fluctuations in arrivals of large aircraft can result in wild fluctuations in the percentage of operations trimmed.

The figures below show 2015 and 2025 throughput for the top 10 busiest airports based on this analysis. In 2015 , most of the top 10 airports are only slightly affected by gate limitations, although both LAS and LAX experience some significant trimming of operations. By 2025, gate constraints still affect a relatively narrow set of these airports. However, at these affected airports, the impact is much greater, especially at LAX and ORD. Figure 5-35 shows the relative and absolute number of gate operations that were accommodated by each 
airport out of all scheduled gate operations. This gives a good idea of the severity of gate capacity relative to the actual number of operations that require gate use.

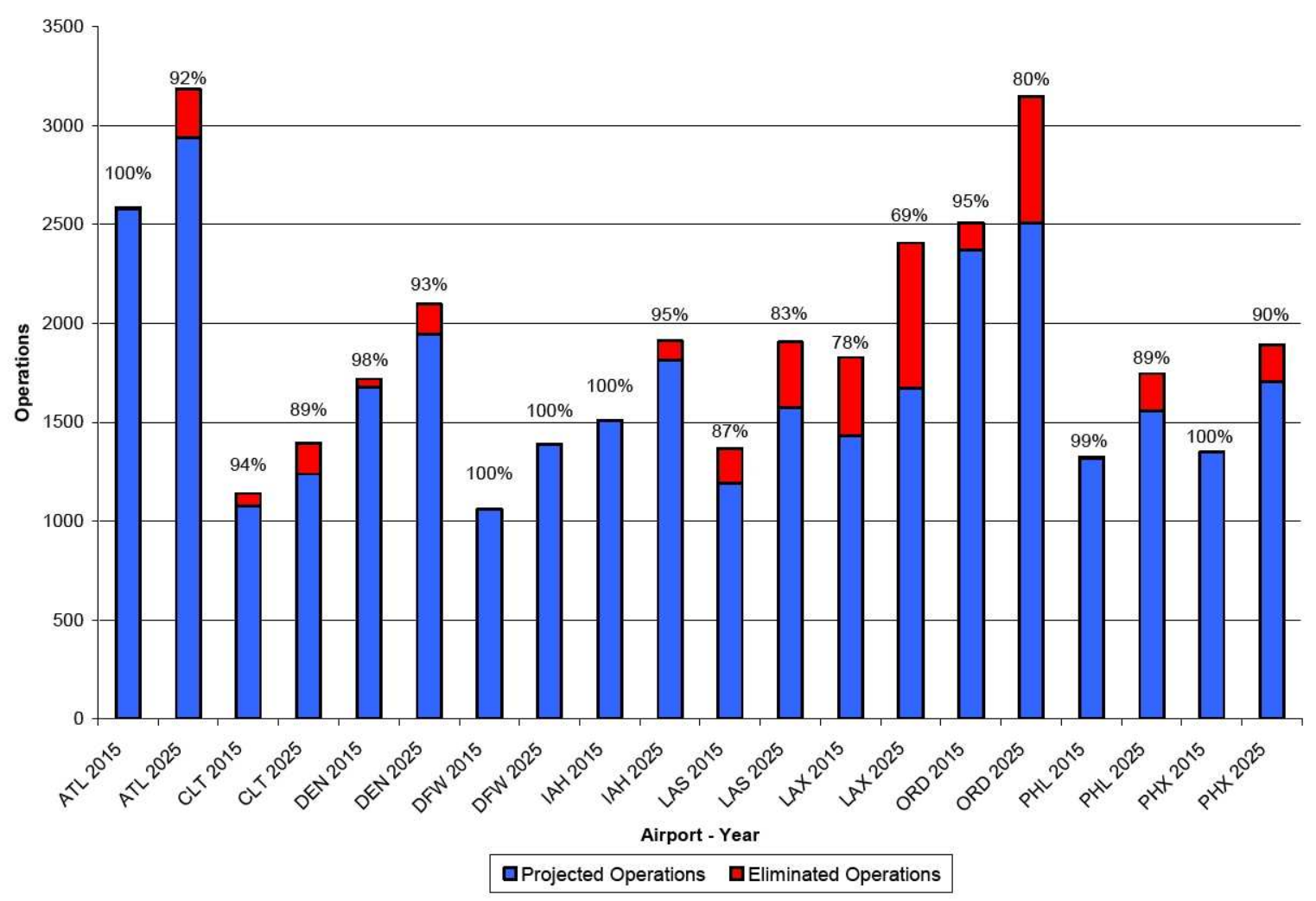

Figure 5-35. 2015 and 2025 unconstrained and gate-constrained throughput out of eligible operations for 10 busiest airports.

Figure 5-36 shows these same figures but in comparison to all operations, regardless of whether the operations did or did not require a gate. Predictably, the proportion of operations accommodated rises considerably when all operations are included. 


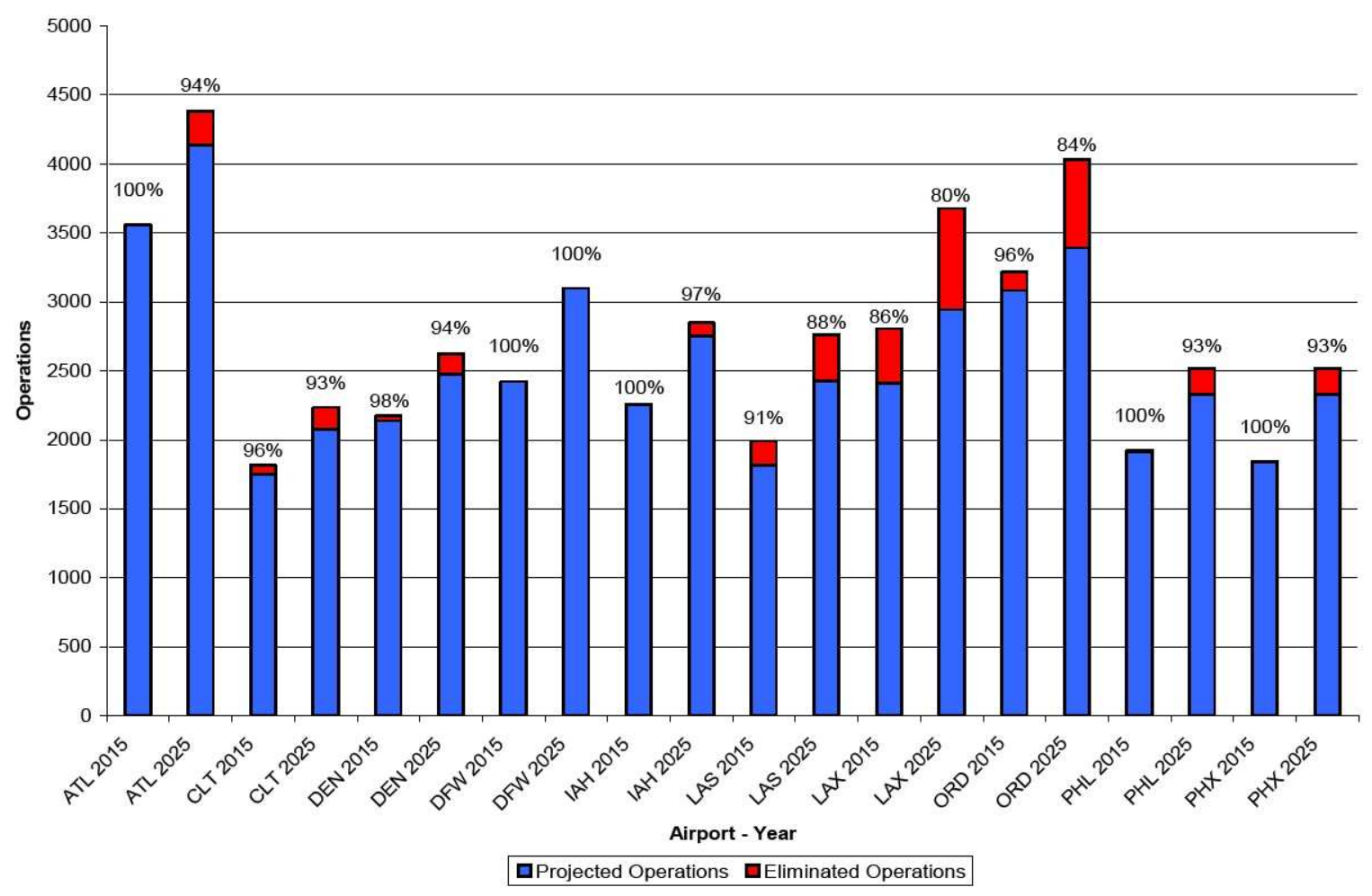

Figure 5-36. 2015 and 2025 unconstrained and gate-constrained throughput out of all operations for 10 busiest airports.

Table 5-9 shows the full results for all 310 airports.

Table 5-9. Gate Throughput for 310 Airports, 2015 and 2025

\begin{tabular}{|c|r|c|r|r|r|r|c|}
\hline \multirow{2}{*}{ Airport } & \multirow{2}{*}{ Gates } & \multicolumn{3}{|c|}{2015 operations } & \multicolumn{3}{|c|}{2025 operations } \\
\cline { 3 - 8 } & Unconstrained & Constrained & Reduction & Unconstrained & Constrained & Reduction \\
\hline ABQ & 23 & 264 & 256 & $3.0 \%$ & 324 & 296 & $8.6 \%$ \\
\hline ANC & 28 & 354 & 274 & $22.6 \%$ & 504 & 306 & $39.3 \%$ \\
\hline ATL & 179 & 2,586 & 2,580 & $0.2 \%$ & 3,184 & 2,938 & $7.7 \%$ \\
\hline AUS & 25 & 274 & 252 & $8.0 \%$ & 342 & 298 & $12.9 \%$ \\
\hline BDL & 37 & 226 & 224 & $0.9 \%$ & 304 & 300 & $1.3 \%$ \\
\hline BHM & 19 & 156 & 150 & $3.8 \%$ & 196 & 184 & $6.1 \%$ \\
\hline BOS & 102 & 842 & 832 & $1.2 \%$ & 998 & 952 & $4.6 \%$ \\
\hline BTR & 7 & 58 & 58 & $0.0 \%$ & 76 & 66 & $13.2 \%$ \\
\hline BUF & 24 & 208 & 204 & $1.9 \%$ & 258 & 236 & $8.5 \%$ \\
\hline BUR & 14 & 220 & 196 & $10.9 \%$ & 260 & 222 & $14.6 \%$ \\
\hline BWI & 78 & 778 & 774 & $0.5 \%$ & 1,002 & 964 & $3.8 \%$ \\
\hline CHS & 10 & 114 & 104 & $8.8 \%$ & 136 & 108 & $20.6 \%$ \\
\hline CLT & 91 & 1,140 & 1,076 & $5.6 \%$ & 1,394 & 1,238 & $11.2 \%$ \\
\hline CMH & 39 & 358 & 338 & $5.6 \%$ & 440 & 400 & $9.1 \%$ \\
\hline CRP & 6 & 54 & 52 & $3.7 \%$ & 62 & 54 & $12.9 \%$ \\
\hline
\end{tabular}


Table 5-9. Gate Throughput for 310 Airports, 2015 and 2025

\begin{tabular}{|c|c|c|c|c|c|c|c|}
\hline \multirow[b]{2}{*}{ Airport } & \multirow[b]{2}{*}{ Gates } & \multicolumn{3}{|c|}{2015 operations } & \multicolumn{3}{|c|}{2025 operations } \\
\hline & & Unconstrained & Constrained & Reduction & Unconstrained & Constrained & Reduction \\
\hline $\mathrm{DAL}$ & 20 & 404 & 350 & $13.4 \%$ & 530 & 408 & $23.0 \%$ \\
\hline DAY & 21 & 158 & 150 & $5.1 \%$ & 180 & 162 & $10.0 \%$ \\
\hline $\mathrm{DCA}$ & 44 & 668 & 638 & $4.5 \%$ & 688 & 630 & $8.4 \%$ \\
\hline DEN & 143 & 1,718 & 1,680 & $2.2 \%$ & 2,096 & 1,946 & $7.2 \%$ \\
\hline DSM & 12 & 114 & 106 & $7.0 \%$ & 134 & 118 & $11.9 \%$ \\
\hline EWR & 121 & 1,182 & 1,136 & $3.9 \%$ & 1,488 & 1,330 & $10.6 \%$ \\
\hline FAT & 8 & 38 & 38 & $0.0 \%$ & 54 & 50 & $7.4 \%$ \\
\hline FLL & 57 & 588 & 570 & $3.1 \%$ & 760 & 716 & $5.8 \%$ \\
\hline FNT & 6 & 42 & 42 & $0.0 \%$ & 56 & 54 & $3.6 \%$ \\
\hline GRR & 12 & 100 & 94 & $6.0 \%$ & 124 & 104 & $16.1 \%$ \\
\hline GSO & 26 & 186 & 184 & $1.1 \%$ & 226 & 224 & $0.9 \%$ \\
\hline $\mathrm{HNL}$ & 29 & 550 & 308 & $44.0 \%$ & 668 & 358 & $46.4 \%$ \\
\hline $\mathrm{HOU}$ & 25 & 400 & 382 & $4.5 \%$ & 482 & 438 & $9.1 \%$ \\
\hline HPN & 4 & 96 & 80 & $16.7 \%$ & 118 & 92 & $22.0 \%$ \\
\hline IAD & 143 & 940 & 940 & $0.0 \%$ & 1,344 & 1,240 & $7.7 \%$ \\
\hline $\mathrm{IAH}$ & 135 & 1,508 & 1,508 & $0.0 \%$ & 1,912 & 1,816 & $5.0 \%$ \\
\hline ICT & 12 & 76 & 74 & $2.6 \%$ & 82 & 76 & $7.3 \%$ \\
\hline IND & 34 & 346 & 316 & $8.7 \%$ & 412 & 360 & $12.6 \%$ \\
\hline ISP & 16 & 98 & 98 & $0.0 \%$ & 128 & 116 & $9.4 \%$ \\
\hline JAX & 29 & 258 & 256 & $0.8 \%$ & 328 & 318 & $3.0 \%$ \\
\hline JFK & 119 & 1,352 & 1,146 & $15.2 \%$ & 1,766 & 1,366 & $22.7 \%$ \\
\hline JNU & 3 & 42 & 40 & $4.8 \%$ & 46 & 40 & $13.0 \%$ \\
\hline LAS & 85 & 1,368 & 1,190 & $13.0 \%$ & 1,906 & 1,574 & $17.4 \%$ \\
\hline LAX & 118 & 1,828 & 1,430 & $21.8 \%$ & 2,406 & 1,670 & $30.6 \%$ \\
\hline LGB & 12 & 98 & 98 & $0.0 \%$ & 122 & 118 & $3.3 \%$ \\
\hline LIT & 20 & 130 & 130 & $0.0 \%$ & 152 & 148 & $2.6 \%$ \\
\hline $\mathrm{MCO}$ & 129 & 1,028 & 1,028 & $0.0 \%$ & 1,304 & 1,284 & $1.5 \%$ \\
\hline MDW & 48 & 680 & 676 & $0.6 \%$ & 858 & 802 & $6.5 \%$ \\
\hline MEM & 83 & 440 & 440 & $0.0 \%$ & 524 & 518 & $1.1 \%$ \\
\hline MHT & 16 & 160 & 148 & $7.5 \%$ & 190 & 180 & $5.3 \%$ \\
\hline MIA & 101 & 698 & 698 & $0.0 \%$ & 892 & 882 & $1.1 \%$ \\
\hline MKE & 45 & 320 & 320 & $0.0 \%$ & 406 & 404 & $0.5 \%$ \\
\hline MSN & 13 & 100 & 96 & $4.0 \%$ & 120 & 106 & $11.7 \%$ \\
\hline $\mathrm{MSY}$ & 29 & 256 & 256 & $0.0 \%$ & 312 & 298 & $4.5 \%$ \\
\hline OAK & 34 & 558 & 506 & $9.3 \%$ & 742 & 658 & $11.3 \%$ \\
\hline OKC & 17 & 164 & 146 & $11.0 \%$ & 184 & 156 & $15.2 \%$ \\
\hline OMA & 21 & 200 & 184 & $8.0 \%$ & 270 & 238 & $11.9 \%$ \\
\hline ONT & 28 & 218 & 218 & $0.0 \%$ & 252 & 246 & $2.4 \%$ \\
\hline ORD & 170 & 2,508 & 2,372 & $5.4 \%$ & 3,148 & 2,508 & $20.3 \%$ \\
\hline ORF & 24 & 168 & 162 & $3.6 \%$ & 186 & 174 & $6.5 \%$ \\
\hline PBI & 25 & 134 & 134 & $0.0 \%$ & 156 & 152 & $2.6 \%$ \\
\hline PDX & 67 & 396 & 396 & $0.0 \%$ & 496 & 482 & $2.8 \%$ \\
\hline $\mathrm{PHL}$ & 120 & 1,326 & 1,318 & $0.6 \%$ & 1,746 & 1,558 & $10.8 \%$ \\
\hline $\mathrm{PHX}$ & 119 & 1,348 & 1,348 & $0.0 \%$ & 1,892 & 1,706 & $9.8 \%$ \\
\hline PVD & 17 & 184 & 172 & $6.5 \%$ & 214 & 190 & $11.2 \%$ \\
\hline
\end{tabular}


Table 5-9. Gate Throughput for 310 Airports, 2015 and 2025

\begin{tabular}{|c|c|c|c|c|c|c|c|}
\hline \multirow[b]{2}{*}{ Airport } & \multirow[b]{2}{*}{ Gates } & \multicolumn{3}{|c|}{2015 operations } & \multicolumn{3}{|c|}{2025 operations } \\
\hline & & Unconstrained & Constrained & Reduction & Unconstrained & Constrained & Reduction \\
\hline RDU & 49 & 410 & 386 & $5.9 \%$ & 500 & 462 & $7.6 \%$ \\
\hline RIC & 16 & 192 & 166 & $13.5 \%$ & 248 & 204 & $17.7 \%$ \\
\hline RNO & 23 & 182 & 178 & $2.2 \%$ & 226 & 216 & $4.4 \%$ \\
\hline $\mathrm{ROC}$ & 22 & 140 & 136 & $2.9 \%$ & 168 & 154 & $8.3 \%$ \\
\hline SAN & 41 & 578 & 498 & $13.8 \%$ & 726 & 598 & $17.6 \%$ \\
\hline SAT & 27 & 276 & 268 & $2.9 \%$ & 360 & 326 & $9.4 \%$ \\
\hline SDF & 23 & 204 & 190 & $6.9 \%$ & 246 & 228 & $7.3 \%$ \\
\hline SEA & 96 & 742 & 718 & $3.2 \%$ & 926 & 862 & $6.9 \%$ \\
\hline SFO & 98 & 996 & 908 & $8.8 \%$ & 1,258 & 1,026 & $18.4 \%$ \\
\hline SJC & 32 & 374 & 352 & $5.9 \%$ & 530 & 480 & $9.4 \%$ \\
\hline SLC & 83 & 916 & 898 & $2.0 \%$ & 1,110 & 1,018 & $8.3 \%$ \\
\hline SMF & 27 & 394 & 356 & $9.6 \%$ & 512 & 446 & $12.9 \%$ \\
\hline SNA & 16 & 310 & 254 & $18.1 \%$ & 380 & 302 & $20.5 \%$ \\
\hline SWF & 7 & 42 & 42 & $0.0 \%$ & 46 & 44 & $4.3 \%$ \\
\hline TPA & 59 & 474 & 474 & $0.0 \%$ & 576 & 558 & $3.1 \%$ \\
\hline TUL & 19 & 146 & 140 & $4.1 \%$ & 162 & 150 & $7.4 \%$ \\
\hline TUS & 20 & 128 & 126 & $1.6 \%$ & 142 & 136 & $4.2 \%$ \\
\hline TVC & 4 & 38 & 32 & $15.8 \%$ & 44 & 38 & $13.6 \%$ \\
\hline TYS & 12 & 138 & 120 & $13.0 \%$ & 168 & 136 & $19.0 \%$ \\
\hline AMA & 4 & 28 & 26 & $7.1 \%$ & 34 & 32 & $5.9 \%$ \\
\hline FAI & 4 & 32 & 28 & $12.5 \%$ & 40 & 32 & $20.0 \%$ \\
\hline ITO & 7 & 42 & 30 & $28.6 \%$ & 46 & 38 & $17.4 \%$ \\
\hline $\mathrm{KOA}$ & 0 & 84 & 4 & $95.2 \%$ & 98 & 4 & $95.9 \%$ \\
\hline KTN & 2 & 20 & 16 & $20.0 \%$ & 20 & 16 & $20.0 \%$ \\
\hline $\mathrm{LIH}$ & 8 & 76 & 54 & $28.9 \%$ & 88 & 62 & $29.5 \%$ \\
\hline OGG & 18 & 150 & 112 & $25.3 \%$ & 184 & 136 & $26.1 \%$ \\
\hline PAE & 0 & 6 & 2 & $66.7 \%$ & 6 & 2 & $66.7 \%$ \\
\hline SFB & 7 & 22 & 22 & $0.0 \%$ & 26 & 22 & $15.4 \%$ \\
\hline
\end{tabular}





\section{Chapter 6}

\section{Analyze Airport Environmental Constraints}

In this chapter, we explain our approach to the environmental analysis and show how the results dovetail with the capacity analysis results to provide an integrated constraint analysis. The environmental analysis addresses fuel efficiency, emissions, and noise, which we have modeled for current and future conditions in an effort to describe and rank environmental factors associated with growth at U.S. airports. The following sections describe environmental metrics and goals, our analytical methods, and the results of the analysis.

\section{ENVIRONMENTAL METRICS AND GOALS}

FAA's Office of Environment and Energy and FAA's Air Traffic Office have many policies and orders clearly defining FAA's responsibility in regard to disclosing potential changes to the environment caused by an FAA action. Air quality and noise are of particular concern and have been used for many years to gauge the environmental significance of a proposed action.

Air quality assessments must use certain metrics, as specified in FAA Order 1050.1E:

2.1a. Two primary laws apply to air quality: [National Environmental Policy Act (NEPA)], and the Clean Air Act (CAA). As a Federal agency, the FAA is required under NEPA to prepare an environmental document (e.g., environmental impact statement (EIS) or environmental assessment (EA)) for major Federal actions that have the potential to affect the quality including air quality of the human environment. An air quality assessment prepared for inclusion in a NEPA environmental document should include an analysis and conclusions of a proposed action's impacts on air quality.

2.1b. The CAA established National Ambient Air Quality Standards (NAAQS) for six pollutants, termed "criteria pollutants." The six pollutants are: carbon monoxide $(\mathrm{CO})$, lead $(\mathrm{Pb})$, nitrogen dioxide (NO2), ozone (O3), particulate matter (PM-10 and PM-2.5), and sulfur dioxide (SO2). The CAA requires each State to adopt a plan to achieve the NAAQS for each pollutant within timeframes established under the CAA. These air quality plans, known as State implementation plans (SIP), are subject to Environmental Protection Agency (EPA) approval. In default of an approved SIP, the EPA is required to promulgate a Federal implementation plan (FIP). 
For large-scale analysis, fuel efficiency is often used as a surrogate for impacts on local air quality; however, for this analysis we compute fuel and many of the pollutants described above. We calculated fuel efficiency by operation and express it in units of kilograms of fuel per kilometer flown. Although all phases of flight could be calculated, our focus is on the fuel, emissions, and noise near each airport.

The FAA has also specified metrics for aircraft noise, notably, day-night levels (DNLs) of sound. DNL is the total noise level averaged over a 24-hour period, except that a $10 \mathrm{~dB}$ penalty is added to noise events occurring at night (between 10 p.m. and 7 a.m.). Community background noise levels typically decrease by about $10 \mathrm{~dB}$ during those nighttime hours, so adding $10 \mathrm{~dB}$ to the nighttime metric reflects the added intrusiveness of nighttime noise events. DNL does not represent the sound level heard at any particular time, but rather represents the average (and partially weighted) sound exposure.

As a result of the DNL metric's correlation with the degree of community annoyance from aircraft noise, DNL has been formally adopted by most federal agencies for measuring and evaluating aircraft noise for land-use planning and noise impact assessment. Committees such as the Federal Interagency Committee on Urban Noise and the Federal Interagency Committee on Noise - which have representatives from the EPA, FAA, Department of Defense, Department of Housing and Urban Development, and Department of Veterans Affairs - found DNL to be the best metric for land-use planning. They also found no new cumulative sound descriptors or metrics of sufficient scientific standing to substitute for DNL. Other cumulative metrics could be used to supplement, but not replace DNL. Furthermore, FAA Order 1050.1E requires that DNL be used in environmental studies to describe cumulative noise exposure and to identify aircraft noise/land-use compatibility issues. ${ }^{1}$

The JPDO defines environmental goals in terms of annual improvements (e.g., 1 percent per year). To remain consistent with this strategy, we first estimate the current conditions for fuel efficiency, emissions, and noise using JPDO's metrics:

- Fuel efficiency is computed as fuel burned divided by distance flown.

- Emissions are calculated as the emissions inventories of carbon monoxide (CO), hydrocarbons (HC), nitrogen oxide (NOx), and sulfur oxide (SOx).

- Noise is computed as the number of people exposed to $65 \mathrm{~dB}$ DNL or greater.

\footnotetext{
${ }^{1}$ Federal Aviation Administration, Draft Environmental Impact Statement: New York/New Jersey/Philadelphia Airspace Redesign, December 2005.
} 
The goals for fuel efficiency, emissions, and noise are as follows:

- Fuel efficiency - improve the average fuel efficiency by 1 percent per year (compounded) relative to a reference year. Again fuel efficiency for this analysis is defined as fuel burned divided by distance flown.

- Emissions - reduce NOx emissions by 1 percent per year (compounded) relative to a reference year. (The FAA's Flight Plan ${ }^{2}$ does not define emission-reduction goals; instead, it uses fuel efficiency as a surrogate. We chose to focus on NOx emissions because of the recent attention to greenhouse gases and growing concerns about climate change.)

- Noise - reduce the number of people exposed to $65 \mathrm{~dB}$ DNL by 4 percent per year (compounded) relative to a reference year.

The goals for fuel efficiency and noise were provided to the JPDO by the JPDO's Environmental Working Group and were used for this project to be consistent with the JPDO. It should be noted that these are not yet official goals for the JPDO but are surrogates until policy and future goals are defined.

Using 2007 to set current environmental conditions, we then applied the environmental goals for fuel efficiency, emissions, and noise (as an annual improvement rate) to define the environmental targets for future years. Table 6-1 shows the desired improvement by metric for 2015 and 2025 relative to the 2007 baseline.

Table 6-1. Environmental Targets for 2015 and 2025

\begin{tabular}{|l|l|c|c|}
\hline \multicolumn{1}{|c|}{ Metric } & \multicolumn{1}{|c|}{ Description } & 2015 target & 2025 target \\
\hline Fuel efficiency & $\begin{array}{l}\text { Fuel burned below 3,000 feet divided by distance } \\
\text { flown }\end{array}$ & $92 \%$ & $83 \%$ \\
\hline Emissions & Total NOx emitted below 3,000 feet & $92 \%$ & $83 \%$ \\
\hline Noise & Number of people exposed to 65 dB DNL & $72 \%$ & $48 \%$ \\
\hline
\end{tabular}

\section{FORECASTING FLEET EVOLUTION}

Although the schedule forecast from FAA looks like a real schedule including the equipment types, it does not consider the change in the aircraft fleet, which is an essential component needed for the environmental constraints analyses. The current aircraft will be gradually replaced by quieter and more efficient aircraft. An evolving picture of fleet mix is more correctly described as fleet evolution, to convey the fact that forecasts of the U.S. and global future fleet are influenced by a number of factors, many of which have a time-dependent component. Point-in-time

\footnotetext{
${ }^{2}$ FAA Flight Plan performance reports are available at http://www.faa.gov/about/plans_reports/Performance/.
} 
fleet mix is one of the characteristics that must be captured in projections of NAS demand to realistically model concepts dependent on the composition of the fleet.

Several aspects of the future NAS, especially ATM and environmental impact, are significantly affected by fleet composition. For example, ATM is sensitive to such factors as preferred flight levels and routing, while environmental impact is sensitive to aircraft size and engine characteristics.

For basic modeling of the evolution of the U.S. air transport fleet, we used MITRE's 2007-2035 forecast. ${ }^{3}$ We modeled the evolution by seat category and engine category using percentages of MITRE's forecast, and we modeled the evolution of cargo and passenger flights independently. We did not predict the evolution of flights by international carriers or GA operations. Figure 6-1 depicts the high-level process used to model the evolution of the fleet. Note that seat category (Cat-N), noise power distance (NPD), and emissions index (EI) are referenced in the figure below. Noise power distance curves and emissions indexes are used during the environmental modeling to produce impacts to the environment in regard to noise and air quality.

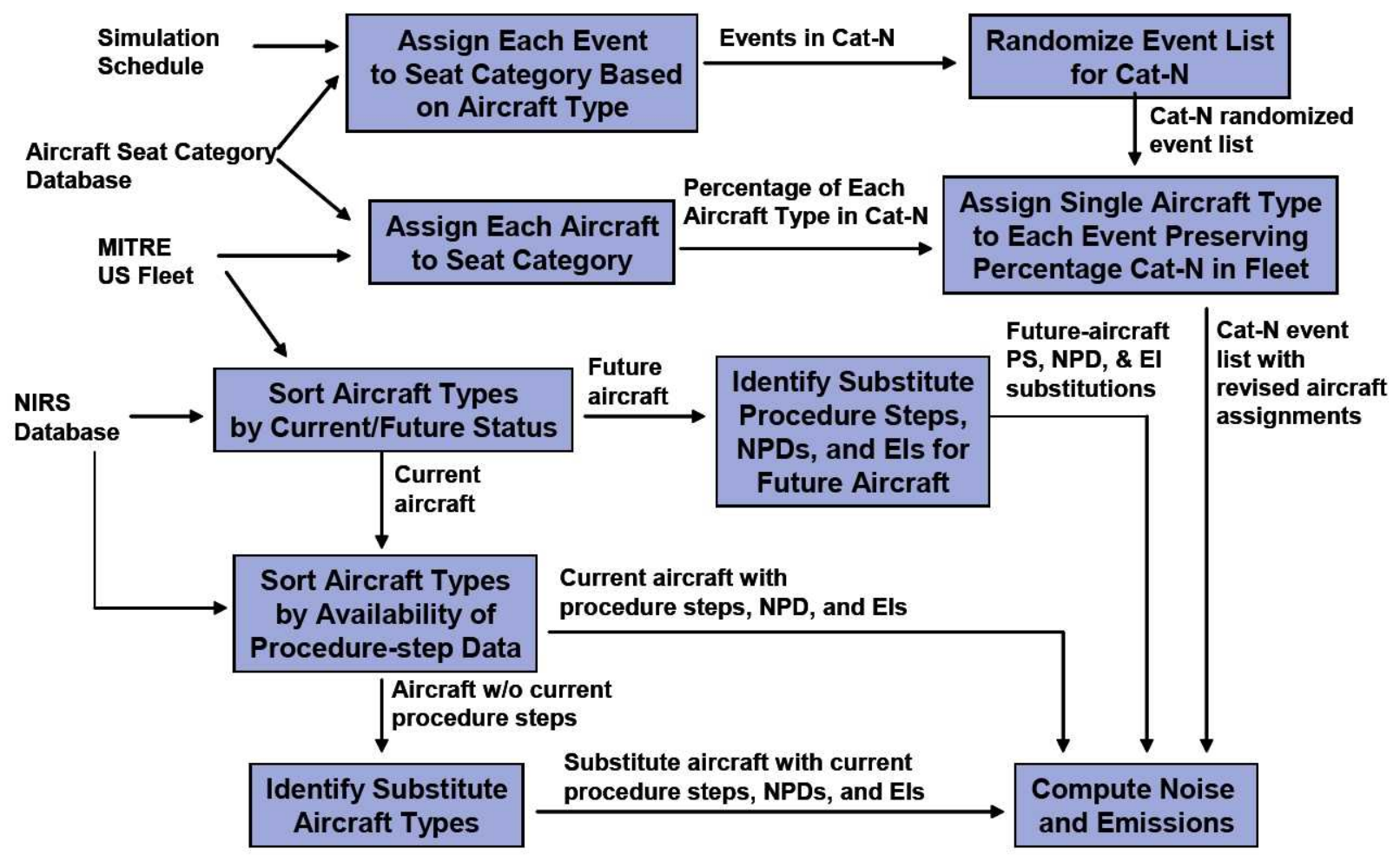

Figure 6-1. Fleet evolution process.

\footnotetext{
${ }^{3}$ Kent V. Hollinger, U.S. Air Transport Fleet Forecast 2007-2035 (McLean, VA: The MITRE Corporation, 2007).
} 
To support the process, we used an aircraft dictionary categorizing each of the aircraft identified in the schedule and MITRE fleet forecast to assign a corresponding seat class and engine category. The engine categories were jet, turbo-prop, and piston, while the seat categories were defined as follows:

- Fewer than 20 seats

- 20-49 seats

- 50-99 seats

- 100-150 seats

- 151-210 seats

- 211-300 seats

- 301-400 seats

- 401-500 seats

- More than 500 seats.

For each aircraft within a seat class, we used the MITRE fleet forecast to define the proportion of aircraft that would be in the proposed schedule. For example, if the MITRE forecast showed that 40 percent of the jets in the 151-210 seat class were Boeing 737-800s, then the proportion of aircraft in the schedule for that engine and seat class would be 40 percent Boeing 737-800s. We used this approach to predict fleet evolution for each future schedule.

The MITRE forecast identified five new aircraft introduced to the U.S. fleet. In coordination with the JPDO's Environmental Working Group, and after review of existing environmental aircraft mappings, we defined the following substitutes to account for those aircraft in the current environmental models. Table 6-2 lists the aircraft substitutions.

Table 6-2. Environmental Substitutes for New Aircraft

\begin{tabular}{|l|l|}
\hline \multicolumn{1}{|c|}{ New aircraft } & \multicolumn{1}{|c|}{ Environmental substitute } \\
\hline Airbus A380 & Boeing 747-400 \\
\hline Boeing 747-800 & Boeing 747-400 \\
\hline Boeing 787 & Boeing 767-400 \\
\hline Embraer 175 & Gulfstream GV \\
\hline Embraer 195 & Gulfstream GV \\
\hline
\end{tabular}

We also assessed a second form of fleet evolution, one that includes long-range projected aircraft performance improvements. We considered the criteria established or being developed by three entities. The first, FAA, is developing the 
Continuous Lower Energy, Emissions and Noise (CLEEN) program, which requires all new aircraft to have the following performance by 2016 :

- Fuel efficiency-25 percent improvement relative to 1997 subsonic aircraft technology ${ }^{4}$

- Emissions -73 percent decrease in NOx relative to Committee on Aviation Environmental Protection (CAEP)/6 (63 percent of CAEP/2)

- Noise-32 dB decrease relative to ICAO Chapter 4 noise certifications.

Second, the JPDO is also considering various CLEEN levels, using the 2007 National Aeronautics Research and Development Plan as a guide. This plan projects environmental technology goals in three time periods, shown in Table 6-3.

Table 6-3. Environmental Projections from the National Aeronautics Research and Development Plan

\begin{tabular}{|l|c|c|c|}
\hline \multicolumn{1}{|c|}{ Time period } & Noise $^{\mathrm{a}}$ & ${\text { Emissions }(\mathrm{NOx})^{\mathrm{b}}}$ & Fuel efficiency \\
\hline $2015(\mathrm{~N}+1)$ & $-32 \mathrm{~dB}$ & $-70 \%$ & $-33 \%$ \\
\hline $2020-2025(\mathrm{~N}+2)$ & $-42 \mathrm{~dB}$ & $-80 \%$ & $-40 \%$ \\
\hline $2030-2035(\mathrm{~N}+3)$ & $-62 \mathrm{~dB}$ & $-80 \%$ & $-70 \%$ \\
\hline
\end{tabular}

Note: $\mathrm{N}$ is the current period (2008).

${ }^{a}$ Noise decreases are relative to Chapter 4 certifications.

${ }^{\mathrm{b}}$ Emissions decreases in $\mathrm{NOx}$ are relative to $\mathrm{CAEP} / 2$.

Finally, the Technology Standing Committee of the Environmental Working Group in the JPDO has provided a market-driven scenario that assumes no new regulations will be defined. Table 6-4 looks at multiple time horizons and also includes aircraft size.

Table 6-4. Environmental Projections from the Environmental Working Group's Technology Standing Committee

\begin{tabular}{|l|c|c|c|c|}
\hline \multicolumn{1}{|c|}{ Time period } & $\begin{array}{c}\text { Regional bizjet } \\
\text { and GA turbofans } \\
(20-49 \text { seats })\end{array}$ & $\begin{array}{c}\text { Regional } \\
\text { turbofans } \\
(50-99 \text { seats })\end{array}$ & $\begin{array}{c}\text { Single aisle } \\
(100-200 \text { seats })\end{array}$ & $\begin{array}{c}\text { Twin aisle } \\
(>200 \text { seats })\end{array}$ \\
\hline $2008-2015$ & $-5 \mathrm{~dB}$ & $-5 \mathrm{~dB}$ & $-5 \mathrm{~dB}$ & $-10 \mathrm{~dB}$ \\
\cline { 1 - 4 } $2016-2021$ & $-10 \mathrm{~dB}$ & $-10 \mathrm{~dB}$ & $-10 \mathrm{~dB}$ & $-15 \mathrm{~dB}$ \\
\hline 2022 and beyond & & &
\end{tabular}

For all seat classes that received new aircraft during the period, we selected a surrogate aircraft that would meet the desired environmental performance. Between 2016 and 2025, six jet and two turboprop seat classes received new aircraft. The A380 is a new seat class that had no operations in the future

\footnotetext{
${ }^{4}$ FAA Market Research Conference, March 2008, http://www.jpdo.gov/events.asp?id=12.
} 
schedules; we mapped those to the 401-500 seat class. Table 6-5 lists the surrogate aircraft.

Table 6-5. Surrogate Aircraft for Future Fleet Performance

\begin{tabular}{|l|c|c|}
\hline \multicolumn{1}{|c|}{ Surrogate } & Seat class & Engine \\
\hline Canadair RJ-900 & $050-099$ & $\mathrm{~J}$ \\
\hline Boeing 737-700 & $100-150$ & $\mathrm{~J}$ \\
\hline Boeing 737-800 & $151-210$ & $\mathrm{~J}$ \\
\hline Boeing 777-200 & $211-300$ & $\mathrm{~J}$ \\
\hline Boeing 747-400 & $401-500$ & $\mathrm{~J}$ \\
\hline Boeing 747-400 & $500-601$ & $\mathrm{~J}$ \\
\hline DeHavilland DASH 8/DHC8-100 & $020-049$ & $\mathrm{~T}$ \\
\hline Aerospatiale ATR072 & $050-099$ & $\mathrm{~T}$ \\
\hline
\end{tabular}

For this analysis, we applied the CLEEN projections to the future aircraft. It should also be noted that by the time of this analysis, the CLEEN and N+1 goals were harmonized between the FAA and NASA. From this point forward, we will reference the future fleet performance as $\mathrm{N}+1$. Because we assume these new aircraft are introduced in 2016, the 2015 forecast will not include these new aircraft. The 2015 demand is evolved using existing or known aircraft, while older aircraft are retired during the 2007-2015 period. As described above, we mapped all aircraft in the 2025 forecast - including the new aircraft types created to represent improved environmental performance - to engine categories and seat classes. Once this was completed, we again distributed the fleet's 2025 scheduled operations by proportion and seat class.

\section{Modeling Of Fuel EfFICIENCY, EMISSIONS, AND NOISE}

\section{Overview}

To analyze fuel efficiency, emissions, and noise, a series of models and associated inputs for each scenario were developed. Because our effort included such a large number of airports, we used a multitiered approach for computing the desired metrics. Each tier makes fewer assumptions, requires additional modeling inputs, and provides more detailed results. In the first tier, we apply a spreadsheet-based method to compute the environmental performance for all 310 airports. The second tier adds information specific to each airport and operation to provide additional detail. Finally, a high-fidelity modeling approach considers existing terminal area routing and runway use at only the 56 FACT2 airports. All 310 airports were modeled in the first two tiers to determine whether trimming was necessary to meet the environmental targets. If an airport required trimming and was a FACT2 airport, we then considered the use of the high-fidelity method to verify that the suggested trimming will meet the desired target. Although this 
approach provides higher fidelity modeling at those airports, it also requires significant additional resources to produce the inputs and derive the environmental impacts. Therefore, it was applied only at select airports. Figure 6-2 shows the general process supporting the constraints modeling approach. Although additional feedback loops could improve the process, it was not feasible to implement them in the time frame provided.

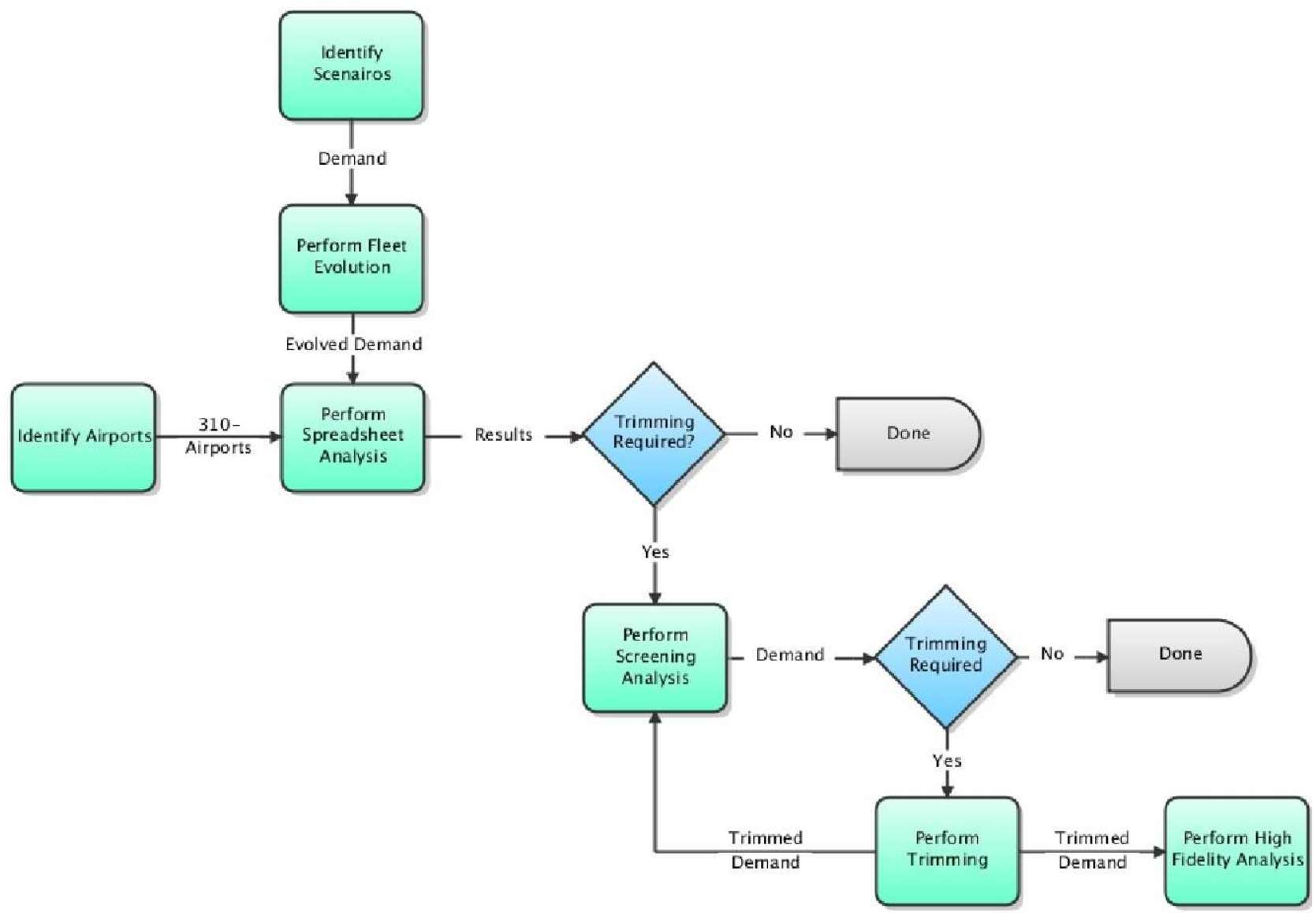

Figure 6-2. Environmental constraints modeling.

\section{Environmental Models}

For this analysis, the LMI team applied models created to support FAA regulatory modeling, JPDO policy modeling, and NASA research. The following subsections briefly describe the models.

\section{Tier 1-Spreadsheet-BAsed Model}

The spreadsheet model was used to develop first-cut estimates of the environmental impact at each airport and the magnitude of trimming required to 
meet environmental goals. We computed standard values for a takeoff and landing operation for each aircraft type. Summing the environmental impact of each operation provided a measure of the impact at each airport:

- Fuel efficiency and emissions. Using version 4.2 of the FAA Emissions and Dispersion Modeling System (EDMS), we estimated flight times in mode, applying defaults established by EPA and the International Civil Aviation Organization (ICAO). Within this model, the default times in mode were used to estimate both fuel burn and emissions produced. Because fuel efficiency requires flight distance, we used a nominal distance for departures to 3,000 feet and arrivals from 3,000 feet. From these, we could assign a standard value for fuel burn and local NOx emissions to each aircraft type.

- Noise. In coordination with the FAA the screening method known as the Area Equivalency Method (AEM) was developed and integrated with the spreadsheet based model. Rather than focusing on the number of people exposed to noise, this method computes the area exposed to noise. AEM applies a logarithmic function based on aircraft type, number of operations, and daytime or nighttime flights to compute the area of the $65 \mathrm{~dB}$ DNL contour.

We used the results of the spreadsheet-based model to inform and refine the higher-fidelity approaches.

\section{TIER 2-ENVIRONMENTAL SCREENING MODEL}

The environmental screening model leverages data available in both the screening and high-fidelity models, but makes assumptions about flight tracks and headings served by each airport. The flight tracks, when combined with runway layout and usage, allow the screening model to estimate not only fuel and emissions but also noise exposure to surrounding communities. For each operation, the aircraft, origin/destination, and time of day are used to better estimate the environmental effects. Time of day is particularly important to noise given the nighttime noise penalty associated with flights that occur after 10 p.m. and before 7 a.m. Likewise, by using the origin and destination of a flight, an aircraft's weight can be estimated, improving the fidelity of the associated impacts related to fuel efficiency, emissions, and noise. For example, a 737 flying from SFO to ATL would burn more fuel than one flying from SFO to PHX. The added fuel for the flight from SFO to ATL would keep the departure from climbing as quickly as the SFO-to-PHX flight and therefore would burn additional fuel, generate additional emissions, and potentially generate more noise.

The following is additional information related to the modeling of each metric:

- Fuel efficiency and emissions. The screening model takes advantage of a database of precalculated flight profiles in which all aircraft available to 
the high-fidelity model are flown on straight-in/straight-out ground tracks using all available stage lengths. The database contains times in mode and distances for all aircraft, and it applies the times in mode to the EDMS fuel flow and emissions indexes to compute fuel burn and emissions produced. Fuel efficiency is estimated by using the fuel burn and flight distance to support the operation.

- Noise. The database also contains a noise grid for each aircraft, created using the flight tracks described above for fuel efficiency and emissions. To compute DNL, we aggregated the noise grids for each operation. Once the grids were aggregated, we aligned them with a given airport's runways to suggest a noise contour. We then applied the contour to population locations to compute the population exposed to certain noise thresholds.

\section{TIER 3-HIGH-FIDELITY ENVIRONMENTAL MODEL}

The high-fidelity environmental models were used as a mechanism to validate the required trimming calculated by the spreadsheet-based or screening models. The models used for this level of analysis are consistent with those used by the federal government for regulatory environmental modeling. For fuel and emissions calculations, the methods and data defined by the FAA's EDMS have been incorporated. For noise, the FAA's Noise Integrated Routing System (NIRS) has been applied:

- Fuel efficiency and emissions. The high-fidelity environmental model for fuel and emissions was developed under a NASA SBIR and is named NAS-wide Environmental Impact Model (NASEIM). The model takes high-fidelity trajectories and performs flight simulation to estimate times in mode. Fuel burn and emissions are then calculated as described above.

- Noise. For noise calculations, we used the FAA's NIRS. We submitted the high-fidelity trajectory calculated for fuel efficiency and emissions, described above, to NIRS to compute DNL exposure to a set of population locations.

\section{Model Inputs}

The next several subsections define the basic inputs to all three models as well as the different requirements for each tier in the process.

\section{POPULATION DATA}

Population distribution is a key input primarily for noise modeling in order to estimate the number of people exposed to various levels of noise. The current analysis evaluates noise conditions for specific locations on the ground based on population centroids (centers of census blocks) surrounding each of the modeled airports. Census blocks are the smallest geographic unit for which the Census 
Bureau tabulates data; they are generally bounded by streets, legal boundaries, and other features. The noise exposure at the centroid is assumed to apply to all people residing in the census block. (In reality, noise levels can vary throughout a census block because the blocks vary in shape and centroid location.)

For this analysis, we used data from the most recent U.S. Census (2000) as our primary source of information. Because the noise metric uses the number of people exposed to $65 \mathrm{~dB}$ DNL, only those centroids within a $20 \mathrm{~nm}$ radius of each airport were considered for noise calculation. Use of those data is consistent with current JPDO analyses, but because the data are now 9 years old, they may not be an adequate reference point. (In some environmental analyses, population projections are obtained from a commercial source that provides projections for a broader area than a census block. Using those data requires in-depth review of land-use policies in each locale and identification of residential and nonresidential areas. Projections are then extrapolated from the broader areas down to the census blocks.)

Population data are not used in the spreadsheet-based model but are used in the screening and high-fidelity models.

\section{RUNWAY CONFIGURATION AND RUNWAY USE}

Data on an airport's runway configuration, or layout, and runway use, when coupled with data on the population near the airport, are critical inputs for supporting the noise analysis and attempting to estimate the population exposed to significant levels of noise.

The spreadsheet-based model ignores runway configuration and runway use. However, the screening model requires those data. To define configuration and runway use for all 310 airports, we applied a layered approach that considered the quality and availability of data. Runway layout and runway use is predominantly driven by local weather conditions and is also driven by many other factors such as demand, airspace restrictions, or even strategies for mitigating environmental impacts. Radar data are the best source for understanding runway use. However, a detailed analysis of radar data was not practical for the scope of the study. Instead, for the 56 FACT2 airports, we used a radar sample of roughly 30 days to determine runway use. We assumed that the sample represented annual conditions. We used the data on FACT2 airports for both the screening model and the high-fidelity model.

For those airports that report runway configuration data to the FAA's Aviation System Performance Metrics (ASPM) database, those data was used to define dominant runway configurations and their use. The ASPM database was used to generate an airport efficiency report for 2007, which is consistent with our baseline year. It is worth noting that the validity of the information depends on human accuracy. Throughout the year, many airports can use several runway configurations to support the given airport's needs. This analysis used the most 
common configurations that together accounted for at least 70 percent of the year. These configurations were then scaled to 100 percent to account for all of the airports' activity.

For airports that do not report data to the ASPM database or are not FACT2 airports, we checked individual airport websites for master plans, including information on runway configuration and use, and noise abatement reports. If the information was not available, we used weather data and two simplifying assumptions to guide the selection of runway configurations and use. The assumptions are as follows:

- Aircraft operations are generally performed into the wind.

- If multiple runways available, the longer runway will be used more frequently.

The U.S. Department of Agriculture provides 30-year "wind rose" data presented as a graph. Each graph shows the distribution of wind speed and direction at a specific location for a specific month. The data are based on an examination, for each month over 30 years, to determine the most influential wind direction and the highest wind speed for the greatest percentage of time. Figure 6-3 displays a sample wind rose graph for Sterling, VA, from January 1961. We used this information, in conjunction with the assumptions described above, to select the top two runway configurations for each airport. 


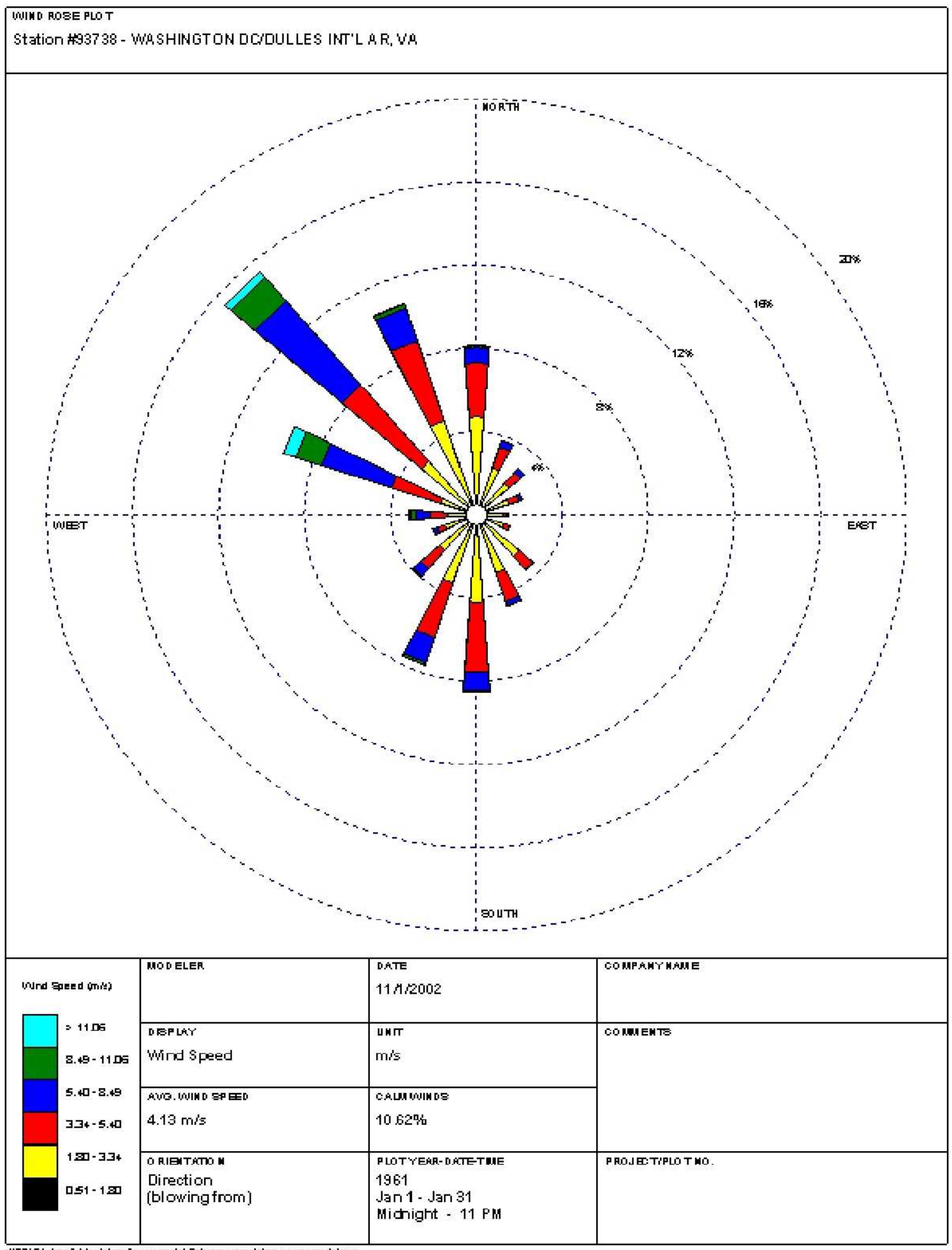

Figure 6-3. Wind rose sample for Sterling, Virginia. 


\section{TERMINAL AREA TRAJECTORIES}

The spreadsheet-based model does not use trajectories for any of its calculations. As mentioned above, the screening model uses predefined trajectories to populate a database of flight performance data that be looked up rather than computed each time. The screening method predefines straight in/out flight trajectories that either originate or terminate at the desired airport's runway. The vertical profile for arrivals is a constant rate of descent from 3,000 feet above field elevation (AFE), while departures are an unrestricted climb to 3,000 feet AFE. No vertical or lateral dispersion is defined.

The high-fidelity model needs to account for annual use of the airport and the airspace surrounding the airport. Of the three desired metrics, noise analysis is typically performed for an average annual day, which represents the spatial variability in traffic patterns throughout the year, and is based on a process of relatively large-scale data sampling and analysis. To capture the needed level of detail without running an entire year's worth of flight data through the model, we sampled radar data and used it to capture actual flight routes and their dispersion characteristics.

With those data, the models can predict fuel efficiency, emissions, and noise more realistically by capturing variations that may be caused by vectoring, changes in runway use or configurations, or other things that produce variability within the terminal area.

Below, we describe the method used to identify terminal-area traffic patterns for the airports considered for the high-fidelity analysis. Ultimately, the process created a large number of flight-route data structures referred to as backbones. The backbones capture information related to operation (arrival or departure), location (fix, airport configuration, and runway), and frequency of use (by time and aircraft category). In addition, each such data structure contains information on the spatial dispersion of routes associated with each backbone. These data were later used in conjunction with the flight schedule to generate the inputs for the environmental models.

Our first step was to determine the scope or boundaries of the analysis. As in the capacity constraint analysis, we considered 310 airports. However, for the environmental analysis, we developed high-fidelity inputs for the 56 FACT2 airports, and for all 310 airports, we developed screening-level inputs. For each of the FACT2 airports, we generated trajectories from the airport to roughly $40 \mathrm{~nm}$. However, for the noise metric, we needed to address only the population exposed to $65 \mathrm{~dB}$ DNL; because that exposure level generally occurs close to the airport, we considered only population residing within $20 \mathrm{~nm}$ of the airport.

Our next step was to identify a representative data sample and use it to generate the backbones to be used as input to the environmental model. We began by 
reviewing terminal area data developed to support JPDO environmental analyses of the 34 OEP airports in the continental United States (CONUS):

Because the operational data modeled by JPDO was characterized as a "good" weather day in the NAS, the assumption was made that the radar data should also represent a good-weather period. Use of a good-weather period also seems appropriate since there is some expectation that future capabilities will push the IMC capacity restrictions to VMC levels. Using the period of September 2004, through September 2005, as a basis, Aviation System Performance Metrics (ASPM) and Ground Delay Programs (GDP) data for the airports within the study were reviewed. This analysis identified April 2005, as a period when good weather occurred for a significant number of the airports. The source data for this 30-day sample was an extraction from the ATA-Lab Offload archive which provides detailed terminal data for most modeled airports.

We supplemented the data to incorporate the remaining FACT2 airports and, in some cases, provided an update to OEP airports to include new runways that had become operational. We also selected radar samples that spanned multiple months in an effort to include more seasonal variation and demand. We assumed that the radar data sample represented appropriate traffic variability, and we used the data to derive time-of-day usage, fix loadings, runway use, and primary airport configurations.

For the detailed analysis of the radar track data for all modeled airports, we used Metron Aviation's Airspace Design Tool (ADT). We first separated the data by airport, then by operation type (arrival, departure), and then by runway. We then grouped the tracks using unique characteristics such as departure headings, arrival intersections, and altitude. Key arrival and departure fixes were also used to identify unique traffic flows. Once the traffic flows were identified, we calculated a statistically determined center track (or backbone) for each group based on track density within each flow. To depict the observed lateral dispersion of operations within a flight corridor, we also defined a set of subtracks associated with each center track. The width and density of the flow determined the number of dispersed subtracks within a corridor, and the distribution of radar tracks within a corridor determined the percentage use or weighting of each subtrack. In addition, we reviewed each backbone's profile to identify any deviations from a 3-degree angle of descent or an unrestricted climb. If sufficient deviations were identified, altitude controls recognized by the flight state generator were placed on the backbone in order to better emulate the performance and flight profiles. Finally, we transferred the operations from the flight schedule to the resulting backbones and used them in an event-weighting process.

Figure 6-4 presents an example of the method applied to identify and generate arrival backbones. The figure shows a portion of the traffic at ORD, with further refinements in the analysis that resulted in the backbones and associated subtracks. 


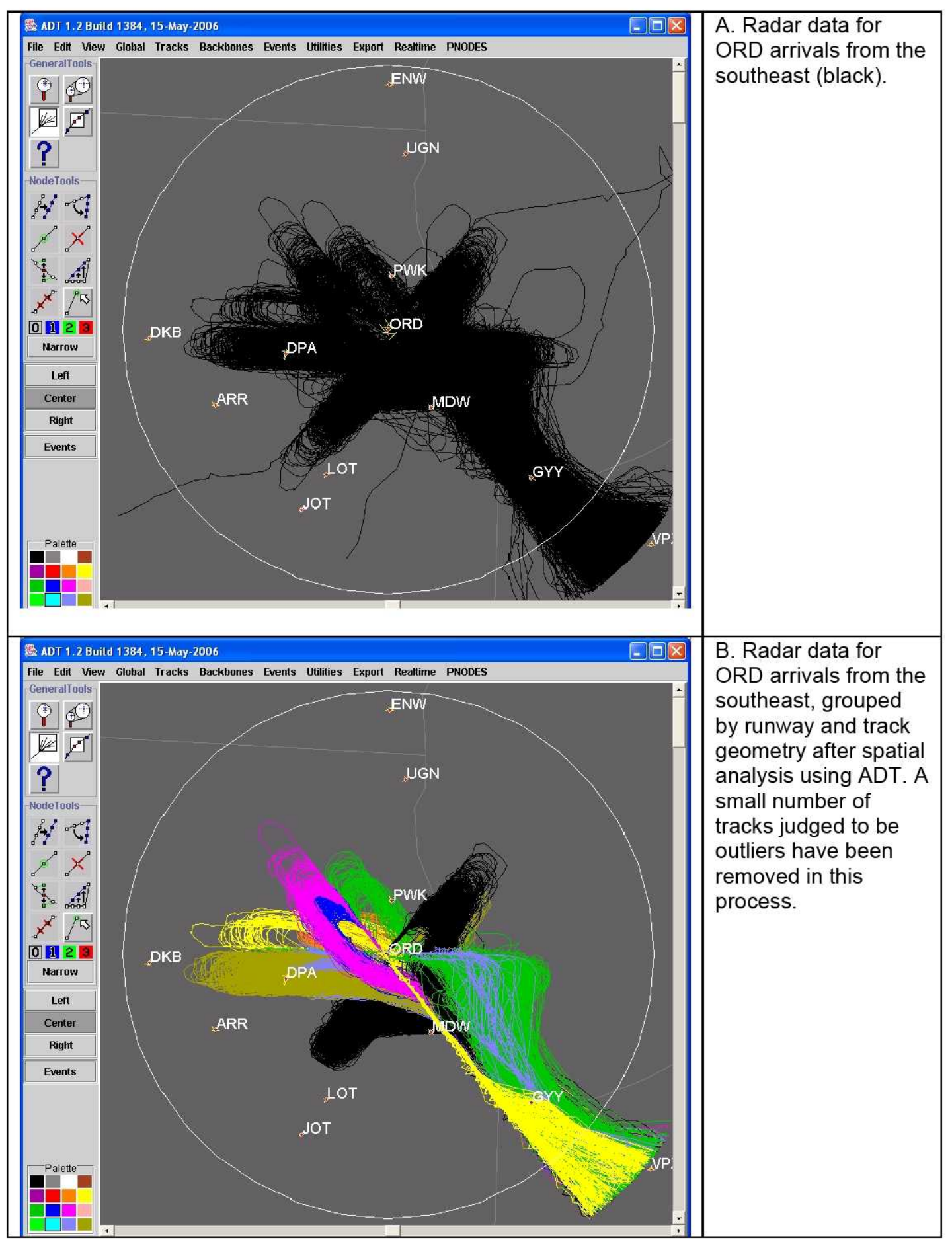




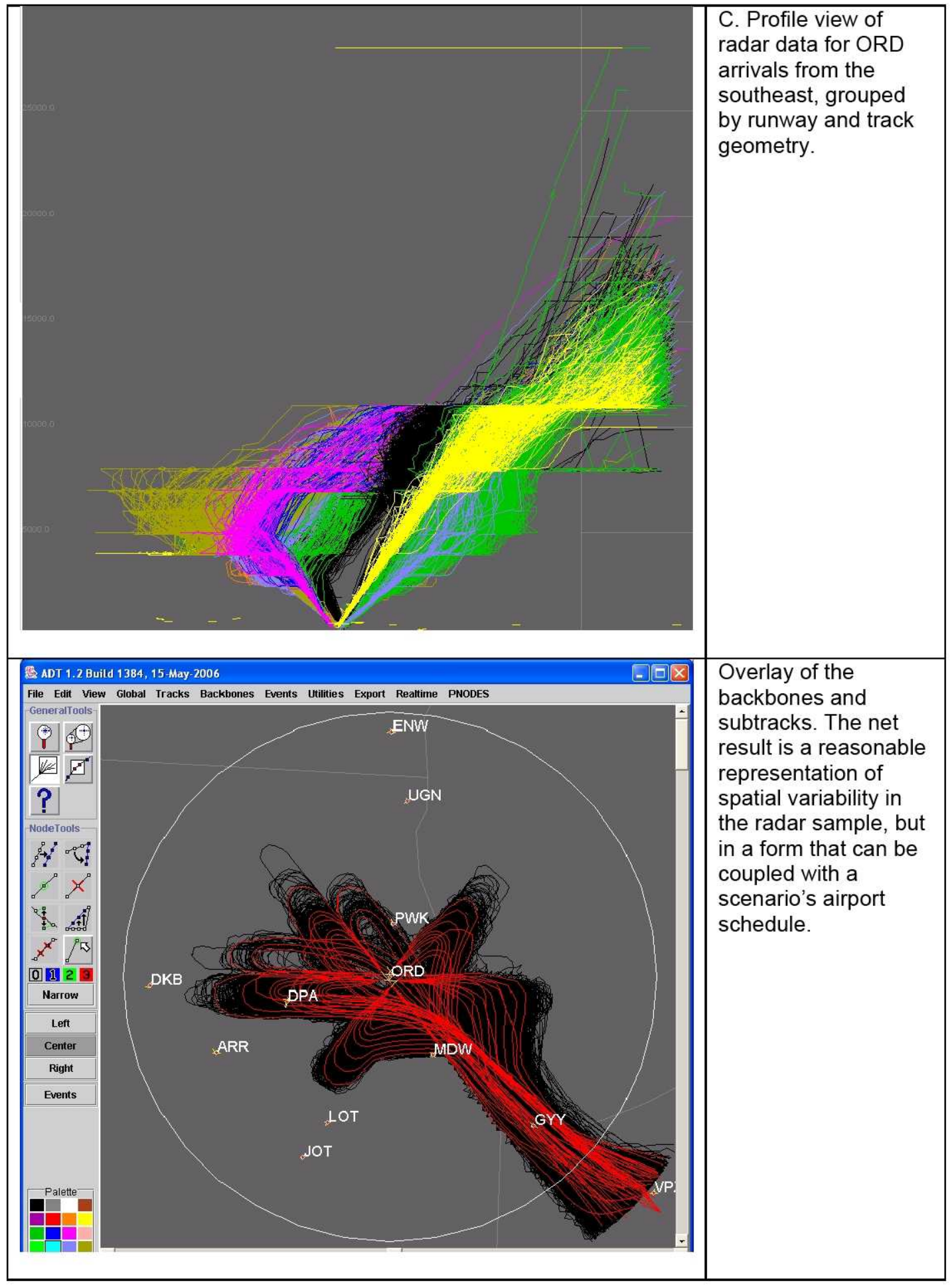

Figure 6-4. Terminal area development. 
This process was applied to each of the FACT2 airports. Environmental input files were defined by airport and operation and were loaded into environmental models for flight performance, fuel efficiency, emissions, and noise modeling.

\section{SCHEDULED DEMAND}

The scheduled demand for all 310 airports was extracted from the 2007, 2015, and 2025 schedules. We converted all times to local times to account for noise penalties assigned to flights occurring during nighttime periods. Both the spreadsheet-based and screening models require minimal schedule input: aircraft, origin/

destination, and time of operation. For the high-fidelity model, we needed additional spatial information related to departure and arrival fixes at the FACT2 airports. This information enabled us to assign flight operations to particular runways; it also helped in assigning potential heading changes near the runways.

One challenge was mapping the schedules to aircraft. In many cases, the schedules included the aircraft required for modeling fuel efficiency and emissions, but the set of aircraft needed for modeling noise was much smaller. The original schedules had aircraft names defined by the Enhanced Traffic Management System (ETMS), while fuel and emissions calculations were limited to aircraft and engines defined by EDMS, and noise calculations were limited to aircraft defined by NIRS. Furthermore, because the schedules did not include information related to an aircraft's engine, default engines were assigned considering use in the current fleet.

\section{Fuel Efficiency and Emissions Computations}

We calculated fuel efficiency and emissions for each flight using a combination of fuel-flow values below 3,000 feet AFE from EDMS 4.3 and fuel-flow values above 3,000 feet AFE from the Base of Aircraft Data (BADA). The basic fuel burn equation is given by

$$
\text { Fuel Burned }=t_{\mathrm{m}} * R_{\mathrm{m}}
$$

where

$t_{\mathrm{m}}$ is the time in minutes for a mode of operation $m$, and

$R_{\mathrm{m}}$ is the rate of fuel flow in $\mathrm{kg} /$ minute for a mode of operation $m$.

BADA fuel flow is expressed in $\mathrm{kg} / \mathrm{sec}$ for the aircraft during the phases of climb, cruise, and descent at different altitudes. EDMS fuel flow is expressed in $\mathrm{kg} / \mathrm{sec}$ for each engine of the aircraft during the phases of taxi/idle, takeoff (to 1,000 feet above ground level, or AGL), climb (1,000 feet AGL to 3,000 feet AGL), and approach (3,000 feet AGL to touchdown). Because flow is for a single engine, the EDMS rate must be multiplied by the number of engines on the airframe. 
The fuel efficiency of a flight is calculated from the total fuel burned and the distance traveled during flight. The basic fuel efficiency equation is given by

$$
\text { Efficiency }=(C * d) / F \text {, }
$$

where

$F$ is the mass in $\mathrm{kg}$ of fuel burned,

$C$ is the seat capacity of the aircraft in number of seats, and

$d$ is the ground-track distance traveled in $\mathrm{km}$.

Emissions calculations utilize the value of fuel burned in each phase to compute the mass of pollutants - $\mathrm{CO}, \mathrm{HC}, \mathrm{NOx}$, and $\mathrm{SOx}$ - concurrently generated. The basic emissions equation is given by

$$
\text { Pollutant Mass }=F_{m} * E I_{m} \text {, }
$$

where

$F_{m}$ is the mass in $\mathrm{kg}$ of fuel burned in mode $\mathrm{m}$, and

$E I_{m}$ is the emissions index in grams $/ \mathrm{kg}$ for pollutant generated in mode $\mathrm{m}$.

The following are the processing steps for computing fuel burn, emissions inventory, and flight ground track distance:

- The taxi in/out time is provided by analysis of ASPM data describing average taxi times, and EDMS taxi/idle fuel-flow values are used to derive the fuel burn during the taxi phase. Because aircraft ground movement is not modeled, zero distance is attributed to this phase of operation.

- The airborne aircraft trajectory is broken into several phases for fuel burn and emissions computation:

$>$ EDMS takeoff fuel flow from takeoff to 1,000 feet AGL.

> EDMS climb fuel flow from 1,000 feet AGL to 3,000 feet AGL.

$>$ EDMS approach fuel flow from 3,000 feet AGL to touchdown.

$>$ BADA fuel flow for all portions of the trajectory above 3,000 feet AFE. To apply BADA fuel-flow factors, we classified each distinct segment as either a climb segment, a cruise segment, or a descent segment. 
- The trajectory's ground-track distance is computed in kilometers from the beginning of takeoff roll to the end of touchdown on a segment-bysegment basis.

The key difference in how these equations were applied for each model lies in the inputs or assumptions used. For the spreadsheet-based model, we used nominal times in mode and flight distances provided by ICAO; nominal times are typically used for this level of analysis. For the screening model, a simple extension leveraging the origin/destination information provided additional stage-length or weight data, which improved the times in mode as well as the track distances. For the high-fidelity model, terminal area trajectories are shared with the noise model, providing identical fidelity for trajectory-based calculations.

\section{Noise Computations}

The FAA requires that all detailed noise analyses use the most current version of the FAA's Integrated Noise Model (INM), Heliport Noise Model, or NIRS. In addition, the FAA has determined that NIRS must be used for modeling noise impacts from the ground to 10,000 feet AFE when the study area is larger than the immediate vicinity of an airport, incorporates more than one airport, or includes air traffic airspace actions that are more than 3,000 feet AFE.

Another contributing factor to the analysis is the increased scope of the number of airports to be considered for noise analysis. We developed high-fidelity model inputs for the 56 FACT2 airports in our set of 310 airports. For all 310 airports in the analysis, we applied two additional noise modeling strategies.

For the spreadsheet-based model, we used the FAA's AEM. This method, rather than focusing on the number of people exposed to noise, computes the area exposed to noise and therefore does not map well to the desired metric. The AEM, which has been available for some years, uses fitted exponential functions to provide a rapid means of estimating the area within a DNL contour (as modeled within INM) based on the number of flights, event times, and aircraft types.

In order to compute a noise level that was based on the required noise metric of population exposed to a certain threshold of noise, a new screening model was developed. The environmental screener takes a slightly different approach of populating a database of flight performance and noise information related to each aircraft than the higher-fidelity approach. We used several tools, including NIRS, to create a set of densely spaced grid locations with noise contributions from a single arrival or departure for each aircraft. This is done by defining a simple runway layout and by applying default assumptions for weather conditions, terrain, and flight track geometry. The locations grid can then be oriented to a specific airport's runway and aggregated with other grids to compute a noise contour. The population's noise exposure is produced when the noise contour is combined with the set of population locations. As described earlier, the screening model requires several inputs: aircraft, operation (arrival and departure), origin 
and destination, operation time (local), population, and runway configurations and use.

In preparation for modeling, we identified the two primary runway configurations and the corresponding runway usage when radar data were not available. Below are the tools and data sources used to identify runway configurations and runway use:

- ASPM. ASPM contains runway configuration data for 77 reported airports, which intersect those for which we have radar data.

- Airport reports. Some airports report primary runway configurations and usage on their websites in master plans or noise abatement documents.

- Historical weather data. When radar data and other sources were not readily available, wind rose data - which provide 30 years worth of historical wind speed and direction at many U.S. locations - were used to identify runway configurations.

- Post-Operations Evaluation Tool (POET). POET is a visualization tool that allows for the review of flight tracks arriving and departing from the airport in question.

- Radar data. Radar data used to develop the high-fidelity terminal area trajectories were used for defining runway use at the FACT2 airports.

ASPM calculates a percentage of hourly use for runway configurations at the reported airports. We chose the two longest-held configurations for 2007 as the primary and secondary configurations. Select airport websites contain runway use, prevailing wind directions, and runway configurations; they were useful when ASPM or radar data were not available. We used wind rose data at the airport's location or the closest city to determine the direction of the prevailing winds. Assuming that most aircraft operations are performed into the wind, we were able to determine configurations and usage. When airport and wind data were not available, we used POET to assist with determining the primary and secondary configurations and runway usage by the number of arrival and departure routes from each runway end. All of the above tools enabled us to gather the most accurate information possible.

NIRS, which we used to calculate noise levels, requires the following principal inputs:

- Study area - study center location, size, elevation at the center, maximum altitude, and average meteorological conditions.

- Population - set of point locations (latitude/longitude) that describe the population location and density. Typically, these data are developed from the Census Bureau, as described above. 
- Terrain - data extracted from the U.S. Geological Survey. These data are used in conjunction with the population locations to determine the actual distance between a flight route and the population.

- Runways - list of runways and their location information.

- Traffic files - set of files that define flight operations within the study. These files contain flight track geometry and flight operations (aircraft type, origin, destination, time of day and weighting) for each route.

For this noise analysis, we assumed the following:

- A single NIRS study was defined to cover the entire country.

- The default meteorological conditions were used for the entire study area.

- Detailed study-area terrain data were used for each of the airports to properly account for changes in elevation.

- The NIRS default altitude of 18,000 feet MSL was used as a study ceiling.

- Population was assumed to stay constant at 2000 levels for all scenarios and was defined to include people located within $20 \mathrm{~nm}$ of a study airport. Although the current metric for noise is the number of people exposed to $65 \mathrm{DNL}$ or greater, it was anticipated that exposures at lower levels may be of interest.

- Runways were consistent with the traffic sample used to generate the terminal area trajectories. No future runways were added to the model for any of the FACT2 airports, but data on future runways could be incorporated. ORD, where massive runway construction and realignment are planned, is a prime example.

- No operational changes were considered. This means that the terminal area trajectories were not augmented to suggest improvements or changes in scenarios.

- The fleet mix for future scenarios was incorporated into the analysis.

- Time of day was extracted from the flight schedules and used to define day/night split.

- Runway use and airport configurations were assumed to be accurately captured from the radar sample collected. When the data were not good enough to define the routes, the analysts used their professional judgment.

- Traffic files were divided by airport and operation. Traffic files could be regenerated in different ways to support more detailed scaling. 
For each scenario $(2007,2015$, and 2025), we produced two NIRS traffic files for each airport, one for arrivals and one for departures. For each of the traffic files, we did the following:

- Imported the traffic file into NIRS.

- Used the NIRS Flight Segment Generator (FSG) to apply SAE 1845 equations and the NIRS aircraft performance data to simulate aircraft performance to meet the trajectories defined within the traffic file. For more detail about FSG, please refer to the NIRS User Guide.

- Computed noise using the FSG results for points within a $20 \mathrm{~nm}$ radius of the airport servicing the traffic in each file. For example, if the traffic file was for ORD departures, we computed noise for all population locations within a $20 \mathrm{~nm}$ radius of ORD. If the $20 \mathrm{~nm}$ rings of multiple airports intersected, we combined the population for the noise computations. For example, ORD and Midway airport (MDW) are within $40 \mathrm{~nm}$; therefore, the population surrounding MDW may be affected by operations from ORD. To consider the cumulative effect of noise, we combined the locations for both MDW and ORD into a single population file for both airports.

After computing noise for all flights and airports, we aggregated the resulting noise exposure for each population centroid. We then calculated the number of people exposed to greater than $65 \mathrm{~dB}$ DNL.

\section{ANALYSIS OF ENVIRONMENTAL CONSTRAINTS AT CRITICAL AIRPORTS}

We determined the size and specificity of environmental constraints on airport operations based on the estimated environmental metrics and goals. One of the key distinguishing features of our overall technical approach is that we are able to analyze capacity and environmental constraints concurrently, giving them equal weight, or independently to evaluate each environmental constraint.

Our analysis of airport environmental constraints began with an analysis of the environmental impacts for a future without NextGen. Figure 6-5 shows our approach to modeling a scenario to characterize future environmental conditions. 


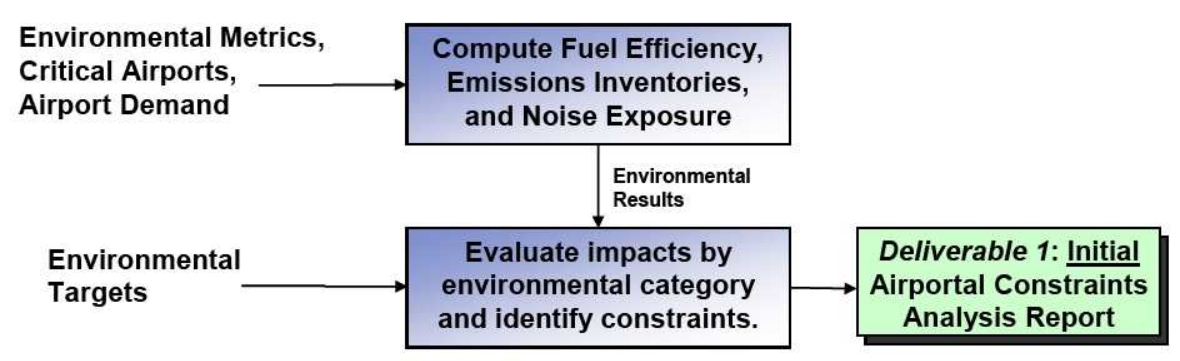

Figure 6-5. Approach to airport environmental constraints analysis.

We began by calculating impacts in terms of fuel efficiency, emissions, and noise using standard metrics and compared them to those same metrics for a baseline year. We then defined the constraints on each airport in terms of the size of these impacts relative to a future-year goal.

Next, we analyzed the gaps in terms of an "unsatisfied demand" metric: the number of flights that need to be trimmed from the projected demand to meet the environmental goals. Thus, we directly compared capacity and environmental constraints in an innovative integrated analysis to determine which constraints are most binding.

More realistic methods of flight trimming to achieve environmental goals would require an equitable distribution of trimmed flights. The definition of "equitable" would include a combination of policy and economic considerations such as the distribution of trimmed flights among carriers, aircraft and engine manufacturers, and the frequency of operations at each airport. Future studies may explore various equitable trimming scenarios with the goal of assessing the impact of specific policy proposals.

\section{Fuel Efficiency}

For our constraints analysis related to fuel efficiency, we focused on a method that allowed for trimming individual flights to reach the defined environmental target. Fuel efficiency was measured on a per flight basis as previously defined. This method is extensible to several forms of the fuel efficiency metric (for example, fuel per unit of payload distance).

We explored several methods of trimming flights from the 2015 and 2025 forecast schedules until the fuel efficiency goals were achieved. We ranked flights in order of various metrics such as greatest fuel burned per flight, per seat, or per kilogram of payload. We then removed individual flights in order, starting with the "worst first" in terms of fuel burned produced per landing and takeoff operation (LTO) until the fuel efficiency produced at each airport met the target. 
The worst-first method trims the schedule by the least amount because the greatest share of fuel burned is generated by the trimmed flights. Other methods trim more flights because the constraints are spread across a wider variety of aircraft. Additional metrics such as a payload-based metric could yield different results.

\section{Emissions}

For emissions, we calculated NOx produced. We chose to focus on NOx because of its role in forming ground-level ozone, which, together with particulates, is a common measure of local air quality. The quantity of NOx produced is expressed in kilograms per LTO. We calculated values for total NOx produced daily at each airport by summing the NOx produced by each LTO in the 2007, 2015, and 2025 schedules.

The ICAO method for measuring emissions applies an emissions index (EI) value for the amount of NOx produced by an engine during four phases of flight: takeoff, climb-out to 3,000 feet AGL, approach from 3,000 feet AGL, and taxi/idle time. The emission indices are based on testing data provided by the engine manufacturer under different throttle conditions.

Calculating NOx per engine is then a matter of multiplying the emissions index by the fuel flow for the typical throttle setting in each flight phase, multiplied by the time spent in that mode. The NOx per engine is multiplied by the number of engines, and the NOx from the four phases of flight are summed to find the NOx per LTO:

$$
N O x \text { per } L T O=E n * \sum(E I * F F * T)_{i},
$$

where

$E n=$ the number of engines on the airframe,

$E I=$ the emissions index for grams of pollutant per $\mathrm{kg}$ of fuel burned,

$F F=$ the fuel flow rate in the flight mode $(\mathrm{kg} / \mathrm{s})$,

$T=$ time in mode, and

$i=$ the four modes of flight (takeoff, climb-out, approach, and idle).

To compare NOx values between aircraft, NOx per LTO can be normalized in terms of NOx per seat or kilogram of payload. 
This method, while long used as a standard for computing aircraft emissions, is very sensitive to the actual time spent in each of the flight modes. Recent studies ${ }^{5}$ have worked to quantify how fleet evolution since the development of the ICAO method in the 1970 s affects emission calculations. In particular, the default time in mode specified in the ICAO standard tends to overestimate the amount of emissions produced by today's twin engine aircraft compared to the four-engine aircraft typical nearly 40 years ago. Also, real-world flight data show significant variability in actual time in mode and throttle settings. We used time-in-mode values from the EDMS where available, which typically specify shorter times for small aircraft. These values help to reduce the overestimation in the ICAO standard profile, but EDMS times are identical to the standard ICAO times for many common aircraft. Table 6-6 shows the standard ICAO times-in-mode.

Table 6-6. ICAO Default Times in Mode

\begin{tabular}{|l|c|}
\hline \multicolumn{1}{|c|}{ ICAO standard profile } & Time in mode (minutes) \\
\hline Takeoff & 0.7 \\
\hline Climb-out to 3,000 feet AGL & 2.2 \\
\hline Approach from 3,000 feet AGL & 4.0 \\
\hline Taxi/idle & 26.0 \\
\hline
\end{tabular}

Because each aircraft that arrives at an airport eventually departs, we calculated NOx per LTO for flights in the arrival dataset. The baseline and forecast data did not identify aircraft types for VFR flights, so we applied the JPDO's method of using a 2-to-1 ratio for turboprop and piston aircraft. The assigned turboprops were the Beechcraft BE20 and assigned pistons were the Cessna C421.

Data on military flights (about 1 percent of flights in the data set) also did not identify an aircraft type. Future analyses may need to examine separately the role of military flights in total NOx produced when they represent a significant fraction of an airport's operations.

We explored several methods of trimming flights from the 2015 and 2025 forecast schedules until the NOx goals were achieved. We ranked flights in order of various metrics such as greatest emissions per flight, per seat, or per kilogram of payload. We then removed individual flights in order, starting with the worst first in terms of NOx produced per LTO until the NOx produced at each airport met the target goal.

The worst-first method trims the schedule by the least amount because the greatest share of emissions is generated by the trimmed flights. Other methods trim more flights because the emissions are spread across a wider variety of

\footnotetext{
${ }^{5}$ Judith Patterson, George Noel, David Senzig, Chris Roof, and Gregg Fleming, "Analysis of the ICAO Departure Profile Using Real Time Cockpit Flight Data Recorder Information" (paper, Transportation Research Board, 2008 annual meeting).
} 
aircraft. By exploring different trimming methods and worst-first rankings, we could develop a trimmed schedule that considers the distribution of flights at an airport and different payload configurations.

\section{Noise}

In our analysis of constraints related to noise, we sought to develop a method that would not only provide the metric of interest but allow for an iterative approach to trimming individual flights to meet the defined target. Again, we used a worstfirst method of ranking aircraft to identify which flights in the schedules should be trimmed. Other metrics that could be considered include the following:

- Normalize by weight. Heavier aircraft usually will produce more noise and usually carry more passengers or more freight. Therefore, to identify the worst offenders independent of how heavy they are, we divided the values of AEM areas of the aircraft by their maximum takeoff weight.

- Normalize by number of seats. Similarly, to see which aircraft were the worst offenders independent of how many seats (passengers) they could carry, we normalized by number of seats. This method does not translate well to freight flights even though the aircraft may have a typical seat configuration.

We parsed the 2007, 2015, and 2025 flight schedules by airport, converting them into schedules that both the spreadsheet-based and screening models could handle. Specifically, we created a simple text file that has the aircraft name, origin and destination, and local time of the flight. Because finding the AEM area or the screening model's population exposed for a certain schedule involves a nonlinear operation, the algorithm has to be rerun every time the schedule is changed.

We trimmed the schedules by going down the ranking list and removing a day and night operation from arrivals and departures. In the spreadsheet-based model, we then recomputed the area and, if it was not at or below the baseline value for that airport, we trimmed the operation of the next aircraft on the list. We continued this process until the baseline and future AEM areas matched. In the screening model, we recomputed the population exposed to $65 \mathrm{~dB}$ DNL and, if it was not at or below the desired target for that airport, we trimmed the next pair of operations until the future scenario met the target.

The results from the screening model show some airports with an extreme level of trimming required to meet the target. In most cases, this was a result of census locations with high population placed either on or near the airport. For example, Figure 6-6 shows a large population point representing 838 people very close to the new runway at Cincinnati/Northern Kentucky International Airport (CVG). Not even the Google satellite image shows the new runway, but a review of the airport's layout provides latitude and longitude information such that the runway 
can be plotted. For comparison, Figure 6-7 displays the current airport diagram for CVG.

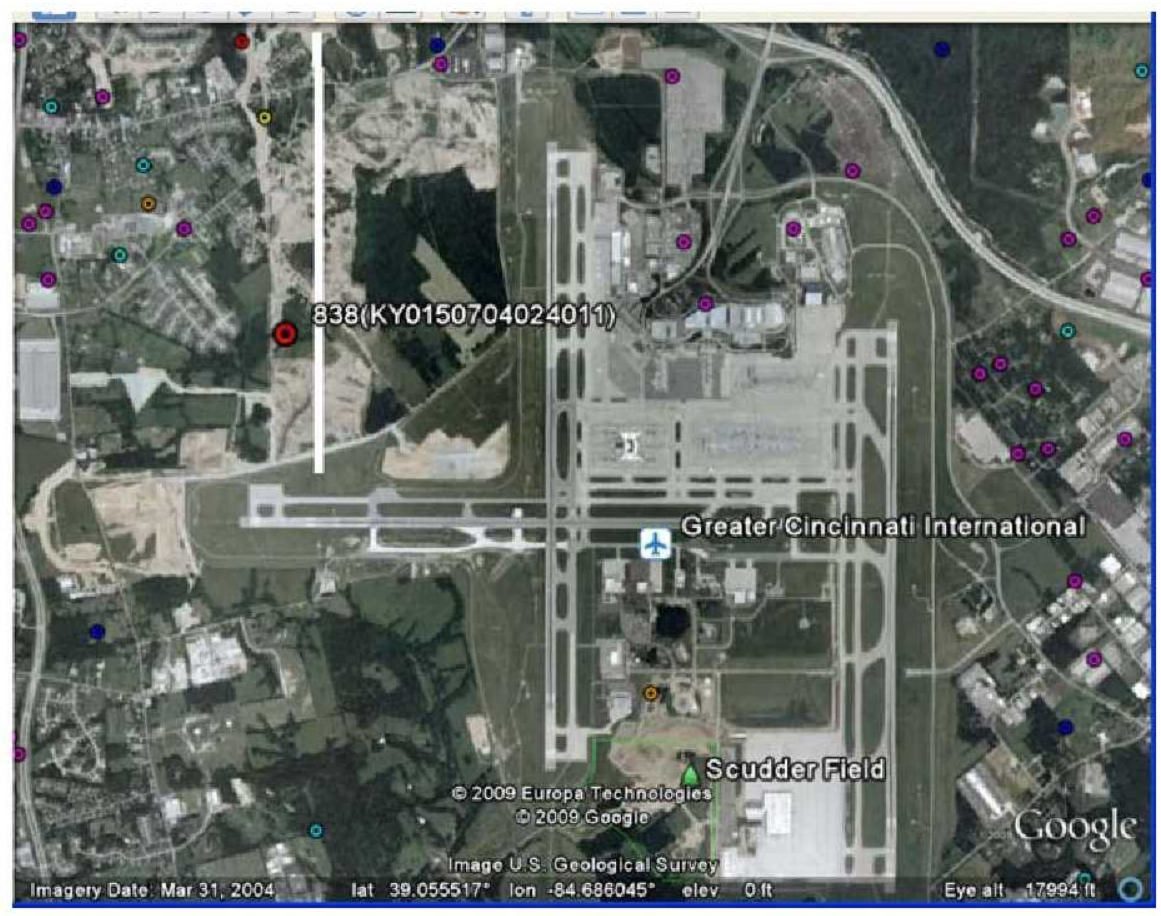

Figure 6-6. Cincinnati/Northern Kentucky International Airport (CVG) with a census population point on the airport grounds. 
08325

AIRPORT DIAGRAM

COVINGTON/CINCINNAT//NORTHERN KENTUCKY INTL (CVG)

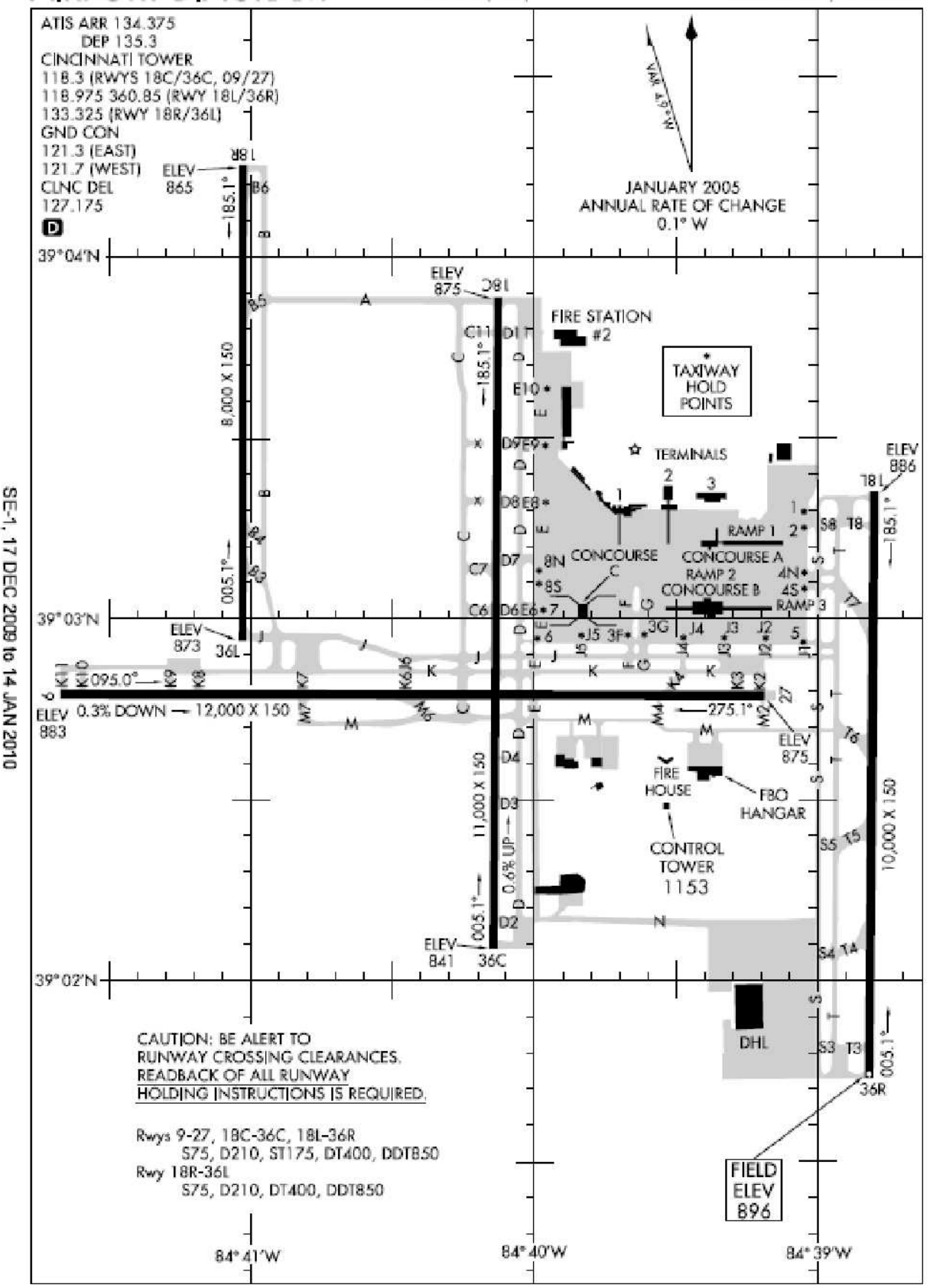

AIRPORT DIAGRAM COVINGTON/ CINCINNATI/NORTHERN KENTUCKY INTL (CVG)

Figure 6-7. Airport diagram of CVG. 
Hayward Executive Airport (HWD), in Hayward, California shows a similarly large census population point, with 354 people near the runway (Figure 6-8).

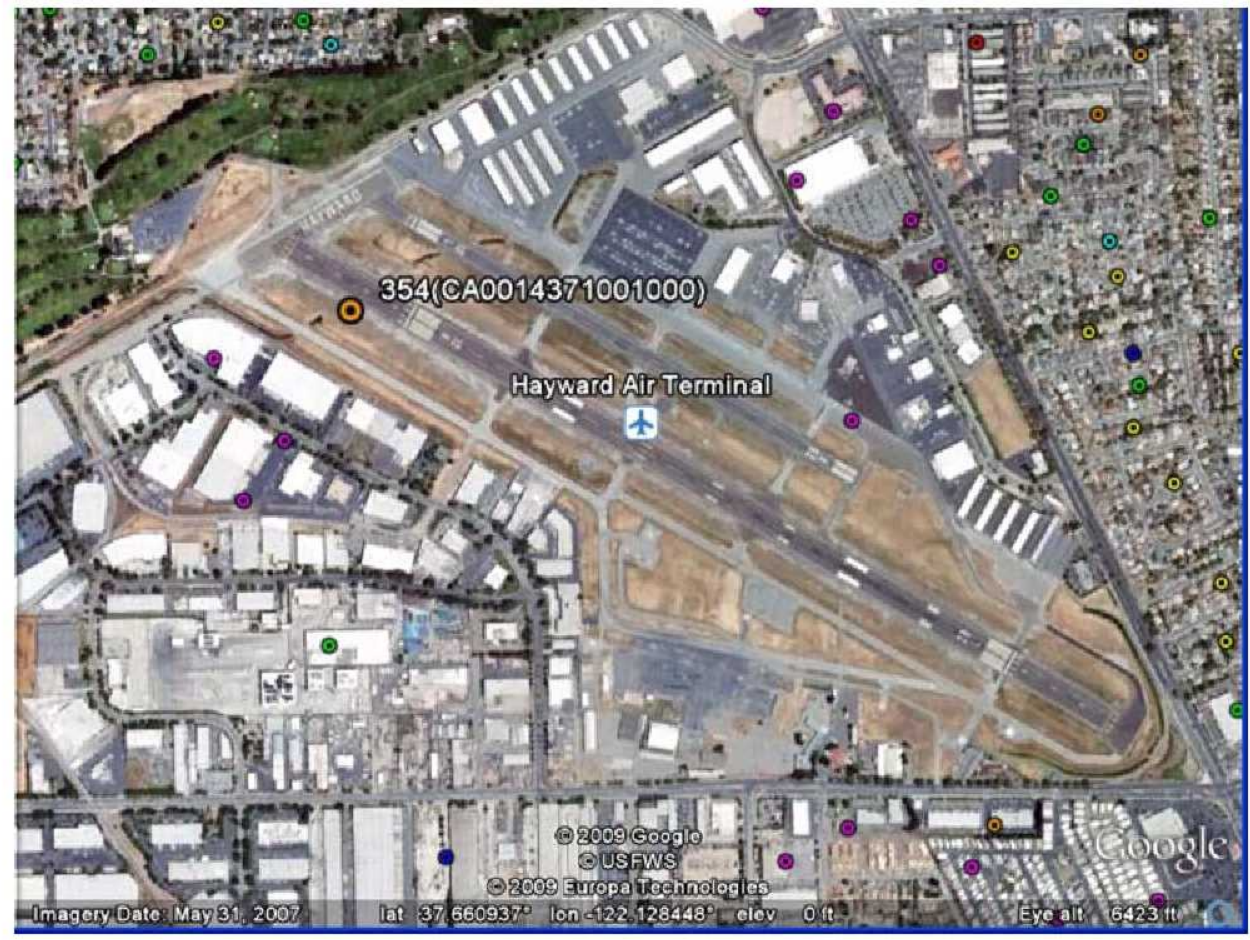

Figure 6-8. Hayward Executive Airport (HWD) with a census population point near the runway.

Future noise modeling could correct for population distribution. The population point represents the weighted centroid of the census tract as determined by the U.S. Census Bureau. But concentrating the population on the centroid is exaggerating the level of noise experienced by people who live in census tracts that incorporate the airport property when the centroid is near a runway.

\section{RESULTS}

Figures 6-9 through 6-11 display the 2015 and 2025 aggregate demand and percentage throughput under the constraints of fuel efficiency, NOx, and noise for the busiest 10, OEP 35, LMI 110, and LMI 310 airport groups.

In Figure 6-9, one can see that the feasible throughput under the fuel-efficiency constraint is roughly 93 to 97 percent for all airport groups and for both years. The fuel-efficiency constraint becomes slightly more binding in 2025 than in 2015 for all 310 airports in total, but slightly less binding for the busiest airports. 


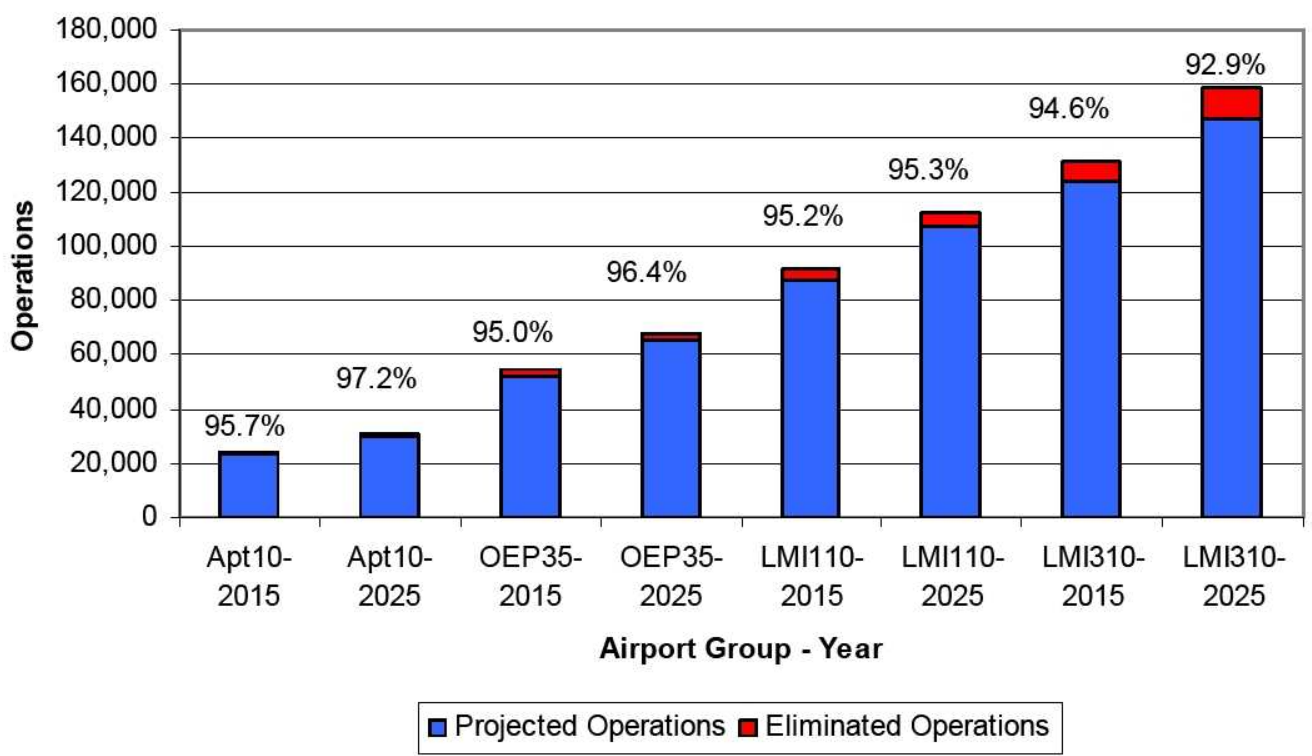

Figure 6-9. Unconstrained and fuel-constrained throughput by airport groups.

In Figure 6-10, one can see that the feasible throughput under the NOx constraint ranges from about 92 to 94 percent for all airport groups and for both years. Its constraint changes by 1 to 3 percent points when we compare the same airport group in the 2 years. There is, however, no general trend when comparing the airport groups.

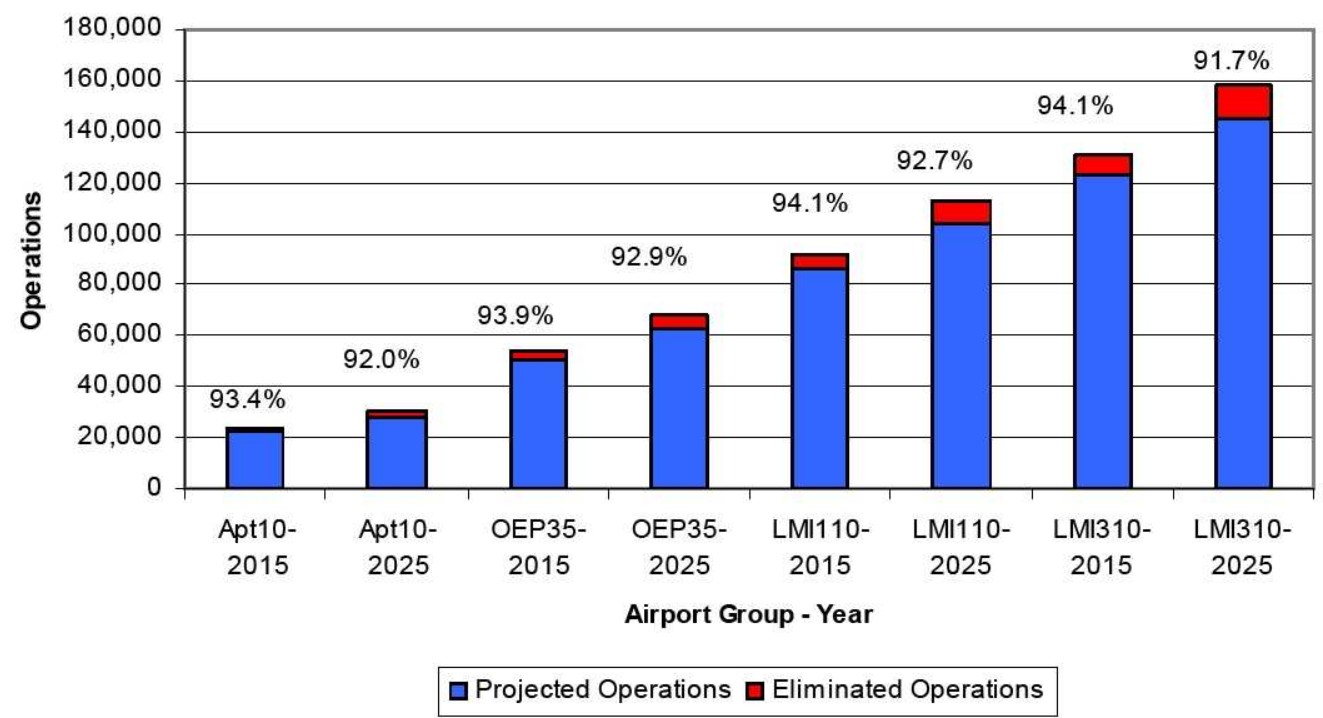

Figure 6-10. 2015 and 2025 unconstrained and NOx-constrained throughput by airport groups. 
In Figure 6-11, one cannot find any pattern when comparing the feasible throughput percentages for the same airport group in the 2 years or for different airport groups in the same year. In general, the feasible throughput percentages are roughly 85 percent for the OEP 35, LMI 110, and LMI 310 airport groups, but higher (around 90 percent) for the busiest 10 airports.

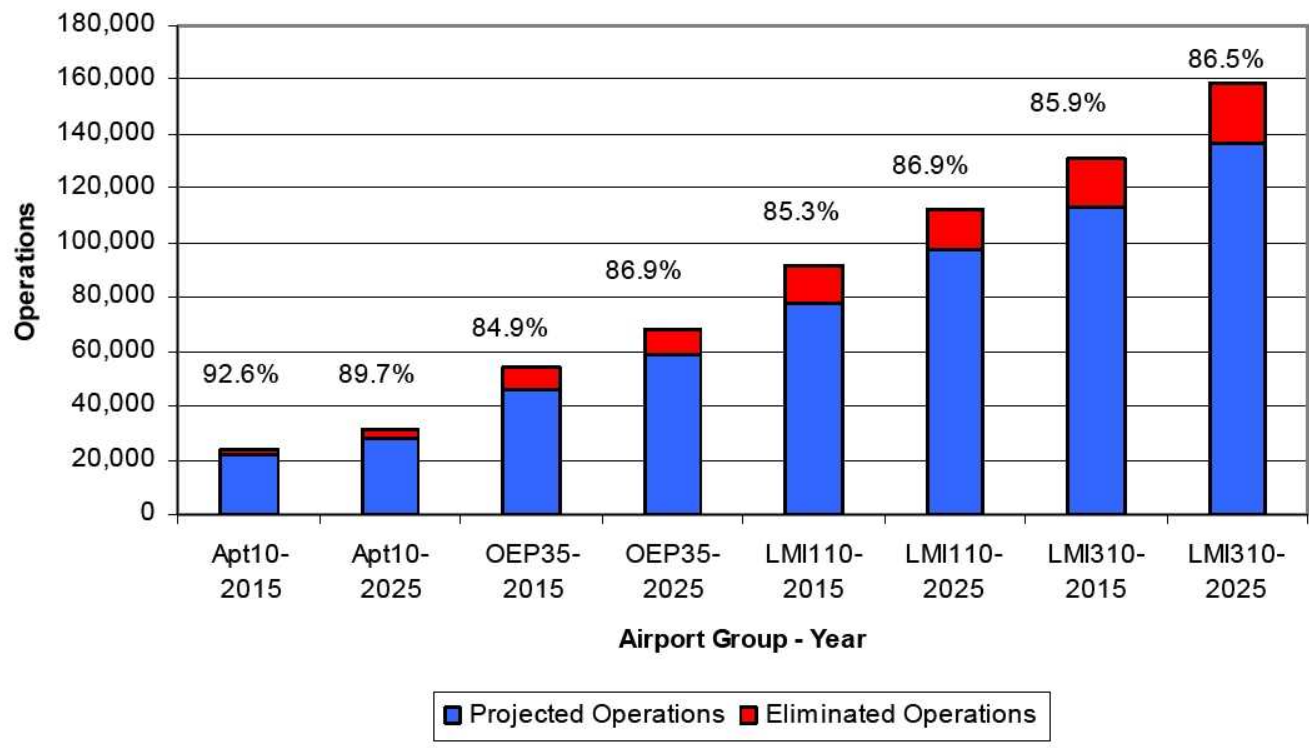

Figure 6-11. 2015 and 2025 unconstrained and noise-constrained throughput by airport groups.

In general, of the three environmental constraints, noise is the most binding, followed by NOx and fuel efficiency. 
Because of the traffic volume at the busiest 10 airports, we analyzed that airport group in more detail. Figures 6-12 through 6-14 show the results.

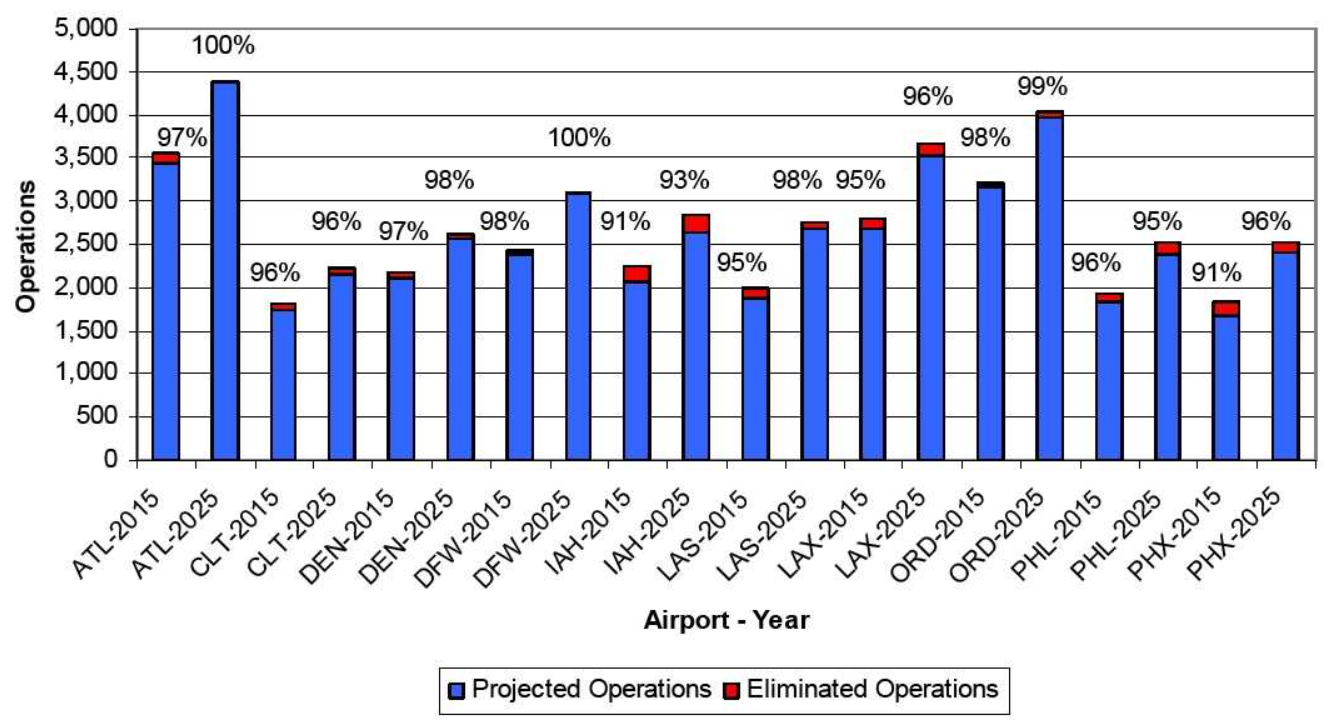

Figure 6-12. 2015 and 2025 unconstrained and fuel-constrained throughput for the 10 busiest airports.

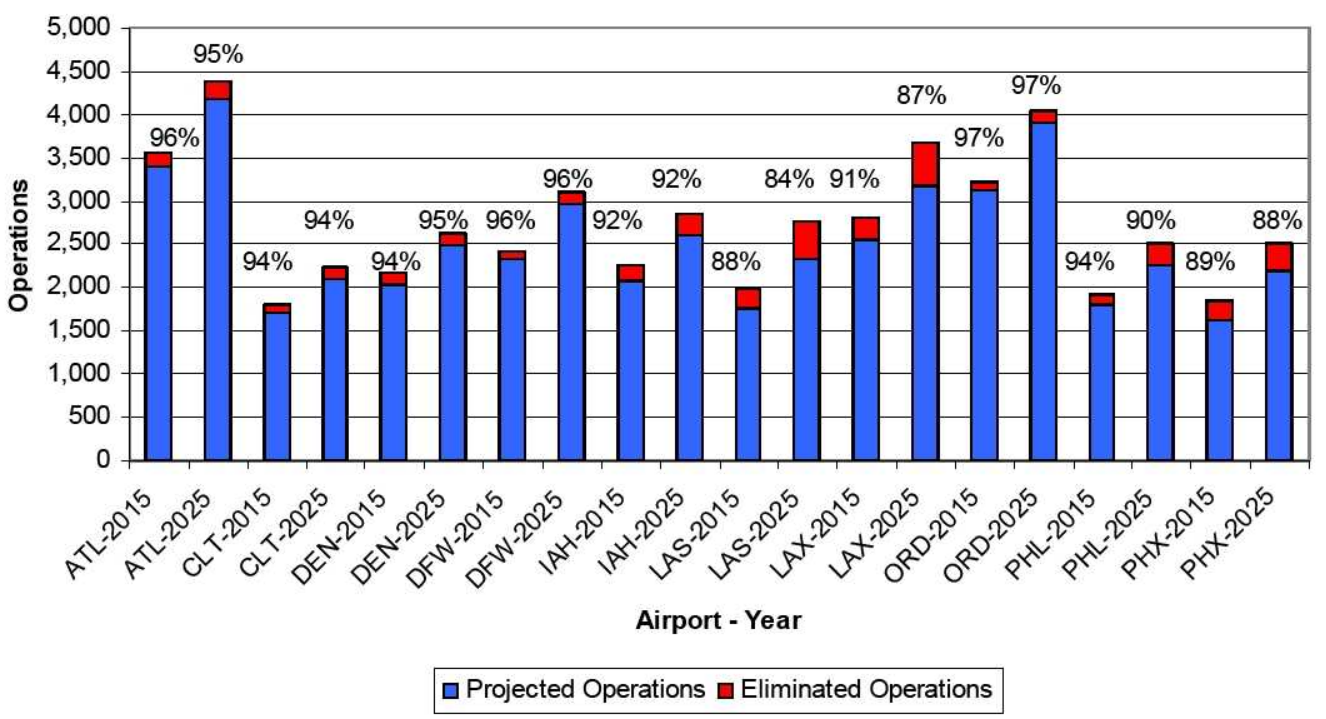

Figure 6-13. 2015 and 2025 unconstrained and NOx-constrained throughput for the 10 busiest airports. 


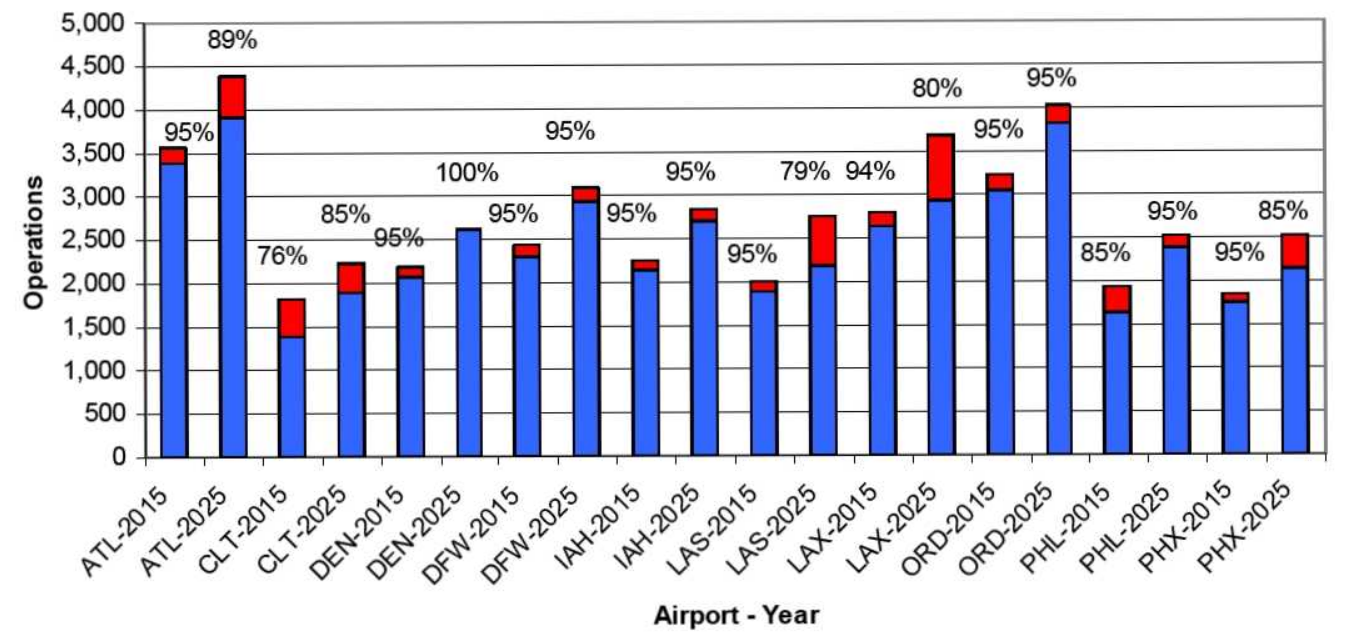

口Projected Operations a Eliminated Operations

Figure 6-14. 2015 and 2025 unconstrained and noise-constrained throughput for the 10 busiest airports. 


\section{Chapter 7 \\ Catalog the Primary and Secondary Airport Constraints}

This chapter summarizes the results of Chapters 5 and 6 and identifies the primary and secondary constraints. Again, for ease of identification, the results are presented by airport groups: busiest 10 airports, OEP 35 airports, LMI 110 airports, and LMI 310 airports. For each group, we provide two graphs, one for 2015 and the other for 2025 , comparing the unconstrained throughput with the throughput if airports are subject to capacity or environmental constraints: runway, taxiway, gate, fuel efficiency, NOx, and noise. We also provide tables showing details about the effects of constraints on each individual airport's feasible throughput.

Our results indicate that some airports will face constraints even with the implementation of NextGen. The runway and taxi constraints are more concentrated in the large airports and the environmental constraints are present at almost every airport regardless of its size. The environmental goals are quite aggressive and directly affect the results of this study; if the goals were not so ambitious then the environmental constraints assessed by this study would be correspondingly less binding. More revolutionary concepts and technologies, in airframes, engines, and ATM, ought to be explored as ways to address the constraints and to ultimately satisfy the forecast traffic growth.

\section{UNCERTAINTIES AND LIMITATIONS}

While we believe our analysis methodology is sound, our approach of decomposing the system into separate constraints is an analytical technique; we recognize that in the real world, everything is interconnected. We also note the uncertainties involved in our analysis. First, demand forecasts of air travel are constantly changing and imperfect. The economic slow-down of 2008-2009 will lower the air traffic volume compared to the demand forecasts we used in the study which were from 20062007. Second, the NextGen capacities are assumed at their planned capability level and schedule which may eventually be delayed or degraded due to budgetary constraints or implementation risks. Finally, our analysis did not consider possible changes to airline operation policies such as schedule smoothing or secondary airports; neither did we consider the interaction between the individual constraints. But we believe that these factors would only modify the relative magnitude of the results or revise the timeframe when the same kind of results would be manifested we believe the overall conclusions of the study remain valid. The revised demand forecast will only depress the traffic by a few percentage points in the target year or the traffic growth will be delayed by a few years before achieving the same volume. For the projected capacities, we have reflected the latest official plans and analysis 
of the JPDO. As for our analysis approach, we believe we have captured the dominant effects even without explicit consideration of the constraint interactions. Finally, although smoothing and spreading of the traffic are strategies that airline operators may use to accommodate traffic growth, they carry associated costs to the airlines and passengers because the current flight schedules are structured according to passenger time-of-day preferences.

\section{BUSIEST 10 AIRPORTS}

For the 10 busiest airports, runway and noise are the most binding constraints in both 2015 and 2025. From 2015 to 2025, their feasible throughput percentages drop by about 4 percent, from the low 90 s to high 80 s. The drop in throughput percentage under the runway constraint is due to increasing traffic compared to a relatively stable NAS capacity between the 2 years. The feasible throughput for airports under the other four constraints (taxiway, gate, fuel efficiency, and NOx) are comparable (in the 90 s for both years), but is generally lower in 2025. Figures 7-1 and 7-2 show the throughput at the busiest 10 airports in 2015 and 2025 , respectively.

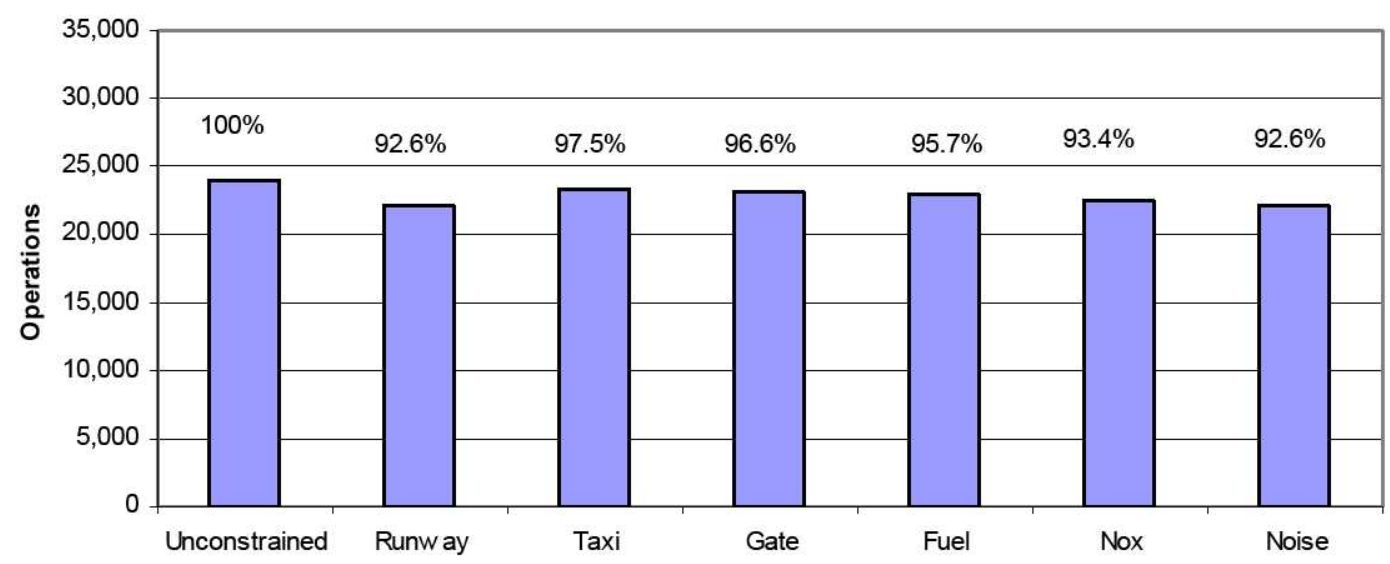

Figure 7-1. Comparison of throughput in 2015 under constraints at busiest 10 airports.

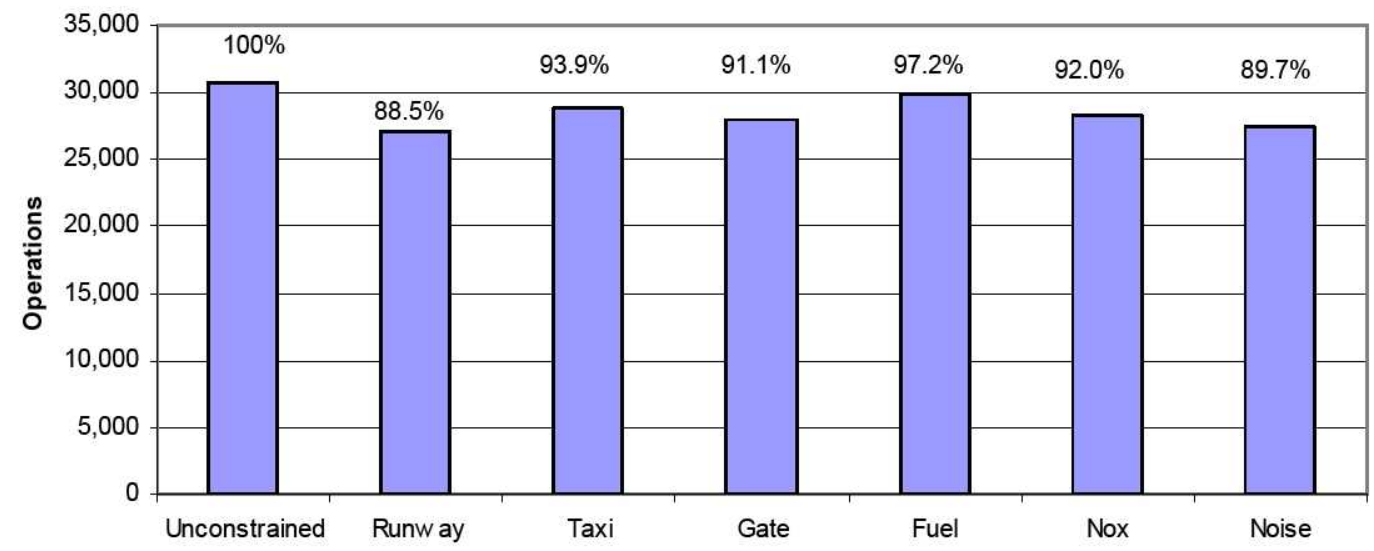

Figure 7-2. Comparison of throughput in 2025 under constraints at busiest 10 airports. 
Tables 7-1 and 7-2 show the results for each individual airport in this airport group. The red highlight indicates the primary constraint, while the orange highlight indicates the secondary constraint. If there are ties among the categories, such as runway and gate for LAX in Table 7-1, all tied categories will be shown. Tables 7-5 and 7-6 will also follow the same convention later in this chapter.

Table 7-1. 2015 Throughput under Constraints at Busiest 10 Airports

\begin{tabular}{|c|c|c|c|c|c|c|c|c|c|c|c|c|c|}
\hline \multirow[b]{4}{*}{ Airport } & \multirow[b]{4}{*}{$\begin{array}{l}\text { Uncon- } \\
\text { strained }\end{array}$} & \multicolumn{5}{|c|}{$=$ highest percentage reduction category in airport. } & \multicolumn{7}{|c|}{ = second highest percentage reduction category in airport. } \\
\hline & & \multicolumn{6}{|c|}{ Capacity constraints } & \multicolumn{6}{|c|}{ Environmental constraints } \\
\hline & & \multicolumn{2}{|c|}{ Runway } & \multicolumn{2}{|c|}{ Taxi } & \multicolumn{2}{|c|}{ Gate } & \multicolumn{2}{|c|}{ Fuel } & \multicolumn{2}{|c|}{ NOx } & \multicolumn{2}{|c|}{ Noise } \\
\hline & & $\begin{array}{l}\text { Daily } \\
\text { ops. }\end{array}$ & $\begin{array}{l}\text { Reduc- } \\
\text { tion }\end{array}$ & $\begin{array}{l}\text { Daily } \\
\text { ops. }\end{array}$ & $\begin{array}{l}\text { Reduc- } \\
\text { tion }\end{array}$ & $\begin{array}{l}\text { Daily } \\
\text { ops. }\end{array}$ & $\begin{array}{l}\text { Reduc- } \\
\text { tion }\end{array}$ & $\begin{array}{l}\text { Daily } \\
\text { ops. }\end{array}$ & $\begin{array}{l}\text { Reduc- } \\
\text { tion }\end{array}$ & $\begin{array}{l}\text { Daily } \\
\text { ops. }\end{array}$ & $\begin{array}{l}\text { Reduc- } \\
\text { tion }\end{array}$ & $\begin{array}{l}\text { Daily } \\
\text { ops. }\end{array}$ & $\begin{array}{l}\text { Reduc- } \\
\text { tion }\end{array}$ \\
\hline ATL & 3,561 & 3,063 & $14.0 \%$ & 3,269 & $8.2 \%$ & 3,555 & $0.2 \%$ & 3,450 & $3.1 \%$ & 3,404 & $4.4 \%$ & 3,380 & $5.1 \%$ \\
\hline CLT & 1,813 & 1,813 & $0.0 \%$ & 1,668 & $8.0 \%$ & 1,749 & $3.5 \%$ & 1,734 & $4.4 \%$ & 1,710 & $5.7 \%$ & 1,376 & $24.1 \%$ \\
\hline DEN & 2,172 & 2,172 & $0.0 \%$ & 2,172 & $0.0 \%$ & 2,134 & $1.7 \%$ & 2,103 & $3.2 \%$ & 2,041 & $6.0 \%$ & 2,059 & $5.2 \%$ \\
\hline DFW & 2,421 & 2,421 & $0.0 \%$ & 2,420 & $0.0 \%$ & 2,421 & $0.0 \%$ & 2,375 & $1.9 \%$ & 2,331 & $3.7 \%$ & 2,299 & $5.0 \%$ \\
\hline $\mathrm{IAH}$ & 2,256 & 2,244 & $0.5 \%$ & 2,256 & $0.0 \%$ & 2,256 & $0.0 \%$ & 2,053 & $9.0 \%$ & 2,085 & $7.6 \%$ & 2,137 & $5.3 \%$ \\
\hline LAS & 1,992 & 1,494 & $25.0 \%$ & 1,992 & $0.0 \%$ & 1,814 & $8.9 \%$ & 1,886 & $5.3 \%$ & 1,750 & $12.1 \%$ & 1,892 & $5.0 \%$ \\
\hline LAX & 2,805 & 2,407 & $14.2 \%$ & 2,768 & $1.3 \%$ & 2,407 & $14.2 \%$ & 2,673 & $4.7 \%$ & 2,549 & $9.1 \%$ & 2,639 & $5.9 \%$ \\
\hline ORD & 3,217 & 3,121 & $3.0 \%$ & 3,216 & $0.0 \%$ & 3,081 & $4.2 \%$ & 3,167 & $1.6 \%$ & 3,123 & $2.9 \%$ & 3,055 & $5.0 \%$ \\
\hline $\mathrm{PHL}$ & 1,923 & 1,763 & $8.3 \%$ & 1,923 & $0.0 \%$ & 1,915 & $0.4 \%$ & 1,845 & $4.1 \%$ & 1,799 & $6.4 \%$ & 1,633 & $15.1 \%$ \\
\hline PHX & 1,840 & 1,718 & $6.6 \%$ & 1,727 & $6.1 \%$ & 1,840 & $0.0 \%$ & 1,670 & $9.2 \%$ & 1,628 & $11.5 \%$ & 1,743 & $5.3 \%$ \\
\hline Total & 24,000 & 22,216 & $92.6 \%$ & 23,411 & $97.5 \%$ & 23,172 & $96.6 \%$ & 22,956 & $95.7 \%$ & 22,420 & $93.4 \%$ & 22,213 & $92.6 \%$ \\
\hline
\end{tabular}

Table 7-2. 2025 Throughput under Constraints at Busiest 10 Airports

\begin{tabular}{|c|c|c|c|c|c|c|c|c|c|c|c|c|c|}
\hline \multirow[b]{4}{*}{ Airport } & \multirow[b]{4}{*}{$\begin{array}{l}\text { Uncon- } \\
\text { strained }\end{array}$} & \multirow{2}{*}{\multicolumn{6}{|c|}{$\begin{array}{l}=\text { highest percentage reduction category in airport. } \\
\text { Capacity constraints }\end{array}$}} & & & & & & \\
\hline & & & & & & & & \multicolumn{6}{|c|}{ Environmental constraints } \\
\hline & & \multicolumn{2}{|c|}{ Runway } & \multicolumn{2}{|c|}{ Taxi } & \multicolumn{2}{|c|}{ Gate } & \multicolumn{2}{|c|}{ Fuel } & \multicolumn{2}{|c|}{$\mathrm{NOx}$} & \multicolumn{2}{|c|}{ Noise } \\
\hline & & $\begin{array}{l}\text { Daily } \\
\text { ops. }\end{array}$ & $\begin{array}{l}\text { Reduc- } \\
\text { tion }\end{array}$ & $\begin{array}{l}\text { Daily } \\
\text { ops. }\end{array}$ & $\begin{array}{l}\text { Reduc- } \\
\text { tion }\end{array}$ & $\begin{array}{l}\text { Daily } \\
\text { ops. }\end{array}$ & $\begin{array}{l}\text { Reduc- } \\
\text { tion }\end{array}$ & $\begin{array}{l}\text { Daily } \\
\text { ops. }\end{array}$ & $\begin{array}{c}\text { Reduc- } \\
\text { tion }\end{array}$ & $\begin{array}{l}\text { Daily } \\
\text { ops. }\end{array}$ & $\begin{array}{l}\text { Reduc- } \\
\text { tion }\end{array}$ & $\begin{array}{l}\text { Daily } \\
\text { ops. }\end{array}$ & $\begin{array}{c}\text { Reduc- } \\
\text { tion }\end{array}$ \\
\hline ATL & 4,383 & 3,605 & $17.8 \%$ & 3,481 & $20.6 \%$ & 4,137 & $5.6 \%$ & 4,371 & $0.3 \%$ & 4,167 & $4.9 \%$ & 3,901 & $11.0 \%$ \\
\hline CLT & 2,232 & 2,232 & $0.0 \%$ & 1,987 & $11.0 \%$ & 2,076 & $7.0 \%$ & 2,148 & $3.8 \%$ & 2,108 & $5.6 \%$ & 1,896 & $15.1 \%$ \\
\hline DEN & 2,621 & 2,621 & $0.0 \%$ & 2,621 & $0.0 \%$ & 2,471 & $5.7 \%$ & 2,564 & $2.2 \%$ & 2,486 & $5.2 \%$ & 2,616 & $0.2 \%$ \\
\hline DFW & 3,099 & 3,099 & $0.0 \%$ & 3,050 & $1.6 \%$ & 3,099 & $0.0 \%$ & 3,087 & $0.4 \%$ & 2,971 & $4.1 \%$ & 2,941 & $5.1 \%$ \\
\hline $\mathrm{IAH}$ & 2,848 & 2,810 & $1.3 \%$ & 2,848 & $0.0 \%$ & 2,752 & $3.4 \%$ & 2,639 & $7.3 \%$ & 2,609 & $8.4 \%$ & 2,697 & $5.3 \%$ \\
\hline LAS & 2,760 & 1,684 & $39.0 \%$ & 2,760 & $0.0 \%$ & 2,428 & $12.0 \%$ & 2,690 & $2.5 \%$ & 2,330 & $15.6 \%$ & 2,188 & $20.7 \%$ \\
\hline LAX & 3,678 & 2,834 & $22.9 \%$ & 3,362 & $8.6 \%$ & 2,942 & $20.0 \%$ & 3,531 & $4.0 \%$ & 3,181 & $13.5 \%$ & 2,929 & $20.4 \%$ \\
\hline ORD & 4,031 & 4,031 & $0.0 \%$ & 3,892 & $3.4 \%$ & 3,391 & $15.9 \%$ & 3,979 & $1.3 \%$ & 3,903 & $3.2 \%$ & 3,829 & $5.0 \%$ \\
\hline $\mathrm{PHL}$ & 2,518 & 2,002 & $20.5 \%$ & 2,518 & $0.0 \%$ & 2,330 & $7.5 \%$ & 2,395 & $4.9 \%$ & 2,269 & $9.9 \%$ & 2,389 & $5.1 \%$ \\
\hline PHX & 2,516 & 2,230 & $11.4 \%$ & 2,293 & $8.9 \%$ & 2,330 & $7.4 \%$ & 2,419 & $3.9 \%$ & 2,203 & $12.4 \%$ & 2,147 & $14.7 \%$ \\
\hline Total & 30,686 & 27,148 & $88.5 \%$ & 28,812 & $93.9 \%$ & 27,956 & $91.1 \%$ & 29,823 & $97.2 \%$ & 28,227 & $92.0 \%$ & 27,533 & $89.7 \%$ \\
\hline
\end{tabular}




\section{OEP 35 AIRPORTS}

The most binding constraint for OEP 35 airports is noise. The feasible throughput percentages for both years are in the mid 80 s. In contrast, the throughput percentages for the other five constraints are in the mid to upper 90s. For the three capacity constraints, the feasible throughput percentages drop by a few points from 2015 to 2025 . For airports under the NOx constraint, the throughput percentage drops about one point, but under the emissions constraint, the throughput percentage increases slightly from 2015 to 2025 .

Figures 7-3 and 7-4 show throughput at the OEP 35 airports in 2015 and 2025, respectively.

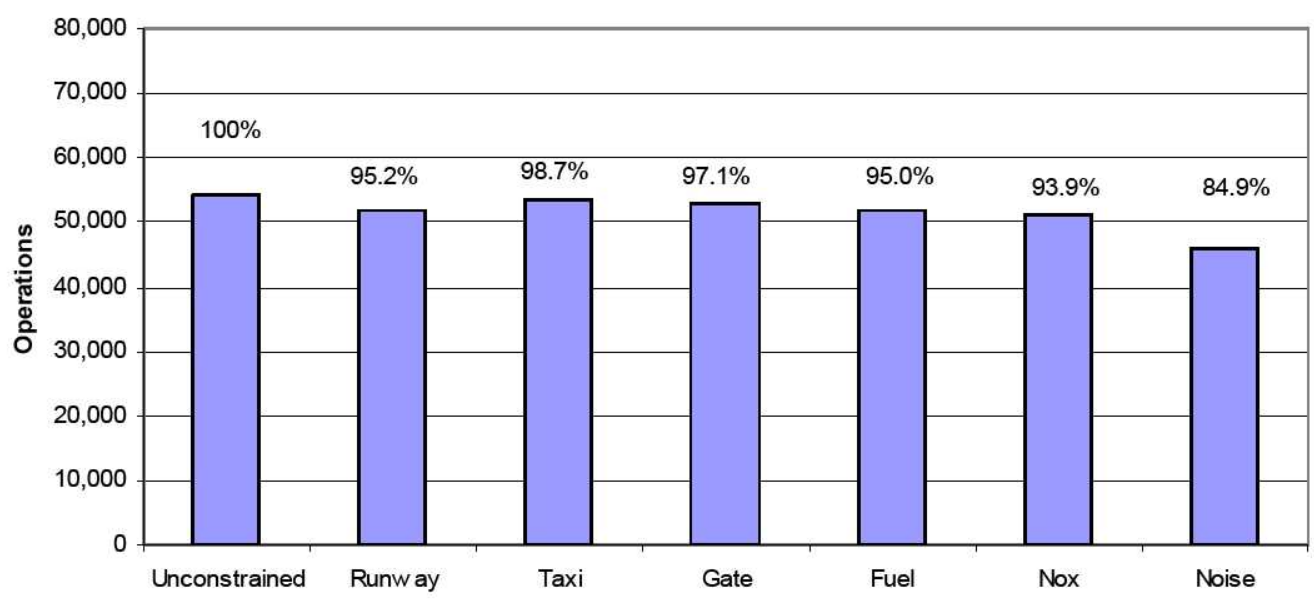

Figure 7-3. Comparison of throughput in 2015 under constraints at OEP 35 airports.

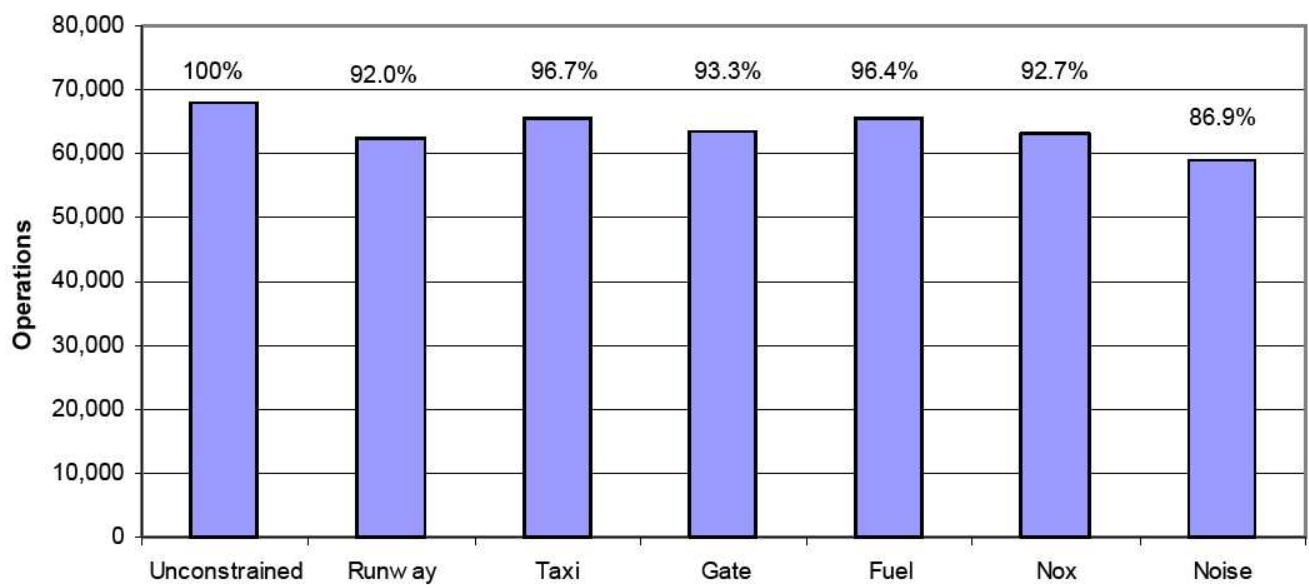

Figure 7-4. Comparison of throughput in 2025 under constraints at OEP 35 airports. 


\section{LMI 110 AIRPORTS}

Like the OEP 35 airport group, the LMI 110 airport group is constrained primarily by noise. The pattern from 2015 to 2025 also is comparable to that seen in the OEP 35 airports.

Figures 7-5 and 7-6 show throughput at the LMI 110 airports in 2015 and 2025, respectively.

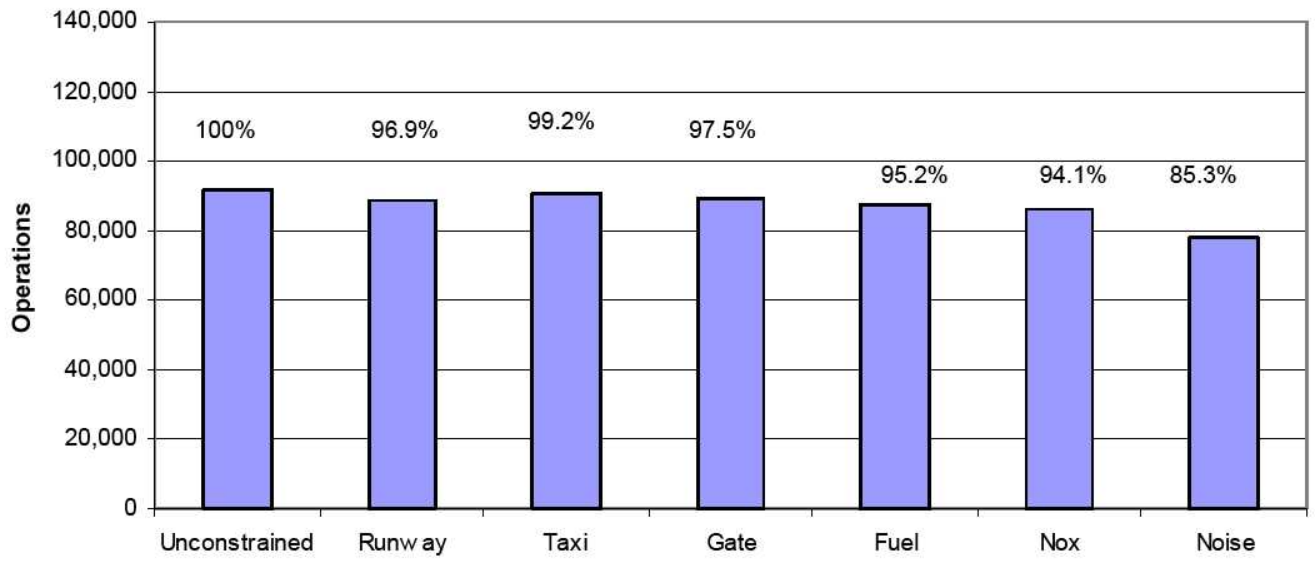

Figure 7-5. Comparison of throughput in 2015 under constraints at LMI 110 airports.

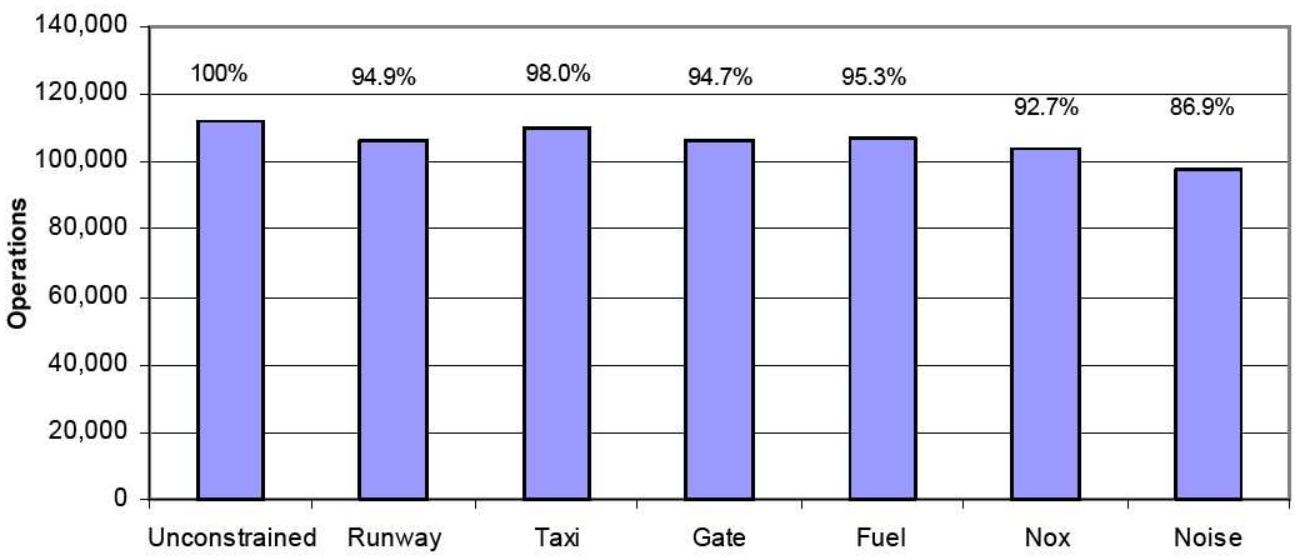

Figure 7-6. Comparison of throughput in 2025 under constraints at LMI 110 airports. 


\section{LMI 310 AIRPORTS}

The results for this airport group are comparable to the results for the OEP 35 and LMI 110 airport groups and follow a similar pattern as well. Noise is still the most binding constraint in both 2015 and 2025. The detailed results for each individual airport in this airport group are included in the tables for the LMI 310 airport group at the end of this chapter.

Figures 7-7 and 7-8 compare throughput at the LMI 310 airports in 2015 and 2025 , respectively.

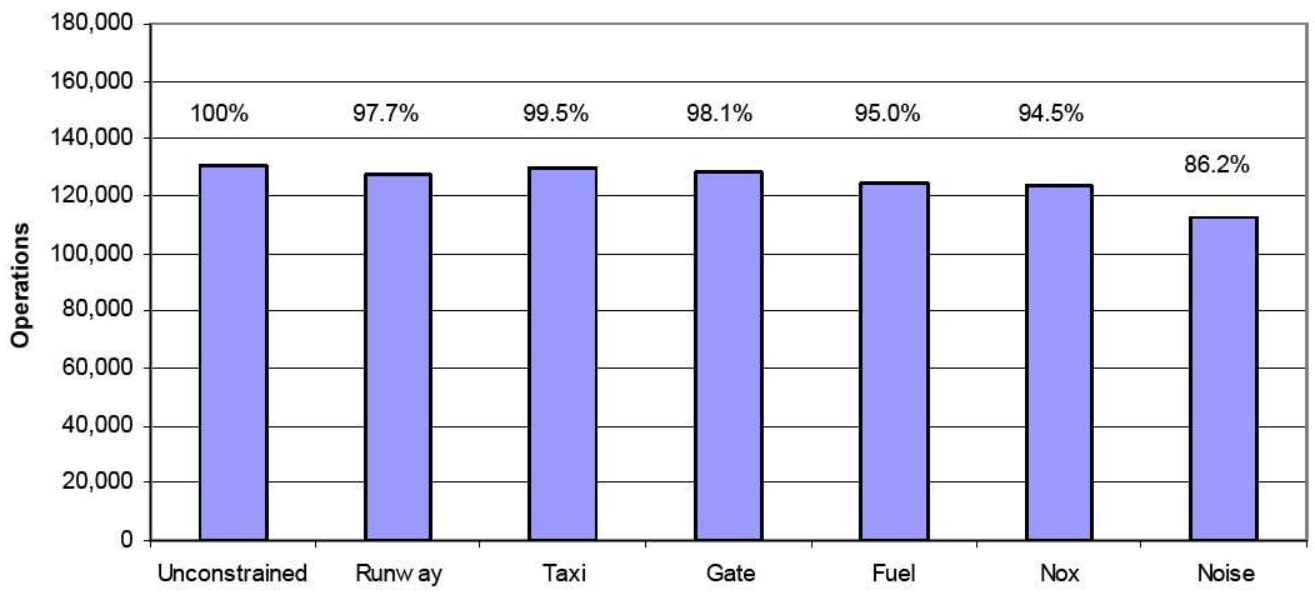

Figure 7-7. Comparison of throughput in 2015 under constraints at LMI 310 airports.

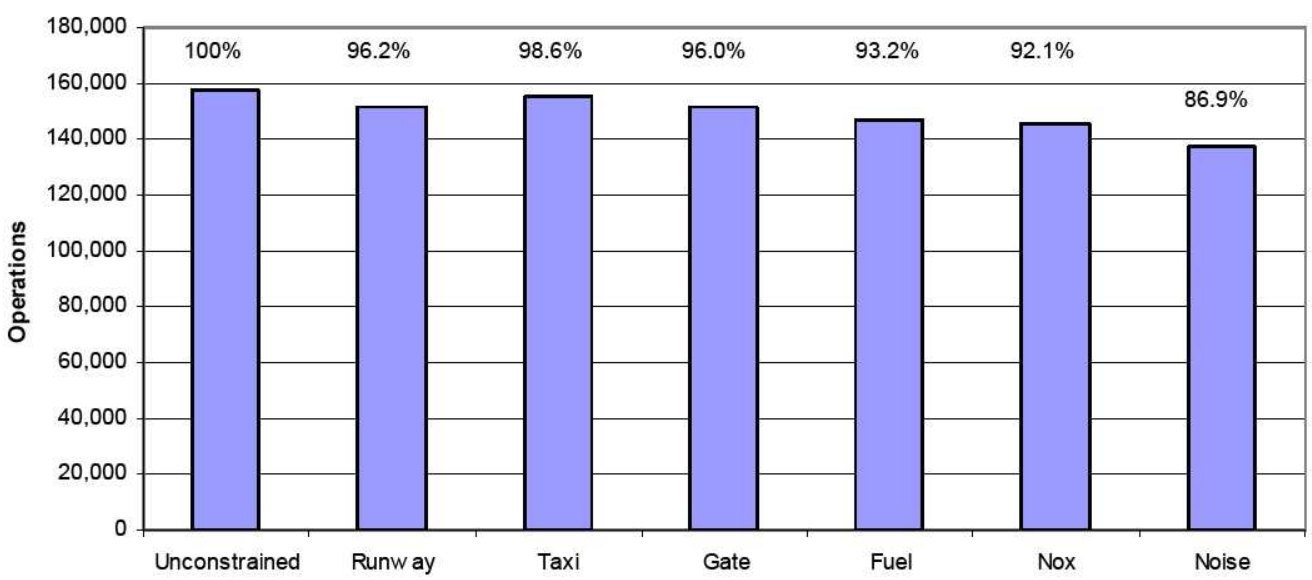

Figure 7-8. Comparison of throughput in 2025 under constraints at LMI 310 airports. 
Although the throughput results for the LMI 310 group are similar to the results for the OEP 35 and LMI 110 groups, it is for different reasons. As shown in Tables 7-3 and 7-4, capacity constraints (runway, taxiway, and gate) have a greater effect on large airports, while environmental constraints spread more to small airports. In these two tables, the cells by the primary and secondary rows show the numbers of airports with the primary and secondary constraint, respectively, under the corresponding column. The cells by the total row show the total numbers of constrained airports under the column regardless. In the case of runway constraint for the busiest 10 airport group, 3 list runway as the primary constraint, 1 lists runway as the secondary constraint, and 6 list runway as a constraint.

Table 7-3. Number of Constrained Airports by Category in 2015

\begin{tabular}{|c|c|c|c|c|c|c|c|}
\hline Airport Group & Constrained & Runway & Taxi & Gate & Fuel & NOx & Noise \\
\hline \multirow{3}{*}{ Busiest10 } & Primary & 3 & 0 & 1 & 1 & 2 & 4 \\
\cline { 2 - 8 } & Secondary & 1 & 2 & 1 & 1 & 4 & 1 \\
\cline { 2 - 8 } & Total & 6 & 4 & 7 & 10 & 10 & 10 \\
\hline \multirow{3}{*}{ OEP35 } & Primary & 3 & 0 & 2 & 4 & 3 & 24 \\
\cline { 2 - 8 } & Secondary & 5 & 2 & 1 & 5 & 19 & 2 \\
\cline { 2 - 8 } & Total & 20 & 10 & 19 & 35 & 35 & 35 \\
\hline \multirow{3}{*}{ LMI110 } & Primary & 4 & 0 & 3 & 19 & 16 & 74 \\
\cline { 2 - 8 } & Secondary & 6 & 1 & 7 & 36 & 57 & 4 \\
\cline { 2 - 8 } & Total & 27 & 10 & 60 & 110 & 110 & 101 \\
\hline \multirow{3}{*}{ LMI310 } & Primary & 6 & 0 & 11 & 104 & 81 & 147 \\
\cline { 2 - 8 } & Secondary & 6 & 1 & 9 & 113 & 157 & 21 \\
\cline { 2 - 8 } & Total & 31 & 10 & 75 & 307 & 308 & 236 \\
\hline
\end{tabular}

Table 7-4. Number of Constrained Airports by Category in 2025

\begin{tabular}{|c|c|c|c|c|c|c|c|}
\hline Airport Group & Constrained & Runway & Taxi & Gate & Fuel & NOx & Noise \\
\hline \multirow{3}{*}{ Busiest10 } & Primary & 3 & 1 & 1 & 0 & 1 & 3 \\
\cline { 2 - 8 } & Secondary & 0 & 1 & 1 & 1 & 4 & 4 \\
\cline { 2 - 8 } & Total & 6 & 6 & 9 & 10 & 10 & 10 \\
\hline \multirow{3}{*}{ OEP35 } & Primary & 4 & 2 & 4 & 3 & 3 & 19 \\
\cline { 2 - 8 } & Secondary & 4 & 1 & 0 & 5 & 17 & 7 \\
\cline { 2 - 8 } & Total & 21 & 12 & 27 & 35 & 34 & 35 \\
\hline \multirow{3}{*}{ LMI110 } & Primary & 5 & 2 & 7 & 21 & 18 & 63 \\
\cline { 2 - 8 } & Secondary & 5 & 1 & 7 & 34 & 58 & 9 \\
\cline { 2 - 8 } & Total & 28 & 12 & 79 & 110 & 109 & 103 \\
\hline \multirow{3}{*}{ LMI310 } & Primary & 5 & 2 & 13 & 111 & 76 & 132 \\
\cline { 2 - 8 } & Secondary & 6 & 1 & 10 & 106 & 149 & 18 \\
\cline { 2 - 8 } & Total & 32 & 12 & 95 & 303 & 305 & 237 \\
\hline
\end{tabular}


Tables 7-5 and 7-6 show the 2015 and 2025 results, respectively, for each individual airport in this airport group. The red highlight indicates the primary constraint, while the orange highlight indicates the secondary constraint.

Table 7-5. 2015 Throughput under Constraints at 310 Critical Airports $=$ highest percentage reduction category in airport.

$=$ second highest percentage reduction category in airport.

\begin{tabular}{|c|c|c|c|c|c|c|c|c|c|c|c|c|c|}
\hline \multirow[b]{3}{*}{$\begin{array}{c}\text { Airpor } \\
t\end{array}$} & \multirow[b]{3}{*}{$\begin{array}{l}\text { Uncon- } \\
\text { strained }\end{array}$} & \multicolumn{6}{|c|}{ Capacity constraints } & \multicolumn{6}{|c|}{ Environmental constraints } \\
\hline & & \multicolumn{2}{|c|}{ Runway } & \multicolumn{2}{|c|}{ Taxi } & \multicolumn{2}{|c|}{ Gate } & \multicolumn{2}{|c|}{ Fuel } & \multicolumn{2}{|c|}{ NOx } & \multicolumn{2}{|c|}{ Noise } \\
\hline & & $\begin{array}{l}\text { Daily } \\
\text { ops. }\end{array}$ & $\begin{array}{l}\text { Reduc- } \\
\text { tion }\end{array}$ & $\begin{array}{l}\text { Daily } \\
\text { ops. }\end{array}$ & $\begin{array}{l}\text { Reduc- } \\
\text { tion }\end{array}$ & $\begin{array}{l}\text { Daily } \\
\text { ops. }\end{array}$ & $\begin{array}{l}\text { Reduc- } \\
\text { tion }\end{array}$ & $\begin{array}{l}\text { Daily } \\
\text { ops. }\end{array}$ & $\begin{array}{l}\text { Reduc- } \\
\text { tion }\end{array}$ & $\begin{array}{l}\text { Daily } \\
\text { ops. }\end{array}$ & $\begin{array}{l}\text { Reduc- } \\
\text { tion }\end{array}$ & $\begin{array}{l}\text { Daily } \\
\text { ops. }\end{array}$ & $\begin{array}{l}\text { Reduc- } \\
\text { tion }\end{array}$ \\
\hline$A B Q$ & 772 & 772 & $0.0 \%$ & 772 & $0.0 \%$ & 764 & $1.0 \%$ & 751 & $2.7 \%$ & 735 & $4.8 \%$ & 679 & $12.0 \%$ \\
\hline ALB & 398 & 398 & $0.0 \%$ & 398 & $0.0 \%$ & 398 & $0.0 \%$ & 386 & $3.0 \%$ & 378 & $5.0 \%$ & 398 & $0.0 \%$ \\
\hline ANC & 1,234 & 1,104 & $10.5 \%$ & 1,234 & $0.0 \%$ & 1,154 & $6.5 \%$ & 1146 & $7.1 \%$ & 1116 & $9.6 \%$ & 1090 & $11.7 \%$ \\
\hline ATL & 3,561 & 3,063 & $14.0 \%$ & 3,269 & $8.2 \%$ & 3,555 & $0.2 \%$ & 3450 & $3.1 \%$ & 3404 & $4.4 \%$ & 3380 & $5.1 \%$ \\
\hline AUS & 837 & 837 & $0.0 \%$ & 837 & $0.0 \%$ & 815 & $2.6 \%$ & 824 & $1.6 \%$ & 804 & $3.9 \%$ & 644 & $23.1 \%$ \\
\hline BDL & 506 & 506 & $0.0 \%$ & 506 & $0.0 \%$ & 504 & $0.4 \%$ & 480 & $5.1 \%$ & 468 & $7.5 \%$ & 390 & $22.9 \%$ \\
\hline BFL & 269 & 269 & $0.0 \%$ & 269 & $0.0 \%$ & 269 & $0.0 \%$ & 252 & $6.3 \%$ & 254 & $5.6 \%$ & 254 & $5.6 \%$ \\
\hline $\mathrm{BHM}$ & 493 & 493 & $0.0 \%$ & 493 & $0.0 \%$ & 487 & $1.2 \%$ & 474 & $3.9 \%$ & 466 & $5.5 \%$ & 490 & $0.6 \%$ \\
\hline BNA & 764 & 764 & $0.0 \%$ & 764 & $0.0 \%$ & 764 & $0.0 \%$ & 723 & $5.4 \%$ & 697 & $8.8 \%$ & 739 & $3.3 \%$ \\
\hline $\mathrm{BOI}$ & 573 & 573 & $0.0 \%$ & 573 & $0.0 \%$ & 573 & $0.0 \%$ & 553 & $3.5 \%$ & 541 & $5.6 \%$ & 503 & $12.2 \%$ \\
\hline BOS & 1,518 & 1,516 & $0.1 \%$ & 1,518 & $0.0 \%$ & 1,508 & $0.7 \%$ & 1481 & $2.4 \%$ & 1469 & $3.2 \%$ & 1091 & $28.1 \%$ \\
\hline BTR & 317 & 317 & $0.0 \%$ & 317 & $0.0 \%$ & 317 & $0.0 \%$ & 309 & $2.5 \%$ & 309 & $2.5 \%$ & 315 & $0.6 \%$ \\
\hline BUF & 439 & 439 & $0.0 \%$ & 439 & $0.0 \%$ & 435 & $0.9 \%$ & 412 & $6.2 \%$ & 412 & $6.2 \%$ & 386 & $12.1 \%$ \\
\hline BUR & 625 & 625 & $0.0 \%$ & 625 & $0.0 \%$ & 601 & $3.8 \%$ & 605 & $3.2 \%$ & 595 & $4.8 \%$ & 541 & $13.4 \%$ \\
\hline BWI & 1,072 & 1,072 & $0.0 \%$ & 1,072 & $0.0 \%$ & 1,068 & $0.4 \%$ & 989 & $7.7 \%$ & 1003 & $6.4 \%$ & 967 & $9.8 \%$ \\
\hline $\mathrm{CHS}$ & 364 & 364 & $0.0 \%$ & 364 & $0.0 \%$ & 354 & $2.7 \%$ & 353 & $3.0 \%$ & 353 & $3.0 \%$ & 325 & $10.7 \%$ \\
\hline CLE & 952 & 952 & $0.0 \%$ & 891 & $6.4 \%$ & 952 & $0.0 \%$ & 858 & $9.9 \%$ & 888 & $6.7 \%$ & 882 & $7.4 \%$ \\
\hline CLT & 1,813 & 1,813 & $0.0 \%$ & 1,668 & $8.0 \%$ & 1,749 & $3.5 \%$ & 1734 & $4.4 \%$ & 1710 & $5.7 \%$ & 1376 & $24.1 \%$ \\
\hline $\mathrm{CMH}$ & 711 & 711 & $0.0 \%$ & 711 & $0.0 \%$ & 691 & $2.8 \%$ & 646 & $9.1 \%$ & 642 & $9.7 \%$ & 608 & $14.5 \%$ \\
\hline cos & 308 & 308 & $0.0 \%$ & 308 & $0.0 \%$ & 308 & $0.0 \%$ & 302 & $1.9 \%$ & 302 & $1.9 \%$ & 308 & $0.0 \%$ \\
\hline CRP & 171 & 171 & $0.0 \%$ & 171 & $0.0 \%$ & 169 & $1.2 \%$ & 159 & $7.0 \%$ & 159 & $7.0 \%$ & 171 & $0.0 \%$ \\
\hline CVG & 1,222 & 1,222 & $0.0 \%$ & 1,222 & $0.0 \%$ & 1,222 & $0.0 \%$ & 1116 & $8.7 \%$ & 1126 & $7.9 \%$ & 922 & $24.5 \%$ \\
\hline DAB & 498 & 498 & $0.0 \%$ & 498 & $0.0 \%$ & 498 & $0.0 \%$ & 493 & $1.0 \%$ & 493 & $1.0 \%$ & 491 & $1.4 \%$ \\
\hline DAL & 913 & 883 & $3.3 \%$ & 913 & $0.0 \%$ & 859 & $5.9 \%$ & 858 & $6.0 \%$ & 806 & $11.7 \%$ & 684 & $25.1 \%$ \\
\hline DAY & 307 & 307 & $0.0 \%$ & 307 & $0.0 \%$ & 299 & $2.6 \%$ & 281 & $8.5 \%$ & 293 & $4.6 \%$ & 183 & $40.4 \%$ \\
\hline DCA & 874 & 864 & $1.1 \%$ & 860 & $1.6 \%$ & 844 & $3.4 \%$ & 789 & $9.7 \%$ & 851 & $2.6 \%$ & 659 & $24.6 \%$ \\
\hline DEN & 2,172 & 2,172 & $0.0 \%$ & 2,172 & $0.0 \%$ & 2,134 & $1.7 \%$ & 2103 & $3.2 \%$ & 2041 & $6.0 \%$ & 2059 & $5.2 \%$ \\
\hline DFW & 2,421 & 2,421 & $0.0 \%$ & 2,420 & $0.0 \%$ & 2,421 & $0.0 \%$ & 2375 & $1.9 \%$ & 2331 & $3.7 \%$ & 2299 & $5.0 \%$ \\
\hline DSM & 348 & 348 & $0.0 \%$ & 348 & $0.0 \%$ & 340 & $2.3 \%$ & 330 & $5.2 \%$ & 334 & $4.0 \%$ & 332 & $4.6 \%$ \\
\hline DTW & 1,625 & 1,625 & $0.0 \%$ & 1,625 & $0.0 \%$ & 1,625 & $0.0 \%$ & 1581 & $2.7 \%$ & 1525 & $6.2 \%$ & 1453 & $10.6 \%$ \\
\hline ELP & 286 & 286 & $0.0 \%$ & 286 & $0.0 \%$ & 286 & $0.0 \%$ & 283 & $1.0 \%$ & 283 & $1.0 \%$ & 273 & $4.5 \%$ \\
\hline EUG & 234 & 234 & $0.0 \%$ & 234 & $0.0 \%$ & 234 & $0.0 \%$ & 222 & $5.1 \%$ & 224 & $4.3 \%$ & 116 & $50.4 \%$ \\
\hline EWR & 1,671 & 1,421 & $15.0 \%$ & 1,643 & $1.7 \%$ & 1,625 & $2.8 \%$ & 1583 & $5.3 \%$ & 1577 & $5.6 \%$ & 1061 & $36.5 \%$ \\
\hline FAT & 527 & 527 & $0.0 \%$ & 527 & $0.0 \%$ & 527 & $0.0 \%$ & 521 & $1.1 \%$ & 521 & $1.1 \%$ & 527 & $0.0 \%$ \\
\hline FLL & 1,021 & 1,021 & $0.0 \%$ & 1,021 & $0.0 \%$ & 1,003 & $1.8 \%$ & 965 & $5.5 \%$ & 947 & $7.2 \%$ & 673 & $34.1 \%$ \\
\hline FNT & 168 & 168 & $0.0 \%$ & 168 & $0.0 \%$ & 168 & $0.0 \%$ & 164 & $2.4 \%$ & 162 & $3.6 \%$ & 168 & $0.0 \%$ \\
\hline FXE & 616 & 616 & $0.0 \%$ & 616 & $0.0 \%$ & 616 & $0.0 \%$ & 601 & $2.4 \%$ & 605 & $1.8 \%$ & 521 & $15.4 \%$ \\
\hline GFK & 338 & 338 & $0.0 \%$ & 338 & $0.0 \%$ & 338 & $0.0 \%$ & 336 & $0.6 \%$ & 336 & $0.6 \%$ & 338 & $0.0 \%$ \\
\hline GRR & 333 & 333 & $0.0 \%$ & 333 & $0.0 \%$ & 327 & $1.8 \%$ & 317 & $4.8 \%$ & 315 & $5.4 \%$ & 301 & $9.6 \%$ \\
\hline GSO & 421 & 421 & $0.0 \%$ & 421 & $0.0 \%$ & 419 & $0.5 \%$ & 401 & $4.8 \%$ & 385 & $8.6 \%$ & 357 & $15.2 \%$ \\
\hline
\end{tabular}


Table 7-5. 2015 Throughput under Constraints at 310 Critical Airports

$=$ highest percentage reduction category in airport. $\quad=$ second highest percentage reduction category in airport.

\begin{tabular}{|c|c|c|c|c|c|c|c|c|c|c|c|c|c|}
\hline \multirow[b]{3}{*}{$\begin{array}{c}\text { Airpor } \\
\mathrm{t}\end{array}$} & \multirow[b]{3}{*}{$\begin{array}{l}\text { Uncon- } \\
\text { strained }\end{array}$} & \multicolumn{6}{|c|}{ Capacity constraints } & \multicolumn{6}{|c|}{ Environmental constraints } \\
\hline & & \multicolumn{2}{|c|}{ Runway } & \multicolumn{2}{|c|}{ Taxi } & \multicolumn{2}{|c|}{ Gate } & \multicolumn{2}{|c|}{ Fuel } & \multicolumn{2}{|c|}{$\mathrm{NOx}$} & \multicolumn{2}{|c|}{ Noise } \\
\hline & & $\begin{array}{l}\text { Daily } \\
\text { ops. }\end{array}$ & $\begin{array}{l}\text { Reduc- } \\
\text { tion }\end{array}$ & $\begin{array}{l}\text { Daily } \\
\text { ops. }\end{array}$ & $\begin{array}{l}\text { Reduc- } \\
\text { tion }\end{array}$ & $\begin{array}{l}\text { Daily } \\
\text { ops. }\end{array}$ & $\begin{array}{l}\text { Reduc- } \\
\text { tion }\end{array}$ & $\begin{array}{l}\text { Daily } \\
\text { ops. }\end{array}$ & $\begin{array}{l}\text { Reduc- } \\
\text { tion }\end{array}$ & $\begin{array}{l}\text { Daily } \\
\text { ops. }\end{array}$ & $\begin{array}{l}\text { Reduc- } \\
\text { tion }\end{array}$ & $\begin{array}{l}\text { Daily } \\
\text { ops. }\end{array}$ & $\begin{array}{l}\text { Reduc- } \\
\text { tion }\end{array}$ \\
\hline GYY & 153 & 153 & $0.0 \%$ & 153 & $0.0 \%$ & 153 & $0.0 \%$ & 146 & $4.6 \%$ & 144 & $5.9 \%$ & 152 & $0.7 \%$ \\
\hline $\mathrm{HNL}$ & 1,067 & 1,061 & $0.6 \%$ & 1,067 & $0.0 \%$ & 825 & $22.7 \%$ & 1002 & $6.1 \%$ & 1000 & $6.3 \%$ & 1064 & $0.3 \%$ \\
\hline $\mathrm{HOU}$ & 894 & 870 & $2.7 \%$ & 894 & $0.0 \%$ & 876 & $2.0 \%$ & 822 & $8.1 \%$ & 798 & $10.7 \%$ & 638 & $28.6 \%$ \\
\hline HPN & 757 & 741 & $2.1 \%$ & 757 & $0.0 \%$ & 741 & $2.1 \%$ & 719 & $5.0 \%$ & 731 & $3.4 \%$ & 633 & $16.4 \%$ \\
\hline $\mathrm{IAD}$ & 1,490 & 1,484 & $0.4 \%$ & 1,490 & $0.0 \%$ & 1,490 & $0.0 \%$ & 1423 & $4.5 \%$ & 1407 & $5.6 \%$ & 1213 & $18.6 \%$ \\
\hline $\mathrm{IAH}$ & 2,256 & 2,244 & $0.5 \%$ & 2,256 & $0.0 \%$ & 2,256 & $0.0 \%$ & 2053 & $9.0 \%$ & 2085 & $7.6 \%$ & 2137 & $5.3 \%$ \\
\hline ICT & 408 & 408 & $0.0 \%$ & 408 & $0.0 \%$ & 406 & $0.5 \%$ & 384 & $5.9 \%$ & 388 & $4.9 \%$ & 396 & $2.9 \%$ \\
\hline IND & 744 & 744 & $0.0 \%$ & 744 & $0.0 \%$ & 714 & $4.0 \%$ & 718 & $3.5 \%$ & 714 & $4.0 \%$ & 706 & $5.1 \%$ \\
\hline ISP & 461 & 461 & $0.0 \%$ & 461 & $0.0 \%$ & 461 & $0.0 \%$ & 441 & $4.3 \%$ & 437 & $5.2 \%$ & 459 & $0.4 \%$ \\
\hline JAX & 429 & 429 & $0.0 \%$ & 429 & $0.0 \%$ & 427 & $0.5 \%$ & 392 & $8.6 \%$ & 394 & $8.2 \%$ & 212 & $50.6 \%$ \\
\hline JFK & 1,775 & 1,591 & $10.4 \%$ & 1,775 & $0.0 \%$ & 1,569 & $11.6 \%$ & 1674 & $5.7 \%$ & 1612 & $9.2 \%$ & 1246 & $29.8 \%$ \\
\hline $\mathrm{JNU}$ & 650 & 628 & $3.4 \%$ & 650 & $0.0 \%$ & 648 & $0.3 \%$ & 640 & $1.5 \%$ & 636 & $2.2 \%$ & 648 & $0.3 \%$ \\
\hline LAN & 190 & 190 & $0.0 \%$ & 190 & $0.0 \%$ & 190 & $0.0 \%$ & 184 & $3.2 \%$ & 186 & $2.1 \%$ & 94 & $50.5 \%$ \\
\hline LAS & 1,992 & 1,494 & $25.0 \%$ & 1,992 & $0.0 \%$ & 1,814 & $8.9 \%$ & 1886 & $5.3 \%$ & 1750 & $12.1 \%$ & 1892 & $5.0 \%$ \\
\hline LAX & 2,805 & 2,407 & $14.2 \%$ & 2,768 & $1.3 \%$ & 2,407 & $14.2 \%$ & 2673 & $4.7 \%$ & 2549 & $9.1 \%$ & 2639 & $5.9 \%$ \\
\hline LGA & 1,277 & 1,129 & $11.6 \%$ & 1,277 & $0.0 \%$ & 1,277 & $0.0 \%$ & 1213 & $5.0 \%$ & 1265 & $0.9 \%$ & 989 & $22.6 \%$ \\
\hline LGB & 531 & 531 & $0.0 \%$ & 531 & $0.0 \%$ & 531 & $0.0 \%$ & 512 & $3.6 \%$ & 512 & $3.6 \%$ & 446 & $16.0 \%$ \\
\hline LIT & 396 & 396 & $0.0 \%$ & 396 & $0.0 \%$ & 396 & $0.0 \%$ & 384 & $3.0 \%$ & 384 & $3.0 \%$ & 370 & $6.6 \%$ \\
\hline $\mathrm{MCl}$ & 733 & 733 & $0.0 \%$ & 733 & $0.0 \%$ & 733 & $0.0 \%$ & 689 & $6.0 \%$ & 679 & $7.4 \%$ & 529 & $27.8 \%$ \\
\hline $\mathrm{MCO}$ & 1,248 & 1,248 & $0.0 \%$ & 1,248 & $0.0 \%$ & 1,248 & $0.0 \%$ & 1179 & $5.5 \%$ & 1169 & $6.3 \%$ & 867 & $30.5 \%$ \\
\hline MDW & 1,058 & 1,028 & $2.8 \%$ & 1,058 & $0.0 \%$ & 1,054 & $0.4 \%$ & 882 & $16.6 \%$ & 966 & $8.7 \%$ & 1052 & $0.6 \%$ \\
\hline MEM & 1,345 & 1,281 & $4.8 \%$ & 1,345 & $0.0 \%$ & 1,345 & $0.0 \%$ & 1336 & $0.7 \%$ & 1310 & $2.6 \%$ & 1278 & $5.0 \%$ \\
\hline MHT & 326 & 326 & $0.0 \%$ & 326 & $0.0 \%$ & 314 & $3.7 \%$ & 316 & $3.1 \%$ & 316 & $3.1 \%$ & 284 & $12.9 \%$ \\
\hline MIA & 1,213 & 1,213 & $0.0 \%$ & 1,213 & $0.0 \%$ & 1,213 & $0.0 \%$ & 1117 & $7.9 \%$ & 1107 & $8.7 \%$ & 1139 & $6.1 \%$ \\
\hline MKE & 827 & 827 & $0.0 \%$ & 827 & $0.0 \%$ & 827 & $0.0 \%$ & 771 & $6.8 \%$ & 799 & $3.4 \%$ & 725 & $12.3 \%$ \\
\hline MLB & 192 & 192 & $0.0 \%$ & 192 & $0.0 \%$ & 192 & $0.0 \%$ & 189 & $1.6 \%$ & 189 & $1.6 \%$ & 139 & $27.6 \%$ \\
\hline MSN & 298 & 298 & $0.0 \%$ & 298 & $0.0 \%$ & 294 & $1.3 \%$ & 280 & $6.0 \%$ & 282 & $5.4 \%$ & 298 & $0.0 \%$ \\
\hline MSP & 1,662 & 1,646 & $1.0 \%$ & 1,662 & $0.0 \%$ & 1,662 & $0.0 \%$ & 1601 & $3.7 \%$ & 1549 & $6.8 \%$ & 1215 & $26.9 \%$ \\
\hline MSY & 507 & 507 & $0.0 \%$ & 507 & $0.0 \%$ & 507 & $0.0 \%$ & 487 & $3.9 \%$ & 457 & $9.9 \%$ & 393 & $22.5 \%$ \\
\hline OAK & 940 & 940 & $0.0 \%$ & 940 & $0.0 \%$ & 888 & $5.5 \%$ & 918 & $2.3 \%$ & 906 & $3.6 \%$ & 596 & $36.6 \%$ \\
\hline OKC & 303 & 303 & $0.0 \%$ & 303 & $0.0 \%$ & 285 & $5.9 \%$ & 292 & $3.6 \%$ & 292 & $3.6 \%$ & 278 & $8.3 \%$ \\
\hline OMA & 491 & 491 & $0.0 \%$ & 491 & $0.0 \%$ & 475 & $3.3 \%$ & 465 & $5.3 \%$ & 461 & $6.1 \%$ & 379 & $22.8 \%$ \\
\hline ONT & 474 & 474 & $0.0 \%$ & 474 & $0.0 \%$ & 474 & $0.0 \%$ & 457 & $3.6 \%$ & 463 & $2.3 \%$ & 447 & $5.7 \%$ \\
\hline ORD & 3,217 & 3,121 & $3.0 \%$ & 3,216 & $0.0 \%$ & 3,081 & $4.2 \%$ & 3167 & $1.6 \%$ & 3123 & $2.9 \%$ & 3055 & $5.0 \%$ \\
\hline ORF & 364 & 364 & $0.0 \%$ & 364 & $0.0 \%$ & 358 & $1.6 \%$ & 344 & $5.5 \%$ & 346 & $4.9 \%$ & 364 & $0.0 \%$ \\
\hline OXR & 113 & 113 & $0.0 \%$ & 113 & $0.0 \%$ & 113 & $0.0 \%$ & 94 & $16.8 \%$ & 96 & $15.0 \%$ & 102 & $9.7 \%$ \\
\hline PBI & 436 & 436 & $0.0 \%$ & 436 & $0.0 \%$ & 436 & $0.0 \%$ & 420 & $3.7 \%$ & 414 & $5.0 \%$ & 324 & $25.7 \%$ \\
\hline PDX & 989 & 989 & $0.0 \%$ & 989 & $0.0 \%$ & 989 & $0.0 \%$ & 965 & $2.4 \%$ & 957 & $3.2 \%$ & 821 & $17.0 \%$ \\
\hline $\mathrm{PHF}$ & 618 & 618 & $0.0 \%$ & 618 & $0.0 \%$ & 618 & $0.0 \%$ & 601 & $2.8 \%$ & 605 & $2.1 \%$ & 611 & $1.1 \%$ \\
\hline $\mathrm{PHL}$ & 1,923 & 1,763 & $8.3 \%$ & 1,923 & $0.0 \%$ & 1,915 & $0.4 \%$ & 1845 & $4.1 \%$ & 1799 & $6.4 \%$ & 1633 & $15.1 \%$ \\
\hline $\mathrm{PHX}$ & 1,840 & 1,718 & $6.6 \%$ & 1,727 & $6.1 \%$ & 1,840 & $0.0 \%$ & 1670 & $9.2 \%$ & 1628 & $11.5 \%$ & 1743 & $5.3 \%$ \\
\hline PIE & 172 & 172 & $0.0 \%$ & 172 & $0.0 \%$ & 172 & $0.0 \%$ & 168 & $2.3 \%$ & 164 & $4.7 \%$ & 170 & $1.2 \%$ \\
\hline PIT & 572 & 572 & $0.0 \%$ & 572 & $0.0 \%$ & 572 & $0.0 \%$ & 522 & $8.7 \%$ & 568 & $0.7 \%$ & 570 & $0.3 \%$ \\
\hline PVD & 373 & 373 & $0.0 \%$ & 373 & $0.0 \%$ & 361 & $3.2 \%$ & 351 & $5.9 \%$ & 353 & $5.4 \%$ & 299 & $19.8 \%$ \\
\hline
\end{tabular}


Table 7-5. 2015 Throughput under Constraints at 310 Critical Airports

$=$ highest percentage reduction category in airport. = second highest percentage reduction category in airport.

\begin{tabular}{|c|c|c|c|c|c|c|c|c|c|c|c|c|c|}
\hline \multirow[b]{3}{*}{$\begin{array}{c}\text { Airpor } \\
\mathrm{t}\end{array}$} & \multirow[b]{3}{*}{$\begin{array}{l}\text { Uncon- } \\
\text { strained }\end{array}$} & \multicolumn{6}{|c|}{ Capacity constraints } & \multicolumn{6}{|c|}{ Environmental constraints } \\
\hline & & \multicolumn{2}{|c|}{ Runway } & \multicolumn{2}{|c|}{ Taxi } & \multicolumn{2}{|c|}{ Gate } & \multicolumn{2}{|c|}{ Fuel } & \multicolumn{2}{|c|}{ NOx } & \multicolumn{2}{|c|}{ Noise } \\
\hline & & $\begin{array}{l}\text { Daily } \\
\text { ops. }\end{array}$ & $\begin{array}{l}\text { Reduc- } \\
\text { tion }\end{array}$ & $\begin{array}{l}\text { Daily } \\
\text { ops. }\end{array}$ & $\begin{array}{l}\text { Reduc- } \\
\text { tion }\end{array}$ & $\begin{array}{l}\text { Daily } \\
\text { ops. }\end{array}$ & $\begin{array}{l}\text { Reduc- } \\
\text { tion }\end{array}$ & $\begin{array}{l}\text { Daily } \\
\text { ops. }\end{array}$ & $\begin{array}{l}\text { Reduc- } \\
\text { tion }\end{array}$ & $\begin{array}{l}\text { Daily } \\
\text { ops. }\end{array}$ & $\begin{array}{l}\text { Reduc- } \\
\text { tion }\end{array}$ & $\begin{array}{l}\text { Daily } \\
\text { ops. }\end{array}$ & $\begin{array}{l}\text { Reduc- } \\
\text { tion }\end{array}$ \\
\hline RDU & 943 & 943 & $0.0 \%$ & 943 & $0.0 \%$ & 919 & $2.5 \%$ & 893 & $5.3 \%$ & 885 & $6.2 \%$ & 801 & $15.1 \%$ \\
\hline RFD & 249 & 249 & $0.0 \%$ & 249 & $0.0 \%$ & 249 & $0.0 \%$ & 231 & $7.2 \%$ & 225 & $9.6 \%$ & 209 & $16.1 \%$ \\
\hline $\mathrm{RIC}$ & 467 & 467 & $0.0 \%$ & 467 & $0.0 \%$ & 441 & $5.6 \%$ & 435 & $6.9 \%$ & 441 & $5.6 \%$ & 415 & $11.1 \%$ \\
\hline $\mathrm{RNO}$ & 514 & 514 & $0.0 \%$ & 514 & $0.0 \%$ & 510 & $0.8 \%$ & 506 & $1.6 \%$ & 492 & $4.3 \%$ & 488 & $5.1 \%$ \\
\hline $\mathrm{ROC}$ & 378 & 378 & $0.0 \%$ & 378 & $0.0 \%$ & 374 & $1.1 \%$ & 365 & $3.4 \%$ & 367 & $2.9 \%$ & 369 & $2.4 \%$ \\
\hline RSW & 292 & 292 & $0.0 \%$ & 292 & $0.0 \%$ & 292 & $0.0 \%$ & 276 & $5.5 \%$ & 262 & $10.3 \%$ & 196 & $32.9 \%$ \\
\hline SAN & 890 & 838 & $5.8 \%$ & 890 & $0.0 \%$ & 810 & $9.0 \%$ & 838 & $5.8 \%$ & 808 & $9.2 \%$ & 580 & $34.8 \%$ \\
\hline SAT & 864 & 864 & $0.0 \%$ & 864 & $0.0 \%$ & 856 & $0.9 \%$ & 837 & $3.1 \%$ & 817 & $5.4 \%$ & 725 & $16.1 \%$ \\
\hline SBA & 251 & 251 & $0.0 \%$ & 251 & $0.0 \%$ & 251 & $0.0 \%$ & 236 & $6.0 \%$ & 238 & $5.2 \%$ & 210 & $16.3 \%$ \\
\hline SDF & 714 & 714 & $0.0 \%$ & 714 & $0.0 \%$ & 700 & $2.0 \%$ & 626 & $12.3 \%$ & 622 & $12.9 \%$ & 640 & $10.4 \%$ \\
\hline SEA & 1,297 & 1,297 & $0.0 \%$ & 1,297 & $0.0 \%$ & 1,273 & $1.9 \%$ & 1261 & $2.8 \%$ & 1227 & $5.4 \%$ & 861 & $33.6 \%$ \\
\hline SFO & 1,443 & 1,439 & $0.3 \%$ & 1,443 & $0.0 \%$ & 1,355 & $6.1 \%$ & 1388 & $3.8 \%$ & 1338 & $7.3 \%$ & 796 & $44.8 \%$ \\
\hline SJC & 733 & 733 & $0.0 \%$ & 733 & $0.0 \%$ & 711 & $3.0 \%$ & 698 & $4.8 \%$ & 648 & $11.6 \%$ & 514 & $29.9 \%$ \\
\hline SLC & 1,443 & 1,399 & $3.0 \%$ & 1,441 & $0.1 \%$ & 1,425 & $1.2 \%$ & 1361 & $5.7 \%$ & 1357 & $6.0 \%$ & 1013 & $29.8 \%$ \\
\hline SMF & 618 & 618 & $0.0 \%$ & 618 & $0.0 \%$ & 580 & $6.1 \%$ & 586 & $5.2 \%$ & 570 & $7.8 \%$ & 476 & $23.0 \%$ \\
\hline SNA & 828 & 828 & $0.0 \%$ & 828 & $0.0 \%$ & 772 & $6.8 \%$ & 786 & $5.1 \%$ & 792 & $4.3 \%$ & 816 & $1.4 \%$ \\
\hline STL & 879 & 879 & $0.0 \%$ & 879 & $0.0 \%$ & 879 & $0.0 \%$ & 849 & $3.4 \%$ & 853 & $3.0 \%$ & 835 & $5.0 \%$ \\
\hline SWF & 306 & 306 & $0.0 \%$ & 306 & $0.0 \%$ & 306 & $0.0 \%$ & 297 & $2.9 \%$ & 299 & $2.3 \%$ & 305 & $0.3 \%$ \\
\hline SYR & 302 & 302 & $0.0 \%$ & 302 & $0.0 \%$ & 302 & $0.0 \%$ & 286 & $5.3 \%$ & 290 & $4.0 \%$ & 302 & $0.0 \%$ \\
\hline TEB & 812 & 806 & $0.7 \%$ & 812 & $0.0 \%$ & 812 & $0.0 \%$ & 766 & $5.7 \%$ & 768 & $5.4 \%$ & 682 & $16.0 \%$ \\
\hline TPA & 794 & 794 & $0.0 \%$ & 794 & $0.0 \%$ & 794 & $0.0 \%$ & 765 & $3.7 \%$ & 759 & $4.4 \%$ & 705 & $11.2 \%$ \\
\hline TUL & 407 & 407 & $0.0 \%$ & 407 & $0.0 \%$ & 401 & $1.5 \%$ & 395 & $2.9 \%$ & 401 & $1.5 \%$ & 345 & $15.2 \%$ \\
\hline TUS & 430 & 430 & $0.0 \%$ & 430 & $0.0 \%$ & 428 & $0.5 \%$ & 408 & $5.1 \%$ & 414 & $3.7 \%$ & 404 & $6.0 \%$ \\
\hline TVC & 253 & 253 & $0.0 \%$ & 253 & $0.0 \%$ & 247 & $2.4 \%$ & 242 & $4.3 \%$ & 244 & $3.6 \%$ & 126 & $50.2 \%$ \\
\hline TYS & 419 & 419 & $0.0 \%$ & 419 & $0.0 \%$ & 401 & $4.3 \%$ & 402 & $4.1 \%$ & 404 & $3.6 \%$ & 354 & $15.5 \%$ \\
\hline VNY & 827 & 815 & $1.5 \%$ & 827 & $0.0 \%$ & 827 & $0.0 \%$ & 816 & $1.3 \%$ & 822 & $0.6 \%$ & 702 & $15.1 \%$ \\
\hline $40 \mathrm{~N}$ & 0 & 0 & $0.0 \%$ & 0 & 0 & 0 & 0 & 0 & 0 & 0 & 0 & 0 & 0 \\
\hline $55 \mathrm{~J}$ & 11 & 11 & $0.0 \%$ & 11 & $0.0 \%$ & 11 & $0.0 \%$ & 3 & $72.7 \%$ & 7 & $36.4 \%$ & 11 & $0.0 \%$ \\
\hline $\mathrm{ABE}$ & 331 & 331 & $0.0 \%$ & 331 & $0.0 \%$ & 331 & $0.0 \%$ & 327 & $1.2 \%$ & 327 & $1.2 \%$ & 315 & $4.8 \%$ \\
\hline $\mathrm{ABI}$ & 102 & 102 & $0.0 \%$ & 102 & $0.0 \%$ & 102 & $0.0 \%$ & 86 & $15.7 \%$ & 94 & $7.8 \%$ & 102 & $0.0 \%$ \\
\hline ABR & 34 & 34 & $0.0 \%$ & 34 & $0.0 \%$ & 34 & $0.0 \%$ & 22 & $35.3 \%$ & 22 & $35.3 \%$ & 34 & $0.0 \%$ \\
\hline$A B Y$ & 61 & 61 & $0.0 \%$ & 61 & $0.0 \%$ & 61 & $0.0 \%$ & 56 & $8.2 \%$ & 56 & $8.2 \%$ & 30 & $50.8 \%$ \\
\hline ACK & 865 & 819 & $5.3 \%$ & 865 & $0.0 \%$ & 865 & $0.0 \%$ & 831 & $3.9 \%$ & 839 & $3.0 \%$ & 859 & $0.7 \%$ \\
\hline ACT & 74 & 74 & $0.0 \%$ & 74 & $0.0 \%$ & 74 & $0.0 \%$ & 48 & $35.1 \%$ & 54 & $27.0 \%$ & 72 & $2.7 \%$ \\
\hline ACV & 48 & 48 & $0.0 \%$ & 48 & $0.0 \%$ & 48 & $0.0 \%$ & 42 & $12.5 \%$ & 44 & $8.3 \%$ & 24 & $50.0 \%$ \\
\hline $\mathrm{ACY}$ & 166 & 166 & $0.0 \%$ & 166 & $0.0 \%$ & 166 & $0.0 \%$ & 159 & $4.2 \%$ & 161 & $3.0 \%$ & 165 & $0.6 \%$ \\
\hline $\mathrm{ADQ}$ & 67 & 67 & $0.0 \%$ & 67 & $0.0 \%$ & 63 & $6.0 \%$ & 65 & $3.0 \%$ & 67 & $0.0 \%$ & 67 & $0.0 \%$ \\
\hline ADS & 286 & 286 & $0.0 \%$ & 286 & $0.0 \%$ & 286 & $0.0 \%$ & 267 & $6.6 \%$ & 273 & $4.5 \%$ & 215 & $24.8 \%$ \\
\hline$A E G$ & 11 & 11 & $0.0 \%$ & 11 & $0.0 \%$ & 11 & $0.0 \%$ & 9 & $18.2 \%$ & 7 & $36.4 \%$ & 11 & $0.0 \%$ \\
\hline AEX & 69 & 69 & $0.0 \%$ & 69 & $0.0 \%$ & 65 & $5.8 \%$ & 61 & $11.6 \%$ & 63 & $8.7 \%$ & 69 & $0.0 \%$ \\
\hline AFW & 261 & 261 & $0.0 \%$ & 261 & $0.0 \%$ & 255 & $2.3 \%$ & 258 & $1.1 \%$ & 252 & $3.4 \%$ & 258 & $1.1 \%$ \\
\hline AGS & 60 & 60 & $0.0 \%$ & 60 & $0.0 \%$ & 60 & $0.0 \%$ & 54 & $10.0 \%$ & 52 & $13.3 \%$ & 60 & $0.0 \%$ \\
\hline $\mathrm{AHN}$ & 80 & 80 & $0.0 \%$ & 80 & $0.0 \%$ & 80 & $0.0 \%$ & 76 & $5.0 \%$ & 74 & $7.5 \%$ & 80 & $0.0 \%$ \\
\hline ALN & 129 & 129 & $0.0 \%$ & 129 & $0.0 \%$ & 129 & $0.0 \%$ & 105 & $18.6 \%$ & 115 & $10.9 \%$ & 65 & $49.6 \%$ \\
\hline
\end{tabular}


Table 7-5. 2015 Throughput under Constraints at 310 Critical Airports

$=$ highest percentage reduction category in airport. $\quad=$ second highest percentage reduction category in airport.

\begin{tabular}{|c|c|c|c|c|c|c|c|c|c|c|c|c|c|}
\hline \multirow[b]{3}{*}{$\begin{array}{c}\text { Airpor } \\
\mathrm{t}\end{array}$} & \multirow[b]{3}{*}{$\begin{array}{l}\text { Uncon- } \\
\text { strained }\end{array}$} & \multicolumn{6}{|c|}{ Capacity constraints } & \multicolumn{6}{|c|}{ Environmental constraints } \\
\hline & & \multicolumn{2}{|c|}{ Runway } & \multicolumn{2}{|c|}{ Taxi } & \multicolumn{2}{|c|}{ Gate } & \multicolumn{2}{|c|}{ Fuel } & \multicolumn{2}{|c|}{$\mathrm{NOx}$} & \multicolumn{2}{|c|}{ Noise } \\
\hline & & $\begin{array}{l}\text { Daily } \\
\text { ops. }\end{array}$ & $\begin{array}{l}\text { Reduc- } \\
\text { tion }\end{array}$ & $\begin{array}{l}\text { Daily } \\
\text { ops. }\end{array}$ & $\begin{array}{l}\text { Reduc- } \\
\text { tion }\end{array}$ & $\begin{array}{l}\text { Daily } \\
\text { ops. }\end{array}$ & $\begin{array}{l}\text { Reduc- } \\
\text { tion }\end{array}$ & $\begin{array}{l}\text { Daily } \\
\text { ops. }\end{array}$ & $\begin{array}{c}\text { Reduc- } \\
\text { tion }\end{array}$ & $\begin{array}{l}\text { Daily } \\
\text { ops. }\end{array}$ & $\begin{array}{l}\text { Reduc- } \\
\text { tion }\end{array}$ & $\begin{array}{l}\text { Daily } \\
\text { ops. }\end{array}$ & $\begin{array}{c}\text { Reduc- } \\
\text { tion }\end{array}$ \\
\hline ALO & 93 & 93 & $0.0 \%$ & 93 & $0.0 \%$ & 93 & $0.0 \%$ & 80 & $14.0 \%$ & 86 & $7.5 \%$ & 46 & $50.5 \%$ \\
\hline AMA & 115 & 115 & $0.0 \%$ & 115 & $0.0 \%$ & 113 & $1.7 \%$ & 104 & $9.6 \%$ & 106 & $7.8 \%$ & 56 & $51.3 \%$ \\
\hline APA & 606 & 606 & $0.0 \%$ & 606 & $0.0 \%$ & 606 & $0.0 \%$ & 565 & $6.8 \%$ & 575 & $5.1 \%$ & 513 & $15.3 \%$ \\
\hline APF & 281 & 281 & $0.0 \%$ & 281 & $0.0 \%$ & 281 & $0.0 \%$ & 270 & $3.9 \%$ & 270 & $3.9 \%$ & 280 & $0.4 \%$ \\
\hline ARR & 193 & 193 & $0.0 \%$ & 193 & $0.0 \%$ & 193 & $0.0 \%$ & 187 & $3.1 \%$ & 175 & $9.3 \%$ & 193 & $0.0 \%$ \\
\hline ASE & 246 & 246 & $0.0 \%$ & 246 & $0.0 \%$ & 246 & $0.0 \%$ & 243 & $1.2 \%$ & 243 & $1.2 \%$ & 231 & $6.1 \%$ \\
\hline ATW & 152 & 152 & $0.0 \%$ & 152 & $0.0 \%$ & 152 & $0.0 \%$ & 141 & $7.2 \%$ & 141 & $7.2 \%$ & 73 & $52.0 \%$ \\
\hline ATY & 19 & 19 & $0.0 \%$ & 19 & $0.0 \%$ & 19 & $0.0 \%$ & 11 & $42.1 \%$ & 13 & $31.6 \%$ & 19 & $0.0 \%$ \\
\hline AUG & 24 & 24 & $0.0 \%$ & 24 & $0.0 \%$ & 24 & $0.0 \%$ & 22 & $8.3 \%$ & 22 & $8.3 \%$ & 12 & $50.0 \%$ \\
\hline AVL & 248 & 248 & $0.0 \%$ & 248 & $0.0 \%$ & 248 & $0.0 \%$ & 237 & $4.4 \%$ & 237 & $4.4 \%$ & 121 & $51.2 \%$ \\
\hline AVP & 233 & 233 & $0.0 \%$ & 233 & $0.0 \%$ & 233 & $0.0 \%$ & 213 & $8.6 \%$ & 215 & $7.7 \%$ & 117 & $49.8 \%$ \\
\hline AWM & 10 & 10 & $0.0 \%$ & 10 & $0.0 \%$ & 10 & $0.0 \%$ & 8 & $20.0 \%$ & 6 & $40.0 \%$ & 10 & $0.0 \%$ \\
\hline $\mathrm{AZO}$ & 133 & 133 & $0.0 \%$ & 133 & $0.0 \%$ & 133 & $0.0 \%$ & 123 & $7.5 \%$ & 125 & $6.0 \%$ & 133 & $0.0 \%$ \\
\hline BCT & 159 & 159 & $0.0 \%$ & 159 & $0.0 \%$ & 159 & $0.0 \%$ & 153 & $3.8 \%$ & 149 & $6.3 \%$ & 155 & $2.5 \%$ \\
\hline BED & 459 & 449 & $2.2 \%$ & 459 & $0.0 \%$ & 459 & $0.0 \%$ & 447 & $2.6 \%$ & 449 & $2.2 \%$ & 227 & $50.5 \%$ \\
\hline BET & 390 & 390 & $0.0 \%$ & 390 & $0.0 \%$ & 382 & $2.1 \%$ & 386 & $1.0 \%$ & 384 & $1.5 \%$ & 390 & $0.0 \%$ \\
\hline BFF & 26 & 26 & $0.0 \%$ & 26 & $0.0 \%$ & 26 & $0.0 \%$ & 22 & $15.4 \%$ & 24 & $7.7 \%$ & 24 & $7.7 \%$ \\
\hline $\mathrm{BFI}$ & 907 & 837 & $7.7 \%$ & 907 & $0.0 \%$ & 907 & $0.0 \%$ & 885 & $2.4 \%$ & 885 & $2.4 \%$ & 851 & $6.2 \%$ \\
\hline BFM & 176 & 176 & $0.0 \%$ & 176 & $0.0 \%$ & 176 & $0.0 \%$ & 175 & $0.6 \%$ & 175 & $0.6 \%$ & 175 & $0.6 \%$ \\
\hline BGM & 93 & 93 & $0.0 \%$ & 93 & $0.0 \%$ & 93 & $0.0 \%$ & 83 & $10.8 \%$ & 85 & $8.6 \%$ & 93 & $0.0 \%$ \\
\hline BGR & 96 & 96 & $0.0 \%$ & 96 & $0.0 \%$ & 96 & $0.0 \%$ & 90 & $6.3 \%$ & 92 & $4.2 \%$ & 96 & $0.0 \%$ \\
\hline BIL & 348 & 348 & $0.0 \%$ & 348 & $0.0 \%$ & 348 & $0.0 \%$ & 344 & $1.1 \%$ & 342 & $1.7 \%$ & 320 & $8.0 \%$ \\
\hline BIS & 149 & 149 & $0.0 \%$ & 149 & $0.0 \%$ & 149 & $0.0 \%$ & 141 & $5.4 \%$ & 141 & $5.4 \%$ & 149 & $0.0 \%$ \\
\hline BJC & 101 & 101 & $0.0 \%$ & 101 & $0.0 \%$ & 101 & $0.0 \%$ & 94 & $6.9 \%$ & 92 & $8.9 \%$ & 98 & $3.0 \%$ \\
\hline $\mathrm{BKL}$ & 242 & 242 & $0.0 \%$ & 242 & $0.0 \%$ & 242 & $0.0 \%$ & 205 & $15.3 \%$ & 221 & $8.7 \%$ & 121 & $50.0 \%$ \\
\hline $\mathrm{BLI}$ & 287 & 287 & $0.0 \%$ & 287 & $0.0 \%$ & 287 & $0.0 \%$ & 281 & $2.1 \%$ & 281 & $2.1 \%$ & 143 & $50.2 \%$ \\
\hline BLV & 8 & 8 & $0.0 \%$ & 8 & $0.0 \%$ & 8 & $0.0 \%$ & 8 & $0.0 \%$ & 6 & $25.0 \%$ & 8 & $0.0 \%$ \\
\hline BMI & 111 & 111 & $0.0 \%$ & 111 & $0.0 \%$ & 111 & $0.0 \%$ & 105 & $5.4 \%$ & 105 & $5.4 \%$ & 97 & $12.6 \%$ \\
\hline BPT & 101 & 101 & $0.0 \%$ & 101 & $0.0 \%$ & 101 & $0.0 \%$ & 91 & $9.9 \%$ & 91 & $9.9 \%$ & 101 & $0.0 \%$ \\
\hline BTM & 20 & 20 & $0.0 \%$ & 20 & $0.0 \%$ & 20 & $0.0 \%$ & 16 & $20.0 \%$ & 18 & $10.0 \%$ & 10 & $50.0 \%$ \\
\hline BTV & 271 & 271 & $0.0 \%$ & 271 & $0.0 \%$ & 271 & $0.0 \%$ & 267 & $1.5 \%$ & 265 & $2.2 \%$ & 259 & $4.4 \%$ \\
\hline BVY & 176 & 176 & $0.0 \%$ & 176 & $0.0 \%$ & 176 & $0.0 \%$ & 148 & $15.9 \%$ & 154 & $12.5 \%$ & 174 & $1.1 \%$ \\
\hline BZN & 259 & 259 & $0.0 \%$ & 259 & $0.0 \%$ & 259 & $0.0 \%$ & 252 & $2.7 \%$ & 250 & $3.5 \%$ & 258 & $0.4 \%$ \\
\hline CAE & 361 & 361 & $0.0 \%$ & 361 & $0.0 \%$ & 361 & $0.0 \%$ & 343 & $5.0 \%$ & 343 & $5.0 \%$ & 351 & $2.8 \%$ \\
\hline CAK & 317 & 317 & $0.0 \%$ & 317 & $0.0 \%$ & 317 & $0.0 \%$ & 304 & $4.1 \%$ & 310 & $2.2 \%$ & 292 & $7.9 \%$ \\
\hline CCR & 207 & 207 & $0.0 \%$ & 207 & $0.0 \%$ & 207 & $0.0 \%$ & 191 & $7.7 \%$ & 203 & $1.9 \%$ & 207 & $0.0 \%$ \\
\hline CDC & 24 & 24 & $0.0 \%$ & 24 & $0.0 \%$ & 24 & $0.0 \%$ & 20 & $16.7 \%$ & 22 & $8.3 \%$ & 24 & $0.0 \%$ \\
\hline CGF & 113 & 113 & $0.0 \%$ & 113 & $0.0 \%$ & 113 & $0.0 \%$ & 109 & $3.5 \%$ & 107 & $5.3 \%$ & 57 & $49.6 \%$ \\
\hline $\mathrm{CGI}$ & 5 & 5 & $0.0 \%$ & 5 & $0.0 \%$ & 5 & $0.0 \%$ & 3 & $40.0 \%$ & 0 & $100.0 \%$ & 3 & $40.0 \%$ \\
\hline $\mathrm{CHA}$ & 239 & 239 & $0.0 \%$ & 239 & $0.0 \%$ & 239 & $0.0 \%$ & 230 & $3.8 \%$ & 228 & $4.6 \%$ & 118 & $50.6 \%$ \\
\hline $\mathrm{CHO}$ & 211 & 211 & $0.0 \%$ & 211 & $0.0 \%$ & 211 & $0.0 \%$ & 193 & $8.5 \%$ & 191 & $9.5 \%$ & 211 & $0.0 \%$ \\
\hline CID & 242 & 242 & $0.0 \%$ & 242 & $0.0 \%$ & 242 & $0.0 \%$ & 226 & $6.6 \%$ & 226 & $6.6 \%$ & 238 & $1.7 \%$ \\
\hline CKB & 68 & 68 & $0.0 \%$ & 68 & $0.0 \%$ & 68 & $0.0 \%$ & 62 & $8.8 \%$ & 62 & $8.8 \%$ & 68 & $0.0 \%$ \\
\hline CLL & 75 & 75 & $0.0 \%$ & 75 & $0.0 \%$ & 75 & $0.0 \%$ & 51 & $32.0 \%$ & 57 & $24.0 \%$ & 75 & $0.0 \%$ \\
\hline
\end{tabular}


Table 7-5. 2015 Throughput under Constraints at 310 Critical Airports

$=$ highest percentage reduction category in airport. $\quad$ = second highest percentage reduction category in airport.

\begin{tabular}{|c|c|c|c|c|c|c|c|c|c|c|c|c|c|}
\hline \multirow[b]{3}{*}{$\begin{array}{c}\text { Airpor } \\
\mathrm{t}\end{array}$} & \multirow[b]{3}{*}{$\begin{array}{l}\text { Uncon- } \\
\text { strained }\end{array}$} & \multicolumn{6}{|c|}{ Capacity constraints } & \multicolumn{6}{|c|}{ Environmental constraints } \\
\hline & & \multicolumn{2}{|c|}{ Runway } & \multicolumn{2}{|c|}{ Taxi } & \multicolumn{2}{|c|}{ Gate } & \multicolumn{2}{|c|}{ Fuel } & \multicolumn{2}{|c|}{ NOx } & \multicolumn{2}{|c|}{ Noise } \\
\hline & & $\begin{array}{l}\text { Daily } \\
\text { ops. }\end{array}$ & $\begin{array}{c}\text { Reduc- } \\
\text { tion }\end{array}$ & $\begin{array}{l}\text { Daily } \\
\text { ops. }\end{array}$ & $\begin{array}{c}\text { Reduc- } \\
\text { tion }\end{array}$ & $\begin{array}{l}\text { Daily } \\
\text { ops. }\end{array}$ & $\begin{array}{c}\text { Reduc- } \\
\text { tion }\end{array}$ & $\begin{array}{l}\text { Daily } \\
\text { ops. }\end{array}$ & $\begin{array}{l}\text { Reduc- } \\
\text { tion }\end{array}$ & $\begin{array}{l}\text { Daily } \\
\text { ops. }\end{array}$ & $\begin{array}{c}\text { Reduc- } \\
\text { tion }\end{array}$ & $\begin{array}{l}\text { Daily } \\
\text { ops. }\end{array}$ & $\begin{array}{c}\text { Reduc- } \\
\text { tion }\end{array}$ \\
\hline $\mathrm{CMI}$ & 127 & 127 & $0.0 \%$ & 127 & $0.0 \%$ & 127 & $0.0 \%$ & 119 & $6.3 \%$ & 123 & $3.1 \%$ & 127 & $0.0 \%$ \\
\hline $\mathrm{COU}$ & 32 & 32 & $0.0 \%$ & 32 & $0.0 \%$ & 32 & $0.0 \%$ & 30 & $6.3 \%$ & 30 & $6.3 \%$ & 16 & $50.0 \%$ \\
\hline CPR & 122 & 122 & $0.0 \%$ & 122 & $0.0 \%$ & 122 & $0.0 \%$ & 120 & $1.6 \%$ & 120 & $1.6 \%$ & 122 & $0.0 \%$ \\
\hline CPS & 248 & 248 & $0.0 \%$ & 248 & $0.0 \%$ & 248 & $0.0 \%$ & 230 & $7.3 \%$ & 230 & $7.3 \%$ & 208 & $16.1 \%$ \\
\hline CRW & 237 & 237 & $0.0 \%$ & 237 & $0.0 \%$ & 237 & $0.0 \%$ & 208 & $12.2 \%$ & 212 & $10.5 \%$ & 236 & $0.4 \%$ \\
\hline CSG & 142 & 142 & $0.0 \%$ & 142 & $0.0 \%$ & 142 & $0.0 \%$ & 135 & $4.9 \%$ & 131 & $7.7 \%$ & 123 & $13.4 \%$ \\
\hline CWA & 99 & 99 & $0.0 \%$ & 99 & $0.0 \%$ & 99 & $0.0 \%$ & 80 & $19.2 \%$ & 84 & $15.2 \%$ & 99 & $0.0 \%$ \\
\hline CYS & 58 & 58 & $0.0 \%$ & 58 & $0.0 \%$ & 58 & $0.0 \%$ & 56 & $3.4 \%$ & 54 & $6.9 \%$ & 58 & $0.0 \%$ \\
\hline DLH & 189 & 189 & $0.0 \%$ & 189 & $0.0 \%$ & 189 & $0.0 \%$ & 181 & $4.2 \%$ & 183 & $3.2 \%$ & 187 & $1.1 \%$ \\
\hline DPA & 350 & 350 & $0.0 \%$ & 350 & $0.0 \%$ & 350 & $0.0 \%$ & 343 & $2.0 \%$ & 333 & $4.9 \%$ & 345 & $1.4 \%$ \\
\hline DTO & 89 & 89 & $0.0 \%$ & 89 & $0.0 \%$ & 89 & $0.0 \%$ & 75 & $15.7 \%$ & 85 & $4.5 \%$ & 89 & $0.0 \%$ \\
\hline DVT & 444 & 444 & $0.0 \%$ & 444 & $0.0 \%$ & 444 & $0.0 \%$ & 428 & $3.6 \%$ & 440 & $0.9 \%$ & 444 & $0.0 \%$ \\
\hline EAU & 126 & 126 & $0.0 \%$ & 126 & $0.0 \%$ & 126 & $0.0 \%$ & 110 & $12.7 \%$ & 118 & $6.3 \%$ & 62 & $50.8 \%$ \\
\hline EFD & 23 & 23 & $0.0 \%$ & 23 & $0.0 \%$ & 23 & $0.0 \%$ & 20 & $13.0 \%$ & 20 & $13.0 \%$ & 22 & $4.3 \%$ \\
\hline EGE & 142 & 142 & $0.0 \%$ & 142 & $0.0 \%$ & 142 & $0.0 \%$ & 139 & $2.1 \%$ & 137 & $3.5 \%$ & 71 & $50.0 \%$ \\
\hline EQY & 29 & 29 & $0.0 \%$ & 29 & $0.0 \%$ & 29 & $0.0 \%$ & 27 & $6.9 \%$ & 27 & $6.9 \%$ & 29 & $0.0 \%$ \\
\hline ERI & 154 & 154 & $0.0 \%$ & 154 & $0.0 \%$ & 154 & $0.0 \%$ & 135 & $12.3 \%$ & 137 & $11.0 \%$ & 101 & $34.4 \%$ \\
\hline EVV & 202 & 202 & $0.0 \%$ & 202 & $0.0 \%$ & 202 & $0.0 \%$ & 182 & $9.9 \%$ & 184 & $8.9 \%$ & 202 & $0.0 \%$ \\
\hline $\mathrm{FAl}$ & 376 & 376 & $0.0 \%$ & 376 & $0.0 \%$ & 372 & $1.1 \%$ & 355 & $5.6 \%$ & 355 & $5.6 \%$ & 363 & $3.5 \%$ \\
\hline FAR & 174 & 174 & $0.0 \%$ & 174 & $0.0 \%$ & 174 & $0.0 \%$ & 170 & $2.3 \%$ & 166 & $4.6 \%$ & 174 & $0.0 \%$ \\
\hline FAY & 65 & 65 & $0.0 \%$ & 65 & $0.0 \%$ & 65 & $0.0 \%$ & 51 & $21.5 \%$ & 49 & $24.6 \%$ & 59 & $9.2 \%$ \\
\hline FDK & 34 & 34 & $0.0 \%$ & 34 & $0.0 \%$ & 34 & $0.0 \%$ & 30 & $11.8 \%$ & 30 & $11.8 \%$ & 16 & $52.9 \%$ \\
\hline FFZ & 519 & 519 & $0.0 \%$ & 519 & $0.0 \%$ & 519 & $0.0 \%$ & 423 & $18.5 \%$ & 457 & $11.9 \%$ & 519 & $0.0 \%$ \\
\hline FNL & 14 & 14 & $0.0 \%$ & 14 & $0.0 \%$ & 14 & $0.0 \%$ & 13 & $7.1 \%$ & 11 & $21.4 \%$ & 13 & $7.1 \%$ \\
\hline FOE & 60 & 60 & $0.0 \%$ & 60 & $0.0 \%$ & 60 & $0.0 \%$ & 58 & $3.3 \%$ & 58 & $3.3 \%$ & 60 & $0.0 \%$ \\
\hline FRG & 432 & 432 & $0.0 \%$ & 432 & $0.0 \%$ & 432 & $0.0 \%$ & 416 & $3.7 \%$ & 420 & $2.8 \%$ & 366 & $15.3 \%$ \\
\hline FSD & 315 & 315 & $0.0 \%$ & 315 & $0.0 \%$ & 315 & $0.0 \%$ & 307 & $2.5 \%$ & 305 & $3.2 \%$ & 311 & $1.3 \%$ \\
\hline FTG & 163 & 163 & $0.0 \%$ & 163 & $0.0 \%$ & 163 & $0.0 \%$ & 133 & $18.4 \%$ & 151 & $7.4 \%$ & 163 & $0.0 \%$ \\
\hline FTW & 278 & 278 & $0.0 \%$ & 278 & $0.0 \%$ & 278 & $0.0 \%$ & 268 & $3.6 \%$ & 262 & $5.8 \%$ & 136 & $51.1 \%$ \\
\hline FTY & 402 & 402 & $0.0 \%$ & 402 & $0.0 \%$ & 402 & $0.0 \%$ & 393 & $2.2 \%$ & 375 & $6.7 \%$ & 389 & $3.2 \%$ \\
\hline FWA & 198 & 198 & $0.0 \%$ & 198 & $0.0 \%$ & 198 & $0.0 \%$ & 194 & $2.0 \%$ & 192 & $3.0 \%$ & 188 & $5.1 \%$ \\
\hline GCN & 3 & 3 & $0.0 \%$ & 3 & $0.0 \%$ & 3 & $0.0 \%$ & 0 & $100.0 \%$ & 0 & $100.0 \%$ & 3 & $0.0 \%$ \\
\hline GEG & 324 & 324 & $0.0 \%$ & 324 & $0.0 \%$ & 324 & $0.0 \%$ & 307 & $5.2 \%$ & 301 & $7.1 \%$ & 277 & $14.5 \%$ \\
\hline GEU & 106 & 106 & $0.0 \%$ & 106 & $0.0 \%$ & 106 & $0.0 \%$ & 96 & $9.4 \%$ & 100 & $5.7 \%$ & 106 & $0.0 \%$ \\
\hline GJT & 197 & 197 & $0.0 \%$ & 197 & $0.0 \%$ & 197 & $0.0 \%$ & 189 & $4.1 \%$ & 191 & $3.0 \%$ & 195 & $1.0 \%$ \\
\hline GNV & 133 & 133 & $0.0 \%$ & 133 & $0.0 \%$ & 133 & $0.0 \%$ & 122 & $8.3 \%$ & 122 & $8.3 \%$ & 128 & $3.8 \%$ \\
\hline GPT & 129 & 129 & $0.0 \%$ & 129 & $0.0 \%$ & 129 & $0.0 \%$ & 117 & $9.3 \%$ & 119 & $7.8 \%$ & 65 & $49.6 \%$ \\
\hline GRB & 122 & 122 & $0.0 \%$ & 122 & $0.0 \%$ & 122 & $0.0 \%$ & 116 & $4.9 \%$ & 114 & $6.6 \%$ & 104 & $14.8 \%$ \\
\hline GRI & 53 & 53 & $0.0 \%$ & 53 & $0.0 \%$ & 53 & $0.0 \%$ & 43 & $18.9 \%$ & 47 & $11.3 \%$ & 27 & $49.1 \%$ \\
\hline GSP & 247 & 247 & $0.0 \%$ & 247 & $0.0 \%$ & 247 & $0.0 \%$ & 230 & $6.9 \%$ & 226 & $8.5 \%$ & 244 & $1.2 \%$ \\
\hline GTF & 168 & 168 & $0.0 \%$ & 168 & $0.0 \%$ & 168 & $0.0 \%$ & 164 & $2.4 \%$ & 162 & $3.6 \%$ & 160 & $4.8 \%$ \\
\hline GUM & 111 & 111 & $0.0 \%$ & 111 & $0.0 \%$ & 111 & $0.0 \%$ & 39 & $64.9 \%$ & 41 & $63.1 \%$ & 111 & $0.0 \%$ \\
\hline $\mathrm{HAO}$ & 32 & 32 & $0.0 \%$ & 32 & $0.0 \%$ & 32 & $0.0 \%$ & 27 & $15.6 \%$ & 27 & $15.6 \%$ & 29 & $9.4 \%$ \\
\hline $\mathrm{HEF}$ & 211 & 211 & $0.0 \%$ & 211 & $0.0 \%$ & 211 & $0.0 \%$ & 193 & $8.5 \%$ & 193 & $8.5 \%$ & 201 & $4.7 \%$ \\
\hline
\end{tabular}


Table 7-5. 2015 Throughput under Constraints at 310 Critical Airports

$=$ highest percentage reduction category in airport. $\quad=$ second highest percentage reduction category in airport.

\begin{tabular}{|c|c|c|c|c|c|c|c|c|c|c|c|c|c|}
\hline \multirow[b]{3}{*}{$\begin{array}{c}\text { Airpor } \\
\mathrm{t}\end{array}$} & \multirow[b]{3}{*}{$\begin{array}{l}\text { Uncon- } \\
\text { strained }\end{array}$} & \multicolumn{6}{|c|}{ Capacity constraints } & \multicolumn{6}{|c|}{ Environmental constraints } \\
\hline & & \multicolumn{2}{|c|}{ Runway } & \multicolumn{2}{|c|}{ Taxi } & \multicolumn{2}{|c|}{ Gate } & \multicolumn{2}{|c|}{ Fuel } & \multicolumn{2}{|c|}{ NOx } & \multicolumn{2}{|c|}{ Noise } \\
\hline & & $\begin{array}{l}\text { Daily } \\
\text { ops. }\end{array}$ & \begin{tabular}{|c} 
Reduc- \\
tion
\end{tabular} & $\begin{array}{l}\text { Daily } \\
\text { ops. }\end{array}$ & $\begin{array}{c}\text { Reduc- } \\
\text { tion }\end{array}$ & $\begin{array}{l}\text { Daily } \\
\text { ops. }\end{array}$ & $\begin{array}{c}\text { Reduc- } \\
\text { tion }\end{array}$ & $\begin{array}{l}\text { Daily } \\
\text { ops. }\end{array}$ & $\begin{array}{c}\text { Reduc- } \\
\text { tion }\end{array}$ & $\begin{array}{l}\text { Daily } \\
\text { ops. }\end{array}$ & $\begin{array}{c}\text { Reduc- } \\
\text { tion }\end{array}$ & $\begin{array}{l}\text { Daily } \\
\text { ops. }\end{array}$ & $\begin{array}{c}\text { Reduc- } \\
\text { tion }\end{array}$ \\
\hline $\mathrm{HIO}$ & 405 & 405 & $0.0 \%$ & 405 & $0.0 \%$ & 405 & $0.0 \%$ & 383 & $5.4 \%$ & 385 & $4.9 \%$ & 395 & $2.5 \%$ \\
\hline HQZ & 8 & 8 & $0.0 \%$ & 8 & $0.0 \%$ & 8 & $0.0 \%$ & 6 & $25.0 \%$ & 6 & $25.0 \%$ & 8 & $0.0 \%$ \\
\hline $\mathrm{HRL}$ & 124 & 124 & $0.0 \%$ & 124 & $0.0 \%$ & 124 & $0.0 \%$ & 120 & $3.2 \%$ & 120 & $3.2 \%$ & 118 & $4.8 \%$ \\
\hline HSV & 198 & 198 & $0.0 \%$ & 198 & $0.0 \%$ & 198 & $0.0 \%$ & 194 & $2.0 \%$ & 192 & $3.0 \%$ & 198 & $0.0 \%$ \\
\hline HWD & 269 & 269 & $0.0 \%$ & 269 & $0.0 \%$ & 269 & $0.0 \%$ & 219 & $18.6 \%$ & 251 & $6.7 \%$ & 133 & $50.6 \%$ \\
\hline IAG & 122 & 122 & $0.0 \%$ & 122 & $0.0 \%$ & 122 & $0.0 \%$ & 119 & $2.5 \%$ & 119 & $2.5 \%$ & 113 & $7.4 \%$ \\
\hline IDA & 126 & 126 & $0.0 \%$ & 126 & $0.0 \%$ & 126 & $0.0 \%$ & 116 & $7.9 \%$ & 116 & $7.9 \%$ & 126 & $0.0 \%$ \\
\hline ILG & 231 & 231 & $0.0 \%$ & 231 & $0.0 \%$ & 231 & $0.0 \%$ & 223 & $3.5 \%$ & 225 & $2.6 \%$ & 225 & $2.6 \%$ \\
\hline ILM & 285 & 285 & $0.0 \%$ & 285 & $0.0 \%$ & 285 & $0.0 \%$ & 271 & $4.9 \%$ & 269 & $5.6 \%$ & 283 & $0.7 \%$ \\
\hline ISM & 202 & 202 & $0.0 \%$ & 202 & $0.0 \%$ & 202 & $0.0 \%$ & 182 & $9.9 \%$ & 180 & $10.9 \%$ & 94 & $53.5 \%$ \\
\hline ITO & 273 & 273 & $0.0 \%$ & 273 & $0.0 \%$ & 261 & $4.4 \%$ & 270 & $1.1 \%$ & 270 & $1.1 \%$ & 272 & $0.4 \%$ \\
\hline IWA & 329 & 329 & $0.0 \%$ & 329 & $0.0 \%$ & 329 & $0.0 \%$ & 307 & $6.7 \%$ & 321 & $2.4 \%$ & 327 & $0.6 \%$ \\
\hline JAC & 152 & 152 & $0.0 \%$ & 152 & $0.0 \%$ & 132 & $13.2 \%$ & 148 & $2.6 \%$ & 144 & $5.3 \%$ & 72 & $52.6 \%$ \\
\hline JAN & 185 & 185 & $0.0 \%$ & 185 & $0.0 \%$ & 185 & $0.0 \%$ & 175 & $5.4 \%$ & 173 & $6.5 \%$ & 183 & $1.1 \%$ \\
\hline JST & 15 & 15 & $0.0 \%$ & 15 & $0.0 \%$ & 15 & $0.0 \%$ & 3 & $80.0 \%$ & 9 & $40.0 \%$ & 15 & $0.0 \%$ \\
\hline $\mathrm{KOA}$ & 261 & 261 & $0.0 \%$ & 261 & $0.0 \%$ & 181 & $30.7 \%$ & 257 & $1.5 \%$ & 253 & $3.1 \%$ & 261 & $0.0 \%$ \\
\hline KTN & 35 & 35 & $0.0 \%$ & 35 & $0.0 \%$ & 31 & $11.4 \%$ & 33 & $5.7 \%$ & 31 & $11.4 \%$ & 35 & $0.0 \%$ \\
\hline $\mathrm{LAL}$ & 100 & 100 & $0.0 \%$ & 100 & $0.0 \%$ & 100 & $0.0 \%$ & 92 & $8.0 \%$ & 94 & $6.0 \%$ & 100 & $0.0 \%$ \\
\hline LBB & 193 & 193 & $0.0 \%$ & 193 & $0.0 \%$ & 193 & $0.0 \%$ & 179 & $7.3 \%$ & 183 & $5.2 \%$ & 189 & $2.1 \%$ \\
\hline LBE & 101 & 101 & $0.0 \%$ & 101 & $0.0 \%$ & 101 & $0.0 \%$ & 98 & $3.0 \%$ & 96 & $5.0 \%$ & 101 & $0.0 \%$ \\
\hline LBF & 31 & 31 & $0.0 \%$ & 31 & $0.0 \%$ & 31 & $0.0 \%$ & 18 & $41.9 \%$ & 26 & $16.1 \%$ & 30 & $3.2 \%$ \\
\hline LBL & 28 & 28 & $0.0 \%$ & 28 & $0.0 \%$ & 28 & $0.0 \%$ & 26 & $7.1 \%$ & 26 & $7.1 \%$ & 28 & $0.0 \%$ \\
\hline LCK & 130 & 130 & $0.0 \%$ & 130 & $0.0 \%$ & 130 & $0.0 \%$ & 94 & $27.7 \%$ & 112 & $13.8 \%$ & 92 & $29.2 \%$ \\
\hline LEX & 256 & 256 & $0.0 \%$ & 256 & $0.0 \%$ & 256 & $0.0 \%$ & 233 & $9.0 \%$ & 235 & $8.2 \%$ & 255 & $0.4 \%$ \\
\hline LFT & 234 & 234 & $0.0 \%$ & 234 & $0.0 \%$ & 234 & $0.0 \%$ & 222 & $5.1 \%$ & 222 & $5.1 \%$ & 217 & $7.3 \%$ \\
\hline LIH & 324 & 324 & $0.0 \%$ & 324 & $0.0 \%$ & 302 & $6.8 \%$ & 322 & $0.6 \%$ & 318 & $1.9 \%$ & 324 & $0.0 \%$ \\
\hline LNK & 151 & 151 & $0.0 \%$ & 151 & $0.0 \%$ & 151 & $0.0 \%$ & 145 & $4.0 \%$ & 145 & $4.0 \%$ & 149 & $1.3 \%$ \\
\hline LSE & 99 & 99 & $0.0 \%$ & 99 & $0.0 \%$ & 99 & $0.0 \%$ & 92 & $7.1 \%$ & 90 & $9.1 \%$ & 98 & $1.0 \%$ \\
\hline LWM & 128 & 128 & $0.0 \%$ & 128 & $0.0 \%$ & 128 & $0.0 \%$ & 126 & $1.6 \%$ & 126 & $1.6 \%$ & 64 & $50.0 \%$ \\
\hline MAF & 156 & 156 & $0.0 \%$ & 156 & $0.0 \%$ & 156 & $0.0 \%$ & 144 & $7.7 \%$ & 146 & $6.4 \%$ & 136 & $12.8 \%$ \\
\hline MBS & 98 & 98 & $0.0 \%$ & 98 & $0.0 \%$ & 98 & $0.0 \%$ & 92 & $6.1 \%$ & 92 & $6.1 \%$ & 98 & $0.0 \%$ \\
\hline MDT & 192 & 192 & $0.0 \%$ & 192 & $0.0 \%$ & 192 & $0.0 \%$ & 187 & $2.6 \%$ & 189 & $1.6 \%$ & 191 & $0.5 \%$ \\
\hline MFE & 199 & 199 & $0.0 \%$ & 199 & $0.0 \%$ & 199 & $0.0 \%$ & 199 & $0.0 \%$ & 195 & $2.0 \%$ & 179 & $10.1 \%$ \\
\hline MHK & 55 & 55 & $0.0 \%$ & 55 & $0.0 \%$ & 55 & $0.0 \%$ & 52 & $5.5 \%$ & 52 & $5.5 \%$ & 54 & $1.8 \%$ \\
\hline MLI & 141 & 141 & $0.0 \%$ & 141 & $0.0 \%$ & 141 & $0.0 \%$ & 114 & $19.1 \%$ & 124 & $12.1 \%$ & 68 & $51.8 \%$ \\
\hline MLU & 147 & 147 & $0.0 \%$ & 147 & $0.0 \%$ & 147 & $0.0 \%$ & 127 & $13.6 \%$ & 127 & $13.6 \%$ & 147 & $0.0 \%$ \\
\hline MMU & 446 & 446 & $0.0 \%$ & 446 & $0.0 \%$ & 446 & $0.0 \%$ & 437 & $2.0 \%$ & 437 & $2.0 \%$ & 445 & $0.2 \%$ \\
\hline MOB & 131 & 131 & $0.0 \%$ & 131 & $0.0 \%$ & 131 & $0.0 \%$ & 117 & $10.7 \%$ & 121 & $7.6 \%$ & 65 & $50.4 \%$ \\
\hline MOT & 81 & 81 & $0.0 \%$ & 81 & $0.0 \%$ & 81 & $0.0 \%$ & 75 & $7.4 \%$ & 75 & $7.4 \%$ & 75 & $7.4 \%$ \\
\hline MSO & 196 & 196 & $0.0 \%$ & 196 & $0.0 \%$ & 196 & $0.0 \%$ & 191 & $2.6 \%$ & 187 & $4.6 \%$ & 185 & $5.6 \%$ \\
\hline MTN & 224 & 224 & $0.0 \%$ & 224 & $0.0 \%$ & 224 & $0.0 \%$ & 217 & $3.1 \%$ & 219 & $2.2 \%$ & 223 & $0.4 \%$ \\
\hline MYR & 222 & 222 & $0.0 \%$ & 222 & $0.0 \%$ & 222 & $0.0 \%$ & 209 & $5.9 \%$ & 207 & $6.8 \%$ & 179 & $19.4 \%$ \\
\hline OGG & 514 & 514 & $0.0 \%$ & 514 & $0.0 \%$ & 476 & $7.4 \%$ & 500 & $2.7 \%$ & 498 & $3.1 \%$ & 514 & $0.0 \%$ \\
\hline OPF & 291 & 291 & $0.0 \%$ & 291 & $0.0 \%$ & 291 & $0.0 \%$ & 276 & $5.2 \%$ & 280 & $3.8 \%$ & 144 & $50.5 \%$ \\
\hline
\end{tabular}


Table 7-5. 2015 Throughput under Constraints at 310 Critical Airports

$=$ highest percentage reduction category in airport. = second highest percentage reduction category in airport.

\begin{tabular}{|c|c|c|c|c|c|c|c|c|c|c|c|c|c|}
\hline \multirow[b]{3}{*}{$\begin{array}{c}\text { Airpor } \\
\mathrm{t}\end{array}$} & \multirow[b]{3}{*}{$\begin{array}{l}\text { Uncon- } \\
\text { strained }\end{array}$} & \multicolumn{6}{|c|}{ Capacity constraints } & \multicolumn{6}{|c|}{ Environmental constraints } \\
\hline & & \multicolumn{2}{|c|}{ Runway } & \multicolumn{2}{|c|}{ Taxi } & \multicolumn{2}{|c|}{ Gate } & \multicolumn{2}{|c|}{ Fuel } & \multicolumn{2}{|c|}{ NOx } & \multicolumn{2}{|c|}{ Noise } \\
\hline & & $\begin{array}{l}\text { Daily } \\
\text { ops. }\end{array}$ & $\begin{array}{c}\text { Reduc- } \\
\text { tion }\end{array}$ & $\begin{array}{l}\text { Daily } \\
\text { ops. }\end{array}$ & $\begin{array}{l}\text { Reduc- } \\
\text { tion }\end{array}$ & $\begin{array}{l}\text { Daily } \\
\text { ops. }\end{array}$ & $\begin{array}{l}\text { Reduc- } \\
\text { tion }\end{array}$ & $\begin{array}{l}\text { Daily } \\
\text { ops. }\end{array}$ & $\begin{array}{l}\text { Reduc- } \\
\text { tion }\end{array}$ & $\begin{array}{l}\text { Daily } \\
\text { ops. }\end{array}$ & $\begin{array}{l}\text { Reduc- } \\
\text { tion }\end{array}$ & $\begin{array}{l}\text { Daily } \\
\text { ops. }\end{array}$ & $\begin{array}{l}\text { Reduc- } \\
\text { tion }\end{array}$ \\
\hline ORL & 262 & 262 & $0.0 \%$ & 262 & $0.0 \%$ & 262 & $0.0 \%$ & 245 & $6.5 \%$ & 247 & $5.7 \%$ & 131 & $50.0 \%$ \\
\hline PAE & 351 & 351 & $0.0 \%$ & 351 & $0.0 \%$ & 347 & $1.1 \%$ & 344 & $2.0 \%$ & 344 & $2.0 \%$ & 346 & $1.4 \%$ \\
\hline PDK & 683 & 683 & $0.0 \%$ & 683 & $0.0 \%$ & 683 & $0.0 \%$ & 672 & $1.6 \%$ & 664 & $2.8 \%$ & 340 & $50.2 \%$ \\
\hline $\mathrm{PIA}$ & 172 & 172 & $0.0 \%$ & 172 & $0.0 \%$ & 172 & $0.0 \%$ & 168 & $2.3 \%$ & 168 & $2.3 \%$ & 172 & $0.0 \%$ \\
\hline PIR & 45 & 45 & $0.0 \%$ & 45 & $0.0 \%$ & 45 & $0.0 \%$ & 34 & $24.4 \%$ & 38 & $15.6 \%$ & 42 & $6.7 \%$ \\
\hline PNE & 305 & 305 & $0.0 \%$ & 305 & $0.0 \%$ & 305 & $0.0 \%$ & 262 & $14.1 \%$ & 290 & $4.9 \%$ & 304 & $0.3 \%$ \\
\hline PNS & 252 & 252 & $0.0 \%$ & 252 & $0.0 \%$ & 252 & $0.0 \%$ & 244 & $3.2 \%$ & 246 & $2.4 \%$ & 126 & $50.0 \%$ \\
\hline PRC & 278 & 278 & $0.0 \%$ & 278 & $0.0 \%$ & 278 & $0.0 \%$ & 249 & $10.4 \%$ & 269 & $3.2 \%$ & 277 & $0.4 \%$ \\
\hline PSC & 164 & 164 & $0.0 \%$ & 164 & $0.0 \%$ & 164 & $0.0 \%$ & 163 & $0.6 \%$ & 163 & $0.6 \%$ & 164 & $0.0 \%$ \\
\hline PSM & 32 & 32 & $0.0 \%$ & 32 & $0.0 \%$ & 32 & $0.0 \%$ & 29 & $9.4 \%$ & 29 & $9.4 \%$ & 31 & $3.1 \%$ \\
\hline PSP & 143 & 143 & $0.0 \%$ & 143 & $0.0 \%$ & 143 & $0.0 \%$ & 133 & $7.0 \%$ & 133 & $7.0 \%$ & 67 & $53.1 \%$ \\
\hline PTK & 402 & 402 & $0.0 \%$ & 402 & $0.0 \%$ & 402 & $0.0 \%$ & 396 & $1.5 \%$ & 394 & $2.0 \%$ & 380 & $5.5 \%$ \\
\hline PUB & 198 & 198 & $0.0 \%$ & 198 & $0.0 \%$ & 198 & $0.0 \%$ & 166 & $16.2 \%$ & 188 & $5.1 \%$ & 198 & $0.0 \%$ \\
\hline PWK & 401 & 397 & $1.0 \%$ & 401 & $0.0 \%$ & 401 & $0.0 \%$ & 384 & $4.2 \%$ & 384 & $4.2 \%$ & 366 & $8.7 \%$ \\
\hline PWM & 306 & 306 & $0.0 \%$ & 306 & $0.0 \%$ & 306 & $0.0 \%$ & 287 & $6.2 \%$ & 291 & $4.9 \%$ & 147 & $52.0 \%$ \\
\hline RAP & 192 & 192 & $0.0 \%$ & 192 & $0.0 \%$ & 192 & $0.0 \%$ & 174 & $9.4 \%$ & 172 & $10.4 \%$ & 104 & $45.8 \%$ \\
\hline RBD & 159 & 159 & $0.0 \%$ & 159 & $0.0 \%$ & 159 & $0.0 \%$ & 121 & $23.9 \%$ & 149 & $6.3 \%$ & 159 & $0.0 \%$ \\
\hline RNT & 181 & 181 & $0.0 \%$ & 181 & $0.0 \%$ & 181 & $0.0 \%$ & 177 & $2.2 \%$ & 179 & $1.1 \%$ & 181 & $0.0 \%$ \\
\hline ROA & 171 & 171 & $0.0 \%$ & 171 & $0.0 \%$ & 171 & $0.0 \%$ & 163 & $4.7 \%$ & 165 & $3.5 \%$ & 169 & $1.2 \%$ \\
\hline RST & 154 & 154 & $0.0 \%$ & 154 & $0.0 \%$ & 154 & $0.0 \%$ & 150 & $2.6 \%$ & 148 & $3.9 \%$ & 154 & $0.0 \%$ \\
\hline RYY & 219 & 219 & $0.0 \%$ & 219 & $0.0 \%$ & 219 & $0.0 \%$ & 214 & $2.3 \%$ & 216 & $1.4 \%$ & 219 & $0.0 \%$ \\
\hline SAF & 156 & 156 & $0.0 \%$ & 156 & $0.0 \%$ & 156 & $0.0 \%$ & 138 & $11.5 \%$ & 140 & $10.3 \%$ & 154 & $1.3 \%$ \\
\hline SAV & 281 & 281 & $0.0 \%$ & 281 & $0.0 \%$ & 281 & $0.0 \%$ & 263 & $6.4 \%$ & 257 & $8.5 \%$ & 275 & $2.1 \%$ \\
\hline SBN & 160 & 160 & $0.0 \%$ & 160 & $0.0 \%$ & 160 & $0.0 \%$ & 155 & $3.1 \%$ & 155 & $3.1 \%$ & 159 & $0.6 \%$ \\
\hline SDL & 433 & 433 & $0.0 \%$ & 433 & $0.0 \%$ & 433 & $0.0 \%$ & 416 & $3.9 \%$ & 408 & $5.8 \%$ & 430 & $0.7 \%$ \\
\hline SDM & 110 & 110 & $0.0 \%$ & 110 & $0.0 \%$ & 110 & $0.0 \%$ & 92 & $16.4 \%$ & 104 & $5.5 \%$ & 110 & $0.0 \%$ \\
\hline SEE & 565 & 565 & $0.0 \%$ & 565 & $0.0 \%$ & 565 & $0.0 \%$ & 460 & $18.6 \%$ & 522 & $7.6 \%$ & 562 & $0.5 \%$ \\
\hline SFB & 324 & 324 & $0.0 \%$ & 324 & $0.0 \%$ & 324 & $0.0 \%$ & 286 & $11.7 \%$ & 282 & $13.0 \%$ & 228 & $29.6 \%$ \\
\hline SGF & 256 & 256 & $0.0 \%$ & 256 & $0.0 \%$ & 256 & $0.0 \%$ & 250 & $2.3 \%$ & 252 & $1.6 \%$ & 250 & $2.3 \%$ \\
\hline SGR & 163 & 163 & $0.0 \%$ & 163 & $0.0 \%$ & 163 & $0.0 \%$ & 143 & $12.3 \%$ & 133 & $18.4 \%$ & 159 & $2.5 \%$ \\
\hline SHV & 196 & 196 & $0.0 \%$ & 196 & $0.0 \%$ & 196 & $0.0 \%$ & 185 & $5.6 \%$ & 187 & $4.6 \%$ & 195 & $0.5 \%$ \\
\hline SJU & 608 & 608 & $0.0 \%$ & 608 & $0.0 \%$ & 608 & $0.0 \%$ & 603 & $0.8 \%$ & 559 & $8.1 \%$ & 603 & $0.8 \%$ \\
\hline SMX & 247 & 247 & $0.0 \%$ & 247 & $0.0 \%$ & 247 & $0.0 \%$ & 233 & $5.7 \%$ & 233 & $5.7 \%$ & 243 & $1.6 \%$ \\
\hline SRQ & 172 & 172 & $0.0 \%$ & 172 & $0.0 \%$ & 172 & $0.0 \%$ & 170 & $1.2 \%$ & 168 & $2.3 \%$ & 150 & $12.8 \%$ \\
\hline STP & 461 & 461 & $0.0 \%$ & 461 & $0.0 \%$ & 461 & $0.0 \%$ & 437 & $5.2 \%$ & 443 & $3.9 \%$ & 225 & $51.2 \%$ \\
\hline STT & 230 & 230 & $0.0 \%$ & 230 & $0.0 \%$ & 210 & $8.7 \%$ & 228 & $0.9 \%$ & 228 & $0.9 \%$ & 230 & $0.0 \%$ \\
\hline SUS & 366 & 366 & $0.0 \%$ & 366 & $0.0 \%$ & 366 & $0.0 \%$ & 341 & $6.8 \%$ & 347 & $5.2 \%$ & 319 & $12.8 \%$ \\
\hline SUX & 47 & 47 & $0.0 \%$ & 47 & $0.0 \%$ & 47 & $0.0 \%$ & 39 & $17.0 \%$ & 41 & $12.8 \%$ & 23 & $51.1 \%$ \\
\hline TKI & 97 & 97 & $0.0 \%$ & 97 & $0.0 \%$ & 97 & $0.0 \%$ & 95 & $2.1 \%$ & 91 & $6.2 \%$ & 97 & $0.0 \%$ \\
\hline TLH & 210 & 210 & $0.0 \%$ & 210 & $0.0 \%$ & 210 & $0.0 \%$ & 199 & $5.2 \%$ & 201 & $4.3 \%$ & 205 & $2.4 \%$ \\
\hline TMB & 448 & 448 & $0.0 \%$ & 448 & $0.0 \%$ & 448 & $0.0 \%$ & 404 & $9.8 \%$ & 428 & $4.5 \%$ & 224 & $50.0 \%$ \\
\hline TOA & 267 & 267 & $0.0 \%$ & 267 & $0.0 \%$ & 267 & $0.0 \%$ & 209 & $21.7 \%$ & 227 & $15.0 \%$ & 263 & $1.5 \%$ \\
\hline TOL & 170 & 170 & $0.0 \%$ & 170 & $0.0 \%$ & 170 & $0.0 \%$ & 166 & $2.4 \%$ & 162 & $4.7 \%$ & 170 & $0.0 \%$ \\
\hline TRI & 176 & 176 & $0.0 \%$ & 176 & $0.0 \%$ & 174 & $1.1 \%$ & 165 & $6.3 \%$ & 163 & $7.4 \%$ & 87 & $50.6 \%$ \\
\hline
\end{tabular}


Table 7-5. 2015 Throughput under Constraints at 310 Critical Airports

$=$ highest percentage reduction category in airport. $\quad=$ second highest percentage reduction category in airport.

\begin{tabular}{|c|c|c|c|c|c|c|c|c|c|c|c|c|c|}
\hline \multirow[b]{3}{*}{$\begin{array}{c}\text { Airpor } \\
\mathrm{t}\end{array}$} & \multirow[b]{3}{*}{$\begin{array}{l}\text { Uncon- } \\
\text { strained }\end{array}$} & \multicolumn{6}{|c|}{ Capacity constraints } & \multicolumn{6}{|c|}{ Environmental constraints } \\
\hline & & \multicolumn{2}{|c|}{ Runway } & \multicolumn{2}{|c|}{ Taxi } & \multicolumn{2}{|c|}{ Gate } & \multicolumn{2}{|c|}{ Fuel } & \multicolumn{2}{|c|}{ NOx } & \multicolumn{2}{|c|}{ Noise } \\
\hline & & $\begin{array}{l}\text { Daily } \\
\text { ops. }\end{array}$ & $\begin{array}{l}\text { Reduc- } \\
\text { tion }\end{array}$ & $\begin{array}{l}\text { Daily } \\
\text { ops. }\end{array}$ & $\begin{array}{c}\text { Reduc- } \\
\text { tion }\end{array}$ & $\begin{array}{l}\text { Daily } \\
\text { ops. }\end{array}$ & $\begin{array}{l}\text { Reduc- } \\
\text { tion }\end{array}$ & $\begin{array}{l}\text { Daily } \\
\text { ops. }\end{array}$ & $\begin{array}{l}\text { Reduc- } \\
\text { tion }\end{array}$ & $\begin{array}{l}\text { Daily } \\
\text { ops. }\end{array}$ & $\begin{array}{c}\text { Reduc- } \\
\text { tion }\end{array}$ & $\begin{array}{l}\text { Daily } \\
\text { ops. }\end{array}$ & $\begin{array}{c}\text { Reduc- } \\
\text { tion }\end{array}$ \\
\hline TTD & 203 & 203 & $0.0 \%$ & 203 & $0.0 \%$ & 203 & $0.0 \%$ & 156 & $23.2 \%$ & 176 & $13.3 \%$ & 202 & $0.5 \%$ \\
\hline UGN & 189 & 189 & $0.0 \%$ & 189 & $0.0 \%$ & 189 & $0.0 \%$ & 185 & $2.1 \%$ & 177 & $6.3 \%$ & 161 & $14.8 \%$ \\
\hline VGT & 546 & 546 & $0.0 \%$ & 546 & $0.0 \%$ & 546 & $0.0 \%$ & 448 & $17.9 \%$ & 424 & $22.3 \%$ & 546 & $0.0 \%$ \\
\hline VPS & 58 & 58 & $0.0 \%$ & 58 & $0.0 \%$ & 58 & $0.0 \%$ & 52 & $10.3 \%$ & 52 & $10.3 \%$ & 58 & $0.0 \%$ \\
\hline XNA & 163 & 163 & $0.0 \%$ & 163 & $0.0 \%$ & 163 & $0.0 \%$ & 141 & $13.5 \%$ & 151 & $7.4 \%$ & 161 & $1.2 \%$ \\
\hline YIP & 312 & 312 & $0.0 \%$ & 312 & $0.0 \%$ & 312 & $0.0 \%$ & 306 & $1.9 \%$ & 302 & $3.2 \%$ & 296 & $5.1 \%$ \\
\hline Total & 131,254 & 128,284 & $97.7 \%$ & 130,560 & $99.5 \%$ & 128,752 & $98.1 \%$ & 124,167 & $94.6 \%$ & 123,570 & $94.1 \%$ & 112,720 & $85.9 \%$ \\
\hline
\end{tabular}

Table 7-6. 2025 Throughput under Constraints at 310 Critical Airports

= highest percentage reduction category in airport. = second highest percentage reduction category in airport.

\begin{tabular}{|c|c|c|c|c|c|c|c|c|c|c|c|c|c|}
\hline \multirow[b]{3}{*}{$\begin{array}{c}\text { Airpor } \\
\mathrm{t}\end{array}$} & \multirow[b]{3}{*}{$\begin{array}{l}\text { Uncon- } \\
\text { strained }\end{array}$} & \multicolumn{6}{|c|}{ Capacity constraints } & \multicolumn{6}{|c|}{ Environmental constraints } \\
\hline & & \multicolumn{2}{|c|}{ Runway } & \multicolumn{2}{|c|}{ Taxi } & \multicolumn{2}{|c|}{ Gate } & \multicolumn{2}{|c|}{ Fuel } & \multicolumn{2}{|c|}{$\mathrm{NOx}$} & \multicolumn{2}{|c|}{ Noise } \\
\hline & & $\begin{array}{l}\text { Daily } \\
\text { ops. }\end{array}$ & $\begin{array}{l}\text { Reduc- } \\
\text { tion }\end{array}$ & $\begin{array}{l}\text { Daily } \\
\text { ops. }\end{array}$ & $\begin{array}{c}\text { Reduc- } \\
\text { tion }\end{array}$ & $\begin{array}{l}\text { Daily } \\
\text { ops. }\end{array}$ & $\begin{array}{l}\text { Reduc- } \\
\text { tion }\end{array}$ & $\begin{array}{l}\text { Daily } \\
\text { ops. }\end{array}$ & $\begin{array}{l}\text { Reduc- } \\
\text { tion }\end{array}$ & $\begin{array}{l}\text { Daily } \\
\text { ops. }\end{array}$ & $\begin{array}{l}\text { Reduc- } \\
\text { tion }\end{array}$ & $\begin{array}{l}\text { Daily } \\
\text { ops. }\end{array}$ & $\begin{array}{l}\text { Reduc- } \\
\text { tion }\end{array}$ \\
\hline $\mathrm{ABQ}$ & 934 & 934 & $0.0 \%$ & 934 & $0.0 \%$ & 906 & $3.0 \%$ & 908 & $2.8 \%$ & 892 & $4.5 \%$ & 882 & $5.6 \%$ \\
\hline ALB & 482 & 482 & $0.0 \%$ & 482 & $0.0 \%$ & 482 & $0.0 \%$ & 450 & $6.6 \%$ & 444 & $7.9 \%$ & 436 & $9.5 \%$ \\
\hline$\overline{A N C}$ & 1,563 & 1,333 & $14.7 \%$ & 1,563 & $0.0 \%$ & 1,365 & $12.7 \%$ & 1371 & $12.3 \%$ & 1313 & $16.0 \%$ & 1363 & $12.8 \%$ \\
\hline ATL & 4,383 & 3,605 & $17.8 \%$ & 3,481 & $20.6 \%$ & 4,137 & $5.6 \%$ & 4371 & $0.3 \%$ & 4167 & $4.9 \%$ & 3901 & $11.0 \%$ \\
\hline AUS & 1,017 & 1,017 & $0.0 \%$ & 1,017 & $0.0 \%$ & 973 & $4.3 \%$ & 989 & $2.8 \%$ & 971 & $4.5 \%$ & 835 & $17.9 \%$ \\
\hline $\mathrm{BDL}$ & 666 & 666 & $0.0 \%$ & 666 & $0.0 \%$ & 662 & $0.6 \%$ & 636 & $4.5 \%$ & 606 & $9.0 \%$ & 552 & $17.1 \%$ \\
\hline $\mathrm{BFL}$ & 305 & 305 & $0.0 \%$ & 305 & $0.0 \%$ & 305 & $0.0 \%$ & 264 & $13.4 \%$ & 268 & $12.1 \%$ & 258 & $15.4 \%$ \\
\hline $\mathrm{BHM}$ & 589 & 589 & $0.0 \%$ & 589 & $0.0 \%$ & 577 & $2.0 \%$ & 551 & $6.5 \%$ & 551 & $6.5 \%$ & 503 & $14.6 \%$ \\
\hline BNA & 941 & 941 & $0.0 \%$ & 941 & $0.0 \%$ & 941 & $0.0 \%$ & 895 & $4.9 \%$ & 867 & $7.9 \%$ & 939 & $0.2 \%$ \\
\hline $\mathrm{BOI}$ & 736 & 736 & $0.0 \%$ & 736 & $0.0 \%$ & 736 & $0.0 \%$ & 702 & $4.6 \%$ & 688 & $6.5 \%$ & 694 & $5.7 \%$ \\
\hline BOS & 1,750 & 1,722 & $1.6 \%$ & 1,743 & $0.4 \%$ & 1,704 & $2.6 \%$ & 1721 & $1.7 \%$ & 1703 & $2.7 \%$ & 1457 & $16.7 \%$ \\
\hline BTR & 367 & 367 & $0.0 \%$ & 367 & $0.0 \%$ & 357 & $2.7 \%$ & 345 & $6.0 \%$ & 343 & $6.5 \%$ & 367 & $0.0 \%$ \\
\hline BUF & 523 & 523 & $0.0 \%$ & 523 & $0.0 \%$ & 501 & $4.2 \%$ & 479 & $8.4 \%$ & 483 & $7.6 \%$ & 475 & $9.2 \%$ \\
\hline BUR & 731 & 731 & $0.0 \%$ & 731 & $0.0 \%$ & 693 & $5.2 \%$ & 697 & $4.7 \%$ & 701 & $4.1 \%$ & 621 & $15.0 \%$ \\
\hline BWI & 1,386 & 1,386 & $0.0 \%$ & 1,386 & $0.0 \%$ & 1,348 & $2.7 \%$ & 1323 & $4.5 \%$ & 1277 & $7.9 \%$ & 1165 & $15.9 \%$ \\
\hline $\mathrm{CHS}$ & 415 & 415 & $0.0 \%$ & 415 & $0.0 \%$ & 387 & $6.7 \%$ & 393 & $5.3 \%$ & 395 & $4.8 \%$ & 373 & $10.1 \%$ \\
\hline CLE & 1,194 & 1,194 & $0.0 \%$ & 1,047 & $12.3 \%$ & 1,194 & $0.0 \%$ & 1080 & $9.5 \%$ & 1090 & $8.7 \%$ & 1050 & $12.1 \%$ \\
\hline CLT & 2,232 & 2,232 & $0.0 \%$ & 1,987 & $11.0 \%$ & 2,076 & $7.0 \%$ & 2148 & $3.8 \%$ & 2108 & $5.6 \%$ & 1896 & $15.1 \%$ \\
\hline $\mathrm{CMH}$ & 841 & 841 & $0.0 \%$ & 841 & $0.0 \%$ & 801 & $4.8 \%$ & 714 & $15.1 \%$ & 742 & $11.8 \%$ & 838 & $0.4 \%$ \\
\hline COS & 353 & 353 & $0.0 \%$ & 353 & $0.0 \%$ & 353 & $0.0 \%$ & 337 & $4.5 \%$ & 339 & $4.0 \%$ & 353 & $0.0 \%$ \\
\hline CRP & 198 & 198 & $0.0 \%$ & 198 & $0.0 \%$ & 190 & $4.0 \%$ & 180 & $9.1 \%$ & 182 & $8.1 \%$ & 198 & $0.0 \%$ \\
\hline CVG & 1,542 & 1,526 & $1.0 \%$ & 1,542 & $0.0 \%$ & 1,542 & $0.0 \%$ & 1352 & $12.3 \%$ & 1372 & $11.0 \%$ & 1390 & $9.9 \%$ \\
\hline DAB & 553 & 553 & $0.0 \%$ & 553 & $0.0 \%$ & 553 & $0.0 \%$ & 542 & $2.0 \%$ & 548 & $0.9 \%$ & 552 & $0.2 \%$ \\
\hline DAL & 1,186 & 1,172 & $1.2 \%$ & 1,186 & $0.0 \%$ & 1,064 & $10.3 \%$ & 1105 & $6.8 \%$ & 1029 & $13.2 \%$ & 955 & $19.5 \%$ \\
\hline DAY & 351 & 351 & $0.0 \%$ & 351 & $0.0 \%$ & 333 & $5.1 \%$ & 311 & $11.4 \%$ & 329 & $6.3 \%$ & 257 & $26.8 \%$ \\
\hline DCA & 893 & 889 & $0.4 \%$ & 886 & $0.8 \%$ & 835 & $6.5 \%$ & 818 & $8.4 \%$ & 892 & $0.1 \%$ & 766 & $14.2 \%$ \\
\hline DEN & 2,621 & 2,621 & $0.0 \%$ & 2,621 & $0.0 \%$ & 2,471 & $5.7 \%$ & 2564 & $2.2 \%$ & 2486 & $5.2 \%$ & 2616 & $0.2 \%$ \\
\hline DFW & 3,099 & 3,099 & $0.0 \%$ & 3,050 & $1.6 \%$ & 3,099 & $0.0 \%$ & 3087 & $0.4 \%$ & 2971 & $4.1 \%$ & 2941 & $5.1 \%$ \\
\hline DSM & 390 & 390 & $0.0 \%$ & 390 & $0.0 \%$ & 374 & $4.1 \%$ & 366 & $6.2 \%$ & 368 & $5.6 \%$ & 328 & $15.9 \%$ \\
\hline
\end{tabular}


Table 7-6. 2025 Throughput under Constraints at 310 Critical Airports

= highest percentage reduction category in airport. $\quad=$ second highest percentage reduction category in airport.

\begin{tabular}{|c|c|c|c|c|c|c|c|c|c|c|c|c|c|}
\hline \multirow[b]{3}{*}{$\begin{array}{c}\text { Airpor } \\
\mathrm{t}\end{array}$} & \multirow[b]{3}{*}{$\begin{array}{l}\text { Uncon- } \\
\text { strained }\end{array}$} & \multicolumn{6}{|c|}{ Capacity constraints } & \multicolumn{6}{|c|}{ Environmental constraints } \\
\hline & & \multicolumn{2}{|c|}{ Runway } & \multicolumn{2}{|c|}{ Taxi } & \multicolumn{2}{|c|}{ Gate } & \multicolumn{2}{|c|}{ Fuel } & \multicolumn{2}{|c|}{$\mathrm{NOx}$} & \multicolumn{2}{|c|}{ Noise } \\
\hline & & $\begin{array}{l}\text { Daily } \\
\text { ops. }\end{array}$ & $\begin{array}{c}\text { Reduc- } \\
\text { tion }\end{array}$ & $\begin{array}{l}\text { Daily } \\
\text { ops. }\end{array}$ & $\begin{array}{l}\text { Reduc- } \\
\text { tion }\end{array}$ & $\begin{array}{l}\text { Daily } \\
\text { ops. }\end{array}$ & $\begin{array}{l}\text { Reduc- } \\
\text { tion }\end{array}$ & $\begin{array}{l}\text { Daily } \\
\text { ops. }\end{array}$ & $\begin{array}{l}\text { Reduc- } \\
\text { tion }\end{array}$ & $\begin{array}{l}\text { Daily } \\
\text { ops. }\end{array}$ & $\begin{array}{l}\text { Reduc- } \\
\text { tion }\end{array}$ & $\begin{array}{l}\text { Daily } \\
\text { ops. }\end{array}$ & $\begin{array}{c}\text { Reduc- } \\
\text { tion }\end{array}$ \\
\hline DTW & 1,979 & 1,909 & $3.5 \%$ & 1,979 & $0.0 \%$ & 1,979 & $0.0 \%$ & 1912 & $3.4 \%$ & 1832 & $7.4 \%$ & 1878 & $5.1 \%$ \\
\hline ELP & 316 & 316 & $0.0 \%$ & 316 & $0.0 \%$ & 316 & $0.0 \%$ & 313 & $0.9 \%$ & 313 & $0.9 \%$ & 299 & $5.4 \%$ \\
\hline EUG & 256 & 256 & $0.0 \%$ & 256 & $0.0 \%$ & 256 & $0.0 \%$ & 224 & $12.5 \%$ & 228 & $10.9 \%$ & 126 & $50.8 \%$ \\
\hline EWR & 2,111 & 1,545 & $26.8 \%$ & 1,949 & $7.7 \%$ & 1,953 & $7.5 \%$ & 2047 & $3.0 \%$ & 1931 & $8.5 \%$ & 1585 & $24.9 \%$ \\
\hline FAT & 615 & 615 & $0.0 \%$ & 615 & $0.0 \%$ & 611 & $0.7 \%$ & 569 & $7.5 \%$ & 567 & $7.8 \%$ & 615 & $0.0 \%$ \\
\hline FLL & 1,281 & 1,281 & $0.0 \%$ & 1,281 & $0.0 \%$ & 1,237 & $3.4 \%$ & 1254 & $2.1 \%$ & 1204 & $6.0 \%$ & 1070 & $16.5 \%$ \\
\hline FNT & 201 & 201 & $0.0 \%$ & 201 & $0.0 \%$ & 199 & $1.0 \%$ & 191 & $5.0 \%$ & 191 & $5.0 \%$ & 201 & $0.0 \%$ \\
\hline FXE & 691 & 691 & $0.0 \%$ & 691 & $0.0 \%$ & 691 & $0.0 \%$ & 596 & $13.7 \%$ & 652 & $5.6 \%$ & 584 & $15.5 \%$ \\
\hline GFK & 381 & 381 & $0.0 \%$ & 381 & $0.0 \%$ & 381 & $0.0 \%$ & 371 & $2.6 \%$ & 373 & $2.1 \%$ & 381 & $0.0 \%$ \\
\hline GRR & 414 & 414 & $0.0 \%$ & 414 & $0.0 \%$ & 394 & $4.8 \%$ & 388 & $6.3 \%$ & 382 & $7.7 \%$ & 386 & $6.8 \%$ \\
\hline GSO & 502 & 502 & $0.0 \%$ & 502 & $0.0 \%$ & 500 & $0.4 \%$ & 474 & $5.6 \%$ & 434 & $13.5 \%$ & 476 & $5.2 \%$ \\
\hline GYY & 187 & 187 & $0.0 \%$ & 187 & $0.0 \%$ & 187 & $0.0 \%$ & 172 & $8.0 \%$ & 160 & $14.4 \%$ & 178 & $4.8 \%$ \\
\hline $\mathrm{HNL}$ & 1,270 & 1,260 & $0.8 \%$ & 1,270 & $0.0 \%$ & 960 & $24.4 \%$ & 1213 & $4.5 \%$ & 1207 & $5.0 \%$ & 1267 & $0.2 \%$ \\
\hline $\mathrm{HOU}$ & 1,055 & 1,023 & $3.0 \%$ & 1,055 & $0.0 \%$ & 1,011 & $4.2 \%$ & 995 & $5.7 \%$ & 985 & $6.6 \%$ & 1029 & $2.5 \%$ \\
\hline HPN & 893 & 871 & $2.5 \%$ & 893 & $0.0 \%$ & 867 & $2.9 \%$ & 821 & $8.1 \%$ & 825 & $7.6 \%$ & 437 & $51.1 \%$ \\
\hline$I A D$ & 2,083 & 1,999 & $4.0 \%$ & 2,083 & $0.0 \%$ & 1,979 & $5.0 \%$ & 2013 & $3.4 \%$ & 1859 & $10.8 \%$ & 1785 & $14.3 \%$ \\
\hline $\mathrm{IAH}$ & 2,848 & 2,810 & $1.3 \%$ & 2,848 & $0.0 \%$ & 2,752 & $3.4 \%$ & 2639 & $7.3 \%$ & 2609 & $8.4 \%$ & 2697 & $5.3 \%$ \\
\hline ICT & 444 & 444 & $0.0 \%$ & 444 & $0.0 \%$ & 438 & $1.4 \%$ & 415 & $6.5 \%$ & 423 & $4.7 \%$ & 431 & $2.9 \%$ \\
\hline IND & 891 & 891 & $0.0 \%$ & 891 & $0.0 \%$ & 839 & $5.8 \%$ & 867 & $2.7 \%$ & 843 & $5.4 \%$ & 847 & $4.9 \%$ \\
\hline ISP & 558 & 558 & $0.0 \%$ & 558 & $0.0 \%$ & 546 & $2.2 \%$ & 526 & $5.7 \%$ & 530 & $5.0 \%$ & 556 & $0.4 \%$ \\
\hline JAX & 547 & 547 & $0.0 \%$ & 547 & $0.0 \%$ & 537 & $1.8 \%$ & 510 & $6.8 \%$ & 500 & $8.6 \%$ & 264 & $51.7 \%$ \\
\hline JFK & 2,327 & 1,899 & $18.4 \%$ & 2,327 & $0.0 \%$ & 1,927 & $17.2 \%$ & 2208 & $5.1 \%$ & 2056 & $11.6 \%$ & 1708 & $26.6 \%$ \\
\hline JNU & 784 & 760 & $3.1 \%$ & 784 & $0.0 \%$ & 778 & $0.8 \%$ & 768 & $2.0 \%$ & 772 & $1.5 \%$ & 782 & $0.3 \%$ \\
\hline LAN & 208 & 208 & $0.0 \%$ & 208 & $0.0 \%$ & 208 & $0.0 \%$ & 202 & $2.9 \%$ & 204 & $1.9 \%$ & 102 & $51.0 \%$ \\
\hline LAS & 2,760 & 1,684 & $39.0 \%$ & 2,760 & $0.0 \%$ & 2,428 & $12.0 \%$ & 2690 & $2.5 \%$ & 2330 & $15.6 \%$ & 2188 & $20.7 \%$ \\
\hline LAX & 3,678 & 2,834 & $22.9 \%$ & 3,362 & $8.6 \%$ & 2,942 & $20.0 \%$ & 3531 & $4.0 \%$ & 3181 & $13.5 \%$ & 2929 & $20.4 \%$ \\
\hline LGA & 1,287 & 1,279 & $0.6 \%$ & 1,287 & $0.0 \%$ & 1,287 & $0.0 \%$ & 1215 & $5.6 \%$ & 1287 & $0.0 \%$ & 1175 & $8.7 \%$ \\
\hline LGB & 630 & 630 & $0.0 \%$ & 630 & $0.0 \%$ & 626 & $0.6 \%$ & 592 & $6.0 \%$ & 594 & $5.7 \%$ & 528 & $16.2 \%$ \\
\hline LIT & 446 & 446 & $0.0 \%$ & 446 & $0.0 \%$ & 442 & $0.9 \%$ & 424 & $4.9 \%$ & 428 & $4.0 \%$ & 398 & $10.8 \%$ \\
\hline $\mathrm{MCl}$ & 914 & 914 & $0.0 \%$ & 914 & $0.0 \%$ & 914 & $0.0 \%$ & 886 & $3.1 \%$ & 862 & $5.7 \%$ & 778 & $14.9 \%$ \\
\hline $\mathrm{MCO}$ & 1,577 & 1,577 & $0.0 \%$ & 1,577 & $0.0 \%$ & 1,557 & $1.3 \%$ & 1563 & $0.9 \%$ & 1493 & $5.3 \%$ & 1315 & $16.6 \%$ \\
\hline MDW & 1,333 & 1,229 & $7.8 \%$ & 1,333 & $0.0 \%$ & 1,277 & $4.2 \%$ & 1175 & $11.9 \%$ & 1241 & $6.9 \%$ & 1091 & $18.2 \%$ \\
\hline MEM & 1,611 & 1,457 & $9.6 \%$ & 1,611 & $0.0 \%$ & 1,605 & $0.4 \%$ & 1596 & $0.9 \%$ & 1526 & $5.3 \%$ & 1358 & $15.7 \%$ \\
\hline MHT & 381 & 381 & $0.0 \%$ & 381 & $0.0 \%$ & 371 & $2.6 \%$ & 361 & $5.2 \%$ & 367 & $3.7 \%$ & 381 & $0.0 \%$ \\
\hline MIA & 1,489 & 1,489 & $0.0 \%$ & 1,489 & $0.0 \%$ & 1,479 & $0.7 \%$ & 1444 & $3.0 \%$ & 1302 & $12.6 \%$ & 1358 & $8.8 \%$ \\
\hline MKE & 1,032 & 1,032 & $0.0 \%$ & 1,032 & $0.0 \%$ & 1,030 & $0.2 \%$ & 964 & $6.6 \%$ & 982 & $4.8 \%$ & 878 & $14.9 \%$ \\
\hline MLB & 218 & 218 & $0.0 \%$ & 218 & $0.0 \%$ & 218 & $0.0 \%$ & 213 & $2.3 \%$ & 215 & $1.4 \%$ & 217 & $0.5 \%$ \\
\hline MSN & 358 & 358 & $0.0 \%$ & 358 & $0.0 \%$ & 344 & $3.9 \%$ & 334 & $6.7 \%$ & 328 & $8.4 \%$ & 300 & $16.2 \%$ \\
\hline MSP & 2,026 & 1,964 & $3.1 \%$ & 2,026 & $0.0 \%$ & 2,026 & $0.0 \%$ & 1952 & $3.7 \%$ & 1888 & $6.8 \%$ & 1758 & $13.2 \%$ \\
\hline MSY & 617 & 617 & $0.0 \%$ & 617 & $0.0 \%$ & 603 & $2.3 \%$ & 599 & $2.9 \%$ & 573 & $7.1 \%$ & 529 & $14.3 \%$ \\
\hline OAK & 1,214 & 1,214 & $0.0 \%$ & 1,214 & $0.0 \%$ & 1,130 & $6.9 \%$ & 1185 & $2.4 \%$ & 1135 & $6.5 \%$ & 891 & $26.6 \%$ \\
\hline OKC & 339 & 339 & $0.0 \%$ & 339 & $0.0 \%$ & 311 & $8.3 \%$ & 322 & $5.0 \%$ & 330 & $2.7 \%$ & 330 & $2.7 \%$ \\
\hline OMA & 633 & 633 & $0.0 \%$ & 633 & $0.0 \%$ & 601 & $5.1 \%$ & 577 & $8.8 \%$ & 573 & $9.5 \%$ & 511 & $19.3 \%$ \\
\hline ONT & 526 & 526 & $0.0 \%$ & 526 & $0.0 \%$ & 520 & $1.1 \%$ & 514 & $2.3 \%$ & 518 & $1.5 \%$ & 498 & $5.3 \%$ \\
\hline
\end{tabular}


Table 7-6. 2025 Throughput under Constraints at 310 Critical Airports

= highest percentage reduction category in airport. = second highest percentage reduction category in airport.

\begin{tabular}{|c|c|c|c|c|c|c|c|c|c|c|c|c|c|}
\hline \multirow[b]{3}{*}{$\begin{array}{c}\text { Airpor } \\
\mathrm{t}\end{array}$} & \multirow[b]{3}{*}{$\begin{array}{l}\text { Uncon- } \\
\text { strained }\end{array}$} & \multicolumn{6}{|c|}{ Capacity constraints } & \multicolumn{6}{|c|}{ Environmental constraints } \\
\hline & & \multicolumn{2}{|c|}{ Runway } & \multicolumn{2}{|c|}{ Taxi } & \multicolumn{2}{|c|}{ Gate } & \multicolumn{2}{|c|}{ Fuel } & \multicolumn{2}{|c|}{$\mathrm{NOx}$} & \multicolumn{2}{|c|}{ Noise } \\
\hline & & $\begin{array}{l}\text { Daily } \\
\text { ops. }\end{array}$ & $\begin{array}{c}\text { Reduc- } \\
\text { tion }\end{array}$ & $\begin{array}{l}\text { Daily } \\
\text { ops. }\end{array}$ & $\begin{array}{l}\text { Reduc- } \\
\text { tion }\end{array}$ & $\begin{array}{l}\text { Daily } \\
\text { ops. }\end{array}$ & $\begin{array}{c}\text { Reduc- } \\
\text { tion }\end{array}$ & $\begin{array}{l}\text { Daily } \\
\text { ops. }\end{array}$ & $\begin{array}{l}\text { Reduc- } \\
\text { tion }\end{array}$ & $\begin{array}{l}\text { Daily } \\
\text { ops. }\end{array}$ & $\begin{array}{l}\text { Reduc- } \\
\text { tion }\end{array}$ & $\begin{array}{l}\text { Daily } \\
\text { ops. }\end{array}$ & $\begin{array}{c}\text { Reduc- } \\
\text { tion }\end{array}$ \\
\hline ORD & 4,031 & 4,031 & $0.0 \%$ & 3,892 & $3.4 \%$ & 3,391 & $15.9 \%$ & 3979 & $1.3 \%$ & 3903 & $3.2 \%$ & 3829 & $5.0 \%$ \\
\hline ORF & 404 & 404 & $0.0 \%$ & 404 & $0.0 \%$ & 392 & $3.0 \%$ & 378 & $6.4 \%$ & 386 & $4.5 \%$ & 396 & $2.0 \%$ \\
\hline OXR & 125 & 125 & $0.0 \%$ & 125 & $0.0 \%$ & 125 & $0.0 \%$ & 97 & $22.4 \%$ & 99 & $20.8 \%$ & 113 & $9.6 \%$ \\
\hline $\mathrm{PBI}$ & 519 & 519 & $0.0 \%$ & 519 & $0.0 \%$ & 515 & $0.8 \%$ & 495 & $4.6 \%$ & 487 & $6.2 \%$ & 373 & $28.1 \%$ \\
\hline PDX & 1,191 & 1,191 & $0.0 \%$ & 1,191 & $0.0 \%$ & 1,177 & $1.2 \%$ & 1166 & $2.1 \%$ & 1146 & $3.8 \%$ & 1126 & $5.5 \%$ \\
\hline $\mathrm{PHF}$ & 718 & 692 & $3.6 \%$ & 718 & $0.0 \%$ & 718 & $0.0 \%$ & 685 & $4.6 \%$ & 695 & $3.2 \%$ & 711 & $1.0 \%$ \\
\hline $\mathrm{PHL}$ & 2,518 & 2,002 & $20.5 \%$ & 2,518 & $0.0 \%$ & 2,330 & $7.5 \%$ & 2395 & $4.9 \%$ & 2269 & $9.9 \%$ & 2389 & $5.1 \%$ \\
\hline $\mathrm{PHX}$ & 2,516 & 2,230 & $11.4 \%$ & 2,293 & $8.9 \%$ & 2,330 & $7.4 \%$ & 2419 & $3.9 \%$ & 2203 & $12.4 \%$ & 2147 & $14.7 \%$ \\
\hline PIE & 208 & 208 & $0.0 \%$ & 208 & $0.0 \%$ & 208 & $0.0 \%$ & 200 & $3.8 \%$ & 194 & $6.7 \%$ & 206 & $1.0 \%$ \\
\hline PIT & 646 & 646 & $0.0 \%$ & 646 & $0.0 \%$ & 646 & $0.0 \%$ & 584 & $9.6 \%$ & 644 & $0.3 \%$ & 644 & $0.3 \%$ \\
\hline PVD & 421 & 421 & $0.0 \%$ & 421 & $0.0 \%$ & 397 & $5.7 \%$ & 387 & $8.1 \%$ & 405 & $3.8 \%$ & 375 & $10.9 \%$ \\
\hline $\mathrm{RDU}$ & 1,158 & 1,158 & $0.0 \%$ & 1,158 & $0.0 \%$ & 1,120 & $3.3 \%$ & 1082 & $6.6 \%$ & 1076 & $7.1 \%$ & 984 & $15.0 \%$ \\
\hline RFD & 282 & 282 & $0.0 \%$ & 282 & $0.0 \%$ & 282 & $0.0 \%$ & 254 & $9.9 \%$ & 244 & $13.5 \%$ & 238 & $15.6 \%$ \\
\hline $\mathrm{RIC}$ & 573 & 573 & $0.0 \%$ & 573 & $0.0 \%$ & 529 & $7.7 \%$ & 525 & $8.4 \%$ & 527 & $8.0 \%$ & 535 & $6.6 \%$ \\
\hline RNO & 632 & 632 & $0.0 \%$ & 632 & $0.0 \%$ & 622 & $1.6 \%$ & 622 & $1.6 \%$ & 602 & $4.7 \%$ & 582 & $7.9 \%$ \\
\hline $\mathrm{ROC}$ & 440 & 440 & $0.0 \%$ & 440 & $0.0 \%$ & 426 & $3.2 \%$ & 415 & $5.7 \%$ & 419 & $4.8 \%$ & 431 & $2.0 \%$ \\
\hline RSW & 377 & 377 & $0.0 \%$ & 377 & $0.0 \%$ & 377 & $0.0 \%$ & 368 & $2.4 \%$ & 350 & $7.2 \%$ & 306 & $18.8 \%$ \\
\hline SAN & 1,146 & 978 & $14.7 \%$ & 1,146 & $0.0 \%$ & 1,018 & $11.2 \%$ & 1118 & $2.4 \%$ & 1038 & $9.4 \%$ & 896 & $21.8 \%$ \\
\hline SAT & 1,060 & 1,060 & $0.0 \%$ & 1,060 & $0.0 \%$ & 1,026 & $3.2 \%$ & 1028 & $3.0 \%$ & 990 & $6.6 \%$ & 924 & $12.8 \%$ \\
\hline SBA & 275 & 275 & $0.0 \%$ & 275 & $0.0 \%$ & 275 & $0.0 \%$ & 204 & $25.8 \%$ & 218 & $20.7 \%$ & 258 & $6.2 \%$ \\
\hline SDF & 877 & 877 & $0.0 \%$ & 877 & $0.0 \%$ & 859 & $2.1 \%$ & 780 & $11.1 \%$ & 726 & $17.2 \%$ & 740 & $15.6 \%$ \\
\hline SEA & 1,594 & 1,594 & $0.0 \%$ & 1,578 & $1.0 \%$ & 1,530 & $4.0 \%$ & 1580 & $0.9 \%$ & 1498 & $6.0 \%$ & 1286 & $19.3 \%$ \\
\hline SFO & 1,791 & 1,721 & $3.9 \%$ & 1,791 & $0.0 \%$ & 1,559 & $13.0 \%$ & 1732 & $3.3 \%$ & 1672 & $6.6 \%$ & 1516 & $15.4 \%$ \\
\hline SJC & 1,013 & 1,013 & $0.0 \%$ & 1,013 & $0.0 \%$ & 963 & $4.9 \%$ & 951 & $6.1 \%$ & 843 & $16.8 \%$ & 775 & $23.5 \%$ \\
\hline SLC & 1,698 & 1,598 & $5.9 \%$ & 1,690 & $0.5 \%$ & 1,606 & $5.4 \%$ & 1592 & $6.2 \%$ & 1604 & $5.5 \%$ & 834 & $50.9 \%$ \\
\hline SMF & 790 & 790 & $0.0 \%$ & 790 & $0.0 \%$ & 724 & $8.4 \%$ & 754 & $4.6 \%$ & 726 & $8.1 \%$ & 750 & $5.1 \%$ \\
\hline SNA & 974 & 974 & $0.0 \%$ & 974 & $0.0 \%$ & 896 & $8.0 \%$ & 924 & $5.1 \%$ & 952 & $2.3 \%$ & 852 & $12.5 \%$ \\
\hline STL & 1,044 & 1,044 & $0.0 \%$ & 1,044 & $0.0 \%$ & 1,044 & $0.0 \%$ & 986 & $5.6 \%$ & 1004 & $3.8 \%$ & 1034 & $1.0 \%$ \\
\hline SWF & 341 & 341 & $0.0 \%$ & 341 & $0.0 \%$ & 339 & $0.6 \%$ & 332 & $2.6 \%$ & 332 & $2.6 \%$ & 340 & $0.3 \%$ \\
\hline SYR & 336 & 336 & $0.0 \%$ & 336 & $0.0 \%$ & 336 & $0.0 \%$ & 304 & $9.5 \%$ & 314 & $6.5 \%$ & 304 & $9.5 \%$ \\
\hline TEB & 932 & 932 & $0.0 \%$ & 932 & $0.0 \%$ & 932 & $0.0 \%$ & 839 & $10.0 \%$ & 821 & $11.9 \%$ & 667 & $28.4 \%$ \\
\hline TPA & 954 & 954 & $0.0 \%$ & 954 & $0.0 \%$ & 936 & $1.9 \%$ & 950 & $0.4 \%$ & 942 & $1.3 \%$ & 948 & $0.6 \%$ \\
\hline TUL & 432 & 432 & $0.0 \%$ & 432 & $0.0 \%$ & 420 & $2.8 \%$ & 412 & $4.6 \%$ & 424 & $1.9 \%$ & 366 & $15.3 \%$ \\
\hline TUS & 477 & 477 & $0.0 \%$ & 477 & $0.0 \%$ & 471 & $1.3 \%$ & 442 & $7.3 \%$ & 462 & $3.1 \%$ & 402 & $15.7 \%$ \\
\hline TVC & 289 & 289 & $0.0 \%$ & 289 & $0.0 \%$ & 283 & $2.1 \%$ & 262 & $9.3 \%$ & 266 & $8.0 \%$ & 140 & $51.6 \%$ \\
\hline TYS & 482 & 482 & $0.0 \%$ & 482 & $0.0 \%$ & 450 & $6.6 \%$ & 440 & $8.7 \%$ & 440 & $8.7 \%$ & 406 & $15.8 \%$ \\
\hline VNY & 890 & 884 & $0.7 \%$ & 890 & $0.0 \%$ & 890 & $0.0 \%$ & 859 & $3.5 \%$ & 869 & $2.4 \%$ & 755 & $15.2 \%$ \\
\hline $40 \mathrm{~N}$ & 0 & 0 & 0 & 0 & 0 & 0 & 0 & 0 & 0 & 0 & 0 & 0 & 0 \\
\hline 55J & 11 & 11 & $0.0 \%$ & 11 & $0.0 \%$ & 11 & $0.0 \%$ & 5 & $54.5 \%$ & 9 & $18.2 \%$ & 11 & $0.0 \%$ \\
\hline $\mathrm{ABE}$ & 367 & 367 & $0.0 \%$ & 367 & $0.0 \%$ & 367 & $0.0 \%$ & 359 & $2.2 \%$ & 357 & $2.7 \%$ & 367 & $0.0 \%$ \\
\hline $\mathrm{ABI}$ & 113 & 113 & $0.0 \%$ & 113 & $0.0 \%$ & 113 & $0.0 \%$ & 79 & $30.1 \%$ & 93 & $17.7 \%$ & 113 & $0.0 \%$ \\
\hline ABR & 40 & 40 & $0.0 \%$ & 40 & $0.0 \%$ & 40 & $0.0 \%$ & 22 & $45.0 \%$ & 24 & $40.0 \%$ & 40 & $0.0 \%$ \\
\hline ABY & 68 & 68 & $0.0 \%$ & 68 & $0.0 \%$ & 68 & $0.0 \%$ & 63 & $7.4 \%$ & 63 & $7.4 \%$ & 33 & $51.5 \%$ \\
\hline ACK & 1,030 & 942 & $8.5 \%$ & 1,030 & $0.0 \%$ & 1,030 & $0.0 \%$ & 894 & $13.2 \%$ & 950 & $7.8 \%$ & 1024 & $0.6 \%$ \\
\hline
\end{tabular}


Table 7-6. 2025 Throughput under Constraints at 310 Critical Airports

= highest percentage reduction category in airport. $\quad=$ second highest percentage reduction category in airport.

\begin{tabular}{|c|c|c|c|c|c|c|c|c|c|c|c|c|c|}
\hline \multirow[b]{3}{*}{$\begin{array}{c}\text { Airpor } \\
\mathrm{t}\end{array}$} & \multirow[b]{3}{*}{$\begin{array}{l}\text { Uncon- } \\
\text { strained }\end{array}$} & \multicolumn{6}{|c|}{ Capacity constraints } & \multicolumn{6}{|c|}{ Environmental constraints } \\
\hline & & \multicolumn{2}{|c|}{ Runway } & \multicolumn{2}{|c|}{ Taxi } & \multicolumn{2}{|c|}{ Gate } & \multicolumn{2}{|c|}{ Fuel } & \multicolumn{2}{|c|}{$\mathrm{NOx}$} & \multicolumn{2}{|c|}{ Noise } \\
\hline & & $\begin{array}{l}\text { Daily } \\
\text { ops. }\end{array}$ & $\begin{array}{c}\text { Reduc- } \\
\text { tion }\end{array}$ & $\begin{array}{l}\text { Daily } \\
\text { ops. }\end{array}$ & $\begin{array}{l}\text { Reduc- } \\
\text { tion }\end{array}$ & $\begin{array}{l}\text { Daily } \\
\text { ops. }\end{array}$ & $\begin{array}{l}\text { Reduc- } \\
\text { tion }\end{array}$ & $\begin{array}{l}\text { Daily } \\
\text { ops. }\end{array}$ & $\begin{array}{l}\text { Reduc- } \\
\text { tion }\end{array}$ & $\begin{array}{l}\text { Daily } \\
\text { ops. }\end{array}$ & $\begin{array}{l}\text { Reduc- } \\
\text { tion }\end{array}$ & $\begin{array}{l}\text { Daily } \\
\text { ops. }\end{array}$ & $\begin{array}{c}\text { Reduc- } \\
\text { tion }\end{array}$ \\
\hline $\mathrm{ACT}$ & 85 & 85 & $0.0 \%$ & 85 & $0.0 \%$ & 85 & $0.0 \%$ & 48 & $43.5 \%$ & 56 & $34.1 \%$ & 84 & $1.2 \%$ \\
\hline ACV & 53 & 53 & $0.0 \%$ & 53 & $0.0 \%$ & 53 & $0.0 \%$ & 23 & $56.6 \%$ & 33 & $37.7 \%$ & 27 & $49.1 \%$ \\
\hline $\mathrm{ACY}$ & 183 & 183 & $0.0 \%$ & 183 & $0.0 \%$ & 183 & $0.0 \%$ & 172 & $6.0 \%$ & 176 & $3.8 \%$ & 182 & $0.5 \%$ \\
\hline $\mathrm{ADQ}$ & 70 & 70 & $0.0 \%$ & 70 & $0.0 \%$ & 66 & $5.7 \%$ & 64 & $8.6 \%$ & 68 & $2.9 \%$ & 70 & $0.0 \%$ \\
\hline ADS & 306 & 306 & $0.0 \%$ & 306 & $0.0 \%$ & 306 & $0.0 \%$ & 269 & $12.1 \%$ & 281 & $8.2 \%$ & 221 & $27.8 \%$ \\
\hline AEG & 13 & 13 & $0.0 \%$ & 13 & $0.0 \%$ & 13 & $0.0 \%$ & 11 & $15.4 \%$ & 9 & $30.8 \%$ & 13 & $0.0 \%$ \\
\hline AEX & 82 & 82 & $0.0 \%$ & 82 & $0.0 \%$ & 74 & $9.8 \%$ & 64 & $22.0 \%$ & 62 & $24.4 \%$ & 82 & $0.0 \%$ \\
\hline AFW & 300 & 300 & $0.0 \%$ & 300 & $0.0 \%$ & 294 & $2.0 \%$ & 297 & $1.0 \%$ & 291 & $3.0 \%$ & 291 & $3.0 \%$ \\
\hline AGS & 68 & 68 & $0.0 \%$ & 68 & $0.0 \%$ & 68 & $0.0 \%$ & 42 & $38.2 \%$ & 48 & $29.4 \%$ & 68 & $0.0 \%$ \\
\hline $\mathrm{AHN}$ & 94 & 94 & $0.0 \%$ & 94 & $0.0 \%$ & 94 & $0.0 \%$ & 76 & $19.1 \%$ & 78 & $17.0 \%$ & 94 & $0.0 \%$ \\
\hline ALN & 146 & 146 & $0.0 \%$ & 146 & $0.0 \%$ & 146 & $0.0 \%$ & 102 & $30.1 \%$ & 114 & $21.9 \%$ & 72 & $50.7 \%$ \\
\hline ALO & 102 & 102 & $0.0 \%$ & 102 & $0.0 \%$ & 102 & $0.0 \%$ & 74 & $27.5 \%$ & 88 & $13.7 \%$ & 88 & $13.7 \%$ \\
\hline AMA & 128 & 128 & $0.0 \%$ & 128 & $0.0 \%$ & 126 & $1.6 \%$ & 109 & $14.8 \%$ & 121 & $5.5 \%$ & 61 & $52.3 \%$ \\
\hline APA & 726 & 724 & $0.3 \%$ & 726 & $0.0 \%$ & 726 & $0.0 \%$ & 618 & $14.9 \%$ & 644 & $11.3 \%$ & 614 & $15.4 \%$ \\
\hline APF & 327 & 327 & $0.0 \%$ & 327 & $0.0 \%$ & 327 & $0.0 \%$ & 239 & $26.9 \%$ & 287 & $12.2 \%$ & 325 & $0.6 \%$ \\
\hline ARR & 256 & 256 & $0.0 \%$ & 256 & $0.0 \%$ & 256 & $0.0 \%$ & 216 & $15.6 \%$ & 192 & $25.0 \%$ & 144 & $43.8 \%$ \\
\hline ASE & 280 & 280 & $0.0 \%$ & 280 & $0.0 \%$ & 280 & $0.0 \%$ & 263 & $6.1 \%$ & 257 & $8.2 \%$ & 263 & $6.1 \%$ \\
\hline ATW & 172 & 172 & $0.0 \%$ & 172 & $0.0 \%$ & 172 & $0.0 \%$ & 157 & $8.7 \%$ & 151 & $12.2 \%$ & 81 & $52.9 \%$ \\
\hline ATY & 19 & 19 & $0.0 \%$ & 19 & $0.0 \%$ & 19 & $0.0 \%$ & 9 & $52.6 \%$ & 13 & $31.6 \%$ & 19 & $0.0 \%$ \\
\hline AUG & 24 & 24 & $0.0 \%$ & 24 & $0.0 \%$ & 24 & $0.0 \%$ & 24 & $0.0 \%$ & 22 & $8.3 \%$ & 12 & $50.0 \%$ \\
\hline AVL & 283 & 283 & $0.0 \%$ & 283 & $0.0 \%$ & 283 & $0.0 \%$ & 252 & $11.0 \%$ & 256 & $9.5 \%$ & 140 & $50.5 \%$ \\
\hline AVP & 270 & 270 & $0.0 \%$ & 270 & $0.0 \%$ & 270 & $0.0 \%$ & 234 & $13.3 \%$ & 238 & $11.9 \%$ & 132 & $51.1 \%$ \\
\hline AWM & 10 & 10 & $0.0 \%$ & 10 & $0.0 \%$ & 10 & $0.0 \%$ & 1 & $90.0 \%$ & 6 & $40.0 \%$ & 10 & $0.0 \%$ \\
\hline $\mathrm{AZO}$ & 140 & 140 & $0.0 \%$ & 140 & $0.0 \%$ & 140 & $0.0 \%$ & 122 & $12.9 \%$ & 128 & $8.6 \%$ & 140 & $0.0 \%$ \\
\hline BCT & 201 & 201 & $0.0 \%$ & 201 & $0.0 \%$ & 201 & $0.0 \%$ & 190 & $5.5 \%$ & 172 & $14.4 \%$ & 186 & $7.5 \%$ \\
\hline BED & 526 & 526 & $0.0 \%$ & 526 & $0.0 \%$ & 526 & $0.0 \%$ & 503 & $4.4 \%$ & 503 & $4.4 \%$ & 253 & $51.9 \%$ \\
\hline BET & 461 & 461 & $0.0 \%$ & 461 & $0.0 \%$ & 453 & $1.7 \%$ & 447 & $3.0 \%$ & 447 & $3.0 \%$ & 461 & $0.0 \%$ \\
\hline BFF & 26 & 26 & $0.0 \%$ & 26 & $0.0 \%$ & 26 & $0.0 \%$ & 8 & $69.2 \%$ & 20 & $23.1 \%$ & 24 & $7.7 \%$ \\
\hline $\mathrm{BFI}$ & 1,051 & 949 & $9.7 \%$ & 1,051 & $0.0 \%$ & 1,051 & $0.0 \%$ & 1016 & $3.3 \%$ & 1016 & $3.3 \%$ & 988 & $6.0 \%$ \\
\hline BFM & 185 & 185 & $0.0 \%$ & 185 & $0.0 \%$ & 185 & $0.0 \%$ & 182 & $1.6 \%$ & 182 & $1.6 \%$ & 184 & $0.5 \%$ \\
\hline BGM & 103 & 103 & $0.0 \%$ & 103 & $0.0 \%$ & 103 & $0.0 \%$ & 77 & $25.2 \%$ & 81 & $21.4 \%$ & 103 & $0.0 \%$ \\
\hline BGR & 108 & 108 & $0.0 \%$ & 108 & $0.0 \%$ & 108 & $0.0 \%$ & 90 & $16.7 \%$ & 94 & $13.0 \%$ & 108 & $0.0 \%$ \\
\hline BIL & 409 & 409 & $0.0 \%$ & 409 & $0.0 \%$ & 409 & $0.0 \%$ & 399 & $2.4 \%$ & 393 & $3.9 \%$ & 371 & $9.3 \%$ \\
\hline BIS & 168 & 168 & $0.0 \%$ & 168 & $0.0 \%$ & 168 & $0.0 \%$ & 150 & $10.7 \%$ & 152 & $9.5 \%$ & 168 & $0.0 \%$ \\
\hline BJC & 115 & 115 & $0.0 \%$ & 115 & $0.0 \%$ & 115 & $0.0 \%$ & 103 & $10.4 \%$ & 97 & $15.7 \%$ & 111 & $3.5 \%$ \\
\hline $\mathrm{BKL}$ & 284 & 284 & $0.0 \%$ & 284 & $0.0 \%$ & 284 & $0.0 \%$ & 191 & $32.7 \%$ & 233 & $18.0 \%$ & 139 & $51.1 \%$ \\
\hline $\mathrm{BLI}$ & 322 & 322 & $0.0 \%$ & 322 & $0.0 \%$ & 322 & $0.0 \%$ & 307 & $4.7 \%$ & 311 & $3.4 \%$ & 156 & $51.6 \%$ \\
\hline BLV & 9 & 9 & $0.0 \%$ & 9 & $0.0 \%$ & 9 & $0.0 \%$ & 7 & $22.2 \%$ & 7 & $22.2 \%$ & 9 & $0.0 \%$ \\
\hline BMI & 119 & 119 & $0.0 \%$ & 119 & $0.0 \%$ & 119 & $0.0 \%$ & 109 & $8.4 \%$ & 113 & $5.0 \%$ & 101 & $15.1 \%$ \\
\hline BPT & 122 & 122 & $0.0 \%$ & 122 & $0.0 \%$ & 122 & $0.0 \%$ & 100 & $18.0 \%$ & 100 & $18.0 \%$ & 122 & $0.0 \%$ \\
\hline BTM & 20 & 20 & $0.0 \%$ & 20 & $0.0 \%$ & 20 & $0.0 \%$ & 14 & $30.0 \%$ & 18 & $10.0 \%$ & 10 & $50.0 \%$ \\
\hline BTV & 312 & 312 & $0.0 \%$ & 312 & $0.0 \%$ & 312 & $0.0 \%$ & 302 & $3.2 \%$ & 298 & $4.5 \%$ & 312 & $0.0 \%$ \\
\hline BVY & 206 & 206 & $0.0 \%$ & 206 & $0.0 \%$ & 206 & $0.0 \%$ & 146 & $29.1 \%$ & 156 & $24.3 \%$ & 204 & $1.0 \%$ \\
\hline $\mathrm{BZN}$ & 303 & 303 & $0.0 \%$ & 303 & $0.0 \%$ & 303 & $0.0 \%$ & 286 & $5.6 \%$ & 282 & $6.9 \%$ & 302 & $0.3 \%$ \\
\hline
\end{tabular}


Table 7-6. 2025 Throughput under Constraints at 310 Critical Airports

= highest percentage reduction category in airport. = second highest percentage reduction category in airport.

\begin{tabular}{|c|c|c|c|c|c|c|c|c|c|c|c|c|c|}
\hline \multirow[b]{3}{*}{$\begin{array}{c}\text { Airpor } \\
\mathrm{t}\end{array}$} & \multirow[b]{3}{*}{$\begin{array}{l}\text { Uncon- } \\
\text { strained }\end{array}$} & \multicolumn{6}{|c|}{ Capacity constraints } & \multicolumn{6}{|c|}{ Environmental constraints } \\
\hline & & \multicolumn{2}{|c|}{ Runway } & \multicolumn{2}{|c|}{ Taxi } & \multicolumn{2}{|c|}{ Gate } & \multicolumn{2}{|c|}{ Fuel } & \multicolumn{2}{|c|}{$\mathrm{NOx}$} & \multicolumn{2}{|c|}{ Noise } \\
\hline & & $\begin{array}{l}\text { Daily } \\
\text { ops. }\end{array}$ & $\begin{array}{c}\text { Reduc- } \\
\text { tion }\end{array}$ & $\begin{array}{l}\text { Daily } \\
\text { ops. }\end{array}$ & $\begin{array}{l}\text { Reduc- } \\
\text { tion }\end{array}$ & $\begin{array}{l}\text { Daily } \\
\text { ops. }\end{array}$ & $\begin{array}{l}\text { Reduc- } \\
\text { tion }\end{array}$ & $\begin{array}{l}\text { Daily } \\
\text { ops. }\end{array}$ & $\begin{array}{l}\text { Reduc- } \\
\text { tion }\end{array}$ & $\begin{array}{l}\text { Daily } \\
\text { ops. }\end{array}$ & $\begin{array}{l}\text { Reduc- } \\
\text { tion }\end{array}$ & $\begin{array}{l}\text { Daily } \\
\text { ops. }\end{array}$ & $\begin{array}{c}\text { Reduc- } \\
\text { tion }\end{array}$ \\
\hline CAE & 417 & 417 & $0.0 \%$ & 417 & $0.0 \%$ & 417 & $0.0 \%$ & 393 & $5.8 \%$ & 391 & $6.2 \%$ & 395 & $5.3 \%$ \\
\hline CAK & 359 & 359 & $0.0 \%$ & 359 & $0.0 \%$ & 359 & $0.0 \%$ & 334 & $7.0 \%$ & 344 & $4.2 \%$ & 336 & $6.4 \%$ \\
\hline CCR & 227 & 227 & $0.0 \%$ & 227 & $0.0 \%$ & 227 & $0.0 \%$ & 161 & $29.1 \%$ & 211 & $7.0 \%$ & 225 & $0.9 \%$ \\
\hline CDC & 24 & 24 & $0.0 \%$ & 24 & $0.0 \%$ & 24 & $0.0 \%$ & 24 & $0.0 \%$ & 22 & $8.3 \%$ & 24 & $0.0 \%$ \\
\hline CGF & 134 & 134 & $0.0 \%$ & 134 & $0.0 \%$ & 134 & $0.0 \%$ & 120 & $10.4 \%$ & 118 & $11.9 \%$ & 66 & $50.7 \%$ \\
\hline CGI & 5 & 5 & $0.0 \%$ & 5 & $0.0 \%$ & 5 & $0.0 \%$ & 1 & $80.0 \%$ & 0 & $100.0 \%$ & 3 & $40.0 \%$ \\
\hline $\mathrm{CHA}$ & 269 & 269 & $0.0 \%$ & 269 & $0.0 \%$ & 269 & $0.0 \%$ & 247 & $8.2 \%$ & 243 & $9.7 \%$ & 131 & $51.3 \%$ \\
\hline $\mathrm{CHO}$ & 249 & 249 & $0.0 \%$ & 249 & $0.0 \%$ & 249 & $0.0 \%$ & 197 & $20.9 \%$ & 201 & $19.3 \%$ & 249 & $0.0 \%$ \\
\hline CID & 272 & 272 & $0.0 \%$ & 272 & $0.0 \%$ & 272 & $0.0 \%$ & 254 & $6.6 \%$ & 252 & $7.4 \%$ & 268 & $1.5 \%$ \\
\hline CKB & 76 & 76 & $0.0 \%$ & 76 & $0.0 \%$ & 76 & $0.0 \%$ & 64 & $15.8 \%$ & 66 & $13.2 \%$ & 76 & $0.0 \%$ \\
\hline CLL & 85 & 85 & $0.0 \%$ & 85 & $0.0 \%$ & 85 & $0.0 \%$ & 49 & $42.4 \%$ & 53 & $37.6 \%$ & 85 & $0.0 \%$ \\
\hline $\mathrm{CMI}$ & 146 & 146 & $0.0 \%$ & 146 & $0.0 \%$ & 146 & $0.0 \%$ & 126 & $13.7 \%$ & 130 & $11.0 \%$ & 146 & $0.0 \%$ \\
\hline $\mathrm{COU}$ & 34 & 34 & $0.0 \%$ & 34 & $0.0 \%$ & 34 & $0.0 \%$ & 32 & $5.9 \%$ & 32 & $5.9 \%$ & 16 & $52.9 \%$ \\
\hline CPR & 136 & 136 & $0.0 \%$ & 136 & $0.0 \%$ & 136 & $0.0 \%$ & 126 & $7.4 \%$ & 130 & $4.4 \%$ & 136 & $0.0 \%$ \\
\hline CPS & 300 & 300 & $0.0 \%$ & 300 & $0.0 \%$ & 300 & $0.0 \%$ & 268 & $10.7 \%$ & 272 & $9.3 \%$ & 162 & $46.0 \%$ \\
\hline CRW & 266 & 266 & $0.0 \%$ & 266 & $0.0 \%$ & 266 & $0.0 \%$ & 203 & $23.7 \%$ & 211 & $20.7 \%$ & 265 & $0.4 \%$ \\
\hline CSG & 172 & 172 & $0.0 \%$ & 172 & $0.0 \%$ & 172 & $0.0 \%$ & 159 & $7.6 \%$ & 153 & $11.0 \%$ & 145 & $15.7 \%$ \\
\hline CWA & 113 & 113 & $0.0 \%$ & 113 & $0.0 \%$ & 113 & $0.0 \%$ & 78 & $31.0 \%$ & 86 & $23.9 \%$ & 113 & $0.0 \%$ \\
\hline CYS & 64 & 64 & $0.0 \%$ & 64 & $0.0 \%$ & 64 & $0.0 \%$ & 60 & $6.3 \%$ & 60 & $6.3 \%$ & 64 & $0.0 \%$ \\
\hline DLH & 232 & 232 & $0.0 \%$ & 232 & $0.0 \%$ & 232 & $0.0 \%$ & 222 & $4.3 \%$ & 222 & $4.3 \%$ & 230 & $0.9 \%$ \\
\hline DPA & 440 & 440 & $0.0 \%$ & 440 & $0.0 \%$ & 440 & $0.0 \%$ & 405 & $8.0 \%$ & 379 & $13.9 \%$ & 421 & $4.3 \%$ \\
\hline DTO & 97 & 97 & $0.0 \%$ & 97 & $0.0 \%$ & 97 & $0.0 \%$ & 63 & $35.1 \%$ & 85 & $12.4 \%$ & 97 & $0.0 \%$ \\
\hline DVT & 507 & 507 & $0.0 \%$ & 507 & $0.0 \%$ & 507 & $0.0 \%$ & 393 & $22.5 \%$ & 459 & $9.5 \%$ & 507 & $0.0 \%$ \\
\hline EAU & 141 & 141 & $0.0 \%$ & 141 & $0.0 \%$ & 141 & $0.0 \%$ & 93 & $34.0 \%$ & 119 & $15.6 \%$ & 69 & $51.1 \%$ \\
\hline EFD & 23 & 23 & $0.0 \%$ & 23 & $0.0 \%$ & 23 & $0.0 \%$ & 20 & $13.0 \%$ & 20 & $13.0 \%$ & 22 & $4.3 \%$ \\
\hline EGE & 168 & 168 & $0.0 \%$ & 168 & $0.0 \%$ & 168 & $0.0 \%$ & 155 & $7.7 \%$ & 153 & $8.9 \%$ & 83 & $50.6 \%$ \\
\hline EQY & 29 & 29 & $0.0 \%$ & 29 & $0.0 \%$ & 29 & $0.0 \%$ & 25 & $13.8 \%$ & 27 & $6.9 \%$ & 15 & $48.3 \%$ \\
\hline ERI & 179 & 179 & $0.0 \%$ & 179 & $0.0 \%$ & 179 & $0.0 \%$ & 144 & $19.6 \%$ & 148 & $17.3 \%$ & 132 & $26.3 \%$ \\
\hline EVV & 230 & 230 & $0.0 \%$ & 230 & $0.0 \%$ & 230 & $0.0 \%$ & 200 & $13.0 \%$ & 206 & $10.4 \%$ & 230 & $0.0 \%$ \\
\hline $\mathrm{FAI}$ & 418 & 418 & $0.0 \%$ & 418 & $0.0 \%$ & 410 & $1.9 \%$ & 395 & $5.5 \%$ & 401 & $4.1 \%$ & 405 & $3.1 \%$ \\
\hline FAR & 194 & 194 & $0.0 \%$ & 194 & $0.0 \%$ & 194 & $0.0 \%$ & 186 & $4.1 \%$ & 184 & $5.2 \%$ & 194 & $0.0 \%$ \\
\hline FAY & 75 & 75 & $0.0 \%$ & 75 & $0.0 \%$ & 75 & $0.0 \%$ & 59 & $21.3 \%$ & 57 & $24.0 \%$ & 43 & $42.7 \%$ \\
\hline FDK & 38 & 38 & $0.0 \%$ & 38 & $0.0 \%$ & 38 & $0.0 \%$ & 32 & $15.8 \%$ & 32 & $15.8 \%$ & 18 & $52.6 \%$ \\
\hline FFZ & 607 & 607 & $0.0 \%$ & 607 & $0.0 \%$ & 607 & $0.0 \%$ & 409 & $32.6 \%$ & 455 & $25.0 \%$ & 607 & $0.0 \%$ \\
\hline FNL & 18 & 18 & $0.0 \%$ & 18 & $0.0 \%$ & 18 & $0.0 \%$ & 15 & $16.7 \%$ & 15 & $16.7 \%$ & 8 & $55.6 \%$ \\
\hline FOE & 64 & 64 & $0.0 \%$ & 64 & $0.0 \%$ & 64 & $0.0 \%$ & 62 & $3.1 \%$ & 62 & $3.1 \%$ & 64 & $0.0 \%$ \\
\hline FRG & 495 & 495 & $0.0 \%$ & 495 & $0.0 \%$ & 495 & $0.0 \%$ & 441 & $10.9 \%$ & 461 & $6.9 \%$ & 419 & $15.4 \%$ \\
\hline FSD & 366 & 366 & $0.0 \%$ & 366 & $0.0 \%$ & 366 & $0.0 \%$ & 346 & $5.5 \%$ & 344 & $6.0 \%$ & 362 & $1.1 \%$ \\
\hline FTG & 181 & 181 & $0.0 \%$ & 181 & $0.0 \%$ & 181 & $0.0 \%$ & 119 & $34.3 \%$ & 145 & $19.9 \%$ & 181 & $0.0 \%$ \\
\hline FTW & 351 & 351 & $0.0 \%$ & 351 & $0.0 \%$ & 351 & $0.0 \%$ & 331 & $5.7 \%$ & 309 & $12.0 \%$ & 169 & $51.9 \%$ \\
\hline FTY & 521 & 521 & $0.0 \%$ & 521 & $0.0 \%$ & 521 & $0.0 \%$ & 479 & $8.1 \%$ & 433 & $16.9 \%$ & 493 & $5.4 \%$ \\
\hline FWA & 224 & 224 & $0.0 \%$ & 224 & $0.0 \%$ & 224 & $0.0 \%$ & 220 & $1.8 \%$ & 212 & $5.4 \%$ & 190 & $15.2 \%$ \\
\hline $\mathrm{GCN}$ & 3 & 3 & $0.0 \%$ & 3 & $0.0 \%$ & 3 & $0.0 \%$ & 0 & $100.0 \%$ & 0 & $100.0 \%$ & 3 & $0.0 \%$ \\
\hline GEG & 408 & 408 & $0.0 \%$ & 408 & $0.0 \%$ & 408 & $0.0 \%$ & 381 & $6.6 \%$ & 371 & $9.1 \%$ & 369 & $9.6 \%$ \\
\hline
\end{tabular}


Table 7-6. 2025 Throughput under Constraints at 310 Critical Airports

= highest percentage reduction category in airport. = second highest percentage reduction category in airport.

\begin{tabular}{|c|c|c|c|c|c|c|c|c|c|c|c|c|c|}
\hline \multirow[b]{3}{*}{$\begin{array}{c}\text { Airpor } \\
\mathrm{t}\end{array}$} & \multirow[b]{3}{*}{$\begin{array}{l}\text { Uncon- } \\
\text { strained }\end{array}$} & \multicolumn{6}{|c|}{ Capacity constraints } & \multicolumn{6}{|c|}{ Environmental constraints } \\
\hline & & \multicolumn{2}{|c|}{ Runway } & \multicolumn{2}{|c|}{ Taxi } & \multicolumn{2}{|c|}{ Gate } & \multicolumn{2}{|c|}{ Fuel } & \multicolumn{2}{|c|}{$\mathrm{NOx}$} & \multicolumn{2}{|c|}{ Noise } \\
\hline & & $\begin{array}{l}\text { Daily } \\
\text { ops. }\end{array}$ & $\begin{array}{c}\text { Reduc- } \\
\text { tion }\end{array}$ & $\begin{array}{l}\text { Daily } \\
\text { ops. }\end{array}$ & $\begin{array}{l}\text { Reduc- } \\
\text { tion }\end{array}$ & $\begin{array}{l}\text { Daily } \\
\text { ops. }\end{array}$ & $\begin{array}{l}\text { Reduc- } \\
\text { tion }\end{array}$ & $\begin{array}{l}\text { Daily } \\
\text { ops. }\end{array}$ & $\begin{array}{l}\text { Reduc- } \\
\text { tion }\end{array}$ & $\begin{array}{l}\text { Daily } \\
\text { ops. }\end{array}$ & $\begin{array}{l}\text { Reduc- } \\
\text { tion }\end{array}$ & $\begin{array}{l}\text { Daily } \\
\text { ops. }\end{array}$ & $\begin{array}{c}\text { Reduc- } \\
\text { tion }\end{array}$ \\
\hline GEU & 122 & 122 & $0.0 \%$ & 122 & $0.0 \%$ & 122 & $0.0 \%$ & 92 & $24.6 \%$ & 100 & $18.0 \%$ & 122 & $0.0 \%$ \\
\hline GJT & 216 & 216 & $0.0 \%$ & 216 & $0.0 \%$ & 216 & $0.0 \%$ & 204 & $5.6 \%$ & 204 & $5.6 \%$ & 214 & $0.9 \%$ \\
\hline GNV & 146 & 146 & $0.0 \%$ & 146 & $0.0 \%$ & 146 & $0.0 \%$ & 128 & $12.3 \%$ & 126 & $13.7 \%$ & 140 & $4.1 \%$ \\
\hline GPT & 156 & 156 & $0.0 \%$ & 156 & $0.0 \%$ & 156 & $0.0 \%$ & 136 & $12.8 \%$ & 140 & $10.3 \%$ & 76 & $51.3 \%$ \\
\hline GRB & 143 & 143 & $0.0 \%$ & 143 & $0.0 \%$ & 143 & $0.0 \%$ & 133 & $7.0 \%$ & 129 & $9.8 \%$ & 117 & $18.2 \%$ \\
\hline GRI & 59 & 59 & $0.0 \%$ & 59 & $0.0 \%$ & 59 & $0.0 \%$ & 31 & $47.5 \%$ & 47 & $20.3 \%$ & 29 & $50.8 \%$ \\
\hline GSP & 300 & 300 & $0.0 \%$ & 300 & $0.0 \%$ & 300 & $0.0 \%$ & 267 & $11.0 \%$ & 255 & $15.0 \%$ & 297 & $1.0 \%$ \\
\hline GTF & 198 & 198 & $0.0 \%$ & 198 & $0.0 \%$ & 198 & $0.0 \%$ & 192 & $3.0 \%$ & 188 & $5.1 \%$ & 188 & $5.1 \%$ \\
\hline GUM & 127 & 127 & $0.0 \%$ & 127 & $0.0 \%$ & 127 & $0.0 \%$ & 37 & $70.9 \%$ & 49 & $61.4 \%$ & 127 & $0.0 \%$ \\
\hline $\mathrm{HAO}$ & 32 & 32 & $0.0 \%$ & 32 & $0.0 \%$ & 32 & $0.0 \%$ & 25 & $21.9 \%$ & 27 & $15.6 \%$ & 29 & $9.4 \%$ \\
\hline HEF & 250 & 250 & $0.0 \%$ & 250 & $0.0 \%$ & 250 & $0.0 \%$ & 207 & $17.2 \%$ & 209 & $16.4 \%$ & 237 & $5.2 \%$ \\
\hline $\mathrm{HIO}$ & 493 & 493 & $0.0 \%$ & 493 & $0.0 \%$ & 493 & $0.0 \%$ & 438 & $11.2 \%$ & 440 & $10.8 \%$ & 480 & $2.6 \%$ \\
\hline $\mathrm{HQZ}$ & 10 & 10 & $0.0 \%$ & 10 & $0.0 \%$ & 10 & $0.0 \%$ & 6 & $40.0 \%$ & 8 & $20.0 \%$ & 10 & $0.0 \%$ \\
\hline HRL & 145 & 145 & $0.0 \%$ & 145 & $0.0 \%$ & 145 & $0.0 \%$ & 145 & $0.0 \%$ & 141 & $2.8 \%$ & 143 & $1.4 \%$ \\
\hline HSV & 237 & 237 & $0.0 \%$ & 237 & $0.0 \%$ & 237 & $0.0 \%$ & 233 & $1.7 \%$ & 229 & $3.4 \%$ & 237 & $0.0 \%$ \\
\hline HWD & 302 & 302 & $0.0 \%$ & 302 & $0.0 \%$ & 302 & $0.0 \%$ & 205 & $32.1 \%$ & 243 & $19.5 \%$ & 147 & $51.3 \%$ \\
\hline IAG & 134 & 134 & $0.0 \%$ & 134 & $0.0 \%$ & 134 & $0.0 \%$ & 132 & $1.5 \%$ & 132 & $1.5 \%$ & 132 & $1.5 \%$ \\
\hline IDA & 136 & 136 & $0.0 \%$ & 136 & $0.0 \%$ & 136 & $0.0 \%$ & 120 & $11.8 \%$ & 124 & $8.8 \%$ & 136 & $0.0 \%$ \\
\hline ILG & 260 & 260 & $0.0 \%$ & 260 & $0.0 \%$ & 260 & $0.0 \%$ & 238 & $8.5 \%$ & 244 & $6.2 \%$ & 238 & $8.5 \%$ \\
\hline ILM & 324 & 324 & $0.0 \%$ & 324 & $0.0 \%$ & 324 & $0.0 \%$ & 300 & $7.4 \%$ & 296 & $8.6 \%$ & 322 & $0.6 \%$ \\
\hline ISM & 246 & 246 & $0.0 \%$ & 246 & $0.0 \%$ & 246 & $0.0 \%$ & 184 & $25.2 \%$ & 200 & $18.7 \%$ & 114 & $53.7 \%$ \\
\hline ITO & 294 & 294 & $0.0 \%$ & 294 & $0.0 \%$ & 286 & $2.7 \%$ & 291 & $1.0 \%$ & 293 & $0.3 \%$ & 293 & $0.3 \%$ \\
\hline IWA & 381 & 381 & $0.0 \%$ & 381 & $0.0 \%$ & 381 & $0.0 \%$ & 285 & $25.2 \%$ & 323 & $15.2 \%$ & 379 & $0.5 \%$ \\
\hline JAC & 194 & 194 & $0.0 \%$ & 194 & $0.0 \%$ & 162 & $16.5 \%$ & 182 & $6.2 \%$ & 174 & $10.3 \%$ & 92 & $52.6 \%$ \\
\hline JAN & 224 & 224 & $0.0 \%$ & 224 & $0.0 \%$ & 224 & $0.0 \%$ & 204 & $8.9 \%$ & 202 & $9.8 \%$ & 224 & $0.0 \%$ \\
\hline JST & 15 & 15 & $0.0 \%$ & 15 & $0.0 \%$ & 15 & $0.0 \%$ & 3 & $80.0 \%$ & 5 & $66.7 \%$ & 15 & $0.0 \%$ \\
\hline $\mathrm{KOA}$ & 292 & 292 & $0.0 \%$ & 292 & $0.0 \%$ & 198 & $32.2 \%$ & 292 & $0.0 \%$ & 292 & $0.0 \%$ & 292 & $0.0 \%$ \\
\hline KTN & 35 & 35 & $0.0 \%$ & 35 & $0.0 \%$ & 31 & $11.4 \%$ & 33 & $5.7 \%$ & 35 & $0.0 \%$ & 35 & $0.0 \%$ \\
\hline $\mathrm{LAL}$ & 115 & 115 & $0.0 \%$ & 115 & $0.0 \%$ & 115 & $0.0 \%$ & 81 & $29.6 \%$ & 97 & $15.7 \%$ & 115 & $0.0 \%$ \\
\hline LBB & 213 & 213 & $0.0 \%$ & 213 & $0.0 \%$ & 213 & $0.0 \%$ & 193 & $9.4 \%$ & 205 & $3.8 \%$ & 203 & $4.7 \%$ \\
\hline LBE & 115 & 115 & $0.0 \%$ & 115 & $0.0 \%$ & 115 & $0.0 \%$ & 106 & $7.8 \%$ & 102 & $11.3 \%$ & 115 & $0.0 \%$ \\
\hline LBF & 31 & 31 & $0.0 \%$ & 31 & $0.0 \%$ & 31 & $0.0 \%$ & 12 & $61.3 \%$ & 26 & $16.1 \%$ & 30 & $3.2 \%$ \\
\hline LBL & 28 & 28 & $0.0 \%$ & 28 & $0.0 \%$ & 28 & $0.0 \%$ & 26 & $7.1 \%$ & 26 & $7.1 \%$ & 28 & $0.0 \%$ \\
\hline LCK & 139 & 139 & $0.0 \%$ & 139 & $0.0 \%$ & 139 & $0.0 \%$ & 105 & $24.5 \%$ & 121 & $12.9 \%$ & 93 & $33.1 \%$ \\
\hline LEX & 300 & 300 & $0.0 \%$ & 300 & $0.0 \%$ & 300 & $0.0 \%$ & 263 & $12.3 \%$ & 259 & $13.7 \%$ & 299 & $0.3 \%$ \\
\hline LFT & 276 & 276 & $0.0 \%$ & 276 & $0.0 \%$ & 276 & $0.0 \%$ & 255 & $7.6 \%$ & 251 & $9.1 \%$ & 269 & $2.5 \%$ \\
\hline $\mathrm{LIH}$ & 383 & 383 & $0.0 \%$ & 383 & $0.0 \%$ & 357 & $6.8 \%$ & 383 & $0.0 \%$ & 375 & $2.1 \%$ & 383 & $0.0 \%$ \\
\hline LNK & 166 & 166 & $0.0 \%$ & 166 & $0.0 \%$ & 166 & $0.0 \%$ & 154 & $7.2 \%$ & 156 & $6.0 \%$ & 164 & $1.2 \%$ \\
\hline LSE & 115 & 115 & $0.0 \%$ & 115 & $0.0 \%$ & 115 & $0.0 \%$ & 98 & $14.8 \%$ & 96 & $16.5 \%$ & 114 & $0.9 \%$ \\
\hline LWM & 149 & 149 & $0.0 \%$ & 149 & $0.0 \%$ & 149 & $0.0 \%$ & 145 & $2.7 \%$ & 145 & $2.7 \%$ & 73 & $51.0 \%$ \\
\hline MAF & 170 & 170 & $0.0 \%$ & 170 & $0.0 \%$ & 170 & $0.0 \%$ & 152 & $10.6 \%$ & 162 & $4.7 \%$ & 154 & $9.4 \%$ \\
\hline MBS & 106 & 106 & $0.0 \%$ & 106 & $0.0 \%$ & 106 & $0.0 \%$ & 100 & $5.7 \%$ & 100 & $5.7 \%$ & 106 & $0.0 \%$ \\
\hline MDT & 210 & 210 & $0.0 \%$ & 210 & $0.0 \%$ & 210 & $0.0 \%$ & 199 & $5.2 \%$ & 199 & $5.2 \%$ & 209 & $0.5 \%$ \\
\hline MFE & 236 & 236 & $0.0 \%$ & 236 & $0.0 \%$ & 236 & $0.0 \%$ & 234 & $0.8 \%$ & 232 & $1.7 \%$ & 200 & $15.3 \%$ \\
\hline
\end{tabular}


Table 7-6. 2025 Throughput under Constraints at 310 Critical Airports

= highest percentage reduction category in airport. = second highest percentage reduction category in airport.

\begin{tabular}{|c|c|c|c|c|c|c|c|c|c|c|c|c|c|}
\hline \multirow[b]{3}{*}{$\begin{array}{c}\text { Airpor } \\
\mathrm{t}\end{array}$} & \multirow[b]{3}{*}{$\begin{array}{l}\text { Uncon- } \\
\text { strained }\end{array}$} & \multicolumn{6}{|c|}{ Capacity constraints } & \multicolumn{6}{|c|}{ Environmental constraints } \\
\hline & & \multicolumn{2}{|c|}{ Runway } & \multicolumn{2}{|c|}{ Taxi } & \multicolumn{2}{|c|}{ Gate } & \multicolumn{2}{|c|}{ Fuel } & \multicolumn{2}{|c|}{$\mathrm{NOx}$} & \multicolumn{2}{|c|}{ Noise } \\
\hline & & $\begin{array}{l}\text { Daily } \\
\text { ops. }\end{array}$ & $\begin{array}{c}\text { Reduc- } \\
\text { tion }\end{array}$ & $\begin{array}{l}\text { Daily } \\
\text { ops. }\end{array}$ & $\begin{array}{l}\text { Reduc- } \\
\text { tion }\end{array}$ & $\begin{array}{l}\text { Daily } \\
\text { ops. }\end{array}$ & $\begin{array}{l}\text { Reduc- } \\
\text { tion }\end{array}$ & $\begin{array}{l}\text { Daily } \\
\text { ops. }\end{array}$ & $\begin{array}{l}\text { Reduc- } \\
\text { tion }\end{array}$ & $\begin{array}{l}\text { Daily } \\
\text { ops. }\end{array}$ & $\begin{array}{l}\text { Reduc- } \\
\text { tion }\end{array}$ & $\begin{array}{l}\text { Daily } \\
\text { ops. }\end{array}$ & $\begin{array}{c}\text { Reduc- } \\
\text { tion }\end{array}$ \\
\hline $\mathrm{MHK}$ & 61 & 61 & $0.0 \%$ & 61 & $0.0 \%$ & 61 & $0.0 \%$ & 56 & $8.2 \%$ & 56 & $8.2 \%$ & 60 & $1.6 \%$ \\
\hline $\mathrm{MLI}$ & 162 & 162 & $0.0 \%$ & 162 & $0.0 \%$ & 162 & $0.0 \%$ & 129 & $20.4 \%$ & 139 & $14.2 \%$ & 77 & $52.5 \%$ \\
\hline MLU & 171 & 171 & $0.0 \%$ & 171 & $0.0 \%$ & 171 & $0.0 \%$ & 135 & $21.1 \%$ & 137 & $19.9 \%$ & 171 & $0.0 \%$ \\
\hline MMU & 505 & 505 & $0.0 \%$ & 505 & $0.0 \%$ & 505 & $0.0 \%$ & 479 & $5.1 \%$ & 473 & $6.3 \%$ & 493 & $2.4 \%$ \\
\hline MOB & 144 & 144 & $0.0 \%$ & 144 & $0.0 \%$ & 144 & $0.0 \%$ & 122 & $15.3 \%$ & 134 & $6.9 \%$ & 72 & $50.0 \%$ \\
\hline MOT & 91 & 91 & $0.0 \%$ & 91 & $0.0 \%$ & 91 & $0.0 \%$ & 85 & $6.6 \%$ & 87 & $4.4 \%$ & 89 & $2.2 \%$ \\
\hline MSO & 236 & 236 & $0.0 \%$ & 236 & $0.0 \%$ & 236 & $0.0 \%$ & 225 & $4.7 \%$ & 217 & $8.1 \%$ & 235 & $0.4 \%$ \\
\hline MTN & 241 & 241 & $0.0 \%$ & 241 & $0.0 \%$ & 241 & $0.0 \%$ & 220 & $8.7 \%$ & 232 & $3.7 \%$ & 240 & $0.4 \%$ \\
\hline MYR & 273 & 273 & $0.0 \%$ & 273 & $0.0 \%$ & 273 & $0.0 \%$ & 252 & $7.7 \%$ & 250 & $8.4 \%$ & 234 & $14.3 \%$ \\
\hline OGG & 597 & 587 & $1.7 \%$ & 597 & $0.0 \%$ & 549 & $8.0 \%$ & 597 & $0.0 \%$ & 597 & $0.0 \%$ & 597 & $0.0 \%$ \\
\hline OPF & 345 & 345 & $0.0 \%$ & 345 & $0.0 \%$ & 345 & $0.0 \%$ & 274 & $20.6 \%$ & 308 & $10.7 \%$ & 166 & $51.9 \%$ \\
\hline ORL & 308 & 308 & $0.0 \%$ & 308 & $0.0 \%$ & 308 & $0.0 \%$ & 265 & $14.0 \%$ & 269 & $12.7 \%$ & 147 & $52.3 \%$ \\
\hline PAE & 410 & 410 & $0.0 \%$ & 410 & $0.0 \%$ & 406 & $1.0 \%$ & 403 & $1.7 \%$ & 401 & $2.2 \%$ & 405 & $1.2 \%$ \\
\hline PDK & 803 & 803 & $0.0 \%$ & 803 & $0.0 \%$ & 803 & $0.0 \%$ & 748 & $6.8 \%$ & 730 & $9.1 \%$ & 388 & $51.7 \%$ \\
\hline $\mathrm{PIA}$ & 187 & 187 & $0.0 \%$ & 187 & $0.0 \%$ & 187 & $0.0 \%$ & 183 & $2.1 \%$ & 183 & $2.1 \%$ & 187 & $0.0 \%$ \\
\hline PIR & 45 & 45 & $0.0 \%$ & 45 & $0.0 \%$ & 45 & $0.0 \%$ & 32 & $28.9 \%$ & 34 & $24.4 \%$ & 42 & $6.7 \%$ \\
\hline PNE & 355 & 355 & $0.0 \%$ & 355 & $0.0 \%$ & 355 & $0.0 \%$ & 247 & $30.4 \%$ & 309 & $13.0 \%$ & 353 & $0.6 \%$ \\
\hline PNS & 286 & 286 & $0.0 \%$ & 286 & $0.0 \%$ & 286 & $0.0 \%$ & 272 & $4.9 \%$ & 276 & $3.5 \%$ & 140 & $51.0 \%$ \\
\hline PRC & 305 & 305 & $0.0 \%$ & 305 & $0.0 \%$ & 305 & $0.0 \%$ & 224 & $26.6 \%$ & 264 & $13.4 \%$ & 304 & $0.3 \%$ \\
\hline PSC & 186 & 186 & $0.0 \%$ & 186 & $0.0 \%$ & 186 & $0.0 \%$ & 173 & $7.0 \%$ & 173 & $7.0 \%$ & 186 & $0.0 \%$ \\
\hline PSM & 32 & 32 & $0.0 \%$ & 32 & $0.0 \%$ & 32 & $0.0 \%$ & 27 & $15.6 \%$ & 29 & $9.4 \%$ & 31 & $3.1 \%$ \\
\hline PSP & 167 & 167 & $0.0 \%$ & 167 & $0.0 \%$ & 167 & $0.0 \%$ & 135 & $19.2 \%$ & 137 & $18.0 \%$ & 81 & $51.5 \%$ \\
\hline PTK & 469 & 469 & $0.0 \%$ & 469 & $0.0 \%$ & 469 & $0.0 \%$ & 449 & $4.3 \%$ & 433 & $7.7 \%$ & 233 & $50.3 \%$ \\
\hline PUB & 221 & 221 & $0.0 \%$ & 221 & $0.0 \%$ & 221 & $0.0 \%$ & 145 & $34.4 \%$ & 189 & $14.5 \%$ & 221 & $0.0 \%$ \\
\hline PWK & 487 & 487 & $0.0 \%$ & 487 & $0.0 \%$ & 487 & $0.0 \%$ & 434 & $10.9 \%$ & 436 & $10.5 \%$ & 426 & $12.5 \%$ \\
\hline PWM & 345 & 345 & $0.0 \%$ & 345 & $0.0 \%$ & 345 & $0.0 \%$ & 316 & $8.4 \%$ & 326 & $5.5 \%$ & 200 & $42.0 \%$ \\
\hline RAP & 216 & 216 & $0.0 \%$ & 216 & $0.0 \%$ & 216 & $0.0 \%$ & 189 & $12.5 \%$ & 189 & $12.5 \%$ & 95 & $56.0 \%$ \\
\hline RBD & 185 & 185 & $0.0 \%$ & 185 & $0.0 \%$ & 185 & $0.0 \%$ & 119 & $35.7 \%$ & 147 & $20.5 \%$ & 185 & $0.0 \%$ \\
\hline RNT & 205 & 205 & $0.0 \%$ & 205 & $0.0 \%$ & 205 & $0.0 \%$ & 191 & $6.8 \%$ & 201 & $2.0 \%$ & 205 & $0.0 \%$ \\
\hline ROA & 177 & 177 & $0.0 \%$ & 177 & $0.0 \%$ & 177 & $0.0 \%$ & 163 & $7.9 \%$ & 169 & $4.5 \%$ & 175 & $1.1 \%$ \\
\hline RST & 175 & 175 & $0.0 \%$ & 175 & $0.0 \%$ & 175 & $0.0 \%$ & 165 & $5.7 \%$ & 165 & $5.7 \%$ & 175 & $0.0 \%$ \\
\hline RYY & 251 & 251 & $0.0 \%$ & 251 & $0.0 \%$ & 251 & $0.0 \%$ & 201 & $19.9 \%$ & 237 & $5.6 \%$ & 251 & $0.0 \%$ \\
\hline SAF & 192 & 192 & $0.0 \%$ & 192 & $0.0 \%$ & 192 & $0.0 \%$ & 156 & $18.8 \%$ & 154 & $19.8 \%$ & 190 & $1.0 \%$ \\
\hline SAV & 341 & 341 & $0.0 \%$ & 341 & $0.0 \%$ & 341 & $0.0 \%$ & 321 & $5.9 \%$ & 309 & $9.4 \%$ & 331 & $2.9 \%$ \\
\hline SBN & 185 & 185 & $0.0 \%$ & 185 & $0.0 \%$ & 185 & $0.0 \%$ & 175 & $5.4 \%$ & 171 & $7.6 \%$ & 183 & $1.1 \%$ \\
\hline SDL & 510 & 510 & $0.0 \%$ & 510 & $0.0 \%$ & 510 & $0.0 \%$ & 447 & $12.4 \%$ & 449 & $12.0 \%$ & 505 & $1.0 \%$ \\
\hline SDM & 129 & 129 & $0.0 \%$ & 129 & $0.0 \%$ & 129 & $0.0 \%$ & 79 & $38.8 \%$ & 109 & $15.5 \%$ & 129 & $0.0 \%$ \\
\hline SEE & 631 & 631 & $0.0 \%$ & 631 & $0.0 \%$ & 631 & $0.0 \%$ & 424 & $32.8 \%$ & 508 & $19.5 \%$ & 618 & $2.1 \%$ \\
\hline SFB & 366 & 366 & $0.0 \%$ & 366 & $0.0 \%$ & 362 & $1.1 \%$ & 316 & $13.7 \%$ & 312 & $14.8 \%$ & 160 & $56.3 \%$ \\
\hline SGF & 288 & 288 & $0.0 \%$ & 288 & $0.0 \%$ & 288 & $0.0 \%$ & 276 & $4.2 \%$ & 276 & $4.2 \%$ & 288 & $0.0 \%$ \\
\hline SGR & 211 & 211 & $0.0 \%$ & 211 & $0.0 \%$ & 211 & $0.0 \%$ & 153 & $27.5 \%$ & 157 & $25.6 \%$ & 205 & $2.8 \%$ \\
\hline SHV & 230 & 230 & $0.0 \%$ & 230 & $0.0 \%$ & 230 & $0.0 \%$ & 215 & $6.5 \%$ & 213 & $7.4 \%$ & 229 & $0.4 \%$ \\
\hline SJU & 717 & 717 & $0.0 \%$ & 717 & $0.0 \%$ & 717 & $0.0 \%$ & 711 & $0.8 \%$ & 679 & $5.3 \%$ & 711 & $0.8 \%$ \\
\hline SMX & 292 & 292 & $0.0 \%$ & 292 & $0.0 \%$ & 292 & $0.0 \%$ & 226 & $22.6 \%$ & 248 & $15.1 \%$ & 288 & $1.4 \%$ \\
\hline
\end{tabular}


Table 7-6. 2025 Throughput under Constraints at 310 Critical Airports

= highest percentage reduction category in airport. = second highest percentage reduction category in airport.

\begin{tabular}{|c|c|c|c|c|c|c|c|c|c|c|c|c|c|}
\hline \multirow[b]{3}{*}{$\begin{array}{c}\text { Airpor } \\
\mathrm{t}\end{array}$} & \multirow[b]{3}{*}{$\begin{array}{l}\text { Uncon- } \\
\text { strained }\end{array}$} & \multicolumn{6}{|c|}{ Capacity constraints } & \multicolumn{6}{|c|}{ Environmental constraints } \\
\hline & & \multicolumn{2}{|c|}{ Runway } & \multicolumn{2}{|c|}{ Taxi } & \multicolumn{2}{|c|}{ Gate } & \multicolumn{2}{|c|}{ Fuel } & \multicolumn{2}{|c|}{$\mathrm{NOx}$} & \multicolumn{2}{|c|}{ Noise } \\
\hline & & $\begin{array}{l}\text { Daily } \\
\text { ops. }\end{array}$ & $\begin{array}{c}\text { Reduc- } \\
\text { tion }\end{array}$ & $\begin{array}{l}\text { Daily } \\
\text { ops. }\end{array}$ & $\begin{array}{c}\text { Reduc- } \\
\text { tion }\end{array}$ & $\begin{array}{l}\text { Daily } \\
\text { ops. }\end{array}$ & $\begin{array}{c}\text { Reduc- } \\
\text { tion }\end{array}$ & $\begin{array}{l}\text { Daily } \\
\text { ops. }\end{array}$ & $\begin{array}{c}\text { Reduc- } \\
\text { tion }\end{array}$ & $\begin{array}{l}\text { Daily } \\
\text { ops. }\end{array}$ & $\begin{array}{c}\text { Reduc- } \\
\text { tion }\end{array}$ & $\begin{array}{l}\text { Daily } \\
\text { ops. }\end{array}$ & $\begin{array}{c}\text { Reduc- } \\
\text { tion }\end{array}$ \\
\hline SRQ & 196 & 196 & $0.0 \%$ & 196 & $0.0 \%$ & 196 & $0.0 \%$ & 192 & $2.0 \%$ & 192 & $2.0 \%$ & 180 & $8.2 \%$ \\
\hline STP & 533 & 533 & $0.0 \%$ & 533 & $0.0 \%$ & 533 & $0.0 \%$ & 403 & $24.4 \%$ & 483 & $9.4 \%$ & 267 & $49.9 \%$ \\
\hline STT & 261 & 261 & $0.0 \%$ & 261 & $0.0 \%$ & 233 & $10.7 \%$ & 257 & $1.5 \%$ & 259 & $0.8 \%$ & 261 & $0.0 \%$ \\
\hline SUS & 424 & 424 & $0.0 \%$ & 424 & $0.0 \%$ & 424 & $0.0 \%$ & 348 & $17.9 \%$ & 368 & $13.2 \%$ & 340 & $19.8 \%$ \\
\hline SUX & 50 & 50 & $0.0 \%$ & 50 & $0.0 \%$ & 50 & $0.0 \%$ & 36 & $28.0 \%$ & 40 & $20.0 \%$ & 24 & $52.0 \%$ \\
\hline TKI & 121 & 121 & $0.0 \%$ & 121 & $0.0 \%$ & 121 & $0.0 \%$ & 113 & $6.6 \%$ & 105 & $13.2 \%$ & 121 & $0.0 \%$ \\
\hline TLH & 236 & 236 & $0.0 \%$ & 236 & $0.0 \%$ & 236 & $0.0 \%$ & 223 & $5.5 \%$ & 223 & $5.5 \%$ & 231 & $2.1 \%$ \\
\hline TMB & 520 & 520 & $0.0 \%$ & 520 & $0.0 \%$ & 520 & $0.0 \%$ & 382 & $26.5 \%$ & 424 & $18.5 \%$ & 252 & $51.5 \%$ \\
\hline TOA & 308 & 308 & $0.0 \%$ & 308 & $0.0 \%$ & 308 & $0.0 \%$ & 206 & $33.1 \%$ & 224 & $27.3 \%$ & 150 & $51.3 \%$ \\
\hline TOL & 177 & 177 & $0.0 \%$ & 177 & $0.0 \%$ & 177 & $0.0 \%$ & 175 & $1.1 \%$ & 169 & $4.5 \%$ & 177 & $0.0 \%$ \\
\hline TRI & 207 & 207 & $0.0 \%$ & 207 & $0.0 \%$ & 203 & $1.9 \%$ & 188 & $9.2 \%$ & 180 & $13.0 \%$ & 100 & $51.7 \%$ \\
\hline TTD & 251 & 251 & $0.0 \%$ & 251 & $0.0 \%$ & 251 & $0.0 \%$ & 162 & $35.5 \%$ & 180 & $28.3 \%$ & 250 & $0.4 \%$ \\
\hline UGN & 244 & 244 & $0.0 \%$ & 244 & $0.0 \%$ & 244 & $0.0 \%$ & 230 & $5.7 \%$ & 212 & $13.1 \%$ & 208 & $14.8 \%$ \\
\hline VGT & 767 & 767 & $0.0 \%$ & 767 & $0.0 \%$ & 767 & $0.0 \%$ & 513 & $33.1 \%$ & 465 & $39.4 \%$ & 767 & $0.0 \%$ \\
\hline VPS & 68 & 68 & $0.0 \%$ & 68 & $0.0 \%$ & 68 & $0.0 \%$ & 64 & $5.9 \%$ & 64 & $5.9 \%$ & 68 & $0.0 \%$ \\
\hline XNA & 196 & 196 & $0.0 \%$ & 196 & $0.0 \%$ & 196 & $0.0 \%$ & 158 & $19.4 \%$ & 168 & $14.3 \%$ & 194 & $1.0 \%$ \\
\hline YIP & 379 & 379 & $0.0 \%$ & 379 & $0.0 \%$ & 379 & $0.0 \%$ & 357 & $5.8 \%$ & 349 & $7.9 \%$ & 323 & $14.8 \%$ \\
\hline Total & 158,373 & 152,407 & $96.2 \%$ & 156,152 & $98.6 \%$ & 152,137 & $96.1 \%$ & 147,100 & $92.9 \%$ & 145,236 & $91.7 \%$ & 137,037 & $86.5 \%$ \\
\hline
\end{tabular}




\section{Chapter 8}

\section{Analyze Shift to Metroplex Operations}

In the previous chapters, we documented the methods and results of our studies on airport capacity and environmental constraints in 2015 and 2025, which culminated in the identification of the primary and secondary airport constraints in Chapter 7. One of the major assumptions of the analyses was that the airports operate independently of each other. That assumption, however, may not be valid for the clustered airports within a metroplex. The operations of those airports often are interdependent, resulting in a loss of combined airport capacities in the metroplex. In other words, the feasible throughput numbers reported in Chapter 7 are the theoretical upper bounds without considering the interaction of airport operations in a metroplex.

Metroplex operations are more constrained than are the individual airport operations, because the airports must share the airspace in the vicinity of the metroplex. Obviously, the metroplex constraints can be different depending on the locations of the airports, their runways and facilities, and the airspace volume. Below are three possible scenarios:

- The airspace is limited such that it cannot support the traffic volume when each of the airports is being operated at its capacity, even under good weather.

- The airports are independent in selecting their own configurations and operations, but the airspace is limited only under IMC when flights are more restricted to take certain routes.

- The airports do not have total freedom in selecting their configurations and operations, especially under IMC.

Because airspace constraints link to the airports' configurations and operations, and because each metroplex is unique, we needed to model each metroplex separately. The metroplex constraint analysis required fundamentally different tools than did the analysis of individual airports. Instead of tools for computing airport capacity and environmental capacities, we needed detailed knowledge of the surrounding airspace, such as the locations of "corner posts," navigation aids (NAVAIDs), final common paths, overflow routes, controller workload, runway orientation and length, equipment, terminals, gates, and so on.

This chapter documents the preliminary analysis of the capacity and environmental constraints of metroplexes in the NAS. We present the analysis in two sections. The first addresses metroplex capacity constraints for all U.S. metroplexes. The second section addresses metroplex environmental constraints. The method we used for the 
environmental analysis enabled us to account for all environmental impacts in a metroplex. Due to resource limitations, we applied only one method for the capacity analysis for all metroplexes in the United States, and we analyzed only three metroplexes for the environmental constraints. The results of our survey of metroplex constraints will help NASA set priorities for funding other projects to look into the modeling and operation at some specific metroplexes.

\section{MetRoplex CAPACITY CONSTRAINTS}

\section{Method}

Our method for identifying metroplex capacity constraints in the current NAS was to analyze empirical data. Specifically, we needed to see whether the metroplex throughput is saturated when all the airports in a metroplex can be operated to their capacities. Because the metroplex constraints are mainly caused by the limited airspace capacities, we needed to focus on the IFR flights, which are under positive radar control. To obtain the needed data, we used ASPM, a database maintained by FAA at 77 airports for IFR flights. Figure 8-1 shows their locations.

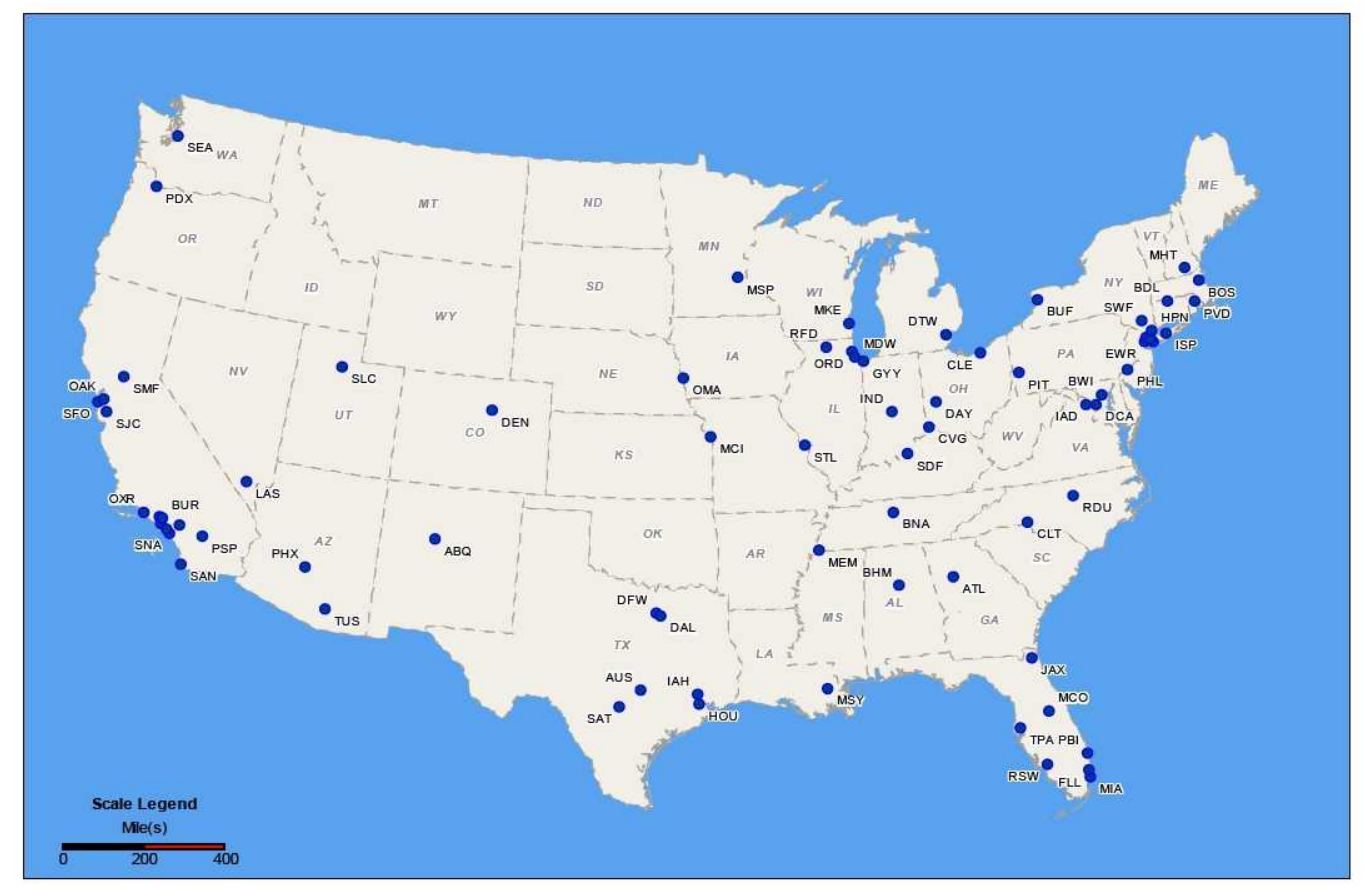

Figure 8-1. Locations of the 77 airports in ASPM.

The ASPM data can be queried for every 15-minute period. For each period, the maximum possible arrival is the lesser of the number of aircraft intending to arrive according to expected time of arrival (ArrDemand data field in ASPM) and AAR. Similarly, the maximum possible departure is the lesser of the number of departure aircraft intending to depart according to the flight schedule 
(DepDemand data filed in ASPM) and ADR. Further, we define the maximum ground operation at an airport as the sum of its maximum possible arrival and maximum possible departure, and we defined the maximum ground operation of a metroplex as the sum of the ground operations of all the airports in the metroplex. By the definition, the maximum ground operation is the largest feasible operation regardless of the airport capacity constraints, be it for the airport or metroplex.

For each airport and for every period of 15 minutes, ASPM can also report the count of IFR arrivals (ETMSArr data field in ASPM) and departures (ETMSDep data field in ASPM). Similarly, ETMS throughput at an individual airport is the sum of ETMSArr and ETMSDep, and the ETMS throughput in a metroplex is the sum of IFR operations of all the airports in the metroplex.

We can see that, for a metroplex, the maximum ground operation is the potential maximum that its constituent airports can support, and the ETMS throughput is the actual throughput in a period of 15 minutes. By their definitions, the ETMS throughput should not exceed the maximum ground operation in general, and it should be equal to the ground operations when the metroplex is not constrained. Such a relationship is ideal, but may not be exact, because the maximum ground operation is the intended demand based on the flight schedule and delay. Therefore, any error in predicting the departure or arrival time will result in the misplacement of a flight in the demand period. This misalignment will put the flight as part of the ground operation in the intended time period and will put it in the actual time period as part of ETMS throughput. To put it another way, there is a probability distribution of ETMS throughput for every ground operation. Because the mismatch is random, we should expect the median of the distribution to be the maximum ground operation. However, we will be mostly interested in the upper tail of the distribution, which is the maximum metroplex throughput or capacity. To avoid possible data error, we have used 99th percentile as the maximum of the distribution.

\section{Results}

This subsection displays the median, or 50th, and 99th percentiles of ETMS throughput as functions of maximum ground operation at all U.S. metroplexes; the airports included are those being tracked by ASPM. The percentiles are based on information recorded in ASPM for every 15-minute period in 2008. To separate the effects from meteorological conditions, the two curves are produced for both VMC and IMC.

\section{POTOMAC TRACON METROPLEX}

The Potomac TRACON metroplex has three airports: Dulles International Airport (IAD), Baltimore Marshall International Airport (BWI), and Ronald Reagan Washington National Airport (DCA). Figure 8-2 shows the throughput median percentile (P50 in the figure's key) and 99th percentile (P99) in this metroplex. When the ground operation is large, which is a relatively rare event, the lines are 
choppy, because they are based on sparse data points. We can draw reliable conclusions only for the range of lines with relatively smooth curves.

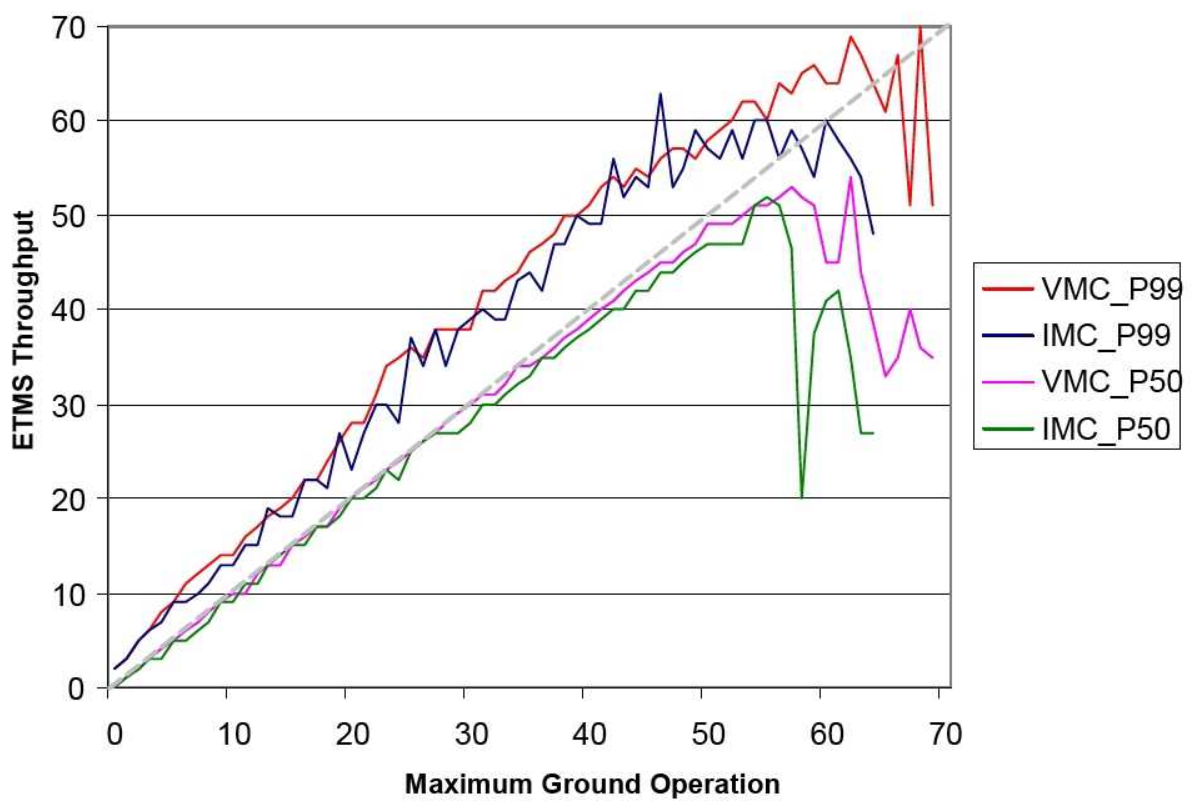

Figure 8-2. Potomac TRACON metroplex.

One can see that the medians of the distributions follow the reference line very well, which is expected because they ought to be close to the ground operation regardless of the meteorological condition. However, one can see that 99th percentile curve under IMC is saturated at about 60 in a period. This implies that the IMC metroplex capacity is about 60 . We cannot draw any conclusion about its VMC capacity, because it is never saturated for the range of observed data, or its VMC capacity is more than 65 in a period.

\section{SOUTHERN FLORIDA METROPLEX}

This metroplex includes Miami (MIA), Fort Lauderdale (FLL), and West Palm Beach (PBI) airports. Figure 8-3 shows the throughput median and 99th percentiles in this metroplex. In this figure, one can see that the two 99th percentile curves are saturated, which leads us to the conclusion that this metroplex's VMC and IMC capacities are about 60 and 50, respectively. 


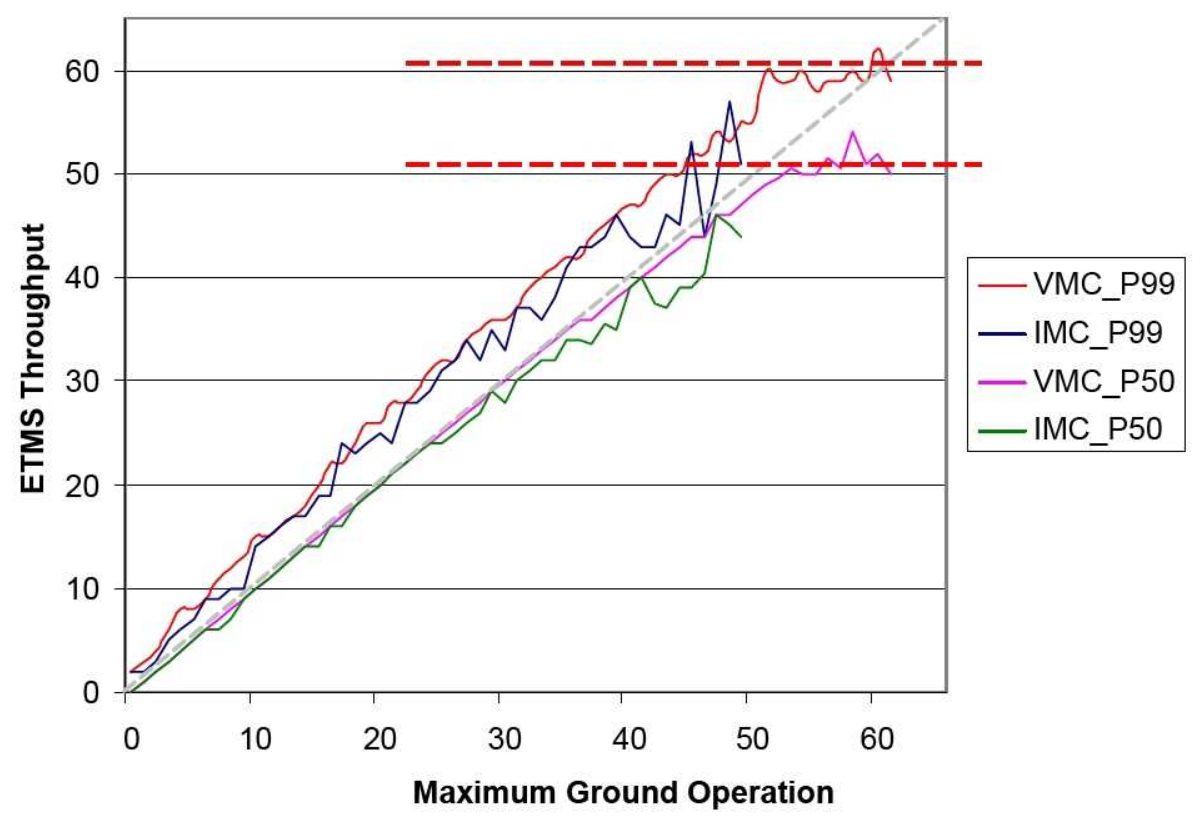

Figure 8-3. Southern Florida metroplex.

\section{SAN FRANCISCO BAy AREA METROPLEX}

Airports tracked by ASPM for this metroplex are San Francisco (SFO), Oakland (OAK), and San Jose (SJC). Figure 8-4 shows the throughput median and 99th percentiles in this metroplex. We do not see any curve saturation in the figure. This result implies that the current airspace in the Bay Area does not impose any additional metroplex constraint or that the metroplex capacity is above 50 in each period. 


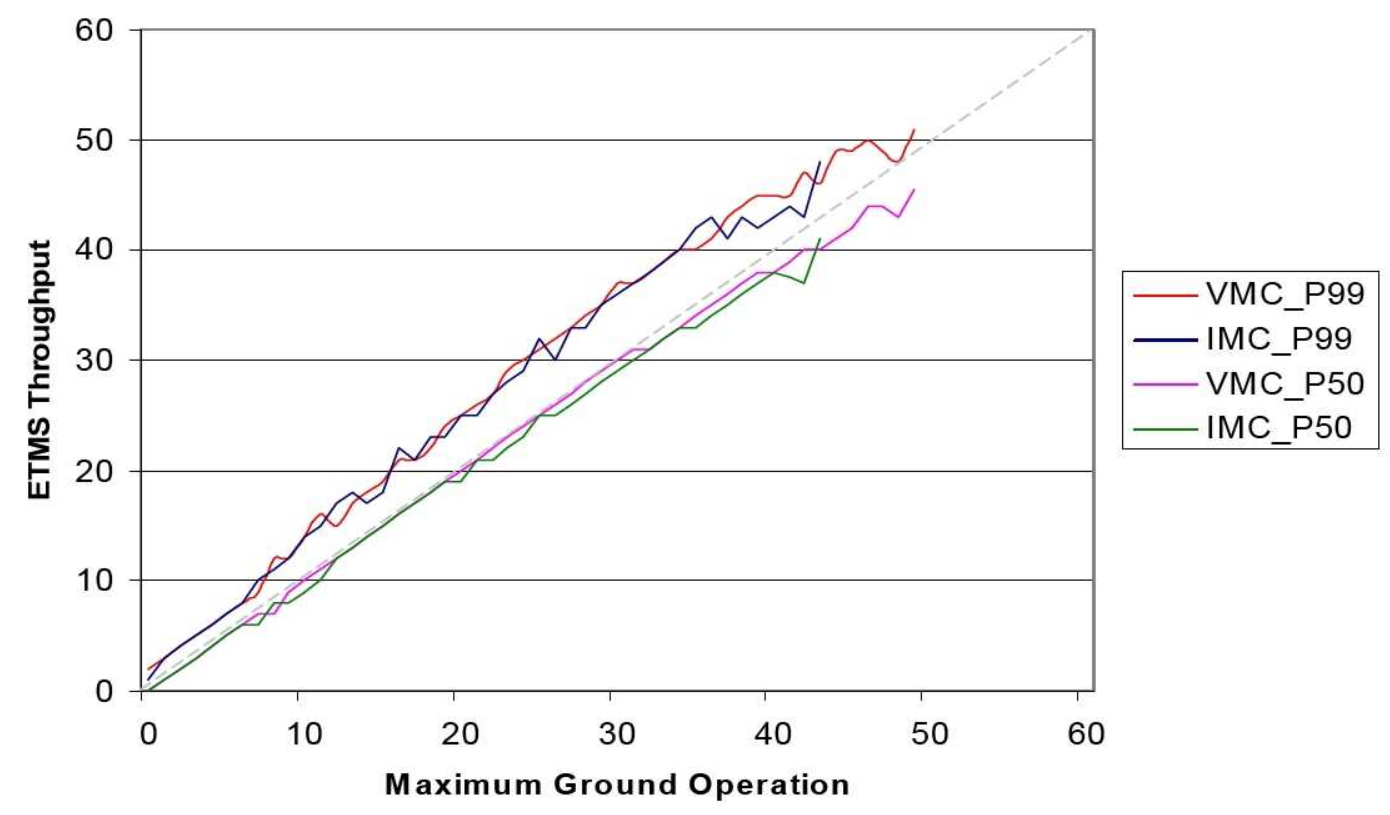

Figure 8-4. San Francisco Bay Area metroplex.

\section{Chicago Area Metroplex}

The Chicago Area metroplex has O'Hare International (ORD), Midway (MDW), and Gary (IN) (GYY) airports. Figure 8-5 shows the throughput median and 99th percentiles in this metroplex, based on ETMS data. IMC throughput seems to be saturated around 62; in other words, this metroplex's IMC capacity is about 62 . We cannot, however, draw any conclusion about VMC capacity.

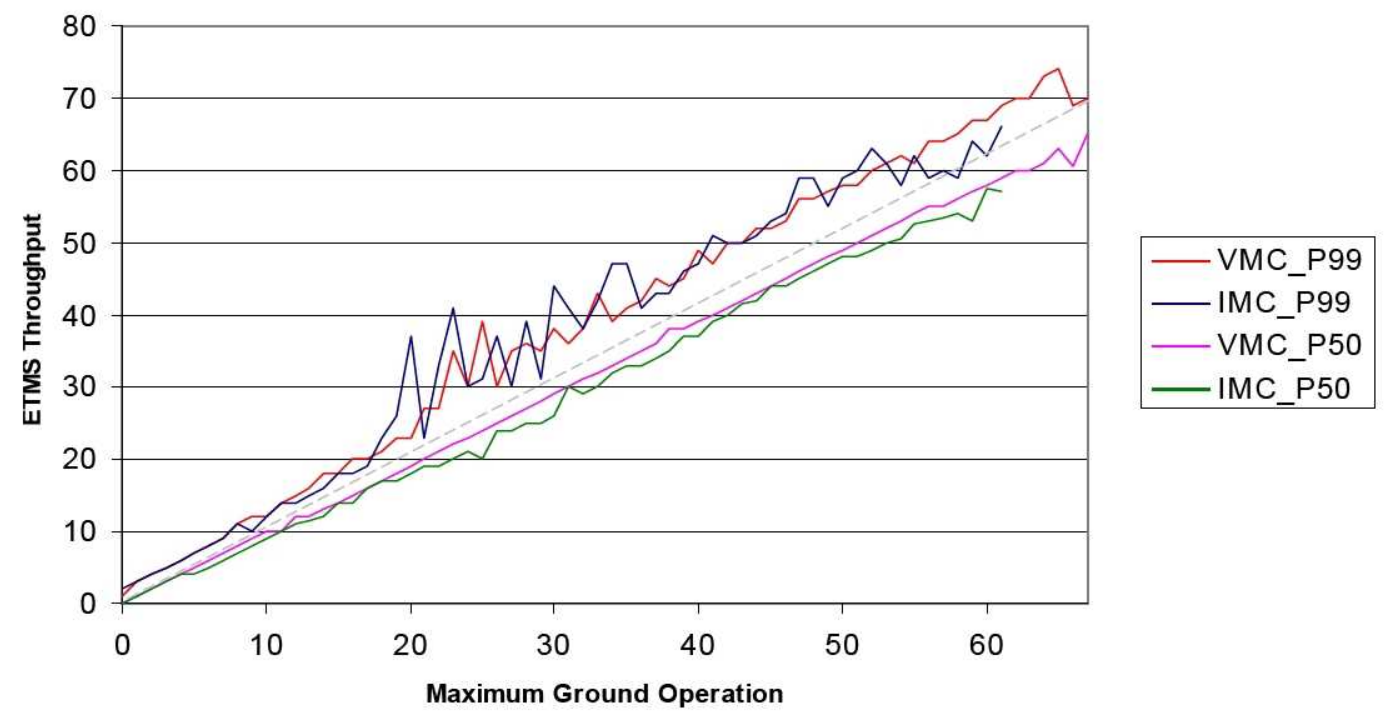

Figure 8-5. Chicago area metroplex. 


\section{LOS ANGELES MeTROPLEX}

ASPM tracks six airports in the Los Angeles metroplex: Los Angeles International (LAX), Van Nuys (VYN), Bob Hope (BUR), Long Beach (LGB), Ontario (ONT), and John Wayne (SNA). Figure 8-6 shows the throughput median and 99th percentiles in this metroplex. The two lines of 99th percentiles seem to be saturated at 70 and 65, respectively, for VMC and IMC. Because the segments of saturated lines are short, we can make only a weak statement in terms of its capacities.

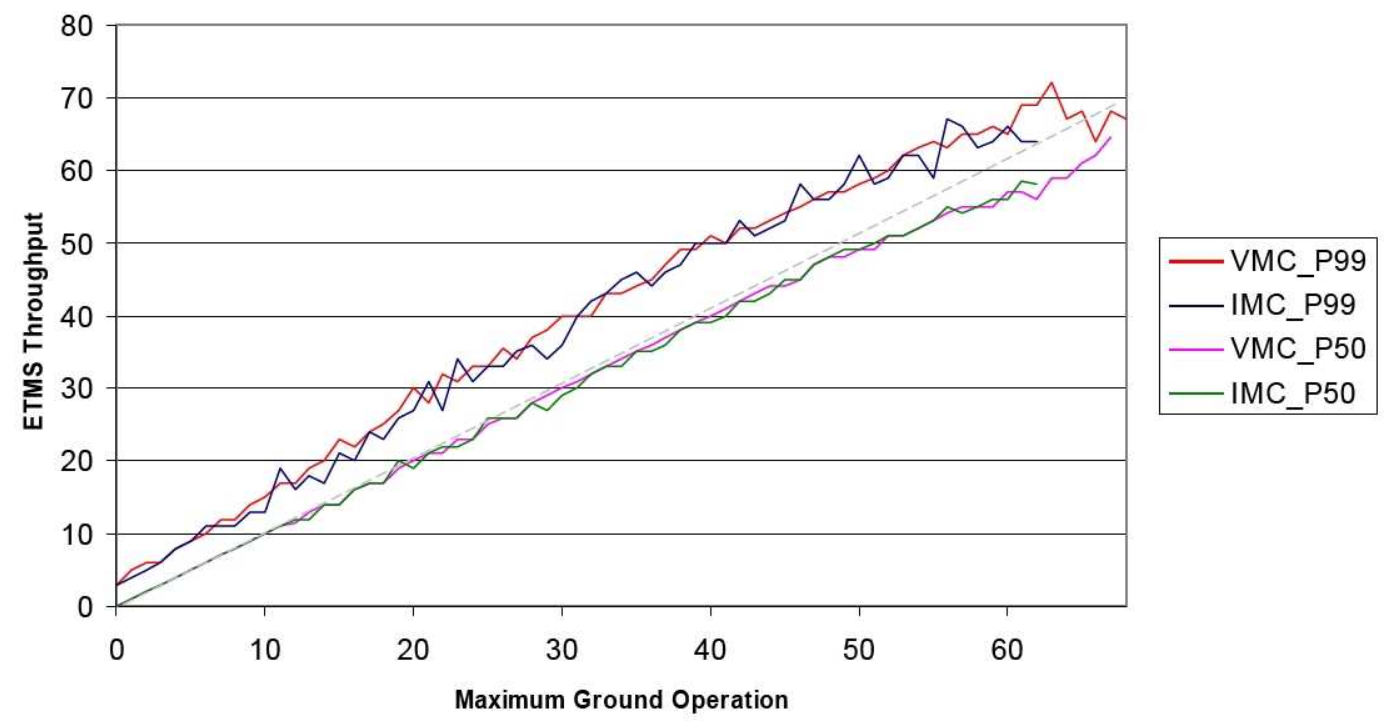

Figure 8-6. Los Angeles metroplex.

\section{HOUSTON METROPLEX}

George Bush Houston Intercontinental (IAH) and Houston Hobby (HOU) are the two airports in Houston metroplex. Figure 8-7 shows the throughput median and 99th percentiles in this metroplex. We can draw no conclusions from this figure. 


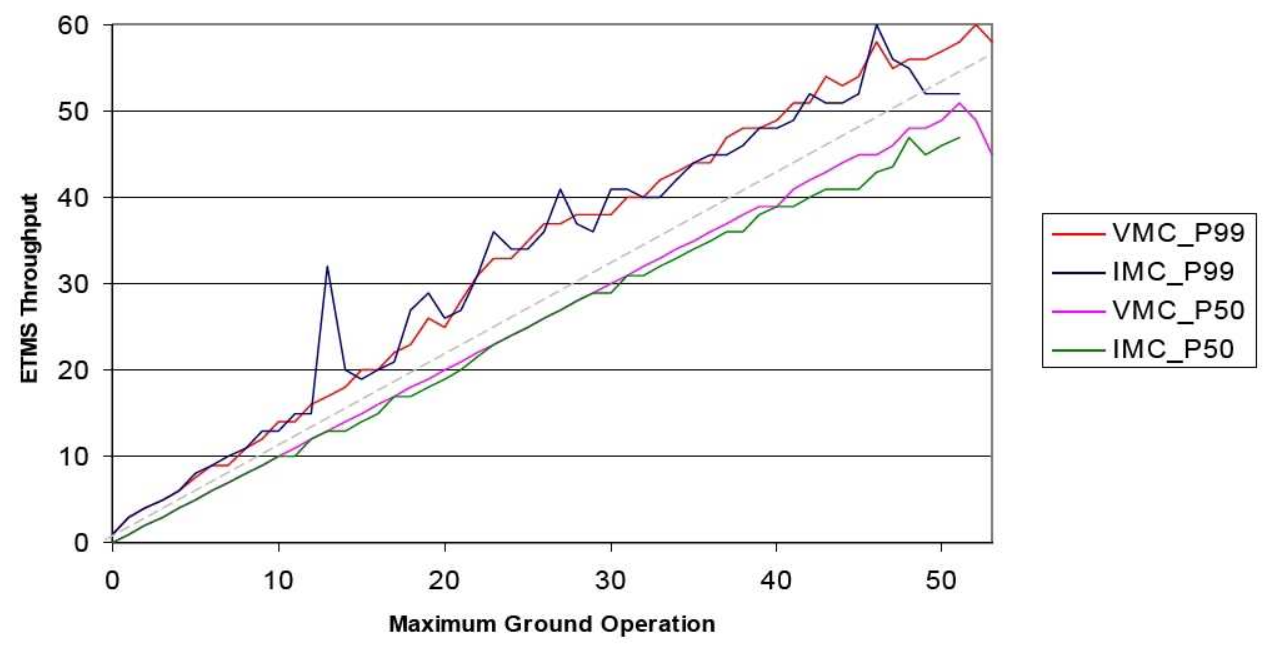

Figure 8-7. Houston metroplex.

\section{DALLAS MetRoplex}

This metroplex includes Dallas-Fort Worth (DFW) and Dallas Love Field (DAL). Figure 8-8 shows the throughput median and 99th percentiles in this metroplex. We can draw no conclusions from this figure.

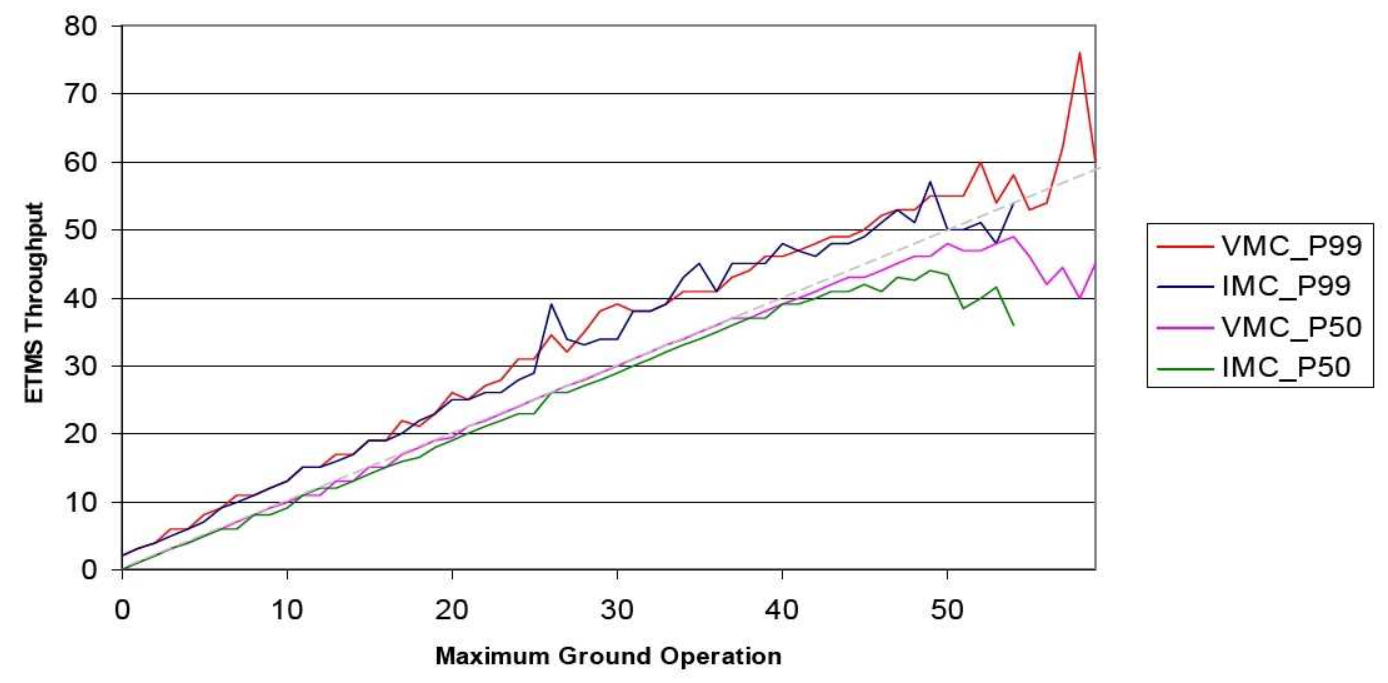

Figure 8-8. Dallas metroplex.

\section{NeW YORK City Metroplex}

This metroplex has six airports: Kennedy International (JFK), La Guardia (LGA), Newark Liberty International (EWR), Teterboro (TEB), Worcester County at White Plains (HPN), and MacArthur Airport at Long Island (ISP). Figure 8-9 shows the throughput median and 99th percentiles in this metroplex. Because of the sparsity of data, the 99th percentile curves had large abruptions. Therefore, we 
used 98th percentile curves, which are quite smooth. One cannot see any curve saturation from this figure.

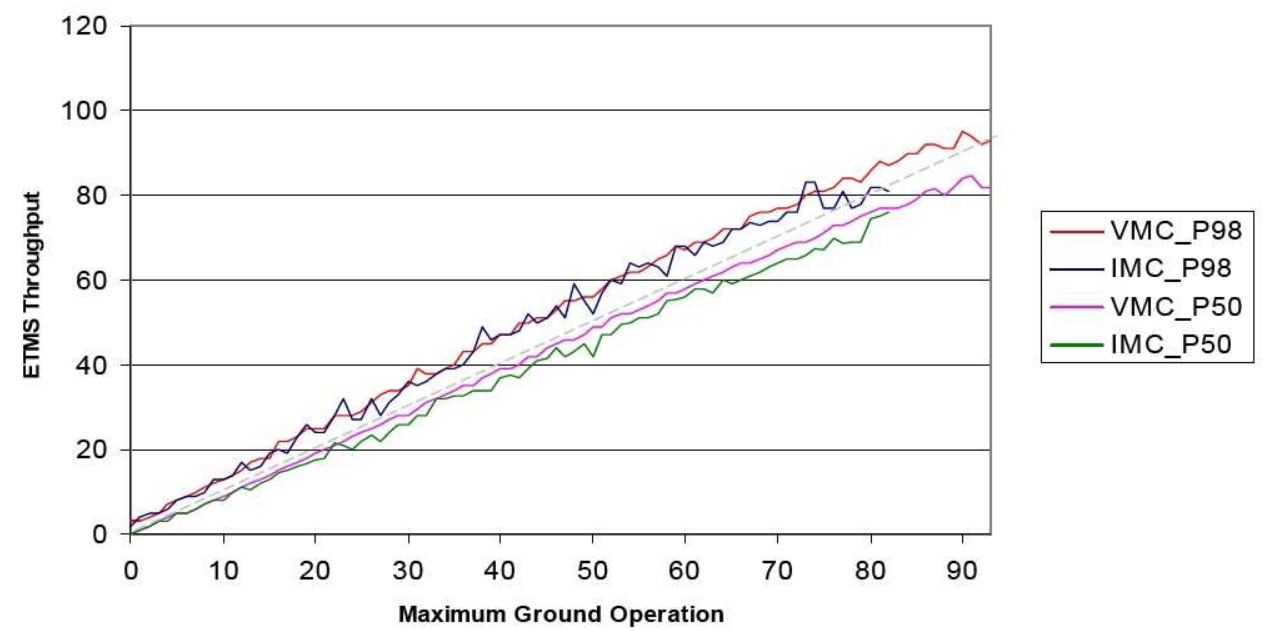

Figure 8-9. New York City metroplex.

\section{Summary of Metroplex Capacity Constraints}

Two of the metroplexes we analyzed have throughput constraints under IMC:

Potomac TRACON and Chicago. Southern Florida is constrained under both IMC and VMC operations.

For the remaining metroplexes analyzed, we see no obvious signs of throughput constraints (although the maximum ground operations are degraded under IMC operations for several metroplexes). One should not infer that these metroplexes can support high traffic volumes in the future. This is because the figures capture traffic congestion only for 2008 . It is entirely possible that these metroplexes will become congested with the growth of traffic.

Another reason could be that the maximum ground operations are set up with metroplex constraints in mind. This could be determined by comparing the optimal with the recorded runway capacity for each airport in the metroplex at every 15-minute period. The optimal runway capacity should be based on the optimal runway configuration at each airport, considering meteorological conditions (VMC or IMC) and wind direction and velocity. In other words, the meteorological condition determines whether a particular runway will be operational given the weather and facility information, and wind direction and velocity will select the runways with maximum headwind and minimum crosswind. Once the runway configuration is determined, its capacity can be determined directly from the FAA airport capacity models or from other models like the LMINET airport capacity model. Of course, the actually implementation of such a model can be complicated by other considerations. For example, the LMINET airport capacity model is capable of selecting the optimal configuration based on the length, orientation, pavement, and information on the meteorological 
condition and wind direction and velocity, but it would need to be augmented to integrate such parameters as noise and other environmental impacts. Such augmentation will require substantial collection of field environmental and operational information and revision of the models, which is beyond the scope of this study.

\section{METROPLEX ENVIRONMENTAL CONSTRAINTS}

\section{Overview}

Like our analysis of environmental constraints at individual airports, our analysis of the cumulative effects of environmental constraints in a metroplex considered both air quality, in terms of emissions, and noise. In this section, we provide additional background when considering the environmental constraints on metroplex regions as well as initial approaches to evaluating these constraints.

\section{EMISSIONS AND GREENHOUSE GASES}

Aircraft emissions are very similar to the emissions produced by other vehicles within the transportation industry. Aircraft jet engines in particular produce carbon dioxide, water vapor, nitrogen oxide, carbon monoxide, sulfur oxide, and various other compounds and particulates. Aircraft emissions are typically categorized as local air quality pollutants (those that occur at or near the ground) or greenhouse gases (those that occur while the aircraft is airborne). According to a report published by the FAA in $2005,{ }^{1}$ roughly 10 percent of the aircraft pollutants, excluding $\mathrm{CO}$ and hydrocarbons, is produced on the surface, and the other 90 percent is produced once the aircraft is airborne. For $\mathrm{CO}$ and hydrocarbons, 30 percent is produced on the surface and the remaining 70 percent is produced when the aircraft is airborne. Many additional sources on or near airports also are typically considered. Examples of such sources are auxiliary power units that produce electricity while the aircraft is parked, various pieces of airport surface equipment that service the aircraft and transport baggage and passengers, additional power sources that supply the airport with electricity, and other forms of vehicles dropping off or picking up passengers at the terminal. Any changes in demand at the airport will most likely result in a change in emissions, affecting local air quality and greenhouse gases.

Greenhouse gases are those gases in the atmosphere that trap the planet's heat on the surface and are often referenced as the cause for climate change or global warming. Greenhouse gases are primarily made up of water vapor, carbon dioxide, methane, nitrous oxide, ozone, and other chlorofluorocarbons. When considering aviation's contribution to greenhouse gases, $\mathrm{CO}_{2}$ and $\mathrm{NOx}$ are typically of primary concern.

\footnotetext{
${ }^{1}$ Federal Aviation Administration, Office of Environment and Energy, Aviation and Emissions: A Primer, January 2005.
} 
Figure 8-10 displays areas that are considered "nonattainment" or "maintenance" by the Environmental Protection Agency. An area designated as "nonattainment" does not meet ambient air quality standards for a particular pollutant. An area designated as "maintenance" was once designated "nonattainment" but has reached attainment status and has developed a plan to maintain containment. As the figure shows, nearly all metropolitan areas that satisfy the definition of a metroplex are in areas of the country that are challenged by air quality issues.

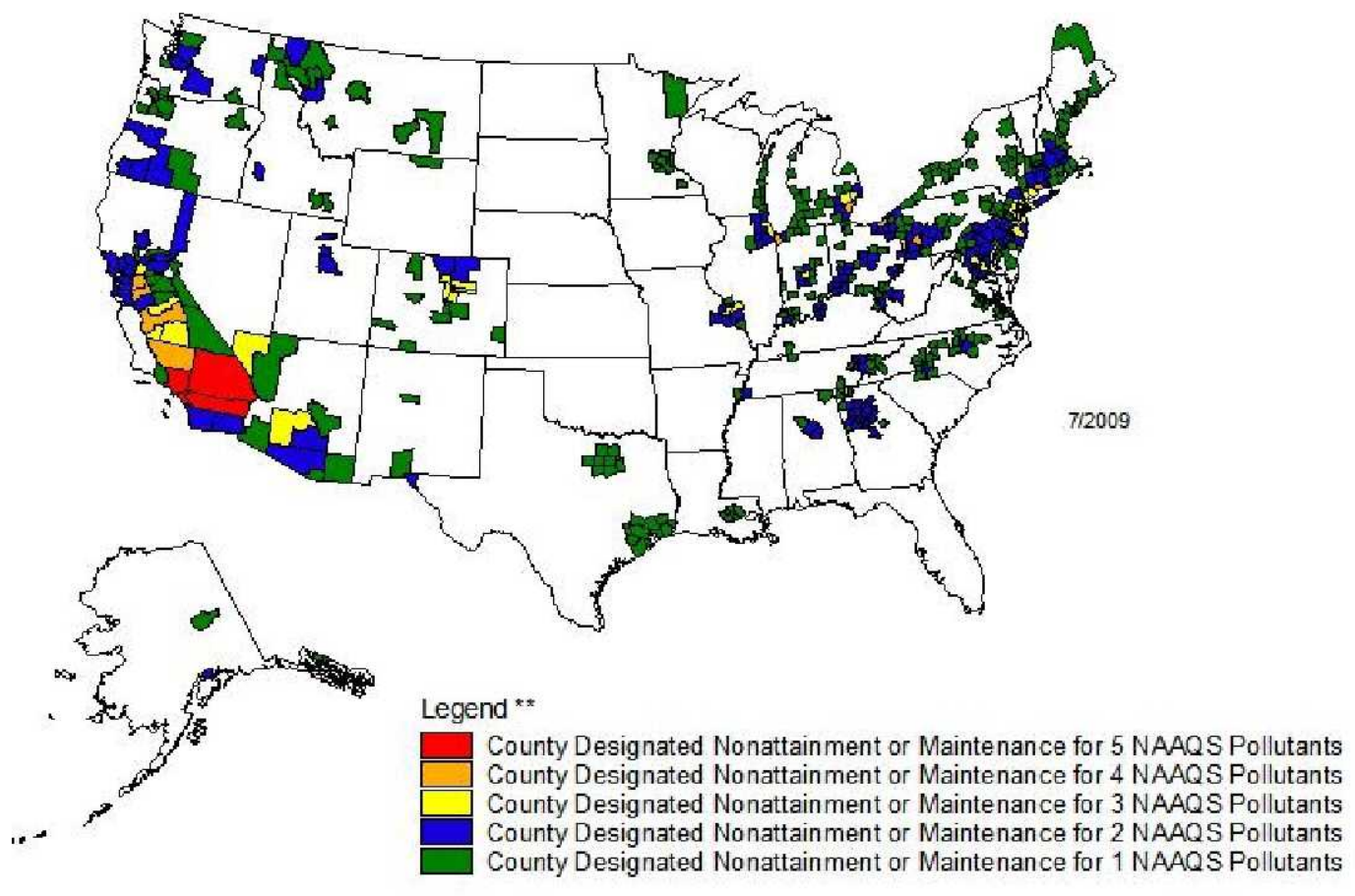

Guam - Piti and Tanguisson Counties are designated nonattainment for the SO2 NAAQS Puerto Rico - Mun. of Guaynamo is designated nonattainment for the PM10 NAAQS

* The National Ambient Air Quality Standards are health standards for lead, carbon monoxide, sulfur dioxide, ground level 8-hr ozone, and particulate matter (PM-10 and PM2.5). There are no nitrogen dioxide nonattainment areas.

* Partial counties, those with part of the county designated non attainment and part attainment, are shown as full counties on the map

Source: EPA Green Book.

Figure 8-10. Counties designated "Nonattainment" or "Maintenance" for NAAQS pollutants.

Aircraft noise has been a highly controversial issue within busy urban or metropolitan areas for more than 40 years. Significant improvements in both airframes and engines over this time period have continued to reduce the number of people exposed to significant noise levels. However, even with the number of people exposed to significant noise levels shrinking, the situation and attention to noise has not subsided. In most cases, the population exposed to significant noise levels has come to accept that living near an airport means hearing aircraft. The 
challenge has become noise mitigation at lower thresholds for those locations farther from the airport where low or no aircraft noise is expected. The advent of RNP and Area Navigation (RNAV), which address efficiency improvements and the leveraging of additional airspace, may only exacerbate the issue. Like exposure to air pollutants, significant levels of noise exposure are predominately found in the densely populated metropolitan areas.

For many years, the FAA focused on single-airport noise modeling, but in the last 15 years, it has expanded its focus to analyze noise in regions with multiple airports. In most cases, these projects are referred to as airspace redesign, which alters the airspace to support a metroplex region. Because the routes to these areas are heavily dependent on each other, one change to improve the operations for an airport often affects the operations at a nearby airport. Likewise, flight routes typically cross each other; when considered independently, they may not cause a significant change, but do when considered cumulatively.

\section{Method}

Similar to the individual airport constraints analysis, two methods were considered to assess potential environmental constraints related to various metroplex regions around the country. The first method involved a screeninglevel analysis that provided a first-order estimate of the interactions associated with nearby airports. Specifically, because both emissions and noise are heavily driven by volume and fleet composition, we assumed a symmetrical distribution around each airport for both emissions and noise. Flight paths are also critical to understanding the potential noise impact, but are not considered in the first method. Likewise, weather conditions would be useful for understanding how the emissions were dispersed but were not considered in this initial method. For example, using the AEM to produce noise area given a fleet mix and schedule of operations produces the result shown in Figure 8-11. 


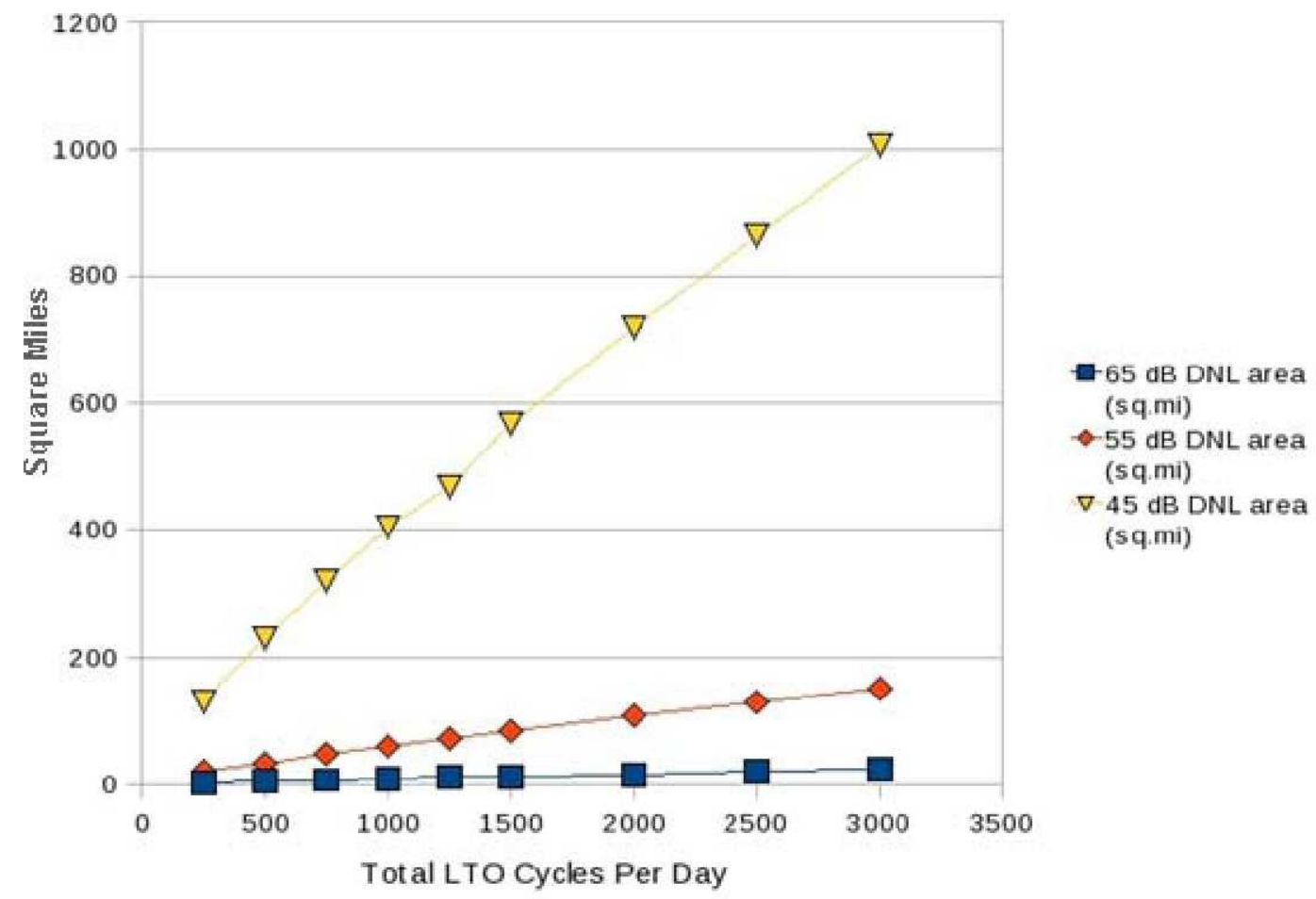

Figure 8-11. Characteristic interaction for noise: area.

Associating an elliptical shape with the noise area allows for the comparison of two airports based on the level of operations and their relative distance. See Figure 8-12.

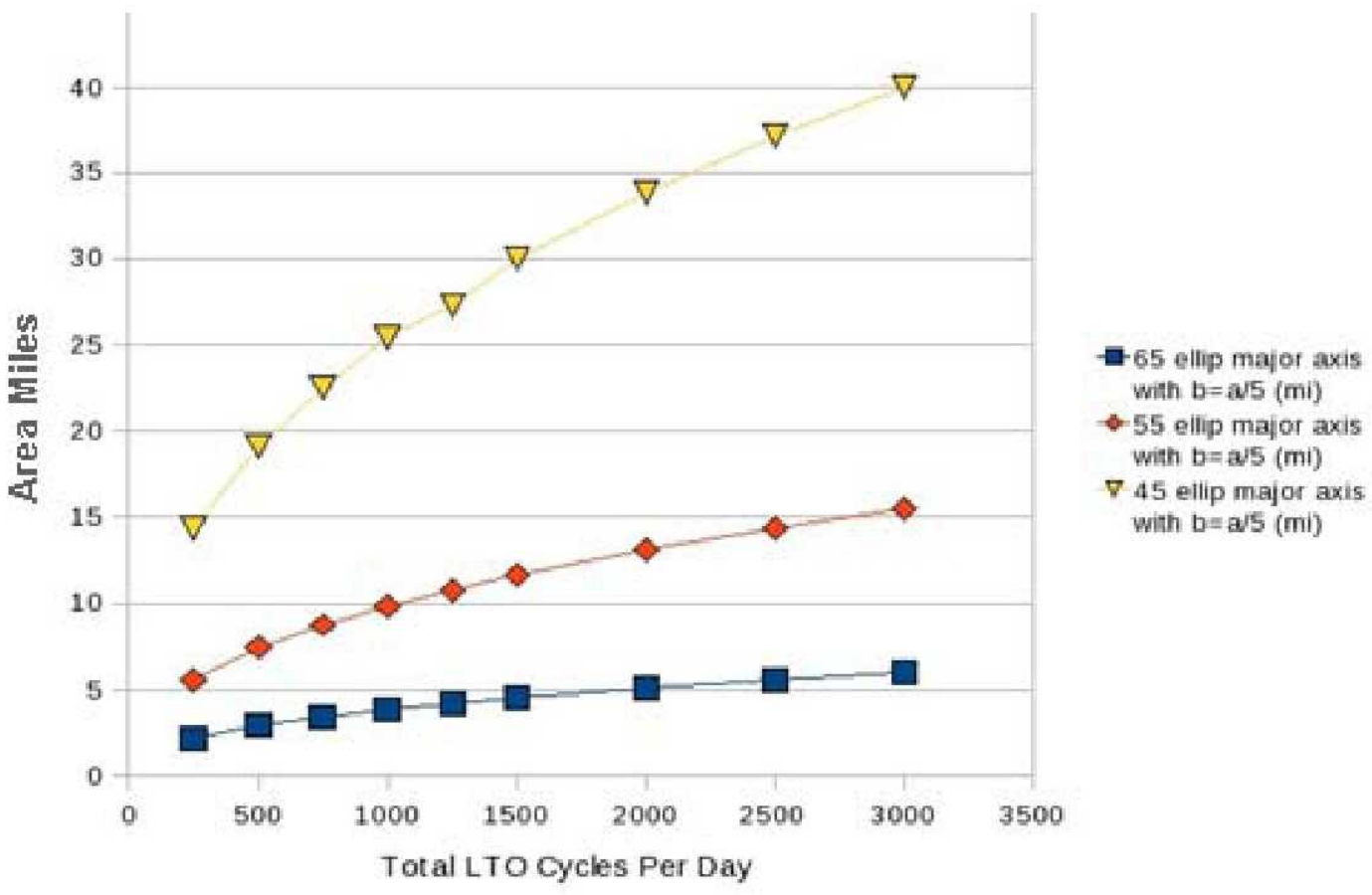

Figure 8-12. Characteristic interaction for noise: elliptical major axis. 
We considered a similar approach to emissions: a volume with the radius determined by 3,000 feet (approximate mixing height for computing emissions) and a 3-degree glide slope. Density is computed for a circular region as

$$
r=\left[\operatorname{mass} /\left(\text { density }{ }^{*} i^{*} \tan (3 \mathrm{deg})\right)\right]^{\wedge}(1 / 3),
$$

with mass determined by LTO-based emissions and density from NAAQS (http://www.epa.gov/air/criteria.html). Figure 8-13 is an example that includes both air quality and noise components used to evaluate metroplex airports.

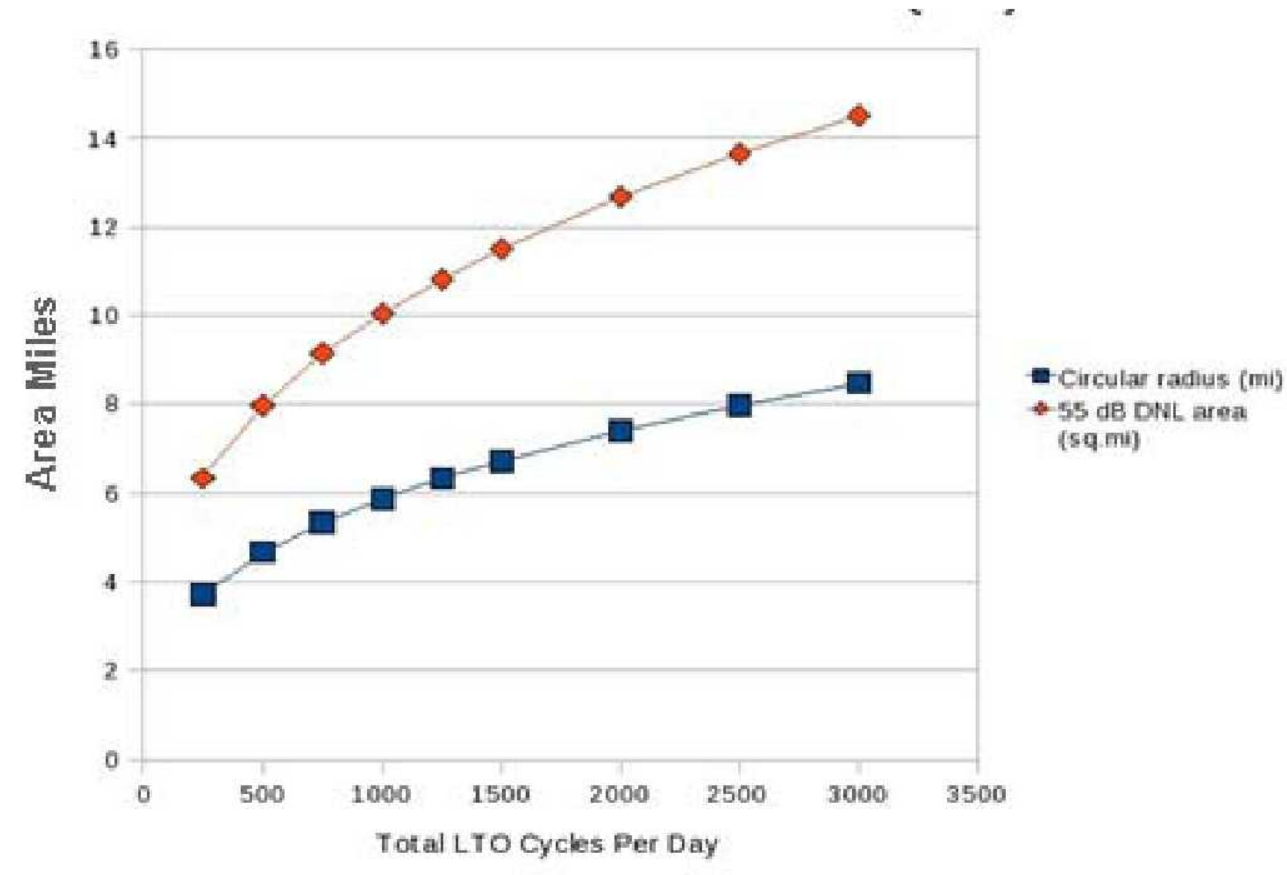

Figure 8-13. Characteristic interaction for air quality and noise.

The second method used to identify potential environmental constraints for metroplex regions was simply a visual inspection. For noise, we considered the noise contours and population impacts generated by the airport environmental constraints described in Chapter 6 of this report. For emissions, we used GIS data and NAAQS county-level information from the EPA to draw similar conclusions about the significance of the emissions impacts on the region.

\section{Results}

We analyzed three metroplex regions, using the following sources of data:

- Noise contours for the metroplex region in 2015

- Current EPA designations for the counties in or near the metroplex region

- An aggregation of individual airport constraints for each metroplex. 
Noise contours are shown as follows:

-65 DNL contour is red.

- $55 \mathrm{DNL}$ contour is green.

- 45 DNL contour is yellow.

EPA designations for counties are as follows:

Legend **

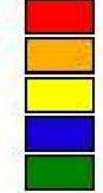

County Designated Nonattainment or Maintenance for 5 NAAQS Pollutants County Designated Nonattainment or Maintenance for 4 NAAQS Pollutants County Designated Nonattainment or Maintenance for 3 NAAQS Pollutants County Designated Nonattainment or Maintenance for 2 NAAQS Pollutants County Designated Nonattainment or Maintenance for 1 NAAQS Pollutants

\section{NEW YORK METROPLEX}

The New York metroplex is known as the most complex and congested airspace region of the world. Of the 310 airports considered in the analysis, 10 airportsrepresenting more than 6 percent of the total NAS operations in both 2015 and 2025 - are in this region. Figure 8-14 shows the noise contours for 2015, and Figure 8-15 shows the counties designated as "nonattainment" or "maintenance" for NAAQS pollutants.

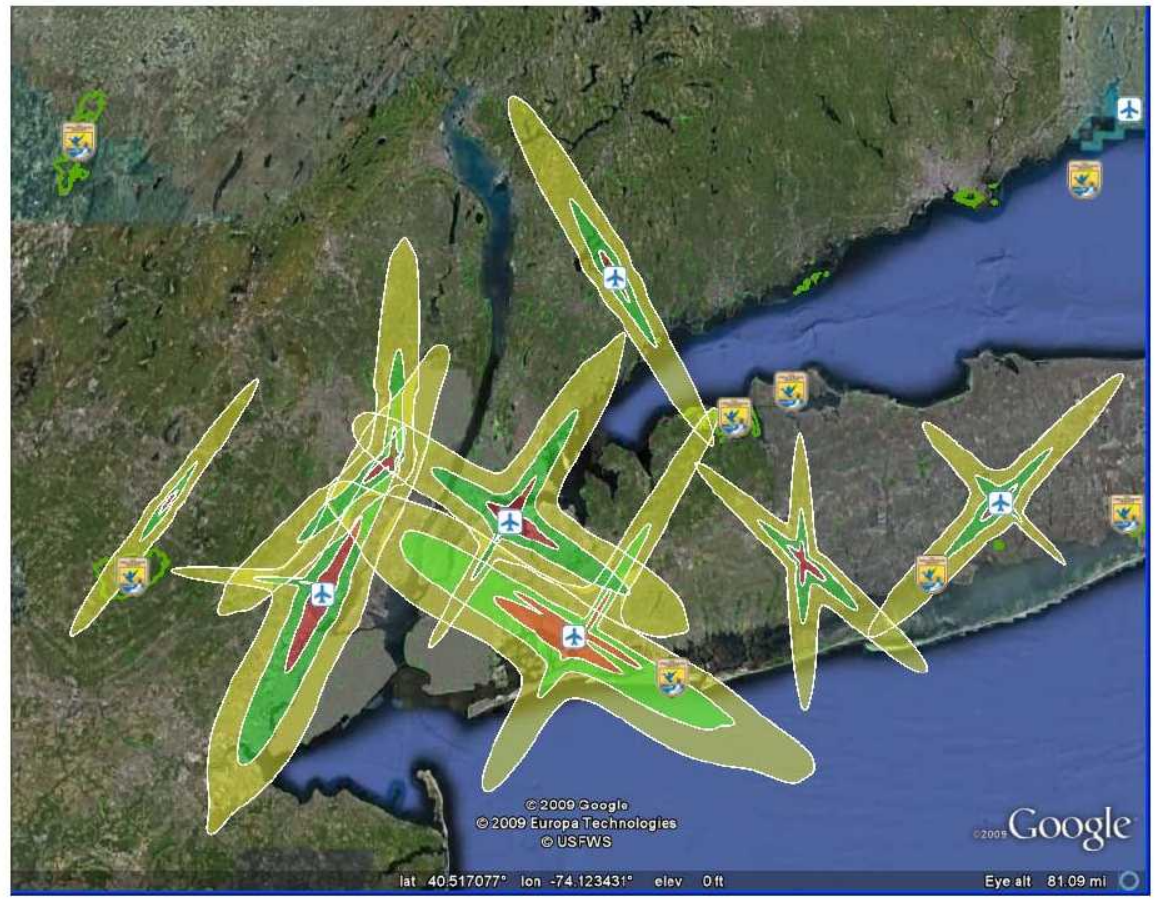

Figure 8-14. New York metroplex: 2015 noise contours. 


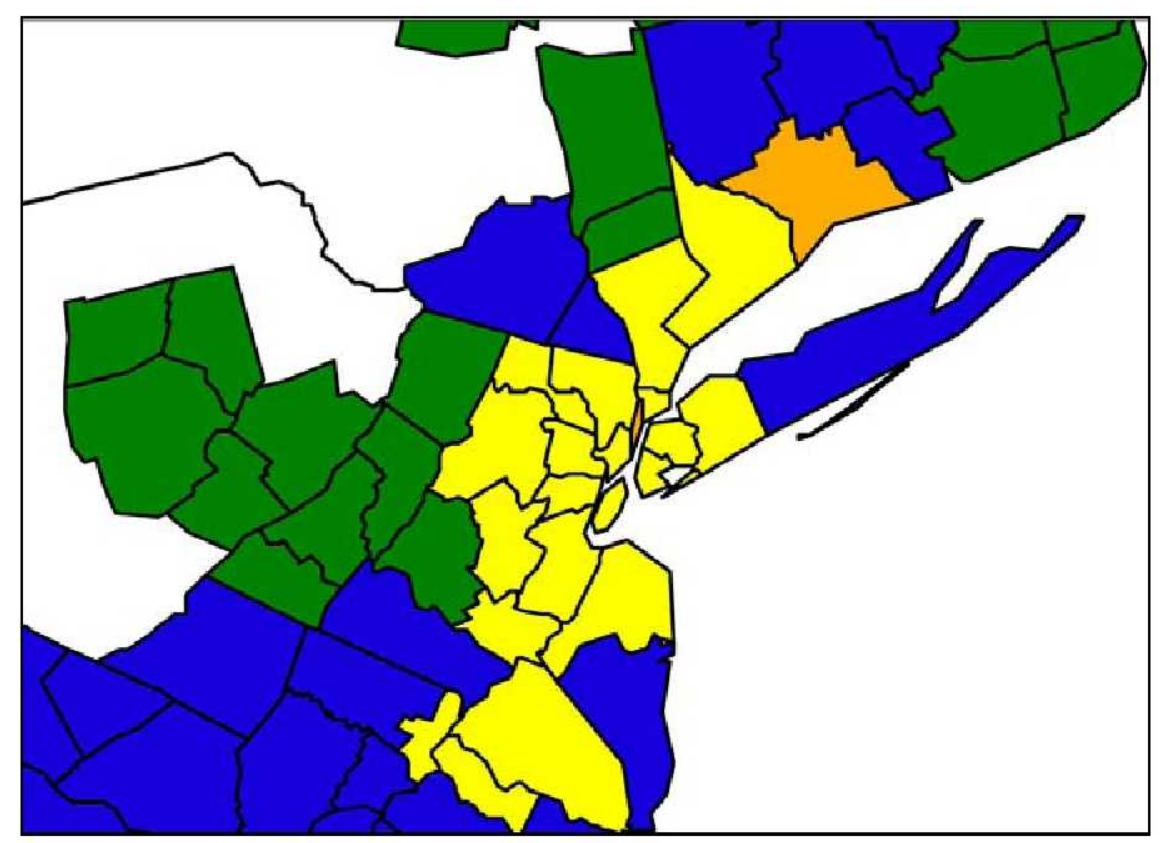

Figure 8-15. New York Region: counties designated "Nonattainment" or "Maintenance" for NAAQS pollutants.

Figure 8-16 shows the additive constraints for the New York metroplex airports.

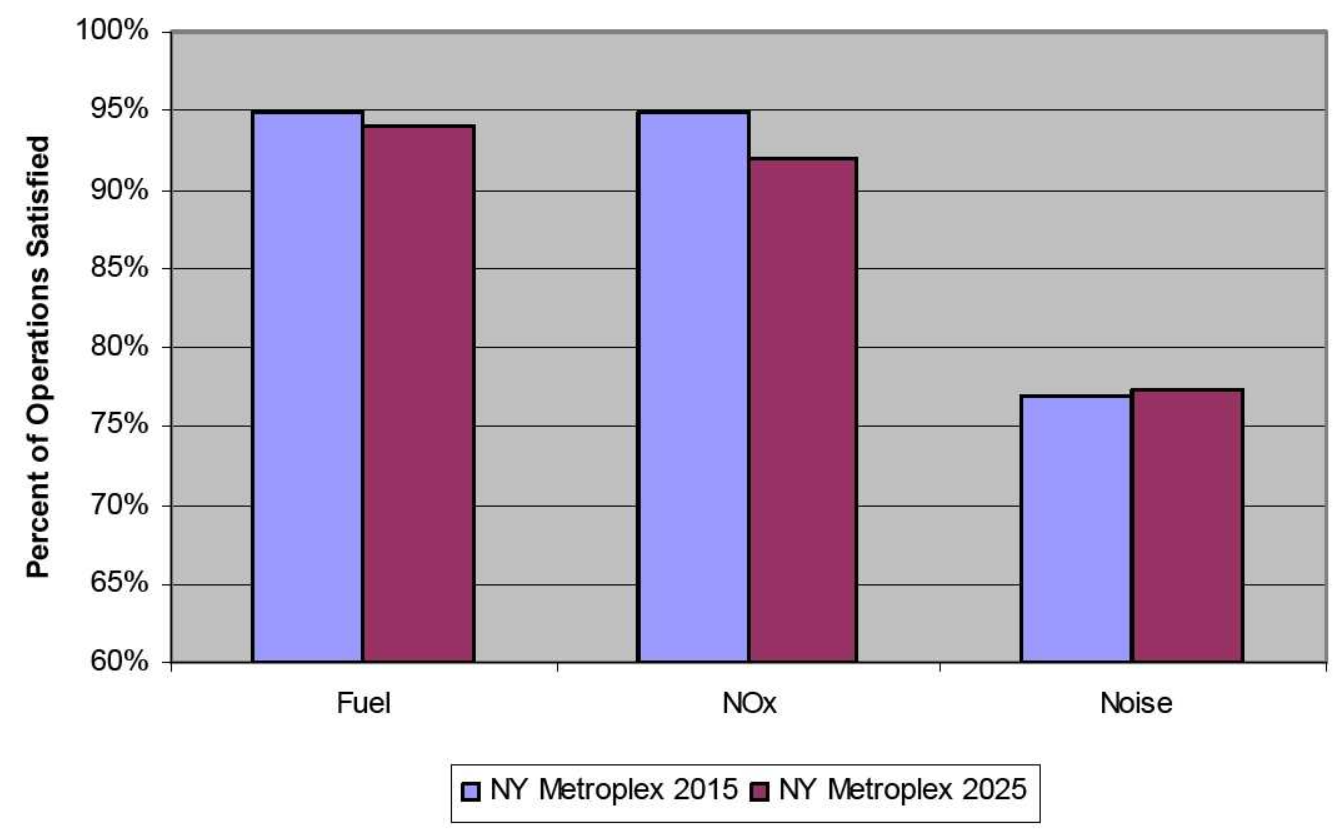

Figure 8-16. New York metroplex: aggregate constraints.

\section{SOUTHERN CALIFORNIA METROPLEX}

The Southern California metroplex region is significantly challenged by terrain and weather patterns that contribute to the overall local air quality conditions. 
When coupled with the densely populated region and existing noise issues, environmental constraints will likely remain a significant issue in the future. Figure 8-17 shows the noise contours for 2015, and Figure 8-18 shows the counties designated as "nonattainment" or "maintenance" for NAAQS pollutants.

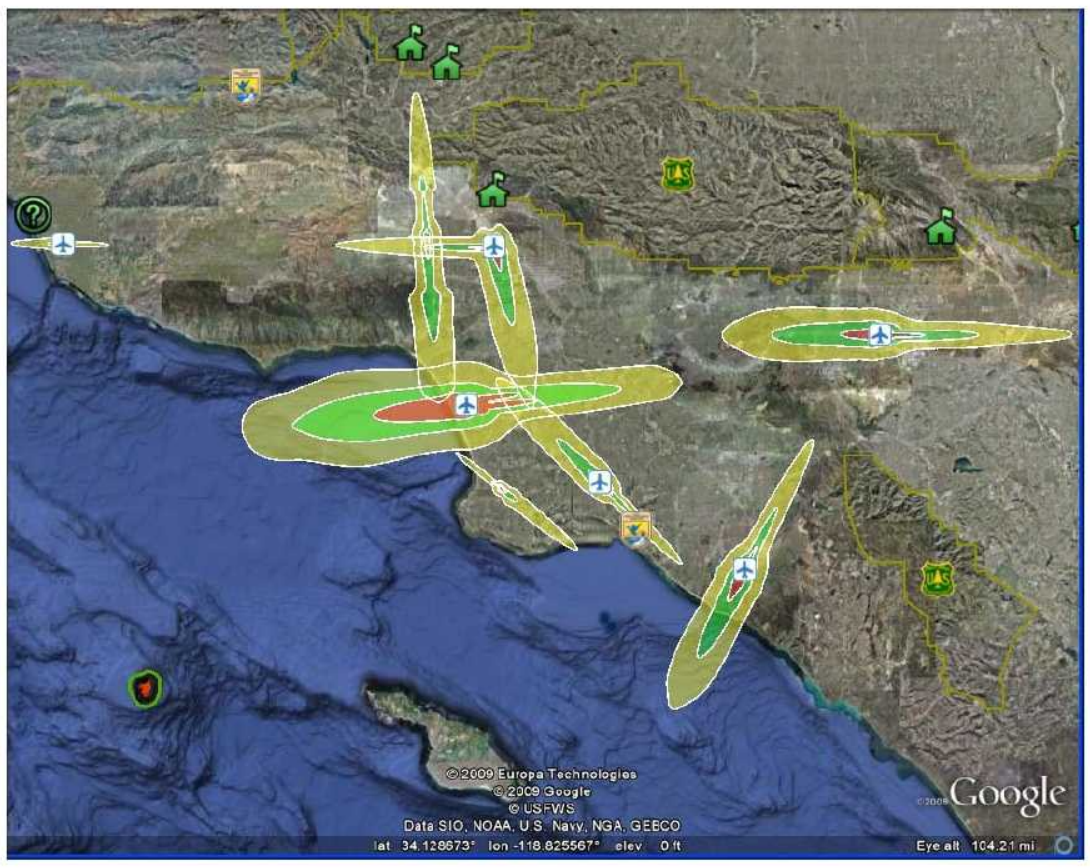

Figure 8-17. Southern California metroplex: 2015 noise contours.

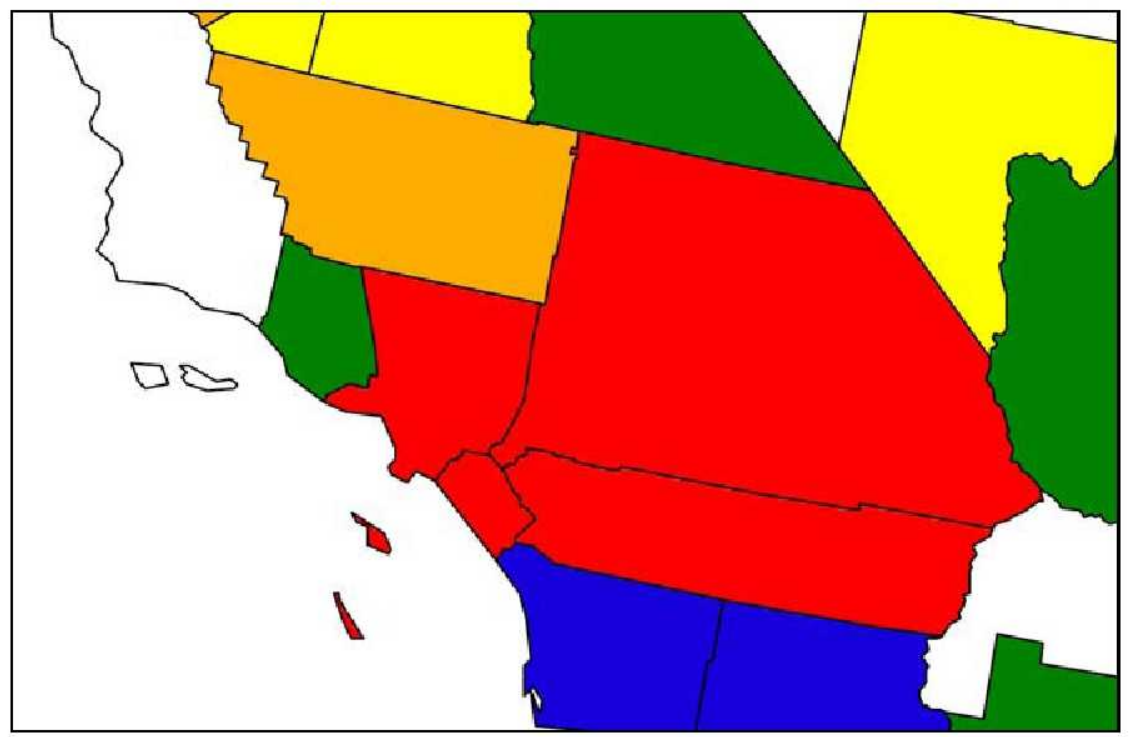

Figure 8-18. Southern California region: counties designated "Nonattainment" or "Maintenance" for NAAQS pollutants. 
Figure 8-19 shows the additive constraints for the Southern California metroplex airports.

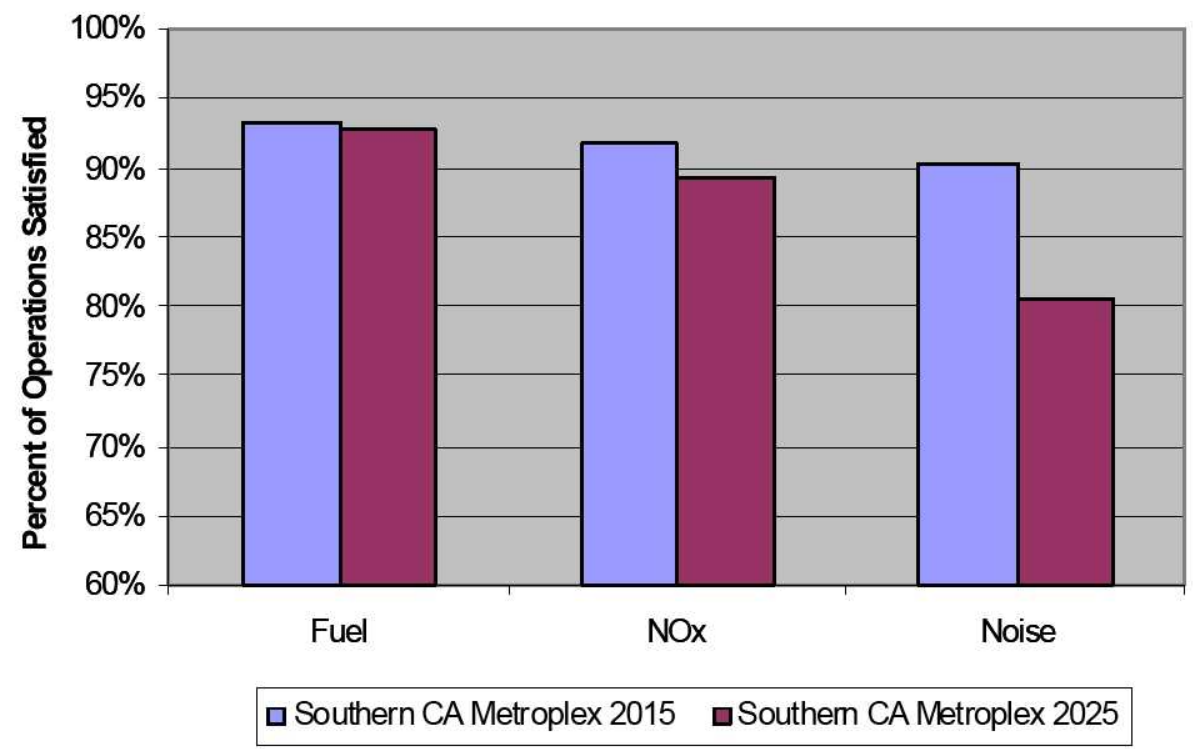

Figure 8-19. Southern California metroplex: aggregate constraints.

\section{ChicAgo Metroplex}

The Chicago metroplex includes two OEP airports, ORD and MDW. ORD, one of the busiest airports in the world, is being significantly restructured to provide increased capacity for the region, but it will more than likely also create new environmental challenges. Figure 8-20 shows the noise contours for 2015, and Figure 8-21 shows the counties designated as "nonattainment" or "maintenance" for NAAQS pollutants. 


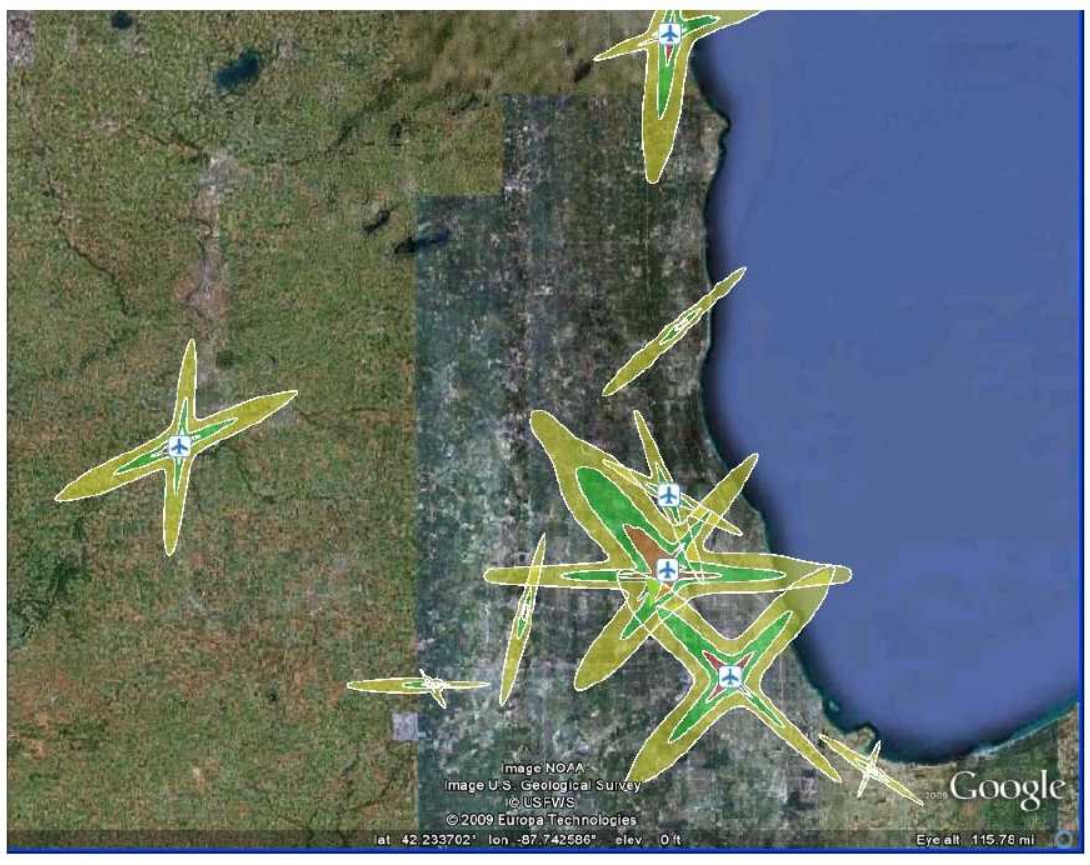

Figure 8-20. Chicago metroplex: 2015 noise contours.

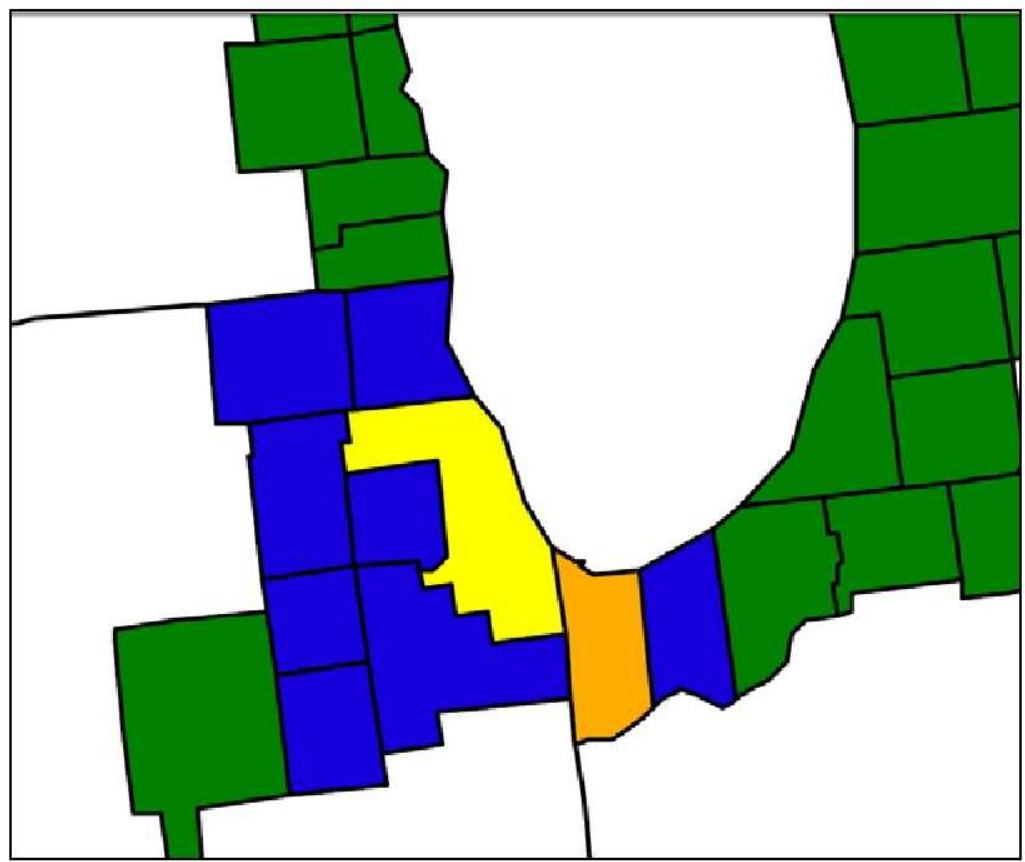

Figure 8-21. Chicago region: counties designated "Nonattainment" or "Maintenance" for NAAQS pollutants. 
Figure 8-22 shows the additive constraints for the Chicago metroplex airports.

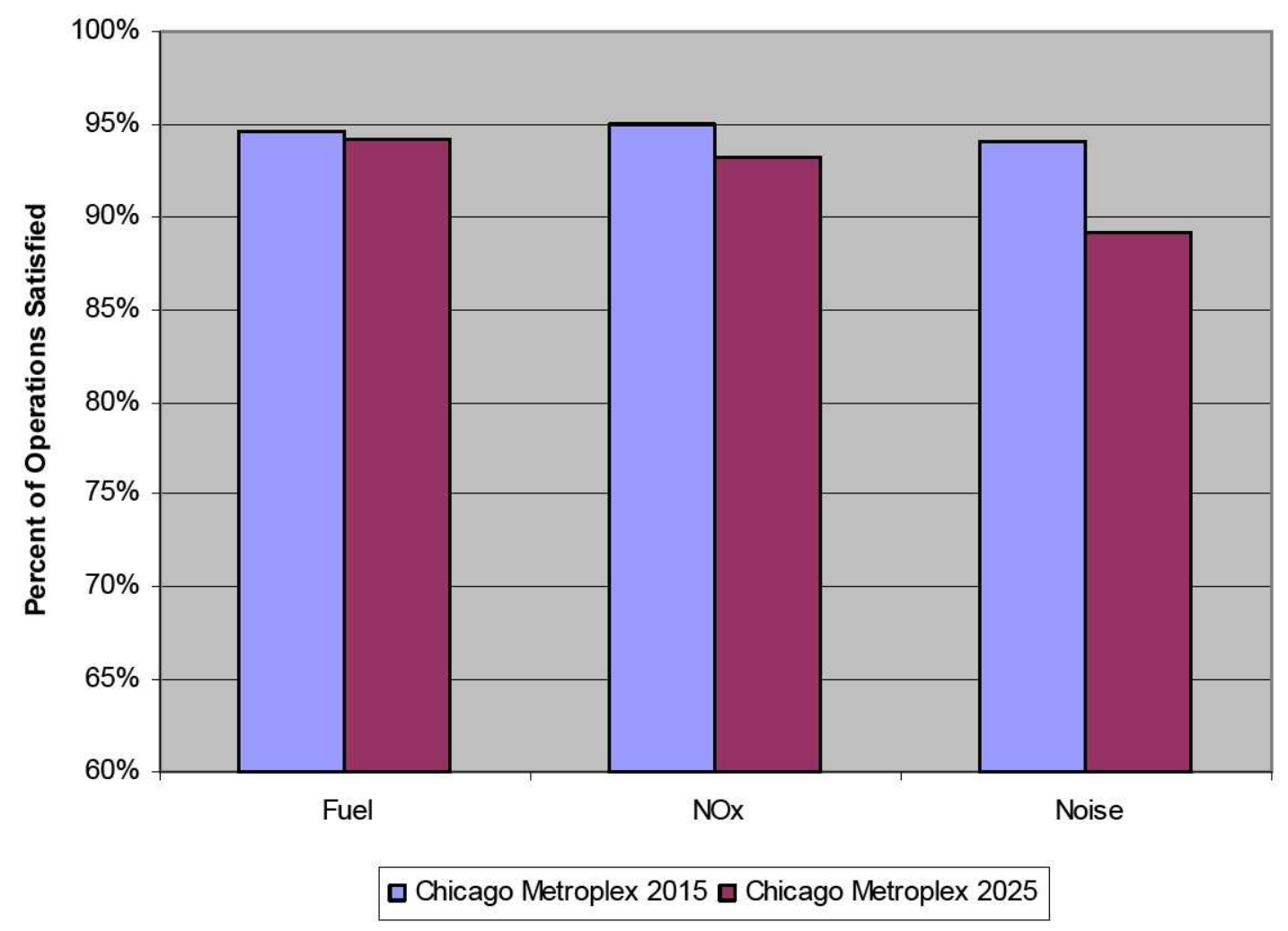

Figure 8-22. Chicago metroplex: aggregate constraints.

\section{Summary of Metroplex Environmental Constraints}

The nation's aviation network comprises not only individual airports but also more complex components known as metroplexes. Typically, metroplexes are in densely populated regions serviced by multiple airports. Any change to one airport will usually have ripple effects on the metroplex. Those effects will change the environmental considerations for the entire region. Thus far, airspace redesign for these regions has been both a technical challenge and a highly controversial issue as the regions understand the ever-changing environmental impacts. These results show the additive environmental effects on a region by simply considering each airport individually and summing their constraints. This describes the potential environmental constraints to each of the regions discussed but does not treat the metroplex as a single entity. A better understanding of the environmental constraints would require a consolidated modeling in more detailed airport interactions within the metroplex and a more detailed environmental analysis of each region in regard to cumulative noise, land use, state and county EPA regulations, and emissions dispersion. 


\section{Appendix A \\ LMI Runway Capacity Model}

This appendix describes the logic and mathematics of the LMI runway capacity model. The mathematics described in this appendix were developed at LMI by Dr. David Lee and Dr. Gerald Shapiro and have been previously published in several NASA contractor reports. The material in this appendix was drawn specifically from two reports:

- The Aviation System Analysis Capability Airport Capacity and Delay Models, Lee, Nelson, and Shapiro, NASA CR-1998-207659, April 1998

- Benefit Estimates of Terminal Area Productivity Program Technologies, Hemm, Shapiro, Lee, Gribko, and Glaser, NASA CR-1999-208989, January 1999.

We first discuss the single runway case and then the case of dependent (staggered) arrivals and departures with parallel runways.

Table A-1 lists the parameters used in the runway capacity model.

Table A-1. Key Airport Modeling Parameters

\begin{tabular}{|l|l|}
\hline \multicolumn{1}{|c|}{ Symbol } & \multicolumn{1}{c|}{ Definition } \\
\hline$c$ & Communications time delay \\
\hline$\delta c, \sigma_{c}$ & Variation in $c$ and standard deviation of $c$ \\
\hline$D$ & Length of common approach path \\
\hline$D_{D}$ & Distance until departures may turn \\
\hline$p_{i}$ & Fraction of operating aircraft that are type $i$ \\
\hline$R_{A i}$ & Arrival runway occupancy time of ith aircraft \\
\hline$\delta R_{A i}, \sigma_{R A l}$ & Variation in and standard deviation of $R_{A i}$ \\
\hline$R_{D i}$ & Departure runway occupancy time of ith aircraft \\
\hline$\delta R_{D i}, \sigma_{R D i}$ & Variation in and standard deviation of $R_{D i}$ \\
\hline$S$ & Miles-in-trail separation minimum \\
\hline$V_{i}$ & Approach speed of aircraft $i$ \\
\hline$\delta V_{i}, \sigma_{V i}$ & $\begin{array}{l}\text { Variation in and standard deviation of approach speed } \\
\text { of aircraft } i\end{array}$ \\
\hline$V_{D i}$ & Departure speed of aircraft $i$ \\
\hline$\delta V_{D i}, \sigma_{V D i}$ & $\begin{array}{l}\text { Variation in and standard deviation of departure } \\
\text { speed of aircraft } i\end{array}$ \\
\hline
\end{tabular}


Table A-1. Key Airport Modeling Parameters

\begin{tabular}{|l|l|}
\hline \multicolumn{1}{|c|}{ Symbol } & \multicolumn{1}{c|}{ Definition } \\
\hline$\delta W_{i}, \sigma_{W i}$ & $\begin{array}{l}\text { Variation in and standard deviation of wind } \\
\text { experienced by aircraft } i\end{array}$ \\
\hline$\delta X_{i}, \sigma_{X i}$ & $\begin{array}{l}\text { Variation in and standard deviation of aircraft } i \\
\text { position }\end{array}$ \\
\hline $1 / \lambda$ & Arrival delivery error or TRACON inefficiency \\
\hline$\mu_{i j}$ & Time increment imposed by controller, $i$ behind $j$ \\
\hline
\end{tabular}

We assume that each of the $\delta c, \delta R_{A_{i}}, \delta R_{D_{i}}, \delta V_{i}, \delta V_{D_{i}}, \delta W_{i}$, and $\delta X_{i}$ variables are independent, normal, and random, with mean of zero and standard deviation of $\sigma_{c}, \sigma_{R A i}, \sigma_{R D i}, \sigma_{V i}, \sigma_{V D i} \sigma_{W i}$, or $\sigma_{X i}$ as appropriate. Both the variations and standard deviations appear in the derivations below, but only the standard deviations are inputs to the model.

\section{SINGLE RUNWAY}

The output of the runway capacity model is an arrival/departure Pareto curve. We calculate all-arrival, all-departure, and equal arrival/departure points, along with a point representing "free" departures available when operating with maximum arrivals. Our analysis takes a controller-based view of operations. That is, we assume that a person controls the aircraft, introducing time (or, equivalently, space) increments in operations streams to meet all applicable rules (e.g., milesin-trail requirements) with specified levels of confidence. For example, consider the arrival-arrival sequence of Figure A-1.

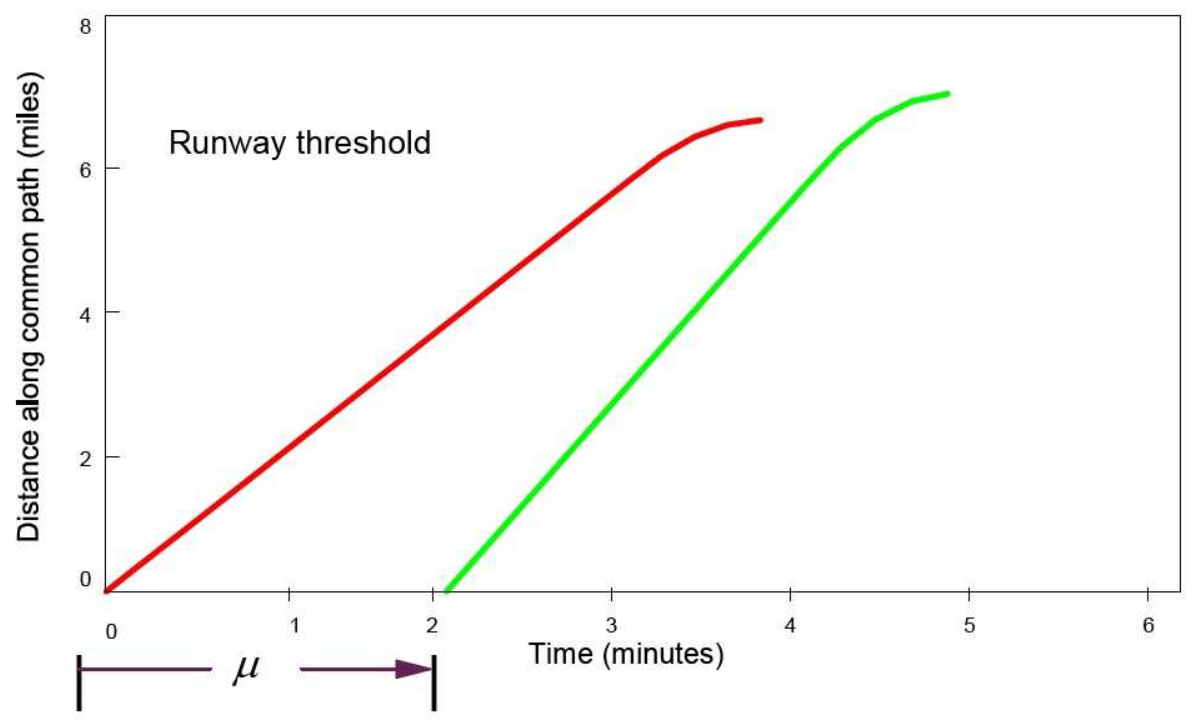

Figure A-1. Time phase for arrivals when follower velocity $>$ leader velocity. 
The figure shows the space-time trajectories of two arrivals. Zero distance is the beginning of the common approach path. In this model, the controller maneuvers the following aircraft so that it enters the common approach path a time $\mu$ after the lead aircraft enters it. (The controller may actually achieve this by bringing the following aircraft onto the common path when the lead aircraft has advanced a specified distance along the path.) The controller chooses the time interval $\mu$ in light of his or her knowledge of typical approach speeds for the two aircraft and of disturbances - winds, position uncertainties, variations in pilot techniqueaffecting their relative positions. The controller does this to ensure that miles-intrail requirements and runway occupancy rules are met, with assigned levels of confidence. This action of the controller, together with information on statistics of aircraft operating parameters and the disturbances to arrival operations (such as winds and position uncertainties), leads directly to statistics of operations and of runway capacity.

This same controller logic, with appropriate input values, applies to a pilot trying to guarantee adequate separation.

\section{Arrivals Only}

We consider first the controller-based paradigm for arrivals only. Two cases are important: follower velocity $\geq$ leader velocity, and follower velocity $<$ leader velocity.

\section{ARRIVAL: FOLLOWER VELOCITY $\geq$ LEADER VELOCITY}

The case, illustrated by Figure A-1, occurs when the mean approach speed of the following aircraft exceeds that of the leader. For this case, the miles-in-trail constraint (distance) applies as the leader crosses the runway threshold. At that time, the leader's position is $D$. We derive a condition on the controller's interval, $\mu$, to guarantee that the miles-in-trail requirement is met (i.e., that at the time the leader crosses the threshold, the follower is at least distance $S$ away from the threshold, with a probability of 95 percent).

The position of the lead aircraft at time $t, X_{L}(t)$, is given by

$$
X_{L}(t)=\delta X_{L}+\left(V_{L}+\delta V_{L}+\delta W_{L}\right) t
$$

and the position of the following aircraft at time $t, X_{F}(t)$, by

$$
X_{F}(t)=\delta X_{F}+\left(V_{F}+\delta V_{F}+\delta W_{F}\right)(t-\mu) \text {. }
$$


The leader crosses the runway threshold at time $t_{L O}$, given by

$$
t_{L O}=\frac{D-\delta X_{L}}{V_{L}+\delta V_{L}+\delta W_{L}} .
$$

At time $t_{L O}$, the follower is at $X_{F}\left(t_{L O}\right)$, given by

$$
X_{F}\left(t_{L O}\right)=\delta X_{F}+\left(V_{F}+\delta V_{F}+\delta W_{F}\right)\left(\frac{D-\delta X_{L}}{V_{L}+\delta V_{L}+\delta W_{L}}-\mu\right)
$$

We wish to derive a condition on $\mu$, to make $D-X_{F}\left(t_{L O}\right) \geq S$ with a probability of at least 95 percent. To keep the problem tractable, we assume that all disturbances are of first order and make Equation A-3 linear. When made linear, Equation A-3 becomes

$$
X_{F}\left(t_{L O}\right)=\delta X_{F}+\frac{D V_{F}}{V_{L}}\left(1+\frac{\delta V_{F}+\delta W_{F}}{V_{F}}-\frac{\delta X_{L}}{D}-\frac{\delta V_{L}+\delta W_{L}}{V_{L}}\right)-\mu V_{F}\left(1+\frac{\delta V_{F}+\delta W_{F}}{V_{F}}\right)
$$

In this linear approximation, $X_{F}\left(t_{L O}\right)$ is a normal random variable of mean

$$
\frac{D V_{F}}{V_{L}}-\mu V_{F}
$$

and variance

$$
\sigma_{1}^{2}=\frac{D^{2} V_{F}^{2}}{V_{L}^{2}}\left(\frac{\sigma_{V F}^{2}+\sigma_{W F}^{2}}{V_{F}^{2}}+\frac{\sigma_{X L}^{2}}{D^{2}}+\frac{\sigma_{V L}^{2}+\sigma_{W L}^{2}}{V_{L}^{2}}\right)+\mu^{2} V_{F}^{2} \frac{\sigma_{V F}^{2}+\sigma_{W F}^{2}}{V_{F}^{2}}+\sigma_{X F}^{2} \text {. [Eq. A-5] }
$$

The condition that $D-X_{F}\left(t_{L O}\right) \geq S$, with probability at least 95 percent, may then be stated as

$$
\frac{D V_{F}}{V_{L}}-\mu V_{F}+1.65 \sigma_{1} \leq D-S
$$

or

$$
\mu \geq \frac{D}{V_{L}}-\frac{D-S}{V_{F}}+\frac{1.65 \sigma_{1}}{V_{F}} .
$$


Equation A-7 gives, in essence, the desired condition. As that equation stands, $\mu$ appears on both sides of the inequality. Straightforward manipulations lead to an explicit condition on $\mu$, which may be written

$$
\mu=\frac{A+\sqrt{A^{2} B^{2}+C^{2}\left(1-B^{2}\right)}}{1-B^{2}},
$$

where

$$
\begin{gathered}
A \equiv \frac{D}{V_{L}}-\frac{D-S}{V_{F}}, \\
B^{2} \equiv 1.65^{2}\left\{\frac{\sigma_{V F}^{2}+\sigma_{W F}^{2}}{V_{F}^{2}}\right\},
\end{gathered}
$$

and

$$
C^{2} \equiv \frac{1.65^{2}}{V_{F}^{2}}\left\{\frac{D^{2} V_{F}^{2}}{V_{L}^{2}}\left(\frac{\sigma_{V F}^{2}+\sigma_{W F}^{2}}{V_{F}^{2}}+\frac{\sigma_{X L}^{2}}{D^{2}}+\frac{\sigma_{V L}^{2}+\sigma_{W L}^{2}}{V_{L}^{2}}\right)+\sigma_{X F}^{2}\right\} .
$$

To determine numerical values of the smallest $\mu$ that meet Equation A-7, the iterative scheme

$$
\mu_{n+1}=\frac{D}{V_{L}}-\frac{D-S}{V_{F}}+\frac{1.65 \sigma_{1}\left(\mu_{n}\right)}{V_{F}},
$$

where $\sigma_{1}(\mu)$ is defined by Equation A-5, is convenient.

Now, let us develop a condition on $\mu$ that will guarantee that the follower aircraft does not cross the runway threshold until the leader has left the runway, with a probability of 98.7 percent. The leader will exit the runway at time

$$
t_{L X}=t_{L O}+R_{A L}
$$

and the follower will cross the threshold at time $t_{F O}$, given by

$$
t_{F O}=\frac{D-\delta X_{F}}{V_{F}+\delta V_{F}+\delta W_{F}}+\mu
$$


Linearizing as above, we find that in the linear approximation $t_{F O}-t_{L X}$ is a normal random variable with mean

$$
\frac{D}{V_{F}}+\mu-\frac{D}{V_{L}}-R_{A L}
$$

and variance

$$
\sigma_{2}^{2}=\frac{D^{2}}{V_{F}^{2}}\left(\frac{\sigma_{X F}^{2}}{D^{2}}+\frac{\sigma_{V F}^{2}+\sigma_{W F}^{2}}{V_{F}^{2}}\right)+\frac{D^{2}}{V_{L}^{2}}\left(\frac{\sigma_{X L}^{2}}{D^{2}}+\frac{\sigma_{V L}^{2}+\sigma_{W L}^{2}}{V_{L}^{2}}\right)+\sigma_{R A L}^{2} \text {. [Eq. A-13] }
$$

It follows that the condition on $\mu$ for the follower not cross the threshold until the leader has exited the runway; that is, $t_{F O}-t_{L X}>0$ with a probability of 98.7 percent, is

$$
\mu \geq \frac{D}{V_{L}}-\frac{D}{V_{F}}+R_{A L}+2.215 \sigma_{2}
$$

The controller will, in effect, impose that value of time interval $\mu$ that is the smallest $\mu$ satisfying both Equations $\mathrm{A}-7$ and $\mathrm{A}-14$.

Given $\mu$, the time between threshold crossings of successive arrivals is, in our approximation, a normal random variable of mean

$$
\frac{D}{V_{F}}-\frac{D}{V_{L}}+\mu
$$

and variance

$$
\sigma_{3}^{2}=\frac{D^{2}}{V_{F}^{2}}\left(\frac{\sigma_{X F}^{2}}{D^{2}}+\frac{\sigma_{V F}^{2}+\sigma_{W F}^{2}}{V_{F}^{2}}\right)+\frac{D^{2}}{V_{L}^{2}}\left(\frac{\sigma_{X L}^{2}}{D^{2}}+\frac{\sigma_{V L}^{2}+\sigma_{W L}^{2}}{V_{L}^{2}}\right) .
$$

\section{ARRIVAL: FOLLOWER VELOCITY < LEADER VELOCITY}

When the follower's approach speed is slower than the leader's, in the controllerbased view, the controller will bring the follower onto the common path after the leader has advanced a distance $S$ along it, as illustrated in Figure A-2. 


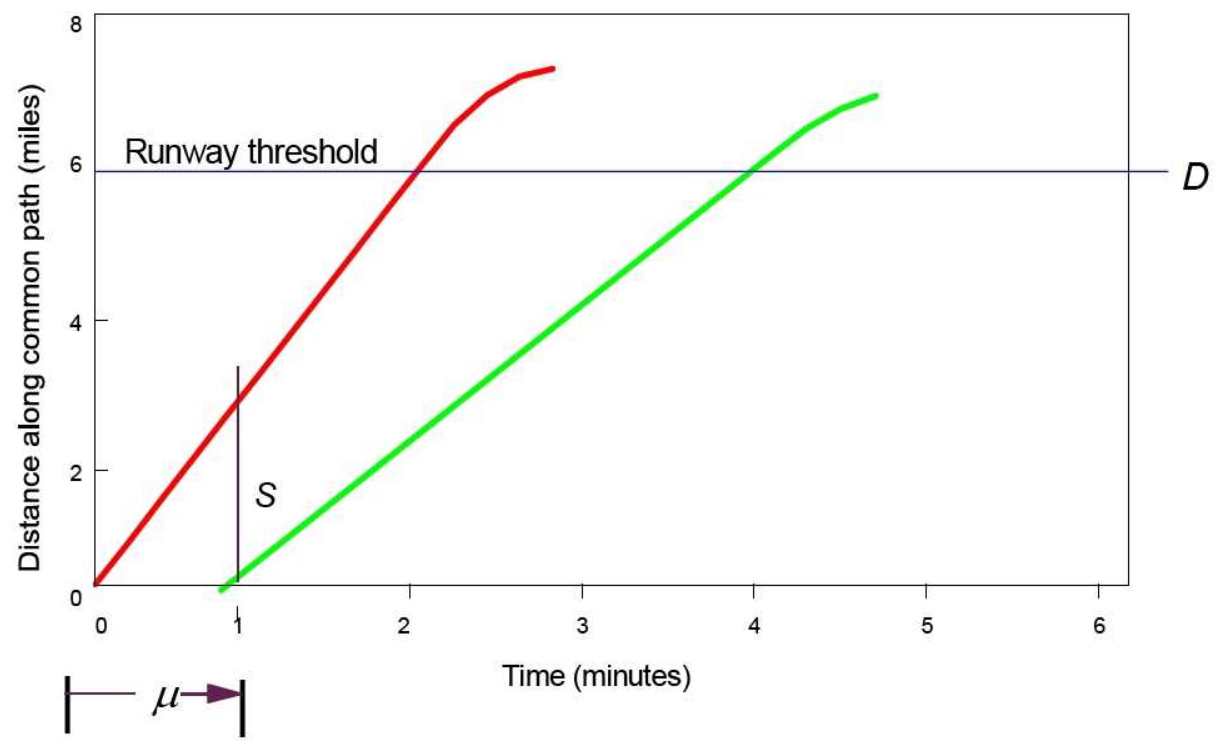

Figure A-2. Time phase of arrivals when follower velocity $\leq$ leader velocity.

In this case, the positions of the two aircraft as functions of time are again given by Equations A-1 and A-2. The miles-in-trail requirement is now that $X_{L}(\mu)-X_{F}(\mu) \geq S$, with a probability of at least 95 percent. Because

$$
X_{L}(\mu)-X_{F}(\mu)=\delta X_{L}+\left(V_{L}+\delta V_{L}+\delta W_{L}\right) \mu-\delta X_{F}
$$

is a normal random variable of mean $V_{L} \mu$ and variance

$$
\sigma_{4}^{2}=\mu^{2}\left(\sigma_{V L}^{2}+\sigma_{W L}^{2}\right)+\sigma_{X F}^{2}+\sigma_{X L}^{2} \text {, }
$$

it follows that the condition that the miles-in-trail requirement is met with a 95 percent confidence is

$$
\mu \geq \frac{S}{V_{L}}+1.65 \frac{\sigma_{4}}{V_{L}}
$$


Equation A-19 may be written as a single condition on $\mu$ using Equation A-8 by replacing Equations A-9, A-10, and A-11 with the following new definitions:

$$
\begin{gathered}
A \equiv \frac{S}{V_{L}}, \\
B^{2} \equiv 1.65^{2} \frac{\sigma_{V L}^{2}+\sigma_{W L}^{2}}{V_{L}^{2}},
\end{gathered}
$$

and

$$
C^{2} \equiv 1.65^{2} \frac{\sigma_{X L}^{2}+\sigma_{X F}^{2}}{V_{L}^{2}} .
$$

The condition that the single-occupant rule is met with 98.7 percent confidence is derived exactly as is that condition for $V_{F} \geq V_{L}$ (the condition expressed in Equation A-14). Also, in the present case, Equations A-15 and A-16 yield the mean and standard deviation of interarrival times, given $\mu$.

\section{ARRIVAL-DEPARTURE-ARRIVAL-DEPARTURE SEQUENCES}

We can readily translate the results of the previous subsection to results for operations when there is a departure between successive arrivals by replacing $R_{A_{L}}$ with $R_{A_{L}}+R_{D F}$, where $R_{D F}$ is the departure runway occupancy time of the intervening departure aircraft. This case is illustrated by Figure A-3.

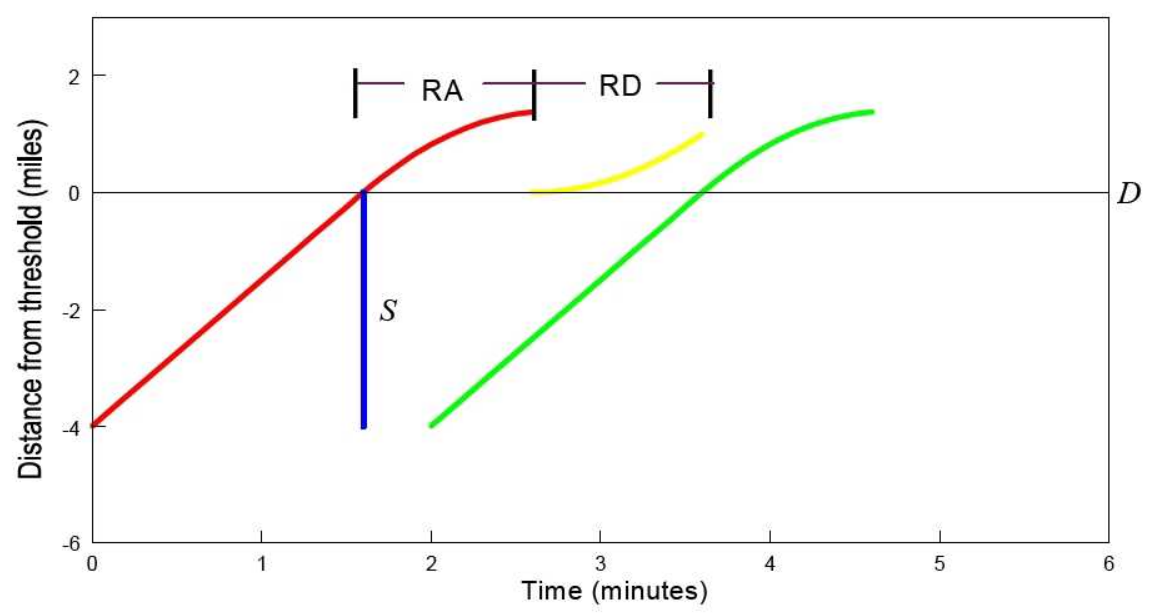

Figure A-3. Time phase of arrivals with intervening departure.

It may be desirable to consider the effect of a communications lag $c$ on the departure. If so, then $R_{A_{L}}$ is replaced by $R_{A_{L}}+c+R_{D F}$. 


\section{StATISTICS OF MULTIPLE OpERATIONS}

At this point, we have expressions for the means and variances of normal random variables representing interarrival times for a variety of cases. Now we will use these to generate statistics of multiple arrivals, or multiple arrivals and departures, that we can then use to produce capacity curves for single runways and combinations of runways.

First, we consider the statistics of sequences of arrivals only. Statistics of the overall interarrival time are determined by the mix of aircraft using the runway, with their individual values for the aircraft parameters of Table A-1. The fraction of the aircraft of type $i$ in the mix is $p_{i}$. The previous results give the interarrival time for each pair as a normal random variable. Let the mean and standard deviation for aircraft type $i$ following aircraft type $j$ be $\mu_{i j}$ and $\sigma_{i j}$, respectively. Then the distribution function for overall interarrival time is

$$
p_{A A}(t)=\sum_{i} \sum_{j} p_{i} p_{j} N\left(t ; \mu_{i j}, \sigma_{i j}\right),
$$

where $N(t ; \mu, \sigma)$ denotes the normal probability distribution function. Obviously, the distribution of interarrival times is not normal. Figure A-4 is an example of an interarrival time distribution of the Equation A-20 type. As the figure shows, the interarrival time distribution is not necessarily monomodal.

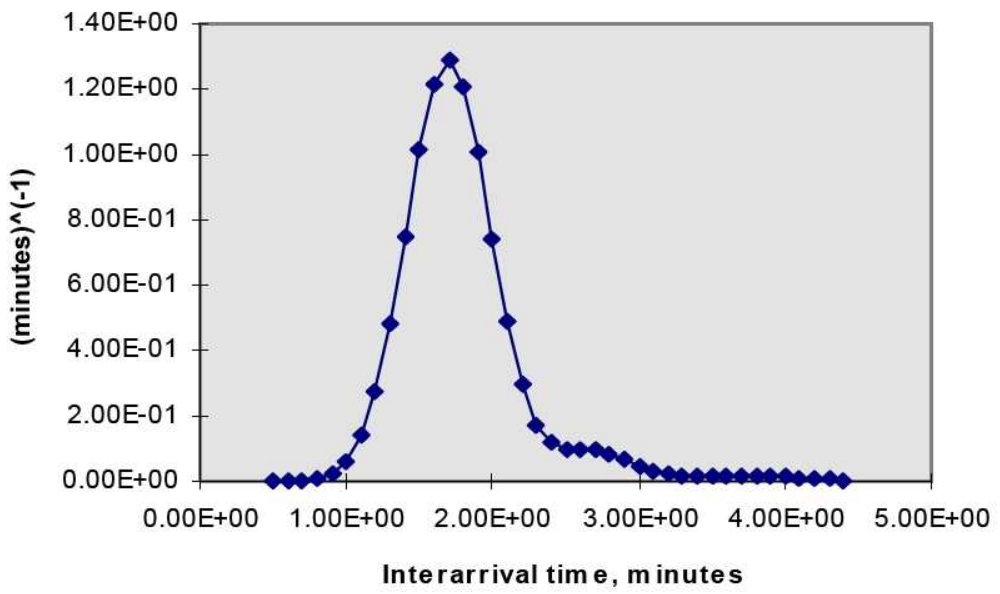

Figure A-4. Interarrival time (minutes). 
The mean and variance of the interarrival time distribution, Equation A-20, can be computed straightforwardly. The results are

$$
<t_{A A}>=\sum_{i} \sum_{j} p_{i} p_{j} \mu_{i j}
$$

and

$$
\operatorname{var}\left(t_{A A}\right)=\sum_{i} \sum_{j} p_{i} p_{j}\left(\sigma_{i j}^{2}+\mu_{i j}^{2}\right)-<t_{A A}>^{2}
$$

In principle, the analyst can compute exactly the distributions of total arrival times for all arrival sequences of arbitrary length and find exact values for the number of arrivals that can, with assigned confidence, be accommodated in 1 hour. These calculations involve sums of many terms, however, and this motivates a search for useful approximations.

Sums of normal random variables are normally distributed, and it is tempting to approximate the distribution of sequences of many arrivals in such a way. An allarrival sequence with $J_{i j}$ cases of aircraft of type $i$ following aircraft of type $j$ has a normal distribution whose parameters are easily computed. If the $J_{i j}$ were chosen so that $J_{i j}=p_{i} p_{j} M$, where $M$ is the sum of the $J_{i j}$, the resulting normal distribution would be a good approximation for the distribution of long arrival sequences. Unfortunately, for the aircraft mixes at some airports, some of the $p_{i}$ values are only a few hundredths, so $M$ would have to be several thousand for this approximation to be accurate.

Nevertheless, because much of our work to this point has been approximate, considering this "very large sequence"-limiting case does not seem unreasonable. In this approximation, then, the time $t_{M}$ of $M$ interarrival times has a normal distribution of mean $M<t_{A A}>$ and variance

$$
\operatorname{var}\left(t_{M}\right)=M \sum_{i} \sum_{j} p_{i} p_{j} \sigma_{i j}^{2}
$$

This result suggests approximating the distribution of interarrival time with a normal distribution of mean $\left\langle t_{A A}>\right.$ and variance $v_{l}$ given by

$$
v_{1}=\sum_{i} \sum_{j} p_{i} p_{j} \sigma_{i j}^{2}
$$


The approximation of Equation A-24 can be used to compute the number of arrivals that can be accommodated in 1 hour with 95 percent confidence. That number $M^{*}$ is determined by the condition

$$
\left(M^{*}-1\right)<t_{A A}>+1.65 \sqrt{\left(M^{*}-1\right) v_{1}} \leq 60
$$

(only $M^{*}-1$ interarrival times are required for $M^{*}$ arrivals), ${ }^{1}$ which leads to the all-arrival capacity of a single runway as $M^{*}=w^{2}+1$, where $w$ is given by

$$
w=-\frac{1.65 \sqrt{v_{1}}}{2<t_{A A}>}+\sqrt{\left(\frac{1.65 \sqrt{v_{1}}}{2<t_{A A}>}\right)^{2}+\frac{60}{<t_{A A}>}} .
$$

To compute the expected number of arrivals we use

$$
<M>=\frac{60}{<t_{A A}>} \text {. }
$$

\section{INPUT-STREAM EFFECTS}

So far, we have developed our model as though the controller could always impose the desired time separation $\mu$, whatever the nature of the stream of arriving aircraft reaching him or her. Because of maneuvering or feeder errors, this may not in fact always be the case. We extend our model to address inputstream effects in this way: we suppose that the controller, wishing to impose separation $\mu$, actually can impose the separation $\mu+v$, where $v$ is a random variable, independent of all others in the analysis, characterizing input-stream effects. We take $v$ to have the exponential distribution with parameter $\lambda$, that is,

$$
v \sim\left\{\begin{array}{c}
\lambda \mathrm{e}^{-\lambda v}, v \geq 0 \\
0, \text { else }
\end{array}\right.
$$

We chose the exponential distribution because it assigns zero probability to negative values, and because its shape resembles patterns of observed data. The mean and standard deviation of $v$ are both equal to $1 / \lambda$.

With the addition of the random variable $v$, the interarrival time for specified leader and follower is the sum of a normal random variable and an exponential random variable. The normal random variable has, in every case, precisely the same mean and variance as in the cases where input stream effects are not considered. It follows straightforwardly that in the present, augmented cases, the

\footnotetext{
${ }^{1}$ This derivation is valid for a single hour, considered in isolation. To compute the long-run average, replace $M^{*}-1$ by $M^{*}$.
} 
mean, variance, and standard deviation of interarrival times for leader $j$ and follower $i$ are

$$
\begin{gathered}
\text { mean }=\mu_{\mathrm{ij}}+\frac{1}{\lambda} \\
\text { variance }=\sigma_{\mathrm{ij}}^{2}+\frac{1}{\lambda^{2}} \\
\text { standard deviation }=\sqrt{\sigma_{i j}^{2}+\frac{1}{\lambda^{2}}} .
\end{gathered}
$$

The distribution function of interarrival time for fixed leader and follower is no longer normal, but, rather, it is the convolution of a normal random variable and an exponential random variable. Specifically, the distribution is

$$
H(t ; \mu, \sigma, \lambda) \equiv \frac{\lambda}{\sqrt{2 \pi} \sigma} \int_{0}^{\infty} e^{-\frac{(t-\tau-\mu)^{2}}{2 \sigma^{2}}-\lambda \tau} d \tau \text {. }
$$

This distribution function may be evaluated conveniently using the expression

$$
H(t ; \mu, \sigma, \lambda)=\lambda e^{-\lambda(t-\mu)+\frac{\lambda^{2} \sigma^{2}}{2}}\left[1-C\left(\mu, t-\lambda \sigma^{2}, \sigma\right)\right]
$$

where $C(x, \mu, \sigma)$ denotes the cumulative normal distribution for mean $\mu$ and standard deviation $\sigma$, evaluated at $x$.

Figure A-5 illustrates this class of distribution, together with the normal distribution that would have been seen absent input-stream effects. For the sake of illustration, the example is somewhat extreme. Typically, input-stream effects would introduce a mean error of 10 seconds or less. 


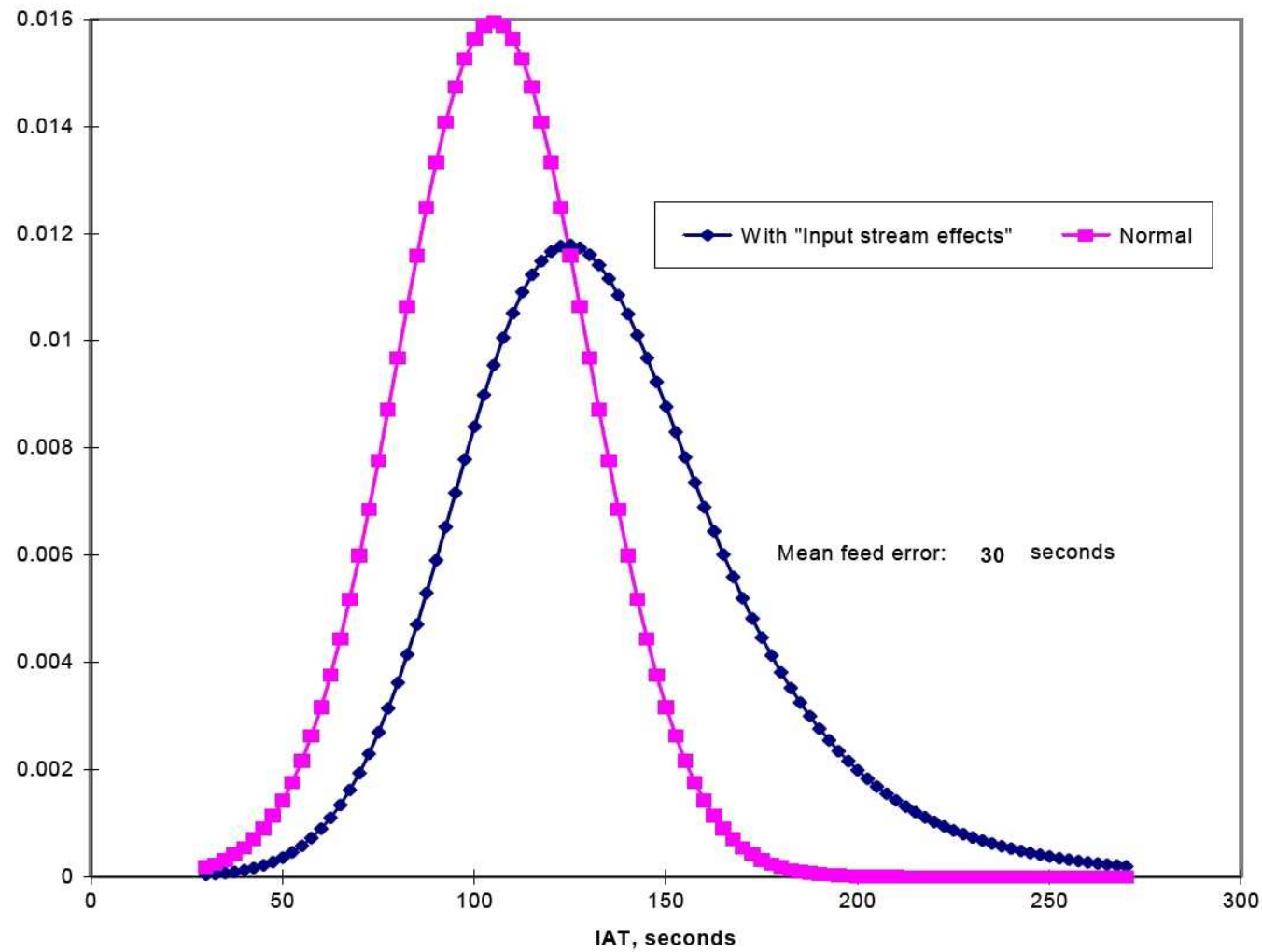

Figure A-5. Example interarrival distribution with input-stream effects.

With the addition to our model of input-stream effects, the distribution of interarrival times changes from that of Equation A-30 to

$$
t_{A A}(1) \sim \sum_{i} \sum_{j} p_{i} p_{j} H\left(t ; \mu_{i j}, \sigma_{i j}, \lambda\right),
$$

and the distribution function of $t_{A A}(M)$ changes from that of Equation A-35 to

$\sum \sum \ldots \sum p_{i} p_{j} \ldots p_{y} p_{z} \hat{H}\left(t ; \mu_{i j}+\mu_{j k}+\ldots+\mu_{y z}, \sqrt{\sigma_{i j}^{2}+\sigma_{j k}^{2}+\ldots+\sigma_{y z}^{2}}, \lambda, M\right)[\mathrm{Eq}$

where

$$
\hat{H}(t ; \mu, \sigma, \lambda, K) \equiv \frac{\lambda^{K}}{\sqrt{2 \pi} \sigma(K-1) !} \int_{0}^{\infty} \tau^{K-1} e^{-\frac{(t-\tau-\mu)^{2}}{2 \sigma^{2}}-\lambda \tau} d \tau .
$$

It is not difficult to show that the mean and variance of $t_{A A}(M)$ may be obtained from the values in Equations A-37 and A-38 simply by adding $M / \lambda$ to $\left\langle t_{A A}(M)>\right.$ and $M /\left(\lambda^{2}\right)$ to $\operatorname{var}\left(t_{A A}(M)\right)$. With these results, and the assumption that the distribution of $t_{A A}(M)$ may be adequately approximated by a normal distribution 
for sufficiently large $M$, we may compute runway capacities with our augmented model of input-stream effects.

For example, taking the value $1 / \lambda=6.3$ seconds, which certain data for operations at DFW suggest, reduces the 95 percent confidence capacity to 28 arrivals/hour, and the "expected-total-arrival-time" capacity to 30 .

\section{FREE DEPARTURES AND EQUALS}

At this point, we have one point on the single runway capacity curve, the one corresponding to all arrivals and no departures. We can generate others.

The distribution function suggests that there is a significant probability of interarrival times being large enough to accommodate a departure. We can compute the number of free departures in a stream of $\langle M\rangle$ arrivals. These are departures that can be accommodated without introducing additional separations into the arrival stream, in this way: the distribution of interarrival time is given by Equation A-20. We assume that departure runway occupancy time, arrival runway occupancy time, and communications delay are normal random variables of means $\left\langle R_{D}>,\left\langle R_{A}\right\rangle,\left\langle c>\right.\right.$ and standard deviations $\sigma_{R D}, \sigma_{R A}$, and $\sigma_{c}$. The difference $t-R_{D}-R_{A}-c$, where $t$ is the interarrival time, is the excess time when a departure is released between two arrivals. (The lead arrival exits the runway in time $R_{A}$, and the departure exits the runway in time $R_{D}+c$.) The distribution of the excess time is

$$
\begin{aligned}
& p\left(t-R_{A}-R_{D}-c\right)=N\left(-<R_{A}>-<R_{D}>-<c>, \sqrt{{\sigma_{R D}}^{2}+\sigma_{R A}{ }^{2}+\sigma_{c}^{2}}\right) \otimes \sum_{i} \sum_{j} p_{i} p_{j} N\left(\mu_{i j}, \sigma_{i j}\right) \\
& =\sum_{i} \sum_{j} p_{i} p_{j} N\left(\mu_{i j}-\left\langle R_{D}>-<R_{A}>-<c>, \sqrt{\sigma_{R D}^{2}+\sigma_{i j}^{2}+\sigma_{R A}^{2}+\sigma_{c}^{2}}\right),\right.
\end{aligned}
$$

where the symbol $\otimes$ denotes convolution. ${ }^{2}$

\footnotetext{
${ }^{2}$ To account for variations in departure runway occupancy time (ROT), the analyst may replace the single normal distribution of ROT with the distribution of departure ROT that would be found with $K$ classes of departing aircraft, each with its own normal distribution of departure ROT. That is, $\sum_{1}^{K} q_{i} N\left(-<R_{D}>_{i}, \sigma_{D i}\right)$, where $q_{i}$ denotes the fraction of departing aircraft that are of type $i$.
} 
The probability that $t-R_{D}-R_{A}-c$ is positive, i.e., the probability that a free departure is possible between any random pair of arrivals, is given by

$$
\begin{gathered}
p_{+}=1-\sum_{i} \sum_{j} p_{i} p_{j} C\left(0, \mu_{i j}-<R_{D}>-<R_{A}>-<c>,\right. \\
\left.\sqrt{\sigma_{R D}^{2}+\sigma_{R A}^{2}+\sigma_{c}^{2}+\sigma_{i j}^{2}}\right)
\end{gathered}
$$

where $C(t, \mu, \sigma)$ is the normal cumulative probability function. This value is readily computed. Then the analyst may determine the number $\langle N\rangle$ of positive values $t-R_{D}-R_{A}-c$ to be expected, in $\langle M\rangle$ draws, from the binomial distribution for probability $p_{+}$.

Under IMC2 or IMC3 weather conditions, current FAA procedures require that departures be held if an arriving aircraft is within a certain distance of the runway threshold. (This distance is now 2 miles.) ${ }^{3}$ In our model, this has the effect of reducing the time available for free departing aircraft. Since the trailing arrival travels a distance less than the full length of the common path, the uncertainties embodied in Equations A-34 and A-35 are also reduced.

The appropriate modifications to Equations A-34 and A-35 are to reduce the mean times available for free departures by

$$
\frac{D_{T}}{V_{F}},
$$

where $D_{T}$ is the distance from threshold after which departures must be held. The variances in Equations A-34 and A-35 are reduced by

$$
\frac{D_{T}\left(2 \times D-D_{T}\right)}{V_{F}^{2}}\left(\frac{\sigma_{V F}^{2}+\sigma_{W F}^{2}}{V_{F}^{2}}\right) \text {. }
$$

A third point on the capacity curve, the point of equal numbers of arrivals and departures, may be computed by considering sequences of repeated arrivaldeparture pairs, as described in the section on arrival-departure-arrival-departure sequences.

Although the expressions for interarrival times and runway capacities developed previously in this appendix are somewhat lengthy, they are readily evaluated numerically.

\footnotetext{
${ }^{3}$ See Federal Aviation Administration, Order JO 7110.65S, Air Traffic Control, Section 5-8-4, Note 1. This distance may be reduced by NextGen technologies.
} 


\section{Departures}

Considerations similar to those for all arrival sequences must be taken into account to develop statistics of departures. Figure A-6 shows the basic departure situation.

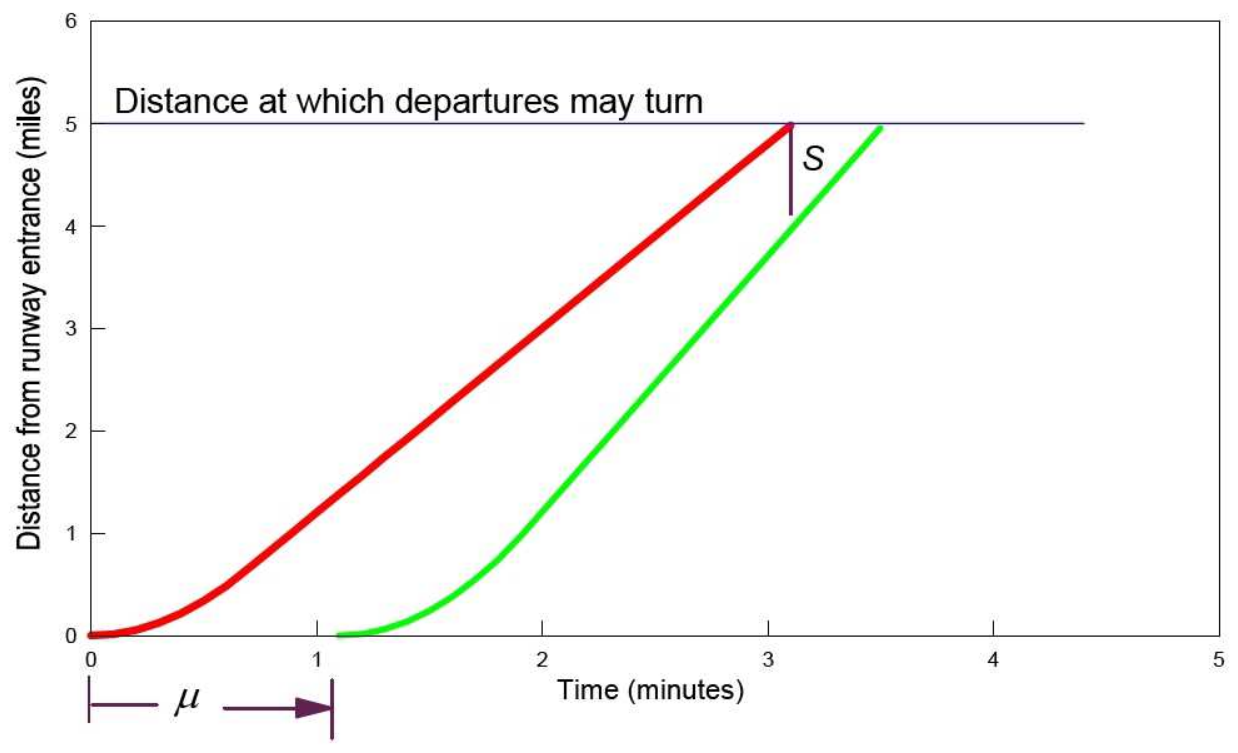

Figure A-6. Time phase of departures.

We model the trajectory of a departing aircraft by specifying its position, $x(t)$, in terms of the parameters $V_{D}$ and $R_{D}$, in this way:

$$
x(t)=\left\{\begin{array}{c}
\frac{1}{2} \frac{V_{D}}{R_{D}} t^{2}, 0<t \leq R_{D} \\
V_{D} t-\frac{1}{2} V_{D} R_{D}, t \geq R_{D}
\end{array} .\right.
$$

This model approximates an actual takeoff roll and climb out by a trajectory with constant acceleration from rest to departure speed $V_{D}$, occurring in time $R_{D}$, followed by continuing departure at constant speed $V_{D}$.

We model controllers' actions on departures by the interdeparture time interval $\mu$, which is the time interval between the start of the lead aircraft's takeoff roll to when a departure clearance is issued to the following aircraft. (The following aircraft begins takeoff role at time $\mu+c$, where $c$ models the delay to move into position.) We assume that, in effect, controllers adjust $\mu$ to give specified confidence that miles-in-trail requirements, and other separation requirements, are met. 
Here again, the required control input varies, depending on whether the following aircraft is faster or slower than the lead aircraft. In the case of a faster follower, the constraining condition is that the separation requirement be met as the lead aircraft exits the system. At that time, the displacement of the lead aircraft is $D_{D}$, the distance to turn on departure. The displacement of the following aircraft must not be greater than $D_{D}-S_{D}$, where $S_{D}$ is the minimum separation. After lengthy but straightforward steps, the controller finds that meeting this condition with 95 percent confidence imposes the condition

$$
\mu \geq \frac{D_{D}}{V_{D L}}+\frac{1}{2}\left(R_{D L}-R_{D F}\right)-\frac{D_{D}-S_{D}}{V_{D F}}-c+\frac{1.65}{V_{D F}} \sqrt{\operatorname{var}}
$$

on $\mu$. The quantity var in the inequality just above is

$$
\begin{aligned}
& \operatorname{var}=\left(\frac{\mathrm{D}_{\mathrm{D}}}{\mathrm{V}_{\mathrm{DL}}}+\frac{1}{2}\left(R_{D L}-R_{D F}\right)-\mu-c\right)^{2}\left(\sigma_{V D F}^{2}+\sigma_{W_{F}}^{2}\right)+\frac{V_{D F}^{2}}{V_{D L}^{2}} D_{D}^{2} \frac{\sigma_{V D L}{ }^{2}+\sigma_{W L}^{2}}{V_{D L}^{2}}+\frac{V_{D F}^{2}}{4} \sigma_{R D L}^{2} \\
& +\frac{1}{4} V_{D F}^{2} \sigma_{R D F}^{2}+V_{D F}^{2} \sigma_{C}^{2} .
\end{aligned}
$$

The inequality may be reduced to an equivalent, explicit condition on $\mu$. For numerical work, we find that iterative methods give the required values of $\mu$ conveniently.

When the follower departs more slowly than the leader, the separation minimums apply as the follower lifts off, unless $D_{D}$ is sufficiently short that the leader can exit the system before the follower completes the takeoff roll. Applying the separation minimum as the follower lifts off leads to the condition (inequality $A$ )

$$
\mu \geq \frac{R_{D L}}{2}+\left(\frac{1}{2} \frac{V_{D F}}{V_{D L}}-1\right) R_{D F}-c+\frac{S_{D}}{V_{D L}}+1.65 \sqrt{\operatorname{var} 1}
$$

where

$$
\begin{aligned}
\operatorname{var} 1=(\mu+ & \left.c+R_{D F}-\frac{1}{2} R_{D L}\right)^{2} \frac{\sigma_{V D L}^{2}+\sigma_{W L}^{2}}{V_{D L}^{2}}+\left(1-\frac{V_{D F}}{2 V_{D L}}\right)^{2} \sigma_{R D F}^{2} \\
& +\frac{1}{4}\left(\sigma_{R D L}^{2}+R_{D F}^{2} \frac{\sigma_{V D F}^{2}+\sigma_{W F}^{2}}{V_{D L}^{2}}\right)+\sigma_{C}^{2} .
\end{aligned}
$$


Alternatively, the controller might impose a value of $\mu$ that caused the follower to lift off just as the leader exited the system. That would lead to inequality $B$ :

$$
\mu \geq \frac{D_{D}}{V_{D L}}+\frac{1}{2} R_{D L}-R_{D F}-c+1.65 \sqrt{\sigma_{R D F}^{2}+\frac{D_{D}^{2}}{V_{D L}^{2}} \frac{\sigma_{V D L}^{2}+\sigma_{W L}^{2}}{V_{D L}^{2}}+\frac{1}{4} \sigma_{R D L}^{2}+\sigma_{C}^{2}} .
$$

Controllers would impose the less restrictive of inequality $A$ or inequality $B$.

Finally, the single-occupant rule must be respected, which leads to inequality $C$ :

$$
\mu \geq R_{D L}-c+2.215 \sqrt{\sigma_{R D L}^{2}+\sigma_{C}^{2}}
$$

For our model, when the follower is slower than the leader, we choose

$$
\mu=\max \left[\min \left(\mu_{A}, \mu_{B}\right), \mu_{C}\right],
$$

where $\mu_{i}$ is the lower bound on $\mu$ resulting from inequality $i$.

\section{PARALLEL RUNWAYS}

The limitations on operations to parallel runways are spelled out in FAA JO 7110.65, The Air Traffic Controller's Handbook. In visual meteorological conditions, when pilots accept visual approaches, simultaneous parallel arrivals can be conducted with runway centerline separations $\geq 700$ feet. For departures, and for approaches under radar control, limitations on simultaneous operations are imposed as runway centerline separations decrease below 4,300 feet. In one case, departures must hold until the arrival on the parallel runway has touched down, but not until it has exited the runway. We call this case closely spaced parallel (CSP) and model it simply by setting the arrival runway occupancy time to zero. In other cases, either or both arrivals and departures must maintain specified diagonal separation from the aircraft on the parallel runway. Modeling these cases requires the algorithms discussed next.

\section{Dependent (Staggered) Parallel Runway Departure and Arrival Models}

We here describe the LMI capacity model algorithm used to estimate the capacity of a parallel runway pair when there are spacing requirements between both aircraft using the same runway and between aircraft using one runway and aircraft using a parallel runway. This can occur when both runways are used for departures or when both runways are used for arrivals.

Unlike separation requirements for single runways, separation requirements in this situation between aircraft approaching the same runway cannot be derived by 
examining aircraft class pairs in isolation; the interdependence of traffic on the two runways requires, in general, knowledge of the entire sequence of operations to determine the separation required between any two aircraft approaching the same runway.

Since exact separations cannot be determined, except for a specific sequence of operations, the algorithm constructs upper and lower bounds on the separation time required between successive operations on one runway of the pair. The bounds are computed for each combination of following aircraft class and leader aircraft class (as in the single runway model). The bounds take into account the interaction with traffic on the other runway.

A user-controllable parameter determines how many historical operations are considered, and thus how much refinement is put into determining the separation bounds, so that capacity can be estimated to any desired degree of precision (at the expense of additional computation time). The capacity bounds of the runway are computed on the basis of the weighted average time between operations; the weighting factors account for the traffic mix on the targeted runway. Since we assume that operations alternate between runways, the capacities of both the targeted runway and the other runway will be the same. We can exploit this symmetry by computing the capacity bounds twice, one using each runway as the target. The computed bounds will generally differ, leading us to identify a best lower bound and a best upper bound on estimated capacity.

Here we discuss the capacity-bounding algorithm from the perspective of departures. The staggered-operations capacity algorithm for arrivals is completely analogous.

\section{Departure Capacity of A PARALlel RunWay Pair}

In modeling the interdeparture times on the target runway, we assume that a departure has just occurred on the other runway. To capture the separation times required between two aircraft on the target runway (aircraft of type $i$, following an aircraft of type $j$, which is next to depart), we need to consider also the aircraft of type $l$, which has just departed on the other runway, and the aircraft of type $k$, which is due to depart the other runway after the aircraft of type $j$ departs the runway under consideration. The departure sequence is $l, j, k, i$. For conciseness, we will refer to an aircraft of type $x$ as simply aircraft $x$.

We define $\mu(i, j, k, l)$ to be the average time separation (in minutes) that the controller will apply to aircraft $i$ following aircraft $j$ on the same runway, when aircraft $l$ has just departed the other runway and aircraft $k$ is next to depart the other runway. We compute both upper and lower bounds on this separation. 
The separation (in minutes) between $i$ and $j$ that we use to compute the runway's capacity is the weighted average

$$
\mu_{P}(i, j)=\sum_{k, l} \mu(i, j, k, l) p_{x k} p_{x l}
$$

where $p_{x k}\left(p_{x l}\right)$ is the probability of aircraft $k(l)$ on the other runway. Upper (lower) bounds on $\mu_{p}(i, j)$ are computed using the upper (lower) bounds on $\mu(i, j, k, l)$.

The hourly runway capacities are estimated by

$$
\text { capacity }=\frac{60}{\sum_{i, j} \mu_{P}(i, j) p_{i} p_{j}},
$$

where $p_{i}$ and $p_{j}$ are the probability of $i$ and $j$ on the targeted runway. Lower (upper) bounds on capacity are derived from the upper (lower) bounds on separation.

To develop the definition of $\mu(i, j, k, l)$, let us define two other separations. $\mu_{S}(i, j)$ is the single runway separation required for aircraft $i$ following aircraft $j$. These are the same separations used in the single runway model; $\mu_{X}(i, k)$ is the separation required between aircraft $i$ following a departure of aircraft $k$ on the other runway. As in the single runway model, these separations are determined from the controller's point of view, including time to account for uncertainties in wind, speed, and position. Let us define $t_{i}$ as the time of departure of aircraft $i$.

Given that aircraft $i$ departs after $j$ on the target runway and $k$ on the other runway, then by definition,

$$
t_{i}=\max \left[t_{j}+\mu_{S}(i, j), t_{k}+\mu_{X}(i, k)\right]
$$

In general, the relative values of $t_{j}$ and $t_{k}$ (and hence $t_{i}$ ) depend on the unspecified history before flight $l$ 's departure; however, under certain conditions, the separation $t_{i}-t_{j}$-i.e., $\mu(i, j, k, l)$-can be computed without knowledge of the prior history:

- Markov property. For any sequence of departures $l, j, k$ such that

$$
\mu_{S}(k, l) \leq \mu_{X}(k, j)+\mu_{X}(j, l),
$$

all prior history is irrelevant in determining

$$
t_{k}=\mu_{X}(k, j)+t_{j}
$$


and

$$
t_{i}-t_{j}=\max \left[\mu_{S}(i, j), \mu_{X}(i, k)+\mu_{X}(k, j)\right] .
$$

- Proof. By definition, $t_{j} \geq t_{l}+\mu_{X}(j, l)$, thus

$$
t_{j}+\mu_{X}(k, j) \geq t_{l}+\mu_{X}(j, l)+\mu_{X}(k, j) .
$$

By hypothesis the right-hand side is greater than $\mu_{S}(k, l)+t_{l}$, leading to

$$
t_{j}+\mu_{X}(k, j) \geq \mu_{S}(k, l)+t_{l} .
$$

The two terms above are those whose maximum defines $t_{k}$, thus the value of $t_{k}$ is known in terms of $t_{j}$. Substituting $t_{j}+\mu_{X}(k, j)$ for $t_{k}$ in the maximum formula for $t_{i}$, and subtracting $t_{j}$ from all terms leads to the final result, QED.

Another useful relationship is the following:

- Parallelogram property. For any departure sequence $l, j, k$ for which the Markov property does not hold, if

$$
\mu_{S}(i, j)+\mu_{X}(j, l) \geq \mu_{S}(k, l)+\mu_{X}(i, k)
$$

then

$$
t_{i}-t_{j}=\mu_{S}(i, j) \text {. }
$$

- Proof. From the defining maximum formula, we note that

$$
t_{k}-t_{j}=\max \left[\mu_{S}(k, l)-\left(t_{j}-t_{l}\right), \mu_{X}(k, j)\right] .
$$

Since $t_{j}-t_{l} \geq \mu_{X}(j, l)$, we have

$$
t_{k}-t_{j} \leq \max \left[\mu_{S}(k, l)-\mu_{X}(j, l), \quad \mu_{X}(k, j)\right],
$$

and the assumption that the Markov property is not true leads to

$$
t_{k}-t_{j} \leq \mu_{S}(k, l)-\mu_{X}(j, l) .
$$

With this result in hand, let us examine the defining relation

$$
t_{i}-t_{j}=\max \left[\mu_{S}(i, j), \quad t_{k}-t_{j}+\mu_{X}(i, k)\right] .
$$


The second term in the maximum is less than

$$
\mu_{S}(k, l)-\mu_{X}(j, l)+\mu_{X}(i, k),
$$

by the inequality just obtained, and by hypothesis, this bound in turn is less than $\mu_{S}(i, j)$, leading to the final result.

\section{BOUNDING SEPARATIONS}

The two properties discussed in the previous section allow direct determination of the separation between $i$ and $j$ for some classes $k$ and $l$. In these cases, we set both the upper and lower bound on separation to the known value. For those cases where neither property is of assistance, we now describe how to establish bounds on the separations.

The maximum separation between $i$ and $j$ occurs if the prior departure on the target runway does not delay flight $j$ by any more than the cross-runway separation from flight $l$. In this case, $j$ is leaving as early as possible, considering that flight $l$ preceded it on the other runway. If we set $t_{j}$ to the lower bound, $t_{l}+\mu_{X}(j, l)$, and choose any arbitrary value for $t_{l}$, then the remaining departure times, including $t_{i}$, can be computed from the defining maximum formulae, and the upper bound on the separation between $i$ and $j$ can be computed.

The minimum separation between $i$ and $j$ occurs when $j$ is forced to lag $l$ by the maximum amount, because of prior history. If

$$
\max \_\operatorname{sep}(j, l)=\max _{m}\left[\mu_{S}(j, m)-\mu_{X}(l, m)\right] \text {, }
$$

the largest value that $t_{j}$ could take on is $t_{l}+\max \_\operatorname{sep}(j, l)$. Assuming an arbitrary value for $t_{l}$ and this maximum value $t_{j}$ allows computation of the remaining departure times and the lower bound on the separation between $i$ and $j$.

Both the lower and the upper bounds computed above depend on $l$ and $k$. The bounds independent of $l$ and $k$ are computed by weighted sums of these $l, k$ dependent terms. 


\section{CONSIDERING MORE HISTORY}

The bounds of the previous section are based on the extreme case for prior history. These bounds can be refined by explicitly considering prior departure sequences. Let us denote the additional flights considered by $f_{1}, f_{2}, f_{3}, \ldots, f_{n}$, each departing earlier than the previous one in the sequence. We will use $F$ to denote the entire sequence. The flights with an odd index depart from the target runway; those with an even index depart from the other runway. The bounds on $\mu_{P}(i, j)$ are calculated as

$$
\text { bound on } \mu_{P}(i, j)=\sum_{l, k, F}(\text { bound due to } l, k, F) p_{x l} p_{x k} \prod_{z=0}^{\left\lfloor\frac{n-1}{2}\right\rfloor} p_{f_{2 z+1}} \prod_{z=1}^{\left\lceil\frac{n-1}{2}\right\rceil} p_{x f_{2 z}} \text {. }
$$

In practice, we may not need to consider the entire sequence $F$ to bound $t_{i}-t_{j}$. If there is any subsequence $f_{z+2}, f_{z+1}, f_{z}$ that satisfies the Markov property, then we can determine $f_{z}$ in terms of $f_{z+1}$. Given $f_{z}$ and $f_{z+1}$ we can determine all subsequent departure times, including the times of interest, $t_{i}$ and $t_{j}$. Any arbitrary value of $f_{z+1}$ will do. The capacity algorithm uses recursive code to add history if the Markov property is not true for the last three flights in the current history $F$. If the Markov property is true, the lower and upper bounds are set to the same (computable) value.

The model user can specify the maximum number of aircraft to add to the history $F$. The larger this maximum, the more accurate the bounds will be, but the longer the computations will take. If a particular history sequence has reached its maximum size without the Markov property being true for some subsequence, then lower and upper bounds due to the sequence are computed.

Before explaining how the bounds are computed, we make the following observation:

- Theorem. If the Markov property does not hold for any subsequence of $k, j, l, F$, then when $f_{n-1}$ is at its earliest time, either all departure times within $k, j, l, F$ are based only on same runway separations, or $t_{i}-t_{j}$ is independent of any further history.

- Proof. Since the Markov property is not true for any subsequence, the cross-runway constraints are not binding on any subsequent flights in $k, j, l, F$ when the last two flights in any subsequence occur at their earliest times. If additional history requires that some flight $f_{x}$ departs later than its unconstrained earliest time, even when $f_{n-1}$ is at its unconstrained earliest time - and at this history-constrained earliest possible time for $f_{x}$, $t_{f_{x}}+\mu_{X}\left(f_{x-1}, f_{x}\right)>t_{f_{x+1}}+\mu_{S}\left(f_{x-1}, f_{x+1}\right)$, then all departure times after $f_{x}$ 
(including $t_{i}$, and $t_{j}$ ) can be determined in terms of $t_{f_{x}}$. Furthermore, in this situation, adding additional history will not change the relative times of departures after $f_{x}$. If additional history would cause $f_{n-1}$ to be later than its earlier time, this would cause $f_{x}$ to be deferred by an equal increment, as by the assumption, it is the accumulated same runway constraints from $f_{n-1}$ back to $f_{x}$ that have determined $t_{f_{x}}$. A later time for $f_{n-1}$ may also activate some other cross-runway constraint, causing $f_{x+1}$ to occur later, but by no more than the additional delay to $f_{x}$; thus, $f_{x}$ would continue to be a point from which later departure times can be computed. If there is no such $f_{x}$ for the current history, $k, j, l, F$, this is equivalent to stating that all separations in $k, j, l, F$ are determined by the same runway separations, $\mu_{S}$, QED.

Now assume that the last flight added is not on the target runway. Then $f_{n-1}$ is on the target runway. When $f_{n-1}$ is at its earliest time, $j$ is also at its earliest time. As the departure time of $f_{n-1}$ is delayed, it may begin to delay flight $j$ via the accumulated same runway separations. Thus, the upper bound on separation between $i$ and $j$ occurs when $t_{f_{n-1}}=t_{f_{n}}+\mu_{X}\left(f_{n-1}, f_{n}\right)$ the lower bound on $t_{f_{n-1}}$; the lower bound on separation occurs when $t_{f_{n-1}}=t_{f_{n}}+\max _{-} \operatorname{sep}\left(f_{n-1}, f_{n}\right)$ and the upper bound on $t_{f_{n-1}}$.

On the other hand, if the last flight added is on the target runway, then $f_{n-1}$ is on the other runway. As the departure of $f_{n-1}$ increases from its earliest time, it may cause flight $k$ to depart later. The cross-runway constraint between $i$ and $k$ may force $i$ to depart later, increasing the time between the departure of $i$ and $j$. (By the theorem, delaying a flight on the other runway either will not change the departure time $j$ or will increase the departure time of $i$ and $j$ equally.) Thus the upper bound on separation occurs when $t_{f_{n-1}}$ is at its upper bound, and the lower bound on separation occurs when $t_{f_{n-1}}$ is at its lower bound.

\section{Implementation}

For this task, we implemented the capacity model algorithms in Pascal code. In addition to the algorithms, the Pascal code includes input/output code to read and write the files generated and used by the Excel models that are discussed in the body of the report. 


\section{Appendix B Airport Capacities}

This appendix contains capacity data in eight tables. Tables B-1 through B-3 contain data for the LMINET 110 airports for the 2007 baseline, 2015 NextGen, and 2025 NextGen, respectively. NextGen benefits are applied only to airports in these tables. Table B-4 contains the capacities for the remaining 200 airports. Capacities for the airports in Table B-4 are not changed by NextGen technologies; however, three of the airports are scheduled to receive new runways before 2025 . Table B-5 contains 2025 capacities for the three airports with new runways.

Tables B-6 through B-8 contain results from an excursion analysis of MIT-limited and ROT-limited capacities for the 110 LMINET airports for 2015 and 2025 NextGen technologies. For reasons discussed in Chapter 5, MIT-limited operations produce no change for 2015 NextGen, so only ROT-limited results are included for 2015.

For all the airports, we examined airport diagrams, instrument procedures, and any other available capacity and operational documentation to identify the best configurations to model. For the 110-airport set, we considered the configurations and capacities modeled by FAA/ATO-F, and for 56 of the 110 , we also considered the configurations and capacities modeled by MITRE for the FAA Future Airport Capacity Task.

Capacities differ for airports with the same configurations due primarily to differences in aircraft mix. Airports with procedures for Land and Hold Short Operations are assumed to use them to support simultaneous operations from intersecting runways in visual meteorological conditions. Airports without towers and radar surveillance are assumed to operate with center-controlled one-on-oneoff procedures in instrument meteorological conditions with a trade between 4 arrivals and 6 departures per hour.

The capacity data in the tables include values for the five capacity points shown in Figure B-1. 


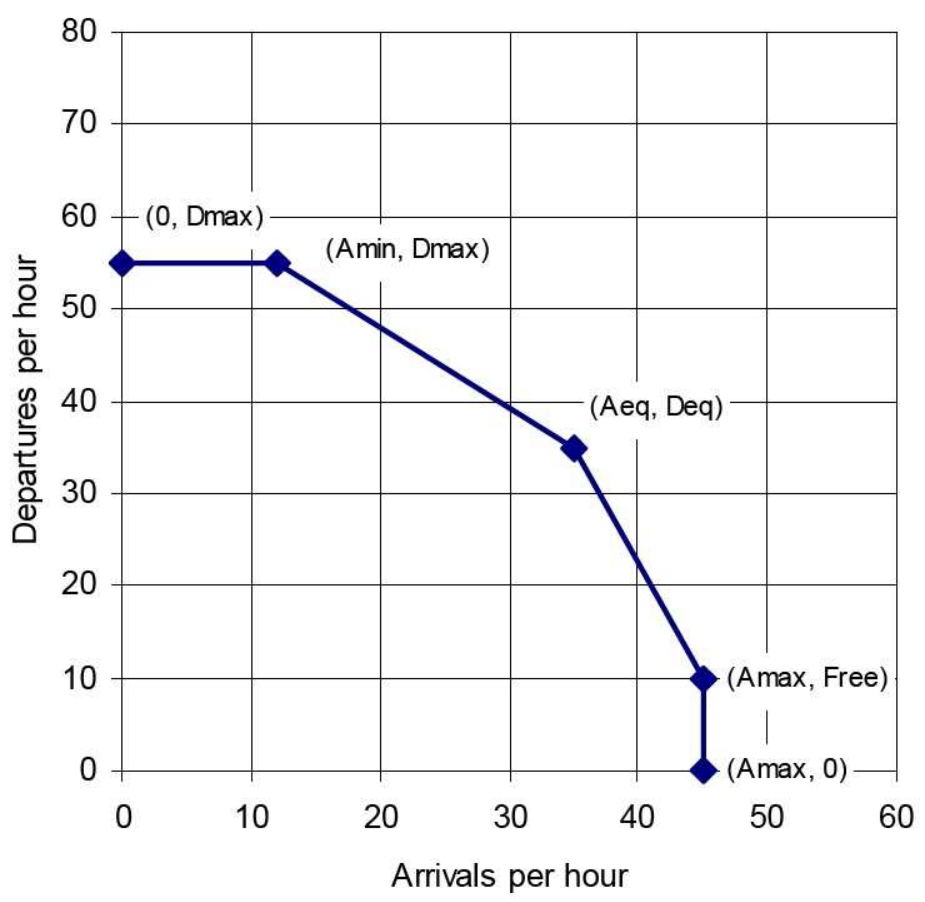

Figure B-1. Capacity points.

The curves for most airports will not include all five points:

- The Amin point occurs only if there is both a dedicated arrival-only runway and a mixed operations runway.

- Airports with dedicated arrival and departure runways will have a 3-point curve with (0, Dmax), (Amax, Dmax), and (Amax, 0). This is often called a single-point curve because the available capacity is always Amax and Dmax.

- Some smaller airports are known to operate all-arrival or all-departure, with no mixed operations. Their capacity curve is a straight-line trade between (0, Dmax) and (Amax, 0). For such airports, the "frees" are set to zero, and the midpoint is arbitrarily set to fall on the tradeoff line.

Table B-1. 110-Airport Capacities in 2007

\begin{tabular}{|c|c|c|c|c|c|c|c|c|c|c|c|c|c|c|}
\hline \multirow[b]{2}{*}{ Apt } & \multicolumn{7}{|c|}{ Visual meteorological conditions } & \multicolumn{7}{|c|}{ Instrument meteorological conditions } \\
\hline & Configuration & Amax & Aeq & Amin & Dmax & Deq & Free & Configuration & Amax & Amin & Aeq & Dmax & Deq & Free \\
\hline$A B Q$ & $\begin{array}{l}1.5 \mathrm{~A} / \mathrm{D} \text { with } \\
2 \mathrm{Amax} \text { with } 1 \\
\text { Free }\end{array}$ & 85 & 28 & 0 & 89 & 54 & 54 & 1.5 A/D with $1 \mathrm{Dmax}$ & 45 & 35 & 0 & 59 & 43 & 43 \\
\hline ALB & $1 \mathrm{~A} / \mathrm{D}$ & 42 & 21 & 0 & 60 & 33 & 33 & $1 \mathrm{~A} / 0.5 \mathrm{D}$ & 31 & 11 & 0 & 30 & 28 & 14 \\
\hline ANC & $\begin{array}{l}1.25 \mathrm{~A} / \mathrm{D} \text { with } \\
1.5^{\star} \mathrm{Dmax}\end{array}$ & 49 & 29 & 0 & 81 & 41 & 41 & $\begin{array}{l}\text { 1 A/D with } \\
1.25^{\star} \mathrm{Dmax}\end{array}$ & 28 & 23 & 0 & 67 & 27 & 27 \\
\hline ATL & $2 A+2 D+1 A / D$ & 122 & 118 & 81 & 150 & 114 & 133 & $2 A+2 D+1 A / D$ & 90 & 122 & 60 & 150 & 88 & 128 \\
\hline AUS & $1.5 \mathrm{~A} / \mathrm{D}$ & 64 & 41 & 0 & 89 & 54 & 54 & $1.5 \mathrm{~A} / \mathrm{D}$ & 44 & 36 & 0 & 85 & 41 & 41 \\
\hline
\end{tabular}


Table B-1. 110-Airport Capacities in 2007

\begin{tabular}{|c|c|c|c|c|c|c|c|c|c|c|c|c|c|c|}
\hline \multirow[b]{2}{*}{ Apt } & \multicolumn{7}{|c|}{ Visual meteorological conditions } & \multicolumn{7}{|c|}{ Instrument meteorological conditions } \\
\hline & Configuration & Amax & Aeq & Amin & Dmax & Deq & Free & Configuration & Amax & Amin & Aeq & Dmax & Deq & Free \\
\hline BDL & $\begin{array}{l}\text { 1.5 Amax with } \\
0.5 \text { Free } \\
\text { alternate with } \\
\text { 1.25Dmax }\end{array}$ & 61 & 12 & 0 & 71 & 30 & 42 & $\begin{array}{l}\text { 1.5 Amax with } 0.5 \\
\text { Free alternate with } \\
\text { 1.25Dmax }\end{array}$ & 44 & 12 & 0 & 70 & 22 & 41 \\
\hline $\mathrm{BFL}$ & $1 \mathrm{~A} / \mathrm{Dep} \mathrm{D}$ & 43 & 28 & 0 & 83 & 36 & 36 & $1 \mathrm{~A} / \mathrm{D}$ & 30 & 26 & 0 & 60 & 29 & 29 \\
\hline $\mathrm{BHM}$ & $\begin{array}{l}1 \mathrm{~A} / \mathrm{D} \text { with } 1.25 \\
\text { Amax } 1.25 \\
\text { Dmax } 0.5 \text { Free }\end{array}$ & 53 & 14 & 0 & 74 & 36 & 36 & $1 \mathrm{~A} / \mathrm{D}$ & 30 & 26 & 0 & 59 & 29 & 29 \\
\hline BNA & $\begin{array}{l}0.75 \mathrm{~A}+1 \mathrm{D} \\
+1 \mathrm{~A} / \mathrm{D} \\
\end{array}$ & 72 & 77 & 31 & 120 & 63 & 92 & $1 A / D+1.5 A+0.5 D$ & 79 & 51 & 47 & 90 & 75 & 58 \\
\hline $\mathrm{BOI}$ & $\begin{array}{l}\text { 1.75A/D with } \\
\text { 2Dmax }\end{array}$ & 73 & 38 & 0 & 120 & 59 & 59 & $1 \mathrm{~A} / \mathrm{D}$ & 31 & 23 & 0 & 60 & 29 & 29 \\
\hline BOS & $\begin{array}{l}\text { 2A/D with } 1 \\
\text { Amin } \\
\end{array}$ & 79 & 46 & 40 & 111 & 66 & 66 & $1 \mathrm{~A} / \mathrm{D}+\mathrm{Dmax}$ & 29 & 78 & 0 & 110 & 29 & 83 \\
\hline BTR & $\begin{array}{l}1.5 \mathrm{~A} \text { alternate } \\
\text { with } 1.5 \mathrm{D}\end{array}$ & 66 & 32 & 0 & 90 & 33 & 61 & $1 \mathrm{~A} / \mathrm{D}$ & 30 & 28 & 0 & 60 & 30 & 30 \\
\hline BUF & 1.5 A/1Dmax & 62 & 26 & 0 & 60 & 48 & 48 & $1 \mathrm{~A} / \mathrm{D}$ & 32 & 21 & 0 & 60 & 28 & 28 \\
\hline BUR & $1.25 \mathrm{~A} / \mathrm{D}$ & 52 & 36 & 0 & 75 & 46 & 46 & $1 \mathrm{~A} / \mathrm{D}$ & 30 & 24 & 0 & 59 & 29 & 29 \\
\hline BWI & $1.5 \mathrm{~A} / \mathrm{D}$ & 60 & 31 & 0 & 85 & 49 & 49 & $1.5 \mathrm{~A} / \mathrm{D}$ & 45 & 33 & 0 & 85 & 42 & 42 \\
\hline $\mathrm{CHS}$ & $\begin{array}{l}1.75^{*} \mathrm{~A} \text { alternate } \\
\text { with } 2 \mathrm{D}\end{array}$ & 72 & 0 & 0 & 120 & 36 & 60 & $1 \mathrm{~A} / \mathrm{D}$ & 31 & 21 & 0 & 60 & 28 & 28 \\
\hline CLE & $2 A / D$ & 82 & 38 & 0 & 119 & 65 & 65 & $1.25 A+1 D$ & 39 & 59 & 39 & 59 & 39 & 59 \\
\hline$\overline{C L T}$ & $2 A / D$ & 82 & 41 & 0 & 116 & 66 & 66 & $2 A / D$ & 61 & 44 & 0 & 116 & 56 & 56 \\
\hline $\mathrm{CMH}$ & $2 A / D$ & 82 & 34 & 0 & 120 & 64 & 64 & $\operatorname{Dep} A / D$ & 52 & 51 & 0 & 115 & 52 & 52 \\
\hline $\cos$ & $\begin{array}{l}1.5 \mathrm{~A} \text { alternate } \\
\text { with } 2 \mathrm{D} \text { with } \\
\text { Free } \mathrm{D} \\
\end{array}$ & 62 & 19 & 0 & 120 & 31 & 69 & $1 \mathrm{~A} / \mathrm{D}$ & 31 & 21 & 0 & 60 & 28 & 28 \\
\hline CRP & $\begin{array}{l}1.5 \mathrm{~A} \text { alternate } \\
\text { with } 1.5 \mathrm{Dmax} \\
\text { with } 0.75 \mathrm{Amin}\end{array}$ & 62 & 26 & 31 & 90 & 46 & 58 & $1 \mathrm{~A} / \mathrm{D}$ & 32 & 21 & 0 & 60 & 28 & 28 \\
\hline CVG & $\begin{array}{l}2 \mathrm{~A} / \mathrm{D}+1 \mathrm{~A}+ \\
0.5 \mathrm{Dmax}\end{array}$ & 121 & 40 & 40 & 143 & 106 & 65 & $2 A / D+1 A$ & 91 & 44 & 30 & 115 & 86 & 56 \\
\hline $\mathrm{DAB}$ & $\begin{array}{l}1.75 \mathrm{~A} \text { alternate } \\
\text { with } 2 \mathrm{Dmax}\end{array}$ & 74 & 52 & 0 & 120 & 37 & 86 & $1 \mathrm{~A} / \mathrm{D}$ & 30 & 25 & 0 & 60 & 29 & 29 \\
\hline DAL & $1 A+1 D$ & 41 & 59 & 41 & 59 & 41 & 59 & $1 A+1 D$ & 32 & 59 & 32 & 59 & 32 & 59 \\
\hline DAY & $1.75 \mathrm{~A} / \mathrm{D}$ & 71 & 30 & 0 & 103 & 56 & 56 & $1.75 \mathrm{~A} / \mathrm{D}$ & 55 & 36 & 0 & 103 & 49 & 49 \\
\hline$\overline{\mathrm{DCA}}$ & $1.25 \mathrm{~A}+1 \mathrm{D}$ & 52 & 60 & 52 & 60 & 52 & 60 & $1.25 \mathrm{~A} / 1 \mathrm{D}$ & 39 & 21 & 0 & 60 & 35 & 35 \\
\hline DEN & $3 A+3 D$ & 120 & 168 & 120 & 168 & 120 & 168 & $3 A+3 D$ & 88 & 168 & 88 & 168 & 88 & 168 \\
\hline DFW & $3 A+2 D+1 A / D$ & 159 & 131 & 119 & 167 & 152 & 143 & $3 A+2 D$ & 90 & 111 & 90 & 111 & 90 & 111 \\
\hline DSM & $\begin{array}{l}\text { 1A/1D with } 0.75 \\
\text { Amin }\end{array}$ & 41 & 18 & 31 & 60 & 36 & 39 & $1 \mathrm{~A} / \mathrm{D}$ & 31 & 21 & 0 & 60 & 28 & 28 \\
\hline$\overline{D T W}$ & $2 A+1 D+1 A / D$ & 120 & 75 & 80 & 113 & 112 & 89 & $2 A+2 D$ & 61 & 112 & 61 & 112 & 61 & 112 \\
\hline ELP & $1 A+1 D$ & 41 & 60 & 41 & 60 & 41 & 60 & $1 \mathrm{~A} / \mathrm{D}$ & 32 & 20 & 0 & 60 & 28 & 28 \\
\hline EUG & $1 \mathrm{~A} / \mathrm{D}$ & 42 & 23 & 0 & 60 & 34 & 34 & $1 \mathrm{~A} / \mathrm{D}$ & 30 & 24 & 0 & 60 & 29 & 29 \\
\hline EWR & $\begin{array}{l}1 A+1 D+0.25 \\
A / D \text { with Dmax } \\
\text { limit }\end{array}$ & 49 & 53 & 39 & 53 & 47 & 53 & $1 A+1 D$ & 30 & 21 & 30 & 53 & 30 & 53 \\
\hline FAT & $\begin{array}{l}1.5 \mathrm{~A} / \mathrm{D} \text { with } \\
1.5 \mathrm{Dmax}\end{array}$ & 63 & 36 & 0 & 89 & 52 & 52 & $1 \mathrm{~A} / \mathrm{D}$ & 30 & 24 & 0 & 59 & 29 & 29 \\
\hline FLL & $1.5 \mathrm{~A} / \mathrm{D}$ & 60 & 36 & 0 & 84 & 50 & 50 & $1 \mathrm{~A} / \mathrm{D}$ & 29 & 24 & 0 & 56 & 28 & 28 \\
\hline FNT & $1.25 \mathrm{~A} / \mathrm{D}$ & 50 & 26 & 0 & 75 & 42 & 42 & $1 \mathrm{~A} / 1.25 \mathrm{D}$ & 31 & 28 & 0 & 75 & 28 & 36 \\
\hline FXE & 1 A/1.4Dmax & 44 & 32 & 0 & 84 & 39 & 39 & $1 \mathrm{~A} / \mathrm{D}$ & 30 & 28 & 0 & 60 & 30 & 30 \\
\hline GFK & $1.25 \mathrm{~A} / \mathrm{D}$ & 52 & 24 & 0 & 75 & 41 & 41 & $1 \mathrm{~A} / \mathrm{D}$ & 31 & 22 & 0 & 60 & 28 & 28 \\
\hline GRR & $1.75 \mathrm{~A} / \mathrm{D}$ & 72 & 34 & 0 & 105 & 58 & 58 & $1 \mathrm{~A} / \mathrm{D}$ & 31 & 22 & 0 & 60 & 28 & 28 \\
\hline GSO & $\begin{array}{l}1.25 \mathrm{~A} / 0.5 \text { Free } \\
\text { alternate } \\
1.25 \mathrm{Dmax}\end{array}$ & 52 & 9 & 0 & 75 & 26 & 42 & $1 \mathrm{~A} / \mathrm{D}$ & 31 & 21 & 0 & 60 & 28 & 28 \\
\hline GYY & $\begin{array}{l}1.5 \mathrm{~A} / 1.0 \text { Free } \\
\text { alternate } \\
1.5 \mathrm{Dmax}\end{array}$ & 68 & 17 & 0 & 90 & 34 & 54 & $1 \mathrm{~A} / \mathrm{D}$ & 32 & 26 & 0 & 65 & 32 & 32 \\
\hline $\mathrm{HNL}$ & $0.5 \mathrm{~A} / 2 \mathrm{D}+1 \mathrm{~A}$ & 57 & 41 & 38 & 100 & 54 & 64 & $1 \mathrm{~A}+1 \mathrm{D}$ & 29 & 21 & 29 & 50 & 29 & 52 \\
\hline $\mathrm{HOU}$ & $1 A / 1.5 D, E \& F$ & 42 & 37 & 0 & 89 & 35 & 52 & $1 \mathrm{~A} / \mathrm{D}+0.5 \mathrm{Dmax}$ & 30 & 54 & 0 & 89 & 30 & 58 \\
\hline
\end{tabular}


Table B-1. 110-Airport Capacities in 2007

\begin{tabular}{|c|c|c|c|c|c|c|c|c|c|c|c|c|c|c|}
\hline \multirow[b]{2}{*}{ Apt } & \multicolumn{7}{|c|}{ Visual meteorological conditions } & \multicolumn{7}{|c|}{ Instrument meteorological conditions } \\
\hline & Configuration & Amax & Aeq & Amin & Dmax & Deq & Free & Configuration & Amax & Amin & Aeq & Dmax & Deq & Free \\
\hline HPN & $\begin{array}{l}1.15 \mathrm{~A} / 1.25 \mathrm{D} 1 \mathrm{~F}, \\
1 \mathrm{E}\end{array}$ & 50 & 30 & 0 & 75 & 37 & 43 & $1 \mathrm{~A} / \mathrm{D}$ & 30 & 27 & 0 & 60 & 30 & 30 \\
\hline $\mathrm{IAD}$ & $2 A / D+1 A$ & 120 & 44 & 40 & 112 & 106 & 66 & $2 A / D$ & 59 & 45 & 0 & 112 & 55 & 55 \\
\hline$\overline{\mathrm{IAH}}$ & $1 A / D+2 A+1.5 D$ & 121 & 104 & 81 & 143 & 113 & 118 & $2 A / D+1 A$ & 92 & 42 & 31 & 114 & 86 & 56 \\
\hline ICT & $\begin{array}{l}1 A / D+0.5 A \\
\text { alternate with } \\
0.75 D\end{array}$ & 62 & 62 & 21 & 105 & 41 & 84 & $0.25 A / 1.25 D+1 A$ & 40 & 21 & 32 & 75 & 36 & 48 \\
\hline IND & $2 A / D+0.1 D$ & 82 & 41 & 0 & 123 & 64 & 70 & $2 A / D$ & 63 & 41 & 0 & 118 & 56 & 56 \\
\hline ISP & $\begin{array}{l}\text { 1A/D + 1Amax + } \\
0.5 \mathrm{Dmax}\end{array}$ & 89 & 32 & 0 & 90 & 83 & 39 & $1 \mathrm{~A} / \mathrm{D}$ & 30 & 24 & 0 & 60 & 29 & 29 \\
\hline JAX & $\begin{array}{l}\text { 1A/D + 1D with } \\
\text { 1.25Amax }\end{array}$ & 51 & 76 & 0 & 90 & 32 & 89 & $1.25 \mathrm{~A} / \mathrm{D}+0.75 \mathrm{D}$ & 38 & 58 & 0 & 58 & 35 & 58 \\
\hline JFK & $2 A / D$ & 74 & 42 & 0 & 80 & 63 & 63 & $2 A / D$ & 55 & 44 & 0 & 80 & 52 & 52 \\
\hline JNU & $1 \mathrm{~A} / \mathrm{D}$ & 44 & 31 & 0 & 60 & 38 & 38 & $1 \mathrm{~A} / \mathrm{D}$ & 30 & 27 & 0 & 60 & 30 & 30 \\
\hline LAN & $\begin{array}{l}1 \mathrm{~A} / \mathrm{D}+1.25 \\
\mathrm{Dmax}\end{array}$ & 41 & 19 & 0 & 75 & 33 & 33 & $1 \mathrm{~A} / \mathrm{D}$ & 31 & 21 & 0 & 60 & 28 & 28 \\
\hline LAS & $1 A / D+1 A$ & 80 & 21 & 40 & 56 & 73 & 33 & $1.1 \mathrm{~A}+1 \mathrm{D}$ & 33 & 56 & 33 & 56 & 33 & 56 \\
\hline LAX & $2 A+2 D$ & 76 & 102 & 76 & 102 & 76 & 102 & $2 A+2 D$ & 57 & 102 & 57 & 102 & 57 & 102 \\
\hline LGA & $1 A+1 D$ & 40 & 50 & 40 & 50 & 40 & 50 & $1 A+1 D$ & 30 & 50 & 30 & 50 & 30 & 50 \\
\hline LGB & $\begin{array}{l}1 \mathrm{~A} / \mathrm{D} \text { with } \\
\text { alternating } 1.5 \\
\text { Amax } 1.5 \mathrm{Dmax} \\
0.5 \text { Free } \\
\end{array}$ & 67 & 17 & 0 & 90 & 33 & 53 & $1 \mathrm{~A} / \mathrm{D}$ & 29 & 25 & 0 & 58 & 28 & 28 \\
\hline LIT & $\begin{array}{l}\text { 2A/D with Dmax } \\
\text { limit }\end{array}$ & 82 & 35 & 0 & 80 & 65 & 65 & $1 \mathrm{~A} / \mathrm{D}$ & 32 & 21 & 0 & 60 & 28 & 28 \\
\hline $\mathrm{MCl}$ & $2 A / D$ & 82 & 35 & 0 & 120 & 65 & 65 & $2 A / D$ & 63 & 42 & 0 & 120 & 56 & 56 \\
\hline $\mathrm{MCO}$ & $3 \mathrm{~A} / \mathrm{D}+0.5 \mathrm{D}$ & 117 & 93 & 0 & 188 & 98 & 125 & 1CSP + 1Dep & 80 & 76 & 0 & 141 & 80 & 80 \\
\hline MDW & $1.25 \mathrm{~A} / \mathrm{D}$ & 51 & 27 & 0 & 73 & 42 & 42 & $1 \mathrm{~A} / \mathrm{D}$ & 30 & 23 & 0 & 58 & 28 & 28 \\
\hline MEM & $\begin{array}{l}1 \mathrm{~A} / \mathrm{D}+1.75 \mathrm{~A}+ \\
1 \mathrm{D}\end{array}$ & 107 & 74 & 68 & 106 & 100 & 85 & $1 A+1 D+1 A / D$ & 58 & 74 & 29 & 106 & 56 & 80 \\
\hline MHT & $1 A+1 D$ & 41 & 60 & 41 & 60 & 41 & 60 & $1 \mathrm{~A} / \mathrm{D}$ & 32 & 21 & 0 & 60 & 28 & 28 \\
\hline MIA & $2 A+2 D$ & 76 & 101 & 76 & 101 & 76 & 101 & $2 A / D$ & 57 & 44 & 0 & 101 & 54 & 54 \\
\hline MKE & $1.5 \mathrm{~A} / \mathrm{D}$ & 62 & 36 & 0 & 89 & 52 & 52 & $1 \mathrm{~A} / \mathrm{D}$ & 30 & 24 & 0 & 59 & 29 & 29 \\
\hline MLB & $1.5 \mathrm{~A} / \mathrm{D}+0.25 \mathrm{D}$ & 63 & 50 & 0 & 105 & 51 & 66 & $1 \mathrm{~A} / \mathrm{D}$ & 30 & 24 & 0 & 60 & 29 & 29 \\
\hline MSN & $1 \mathrm{~A} / \mathrm{D}$ & 41 & 20 & 0 & 60 & 33 & 33 & $1 \mathrm{~A} / \mathrm{D}$ & 31 & 22 & 0 & 60 & 28 & 28 \\
\hline MSP & $\begin{array}{l}2 \mathrm{~A} / \mathrm{D}+ \\
\text { alternating } 1 \\
\text { Amax and } 1 \\
\text { Dmax }\end{array}$ & 120 & 39 & 0 & 169 & 60 & 104 & Dep Pair +1D & 52 & 107 & 0 & 145 & 52 & 108 \\
\hline MSY & 1.2 A/1Dmax & 49 & 21 & 0 & 59 & 39 & 39 & 1.2 A/1Dmax & 38 & 24 & 0 & 59 & 34 & 34 \\
\hline OAK & $1.5 \mathrm{~A} / \mathrm{D}$ & 62 & 40 & 0 & 88 & 53 & 53 & $1 \mathrm{~A} / \mathrm{D}$ & 30 & 22 & 0 & 57 & 28 & 28 \\
\hline OKC & $1.5 \mathrm{~A} / \mathrm{D}$ & 66 & 48 & 0 & 90 & 58 & 58 & $1 A+1 D$ & 30 & 60 & 30 & 60 & 30 & 60 \\
\hline OMA & $2 \mathrm{~A} / 1 \mathrm{Dmax}$ & 88 & 32 & 0 & 60 & 77 & 39 & $1.5 \mathrm{~A} / 1 \mathrm{Dmax}$ & 45 & 28 & 0 & 60 & 45 & 30 \\
\hline ONT & $1.75 \mathrm{~A} / \mathrm{D}$ & 71 & 45 & 0 & 100 & 60 & 60 & $1 \mathrm{~A} / \mathrm{D}$ & 29 & 23 & 0 & 56 & 28 & 28 \\
\hline ORD & $1 A / D+2 A+2 D$ & 119 & 128 & 79 & 164 & 111 & 141 & $1 A / D+1 A+2 D$ & 60 & 130 & 30 & 164 & 58 & 137 \\
\hline ORF & $\begin{array}{l}1 \mathrm{~A} / \mathrm{D}+1.25 \\
\mathrm{Dmax}\end{array}$ & 44 & 32 & 0 & 75 & 39 & 39 & $1 \mathrm{~A} / \mathrm{D}$ & 30 & 28 & 0 & 60 & 30 & 30 \\
\hline OXR & $1 \mathrm{~A} / \mathrm{D}$ & 46 & 35 & 0 & 60 & 41 & 60 & $1 \mathrm{~A} / \mathrm{D}$ & 30 & 29 & 0 & 60 & 30 & 30 \\
\hline $\mathrm{PBI}$ & $1 \mathrm{~A} / \mathrm{D}$ & 42 & 29 & 0 & 59 & 36 & 36 & $1 \mathrm{~A} / \mathrm{D}$ & 29 & 25 & 0 & 58 & 28 & 28 \\
\hline PDX & $2 A / D$ & 81 & 45 & 0 & 114 & 67 & 67 & Dep pair & 51 & 51 & 0 & 75 & 51 & 51 \\
\hline PHF & $\begin{array}{l}1 \mathrm{~A} / \mathrm{D} \text { alternate } \\
\text { with } 0.25 \mathrm{Dmax}\end{array}$ & 44 & 32 & 0 & 75 & 22 & 53 & $1 \mathrm{~A} / \mathrm{D}$ & 30 & 28 & 0 & 60 & 30 & 30 \\
\hline PHL & $\begin{array}{l}1 \mathrm{~A}+1 \mathrm{D} \\
+0.5^{\star} \mathrm{A} / \mathrm{D}\end{array}$ & 60 & 67 & 40 & 87 & 58 & 73 & $1 A+1 D+0.5^{\star} A / D$ & 45 & 67 & 30 & 84 & 44 & 70 \\
\hline $\mathrm{PHX}$ & $1 A+1 D+1 A / D$ & 81 & 77 & 41 & 116 & 73 & 90 & $2 \mathrm{~A} / \mathrm{D}$ & 61 & 43 & 0 & 116 & 56 & 56 \\
\hline PIE & $\begin{array}{l}1 \mathrm{~A} / \mathrm{D}+0.75 \mathrm{~A}+ \\
0.5 \mathrm{D}\end{array}$ & 76 & 62 & 33 & 90 & 71 & 68 & $1 \mathrm{~A} / \mathrm{D}$ & 30 & 27 & 0 & 60 & 30 & 30 \\
\hline PIT & $1 A+1 D+1 A / D$ & 82 & 80 & 41 & 118 & 75 & 92 & $2 A / D$ & 61 & 46 & 0 & 118 & 57 & 57 \\
\hline PVD & $1 \mathrm{~A} / \mathrm{D}$ & 42 & 25 & 0 & 59 & 35 & 35 & $1 \mathrm{~A} / \mathrm{D}$ & 30 & 23 & 0 & 59 & 28 & 28 \\
\hline RDU & $2 A / D$ & 82 & 35 & 0 & 119 & 65 & 65 & $\begin{array}{l}\text { Dep pair - with } \\
1.5^{*} \mathrm{Dmax} \mathrm{Sgl}\end{array}$ & 52 & 51 & 0 & 89 & 52 & 52 \\
\hline RFD & $1.75 \mathrm{~A} / \mathrm{D}$ & 73 & 55 & 0 & 103 & 65 & 65 & $1.25 \mathrm{~A} / \mathrm{D}$ & 33 & 30 & 0 & 78 & 33 & 33 \\
\hline
\end{tabular}


Table B-1. 110-Airport Capacities in 2007

\begin{tabular}{|c|c|c|c|c|c|c|c|c|c|c|c|c|c|c|}
\hline \multirow[b]{2}{*}{ Apt } & \multicolumn{7}{|c|}{ Visual meteorological conditions } & \multicolumn{7}{|c|}{ Instrument meteorological conditions } \\
\hline & Configuration & Amax & Aeq & Amin & Dmax & Deq & Free & Configuration & Amax & Amin & Aeq & Dmax & Deq & Free \\
\hline $\mathrm{RIC}$ & $1.25 \mathrm{~A} / \mathrm{D}$ & 55 & 40 & 0 & 75 & 48 & 48 & $1 \mathrm{~A} / \mathrm{D}$ & 30 & 28 & 0 & 60 & 30 & 30 \\
\hline RNO & $1.5 \mathrm{~A} / \mathrm{D}$ & 61 & 29 & 0 & 88 & 49 & 49 & $1 \mathrm{~A} / \mathrm{D}$ & 31 & 22 & 0 & 59 & 28 & 28 \\
\hline $\mathrm{ROC}$ & $1.25 \mathrm{~A} / \mathrm{D}$ & 52 & 23 & 0 & 75 & 41 & 41 & $1 \mathrm{~A} / \mathrm{D}$ & 31 & 21 & 0 & 60 & 28 & 28 \\
\hline RSW & $1 \mathrm{~A} / \mathrm{D}$ & 40 & 20 & 0 & 56 & 33 & 33 & $1 \mathrm{~A} / \mathrm{D}$ & 30 & 22 & 0 & 56 & 28 & 28 \\
\hline SAN & $1 \mathrm{~A} / \mathrm{D}$ & 40 & 21 & 0 & 57 & 33 & 33 & $1 \mathrm{~A} / \mathrm{D}$ & 30 & 22 & 0 & 57 & 28 & 28 \\
\hline SAT & $2 A / D$ & 85 & 55 & 0 & 119 & 72 & 72 & $1 \mathrm{~A} / \mathrm{D}$ & 30 & 24 & 0 & 59 & 28 & 28 \\
\hline SBA & $\begin{array}{l}1 \mathrm{~A} / \mathrm{D}+0.25 \mathrm{~A}+ \\
0.25 \mathrm{D}\end{array}$ & 53 & 40 & 11 & 75 & 45 & 50 & $1 A+1 D$ & 30 & 60 & 30 & 60 & 30 & 60 \\
\hline SDF & $2 A / D$ & 82 & 34 & 0 & 120 & 64 & 64 & $2 A / D$ & 63 & 41 & 0 & 120 & 56 & 56 \\
\hline SEA & 2A/dep Dmax & 80 & 40 & 0 & 86 & 65 & 65 & $1 A+D e p D$ & 30 & 51 & 0 & 86 & 30 & 51 \\
\hline SFO & $2 A / D$ & 71 & 51 & 0 & 103 & 63 & 63 & $1 \mathrm{~A} / \mathrm{D}+0.5 \mathrm{D}$ & 28 & 48 & 0 & 77 & 27 & 53 \\
\hline SJC & $\begin{array}{l}1 \mathrm{~A}+1 \mathrm{D} \\
+0.25 \mathrm{~A} / \mathrm{D} \\
\end{array}$ & 52 & 65 & 41 & 73 & 50 & 67 & CSP w/Sgl Dmax & 30 & 30 & 0 & 58 & 30 & 30 \\
\hline SLC & $\begin{array}{l}\text { 2A/D with } \\
2.25 \mathrm{Amax}\end{array}$ & 90 & 47 & 0 & 113 & 77 & 67 & $2 \mathrm{~A} / \mathrm{D}$ with $2.25 \mathrm{Amax}$ & 66 & 47 & 0 & 113 & 63 & 56 \\
\hline SMF & $2 A / D$ & 82 & 36 & 0 & 118 & 65 & 65 & $2 A / D$ & 62 & 42 & 0 & 118 & 56 & 56 \\
\hline SNA & $1.5 \mathrm{~A} / \mathrm{D}$ & 65 & 45 & 0 & 90 & 56 & 56 & $1 \mathrm{~A} / \mathrm{D}$ & 30 & 24 & 0 & 59 & 28 & 28 \\
\hline STL & $1 A+1 D+1 A / D$ & 81 & 77 & 41 & 115 & 73 & 90 & $2 A / D$ & 61 & 43 & 0 & 115 & 56 & 56 \\
\hline SWF & $1 \mathrm{~A} / \mathrm{D}$ & 44 & 32 & 0 & 60 & 39 & 39 & $1 \mathrm{~A} / \mathrm{D}$ & 30 & 24 & 0 & 58 & 28 & 28 \\
\hline SYR & $1.3 \mathrm{~A} / \mathrm{D}$ & 57 & 41 & 0 & 78 & 50 & 50 & $1 \mathrm{~A} / \mathrm{D}$ & 30 & 28 & 0 & 60 & 30 & 30 \\
\hline TEB & $1 \mathrm{~A} / \mathrm{D}+0.5 \mathrm{D}$ & 44 & 62 & 0 & 90 & 39 & 68 & $1 \mathrm{~A} / \mathrm{D}$ & 30 & 28 & 0 & 60 & 30 & 30 \\
\hline TPA & $\begin{array}{l}\text { 2A/D with Dmax } \\
\text { limit }\end{array}$ & 81 & 46 & 0 & 65 & 67 & 65 & $2 A / D$ with Dmax limit & 59 & 46 & 0 & 65 & 56 & 65 \\
\hline TUL & 1.6 A/D & 70 & 51 & 0 & 96 & 62 & 62 & $1.5 \mathrm{~A} / \mathrm{D}$ & 45 & 41 & 0 & 90 & 45 & 45 \\
\hline TUS & $2 A / D$ & 87 & 60 & 0 & 120 & 75 & 75 & $1 \mathrm{~A} / \mathrm{D}+0.5 \mathrm{D}$ & 31 & 53 & 0 & 90 & 28 & 58 \\
\hline TVC & $\begin{array}{l}1.25 \mathrm{~A} / \mathrm{D} \text { with } \\
\text { interpolated } \mathrm{E}\end{array}$ & 55 & 40 & 0 & 90 & 39 & 60 & $1 \mathrm{~A} / \mathrm{D}$ & 30 & 28 & 0 & 60 & 30 & 30 \\
\hline TYS & $1.25 \mathrm{~A} / \mathrm{D}$ & 55 & 40 & 0 & 75 & 48 & 48 & $1 \mathrm{~A} / \mathrm{D}$ & 30 & 28 & 0 & 60 & 30 & 30 \\
\hline VNY & $1.25 \mathrm{~A} / \mathrm{D}$ & 55 & 40 & 0 & 75 & 48 & 48 & $1 \mathrm{~A} / \mathrm{D}+0.25 \mathrm{D}$ & 30 & 43 & 0 & 75 & 30 & 45 \\
\hline
\end{tabular}

Table B-2. 110-Airport NextGen Capacities in 2015

\begin{tabular}{|c|c|c|c|c|c|c|c|c|c|c|c|c|c|c|}
\hline \multirow[b]{2}{*}{ Apt } & \multicolumn{7}{|c|}{ Visual meteorological conditions } & \multicolumn{7}{|c|}{ Instrument meteorological conditions } \\
\hline & Configuration & Amax & Aeq & Amin & Dmax & Deq & Free & Configuration & Amax & Amin & Aeq & Dmax & Deq & Free \\
\hline$A B Q$ & $\begin{array}{l}1.5 \mathrm{~A} / \mathrm{D} \text { with } \\
2 \mathrm{Amax} \text { with } 1 \\
\text { Free }\end{array}$ & 85 & 28 & 0 & 89 & 54 & 54 & 1.5 A/D with $1 \mathrm{Dmax}$ & 45 & 36 & 0 & 59 & 43 & 43 \\
\hline ALB & $1 \mathrm{~A} / \mathrm{D}$ & 42 & 21 & 0 & 60 & 33 & 33 & $1 \mathrm{~A} / 0.5 \mathrm{D}$ & 31 & 11 & 0 & 30 & 28 & 14 \\
\hline ANC & $\begin{array}{l}\text { 1.25 A/D with } \\
1.5^{*} \mathrm{Dmax}\end{array}$ & 49 & 30 & 0 & 81 & 42 & 42 & $\begin{array}{l}\text { 1 A/D with } \\
1.25^{\star} \mathrm{Dmax}\end{array}$ & 28 & 23 & 0 & 67 & 28 & 28 \\
\hline ATL & $2 A+2 D+1 A / D$ & 122 & 119 & 81 & 150 & 114 & 133 & $2 A+2 D+1 A / D$ & 90 & 123 & 60 & 150 & 88 & 128 \\
\hline AUS & $1.5 \mathrm{~A} / \mathrm{D}$ & 64 & 41 & 0 & 89 & 54 & 54 & $1.5 \mathrm{~A} / \mathrm{D}$ & 44 & 36 & 0 & 85 & 41 & 41 \\
\hline BDL & $\begin{array}{l}\text { !.5Amax with } 0.5 \\
\text { Free alternate } \\
\text { with 1.25Dmax }\end{array}$ & 61 & 13 & 0 & 71 & 30 & 42 & $\begin{array}{l}\text { !.5Amax with } 0.5 \\
\text { Free alternate with } \\
\text { 1.25Dmax }\end{array}$ & 44 & 12 & 0 & 70 & 22 & 41 \\
\hline$\overline{B F L}$ & $1 \mathrm{~A} / \mathrm{Dep} \mathrm{D}$ & 43 & 28 & 0 & 83 & 36 & 36 & $1 \mathrm{~A} / \mathrm{D}$ & 30 & 26 & 0 & 60 & 29 & 29 \\
\hline BHM & $\begin{array}{l}1 \mathrm{~A} / \mathrm{D} \text { with } 1.25 \\
\text { Amax } 1.25 \\
\text { Dmax } 0.5 \text { Free }\end{array}$ & 53 & 14 & 0 & 74 & 36 & 36 & $1 \mathrm{~A} / \mathrm{D}$ & 30 & 26 & 0 & 59 & 29 & 29 \\
\hline BNA & $\begin{array}{l}0.75 \mathrm{~A}+1 \mathrm{D} \\
+1 \mathrm{~A} / \mathrm{D}\end{array}$ & 72 & 77 & 31 & 120 & 63 & 92 & $1 A / D+1.5 A+0.5 D$ & 79 & 51 & 47 & 90 & 75 & 58 \\
\hline $\mathrm{BOI}$ & $\begin{array}{l}\text { 1.75A/D with } \\
2 \mathrm{Dmax}\end{array}$ & 73 & 38 & 0 & 120 & 59 & 59 & $1 \mathrm{~A} / \mathrm{D}$ & 31 & 23 & 0 & 60 & 29 & 29 \\
\hline BOS & $\begin{array}{l}\text { 2A/D with } 1 \\
\text { Amin }\end{array}$ & 83 & 41 & 41 & 111 & 68 & 68 & $\operatorname{Dep} A+2 D$ & 53 & 52 & 0 & 110 & 53 & 53 \\
\hline BTR & $\begin{array}{l}1.5 \mathrm{~A} \text { alternate } \\
\text { with } 1.5 \mathrm{D}\end{array}$ & 66 & 32 & 0 & 90 & 33 & 61 & $1 \mathrm{~A} / \mathrm{D}$ & 30 & 28 & 0 & 60 & 30 & 30 \\
\hline BUF & 1.5 A/1Dmax & 62 & 26 & 0 & 60 & 48 & 48 & $1 \mathrm{~A} / \mathrm{D}$ & 32 & 21 & 0 & 60 & 28 & 28 \\
\hline
\end{tabular}


Table B-2. 110-Airport NextGen Capacities in 2015

\begin{tabular}{|c|c|c|c|c|c|c|c|c|c|c|c|c|c|c|}
\hline \multirow[b]{2}{*}{ Apt } & \multicolumn{7}{|c|}{ Visual meteorological conditions } & \multicolumn{7}{|c|}{ Instrument meteorological conditions } \\
\hline & Configuration & Amax & Aeq & Amin & Dmax & Deq & Free & Configuration & Amax & Amin & Aeq & Dmax & Deq & Free \\
\hline$\overline{B U R}$ & $1.25 \mathrm{~A} / \mathrm{D}$ & 52 & 36 & 0 & 75 & 46 & 46 & $1 \mathrm{~A} / \mathrm{D}$ & 30 & 24 & 0 & 59 & 29 & 29 \\
\hline BWI & $1.5 \mathrm{~A} / \mathrm{D}$ & 63 & 27 & 0 & 85 & 50 & 50 & $1.5 \mathrm{~A} / \mathrm{D}$ & 47 & 35 & 0 & 85 & 43 & 43 \\
\hline $\mathrm{CHS}$ & $\begin{array}{l}1.75^{*} \mathrm{~A} \text { alternate } \\
\text { with } 2 \mathrm{D}\end{array}$ & 72 & 0 & 0 & 120 & 36 & 60 & $1 \mathrm{~A} / \mathrm{D}$ & 31 & 21 & 0 & 60 & 28 & 28 \\
\hline CLE & $2 \mathrm{~A} / \mathrm{D}$ & 86 & 31 & 0 & 119 & 66 & 66 & $1.25 A+1 D$ & 40 & 59 & 40 & 59 & 40 & 59 \\
\hline $\mathrm{CLT}$ & $3 \mathrm{~A} / \mathrm{D}$ & 128 & 52 & 0 & 175 & 100 & 100 & $3 \mathrm{~A} / \mathrm{D}$ & 95 & 69 & 0 & 175 & 87 & 87 \\
\hline $\mathrm{CMH}$ & $2 A / D$ & 82 & 34 & 0 & 120 & 64 & 64 & Dep A/D & 52 & 51 & 0 & 115 & 52 & 52 \\
\hline $\cos$ & $\begin{array}{l}1.5 \mathrm{~A} \text { alternate } \\
\text { with } 2 \mathrm{D} \text { with } \\
\text { FreeD } \\
\end{array}$ & 62 & 19 & 0 & 120 & 31 & 69 & $1 \mathrm{~A} / \mathrm{D}$ & 31 & 21 & 0 & 60 & 28 & 28 \\
\hline CRP & $\begin{array}{l}1.5 \mathrm{~A} \text { alternate } \\
\text { with } 1.5 \mathrm{Dmax} \\
\text { with } 0.75 \mathrm{Amin}\end{array}$ & 62 & 26 & 31 & 90 & 46 & 58 & $1 \mathrm{~A} / \mathrm{D}$ & 32 & 21 & 0 & 60 & 28 & 28 \\
\hline CVG & $\begin{array}{l}2 \mathrm{~A} / \mathrm{D}+1 \mathrm{~A}+ \\
0.5 \mathrm{Dmax}\end{array}$ & 127 & 34 & 42 & 143 & 109 & 66 & $2 A / D+1 A$ & 94 & 46 & 31 & 115 & 89 & 58 \\
\hline DAB & $\begin{array}{l}1.75 \mathrm{~A} \text { alternate } \\
\text { with } 2 \mathrm{Dmax}\end{array}$ & 74 & 52 & 0 & 120 & 37 & 86 & $1 \mathrm{~A} / \mathrm{D}$ & 30 & 25 & 0 & 60 & 29 & 29 \\
\hline DAL & $1 A+1 D$ & 41 & 59 & 41 & 59 & 41 & 59 & $1 A+1 D$ & 32 & 59 & 32 & 59 & 32 & 59 \\
\hline DAY & $2 A / D$ & 82 & 35 & 0 & 117 & 64 & 64 & $2 \mathrm{~A} / \mathrm{D}$ & 63 & 41 & 0 & 117 & 56 & 56 \\
\hline $\mathrm{DCA}$ & $1.25 A+1 D$ & 54 & 60 & 54 & 60 & 54 & 60 & $1.25 \mathrm{~A} / 1 \mathrm{D}$ & 41 & 22 & 0 & 60 & 36 & 36 \\
\hline$\overline{D E N}$ & $3 A+3 D$ & 125 & 168 & 125 & 168 & 125 & 168 & $3 A+3 D$ & 91 & 168 & 91 & 168 & 91 & 168 \\
\hline$\overline{D F W}$ & $3 A+2 D+1 A / D$ & 166 & 128 & 125 & 167 & 158 & 144 & $3 A+2 D$ & 93 & 111 & 93 & 111 & 93 & 111 \\
\hline DSM & $\begin{array}{l}1 \mathrm{~A} / 1 \mathrm{D} \text { with } 0.75 \\
\text { Amin }\end{array}$ & 41 & 18 & 31 & 60 & 36 & 39 & $1 \mathrm{~A} / \mathrm{D}$ & 31 & 21 & 0 & 60 & 28 & 28 \\
\hline DTW & $2 A+1 D+1 A / D$ & 126 & 72 & 84 & 113 & 117 & 89 & $2 A+2 D$ & 63 & 112 & 63 & 112 & 63 & 112 \\
\hline ELP & $1 A+1 D$ & 41 & 60 & 41 & 60 & 41 & 60 & $1 \mathrm{~A} / \mathrm{D}$ & 32 & 20 & 0 & 60 & 28 & 28 \\
\hline EUG & $1 \mathrm{~A} / \mathrm{D}$ & 42 & 23 & 0 & 60 & 34 & 34 & $1 \mathrm{~A} / \mathrm{D}$ & 30 & 24 & 0 & 60 & 29 & 29 \\
\hline EWR & $\begin{array}{l}1 A+1 D+0.25 \\
A / D \text { with Dmax } \\
\text { limit }\end{array}$ & 49 & 53 & 39 & 53 & 47 & 53 & $1 A+1 D$ & 30 & 21 & 30 & 53 & 30 & 53 \\
\hline FAT & $\begin{array}{l}\text { 1.5 A/D with } \\
1.5 \mathrm{Dmax}\end{array}$ & 63 & 36 & 0 & 89 & 52 & 52 & $1 \mathrm{~A} / \mathrm{D}$ & 30 & 24 & 0 & 59 & 29 & 29 \\
\hline FLL & $2 \mathrm{~A} / \mathrm{D}$ & 84 & 44 & 0 & 112 & 69 & 69 & $2 A / D$ & 60 & 49 & 0 & 112 & 58 & 58 \\
\hline FNT & $1.25 \mathrm{~A} / \mathrm{D}$ & 52 & 26 & 0 & 75 & 42 & 42 & $1 \mathrm{~A} / 1.25 \mathrm{D}$ & 31 & 28 & 0 & 75 & 28 & 36 \\
\hline FXE & 1 A/1.4Dmax & 44 & 32 & 0 & 84 & 39 & 39 & $1 \mathrm{~A} / \mathrm{D}$ & 30 & 28 & 0 & 60 & 30 & 30 \\
\hline GFK & $1.25 \mathrm{~A} / \mathrm{D}$ & 52 & 24 & 0 & 75 & 41 & 41 & $1 \mathrm{~A} / \mathrm{D}$ & 31 & 22 & 0 & 60 & 28 & 28 \\
\hline GRR & $1.75 \mathrm{~A} / \mathrm{D}$ & 72 & 34 & 0 & 105 & 58 & 58 & $1 \mathrm{~A} / \mathrm{D}$ & 31 & 22 & 0 & 60 & 28 & 28 \\
\hline GSO & $2 A / D$ & 82 & 35 & 0 & 120 & 65 & 65 & $2 A / D$ & 63 & 42 & 0 & 120 & 57 & 57 \\
\hline GYY & $\begin{array}{l}1.5 \mathrm{~A} / 1.0 \text { Free } \\
\text { alternate } \\
1.5 \mathrm{Dmax}\end{array}$ & 68 & 17 & 0 & 90 & 34 & 54 & $1 \mathrm{~A} / \mathrm{D}$ & 32 & 26 & 0 & 65 & 32 & 32 \\
\hline $\mathrm{HNL}$ & $0.5 \mathrm{~A} / 2 \mathrm{D}+1 \mathrm{~A}$ & 60 & 36 & 40 & 100 & 56 & 65 & $1 A+1 D$ & 30 & 22 & 30 & 50 & 30 & 53 \\
\hline $\mathrm{HOU}$ & $1 \mathrm{~A} / 1.5 \mathrm{D}, \mathrm{E} \& \mathrm{~F}$ & 42 & 38 & 0 & 89 & 35 & 52 & $1 \mathrm{~A} / \mathrm{D}+0.5 \mathrm{Dmax}$ & 30 & 54 & 0 & 89 & 30 & 58 \\
\hline HPN & $\begin{array}{l}1.15 \mathrm{~A} / 1.25 \mathrm{D} 1 \mathrm{~F}, \\
1 \mathrm{E}\end{array}$ & 50 & 30 & 0 & 75 & 37 & 43 & $1 \mathrm{~A} / \mathrm{D}$ & 30 & 27 & 0 & 60 & 30 & 30 \\
\hline IAD & $2 A / D+1 A+1 D$ & 125 & 75 & 42 & 168 & 109 & 123 & $2 A / D+1 D$ & 61 & 103 & 0 & 168 & 58 & 114 \\
\hline$\overline{\mathrm{IAH}}$ & $1 A / D+2 A+1.5 D$ & 126 & 101 & 84 & 143 & 117 & 118 & $2 A / D+1 A$ & 95 & 44 & 32 & 114 & 89 & 58 \\
\hline ICT & \begin{tabular}{|l|}
$1 \mathrm{~A} / \mathrm{D}+0.5 \mathrm{~A}$ \\
alternate with \\
$0.75 \mathrm{D}$ \\
\end{tabular} & 62 & 62 & 21 & 105 & 41 & 84 & $0.25 A / 1.25 D+1 A$ & 40 & 21 & 32 & 75 & 36 & 58 \\
\hline IND & $2 A / D+0.1 D$ & 82 & 41 & 0 & 123 & 64 & 70 & $2 \mathrm{~A} / \mathrm{D}$ & 63 & 41 & 0 & 118 & 56 & 56 \\
\hline ISP & $\begin{array}{l}\text { 1A/D + 1Amax + } \\
0.5 \mathrm{Dmax}\end{array}$ & 89 & 32 & 0 & 90 & 83 & 39 & $1 \mathrm{~A} / \mathrm{D}$ & 30 & 24 & 0 & 60 & 29 & 29 \\
\hline JAX & $\begin{array}{l}\text { 1A/D + 1D with } \\
\text { 1.25Amax }\end{array}$ & 51 & 76 & 0 & 90 & 32 & 89 & $1.25 \mathrm{~A} / \mathrm{D}+0.75 \mathrm{D}$ & 38 & 58 & 0 & 58 & 35 & 58 \\
\hline JFK & $2 A / D$ & 77 & 38 & 0 & 80 & 64 & 64 & $2 A / D$ & 56 & 45 & 0 & 80 & 54 & 54 \\
\hline JNU & $1 \mathrm{~A} / \mathrm{D}$ & 44 & 31 & 0 & 60 & 38 & 38 & $1 \mathrm{~A} / \mathrm{D}$ & 30 & 27 & 0 & 60 & 30 & 30 \\
\hline LAN & $\begin{array}{l}1 \mathrm{~A} / \mathrm{D}+1.25 \\
\mathrm{Dmax}\end{array}$ & 41 & 19 & 0 & 75 & 33 & 33 & $1 \mathrm{~A} / \mathrm{D}$ & 31 & 21 & 0 & 60 & 28 & 28 \\
\hline LAS & $1 \mathrm{~A} / \mathrm{D}+1 \mathrm{~A}$ & 83 & 19 & 42 & 56 & 75 & 33 & $1.1 \mathrm{~A}+1 \mathrm{D}$ & 34 & 56 & 34 & 56 & 34 & 56 \\
\hline LAX & $2 A+2 D$ & 80 & 102 & 80 & 102 & 80 & 102 & $2 A+2 D$ & 58 & 102 & 58 & 102 & 58 & 102 \\
\hline LGA & $1 \mathrm{~A}+1 \mathrm{D}$ & 42 & 50 & 42 & 50 & 42 & 50 & $1 A+1 D$ & 31 & 50 & 31 & 50 & 31 & 50 \\
\hline
\end{tabular}


Table B-2. 110-Airport NextGen Capacities in 2015

\begin{tabular}{|c|c|c|c|c|c|c|c|c|c|c|c|c|c|c|}
\hline \multirow[b]{2}{*}{ Apt } & \multicolumn{7}{|c|}{ Visual meteorological conditions } & \multicolumn{7}{|c|}{ Instrument meteorological conditions } \\
\hline & Configuration & Amax & Aeq & Amin & Dmax & Deq & Free & Configuration & Amax & Amin & Aeq & Dmax & Deq & Free \\
\hline LGB & $\begin{array}{l}1 \mathrm{~A} / \mathrm{D} \text { with } \\
\text { alternating } 1.5 \\
\text { Amax } 1.5 \mathrm{Dmax} \\
0.5 \text { Free }\end{array}$ & 67 & 17 & 0 & 90 & 33 & 53 & $1 \mathrm{~A} / \mathrm{D}$ & 29 & 25 & 0 & 58 & 29 & 29 \\
\hline LIT & $\begin{array}{l}\text { 2A/D with Dmax } \\
\text { limit }\end{array}$ & 82 & 35 & 0 & 80 & 65 & 65 & $1 \mathrm{~A} / \mathrm{D}$ & 32 & 21 & 0 & 60 & 28 & 28 \\
\hline $\mathrm{MCl}$ & $2 \mathrm{~A} / \mathrm{D}$ & 82 & 35 & 0 & 120 & 65 & 65 & $2 A / D$ & 63 & 42 & 0 & 120 & 56 & 56 \\
\hline $\mathrm{MCO}$ & $3 \mathrm{~A} / \mathrm{D}+0.5 \mathrm{D}$ & 122 & 87 & 0 & 188 & 100 & 127 & 1CSP + 1Dep & 83 & 79 & 0 & 140 & 83 & 83 \\
\hline MDW & $1.25 \mathrm{~A} / \mathrm{D}$ & 53 & 24 & 0 & 73 & 42 & 42 & $1 \mathrm{~A} / \mathrm{D}$ & 31 & 24 & 0 & 58 & 29 & 29 \\
\hline MEM & $\begin{array}{l}1 \mathrm{~A} / \mathrm{D}+1.75 \mathrm{~A}+ \\
1 \mathrm{D}\end{array}$ & 112 & 72 & 71 & 106 & 104 & 86 & $1 A+1 D+1 A / D$ & 60 & 75 & 30 & 106 & 58 & 81 \\
\hline MHT & $1 A+1 D$ & 41 & 60 & 41 & 60 & 41 & 60 & $1 \mathrm{~A} / \mathrm{D}$ & 32 & 21 & 0 & 60 & 28 & 28 \\
\hline MIA & $2 A+2 D$ & 79 & 101 & 79 & 101 & 79 & 101 & $2 A / D$ & 58 & 46 & 0 & 101 & 56 & 56 \\
\hline MKE & $2 A / D$ & 83 & 48 & 0 & 118 & 69 & 69 & $2 A / D$ & 60 & 48 & 0 & 118 & 57 & 57 \\
\hline MLB & $1.5 \mathrm{~A} / \mathrm{D}+0.25 \mathrm{D}$ & 63 & 50 & 0 & 105 & 51 & 66 & $1 \mathrm{~A} / \mathrm{D}$ & 30 & 24 & 0 & 60 & 29 & 29 \\
\hline MSN & $1 \mathrm{~A} / \mathrm{D}$ & 41 & 20 & 0 & 60 & 33 & 33 & $1 \mathrm{~A} / \mathrm{D}$ & 31 & 22 & 0 & 60 & 28 & 28 \\
\hline MSP & $\begin{array}{l}2 \mathrm{~A} / \mathrm{D}+ \\
\text { alternating } 1 \\
\text { Amax and } 1 \\
\text { Dmax }\end{array}$ & 126 & 33 & 0 & 169 & 63 & 101 & Dep Pair +1D & 53 & 109 & 0 & 145 & 53 & 110 \\
\hline MSY & 1.2 A/1Dmax & 49 & 21 & 0 & 59 & 39 & 39 & 1.2 A/1Dmax & 38 & 24 & 0 & 59 & 34 & 34 \\
\hline OAK & $1.5 \mathrm{~A} / \mathrm{D}$ & 62 & 40 & 0 & 88 & 53 & 53 & $1 \mathrm{~A} / \mathrm{D}$ & 30 & 23 & 0 & 57 & 28 & 28 \\
\hline OKC & $2 A / D$ & 88 & 64 & 0 & 120 & 77 & 77 & $2 A / D$ & 60 & 55 & 0 & 120 & 60 & 60 \\
\hline OMA & $2 \mathrm{~A} / 1 \mathrm{Dmax}$ & 88 & 32 & 0 & 60 & 77 & 39 & $1.5 \mathrm{~A} / 1 \mathrm{Dmax}$ & 45 & 28 & 0 & 60 & 45 & 30 \\
\hline ONT & $1.75 \mathrm{~A} / \mathrm{D}$ & 71 & 46 & 0 & 100 & 61 & 61 & $1 \mathrm{~A} / \mathrm{D}$ & 29 & 23 & 0 & 56 & 28 & 28 \\
\hline ORD & $1 A / D+2 A+2 D$ & 124 & 126 & 83 & 164 & 115 & 142 & $1 A / D+2 A+2 D$ & 93 & 131 & 62 & 164 & 91 & 138 \\
\hline ORF & $\begin{array}{l}1 \mathrm{~A} / \mathrm{D}+1.25 \\
\mathrm{Dmax}\end{array}$ & 44 & 32 & 0 & 75 & 39 & 39 & $1 \mathrm{~A} / \mathrm{D}$ & 30 & 28 & 0 & 60 & 30 & 30 \\
\hline OXR & $1 \mathrm{~A} / \mathrm{D}$ & 46 & 35 & 0 & 60 & 41 & 60 & $1 \mathrm{~A} / \mathrm{D}$ & 30 & 29 & 0 & 60 & 30 & 30 \\
\hline $\mathrm{PBI}$ & $1.5 \mathrm{~A} / \mathrm{D}$ & 63 & 43 & 0 & 89 & 55 & 55 & $1 \mathrm{~A} / \mathrm{D}$ & 29 & 25 & 0 & 58 & 29 & 29 \\
\hline PDX & $2 A / D$ & 84 & 40 & 0 & 114 & 68 & 68 & $2 \mathrm{~A} / \mathrm{D}$ & 61 & 48 & 0 & 114 & 58 & 58 \\
\hline PHF & $\begin{array}{l}1 \mathrm{~A} / \mathrm{D} \text { alternate } \\
\text { with } 0.25 \mathrm{Dmax}\end{array}$ & 44 & 32 & 0 & 75 & 22 & 53 & $1 \mathrm{~A} / \mathrm{D}$ & 30 & 28 & 0 & 60 & 30 & 30 \\
\hline $\mathrm{PHL}$ & $2 \mathrm{~A} / \mathrm{D}$ & 84 & 36 & 0 & 113 & 67 & 67 & $2 \mathrm{~A} / \mathrm{D}$ & 62 & 46 & 0 & 113 & 58 & 58 \\
\hline $\mathrm{PHX}$ & $1 A+1 D+1 A / D$ & 85 & 74 & 42 & 116 & 75 & 91 & $2 A / D$ & 64 & 44 & 0 & 116 & 58 & 58 \\
\hline PIE & $\begin{array}{l}1 \mathrm{~A} / \mathrm{D}+0.75 \mathrm{~A}+ \\
0.5 \mathrm{D}\end{array}$ & 76 & 62 & 33 & 90 & 71 & 68 & $1 \mathrm{~A} / \mathrm{D}$ & 30 & 28 & 0 & 60 & 30 & 30 \\
\hline PIT & $1 A+1 D+1 A / D$ & 86 & 77 & 43 & 118 & 77 & 93 & $2 \mathrm{~A} / \mathrm{D}$ & 63 & 47 & 0 & 118 & 59 & 59 \\
\hline PVD & $1 \mathrm{~A} / \mathrm{D}$ & 42 & 25 & 0 & 59 & 35 & 35 & $1 \mathrm{~A} / \mathrm{D}$ & 30 & 23 & 0 & 59 & 28 & 28 \\
\hline RDU & $2 A / D$ & 82 & 35 & 0 & 119 & 65 & 65 & $\begin{array}{l}\text { Dep pair - with } \\
1.5^{*} \mathrm{Dmax} \mathrm{Sgl}\end{array}$ & 52 & 52 & 0 & 89 & 52 & 52 \\
\hline RFD & $1.75 \mathrm{~A} / \mathrm{D}$ & 73 & 58 & 0 & 103 & 68 & 68 & $1.25 \mathrm{~A} / \mathrm{D}$ & 33 & 32 & 0 & 78 & 35 & 35 \\
\hline RIC & $1.25 \mathrm{~A} / \mathrm{D}$ & 55 & 40 & 0 & 75 & 48 & 48 & $1 \mathrm{~A} / \mathrm{D}$ & 30 & 28 & 0 & 60 & 30 & 30 \\
\hline RNO & $1.5 \mathrm{~A} / \mathrm{D}$ & 61 & 29 & 0 & 88 & 49 & 49 & $1 \mathrm{~A} / \mathrm{D}$ & 31 & 22 & 0 & 59 & 28 & 28 \\
\hline ROC & $1.25 \mathrm{~A} / \mathrm{D}$ & 52 & 23 & 0 & 75 & 41 & 41 & $1 \mathrm{~A} / \mathrm{D}$ & 31 & 21 & 0 & 60 & 28 & 28 \\
\hline RSW & $1 \mathrm{~A} / \mathrm{D}$ & 40 & 20 & 0 & 56 & 33 & 33 & $1 \mathrm{~A} / \mathrm{D}$ & 30 & 22 & 0 & 56 & 28 & 28 \\
\hline SAN & $1 \mathrm{~A} / \mathrm{D}$ & 42 & 18 & 0 & 57 & 33 & 33 & $1 \mathrm{~A} / \mathrm{D}$ & 31 & 23 & 0 & 57 & 29 & 29 \\
\hline SAT & $2 A / D$ & 85 & 55 & 0 & 119 & 72 & 72 & $1 \mathrm{~A} / \mathrm{D}$ & 30 & 24 & 0 & 59 & 29 & 29 \\
\hline SBA & $\begin{array}{l}1 \mathrm{~A} / \mathrm{D}+0.25 \mathrm{~A}+ \\
0.25 \mathrm{D}\end{array}$ & 53 & 40 & 11 & 75 & 45 & 50 & $1 A+1 D$ & 30 & 60 & 30 & 60 & 30 & 60 \\
\hline SDF & $2 A / D$ & 82 & 34 & 0 & 120 & 64 & 64 & $2 A / D$ & 63 & 41 & 0 & 120 & 56 & 56 \\
\hline SEA & $2 A / D$ & 84 & 34 & 0 & 112 & 66 & 66 & Dep pair & 53 & 26 & 0 & 86 & 53 & 53 \\
\hline SFO & $2 A / D$ & 76 & 48 & 0 & 103 & 66 & 66 & $1 \mathrm{~A} / \mathrm{D}+0.5 \mathrm{D}$ & 29 & 50 & 0 & 77 & 28 & 54 \\
\hline SJC & $\begin{array}{l}1 A+1 D \\
+0.25 A / D\end{array}$ & 52 & 65 & 41 & 73 & 50 & 67 & CSP w/Sgl Dmax & 31 & 30 & 0 & 58 & 31 & 31 \\
\hline SLC & $\begin{array}{l}\text { 2A/D with } \\
2.25 \mathrm{Amax}\end{array}$ & 95 & 42 & 0 & 113 & 79 & 69 & $2 \mathrm{~A} / \mathrm{D}$ with $2.25 \mathrm{Amax}$ & 68 & 49 & 0 & 113 & 66 & 58 \\
\hline SMF & $2 A / D$ & 82 & 36 & 0 & 118 & 65 & 65 & $2 A / D$ & 62 & 42 & 0 & 118 & 56 & 56 \\
\hline SNA & $1.5 \mathrm{~A} / \mathrm{D}$ & 65 & 46 & 0 & 90 & 56 & 56 & $1 \mathrm{~A} / \mathrm{D}$ & 30 & 24 & 0 & 59 & 29 & 29 \\
\hline STL & $1 A+1 D+1 A / D$ & 81 & 77 & 41 & 115 & 73 & 90 & $2 A / D$ & 61 & 43 & 0 & 115 & 56 & 56 \\
\hline SWF & $1 \mathrm{~A} / \mathrm{D}$ & 44 & 32 & 0 & 60 & 39 & 39 & $1 \mathrm{~A} / \mathrm{D}$ & 30 & 24 & 0 & 58 & 29 & 29 \\
\hline SYR & $1.3 \mathrm{~A} / \mathrm{D}$ & 57 & 41 & 0 & 78 & 50 & 50 & $1 \mathrm{~A} / \mathrm{D}$ & 30 & 28 & 0 & 60 & 30 & 30 \\
\hline
\end{tabular}


Table B-2. 110-Airport NextGen Capacities in 2015

\begin{tabular}{|c|c|c|c|c|c|c|c|c|c|c|c|c|c|c|}
\hline \multirow[b]{2}{*}{ Apt } & \multicolumn{7}{|c|}{ Visual meteorological conditions } & \multicolumn{7}{|c|}{ Instrument meteorological conditions } \\
\hline & Configuration & Amax & Aeq & Amin & Dmax & Deq & Free & Configuration & Amax & Amin & Aeq & Dmax & Deq & Free \\
\hline TEB & $1 \mathrm{~A} / \mathrm{D}+0.5 \mathrm{D}$ & 44 & 62 & 0 & 90 & 39 & 68 & $1 \mathrm{~A} / \mathrm{D}$ & 30 & 28 & 0 & 60 & 30 & 30 \\
\hline TPA & $\begin{array}{l}\text { 2A/D with Dmax } \\
\text { limit }\end{array}$ & 84 & 41 & 0 & 65 & 68 & 65 & 2A/D with Dmax limit & 61 & 48 & 0 & 65 & 58 & 65 \\
\hline TUL & $1.6 \mathrm{~A} / \mathrm{D}$ & 70 & 51 & 0 & 96 & 62 & 62 & \begin{tabular}{|l|}
$1.5 \mathrm{~A} / \mathrm{D}$ \\
\end{tabular} & 45 & 42 & 0 & 90 & 45 & 45 \\
\hline TUS & $2 A / D$ & 87 & 60 & 0 & 120 & 75 & 75 & $1 \mathrm{~A} / \mathrm{D}+0.5 \mathrm{D}$ & 31 & 53 & 0 & 90 & 28 & 58 \\
\hline TVC & $\begin{array}{l}1.25 \mathrm{~A} / \mathrm{D} \text { with } \\
\text { interpolated } \mathrm{E}\end{array}$ & 55 & 40 & 0 & 90 & 39 & 60 & $1 \mathrm{~A} / \mathrm{D}$ & 30 & 28 & 0 & 60 & 30 & 30 \\
\hline TYS & $1.25 \mathrm{~A} / \mathrm{D}$ & 55 & 40 & 0 & 75 & 48 & 48 & $1 \mathrm{~A} / \mathrm{D}$ & 30 & 28 & 0 & 60 & 30 & 30 \\
\hline VNY & $1.25 \mathrm{~A} / \mathrm{D}$ & 55 & 40 & 0 & 75 & 48 & 48 & $1 \mathrm{~A} / \mathrm{D}+0.25 \mathrm{D}$ & 30 & 43 & 0 & 75 & 30 & 45 \\
\hline
\end{tabular}

Table B-3. 110-Airport NextGen Capacities in 2025

\begin{tabular}{|c|c|c|c|c|c|c|c|c|c|c|c|c|c|c|}
\hline \multirow[b]{2}{*}{ Apt } & \multicolumn{7}{|c|}{ Visual meteorological conditions } & \multicolumn{7}{|c|}{ Instrument meteorological conditions } \\
\hline & Configuration & Amax & Aeq & Amin & Dmax & Deq & Free & Configuration & Amax & Amin & Aeq & Dmax & Deq & Free \\
\hline$A B Q$ & $\begin{array}{l}1.5 \mathrm{~A} / \mathrm{D} \text { with } \\
2 \mathrm{Amax} \text { with } 1 \\
\text { Free }\end{array}$ & 104 & 13 & 0 & 90 & 61 & 61 & 1.5 A/D with $1 \mathrm{Dmax}$ & 78 & 10 & 0 & 59 & 57 & 57 \\
\hline ALB & $1 \mathrm{~A} / \mathrm{D}$ & 54 & 4 & 0 & 60 & 38 & 38 & $1 \mathrm{~A} / 0.5 \mathrm{D}$ & 54 & 2 & 0 & 30 & 38 & 19 \\
\hline ANC & $\begin{array}{l}1.25 \mathrm{~A} / \mathrm{D} \text { with } \\
1.5^{\star} \mathrm{Dmax}\end{array}$ & 59 & 15 & 0 & 81 & 47 & 47 & $\begin{array}{l}\text { A/D with } \\
1.25^{*} \mathrm{Dmax}\end{array}$ & 47 & 10 & 0 & 67 & 38 & 38 \\
\hline ATL & $2 A+2 D+1 A / D$ & 144 & 110 & 96 & 151 & 132 & 137 & $2 A+2 D+1 A / D$ & 144 & 108 & 96 & 151 & 132 & 137 \\
\hline AUS & $1.5 \mathrm{~A} / \mathrm{D}$ & 78 & 19 & 0 & 90 & 61 & 61 & $1.5 \mathrm{~A} / \mathrm{D}$ & 75 & 10 & 0 & 85 & 55 & 55 \\
\hline BDL & $\begin{array}{l}\text { !.5Amax with } 0.5 \\
\text { Free alternate } \\
\text { with } 1.25 \mathrm{Dmax}\end{array}$ & 74 & 6 & 0 & 72 & 37 & 39 & $\begin{array}{l}\text { !.5Amax with } 0.5 \\
\text { Free alternate with } \\
\text { 1.25Dmax }\end{array}$ & 75 & 4 & 0 & 70 & 37 & 37 \\
\hline $\mathrm{BFL}$ & $1 \mathrm{~A} / \mathrm{Dep} \mathrm{D}$ & 52 & 12 & 0 & 87 & 41 & 41 & $1 \mathrm{~A} / \mathrm{D}$ & 52 & 11 & 0 & 60 & 41 & 41 \\
\hline BHM & $\begin{array}{l}1 \mathrm{~A} / \mathrm{D} \text { with } 1.25 \\
\text { Amax } 1.25 \\
\text { Dmax } 0.5 \text { Free }\end{array}$ & 65 & 6 & 0 & 75 & 41 & 41 & $1 \mathrm{~A} / \mathrm{D}$ & 52 & 11 & 0 & 60 & 41 & 41 \\
\hline BNA & $\begin{array}{l}0.75 A+1 D \\
+1 A / D \\
\end{array}$ & 97 & 61 & 42 & 120 & 78 & 96 & $1 A / D+1.5 A+0.5 D$ & 139 & 31 & 83 & 90 & 120 & 66 \\
\hline $\mathrm{BOI}$ & $\begin{array}{l}\text { 1.75A/D with } \\
\text { 2Dmax }\end{array}$ & 93 & 8 & 0 & 120 & 66 & 66 & $1 \mathrm{~A} / \mathrm{D}$ & 53 & 5 & 0 & 60 & 38 & 38 \\
\hline BOS & $\begin{array}{l}\text { 2A/D with } 1 \\
\text { Amin }\end{array}$ & 98 & 19 & 49 & 111 & 75 & 75 & $2 A / D$ & 98 & 17 & 0 & 111 & 75 & 75 \\
\hline BTR & $\begin{array}{l}1.5 \mathrm{~A} \text { alternate } \\
\text { with } 1.5 \mathrm{D}\end{array}$ & 81 & 17 & 0 & 90 & 41 & 54 & $1 \mathrm{~A} / \mathrm{D}$ & 54 & 14 & 0 & 60 & 44 & 44 \\
\hline BUF & 1.5 A/1Dmax & 83 & 1 & 0 & 60 & 55 & 55 & $1 \mathrm{~A} / \mathrm{D}$ & 56 & 1 & 0 & 60 & 36 & 36 \\
\hline BUR & $1.25 \mathrm{~A} / \mathrm{D}$ & 69 & 17 & 0 & 75 & 52 & 52 & $1 \mathrm{~A} / \mathrm{D}$ & 52 & 6 & 0 & 60 & 38 & 38 \\
\hline BWI & $1.75 \mathrm{~A} / \mathrm{D}+1 \mathrm{~A}$ & 142 & 10 & 52 & 99 & 116 & 65 & $1.75 \mathrm{~A} / \mathrm{D}+1 \mathrm{~A}$ & 142 & 9 & 52 & 99 & 116 & 65 \\
\hline $\mathrm{CHS}$ & $\begin{array}{l}1.75^{*} \mathrm{~A} \text { alternate } \\
\text { with } 2 \mathrm{D}\end{array}$ & 97 & 0 & 0 & 120 & 48 & 60 & $1 \mathrm{~A} / \mathrm{D}$ & 55 & 1 & 0 & 60 & 37 & 37 \\
\hline CLE & $2 \mathrm{~A} / \mathrm{D}$ & 108 & 5 & 0 & 119 & 74 & 74 & $1.25 \mathrm{~A}+1 \mathrm{D}$ & 68 & 59 & 68 & 59 & 68 & 59 \\
\hline $\mathrm{CLT}$ & $3 \mathrm{~A} / \mathrm{D}$ & 158 & 13 & 0 & 175 & 111 & 111 & $3 \mathrm{~A} / \mathrm{D}$ & 158 & 13 & 0 & 175 & 111 & 111 \\
\hline $\mathrm{CMH}$ & $1 A / D+1 A+1 D$ & 111 & 60 & 56 & 120 & 92 & 96 & $2 \mathrm{~A} / \mathrm{D}$ & 111 & 1 & 0 & 120 & 73 & 73 \\
\hline $\cos$ & $\begin{array}{l}1.5 \mathrm{~A} \text { alternate } \\
\text { with } 2 \mathrm{D} \text { with } \\
\text { Free } \mathrm{D}\end{array}$ & 82 & 2 & 0 & 120 & 41 & 61 & $1 \mathrm{~A} / \mathrm{D}$ & 55 & 2 & 0 & 60 & 37 & 37 \\
\hline CRP & $\begin{array}{l}1.5 \mathrm{~A} \text { alternate } \\
\text { with } 1.5 \mathrm{Dmax} \\
\text { with } 0.75 \mathrm{Amin} \\
\end{array}$ & 83 & 1 & 42 & 90 & 63 & 45 & $1 \mathrm{~A} / \mathrm{D}$ & 56 & 1 & 0 & 60 & 36 & 36 \\
\hline CVG & $\begin{array}{l}2 \mathrm{~A} / \mathrm{D}+1 \mathrm{~A}+ \\
0.5 \mathrm{Dmax}\end{array}$ & 157 & 10 & 52 & 144 & 126 & 74 & $2 A / D+1 A$ & 157 & 9 & 52 & 115 & 126 & 74 \\
\hline DAB & $\begin{array}{l}1.75 \mathrm{~A} \text { alternate } \\
\text { with } 2 \mathrm{Dmax}\end{array}$ & 92 & 21 & 0 & 120 & 46 & 70 & $1 \mathrm{~A} / \mathrm{D}$ & 52 & 10 & 0 & 60 & 40 & 40 \\
\hline DAL & $1 A+1 D$ & 55 & 59 & 55 & 59 & 55 & 59 & $1 A+1 D$ & 55 & 59 & 55 & 59 & 55 & 59 \\
\hline DAY & $2 A / D$ & 109 & 4 & 0 & 117 & 72 & 72 & $2 A / D$ & 109 & 4 & 0 & 117 & 72 & 72 \\
\hline$\overline{\mathrm{DCA}}$ & $1.25 \mathrm{~A}+1 \mathrm{D}$ & 69 & 60 & 69 & 60 & 69 & 60 & $1.25 \mathrm{~A} / 1 \mathrm{D}$ & 69 & 1 & 0 & 60 & 46 & 46 \\
\hline DEN & $2 A+2 D+2 A / D$ & 199 & 130 & 99 & 225 & 175 & 188 & $2 A+2 D+2 A / D$ & 199 & 129 & 99 & 225 & 175 & 188 \\
\hline DFW & $2 A+2 D+2 A / D$ & 206 & 122 & 103 & 223 & 176 & 184 & $2 A+2 D+2 A / D$ & 206 & 121 & 103 & 223 & 176 & 184 \\
\hline
\end{tabular}


Table B-3. 110-Airport NextGen Capacities in 2025

\begin{tabular}{|c|c|c|c|c|c|c|c|c|c|c|c|c|c|c|}
\hline \multirow[b]{2}{*}{ Apt } & \multicolumn{7}{|c|}{ Visual meteorological conditions } & \multicolumn{7}{|c|}{ Instrument meteorological conditions } \\
\hline & Configuration & Amax & Aeq & Amin & Dmax & Deq & Free & Configuration & Amax & Amin & Aeq & Dmax & Deq & Free \\
\hline DSM & $\begin{array}{l}\text { 1A/1D with } 0.75 \\
\text { Amin }\end{array}$ & 55 & 1 & 41 & 60 & 48 & 31 & $1 \mathrm{~A} / \mathrm{D}$ & 55 & 1 & 0 & 60 & 37 & 37 \\
\hline DTW & $2 A+1 D+1 A / D$ & 158 & 60 & 105 & 113 & 141 & 93 & $2 A+2 D$ & 105 & 113 & 105 & 113 & 105 & 113 \\
\hline ELP & $1 A+1 D$ & 56 & 60 & 56 & 60 & 56 & 60 & $1 \mathrm{~A} / \mathrm{D}$ & 56 & 0 & 0 & 60 & 36 & 36 \\
\hline EUG & $1 \mathrm{~A} / \mathrm{D}$ & 53 & 6 & 0 & 60 & 38 & 38 & $1 \mathrm{~A} / \mathrm{D}$ & 53 & 6 & 0 & 60 & 38 & 38 \\
\hline EWR & $\begin{array}{l}1 A+1 D+0.25 \\
A / D \text { with Dmax } \\
\text { limit }\end{array}$ & 62 & 53 & 50 & 53 & 59 & 53 & $1 A+1 D$ & 50 & 7 & 50 & 53 & 50 & 53 \\
\hline FAT & $\begin{array}{l}\text { 1.5 A/D with } \\
1.5 \mathrm{Dmax}\end{array}$ & 78 & 12 & 0 & 89 & 58 & 58 & $1 \mathrm{~A} / \mathrm{D}$ & 52 & 8 & 0 & 60 & 39 & 39 \\
\hline FLL & $2 A / D$ & 98 & 21 & 0 & 113 & 76 & 76 & $2 A / D$ & 98 & 18 & 0 & 113 & 76 & 76 \\
\hline FNT & $1.25 \mathrm{~A} / \mathrm{D}$ & 62 & 5 & 0 & 75 & 47 & 47 & $1 \mathrm{~A} / 1.25 \mathrm{D}$ & 54 & 5 & 0 & 75 & 38 & 47 \\
\hline FXE & $1 \mathrm{~A} / 1.4 \mathrm{Dmax}$ & 54 & 17 & 0 & 84 & 44 & 44 & $1 \mathrm{~A} / \mathrm{D}$ & 54 & 14 & 0 & 60 & 44 & 44 \\
\hline GFK & $1.25 \mathrm{~A} / \mathrm{D}$ & 68 & 3 & 0 & 75 & 46 & 46 & $1 \mathrm{~A} / \mathrm{D}$ & 54 & 3 & 0 & 60 & 37 & 37 \\
\hline GRR & $1.75 \mathrm{~A} / \mathrm{D}$ & 95 & 4 & 0 & 105 & 65 & 65 & $1 \mathrm{~A} / \mathrm{D}$ & 54 & 3 & 0 & 60 & 37 & 37 \\
\hline GSO & $2 A / D$ & 111 & 2 & 0 & 120 & 73 & 73 & $2 A / D$ & 111 & 2 & 0 & 120 & 73 & 73 \\
\hline GYY & $\begin{array}{l}1.5 \mathrm{~A} / 1.0 \text { Free } \\
\text { alternate } \\
1.5 \mathrm{Dmax}\end{array}$ & 84 & 10 & 0 & 90 & 42 & 50 & $1 \mathrm{~A} / \mathrm{D}$ & 58 & 13 & 0 & 65 & 47 & 47 \\
\hline $\mathrm{HNL}$ & $0.5 \mathrm{~A} / 2 \mathrm{D}+1 \mathrm{~A}$ & 70 & 21 & 47 & 101 & 65 & 71 & $1 A+1 D$ & 47 & 10 & 47 & 50 & 47 & 61 \\
\hline $\mathrm{HOU}$ & $1 \mathrm{~A} / 1.5 \mathrm{D}, \mathrm{E} \& \mathrm{~F}$ & 52 & 14 & 0 & 89 & 39 & 59 & $1 \mathrm{~A} / \mathrm{D}+0.5 \mathrm{Dmax}$ & 52 & 38 & 0 & 89 & 52 & 69 \\
\hline HPN & $\begin{array}{l}1.15 \mathrm{~A} / 1.25 \mathrm{D} 1 \mathrm{~F}, \\
1 \mathrm{E}\end{array}$ & 61 & 15 & 0 & 75 & 43 & 49 & $1 \mathrm{~A} / \mathrm{D}$ & 53 & 13 & 0 & 60 & 43 & 43 \\
\hline $\mathrm{IAD}$ & $2 A / D+1 A+1 D$ & 150 & 64 & 50 & 168 & 124 & 131 & $2 A / D+1 D$ & 100 & 71 & 0 & 168 & 74 & 131 \\
\hline $\mathrm{IAH}$ & $2 A / D+1 A+1 D$ & 159 & 61 & 53 & 171 & 126 & 130 & $2 A / D+1 A+1 D$ & 159 & 61 & 53 & 171 & 126 & 130 \\
\hline ICT & $\begin{array}{l}1 \mathrm{~A} / \mathrm{D}+0.5 \mathrm{~A} \\
\text { alternate with } \\
0.75 \mathrm{D}\end{array}$ & 83 & 46 & 28 & 105 & 56 & 75 & $0.25 A / 1.25 D+1 A$ & 70 & 1 & 56 & 75 & 63 & 66 \\
\hline IND & $2 A / D+1 D$ & 109 & 62 & 0 & 176 & 72 & 131 & $2 A / D+1 D$ & 109 & 62 & 0 & 176 & 72 & 131 \\
\hline ISP & $\begin{array}{l}\text { 1A/D + 1Amax + } \\
0.5 \mathrm{Dmax}\end{array}$ & 109 & 18 & 0 & 90 & 99 & 45 & $1 \mathrm{~A} / \mathrm{D}$ & 53 & 7 & 0 & 60 & 39 & 39 \\
\hline JAX & $2 \mathrm{~A} / \mathrm{D}$ & 106 & 8 & 0 & 114 & 73 & 73 & $2 A / D$ & 106 & 7 & 0 & 114 & 73 & 73 \\
\hline JFK & $2 A / D$ & 87 & 25 & 0 & 80 & 70 & 70 & $2 A / D$ & 87 & 23 & 0 & 80 & 70 & 70 \\
\hline JNU & $1 \mathrm{~A} / \mathrm{D}$ & 53 & 16 & 0 & 60 & 43 & 43 & $1 \mathrm{~A} / \mathrm{D}$ & 53 & 14 & 0 & 60 & 43 & 43 \\
\hline LAN & \begin{tabular}{|l}
$1 \mathrm{~A} / \mathrm{D}+1.25$ \\
$\mathrm{Dmax}$ \\
\end{tabular} & 55 & 2 & 0 & 75 & 37 & 37 & $1 \mathrm{~A} / \mathrm{D}$ & 55 & 2 & 0 & 60 & 37 & 37 \\
\hline LAS & $1 A / D+1 A$ & 101 & 7 & 51 & 56 & 88 & 37 & $1.1 \mathrm{~A}+1 \mathrm{D}$ & 56 & 56 & 56 & 56 & 56 & 56 \\
\hline LAX & $2 A+2 D$ & 93 & 103 & 93 & 103 & 93 & 103 & $2 A+2 D$ & 93 & 103 & 93 & 103 & 93 & 103 \\
\hline LGA & $1 A+1 D$ & 53 & 58 & 53 & 58 & 53 & 57 & $1 A+1 D$ & 53 & 58 & 53 & 58 & 53 & 57 \\
\hline LGB & $\begin{array}{l}1 \mathrm{~A} / \mathrm{D} \text { with } \\
\text { alternating } 1.5 \\
\text { Amax } 1.5 \mathrm{Dmax} \\
0.5 \text { Free } \\
\end{array}$ & 82 & 10 & 0 & 90 & 41 & 50 & $1 \mathrm{~A} / \mathrm{D}$ & 50 & 10 & 0 & 58 & 39 & 39 \\
\hline LIT & $\begin{array}{l}\text { 2A/D with Dmax } \\
\text { limit }\end{array}$ & 111 & 1 & 0 & 80 & 73 & 73 & $1 \mathrm{~A} / \mathrm{D}$ & 56 & 1 & 0 & 60 & 36 & 36 \\
\hline $\mathrm{MCl}$ & $2 \mathrm{~A} / \mathrm{D}$ & 110 & 2 & 0 & 120 & 73 & 73 & $2 A / D$ & 110 & 2 & 0 & 120 & 73 & 73 \\
\hline $\mathrm{MCO}$ & $3 \mathrm{~A} / \mathrm{D}+0.5 \mathrm{D}$ & 146 & 55 & 0 & 189 & 111 & 138 & 1CSP + 1Dep & 127 & 80 & 0 & 145 & 122 & 122 \\
\hline MDW & $1.25 \mathrm{~A} / \mathrm{D}$ & 65 & 7 & 0 & 73 & 47 & 47 & $1 \mathrm{~A} / \mathrm{D}$ & 52 & 6 & 0 & 58 & 38 & 38 \\
\hline MEM & $\begin{array}{l}1 \mathrm{~A} / \mathrm{D}+1.75 \mathrm{~A}+ \\
1 \mathrm{D}\end{array}$ & 133 & 63 & 84 & 106 & 121 & 90 & $1 A+1 D+1 A / D$ & 96 & 62 & 48 & 106 & 85 & 90 \\
\hline$\overline{\mathrm{MHT}}$ & $1 A+1 D$ & 55 & 60 & 55 & 60 & 55 & 60 & $1 \mathrm{~A} / \mathrm{D}$ & 55 & 1 & 0 & 60 & 37 & 37 \\
\hline MIA & $2 A+2 D$ & 93 & 102 & 93 & 102 & 93 & 102 & $2 A / D$ & 93 & 20 & 0 & 102 & 73 & 73 \\
\hline MKE & $2 A / D$ & 104 & 17 & 0 & 119 & 78 & 78 & $2 A / D$ & 104 & 14 & 0 & 119 & 77 & 77 \\
\hline MLB & $1.5 \mathrm{~A} / \mathrm{D}+0.25 \mathrm{D}$ & 79 & 25 & 0 & 105 & 58 & 73 & $1 \mathrm{~A} / \mathrm{D}$ & 53 & 6 & 0 & 60 & 39 & 39 \\
\hline $\mathrm{MSN}$ & $1 \mathrm{~A} / \mathrm{D}$ & 54 & 3 & 0 & 60 & 37 & 37 & $1 \mathrm{~A} / \mathrm{D}$ & 54 & 3 & 0 & 60 & 37 & 37 \\
\hline MSP & $\begin{array}{l}2 \mathrm{~A} / \mathrm{D}+ \\
\text { alternating } 1 \\
\text { Amax and } 1 \\
\text { Dmax } \\
\end{array}$ & 156 & 9 & 0 & 170 & 78 & 89 & Dep Pair +1D & 79 & 96 & 0 & 147 & 72 & 129 \\
\hline MSY & $2 \mathrm{~A} / \mathrm{D}$ & 110 & 3 & 0 & 117 & 72 & 72 & $2 A / D$ & 110 & 3 & 0 & 117 & 72 & 72 \\
\hline OAK & $1.5 \mathrm{~A} / \mathrm{D}$ & 76 & 19 & 0 & 88 & 60 & 60 & $1 \mathrm{~A} / \mathrm{D}$ & 51 & 6 & 0 & 57 & 37 & 37 \\
\hline OKC & $1 A / D+1 A+1 D$ & 108 & 77 & 54 & 120 & 98 & 104 & $1 A / D+1 A+1 D$ & 108 & 74 & 54 & 120 & 98 & 104 \\
\hline
\end{tabular}


Table B-3. 110-Airport NextGen Capacities in 2025

\begin{tabular}{|c|c|c|c|c|c|c|c|c|c|c|c|c|c|c|}
\hline \multirow[b]{2}{*}{ Apt } & \multicolumn{7}{|c|}{ Visual meteorological conditions } & \multicolumn{7}{|c|}{ Instrument meteorological conditions } \\
\hline & Configuration & Amax & Aeq & Amin & Dmax & Deq & Free & Configuration & Amax & Amin & Aeq & Dmax & Deq & Free \\
\hline OMA & $2 \mathrm{~A} / 1 \mathrm{Dmax}$ & 108 & 17 & 0 & 60 & 88 & 44 & 1.5 A/1Dmax & 81 & 14 & 0 & 60 & 66 & 44 \\
\hline ONT & $1.75 \mathrm{~A} / \mathrm{D}$ & 86 & 22 & 0 & 101 & 69 & 69 & $1 \mathrm{~A} / \mathrm{D}$ & 49 & 8 & 0 & 56 & 38 & 38 \\
\hline ORD & $2 A / D+2 A+2 D$ & 204 & 121 & 102 & 219 & 174 & 182 & $2 A / D+2 A+2 D$ & 204 & 121 & 102 & 219 & 174 & 182 \\
\hline ORF & $1.5 \mathrm{~A} / \mathrm{D}$ & 81 & 26 & 0 & 90 & 66 & 66 & $1 \mathrm{CSP}$ & 54 & 45 & 0 & 92 & 54 & 54 \\
\hline OXR & $1 \mathrm{~A} / \mathrm{D}$ & 57 & 20 & 0 & 60 & 48 & 60 & $1 \mathrm{~A} / \mathrm{D}$ & 57 & 15 & 0 & 60 & 48 & 48 \\
\hline $\mathrm{PBI}$ & $1.5 \mathrm{~A} / \mathrm{D}$ & 77 & 22 & 0 & 89 & 62 & 62 & $1 \mathrm{~A} / \mathrm{D}$ & 50 & 11 & 0 & 58 & 39 & 39 \\
\hline PDX & $2 A / D$ & 101 & 16 & 0 & 114 & 75 & 75 & $2 A / D$ & 101 & 15 & 0 & 114 & 75 & 75 \\
\hline PHF & $\begin{array}{l}1 \mathrm{~A} / \mathrm{D} \text { alternate } \\
\text { with } 0.25 \mathrm{Dmax}\end{array}$ & 54 & 17 & 0 & 75 & 27 & 46 & $1 \mathrm{~A} / \mathrm{D}$ & 54 & 14 & 0 & 60 & 44 & 44 \\
\hline $\mathrm{PHL}$ & $2 A / D$ & 102 & 13 & 0 & 113 & 74 & 74 & $2 A / D$ & 102 & 12 & 0 & 113 & 74 & 74 \\
\hline $\mathrm{PHX}$ & $1 A+1 D+1 A / D$ & 107 & 61 & 53 & 116 & 90 & 95 & $2 A / D$ & 107 & 6 & 0 & 116 & 73 & 73 \\
\hline PIE & $\begin{array}{l}1 A / D+0.75 A+ \\
0.5 D\end{array}$ & 93 & 48 & 40 & 90 & 84 & 74 & $1 \mathrm{~A} / \mathrm{D}$ & 53 & 14 & 0 & 60 & 44 & 44 \\
\hline PIT & $1 A+1 D+1 A / D$ & 105 & 64 & 53 & 118 & 90 & 97 & $2 A / D$ & 105 & 10 & 0 & 118 & 75 & 75 \\
\hline PVD & $1 \mathrm{~A} / \mathrm{D}$ & 52 & 9 & 0 & 60 & 39 & 39 & $1 \mathrm{~A} / \mathrm{D}$ & 53 & 5 & 0 & 59 & 38 & 38 \\
\hline RDU & $1 A / D+1 A+1 D$ & 110 & 61 & 55 & 119 & 92 & 96 & $1 A / D+1 A+1 D$ & 110 & 61 & 55 & 119 & 92 & 96 \\
\hline RFD & $1.75 \mathrm{~A} / \mathrm{D}$ & 88 & 35 & 0 & 103 & 78 & 78 & $1.25 \mathrm{~A} / \mathrm{D}$ & 54 & 16 & 0 & 79 & 49 & 49 \\
\hline RIC & $1.25 \mathrm{~A} / \mathrm{D}$ & 68 & 22 & 0 & 75 & 55 & 55 & $1 \mathrm{~A} / \mathrm{D}$ & 54 & 14 & 0 & 60 & 44 & 44 \\
\hline RNO & $1.5 \mathrm{~A} / \mathrm{D}$ & 81 & 4 & 0 & 88 & 55 & 55 & $1 \mathrm{~A} / \mathrm{D}$ & 54 & 3 & 0 & 59 & 37 & 37 \\
\hline $\mathrm{ROC}$ & $1.25 \mathrm{~A} / \mathrm{D}$ & 68 & 2 & 0 & 75 & 46 & 46 & $1 \mathrm{~A} / \mathrm{D}$ & 55 & 2 & 0 & 60 & 37 & 37 \\
\hline RSW & $2 A / D$ & 104 & 10 & 0 & 113 & 73 & 73 & $2 A / D$ & 104 & 9 & 0 & 113 & 73 & 73 \\
\hline SAN & $1 \mathrm{~A} / \mathrm{D}$ & 52 & 6 & 0 & 57 & 37 & 37 & $1 \mathrm{~A} / \mathrm{D}$ & 52 & 6 & 0 & 57 & 37 & 37 \\
\hline SAT & $2 A / D$ & 104 & 25 & 0 & 119 & 81 & 81 & $1 \mathrm{~A} / \mathrm{D}+1 \mathrm{D}$ & 52 & 67 & 0 & 118 & 39 & 98 \\
\hline SBA & $\begin{array}{l}1 A / D+0.25 A+ \\
0.25 D\end{array}$ & 66 & 24 & 13 & 75 & 52 & 54 & $1 A+1 D$ & 52 & 60 & 52 & 60 & 52 & 60 \\
\hline SDF & $2 \mathrm{~A} / \mathrm{D}$ & 112 & 0 & 0 & 120 & 73 & 73 & $2 A / D$ & 112 & 1 & 0 & 120 & 73 & 73 \\
\hline SEA & $2 A / D$ & 104 & 10 & 0 & 113 & 73 & 73 & $2 A / D$ & 104 & 9 & 0 & 113 & 73 & 73 \\
\hline SFO & 2A/D & 80 & 42 & 0 & 103 & 67 & 67 & $1 \mathrm{~A} / \mathrm{D}+0.5 \mathrm{D}$ & 46 & 37 & 0 & 78 & 37 & 63 \\
\hline SJC & $\begin{array}{l}1 \mathrm{~A}+1 \mathrm{D} \\
+0.25 \mathrm{~A} / \mathrm{D} \\
\end{array}$ & 64 & 61 & 51 & 74 & 61 & 69 & CSP w/Sgl Dmax & 53 & 43 & 0 & 58 & 53 & 53 \\
\hline SLC & $\begin{array}{l}\text { 2A/D with } \\
2.25 \mathrm{Amax}\end{array}$ & 112 & 19 & 0 & 114 & 88 & 76 & 2A/D with $2.25 \mathrm{Amax}$ & 112 & 17 & 0 & 114 & 88 & 76 \\
\hline SMF & $2 A / D$ & 109 & 4 & 0 & 118 & 73 & 73 & $2 A / D$ & 109 & 4 & 0 & 118 & 73 & 73 \\
\hline SNA & $1.5 \mathrm{~A} / \mathrm{D}$ & 79 & 24 & 0 & 90 & 64 & 64 & $1 \mathrm{~A} / \mathrm{D}$ & 51 & 8 & 0 & 59 & 39 & 39 \\
\hline STL & $1 A+1 D+1 A / D$ & 106 & 61 & 53 & 116 & 90 & 94 & $2 A / D$ & 106 & 7 & 0 & 116 & 73 & 73 \\
\hline SWF & $1 \mathrm{~A} / \mathrm{D}$ & 54 & 18 & 0 & 60 & 44 & 44 & $1 \mathrm{~A} / \mathrm{D}$ & 51 & 7 & 0 & 59 & 38 & 38 \\
\hline SYR & $1.3 \mathrm{~A} / \mathrm{D}$ & 70 & 23 & 0 & 78 & 57 & 57 & $1 \mathrm{~A} / \mathrm{D}$ & 54 & 14 & 0 & 60 & 44 & 44 \\
\hline TEB & $1 \mathrm{~A} / \mathrm{D}+0.5 \mathrm{D}$ & 54 & 47 & 0 & 90 & 44 & 74 & $1 \mathrm{~A} / \mathrm{D}$ & 54 & 14 & 0 & 60 & 44 & 44 \\
\hline TPA & $\begin{array}{l}1 A / D+1 A+1 D \\
\text { with Dmax limit }\end{array}$ & 101 & 8 & 50 & 80 & 88 & 80 & $\begin{array}{l}\text { 1A/D + 1A+1D with } \\
\text { Dmax limit }\end{array}$ & 101 & 8 & 50 & 80 & 88 & 80 \\
\hline TUL & $3 \mathrm{~A} / \mathrm{D}$ & 161 & 53 & 0 & 180 & 133 & 133 & $2 A / D$ & 108 & 29 & 0 & 120 & 88 & 88 \\
\hline TUS & $2 A / D$ & 106 & 31 & 0 & 120 & 85 & 85 & $1 A / D+0.5 D$ & 54 & 34 & 0 & 90 & 38 & 68 \\
\hline TVC & $\begin{array}{l}1.25 \mathrm{~A} / \mathrm{D} \text { with } \\
\text { interpolated } \mathrm{E}\end{array}$ & 68 & 22 & 0 & 90 & 44 & 55 & $1 \mathrm{~A} / \mathrm{D}$ & 54 & 14 & 0 & 60 & 44 & 44 \\
\hline TYS & $1.25 \mathrm{~A} / \mathrm{D}$ & 68 & 22 & 0 & 75 & 55 & 55 & $1 \mathrm{~A} / \mathrm{D}$ & 54 & 14 & 0 & 60 & 44 & 44 \\
\hline VNY & $1.25 \mathrm{~A} / \mathrm{D}$ & 68 & 22 & 0 & 75 & 55 & 55 & $1 A / D+0.25 D$ & 54 & 29 & 0 & 75 & 44 & 59 \\
\hline
\end{tabular}

Table B-4. Additional 200 Airport Capacities

\begin{tabular}{|c|c|c|c|c|c|c|c|c|c|c|c|c|c|c|}
\hline \multirow[b]{2}{*}{ Apt } & \multicolumn{7}{|c|}{ Visual meteorological conditions } & \multicolumn{7}{|c|}{ Instrument meteorological conditions } \\
\hline & Configuration & Amax & Aeq & Amin & Dmax & Deq & Free & Configuration & Amax & Amin & Aeq & Dmax & Deq & Free \\
\hline $\mathrm{ABE}$ & $1 \mathrm{~A} / \mathrm{D}$ & 44 & 38 & 0 & 60 & 38 & 30 & $1 \mathrm{~A} / \mathrm{D}$ & 30 & 0 & 30 & 60 & 30 & 27 \\
\hline $\mathrm{ACY}$ & $1 \mathrm{~A} / \mathrm{D}+0.5 \mathrm{D}$ & 44 & 39 & 0 & 90 & 68 & 62 & $1 \mathrm{~A} / \mathrm{D}$ & 30 & 0 & 30 & 60 & 30 & 28 \\
\hline AMA & $1 \mathrm{~A} / \mathrm{D}$ & 43 & 37 & 0 & 60 & 37 & 29 & $1 \mathrm{~A} / \mathrm{D}$ & 30 & 0 & 29 & 60 & 29 & 26 \\
\hline AVL & $1 \mathrm{~A} / \mathrm{D}$ & 44 & 38 & 0 & 60 & 38 & 30 & $1 \mathrm{~A} / \mathrm{D}$ & 30 & 0 & 30 & 60 & 30 & 27 \\
\hline AVP & $1 \mathrm{~A} / \mathrm{D}+0.5 \mathrm{D}$ & 43 & 37 & 0 & 90 & 66 & 58 & $1 \mathrm{~A} / \mathrm{D}$ & 30 & 0 & 29 & 60 & 29 & 26 \\
\hline AZO & $1 \mathrm{~A} / \mathrm{D}+0.25 \mathrm{D}$ & 44 & 38 & 0 & 75 & 52 & 45 & $1 \mathrm{~A} / \mathrm{D}$ & 30 & 0 & 30 & 60 & 30 & 27 \\
\hline $\mathrm{BFI}$ & $1 \mathrm{~A} / \mathrm{D}$ & 46 & 41 & 0 & 60 & 41 & 35 & $1 \mathrm{~A} / \mathrm{D}$ & 30 & 0 & 30 & 60 & 30 & 29 \\
\hline $\mathrm{BIL}$ & $1.5 \mathrm{~A} / \mathrm{D}$ & 66 & 58 & 0 & 90 & 58 & 48 & $1 \mathrm{~A} / \mathrm{D}$ & 30 & 0 & 30 & 60 & 30 & 28 \\
\hline
\end{tabular}


Table B-4. Additional 200 Airport Capacities

\begin{tabular}{|c|c|c|c|c|c|c|c|c|c|c|c|c|c|c|}
\hline \multirow[b]{2}{*}{ Apt } & \multicolumn{7}{|c|}{ Visual meteorological conditions } & \multicolumn{7}{|c|}{ Instrument meteorological conditions } \\
\hline & Configuration & Amax & Aeq & Amin & Dmax & Deq & Free & Configuration & Amax & Amin & Aeq & Dmax & Deq & Free \\
\hline BIS & $1 \mathrm{~A} / \mathrm{D}+0.25 \mathrm{D}$ & 44 & 39 & 0 & 75 & 53 & 47 & $1 \mathrm{~A} / \mathrm{D}$ & 30 & 0 & 30 & 60 & 30 & 28 \\
\hline $\mathrm{BLI}$ & $1 \mathrm{~A} / \mathrm{D}$ & 45 & 40 & 0 & 60 & 40 & 34 & $1 \mathrm{~A} / \mathrm{D}$ & 30 & 0 & 30 & 60 & 30 & 28 \\
\hline$\overline{\mathrm{BLV}}$ & $2 A / D$ & 90 & 79 & 0 & 120 & 79 & 67 & $2 A / D$ & 60 & 0 & 60 & 120 & 60 & 56 \\
\hline $\mathrm{BMI}$ & $1 A+1 D$ & 43 & 43 & 43 & 60 & 60 & 60 & $1 \mathrm{~A} / \mathrm{D}$ & 30 & 0 & 29 & 60 & 29 & 26 \\
\hline BTV & $1 \mathrm{~A} / \mathrm{D}+0.25 \mathrm{D}$ & 43 & 36 & 0 & 75 & 51 & 42 & $1 \mathrm{~A} / \mathrm{D}$ & 30 & 0 & 29 & 60 & 29 & 26 \\
\hline $\mathrm{CAE}$ & $1 \mathrm{~A} / \mathrm{D}+0.5 \mathrm{D}$ & 43 & 37 & 0 & 90 & 66 & 58 & $1 \mathrm{~A} / \mathrm{D}$ & 30 & 0 & 29 & 60 & 29 & 26 \\
\hline CAK & $1 A+1 D$ & 43 & 43 & 43 & 60 & 60 & 60 & $1 \mathrm{~A} / \mathrm{D}$ & 30 & 0 & 29 & 60 & 29 & 26 \\
\hline CID & $1 A+1 D$ & 43 & 43 & 43 & 60 & 60 & 60 & $1 \mathrm{~A} / \mathrm{D}$ & 30 & 0 & 29 & 60 & 29 & 26 \\
\hline CRW & $1 \mathrm{~A} / \mathrm{D}$ & 43 & 37 & 0 & 60 & 37 & 29 & $1 \mathrm{~A} / \mathrm{D}$ & 30 & 0 & 29 & 60 & 29 & 26 \\
\hline CSG & $1 \mathrm{~A} / \mathrm{D}+0.25 \mathrm{D}$ & 45 & 40 & 0 & 75 & 55 & 49 & $1 \mathrm{~A} / \mathrm{D}$ & 30 & 0 & 30 & 60 & 30 & 28 \\
\hline CYS & $1 \mathrm{~A} / \mathrm{D}+0.25 \mathrm{D}$ & 46 & 41 & 0 & 75 & 56 & 50 & $1 \mathrm{~A} / \mathrm{D}$ & 30 & 0 & 30 & 60 & 30 & 29 \\
\hline $\mathrm{DLH}$ & $1 \mathrm{~A} / \mathrm{D}+0.25 \mathrm{D}$ & 45 & 40 & 0 & 75 & 55 & 49 & $1 \mathrm{~A} / \mathrm{D}$ & 30 & 0 & 30 & 60 & 30 & 28 \\
\hline FAI & $2 A / D$ & 88 & 77 & 0 & 120 & 77 & 64 & $1 \mathrm{~A} / \mathrm{D}$ & 30 & 0 & 30 & 60 & 30 & 28 \\
\hline FAR & $1.5 \mathrm{~A} / \mathrm{D}$ & 66 & 58 & 0 & 90 & 58 & 48 & $1 \mathrm{~A} / \mathrm{D}$ & 30 & 0 & 30 & 60 & 30 & 28 \\
\hline FSD & $1.5 \mathrm{~A} / \mathrm{D}$ & 65 & 56 & 0 & 90 & 56 & 45 & $1 \mathrm{~A} / \mathrm{D}$ & 30 & 0 & 30 & 60 & 30 & 27 \\
\hline GEG & $1 A+1 D$ & 43 & 43 & 43 & 60 & 60 & 60 & $1 \mathrm{~A} / \mathrm{D}$ & 30 & 0 & 29 & 60 & 29 & 26 \\
\hline GPT & $1 A / D+1 D$ & 44 & 38 & 0 & 120 & 97 & 90 & $1 \mathrm{~A} / \mathrm{D}$ & 30 & 0 & 30 & 60 & 30 & 27 \\
\hline GRB & $1 A+1 D$ & 43 & 43 & 43 & 60 & 60 & 60 & $1 \mathrm{~A} / \mathrm{D}$ & 30 & 0 & 29 & 60 & 29 & 26 \\
\hline GSP & $1 \mathrm{~A} / \mathrm{D}$ & 42 & 33 & 0 & 60 & 33 & 21 & $1 \mathrm{~A} / \mathrm{D}$ & 31 & 0 & 29 & 60 & 29 & 23 \\
\hline $\mathrm{HRL}$ & $2 A / D$ & 85 & 71 & 0 & 120 & 71 & 53 & $1 \mathrm{~A} / \mathrm{D}$ & 30 & 0 & 29 & 60 & 29 & 26 \\
\hline $\mathrm{HSV}$ & $2 A / D$ & 84 & 70 & 0 & 120 & 70 & 50 & $2 A / D$ & 60 & 0 & 58 & 120 & 58 & 49 \\
\hline ILM & $1 \mathrm{~A} / \mathrm{D}$ & 44 & 39 & 0 & 60 & 39 & 32 & $1 \mathrm{~A} / \mathrm{D}$ & 30 & 0 & 30 & 60 & 30 & 28 \\
\hline ITO & $1 \mathrm{~A} / \mathrm{D}+0.25 \mathrm{D}$ & 44 & 39 & 0 & 75 & 53 & 47 & $1 \mathrm{~A} / \mathrm{D}$ & 30 & 0 & 30 & 60 & 30 & 28 \\
\hline JAN & $2 A / D$ & 84 & 70 & 0 & 120 & 70 & 50 & Dep pair & 51 & 0 & 51 & 87 & 51 & 51 \\
\hline KOA & $1 \mathrm{~A} / \mathrm{D}$ & 51 & 44 & 0 & 74 & 44 & 38 & $1 \mathrm{~A} / \mathrm{D}$ & 36 & 0 & 35 & 74 & 35 & 23 \\
\hline LBB & $1 A+1 D$ & 44 & 44 & 44 & 60 & 60 & 60 & $1 \mathrm{~A} / \mathrm{D}$ & 30 & 0 & 30 & 60 & 30 & 27 \\
\hline LEX & $1 \mathrm{~A} / \mathrm{D}$ & 43 & 37 & 0 & 60 & 37 & 29 & $1 \mathrm{~A} / \mathrm{D}$ & 30 & 0 & 29 & 60 & 29 & 26 \\
\hline LIH & $1 A+1 D$ & 52 & 52 & 52 & 74 & 74 & 74 & $1 \mathrm{~A} / \mathrm{D}$ & 36 & 0 & 36 & 74 & 36 & 24 \\
\hline MDT & $\begin{array}{l}\mathrm{A} / \mathrm{D} \\
\end{array}$ & 42 & 35 & 0 & 60 & 35 & 25 & $1 \mathrm{~A} / \mathrm{D}$ & 30 & 0 & 29 & 60 & 29 & 25 \\
\hline MFE & $1 \mathrm{~A} / \mathrm{D}$ & 44 & 39 & 0 & 60 & 39 & 32 & $1 \mathrm{~A} / \mathrm{D}$ & 30 & 0 & 30 & 60 & 30 & 28 \\
\hline $\mathrm{MLI}$ & $1 A+1 D$ & 43 & 43 & 43 & 60 & 60 & 60 & $1 \mathrm{~A} / \mathrm{D}$ & 30 & 0 & 29 & 60 & 29 & 26 \\
\hline MOB & $1 A+1 D$ & 43 & 43 & 43 & 60 & 60 & 60 & $1 \mathrm{~A} / \mathrm{D}$ & 30 & 0 & 29 & 60 & 29 & 26 \\
\hline MYR & $1 \mathrm{~A} / \mathrm{D}$ & 43 & 36 & 0 & 60 & 36 & 27 & $1 \mathrm{~A} / \mathrm{D}$ & 30 & 0 & 29 & 60 & 29 & 26 \\
\hline OGG & $1 \mathrm{~A} / \mathrm{D}+0.25 \mathrm{D}$ & 41 & 36 & 0 & 73 & 50 & 43 & $1 \mathrm{~A} / \mathrm{D}$ & 29 & 0 & 28 & 58 & 28 & 26 \\
\hline PNS & $1 \mathrm{~A} / \mathrm{D}$ & 43 & 36 & 0 & 60 & 36 & 27 & $1 \mathrm{~A} / \mathrm{D}$ & 30 & 0 & 29 & 60 & 29 & 26 \\
\hline PSP & $1.5 \mathrm{~A} / \mathrm{D}$ & 66 & 58 & 0 & 90 & 58 & 48 & $1 \mathrm{~A} / \mathrm{D}$ & 30 & 0 & 30 & 60 & 30 & 28 \\
\hline PWM & $1 \mathrm{~A}+1 \mathrm{D}$ & 43 & 43 & 43 & 60 & 60 & 60 & $1 \mathrm{~A} / \mathrm{D}$ & 30 & 0 & 29 & 60 & 29 & 26 \\
\hline SAV & $1 A+1 D$ & 43 & 43 & 43 & 60 & 60 & 60 & $1 \mathrm{~A} / \mathrm{D}$ & 30 & 0 & 29 & 60 & 29 & 26 \\
\hline SFB & $2 A / D$ & 91 & 82 & 0 & 120 & 82 & 70 & $1 \mathrm{~A} / \mathrm{D}$ & 30 & 0 & 30 & 60 & 30 & 29 \\
\hline SJU & $1 A / D+1 D$ & 40 & 35 & 0 & 113 & 91 & 84 & $1 A / D+1 D$ & 28 & 0 & 28 & 113 & 84 & 81 \\
\hline SRQ & $1.25 \mathrm{~A} / \mathrm{D}$ & 56 & 50 & 0 & 75 & 50 & 42 & $1 \mathrm{~A} / \mathrm{D}$ & 30 & 0 & 30 & 60 & 30 & 28 \\
\hline STT & $1 \mathrm{~A} / \mathrm{D}$ & 43 & 38 & 0 & 59 & 38 & 32 & $1 \mathrm{~A} / \mathrm{D}$ & 29 & 0 & 29 & 59 & 29 & 27 \\
\hline TLH & $1 A / D+1 D$ & 43 & 37 & 0 & 120 & 96 & 88 & $1 \mathrm{~A} / \mathrm{D}$ & 30 & 0 & 29 & 60 & 29 & 26 \\
\hline TOL & $1 \mathrm{~A} / \mathrm{D}$ & 35 & 29 & 0 & 48 & 29 & 19 & $1 \mathrm{~A} / \mathrm{D}$ & 24 & 0 & 24 & 48 & 24 & 28 \\
\hline VPS & $1 A / D+1 D$ & 43 & 36 & 0 & 120 & 96 & 87 & $1 \mathrm{~A} / \mathrm{D}$ & 30 & 0 & 29 & 60 & 29 & 26 \\
\hline $\mathrm{ABI}$ & $1 A+1 D$ & 44 & 44 & 44 & 60 & 60 & 60 & $1 A+1 D$ & 30 & 30 & 30 & 60 & 60 & 60 \\
\hline ABY & $1 \mathrm{~A} / \mathrm{D}$ & 45 & 40 & 0 & 60 & 40 & 34 & $1 \mathrm{~A} / \mathrm{D}$ & 30 & 0 & 30 & 60 & 30 & 28 \\
\hline ACK & $1 \mathrm{~A}+1 \mathrm{D}$ & 46 & 46 & 46 & 60 & 60 & 60 & $1 \mathrm{~A} / \mathrm{D}$ & 30 & 0 & 30 & 60 & 30 & 29 \\
\hline ADS & $1 \mathrm{~A} / \mathrm{D}$ & 46 & 41 & 0 & 60 & 41 & 35 & $1 \mathrm{~A} / \mathrm{D}$ & 30 & 0 & 30 & 60 & 30 & 29 \\
\hline AEX & $1 \mathrm{~A} / \mathrm{D}+0.25 \mathrm{D}$ & 43 & 36 & 0 & 75 & 51 & 42 & $1 \mathrm{~A} / \mathrm{D}$ & 30 & 0 & 29 & 60 & 29 & 26 \\
\hline AGS & $1 \mathrm{~A} / \mathrm{D}+0.25 \mathrm{D}$ & 43 & 37 & 0 & 75 & 51 & 43 & $1 \mathrm{~A} / \mathrm{D}$ & 30 & 0 & 29 & 60 & 29 & 26 \\
\hline APA & $1 A+1 D+0.5 D$ & 46 & 46 & 46 & 90 & 90 & 90 & $1 \mathrm{~A} / \mathrm{D}$ & 30 & 0 & 30 & 60 & 30 & 29 \\
\hline ATW & $1 A+1 D$ & 43 & 43 & 43 & 60 & 60 & 60 & $1 \mathrm{~A} / \mathrm{D}$ & 30 & 0 & 29 & 60 & 29 & 26 \\
\hline BED & $1 A+1 D$ & 46 & 46 & 46 & 60 & 60 & 60 & $1 \mathrm{~A} / \mathrm{D}$ & 30 & 0 & 30 & 60 & 30 & 29 \\
\hline BFF & $1 \mathrm{~A} / \mathrm{D}$ & 46 & 41 & 0 & 60 & 41 & 35 & 1 On - 1 Off & 4 & 0 & 2 & 6 & 3 & 0 \\
\hline BGR & $1 \mathrm{~A} / \mathrm{D}$ & 43 & 37 & 0 & 60 & 37 & 29 & $1 \mathrm{~A} / \mathrm{D}$ & 30 & 0 & 29 & 60 & 29 & 26 \\
\hline BKL & $1 A+1 D$ & 46 & 46 & 46 & 60 & 60 & 60 & $1 \mathrm{~A} / \mathrm{D}$ & 30 & 0 & 30 & 60 & 30 & 29 \\
\hline BPT & $1 \mathrm{~A} / \mathrm{D}+0.25 \mathrm{D}$ & 44 & 39 & 0 & 75 & 53 & 47 & $1 \mathrm{~A} / \mathrm{D}$ & 30 & 0 & 30 & 60 & 30 & 28 \\
\hline BVY & $1 \mathrm{~A}+1 \mathrm{D}$ & 46 & 46 & 46 & 60 & 60 & 60 & $1 \mathrm{~A} / \mathrm{D}$ & 30 & 0 & 30 & 60 & 30 & 29 \\
\hline BZN & $1 \mathrm{~A} / \mathrm{D}$ & 44 & 39 & 0 & 60 & 39 & 32 & $1 \mathrm{~A} / \mathrm{D}$ & 30 & 0 & 30 & 60 & 30 & 28 \\
\hline CGF & $1 \mathrm{~A} / \mathrm{D}$ & 46 & 41 & 0 & 60 & 41 & 35 & $1 \mathrm{~A} / \mathrm{D}$ & 30 & 0 & 30 & 60 & 30 & 29 \\
\hline
\end{tabular}


Table B-4. Additional 200 Airport Capacities

\begin{tabular}{|c|c|c|c|c|c|c|c|c|c|c|c|c|c|c|}
\hline \multirow[b]{2}{*}{ Apt } & \multicolumn{7}{|c|}{ Visual meteorological conditions } & \multicolumn{7}{|c|}{ Instrument meteorological conditions } \\
\hline & Configuration & Amax & Aeq & Amin & Dmax & Deq & Free & Configuration & Amax & Amin & Aeq & Dmax & Deq & Free \\
\hline$\overline{\mathrm{CHA}}$ & $1 \mathrm{~A} / \mathrm{D}+0.25 \mathrm{D}$ & 44 & 38 & 0 & 75 & 52 & 45 & $1 \mathrm{~A} / \mathrm{D}$ & 30 & 0 & 30 & 60 & 30 & 27 \\
\hline $\mathrm{CHO}$ & $1 \mathrm{~A} / \mathrm{D}$ & 43 & 37 & 0 & 60 & 37 & 29 & $1 \mathrm{~A} / \mathrm{D}$ & 30 & 0 & 29 & 60 & 29 & 26 \\
\hline CMI & $1.5 \mathrm{~A}+1.5 \mathrm{D}$ & 66 & 66 & 66 & 90 & 90 & 90 & $1 \mathrm{~A} / \mathrm{D}$ & 30 & 0 & 30 & 60 & 30 & 28 \\
\hline CPS & $2 \mathrm{~A} / \mathrm{D}$ & 91 & 82 & 0 & 120 & 82 & 70 & $1 \mathrm{~A} / \mathrm{D}$ & 30 & 0 & 30 & 60 & 30 & 29 \\
\hline DPA & $2 A / D$ & 91 & 82 & 0 & 120 & 82 & 70 & $1 \mathrm{~A} / \mathrm{D}$ & 30 & 0 & 30 & 60 & 30 & 29 \\
\hline ERI & $1 A+1 D$ & 44 & 44 & 44 & 60 & 60 & 60 & $1 \mathrm{~A} / \mathrm{D}$ & 30 & 0 & 30 & 60 & 30 & 27 \\
\hline FAY & $1 \mathrm{~A} / \mathrm{D}+0.25 \mathrm{D}$ & 44 & 38 & 0 & 75 & 52 & 45 & $1 \mathrm{~A} / \mathrm{D}$ & 30 & 0 & 30 & 60 & 30 & 27 \\
\hline FNL & $1 \mathrm{~A} / \mathrm{D}$ & 46 & 41 & 0 & 60 & 41 & 35 & $1 \mathrm{~A} / \mathrm{D}$ & 30 & 0 & 30 & 60 & 30 & 29 \\
\hline FOE & $1 A+1 D$ & 46 & 46 & 46 & 60 & 60 & 60 & $1 \mathrm{~A} / \mathrm{D}$ & 30 & 0 & 30 & 60 & 30 & 29 \\
\hline FRG & $1 A+1 D$ & 46 & 46 & 46 & 60 & 60 & 60 & $1 \mathrm{~A} / \mathrm{D}$ & 30 & 0 & 30 & 60 & 30 & 29 \\
\hline FTW & $2 A / D$ & 91 & 82 & 0 & 120 & 82 & 70 & $1 \mathrm{~A} / \mathrm{D}$ & 30 & 0 & 30 & 60 & 30 & 29 \\
\hline GJT & $1.5 \mathrm{~A} / \mathrm{D}$ & 67 & 60 & 0 & 90 & 60 & 50 & $1 \mathrm{~A} / \mathrm{D}$ & 30 & 0 & 30 & 60 & 30 & 28 \\
\hline GNV & $1 A+1 D$ & 44 & 44 & 44 & 60 & 60 & 60 & $1 \mathrm{~A} / \mathrm{D}$ & 30 & 0 & 30 & 60 & 30 & 28 \\
\hline GTF & $1.5 \mathrm{~A} / \mathrm{D}$ & 65 & 56 & 0 & 90 & 56 & 45 & $1 \mathrm{~A} / \mathrm{D}$ & 30 & 0 & 30 & 60 & 30 & 27 \\
\hline GUM & $1 \mathrm{~A}+1 \mathrm{D}$ & 47 & 47 & 47 & 67 & 67 & 67 & $1 \mathrm{~A} / \mathrm{D}$ & 34 & 0 & 33 & 67 & 33 & 20 \\
\hline IAG & $2 A / D$ & 90 & 79 & 0 & 120 & 79 & 67 & $1 \mathrm{~A} / \mathrm{D}$ & 30 & 0 & 30 & 60 & 30 & 28 \\
\hline ILG & $1 A+1 D$ & 46 & 46 & 46 & 60 & 60 & 60 & $1 \mathrm{~A} / \mathrm{D}$ & 30 & 0 & 30 & 60 & 30 & 29 \\
\hline KTN & $1 \mathrm{~A} / \mathrm{D}$ & 44 & 39 & 0 & 60 & 39 & 32 & $1 \mathrm{~A} / \mathrm{D}$ & 30 & 0 & 30 & 60 & 30 & 28 \\
\hline LNK & $2 A / D$ & 88 & 77 & 0 & 120 & 77 & 64 & $1 \mathrm{~A} / \mathrm{D}$ & 30 & 0 & 30 & 60 & 30 & 28 \\
\hline MAF & $2 A / D$ & 87 & 75 & 0 & 120 & 75 & 60 & $1 \mathrm{~A} / \mathrm{D}$ & 30 & 0 & 30 & 60 & 30 & 27 \\
\hline MSO & $1 \mathrm{~A} / \mathrm{D}+0.25 \mathrm{D}$ & 44 & 39 & 0 & 75 & 53 & 47 & $1 \mathrm{~A} / \mathrm{D}$ & 30 & 0 & 30 & 60 & 30 & 28 \\
\hline PTK & $1 A+1 D$ & 46 & 46 & 46 & 60 & 60 & 60 & $1 \mathrm{~A} / \mathrm{D}$ & 30 & 0 & 30 & 60 & 30 & 29 \\
\hline RAP & $1 \mathrm{~A} / \mathrm{D}$ & 44 & 39 & 0 & 60 & 39 & 32 & $1 \mathrm{~A} / \mathrm{D}$ & 30 & 0 & 30 & 60 & 30 & 28 \\
\hline SHV & $1 \mathrm{~A} / \mathrm{D}$ & 43 & 36 & 0 & 60 & 36 & 27 & $1 \mathrm{~A} / \mathrm{D}$ & 30 & 0 & 29 & 60 & 29 & 26 \\
\hline $40 \mathrm{~N}$ & $1 \mathrm{~A} / \mathrm{D}$ & 46 & 41 & 0 & 60 & 41 & 35 & 1 On-1 Off & 4 & 0 & 2 & 6 & 3 & 0 \\
\hline $55 \mathrm{~J}$ & $1 A+1 D$ & 46 & 46 & 46 & 60 & 60 & 60 & 1 On-1 Off & 4 & 0 & 2 & 6 & 3 & 0 \\
\hline ABR & $1 \mathrm{~A} / \mathrm{D}$ & 44 & 39 & 0 & 60 & 39 & 32 & 1 On -1 Off & 4 & 0 & 2 & 6 & 3 & 0 \\
\hline ACT & $1 A+1 D$ & 44 & 44 & 44 & 60 & 60 & 60 & $1 \mathrm{~A} / \mathrm{D}$ & 30 & 0 & 30 & 60 & 30 & 28 \\
\hline ACV & $1 \mathrm{~A} / \mathrm{D}$ & 46 & 41 & 0 & 60 & 41 & 35 & 1 On-1 Off & 4 & 0 & 2 & 6 & 3 & 0 \\
\hline $\mathrm{ADQ}$ & $1.25 \mathrm{~A} / \mathrm{D}$ & 54 & 47 & 0 & 75 & 47 & 38 & $1 \mathrm{~A} / \mathrm{D}$ & 30 & 0 & 30 & 60 & 30 & 27 \\
\hline AEG & $1 \mathrm{~A} / \mathrm{D}$ & 46 & 41 & 0 & 60 & 41 & 35 & 1 On - 1 Off & 4 & 0 & 2 & 6 & 3 & 0 \\
\hline AFW & $1 A+1 D$ & 46 & 46 & 46 & 60 & 60 & 60 & $1 \mathrm{~A} / \mathrm{D}$ & 30 & 0 & 30 & 60 & 30 & 29 \\
\hline $\mathrm{AHN}$ & $1 \mathrm{~A} / \mathrm{D}$ & 46 & 41 & 0 & 60 & 41 & 35 & $1 \mathrm{~A} / \mathrm{D}$ & 30 & 0 & 30 & 60 & 30 & 29 \\
\hline ALN & $1 A+1 D$ & 46 & 46 & 46 & 60 & 60 & 60 & $1 \mathrm{~A} / \mathrm{D}$ & 30 & 0 & 30 & 60 & 30 & 29 \\
\hline ALO & $1 \mathrm{~A}+1 \mathrm{D}$ & 44 & 44 & 44 & 60 & 60 & 60 & $1 \mathrm{~A} / \mathrm{D}$ & 30 & 0 & 30 & 60 & 30 & 28 \\
\hline APF & $1 \mathrm{~A} / \mathrm{D}$ & 46 & 41 & $\frac{17}{0}$ & 60 & 41 & 35 & $1 \mathrm{~A} / \mathrm{D}$ & 30 & 0 & 30 & 60 & 30 & $\frac{29}{29}$ \\
\hline ARR & $1 A+1 D$ & 46 & 46 & 46 & 60 & 60 & 60 & $1 \mathrm{~A} / \mathrm{D}$ & 30 & 0 & 30 & 60 & 30 & $\frac{29}{29}$ \\
\hline ASE & $1 \mathrm{~A} / \mathrm{D}$ & 45 & 40 & 0 & 60 & 40 & 34 & $1 \mathrm{~A}$ alternate with $1 \mathrm{D}$ & 30 & 0 & 15 & 60 & 30 & $\frac{0}{0}$ \\
\hline ATY & $1 \mathrm{~A} / \mathrm{D}$ & 44 & 38 & 0 & 60 & 38 & 30 & 1 On-1 off & 4 & 0 & $\frac{2}{2}$ & 6 & 3 & 0 \\
\hline AUG & $1 \mathrm{~A} / \mathrm{D}$ & 46 & 41 & 0 & 60 & 41 & 35 & 1 On-1 Off & 4 & 0 & 2 & 6 & 3 & 0 \\
\hline AWM & $1 \mathrm{~A} / \mathrm{D}$ & 46 & 41 & 0 & 60 & 41 & 35 & 1 On-1 Off & 4 & 0 & 2 & 6 & 3 & 0 \\
\hline BCT & $1 \mathrm{~A} / \mathrm{D}$ & 46 & 41 & 0 & 60 & 41 & 35 & $1 \mathrm{~A} / \mathrm{D}$ & 30 & 0 & 30 & 60 & 30 & 29 \\
\hline BET & $1 \mathrm{~A} / \mathrm{D}$ & 46 & 41 & 0 & 60 & 41 & 35 & $1 \mathrm{~A} / \mathrm{D}$ & 30 & 0 & 30 & 60 & 30 & 29 \\
\hline BFM & $1 A+1 D$ & 46 & 46 & 46 & 60 & 60 & 60 & $1 \mathrm{~A} / \mathrm{D}$ & 30 & 0 & 30 & 60 & 30 & 29 \\
\hline BGM & $1 \mathrm{~A} / \mathrm{D}$ & 43 & 36 & 0 & 60 & 36 & 27 & $1 \mathrm{~A} / \mathrm{D}$ & 30 & 0 & 29 & 60 & 29 & 26 \\
\hline BJC & $2 \mathrm{~A} / \mathrm{D}$ & 91 & 82 & 0 & 120 & 82 & 70 & $1 \mathrm{~A} / \mathrm{D}$ & 30 & 0 & 30 & 60 & 30 & 29 \\
\hline BTM & $1 \mathrm{~A} / \mathrm{D}$ & 44 & 39 & 0 & 60 & 39 & 32 & 1 On - 1 Off & 4 & 0 & 2 & 6 & 3 & 0 \\
\hline CCR & $1 \mathrm{~A} / \mathrm{D}+0.25 \mathrm{D}$ & 46 & 41 & 0 & 75 & 56 & 50 & $1 \mathrm{~A} / \mathrm{D}$ & 30 & 0 & 30 & 60 & 30 & 29 \\
\hline CDC & $1 \mathrm{~A} / \mathrm{D}$ & 46 & 41 & 0 & 60 & 41 & 35 & 1 On - 1 Off & 4 & 0 & 2 & 6 & 3 & 0 \\
\hline CGI & $1 \mathrm{~A} / \mathrm{D}$ & 46 & 41 & 0 & 60 & 41 & 35 & $1 \mathrm{~A} / \mathrm{D}$ & 30 & 0 & 30 & 60 & 30 & 29 \\
\hline CKB & $1 \mathrm{~A} / \mathrm{D}$ & 46 & 41 & 0 & 60 & 41 & 35 & $1 \mathrm{~A} / \mathrm{D}$ & 30 & 0 & 30 & 60 & 30 & 29 \\
\hline $\mathrm{CLL}$ & $1 \mathrm{~A} / \mathrm{D}+0.25 \mathrm{D}$ & 44 & 39 & 0 & 75 & 53 & 47 & $1 \mathrm{~A} / \mathrm{D}$ & 30 & 0 & 30 & 60 & 30 & 28 \\
\hline $\operatorname{coU}$ & $1 A+1 D$ & 46 & 46 & 46 & 60 & 60 & 60 & $1 \mathrm{~A} / \mathrm{D}$ & 30 & 0 & 30 & 60 & 30 & 29 \\
\hline CPR & $1.5 \mathrm{~A} / \mathrm{D}$ & 67 & 60 & 0 & 90 & 60 & 50 & $1 \mathrm{~A} / \mathrm{D}$ & 30 & 0 & 30 & 60 & 30 & 28 \\
\hline CWA & $1 A+1 D$ & 43 & 43 & 43 & 60 & 60 & 60 & $1 \mathrm{~A} / \mathrm{D}$ & 30 & 0 & 29 & 60 & 29 & 26 \\
\hline DTO & $1 \mathrm{~A} / \mathrm{D}$ & 46 & 41 & 0 & 60 & 41 & 35 & $1 \mathrm{~A} / \mathrm{D}$ & 30 & 0 & 30 & 60 & 30 & 29 \\
\hline DVT & $1.5 \mathrm{~A} / \mathrm{D}$ & 69 & 62 & 0 & 90 & 62 & 53 & $1 \mathrm{~A} / \mathrm{D}$ & 30 & 0 & 30 & 60 & 30 & 29 \\
\hline EAU & $1 \mathrm{~A} / \mathrm{D}$ & 45 & 40 & 0 & 60 & 40 & 34 & $1 \mathrm{~A} / \mathrm{D}$ & 30 & 0 & 30 & 60 & 30 & 28 \\
\hline EFD & $2 A / D$ & 91 & 82 & 0 & 120 & 82 & 70 & $1 \mathrm{~A} / \mathrm{D}$ & 30 & 0 & 30 & 60 & 30 & 29 \\
\hline EGE & $1 \mathrm{~A} / \mathrm{D}$ & 44 & 39 & 0 & 60 & 39 & 34 & $1 \mathrm{~A} / \mathrm{D}$ & 29 & 0 & 29 & 60 & 29 & $\frac{28}{28}$ \\
\hline EQY & $1 \mathrm{~A} / \mathrm{D}$ & 46 & 41 & 0 & 60 & 41 & 35 & 1 On - 1 Off & 4 & 0 & 2 & 6 & 3 & 0 \\
\hline
\end{tabular}


Table B-4. Additional 200 Airport Capacities

\begin{tabular}{|c|c|c|c|c|c|c|c|c|c|c|c|c|c|c|}
\hline \multirow[b]{2}{*}{ Apt } & \multicolumn{7}{|c|}{ Visual meteorological conditions } & \multicolumn{7}{|c|}{ Instrument meteorological conditions } \\
\hline & Configuration & Amax & Aeq & Amin & Dmax & Deq & Free & Configuration & Amax & Amin & Aeq & Dmax & Deq & Free \\
\hline EVV & $1 \mathrm{~A} / \mathrm{D}+0.25 \mathrm{D}$ & 43 & 37 & 0 & 75 & 51 & 43 & $1 \mathrm{~A} / \mathrm{D}$ & 30 & 0 & 29 & 60 & 29 & 26 \\
\hline FDK & $1 \mathrm{~A} / \mathrm{D}$ & 46 & 41 & 0 & 60 & 41 & 35 & $10 \mathrm{O}-1 \mathrm{Off}$ & 4 & 0 & 2 & 6 & 3 & 0 \\
\hline FFZ & $2 A / D$ & 91 & 82 & 0 & 120 & 82 & 70 & $1 \mathrm{~A} / \mathrm{D}$ & 30 & 0 & 30 & 60 & 30 & 29 \\
\hline FTG & $1 A+1 D$ & 46 & 46 & 46 & 60 & 60 & 60 & $1 \mathrm{~A} / \mathrm{D}$ & 30 & 0 & 30 & 60 & 30 & 29 \\
\hline FTY & $1.5 \mathrm{~A} / \mathrm{D}$ & 69 & 62 & 0 & 90 & 62 & 53 & $1 \mathrm{~A} / \mathrm{D}$ & 30 & 0 & 30 & 60 & 30 & 29 \\
\hline FWA & $1 A+1 D$ & 43 & 43 & 43 & 60 & 60 & 60 & $1 \mathrm{~A} / \mathrm{D}$ & 30 & 0 & 29 & 60 & 29 & 26 \\
\hline $\mathrm{GCN}$ & $1 \mathrm{~A} / \mathrm{D}$ & 46 & 41 & 0 & 60 & 41 & 35 & $1 \mathrm{~A} / \mathrm{D}$ & 30 & 0 & 30 & 60 & 30 & 29 \\
\hline GEU & $1 \mathrm{~A} / \mathrm{D}$ & 46 & 41 & 0 & 60 & 41 & 35 & $1 \mathrm{~A} / \mathrm{D}$ & 30 & 0 & 30 & 60 & 30 & 29 \\
\hline GRI & $1.5 \mathrm{~A} / \mathrm{D}$ & 69 & 62 & 0 & 90 & 62 & 53 & $1 \mathrm{~A} / \mathrm{D}$ & 30 & 0 & 30 & 60 & 30 & 29 \\
\hline $\mathrm{HAO}$ & $1 A+1 D$ & 46 & 46 & 46 & 60 & 60 & 60 & $10 \mathrm{O}-1 \mathrm{Off}$ & 4 & 0 & 2 & 6 & 3 & 0 \\
\hline HEF & $2 \mathrm{~A} / \mathrm{D}$ & 91 & 82 & 0 & 120 & 82 & 70 & $1 \mathrm{~A} / \mathrm{D}$ & 30 & 0 & 30 & 60 & 30 & 29 \\
\hline $\mathrm{HIO}$ & $1 A+1 D$ & 46 & 46 & 46 & 60 & 60 & 60 & $1 \mathrm{~A} / \mathrm{D}$ & 30 & 0 & 30 & 60 & 30 & 29 \\
\hline $\mathrm{HQZ}$ & $1 \mathrm{~A} / \mathrm{D}$ & 46 & 41 & 0 & 60 & 41 & 35 & $10 n-10 f f$ & 4 & 0 & 2 & 6 & 3 & 0 \\
\hline HWD & $2 A / D$ & 91 & 82 & 0 & 120 & 82 & 70 & $1 \mathrm{~A}$ alternate with $1 \mathrm{D}$ & 30 & 0 & 15 & 60 & 30 & 0 \\
\hline IDA & $1 \mathrm{~A} / \mathrm{D}+0.25 \mathrm{D}$ & 44 & 39 & 0 & 75 & 53 & 47 & $1 \mathrm{~A} / \mathrm{D}$ & 30 & 0 & 30 & 60 & 30 & 28 \\
\hline ISM & $1 \mathrm{~A} / \mathrm{D}$ & 46 & 41 & 0 & 60 & 41 & 35 & $1 \mathrm{~A} / \mathrm{D}$ & 30 & 0 & 30 & 60 & 30 & 29 \\
\hline IWA & $2 A / D$ & 91 & 82 & 0 & 120 & 82 & 70 & $1 \mathrm{~A} / \mathrm{D}$ & 30 & 0 & 30 & 60 & 30 & 29 \\
\hline JAC & $1 \mathrm{~A} / \mathrm{D}$ & 45 & 40 & 0 & 60 & 40 & 34 & $1 \mathrm{~A} / \mathrm{D}$ & 30 & 0 & 30 & 60 & 30 & 28 \\
\hline JST & $1 \mathrm{~A} / \mathrm{D}$ & 44 & 39 & 0 & 60 & 39 & 32 & $1 \mathrm{~A} / \mathrm{D}$ & 30 & 0 & 30 & 60 & 30 & 28 \\
\hline$\overline{L A L}$ & $1 \mathrm{~A} / \mathrm{D}+0.25 \mathrm{D}$ & 46 & 41 & 0 & 75 & 56 & 50 & $1 \mathrm{~A} / \mathrm{D}$ & 30 & 0 & 30 & 60 & 30 & 29 \\
\hline$\overline{\mathrm{LBE}}$ & $1 \mathrm{~A} / \mathrm{D}+0.25 \mathrm{D}$ & 45 & 40 & 0 & 75 & 55 & 49 & $1 \mathrm{~A} / \mathrm{D}$ & 30 & 0 & 30 & 60 & 30 & 28 \\
\hline LBF & $1 \mathrm{~A} / \mathrm{D}$ & 46 & 41 & 0 & 60 & 41 & 35 & $10 n-10 f f$ & 4 & 0 & 2 & 6 & 3 & 0 \\
\hline LBL & $1 \mathrm{~A} / \mathrm{D}+0.25 \mathrm{D}$ & 46 & 41 & 0 & 75 & 56 & 50 & 1 On - 1 Off & 4 & 0 & 2 & 6 & 3 & 0 \\
\hline LCK & $2 A / D$ & 91 & 82 & 0 & 120 & 82 & 70 & $1 \mathrm{~A} / \mathrm{D}$ & 30 & 0 & 30 & 60 & 30 & 29 \\
\hline LFT & $2 A / D$ & 88 & 77 & 0 & 120 & 77 & 64 & $1 \mathrm{~A} / \mathrm{D}$ & 30 & 0 & 30 & 60 & 30 & 28 \\
\hline LSE & $1 \mathrm{~A} / \mathrm{D}+0.25 \mathrm{D}$ & 44 & 38 & 0 & 75 & 52 & 45 & $1 \mathrm{~A} / \mathrm{D}$ & 30 & 0 & 30 & 60 & 30 & 27 \\
\hline LWM & $1 \mathrm{~A} / \mathrm{D}$ & 46 & 41 & 0 & 60 & 41 & 35 & $1 \mathrm{~A} / \mathrm{D}$ & 30 & 0 & 30 & 60 & 30 & 29 \\
\hline MBS & $1 \mathrm{~A} / \mathrm{D}$ & 44 & 38 & 0 & 60 & 38 & 30 & $1 \mathrm{~A} / \mathrm{D}$ & 30 & 0 & 30 & 60 & 30 & 27 \\
\hline MHK & $1 \mathrm{~A} / \mathrm{D}$ & 46 & 41 & 0 & 60 & 41 & 35 & $1 \mathrm{~A} / \mathrm{D}$ & 30 & 0 & 30 & 60 & 30 & 29 \\
\hline $\mathrm{MLU}$ & $1 \mathrm{~A} / \mathrm{D}+0.25 \mathrm{D}$ & 44 & 39 & 0 & 75 & 53 & 47 & $1 \mathrm{~A} / \mathrm{D}$ & 30 & 0 & 30 & 60 & 30 & 28 \\
\hline $\mathrm{MMU}$ & $1 \mathrm{~A} / \mathrm{D}$ & 46 & 41 & 0 & 60 & 41 & 35 & $1 \mathrm{~A} / \mathrm{D}$ & 30 & 0 & 30 & 60 & 30 & 29 \\
\hline MOT & $1 \mathrm{~A} / \mathrm{D}$ & 45 & 40 & 0 & 60 & 40 & 34 & $1 \mathrm{~A} / \mathrm{D}$ & 30 & 0 & 30 & 60 & 30 & 28 \\
\hline MTN & $1 \mathrm{~A} / \mathrm{D}$ & 46 & 41 & 0 & 60 & 41 & 35 & $1 \mathrm{~A} / \mathrm{D}$ & 30 & 0 & 30 & 60 & 30 & 29 \\
\hline OPF & $2 A / D$ & 91 & 82 & 0 & 120 & 82 & 70 & $1 A+1 D$ & 30 & 0 & 30 & 60 & 60 & 60 \\
\hline ORL & $1 \mathrm{~A} / \mathrm{D}+0.25 \mathrm{D}$ & 46 & 41 & 0 & 75 & 56 & 50 & $1 \mathrm{~A} / \mathrm{D}$ & 30 & 0 & 30 & 60 & 30 & 29 \\
\hline PAE & $2 A / D$ & 91 & 82 & 0 & 120 & 82 & 70 & $1 \mathrm{~A} / \mathrm{D}$ & 30 & 0 & 30 & 60 & 30 & 29 \\
\hline PDK & $2 \mathrm{~A} / \mathrm{D}$ & 91 & 82 & 0 & 120 & 82 & 70 & $1 \mathrm{~A} / \mathrm{D}$ & 30 & 0 & 30 & 60 & 30 & 29 \\
\hline $\mathrm{PIA}$ & $1 \mathrm{~A} / \mathrm{D}$ & 44 & 38 & 0 & 60 & 38 & 30 & $1 \mathrm{~A} / \mathrm{D}$ & 30 & 0 & 30 & 60 & 30 & 27 \\
\hline PIR & $1 \mathrm{~A} / \mathrm{D}+0.25 \mathrm{D}$ & 45 & 40 & 0 & 75 & 55 & 49 & $1 \mathrm{On}-1 \mathrm{Off}$ & 4 & 0 & 2 & 6 & 3 & 0 \\
\hline PNE & $1 A+1 D$ & 46 & 46 & 46 & 60 & 60 & 60 & $1 \mathrm{~A} / \mathrm{D}$ & 30 & 0 & 30 & 60 & 30 & 29 \\
\hline PRC & $2 \mathrm{~A} / \mathrm{D}$ & 91 & 82 & 0 & 120 & 82 & 70 & $1 \mathrm{~A} / \mathrm{D}$ & 30 & 0 & 30 & 60 & 30 & 29 \\
\hline PSC & $2 A / D$ & 87 & 75 & 0 & 120 & 75 & 60 & $1 \mathrm{~A} / \mathrm{D}$ & 30 & 0 & 30 & 60 & 30 & 27 \\
\hline PSM & $1 \mathrm{~A} / \mathrm{D}$ & 45 & 40 & 0 & 60 & 40 & 34 & $1 \mathrm{~A} / \mathrm{D}$ & 30 & 0 & 30 & 60 & 30 & 28 \\
\hline PUB & $2 \mathrm{~A} / \mathrm{D}$ & 91 & 82 & 0 & 120 & 82 & 70 & $1 \mathrm{~A} / \mathrm{D}$ & 30 & 0 & 30 & 60 & 30 & 29 \\
\hline PWK & $1 A+1 D$ & 46 & 46 & 46 & 60 & 60 & 60 & $1 \mathrm{~A} / \mathrm{D}$ & 30 & 0 & 30 & 60 & 30 & 29 \\
\hline RBD & $1 \mathrm{~A} / \mathrm{D}$ & 46 & 41 & 0 & 60 & 41 & 35 & $1 \mathrm{~A} / \mathrm{D}$ & 30 & 0 & 30 & 60 & 30 & 29 \\
\hline RNT & $1 \mathrm{~A} / \mathrm{D}$ & 46 & 41 & 0 & 60 & 41 & 35 & $1 \mathrm{~A} / \mathrm{D}$ & 30 & 0 & 30 & 60 & 30 & 29 \\
\hline ROA & $1 \mathrm{~A} / \mathrm{D}+0.25 \mathrm{D}$ & 43 & 37 & 0 & 75 & 51 & 43 & $1 \mathrm{~A} / \mathrm{D}$ & 30 & 0 & 29 & 60 & 29 & 26 \\
\hline RST & $1 A+1 D$ & 44 & 44 & 44 & 60 & 60 & 60 & $1 \mathrm{~A} / \mathrm{D}$ & 30 & 0 & 30 & 60 & 30 & 27 \\
\hline RYY & $1 \mathrm{~A} / \mathrm{D}$ & 46 & 41 & 0 & 60 & 41 & 35 & $1 \mathrm{~A} / \mathrm{D}$ & 30 & 0 & 30 & 60 & 30 & 29 \\
\hline SAF & $1 \mathrm{~A} / \mathrm{D}+0.25 \mathrm{D}$ & 46 & 41 & 0 & 75 & 56 & 50 & $1 \mathrm{~A} / \mathrm{D}$ & 30 & 0 & 30 & 60 & 30 & 29 \\
\hline SBN & $2 A / D$ & 86 & 73 & 0 & 120 & 73 & 57 & $1 \mathrm{~A} / \mathrm{D}$ & 30 & 0 & 29 & 60 & 29 & 26 \\
\hline SDL & $1 \mathrm{~A} / \mathrm{D}$ & 46 & 41 & 0 & 60 & 41 & 35 & $1 \mathrm{~A} / \mathrm{D}$ & 30 & 0 & 30 & 60 & 30 & 29 \\
\hline SDM & $1 \mathrm{~A} / \mathrm{D}$ & 46 & 41 & 0 & 60 & 41 & 35 & $1 \mathrm{~A}$ alternate with $1 \mathrm{D}$ & 30 & 0 & 15 & 60 & 30 & 0 \\
\hline SEE & $1 \mathrm{~A} / \mathrm{D}+0.5 \mathrm{D}$ & 46 & 41 & 0 & 90 & 71 & 65 & $1 \mathrm{~A} / \mathrm{D}$ & 30 & 0 & 30 & 60 & 30 & 29 \\
\hline SGF & $1 \mathrm{~A} / \mathrm{D}+0.25 \mathrm{D}$ & 43 & 37 & 0 & 75 & 51 & 43 & $1 \mathrm{~A} / \mathrm{D}$ & 30 & 0 & 29 & 60 & 29 & 26 \\
\hline SGR & $1 \mathrm{~A} / \mathrm{D}$ & 46 & 41 & 0 & 60 & 41 & 35 & $1 \mathrm{~A} / \mathrm{D}$ & 30 & 0 & 30 & 60 & 30 & 29 \\
\hline SMX & $1 \mathrm{~A} / \mathrm{D}$ & 46 & 41 & 0 & 60 & 41 & 35 & $1 \mathrm{~A} / \mathrm{D}$ & 30 & 0 & 30 & 60 & 30 & 29 \\
\hline STP & $1 \mathrm{~A} / \mathrm{D}+0.25 \mathrm{D}$ & 46 & 41 & 0 & 75 & 56 & 50 & $1 \mathrm{~A} / \mathrm{D}$ & 30 & 0 & 30 & 60 & 30 & 29 \\
\hline SUS & $2 A / D$ & 91 & 82 & 0 & 120 & 82 & 70 & $1 \mathrm{~A} / \mathrm{D}$ & 30 & 0 & 30 & 60 & 30 & 29 \\
\hline SUX & $1 \mathrm{~A} / \mathrm{D}+0.25 \mathrm{D}$ & 44 & 39 & 0 & 75 & 53 & 47 & $1 \mathrm{~A} / \mathrm{D}$ & 30 & 0 & 30 & 60 & 30 & 28 \\
\hline
\end{tabular}


Table B-4. Additional 200 Airport Capacities

\begin{tabular}{|c|c|c|c|c|c|c|c|c|c|c|c|c|c|c|}
\hline \multirow[b]{2}{*}{ Apt } & \multicolumn{7}{|c|}{ Visual meteorological conditions } & \multicolumn{7}{|c|}{ Instrument meteorological conditions } \\
\hline & Configuration & Amax & Aeq & Amin & Dmax & Deq & Free & Configuration & Amax & Amin & Aeq & Dmax & Deq & Free \\
\hline TKI & $1 \mathrm{~A} / \mathrm{D}$ & 46 & 41 & 0 & 60 & $\overline{41}$ & 35 & $1 \mathrm{~A} / \mathrm{D}$ & 30 & 0 & 30 & 60 & 30 & 29 \\
\hline TMB & $2 A / D$ & 91 & 82 & 0 & 120 & 82 & 70 & $1 \mathrm{~A} / \mathrm{D}$ & 30 & 0 & 30 & 60 & 30 & 29 \\
\hline TOA & $1 \mathrm{~A} / \mathrm{D}+0.25 \mathrm{D}$ & 46 & 41 & 0 & 75 & 56 & 50 & $1 \mathrm{~A} / \mathrm{D}$ & 30 & 0 & 30 & 60 & 30 & 29 \\
\hline TRI & $1 \mathrm{~A} / \mathrm{D}$ & 44 & 38 & 0 & 60 & 38 & 30 & $1 \mathrm{~A} / \mathrm{D}$ & 30 & 0 & 30 & 60 & 30 & 27 \\
\hline TTD & $1 \mathrm{~A} / \mathrm{D}$ & 46 & 41 & 0 & 60 & 41 & 35 & $1 \mathrm{~A} / \mathrm{D}$ & 30 & 0 & 30 & 60 & 30 & 29 \\
\hline UGN & $1 \mathrm{~A} / \mathrm{D}$ & 46 & 41 & 0 & 60 & 41 & 35 & $1 \mathrm{~A} / \mathrm{D}$ & 30 & 0 & 30 & 60 & 30 & 29 \\
\hline VGT & $2 A / D$ & 91 & 82 & 0 & 120 & 82 & 70 & $1 \mathrm{~A} / \mathrm{D}$ & 30 & 0 & 30 & 60 & 30 & 29 \\
\hline XNA & $1 \mathrm{~A} / \mathrm{D}$ & 42 & 33 & 0 & 60 & 33 & 21 & $1 \mathrm{~A} / \mathrm{D}$ & 31 & 0 & 29 & 60 & 29 & 23 \\
\hline YIP & $2 A / D$ & 91 & 82 & 0 & 120 & 82 & 70 & $1 \mathrm{~A} / \mathrm{D}$ & 30 & 0 & 30 & 60 & 30 & 29 \\
\hline
\end{tabular}

Table B-5. 2025 Capacities for Airports with New Runways in the Additional 200 Airport Set

\begin{tabular}{|c|c|c|c|c|c|c|c|c|c|c|c|c|c|c|}
\hline \multirow[b]{2}{*}{ Apt } & \multicolumn{7}{|c|}{ Visual meteorological conditions } & \multicolumn{7}{|c|}{ Instrument meteorological conditions } \\
\hline & Configuration & Amax & Aeq & Amin & Dmax & Deq & Free & Configuration & Amax & Amin & Aeq & Dmax & Deq & Free \\
\hline GEG & $2 \mathrm{~A} / \mathrm{D}$ & 86 & 57 & 0 & 120 & 73 & 73 & $2 A / D$ & 60 & 53 & 0 & 120 & 58 & 58 \\
\hline GSP & $2 \mathrm{~A} / \mathrm{D}$ & 83 & 42 & 0 & 120 & 67 & 67 & $2 A / D$ & 61 & 45 & 0 & 120 & 57 & 57 \\
\hline SAV & $2 A / D$ & 86 & 57 & 0 & 120 & 73 & 73 & $2 A / D$ & 60 & 53 & 0 & 120 & 58 & 58 \\
\hline
\end{tabular}

Table B-6. 110-Airport ROT-Limited Capacities for 2015 NextGen

\begin{tabular}{|c|c|c|c|c|c|c|c|c|c|c|c|c|c|c|}
\hline \multirow[b]{2}{*}{ Apt } & \multicolumn{7}{|c|}{ Visual meteorological conditions } & \multicolumn{7}{|c|}{ Instrument meteorological conditions } \\
\hline & Configuration & Amax & Aeq & Amin & Dmax & Deq & Free & Configuration & Amax & Amin & Aeq & Dmax & Deq & Free \\
\hline$A B Q$ & $\begin{array}{l}1.5 \mathrm{~A} / \mathrm{D} \text { with } \\
2 \mathrm{Amax} \text { with } 1 \\
\text { Free }\end{array}$ & 90 & 25 & 0 & 90 & 54 & 54 & $1.5 \mathrm{~A} / \mathrm{D}$ with $1 \mathrm{Dmax}$ & 57 & 14 & 0 & 60 & 44 & 44 \\
\hline ALB & $1 \mathrm{~A} / \mathrm{D}$ & 42 & 19 & 0 & 60 & 33 & 33 & $1 \mathrm{~A} / 0.5 \mathrm{D}$ & 38 & 5 & 0 & 30 & 29 & 14 \\
\hline ANC & $\begin{array}{l}1.25 \mathrm{~A} / \mathrm{D} \text { with } \\
1.5^{*} \mathrm{Dmax}\end{array}$ & 53 & 25 & 0 & 90 & 42 & 41 & $\begin{array}{l}\text { 1 A/D with } \\
1.25^{*} \mathrm{Dmax}\end{array}$ & 38 & 9 & 0 & 75 & 29 & 29 \\
\hline ATL & $2 A+2 D+1 A / D$ & 130 & 132 & 87 & 178 & 120 & 133 & $2 A+2 D+1 A / D$ & 116 & 128 & 77 & 178 & 107 & 148 \\
\hline AUS & $1.5 \mathrm{~A} / \mathrm{D}$ & 68 & 37 & 0 & 90 & 54 & 54 & $1.5 \mathrm{~A} / \mathrm{D}$ & 56 & 14 & 0 & 87 & 42 & 42 \\
\hline BDL & $\begin{array}{l}\text { 1.5 Amax with } \\
0.5 \text { Free } \\
\text { alternate with } \\
\text { 1.25Dmax }\end{array}$ & 65 & 11 & 0 & 75 & 33 & 42 & $\begin{array}{l}\text { 1.5 Amax with } 0.5 \\
\text { Free alternate with } \\
\text { 1.25Dmax }\end{array}$ & 57 & 5 & 0 & 75 & 29 & 40 \\
\hline $\mathrm{BFL}$ & $1 \mathrm{~A} / \mathrm{Dep} \mathrm{D}$ & 45 & 25 & 0 & 83 & 36 & 36 & $1 \mathrm{~A} / \mathrm{D}$ & 40 & 10 & 0 & 60 & 30 & 30 \\
\hline BHM & $\begin{array}{l}\mathrm{A} / \mathrm{D} \text { with } 1.25 \\
\text { Amax } 1.25 \\
\text { Dmax } 0.5 \text { Free }\end{array}$ & 56 & 12 & 0 & 75 & 36 & 36 & $1 \mathrm{~A} / \mathrm{D}$ & 40 & 10 & 0 & 60 & 30 & 30 \\
\hline BNA & $\begin{array}{l}0.75 \mathrm{~A}+1 \mathrm{D} \\
+1 \mathrm{~A} / \mathrm{D} \\
\end{array}$ & 72 & 77 & 31 & 120 & 63 & 92 & $1 A / D+1.5 A+0.5 D$ & 93 & 39 & 56 & 90 & 84 & 58 \\
\hline $\mathrm{BOI}$ & $\begin{array}{l}\text { 1.75A/D with } \\
\text { 2Dmax }\end{array}$ & 75 & 35 & 0 & 120 & 59 & 59 & $1 \mathrm{~A} / \mathrm{D}$ & 38 & 9 & 0 & 60 & 29 & 29 \\
\hline BOS & $\begin{array}{l}\text { 2A/D with } 1 \\
\text { Amin }\end{array}$ & 89 & 32 & 45 & 120 & 68 & 66 & $1 \mathrm{~A} / \mathrm{D}+\mathrm{Dmax}$ & 55 & 53 & 0 & 120 & 55 & 55 \\
\hline BTR & $\begin{array}{l}1.5 \mathrm{~A} \text { alternate } \\
\text { with } 1.5 \mathrm{D}\end{array}$ & 71 & 29 & 0 & 90 & 35 & 61 & $1 \mathrm{~A} / \mathrm{D}$ & 41 & 11 & 0 & 60 & 31 & 31 \\
\hline BUF & 1.5 A/1Dmax & 62 & 26 & 0 & 60 & 48 & 48 & $1 \mathrm{~A} / \mathrm{D}$ & 37 & 9 & 0 & 60 & 28 & 28 \\
\hline BUR & $1.25 \mathrm{~A} / \mathrm{D}$ & 52 & 32 & 0 & 75 & 46 & 46 & $1 \mathrm{~A} / \mathrm{D}$ & 38 & 9 & 0 & 60 & 29 & 29 \\
\hline BWI & $1.5 \mathrm{~A} / \mathrm{D}$ & 66 & 22 & 0 & 90 & 50 & 49 & $1.5 \mathrm{~A} / \mathrm{D}$ & 59 & 14 & 0 & 90 & 44 & 44 \\
\hline $\mathrm{CHS}$ & $\begin{array}{l}1.75^{\star} \mathrm{A} \text { alternate } \\
\text { with } 2 \mathrm{D} \\
\end{array}$ & 73 & 0 & 0 & 120 & 36 & 60 & $1 \mathrm{~A} / \mathrm{D}$ & 37 & 9 & 0 & 60 & 28 & 28 \\
\hline CLE & $2 A / D$ & 88 & 29 & 0 & 120 & 66 & 65 & $1.25 \mathrm{~A}+1 \mathrm{D}$ & 49 & 60 & 49 & 60 & 49 & 60 \\
\hline $\mathrm{CLT}$ & $2 \mathrm{~A} / \mathrm{D}$ & 132 & 45 & 0 & 180 & 100 & 66 & $2 A / D$ & 118 & 28 & 0 & 180 & 89 & 89 \\
\hline $\mathrm{CMH}$ & $2 \mathrm{~A} / \mathrm{D}$ & 83 & 34 & 0 & 120 & 64 & 64 & Dep A/D & 52 & 51 & 0 & 115 & 52 & 52 \\
\hline
\end{tabular}


Table B-6. 110-Airport ROT-Limited Capacities for 2015 NextGen

\begin{tabular}{|c|c|c|c|c|c|c|c|c|c|c|c|c|c|c|}
\hline \multirow[b]{2}{*}{ Apt } & \multicolumn{7}{|c|}{ Visual meteorological conditions } & \multicolumn{7}{|c|}{ Instrument meteorological conditions } \\
\hline & Configuration & Amax & Aeq & Amin & Dmax & Deq & Free & Configuration & Amax & Amin & Aeq & Dmax & Deq & Free \\
\hline $\cos$ & $\begin{array}{l}1.5 \mathrm{~A} \text { alternate } \\
\text { with } 2 \mathrm{D} \text { with } \\
\text { Free } \mathrm{D} \\
\end{array}$ & 63 & 18 & 0 & 120 & 31 & 69 & $1 \mathrm{~A} / \mathrm{D}$ & 37 & 9 & 0 & 60 & 28 & 28 \\
\hline CRP & $\begin{array}{l}1.5 \mathrm{~A} \text { alternate } \\
\text { with } 1.5 \mathrm{Dmax} \\
\text { with } 0.75 \mathrm{Amin} \\
\end{array}$ & 62 & 25 & 31 & 90 & 47 & 58 & $1 \mathrm{~A} / \mathrm{D}$ & 37 & 9 & 0 & 60 & 28 & 28 \\
\hline CVG & $\begin{array}{l}2 \mathrm{~A} / \mathrm{D}+1 \mathrm{~A}+ \\
0.5 \mathrm{Dmax}\end{array}$ & 132 & 29 & 44 & 150 & 110 & 65 & $2 A / D+1 A$ & 117 & 19 & 39 & 120 & 98 & 59 \\
\hline DAB & $\begin{array}{l}1.75 \mathrm{~A} \text { alternate } \\
\text { with } 2 \mathrm{Dmax}\end{array}$ & 78 & 47 & 0 & 120 & 39 & 86 & $1 \mathrm{~A} / \mathrm{D}$ & 39 & 10 & 0 & 60 & 30 & 30 \\
\hline DAL & $1 A+1 D$ & 41 & 60 & 41 & 60 & 41 & 59 & $1 A+1 D$ & 37 & 60 & 37 & 60 & 37 & 60 \\
\hline DAY & $1.75 \mathrm{~A} / \mathrm{D}$ & 83 & 33 & 0 & 120 & 64 & 56 & $1.75 \mathrm{~A} / \mathrm{D}$ & 74 & 17 & 0 & 120 & 56 & 56 \\
\hline$\overline{D C A}$ & $1.25 A+1 D$ & 54 & 60 & 54 & 60 & 54 & 60 & $1.25 \mathrm{~A} / 1 \mathrm{D}$ & 48 & 9 & 0 & 60 & 37 & 37 \\
\hline DEN & $3 A+3 D$ & 134 & 180 & 134 & 180 & 134 & 168 & $3 A+3 D$ & 119 & 180 & 119 & 180 & 119 & 180 \\
\hline$\overline{D F W}$ & $3 A+2 D+1 A / D$ & 174 & 134 & 131 & 180 & 163 & 143 & $3 A+2 D$ & 116 & 120 & 116 & 120 & 116 & 120 \\
\hline DSM & $\begin{array}{l}\text { 1A/1D with } 0.75 \\
\text { Amin }\end{array}$ & 42 & 17 & 31 & 60 & 36 & 39 & $1 \mathrm{~A} / \mathrm{D}$ & 37 & 9 & 0 & 60 & 28 & 28 \\
\hline DTW & $2 A+1 D+1 A / D$ & 130 & 73 & 87 & 120 & 119 & 89 & $2 A+2 D$ & 77 & 120 & 77 & 120 & 77 & 120 \\
\hline ELP & $1 A+1 D$ & 41 & 60 & 41 & 60 & 41 & 60 & $1 \mathrm{~A} / \mathrm{D}$ & 37 & 9 & 0 & 60 & 28 & 28 \\
\hline EUG & $1 \mathrm{~A} / \mathrm{D}$ & 43 & 21 & 0 & 60 & 34 & 34 & $1 \mathrm{~A} / \mathrm{D}$ & 38 & 9 & 0 & 60 & 29 & 29 \\
\hline EWR & $\begin{array}{l}1 A+1 D+0.25 \\
A / D \text { with Dmax } \\
\text { limit }\end{array}$ & 52 & 64 & 41 & 75 & 49 & 53 & $1 A+1 D$ & 37 & 9 & 37 & 60 & 37 & 60 \\
\hline FAT & $\begin{array}{l}\text { 1.5 A/D with } \\
\text { 1.5Dmax }\end{array}$ & 65 & 33 & 0 & 90 & 52 & 52 & $1 \mathrm{~A} / \mathrm{D}$ & 39 & 10 & 0 & 60 & 29 & 29 \\
\hline FLL & $1.5 \mathrm{~A} / \mathrm{D}$ & 90 & 34 & 0 & 120 & 69 & 50 & $1 \mathrm{~A} / \mathrm{D}$ & 80 & 20 & 0 & 120 & 60 & 60 \\
\hline FNT & $1.25 \mathrm{~A} / \mathrm{D}$ & 56 & 24 & 0 & 75 & 42 & 42 & $1 \mathrm{~A} / 1.25 \mathrm{D}$ & 38 & 11 & 0 & 75 & 29 & 36 \\
\hline FXE & 1 A/1.4Dmax & 47 & 29 & 0 & 84 & 39 & 39 & $1 \mathrm{~A} / \mathrm{D}$ & 41 & 11 & 0 & 60 & 31 & 31 \\
\hline GFK & $1.25 \mathrm{~A} / \mathrm{D}$ & 53 & 23 & 0 & 75 & 41 & 41 & $1 \mathrm{~A} / \mathrm{D}$ & 38 & 9 & 0 & 60 & 29 & 29 \\
\hline GRR & $1.75 \mathrm{~A} / \mathrm{D}$ & 74 & 32 & 0 & 105 & 58 & 58 & $1 \mathrm{~A} / \mathrm{D}$ & 38 & 9 & 0 & 60 & 29 & 29 \\
\hline GSO & $\begin{array}{l}1.25 \mathrm{~A} / 0.5 \text { Free } \\
\text { alternate } \\
1.25 \mathrm{Dmax}\end{array}$ & 83 & 34 & 0 & 120 & 65 & 42 & $1 \mathrm{~A} / \mathrm{D}$ & 74 & 17 & 0 & 120 & 57 & 57 \\
\hline GYY & $\begin{array}{l}1.5 \mathrm{~A} / 1.0 \text { Free } \\
\text { alternate } \\
1.5 \mathrm{Dmax}\end{array}$ & 72 & 16 & 0 & 90 & 36 & 54 & $1 \mathrm{~A} / \mathrm{D}$ & 44 & 10 & 0 & 65 & 33 & 33 \\
\hline $\mathrm{HNL}$ & $0.5 \mathrm{~A} / 2 \mathrm{D}+1 \mathrm{~A}$ & 64 & 26 & 43 & 120 & 59 & 64 & $1 \mathrm{~A}+1 \mathrm{D}$ & 38 & 9 & 38 & 60 & 38 & 59 \\
\hline $\mathrm{HOU}$ & $1 \mathrm{~A} / 1.5 \mathrm{D}, \mathrm{E} \& \mathrm{~F}$ & 44 & 33 & 0 & 90 & 35 & 52 & $1 \mathrm{~A} / \mathrm{D}+0.5 \mathrm{Dmax}$ & 39 & 40 & 0 & 90 & 39 & 59 \\
\hline HPN & $\begin{array}{l}1.15 \mathrm{~A} / 1.25 \mathrm{D} 1 \mathrm{~F}, \\
1 \mathrm{E}\end{array}$ & 53 & 27 & 0 & 75 & 37 & 43 & $1 \mathrm{~A} / \mathrm{D}$ & 41 & 10 & 0 & 60 & 30 & 30 \\
\hline IAD & $2 A / D+1 A$ & 133 & 76 & 44 & 180 & 112 & 66 & $2 A / D$ & 79 & 79 & 0 & 180 & 59 & 119 \\
\hline$\overline{\mathrm{IAH}}$ & $1 A / D+2 A+1.5 D$ & 130 & 104 & 87 & 150 & 120 & 118 & $2 A / D+1 A$ & 116 & 18 & 39 & 120 & 97 & 58 \\
\hline ICT & \begin{tabular}{|l|}
$1 \mathrm{~A} / \mathrm{D}+0.5 \mathrm{~A}$ \\
alternate with \\
$0.75 \mathrm{D}$ \\
\end{tabular} & 62 & 62 & 21 & 105 & 41 & 84 & $0.25 A / 1.25 D+1 A$ & 46 & 9 & 37 & 75 & 42 & 42 \\
\hline IND & $2 \mathrm{~A} / \mathrm{D}+0.1 \mathrm{D}$ & 83 & 39 & 0 & 126 & 64 & 70 & $2 A / D$ & 74 & 17 & 0 & 120 & 56 & 56 \\
\hline ISP & $\begin{array}{l}1 \mathrm{~A} / \mathrm{D}+1 \mathrm{Amax}+ \\
0.5 \mathrm{Dmax}\end{array}$ & 95 & 30 & 0 & 90 & 86 & 39 & $1 \mathrm{~A} / \mathrm{D}$ & 39 & 10 & 0 & 60 & 29 & 29 \\
\hline JAX & $\begin{array}{l}\text { 1A/D + 1D with } \\
1.25 \mathrm{Amax}\end{array}$ & 52 & 77 & 0 & 90 & 32 & 89 & $1.25 \mathrm{~A} / \mathrm{D}+0.75 \mathrm{D}$ & 46 & 56 & 0 & 58 & 35 & 58 \\
\hline JFK & $2 A / D$ & 85 & 25 & 0 & 80 & 64 & 63 & $2 A / D$ & 76 & 18 & 0 & 80 & 58 & 58 \\
\hline JNU & $1 \mathrm{~A} / \mathrm{D}$ & 47 & 28 & 0 & 60 & 38 & 38 & $1 \mathrm{~A} / \mathrm{D}$ & 41 & 10 & 0 & 60 & 30 & 30 \\
\hline LAN & $\begin{array}{l}1 \mathrm{~A} / \mathrm{D}+1.25 \\
\mathrm{Dmax}\end{array}$ & 42 & 18 & 0 & 75 & 33 & 33 & $1 \mathrm{~A} / \mathrm{D}$ & 37 & 9 & 0 & 60 & 28 & 28 \\
\hline LAS & $1 \mathrm{~A} / \mathrm{D}+1 \mathrm{~A}$ & 88 & 15 & 44 & 60 & 77 & 33 & $1.1 \mathrm{~A}+1 \mathrm{D}$ & 43 & 60 & 43 & 60 & 43 & 60 \\
\hline LAX & $2 A+2 D$ & 88 & 120 & 88 & 120 & 88 & 102 & $2 A+2 D$ & 78 & 120 & 78 & 120 & 78 & 120 \\
\hline LGA & $1 \mathrm{~A}+1 \mathrm{D}$ & 44 & 50 & 44 & 50 & 44 & 50 & $1 A+1 D$ & 39 & 50 & 39 & 50 & 39 & 50 \\
\hline LGB & $\begin{array}{l}\mathrm{A} / \mathrm{D} \text { with } \\
\text { alternating } 1.5 \\
\text { Amax } 1.5 \mathrm{Dmax} \\
0.5 \text { Free }\end{array}$ & 72 & 15 & 0 & 90 & 36 & 53 & $1 \mathrm{~A} / \mathrm{D}$ & 39 & 10 & 0 & 60 & 29 & 29 \\
\hline
\end{tabular}


Table B-6. 110-Airport ROT-Limited Capacities for 2015 NextGen

\begin{tabular}{|c|c|c|c|c|c|c|c|c|c|c|c|c|c|c|}
\hline \multirow[b]{2}{*}{ Apt } & \multicolumn{7}{|c|}{ Visual meteorological conditions } & \multicolumn{7}{|c|}{ Instrument meteorological conditions } \\
\hline & Configuration & Amax & Aeq & Amin & Dmax & Deq & Free & Configuration & Amax & Amin & Aeq & Dmax & Deq & Free \\
\hline LIT & $\begin{array}{l}\text { 2A/D with Dmax } \\
\text { limit }\end{array}$ & 83 & 34 & 0 & 80 & 65 & 65 & $1 \mathrm{~A} / \mathrm{D}$ & 37 & 9 & 0 & 60 & 28 & 28 \\
\hline $\mathrm{MCl}$ & $2 \mathrm{~A} / \mathrm{D}$ & 83 & 34 & 0 & 120 & 65 & 65 & $2 A / D$ & 74 & 17 & 0 & 120 & 57 & 57 \\
\hline $\mathrm{MCO}$ & $3 \mathrm{~A} / \mathrm{D}+0.5 \mathrm{D}$ & 132 & 75 & 0 & 210 & 100 & 125 & $1 \mathrm{CSP}+1 \mathrm{Dep}$ & 94 & 92 & 0 & 140 & 94 & 94 \\
\hline MDW & $1.25 \mathrm{~A} / \mathrm{D}$ & 56 & 20 & 0 & 75 & 42 & 42 & $1 \mathrm{~A} / \mathrm{D}$ & 40 & 10 & 0 & 60 & 30 & 30 \\
\hline MEM & $\begin{array}{l}1 \mathrm{~A} / \mathrm{D}+1.75 \mathrm{~A}+ \\
1 \mathrm{D}\end{array}$ & 120 & 74 & 76 & 120 & 109 & 85 & $1 A+1 D+1 A / D$ & 78 & 69 & 39 & 120 & 68 & 89 \\
\hline MHT & $1 \mathrm{~A}+1 \mathrm{D}$ & 42 & 60 & 42 & 60 & 42 & 60 & $1 \mathrm{~A} / \mathrm{D}$ & 37 & 9 & 0 & 60 & 28 & 28 \\
\hline MIA & $2 A+2 D$ & 87 & 120 & 87 & 120 & 87 & 101 & $2 A / D$ & 78 & 19 & 0 & 120 & 59 & 59 \\
\hline MKE & $1.5 \mathrm{~A} / \mathrm{D}$ & 87 & 43 & 0 & 120 & 69 & 52 & $1 \mathrm{~A} / \mathrm{D}$ & 77 & 19 & 0 & 120 & 58 & 58 \\
\hline MLB & $1.5 \mathrm{~A} / \mathrm{D}+0.25 \mathrm{D}$ & 65 & 47 & 0 & 105 & 51 & 66 & $1 \mathrm{~A} / \mathrm{D}$ & 39 & 9 & 0 & 60 & 29 & 29 \\
\hline MSN & $1 \mathrm{~A} / \mathrm{D}$ & 42 & 19 & 0 & 60 & 33 & 33 & $1 \mathrm{~A} / \mathrm{D}$ & 38 & 9 & 0 & 60 & 29 & 29 \\
\hline MSP & $\begin{array}{l}2 \text { A/D + } \\
\text { alternating } 1 \\
\text { Amax and } 1 \\
\text { Dmax }\end{array}$ & 131 & 28 & 0 & 180 & 65 & 104 & Dep Pair +1D & 54 & 113 & 0 & 148 & 54 & 114 \\
\hline MSY & 1.2 A/1Dmax & 49 & 20 & 0 & 60 & 39 & 39 & 1.2 A/1Dmax & 44 & 10 & 0 & 60 & 34 & 34 \\
\hline OAK & $1.5 \mathrm{~A} / \mathrm{D}$ & 67 & 35 & 0 & 90 & 53 & 53 & $1 \mathrm{~A} / \mathrm{D}$ & 38 & 9 & 0 & 60 & 29 & 29 \\
\hline OKC & $1.5 \mathrm{~A} / \mathrm{D}$ & 94 & 58 & 0 & 120 & 77 & 58 & $1 A+1 D$ & 82 & 21 & 0 & 120 & 61 & 61 \\
\hline OMA & $2 \mathrm{~A} / 1 \mathrm{Dmax}$ & 94 & 29 & 0 & 60 & 77 & 39 & 1.5 A/1Dmax & 62 & 11 & 0 & 60 & 46 & 31 \\
\hline ONT & $1.75 \mathrm{~A} / \mathrm{D}$ & 77 & 40 & 0 & 105 & 61 & 60 & $1 \mathrm{~A} / \mathrm{D}$ & 38 & 9 & 0 & 60 & 29 & 29 \\
\hline ORD & $1 A / D+2 A+2 D$ & 130 & 133 & 86 & 180 & 119 & 141 & $1 A / D+1 A+2 D$ & 116 & 129 & 77 & 180 & 106 & 149 \\
\hline ORF & $\begin{array}{l}1 \mathrm{~A} / \mathrm{D}+1.25 \\
\mathrm{Dmax}\end{array}$ & 47 & 29 & 0 & 75 & 39 & 39 & $1 \mathrm{~A} / \mathrm{D}$ & 41 & 11 & 0 & 60 & 31 & 31 \\
\hline OXR & $1 \mathrm{~A} / \mathrm{D}$ & 49 & 33 & 0 & 60 & 41 & 60 & $1 \mathrm{~A} / \mathrm{D}$ & 42 & 11 & 0 & 60 & 31 & 31 \\
\hline $\mathrm{PBI}$ & $1 \mathrm{~A} / \mathrm{D}$ & 68 & 38 & 0 & 90 & 55 & 36 & $1 \mathrm{~A} / \mathrm{D}$ & 39 & 10 & 0 & 60 & 29 & 29 \\
\hline $\mathrm{PDX}$ & $2 \mathrm{~A} / \mathrm{D}$ & 90 & 33 & 0 & 120 & 68 & 67 & Dep pair & 79 & 19 & 0 & 120 & 60 & 60 \\
\hline PHF & $\begin{array}{l}1 \mathrm{~A} / \mathrm{D} \text { alternate } \\
\text { with } 0.25 \mathrm{Dmax}\end{array}$ & 47 & 29 & 0 & 75 & 24 & 53 & $1 \mathrm{~A} / \mathrm{D}$ & 41 & 11 & 0 & 60 & 31 & 31 \\
\hline PHL & $\begin{array}{l}1 \mathrm{~A}+1 \mathrm{D} \\
+0.5^{\star} \mathrm{A} / \mathrm{D} \\
\end{array}$ & 88 & 30 & 0 & 120 & 67 & 73 & $1 A+1 D+0.5^{\star} A / D$ & 78 & 19 & 0 & 120 & 59 & 59 \\
\hline $\mathrm{PHX}$ & $1 A+1 D+1 A / D$ & 87 & 74 & 44 & 120 & 77 & 90 & $2 \mathrm{~A} / \mathrm{D}$ & 78 & 18 & 0 & 120 & 59 & 59 \\
\hline PIE & $\begin{array}{l}1 \mathrm{~A} / \mathrm{D}+0.75 \mathrm{~A}+ \\
0.5 \mathrm{D}\end{array}$ & 82 & 59 & 35 & 90 & 74 & 68 & $1 \mathrm{~A} / \mathrm{D}$ & 41 & 11 & 0 & 60 & 31 & 31 \\
\hline PIT & $1 A+1 D+1 A / D$ & 89 & 76 & 45 & 120 & 78 & 92 & $2 A / D$ & 79 & 19 & 0 & 120 & 59 & 59 \\
\hline PVD & $1 \mathrm{~A} / \mathrm{D}$ & 44 & 22 & 0 & 60 & 35 & 35 & $1 \mathrm{~A} / \mathrm{D}$ & 38 & 9 & 0 & 60 & 29 & 29 \\
\hline RDU & $2 A / D$ & 83 & 34 & 0 & 120 & 65 & 65 & $\begin{array}{l}\text { Dep pair - with } \\
1.5^{\star} \text { Dmax Sgl }\end{array}$ & 53 & 52 & 0 & 90 & 52 & 52 \\
\hline RFD & $1.75 \mathrm{~A} / \mathrm{D}$ & 82 & 51 & 0 & 105 & 68 & 65 & $1.25 \mathrm{~A} / \mathrm{D}$ & 49 & 12 & 0 & 90 & 37 & 37 \\
\hline RIC & $1.25 \mathrm{~A} / \mathrm{D}$ & 59 & 36 & 0 & 75 & 48 & 48 & $1 \mathrm{~A} / \mathrm{D}$ & 41 & 11 & 0 & 60 & 31 & 31 \\
\hline RNO & $1.5 \mathrm{~A} / \mathrm{D}$ & 63 & 26 & 0 & 90 & 49 & 49 & $1 \mathrm{~A} / \mathrm{D}$ & 37 & 9 & 0 & 60 & 28 & 28 \\
\hline $\mathrm{ROC}$ & $1.25 \mathrm{~A} / \mathrm{D}$ & 52 & 22 & 0 & 75 & 41 & 41 & $1 \mathrm{~A} / \mathrm{D}$ & 37 & 9 & 0 & 60 & 28 & 28 \\
\hline RSW & $1 \mathrm{~A} / \mathrm{D}$ & 42 & 18 & 0 & 60 & 33 & 33 & $1 \mathrm{~A} / \mathrm{D}$ & 37 & 9 & 0 & 60 & 28 & 28 \\
\hline SAN & $1 \mathrm{~A} / \mathrm{D}$ & 44 & 15 & 0 & 60 & 33 & 33 & $1 \mathrm{~A} / \mathrm{D}$ & 39 & 9 & 0 & 60 & 30 & 30 \\
\hline SAT & $2 A / D$ & 90 & 49 & 0 & 120 & 72 & 72 & $1 \mathrm{~A} / \mathrm{D}$ & 39 & 10 & 0 & 60 & 29 & 29 \\
\hline SBA & $\begin{array}{l}1 A / D+0.25 A+ \\
0.25 D\end{array}$ & 55 & 37 & 11 & 75 & 46 & 50 & $1 A+1 D$ & 39 & 60 & 39 & 60 & 39 & 60 \\
\hline SDF & $2 A / D$ & 83 & 33 & 0 & 120 & 64 & 64 & $2 A / D$ & 74 & 17 & 0 & 120 & 56 & 56 \\
\hline SEA & 2A/dep Dmax & 87 & 28 & 0 & 120 & 66 & 65 & $1 A+\operatorname{DepD}$ & 54 & 26 & 0 & 86 & 54 & 54 \\
\hline SFO & $2 \mathrm{~A} / \mathrm{D}$ & 84 & 38 & 0 & 120 & 66 & 63 & $1 \mathrm{~A} / \mathrm{D}+0.5 \mathrm{D}$ & 39 & 40 & 0 & 90 & 30 & 60 \\
\hline SJC & $\begin{array}{l}1 \mathrm{~A}+1 \mathrm{D} \\
+0.25 \mathrm{~A} / \mathrm{D}\end{array}$ & 54 & 65 & 44 & 75 & 52 & 67 & CSP w/Sgl Dmax & 38 & 37 & 0 & 60 & 38 & 38 \\
\hline SLC & $\begin{array}{l}\text { 2A/D with } \\
2.25 \mathrm{Amax}\end{array}$ & 101 & 34 & 0 & 120 & 80 & 67 & $2 \mathrm{~A} / \mathrm{D}$ with $2.25 \mathrm{Amax}$ & 90 & 20 & 0 & 120 & 70 & 60 \\
\hline SMF & $2 A / D$ & 83 & 34 & 0 & 120 & 65 & 65 & $2 A / D$ & 74 & 17 & 0 & 120 & 57 & 57 \\
\hline SNA & $1.5 \mathrm{~A} / \mathrm{D}$ & 69 & 41 & 0 & 90 & 56 & 56 & $1 \mathrm{~A} / \mathrm{D}$ & 39 & 9 & 0 & 60 & 29 & 29 \\
\hline STL & $1 A+1 D+1 A / D$ & 83 & 78 & 42 & 120 & 74 & 90 & $2 A / D$ & 75 & 18 & 0 & 120 & 57 & 57 \\
\hline SWF & $1 \mathrm{~A} / \mathrm{D}$ & 47 & 29 & 0 & 60 & 39 & 39 & $1 \mathrm{~A} / \mathrm{D}$ & 38 & 9 & 0 & 60 & 29 & 29 \\
\hline SYR & $1.3 \mathrm{~A} / \mathrm{D}$ & 61 & 38 & 0 & 78 & 50 & 50 & $1 \mathrm{~A} / \mathrm{D}$ & 41 & 11 & 0 & 60 & 31 & 31 \\
\hline TEB & $1 \mathrm{~A} / \mathrm{D}+0.5 \mathrm{D}$ & 47 & 59 & 0 & 90 & 39 & 68 & $1 \mathrm{~A} / \mathrm{D}$ & 41 & 11 & 0 & 60 & 31 & 31 \\
\hline
\end{tabular}


Table B-6. 110-Airport ROT-Limited Capacities for 2015 NextGen

\begin{tabular}{|c|c|c|c|c|c|c|c|c|c|c|c|c|c|c|}
\hline \multirow[b]{2}{*}{ Apt } & \multicolumn{7}{|c|}{ Visual meteorological conditions } & \multicolumn{7}{|c|}{ Instrument meteorological conditions } \\
\hline & Configuration & Amax & Aeq & Amin & Dmax & Deq & Free & Configuration & Amax & Amin & Aeq & Dmax & Deq & Free \\
\hline TPA & $\begin{array}{l}\text { 2A/D with Dmax } \\
\text { limit }\end{array}$ & 90 & 33 & 0 & 65 & 68 & 65 & $2 \mathrm{~A} / \mathrm{D}$ with $\mathrm{Dmax}$ limit & 79 & 19 & 0 & 65 & 60 & 65 \\
\hline TUL & $1.6 \mathrm{~A} / \mathrm{D}$ & 75 & 46 & 0 & 96 & 62 & 62 & $1.5 \mathrm{~A} / \mathrm{D}$ & 62 & 16 & 0 & 90 & 46 & 46 \\
\hline TUS & $2 A / D$ & 93 & 55 & 0 & 120 & 75 & 75 & $1 \mathrm{~A} / \mathrm{D}+0.5 \mathrm{D}$ & 38 & 39 & 0 & 90 & 29 & 59 \\
\hline TVC & $\begin{array}{l}1.25 \mathrm{~A} / \mathrm{D} \text { with } \\
\text { interpolated } \mathrm{E}\end{array}$ & 59 & 36 & 0 & 90 & 39 & 60 & $1 \mathrm{~A} / \mathrm{D}$ & 41 & 11 & 0 & 60 & 31 & 31 \\
\hline TYS & $1.25 \mathrm{~A} / \mathrm{D}$ & 59 & 36 & 0 & 75 & 48 & 48 & $1 \mathrm{~A} / \mathrm{D}$ & 41 & 11 & 0 & 60 & 31 & 31 \\
\hline VNY & $1.25 \mathrm{~A} / \mathrm{D}$ & 59 & 36 & 0 & 75 & 48 & 48 & $1 \mathrm{~A} / \mathrm{D}+0.25 \mathrm{D}$ & 41 & 26 & 0 & 75 & 31 & 46 \\
\hline
\end{tabular}

Table B-7. 110-Airport MIT-Limited Capacities for 2025 NextGen

\begin{tabular}{|c|c|c|c|c|c|c|c|c|c|c|c|c|c|c|}
\hline \multirow[b]{2}{*}{ Apt } & \multicolumn{7}{|c|}{ Visual meteorological conditions } & \multicolumn{7}{|c|}{ Instrument meteorological conditions } \\
\hline & Configuration & Amax & Aeq & Amin & Dmax & Deq & Free & Configuration & Amax & Amin & Aeq & Dmax & Deq & Free \\
\hline$A B Q$ & $\begin{array}{l}1.5 \mathrm{~A} / \mathrm{D} \text { with } \\
2 \mathrm{Amax} \text { with } 1 \\
\text { Free }\end{array}$ & 105 & 13 & 0 & 90 & 61 & 61 & 1.5 A/D with $1 \mathrm{Dmax}$ & 80 & 11 & 0 & 59 & 57 & 57 \\
\hline ALB & $1 \mathrm{~A} / \mathrm{D}$ & 56 & 4 & 0 & 60 & 38 & 38 & $1 \mathrm{~A} / 0.5 \mathrm{D}$ & 56 & 2 & 0 & 30 & 38 & 19 \\
\hline ANC & $\begin{array}{l}1.25 \mathrm{~A} / \mathrm{D} \text { with } \\
1.5^{\star} \mathrm{Dmax}\end{array}$ & 60 & 16 & 0 & 81 & 47 & 47 & $\begin{array}{l}\text { 1 } \mathrm{A} / \mathrm{D} \text { with } \\
1.25^{*} \mathrm{Dmax}\end{array}$ & 48 & 10 & 0 & 67 & 38 & 38 \\
\hline ATL & $2 A+2 D+1 A / D$ & 149 & 110 & 99 & 151 & 135 & 137 & $2 A+2 D+1 A / D$ & 149 & 108 & 99 & 151 & 135 & 137 \\
\hline AUS & $1.5 \mathrm{~A} / \mathrm{D}$ & 79 & 19 & 0 & 90 & 61 & 61 & $1.5 \mathrm{~A} / \mathrm{D}$ & 77 & 10 & 0 & 85 & 55 & 55 \\
\hline BDL & $\begin{array}{l}\text { 1.5 Amax with } \\
0.5 \text { Free } \\
\text { alternate with } \\
\text { 1.25Dmax }\end{array}$ & 75 & 6 & 0 & 72 & 38 & 39 & $\begin{array}{l}1.5 \text { Amax with } 0.5 \\
\text { Free alternate with } \\
\text { 1.25Dmax }\end{array}$ & 76 & 4 & 0 & 70 & 38 & 37 \\
\hline $\mathrm{BFL}$ & $1 \mathrm{~A} / \mathrm{Dep} \mathrm{D}$ & 53 & 13 & 0 & 87 & 41 & 41 & $1 \mathrm{~A} / \mathrm{D}$ & 53 & 11 & 0 & 60 & 41 & 41 \\
\hline BHM & $\begin{array}{l}1 \mathrm{~A} / \mathrm{D} \text { with } 1.25 \\
\text { Amax } 1.25 \\
\text { Dmax } 0.5 \text { Free }\end{array}$ & 65 & 6 & 0 & 75 & 41 & 41 & $1 \mathrm{~A} / \mathrm{D}$ & 52 & 11 & 0 & 60 & 41 & 41 \\
\hline BNA & $\begin{array}{l}0.75 \mathrm{~A}+1 \mathrm{D} \\
+1 \mathrm{~A} / \mathrm{D}\end{array}$ & 102 & 61 & 44 & 120 & 80 & 96 & $1 A / D+1.5 A+0.5 D$ & 146 & 31 & 87 & 90 & 124 & 66 \\
\hline $\mathrm{BOI}$ & $\begin{array}{l}\text { 1.75A/D with } \\
\text { 2Dmax }\end{array}$ & 96 & 8 & 0 & 120 & 66 & 66 & $1 \mathrm{~A} / \mathrm{D}$ & 55 & 5 & 0 & 60 & 38 & 38 \\
\hline BOS & $\begin{array}{l}\text { 2A/D with } 1 \\
\text { Amin }\end{array}$ & 100 & 20 & 50 & 111 & 75 & 75 & $1 \mathrm{~A} / \mathrm{D}+\mathrm{Dmax}$ & 100 & 17 & 0 & 111 & 75 & 75 \\
\hline BTR & $\begin{array}{l}1.5 \mathrm{~A} \text { alternate } \\
\text { with } 1.5 \mathrm{D}\end{array}$ & 81 & 17 & 0 & 90 & 41 & 54 & $1 \mathrm{~A} / \mathrm{D}$ & 54 & 15 & 0 & 60 & 44 & 44 \\
\hline BUF & 1.5 A/1Dmax & 88 & 1 & 0 & 60 & 55 & 55 & $1 \mathrm{~A} / \mathrm{D}$ & 58 & 1 & 0 & 60 & 36 & 36 \\
\hline BUR & $1.25 \mathrm{~A} / \mathrm{D}$ & 73 & 17 & 0 & 75 & 52 & 52 & $1 \mathrm{~A} / \mathrm{D}$ & 54 & 6 & 0 & 60 & 38 & 38 \\
\hline BWI & $1.5 \mathrm{~A} / \mathrm{D}$ & 147 & 10 & 53 & 99 & 118 & 65 & $1.5 \mathrm{~A} / \mathrm{D}$ & 147 & 9 & 53 & 99 & 118 & 65 \\
\hline $\mathrm{CHS}$ & $\begin{array}{l}1.75^{\star} \mathrm{A} \text { alternate } \\
\text { with } 2 \mathrm{D} \\
\end{array}$ & 102 & 0 & 0 & 120 & 51 & 60 & $1 \mathrm{~A} / \mathrm{D}$ & 58 & 1 & 0 & 60 & 37 & 37 \\
\hline CLE & $2 \mathrm{~A} / \mathrm{D}$ & 113 & 5 & 0 & 119 & 74 & 74 & $1.25 A+1 D$ & 71 & 59 & 71 & 59 & 71 & 59 \\
\hline $\mathrm{CLT}$ & $2 A / D$ & 164 & 14 & 0 & 175 & 111 & 111 & $2 A / D$ & 164 & 13 & 0 & 175 & 111 & 111 \\
\hline $\mathrm{CMH}$ & $2 A / D$ & 117 & 60 & 59 & 120 & 95 & 96 & Dep A/D & 117 & 1 & 0 & 120 & 73 & 73 \\
\hline $\cos$ & $\begin{array}{l}1.5 \mathrm{~A} \text { alternate } \\
\text { with } 2 \mathrm{D} \text { with } \\
\text { Free } \mathrm{D}\end{array}$ & 86 & 2 & 0 & 120 & 43 & 61 & $1 \mathrm{~A} / \mathrm{D}$ & 57 & 2 & 0 & 60 & 37 & 37 \\
\hline CRP & $\begin{array}{l}1.5 \mathrm{~A} \text { alternate } \\
\text { with } 1.5 \mathrm{Dmax} \\
\text { with } 0.75 \mathrm{Amin} \\
\end{array}$ & 88 & 1 & 44 & 90 & 66 & 45 & $1 \mathrm{~A} / \mathrm{D}$ & 59 & 1 & 0 & 60 & 36 & 36 \\
\hline CVG & $\begin{array}{l}2 \mathrm{~A} / \mathrm{D}+1 \mathrm{~A}+ \\
0.5 \mathrm{Dmax}\end{array}$ & 162 & 10 & 54 & 144 & 128 & 74 & $2 A / D+1 A$ & 162 & 9 & 54 & 115 & 128 & 74 \\
\hline DAB & $\begin{array}{l}1.75 \mathrm{~A} \text { alternate } \\
\text { with } 2 \mathrm{Dmax}\end{array}$ & 93 & 21 & 0 & 120 & 46 & 70 & $1 \mathrm{~A} / \mathrm{D}$ & 53 & 10 & 0 & 60 & 40 & 40 \\
\hline $\mathrm{DAL}$ & $1 A+1 D$ & 59 & 59 & 59 & 59 & 59 & 59 & $1 A+1 D$ & 59 & 59 & 59 & 59 & 59 & 59 \\
\hline DAY & $1.75 \mathrm{~A} / \mathrm{D}$ & 115 & 4 & 0 & 117 & 72 & 72 & $1.75 \mathrm{~A} / \mathrm{D}$ & 115 & 4 & 0 & 117 & 72 & 72 \\
\hline$\overline{D C A}$ & $1.25 A+1 D$ & 73 & 60 & 73 & 60 & 73 & 60 & $1.25 \mathrm{~A} / 1 \mathrm{D}$ & 73 & 1 & 0 & 60 & 46 & 46 \\
\hline DEN & $3 A+3 D$ & 204 & 131 & 102 & 225 & 177 & 188 & $3 A+3 D$ & 204 & 129 & 102 & 225 & 177 & 188 \\
\hline
\end{tabular}


Table B-7. 110-Airport MIT-Limited Capacities for 2025 NextGen

\begin{tabular}{|c|c|c|c|c|c|c|c|c|c|c|c|c|c|c|}
\hline \multirow[b]{2}{*}{ Apt } & \multicolumn{7}{|c|}{ Visual meteorological conditions } & \multicolumn{7}{|c|}{ Instrument meteorological conditions } \\
\hline & Configuration & Amax & Aeq & Amin & Dmax & Deq & Free & Configuration & Amax & Amin & Aeq & Dmax & Deq & Free \\
\hline$\overline{D F W}$ & $3 A+2 D+1 A / D$ & 214 & 122 & 107 & 223 & 180 & 184 & $3 A+2 D$ & 214 & 122 & 107 & 223 & 180 & 184 \\
\hline DSM & $\begin{array}{l}\text { 1A/1D with } 0.75 \\
\text { Amin }\end{array}$ & 58 & 1 & 43 & 60 & 50 & 31 & $1 \mathrm{~A} / \mathrm{D}$ & 58 & 1 & 0 & 60 & 37 & 37 \\
\hline DTW & $2 A+1 D+1 A / D$ & 164 & 61 & 110 & 113 & 146 & 93 & $2 A+2 D$ & 110 & 113 & 110 & 113 & 110 & 113 \\
\hline ELP & $1 A+1 D$ & 59 & 60 & 59 & 60 & 59 & 60 & $1 \mathrm{~A} / \mathrm{D}$ & 59 & 0 & 0 & 60 & 36 & 36 \\
\hline EUG & $1 \mathrm{~A} / \mathrm{D}$ & 54 & 6 & 0 & 60 & 38 & 38 & $1 \mathrm{~A} / \mathrm{D}$ & 54 & 6 & 0 & 60 & 38 & 38 \\
\hline EWR & $\begin{array}{l}1 A+1 D+0.25 \\
A / D \text { with Dmax } \\
\text { limit }\end{array}$ & 65 & 53 & 52 & 53 & 61 & 53 & $1 A+1 D$ & 52 & 7 & 52 & 53 & 52 & 53 \\
\hline FAT & $\begin{array}{l}1.5 \mathrm{~A} / \mathrm{D} \text { with } \\
\text { 1.5Dmax }\end{array}$ & 80 & 13 & 0 & 89 & 58 & 58 & $1 \mathrm{~A} / \mathrm{D}$ & 53 & 8 & 0 & 60 & 39 & 39 \\
\hline FLL & $1.5 \mathrm{~A} / \mathrm{D}$ & 100 & 21 & 0 & 113 & 76 & 76 & $1 \mathrm{~A} / \mathrm{D}$ & 100 & 19 & 0 & 113 & 76 & 76 \\
\hline FNT & $1.25 \mathrm{~A} / \mathrm{D}$ & 63 & 5 & 0 & 75 & 47 & 47 & $1 \mathrm{~A} / 1.25 \mathrm{D}$ & 56 & 5 & 0 & 75 & 38 & 47 \\
\hline FXE & $1 \mathrm{~A} / 1.4 \mathrm{Dmax}$ & 54 & 17 & 0 & 84 & 44 & 44 & $1 \mathrm{~A} / \mathrm{D}$ & 54 & 15 & 0 & 60 & 44 & 44 \\
\hline GFK & $1.25 \mathrm{~A} / \mathrm{D}$ & 71 & 3 & 0 & 75 & 46 & 46 & $1 \mathrm{~A} / \mathrm{D}$ & 57 & 3 & 0 & 60 & 37 & 37 \\
\hline GRR & $1.75 \mathrm{~A} / \mathrm{D}$ & 99 & 4 & 0 & 105 & 65 & 65 & $1 \mathrm{~A} / \mathrm{D}$ & 57 & 3 & 0 & 60 & 37 & 37 \\
\hline GSO & $\begin{array}{l}1.25 \mathrm{~A} / 0.5 \text { Free } \\
\text { alternate } \\
1.25 \mathrm{Dmax}\end{array}$ & 116 & 2 & 0 & 120 & 73 & 73 & $1 \mathrm{~A} / \mathrm{D}$ & 116 & 2 & 0 & 120 & 73 & 73 \\
\hline GYY & $\begin{array}{l}1.5 \mathrm{~A} / 1.0 \text { Free } \\
\text { alternate } \\
1.5 \mathrm{Dmax}\end{array}$ & 84 & 10 & 0 & 90 & 42 & 50 & $1 \mathrm{~A} / \mathrm{D}$ & 58 & 13 & 0 & 65 & 47 & 47 \\
\hline $\mathrm{HNL}$ & $0.5 \mathrm{~A} / 2 \mathrm{D}+1 \mathrm{~A}$ & 73 & 22 & 48 & 101 & 66 & 71 & $1 A+1 D$ & 48 & 10 & 48 & 50 & 48 & 61 \\
\hline $\mathrm{HOU}$ & $1 \mathrm{~A} / 1.5 \mathrm{D}, \mathrm{E} \& \mathrm{~F}$ & 53 & 14 & 0 & 89 & 39 & 59 & $1 \mathrm{~A} / \mathrm{D}+0.5 \mathrm{Dmax}$ & 53 & 39 & 0 & 89 & 53 & 69 \\
\hline HPN & $\begin{array}{l}1.15 \mathrm{~A} / 1.25 \mathrm{D} 1 \mathrm{~F}, \\
1 \mathrm{E}\end{array}$ & 61 & 15 & 0 & 75 & 43 & 49 & $1 \mathrm{~A} / \mathrm{D}$ & 53 & 13 & 0 & 60 & 43 & 43 \\
\hline $\mathrm{IAD}$ & $2 A / D+1 A$ & 154 & 65 & 51 & 168 & 126 & 131 & $2 A / D$ & 103 & 71 & 0 & 168 & 74 & 131 \\
\hline$\overline{\mathrm{IAH}}$ & $1 A / D+2 A+1.5 D$ & 166 & 61 & 55 & 171 & 128 & 130 & $2 A / D+1 A$ & 166 & 61 & 55 & 171 & 128 & 130 \\
\hline ICT & \begin{tabular}{|l|}
$1 \mathrm{~A} / \mathrm{D}+0.5 \mathrm{~A}$ \\
alternate with \\
$0.75 \mathrm{D}$ \\
\end{tabular} & 88 & 46 & 29 & 105 & 59 & 75 & $0.25 A / 1.25 D+1 A$ & 73 & 1 & 59 & 75 & 66 & 66 \\
\hline IND & $2 \mathrm{~A} / \mathrm{D}+0.1 \mathrm{D}$ & 115 & 62 & 0 & 176 & 72 & 131 & $2 A / D$ & 115 & 62 & 0 & 176 & 72 & 131 \\
\hline ISP & $\begin{array}{l}\text { 1A/D + 1Amax + } \\
0.5 \mathrm{Dmax}\end{array}$ & 109 & 18 & 0 & 90 & 99 & 45 & $1 \mathrm{~A} / \mathrm{D}$ & 54 & 7 & 0 & 60 & 39 & 39 \\
\hline JAX & $\begin{array}{l}\text { 1A/D + 1D with } \\
\text { 1.25Amax }\end{array}$ & 111 & 8 & 0 & 114 & 73 & 73 & $1.25 \mathrm{~A} / \mathrm{D}+0.75 \mathrm{D}$ & 111 & 8 & 0 & 114 & 73 & 73 \\
\hline JFK & $2 A / D$ & 89 & 26 & 0 & 80 & 70 & 70 & $2 A / D$ & 89 & 23 & 0 & 80 & 70 & 70 \\
\hline JNU & $1 \mathrm{~A} / \mathrm{D}$ & 54 & 16 & 0 & 60 & 43 & 43 & $1 \mathrm{~A} / \mathrm{D}$ & 54 & 14 & 0 & 60 & 43 & 43 \\
\hline LAN & $\begin{array}{l}1 \mathrm{~A} / \mathrm{D}+1.25 \\
\mathrm{Dmax}\end{array}$ & 57 & 2 & 0 & 75 & 37 & 37 & $1 \mathrm{~A} / \mathrm{D}$ & 57 & 2 & 0 & 60 & 37 & 37 \\
\hline LAS & $1 \mathrm{~A} / \mathrm{D}+1 \mathrm{~A}$ & 104 & 7 & 52 & 56 & 89 & 37 & $1.1 \mathrm{~A}+1 \mathrm{D}$ & 57 & 56 & 57 & 56 & 57 & 56 \\
\hline LAX & $2 A+2 D$ & 95 & 103 & 95 & 103 & 95 & 103 & $2 A+2 D$ & 95 & 103 & 95 & 103 & 95 & 103 \\
\hline LGA & $1 A+1 D$ & 55 & 58 & 55 & 58 & 55 & 57 & $1 \mathrm{~A}+1 \mathrm{D}$ & 55 & 58 & 55 & 58 & 55 & 57 \\
\hline LGB & $\begin{array}{l}1 \text { A/D with } \\
\text { alternating } 1.5 \\
\text { Amax } 1.5 \text { Dmax } \\
0.5 \text { Free }\end{array}$ & 82 & 10 & 0 & 90 & 41 & 50 & $1 \mathrm{~A} / \mathrm{D}$ & 51 & 10 & 0 & 58 & 39 & 39 \\
\hline LIT & $\begin{array}{l}\text { 2A/D with Dmax } \\
\text { limit }\end{array}$ & 117 & 1 & 0 & 80 & 73 & 73 & $1 \mathrm{~A} / \mathrm{D}$ & 59 & 1 & 0 & 60 & 36 & 36 \\
\hline$\overline{\mathrm{MCl}}$ & $2 \mathrm{~A} / \mathrm{D}$ & 116 & 2 & 0 & 120 & 73 & 73 & $2 A / D$ & 116 & 2 & 0 & 120 & 73 & 73 \\
\hline $\mathrm{MCO}$ & $3 \mathrm{~A} / \mathrm{D}+0.5 \mathrm{D}$ & 150 & 56 & 0 & 189 & 111 & 138 & 1CSP + 1Dep & 128 & 81 & 0 & 145 & 123 & 123 \\
\hline MDW & $1.25 \mathrm{~A} / \mathrm{D}$ & 67 & 8 & 0 & 73 & 47 & 47 & $1 \mathrm{~A} / \mathrm{D}$ & 54 & 6 & 0 & 58 & 38 & 38 \\
\hline MEM & $\begin{array}{l}1 \mathrm{~A} / \mathrm{D}+1.75 \mathrm{~A}+ \\
1 \mathrm{D}\end{array}$ & 137 & 63 & 87 & 106 & 124 & 90 & $1 A+1 D+1 A / D$ & 100 & 62 & 50 & 106 & 86 & 90 \\
\hline MHT & $1 A+1 D$ & 58 & 60 & 58 & 60 & 58 & 60 & $1 \mathrm{~A} / \mathrm{D}$ & 58 & 1 & 0 & 60 & 37 & 37 \\
\hline MIA & $2 A+2 D$ & 95 & 102 & 95 & 102 & 95 & 102 & $2 A / D$ & 95 & 21 & 0 & 102 & 73 & 73 \\
\hline MKE & $1.5 \mathrm{~A} / \mathrm{D}$ & 106 & 17 & 0 & 119 & 78 & 78 & $1 \mathrm{~A} / \mathrm{D}$ & 107 & 14 & 0 & 119 & 77 & 77 \\
\hline MLB & $1.5 \mathrm{~A} / \mathrm{D}+0.25 \mathrm{D}$ & 81 & 25 & 0 & 105 & 58 & 73 & $1 \mathrm{~A} / \mathrm{D}$ & 54 & 7 & 0 & 60 & 39 & 39 \\
\hline MSN & $1 \mathrm{~A} / \mathrm{D}$ & 56 & 3 & 0 & 60 & 37 & 37 & $1 \mathrm{~A} / \mathrm{D}$ & 56 & 3 & 0 & 60 & 37 & 37 \\
\hline
\end{tabular}


Table B-7. 110-Airport MIT-Limited Capacities for 2025 NextGen

\begin{tabular}{|c|c|c|c|c|c|c|c|c|c|c|c|c|c|c|}
\hline \multirow[b]{2}{*}{ Apt } & \multicolumn{7}{|c|}{ Visual meteorological conditions } & \multicolumn{7}{|c|}{ Instrument meteorological conditions } \\
\hline & Configuration & Amax & Aeq & Amin & Dmax & Deq & Free & Configuration & Amax & Amin & Aeq & Dmax & Deq & Free \\
\hline MSP & $\begin{array}{l}2 \text { A/D + } \\
\text { alternating } 1 \\
\text { Amax and } 1 \\
\text { Dmax }\end{array}$ & 163 & 9 & 0 & 170 & 81 & 89 & Dep Pair +1D & 79 & 96 & 0 & 147 & 72 & 129 \\
\hline MSY & $1.2 \mathrm{~A} / 1 \mathrm{Dmax}$ & 116 & 3 & 0 & 117 & 72 & 72 & $1.2 \mathrm{~A} / 1 \mathrm{Dmax}$ & 116 & 3 & 0 & 117 & 72 & 72 \\
\hline OAK & $1.5 \mathrm{~A} / \mathrm{D}$ & 77 & 19 & 0 & 88 & 60 & 60 & $1 \mathrm{~A} / \mathrm{D}$ & 53 & 7 & 0 & 57 & 37 & 37 \\
\hline OKC & $1.5 \mathrm{~A} / \mathrm{D}$ & 108 & 77 & 54 & 120 & 98 & 104 & $1 A+1 D$ & 108 & 75 & 54 & 120 & 98 & 104 \\
\hline OMA & $2 \mathrm{~A} / 1 \mathrm{Dmax}$ & 108 & 17 & 0 & 60 & 88 & 44 & $1.5 \mathrm{~A} / 1 \mathrm{Dmax}$ & 81 & 15 & 0 & 60 & 66 & 44 \\
\hline ONT & $1.75 \mathrm{~A} / \mathrm{D}$ & 87 & 22 & 0 & 101 & 69 & 69 & $1 \mathrm{~A} / \mathrm{D}$ & 51 & 8 & 0 & 56 & 38 & 38 \\
\hline ORD & $1 A / D+2 A+2 D$ & 213 & 122 & 107 & 219 & 179 & 182 & $1 A / D+1 A+2 D$ & 213 & 121 & 107 & 219 & 179 & 182 \\
\hline ORF & $\begin{array}{l}1 \mathrm{~A} / \mathrm{D}+1.25 \\
\mathrm{Dmax}\end{array}$ & 81 & 26 & 0 & 90 & 66 & 66 & $1 \mathrm{~A} / \mathrm{D}$ & 54 & 45 & 0 & 92 & 54 & 54 \\
\hline OXR & $1 \mathrm{~A} / \mathrm{D}$ & 57 & 20 & 0 & 60 & 48 & 60 & $1 \mathrm{~A} / \mathrm{D}$ & 57 & 15 & 0 & 60 & 48 & 48 \\
\hline PBI & $1 \mathrm{~A} / \mathrm{D}$ & 77 & 23 & 0 & 89 & 62 & 62 & $1 \mathrm{~A} / \mathrm{D}$ & 51 & 11 & 0 & 58 & 39 & 39 \\
\hline PDX & $2 \mathrm{~A} / \mathrm{D}$ & 103 & 17 & 0 & 114 & 75 & 75 & Dep pair & 103 & 15 & 0 & 114 & 75 & 75 \\
\hline PHF & $\begin{array}{l}1 \mathrm{~A} / \mathrm{D} \text { alternate } \\
\text { with } 0.25 \mathrm{Dmax}\end{array}$ & 54 & 17 & 0 & 75 & 27 & 46 & $1 \mathrm{~A} / \mathrm{D}$ & 54 & 15 & 0 & 60 & 44 & 44 \\
\hline PHL & $\begin{array}{l}1 \mathrm{~A}+1 \mathrm{D} \\
+0.5^{\star} \mathrm{A} / \mathrm{D}\end{array}$ & 105 & 13 & 0 & 113 & 74 & 74 & $1 \mathrm{~A}+1 \mathrm{D}+0.5^{\star} \mathrm{A} / \mathrm{D}$ & 105 & 12 & 0 & 113 & 74 & 74 \\
\hline $\mathrm{PHX}$ & $1 A+1 D+1 A / D$ & 111 & 61 & 56 & 116 & 92 & 95 & $2 A / D$ & 111 & 6 & 0 & 116 & 73 & 73 \\
\hline PIE & $\begin{array}{l}1 \mathrm{~A} / \mathrm{D}+0.75 \mathrm{~A}+ \\
0.5 \mathrm{D}\end{array}$ & 93 & 48 & 40 & 90 & 84 & 74 & $1 \mathrm{~A} / \mathrm{D}$ & 53 & 14 & 0 & 60 & 44 & 44 \\
\hline PIT & $1 A+1 D+1 A / D$ & 108 & 64 & 54 & 118 & 92 & 97 & $2 A / D$ & 108 & 11 & 0 & 118 & 75 & 75 \\
\hline PVD & $1 \mathrm{~A} / \mathrm{D}$ & 53 & 9 & 0 & 60 & 39 & 39 & $1 \mathrm{~A} / \mathrm{D}$ & 54 & 5 & 0 & 59 & 38 & 38 \\
\hline RDU & $2 A / D$ & 116 & 61 & 58 & 119 & 94 & 96 & $\begin{array}{l}\text { Dep pair - with } \\
1.5^{\star} \mathrm{Dmax} \mathrm{Sgl}\end{array}$ & 116 & 61 & 58 & 119 & 94 & 96 \\
\hline RFD & $1.75 \mathrm{~A} / \mathrm{D}$ & 88 & 35 & 0 & 103 & 78 & 78 & $1.25 \mathrm{~A} / \mathrm{D}$ & 54 & 16 & 0 & 79 & 49 & 49 \\
\hline $\mathrm{RIC}$ & $1.25 \mathrm{~A} / \mathrm{D}$ & 68 & 22 & 0 & 75 & 55 & 55 & $1 \mathrm{~A} / \mathrm{D}$ & 54 & 15 & 0 & 60 & 44 & 44 \\
\hline RNO & $1.5 \mathrm{~A} / \mathrm{D}$ & 84 & 4 & 0 & 88 & 55 & 55 & $1 \mathrm{~A} / \mathrm{D}$ & 56 & 3 & 0 & 59 & 37 & 37 \\
\hline $\mathrm{ROC}$ & $1.25 \mathrm{~A} / \mathrm{D}$ & 72 & 2 & 0 & 75 & 46 & 46 & $1 \mathrm{~A} / \mathrm{D}$ & 57 & 2 & 0 & 60 & 37 & 37 \\
\hline RSW & $1 \mathrm{~A} / \mathrm{D}$ & 108 & 10 & 0 & 113 & 73 & 73 & $1 \mathrm{~A} / \mathrm{D}$ & 108 & 9 & 0 & 113 & 73 & 73 \\
\hline SAN & $1 \mathrm{~A} / \mathrm{D}$ & 53 & 6 & 0 & 57 & 37 & 37 & $1 \mathrm{~A} / \mathrm{D}$ & 53 & 6 & 0 & 57 & 37 & 37 \\
\hline SAT & $2 \mathrm{~A} / \mathrm{D}$ & 105 & 25 & 0 & 119 & 81 & 81 & $1 \mathrm{~A} / \mathrm{D}$ & 53 & 67 & 0 & 118 & 39 & 98 \\
\hline SBA & $\begin{array}{l}1 A / D+0.25 A+ \\
0.25 D\end{array}$ & 67 & 24 & 13 & 75 & 53 & 54 & $1 A+1 D$ & 53 & 60 & 53 & 60 & 53 & 60 \\
\hline SDF & $2 \mathrm{~A} / \mathrm{D}$ & 118 & 1 & 0 & 120 & 73 & 73 & $2 A / D$ & 118 & 1 & 0 & 120 & 73 & 73 \\
\hline SEA & 2A/dep Dmax & 108 & 10 & 0 & 113 & 73 & 73 & $1 A+D e p D$ & 108 & 10 & 0 & 113 & 73 & 73 \\
\hline SFO & $2 A / D$ & 81 & 41 & 0 & 103 & 67 & 67 & $1 \mathrm{~A} / \mathrm{D}+0.5 \mathrm{D}$ & 47 & 37 & 0 & 78 & 37 & 63 \\
\hline SJC & $\begin{array}{l}1 \mathrm{~A}+1 \mathrm{D} \\
+0.25 \mathrm{~A} / \mathrm{D}\end{array}$ & 66 & 61 & 52 & 74 & 62 & 69 & CSP w/Sgl Dmax & 55 & 44 & 0 & 58 & 55 & 55 \\
\hline SLC & $\begin{array}{l}\text { 2A/D with } \\
2.25 \mathrm{Amax}\end{array}$ & 115 & 20 & 0 & 114 & 89 & 76 & $2 \mathrm{~A} / \mathrm{D}$ with $2.25 \mathrm{Amax}$ & 115 & 17 & 0 & 114 & 89 & 76 \\
\hline SMF & $2 A / D$ & 114 & 4 & 0 & 118 & 73 & 73 & $2 A / D$ & 114 & 4 & 0 & 118 & 73 & 73 \\
\hline SNA & $1.5 \mathrm{~A} / \mathrm{D}$ & 79 & 24 & 0 & 90 & 64 & 64 & $1 \mathrm{~A} / \mathrm{D}$ & 53 & 8 & 0 & 59 & 39 & 39 \\
\hline STL & $1 A+1 D+1 A / D$ & 110 & 61 & 55 & 116 & 92 & 94 & $2 A / D$ & 110 & 7 & 0 & 116 & 73 & 73 \\
\hline SWF & $1 \mathrm{~A} / \mathrm{D}$ & 54 & 18 & 0 & 60 & 44 & 44 & $1 \mathrm{~A} / \mathrm{D}$ & 53 & 8 & 0 & 59 & 38 & 38 \\
\hline SYR & $1.3 \mathrm{~A} / \mathrm{D}$ & 70 & 23 & 0 & 78 & 57 & 57 & $1 \mathrm{~A} / \mathrm{D}$ & 54 & 15 & 0 & 60 & 44 & 44 \\
\hline TEB & $1 \mathrm{~A} / \mathrm{D}+0.5 \mathrm{D}$ & 54 & 47 & 0 & 90 & 44 & 74 & $1 \mathrm{~A} / \mathrm{D}$ & 54 & 15 & 0 & 60 & 44 & 44 \\
\hline TPA & $\begin{array}{l}\text { 2A/D with Dmax } \\
\text { limit }\end{array}$ & 103 & 8 & 52 & 80 & 90 & 80 & $2 A / D$ with Dmax limit & 103 & 8 & 52 & 80 & 90 & 80 \\
\hline TUL & $1.6 \mathrm{~A} / \mathrm{D}$ & 162 & 53 & 0 & 180 & 133 & 133 & $1.5 \mathrm{~A} / \mathrm{D}$ & 108 & 29 & 0 & 120 & 88 & 88 \\
\hline TUS & $2 A / D$ & 107 & 31 & 0 & 120 & 85 & 85 & $1 \mathrm{~A} / \mathrm{D}+0.5 \mathrm{D}$ & 56 & 34 & 0 & 90 & 38 & 68 \\
\hline TVC & $\begin{array}{l}1.25 \mathrm{~A} / \mathrm{D} \text { with } \\
\text { interpolated } \mathrm{E}\end{array}$ & 68 & 22 & 0 & 90 & 44 & 55 & $1 \mathrm{~A} / \mathrm{D}$ & 54 & 15 & 0 & 60 & 44 & 44 \\
\hline TYS & $1.25 \mathrm{~A} / \mathrm{D}$ & 68 & 22 & 0 & 75 & 55 & 55 & $1 \mathrm{~A} / \mathrm{D}$ & 54 & 15 & 0 & 60 & 44 & 44 \\
\hline VNY & $1.25 \mathrm{~A} / \mathrm{D}$ & 68 & 22 & 0 & 75 & 55 & 55 & $1 \mathrm{~A} / \mathrm{D}+0.25 \mathrm{D}$ & 54 & 30 & 0 & 75 & 44 & 59 \\
\hline
\end{tabular}


Table B-8. 110-Airport ROT-Limited Capacities for 2025 NextGen

\begin{tabular}{|c|c|c|c|c|c|c|c|c|c|c|c|c|c|c|}
\hline \multirow[b]{2}{*}{ Apt } & \multicolumn{7}{|c|}{ Visual meteorological conditions } & \multicolumn{7}{|c|}{ Instrument meteorological conditions } \\
\hline & Configuration & Amax & Aeq & Amin & Dmax & Deq & Free & Configuration & Amax & Amin & Aeq & Dmax & Deq & Free \\
\hline$A B Q$ & $\begin{array}{l}1.5 \mathrm{~A} / \mathrm{D} \text { with } \\
2 \mathrm{Amax} \text { with } 1 \\
\text { Free }\end{array}$ & 128 & 0 & 0 & 90 & 61 & 128 & $1.5 \mathrm{~A} / \mathrm{D}$ with $1 \mathrm{Dmax}$ & 90 & 0 & 0 & 60 & 57 & 57 \\
\hline ALB & $1 \mathrm{~A} / \mathrm{D}$ & 59 & 0 & 0 & 60 & 38 & 59 & $1 \mathrm{~A} / 0.5 \mathrm{D}$ & 59 & 0 & 0 & 30 & 38 & 19 \\
\hline ANC & $\begin{array}{l}1.25 \mathrm{~A} / \mathrm{D} \text { with } \\
1.5^{*} \mathrm{Dmax}\end{array}$ & 73 & 0 & 0 & 90 & 47 & 73 & $\begin{array}{l}1 \mathrm{~A} / \mathrm{D} \text { with } \\
1.25^{*} \mathrm{Dmax}\end{array}$ & 59 & 0 & 0 & 75 & 38 & 38 \\
\hline ATL & $2 A+2 D+1 A / D$ & 167 & 119 & 111 & 178 & 147 & 167 & $2 A+2 D+1 A / D$ & 167 & 118 & 111 & 178 & 147 & 155 \\
\hline AUS & $1.5 \mathrm{~A} / \mathrm{D}$ & 96 & 0 & 0 & 90 & 61 & 96 & $1.5 \mathrm{~A} / \mathrm{D}$ & 86 & 0 & 0 & 87 & 55 & 55 \\
\hline BDL & $\begin{array}{l}\text { 1.5 Amax with } \\
0.5 \text { Free } \\
\text { alternate with } \\
\text { 1.25Dmax }\end{array}$ & 91 & 0 & 0 & 75 & 45 & 91 & $\begin{array}{l}\text { 1.5 Amax with } 0.5 \\
\text { Free alternate with } \\
\text { 1.25Dmax }\end{array}$ & 88 & 0 & 0 & 75 & 44 & 38 \\
\hline BFL & $1 \mathrm{~A} / \mathrm{Dep} \mathrm{D}$ & 64 & 0 & 0 & 87 & 41 & 64 & $1 \mathrm{~A} / \mathrm{D}$ & 64 & 0 & 0 & 60 & 41 & 41 \\
\hline BHM & $\begin{array}{l}1 \mathrm{~A} / \mathrm{D} \text { with } 1.25 \\
\text { Amax } 1.25 \\
\text { Dmax } 0.5 \text { Free }\end{array}$ & 80 & 0 & 0 & 75 & 41 & 80 & $1 \mathrm{~A} / \mathrm{D}$ & 64 & 0 & 0 & 60 & 41 & 41 \\
\hline BNA & $\begin{array}{l}0.75 \mathrm{~A}+1 \mathrm{D} \\
+1 \mathrm{~A} / \mathrm{D} \\
\end{array}$ & 99 & 60 & 42 & 120 & 79 & 99 & $1 A / D+1.5 A+0.5 D$ & 141 & 30 & 85 & 90 & 121 & 66 \\
\hline $\mathrm{BOI}$ & $\begin{array}{l}\text { 1.75A/D with } \\
\text { 2Dmax }\end{array}$ & 103 & 0 & 0 & 120 & 66 & 103 & $1 \mathrm{~A} / \mathrm{D}$ & 59 & 0 & 0 & 60 & 38 & 38 \\
\hline BOS & $\begin{array}{l}\text { 2A/D with } 1 \\
\text { Amin }\end{array}$ & 117 & 0 & 59 & 120 & 86 & 117 & $1 \mathrm{~A} / \mathrm{D}+\mathrm{Dmax}$ & 117 & 0 & 0 & 120 & 75 & 75 \\
\hline BTR & $\begin{array}{l}1.5 \mathrm{~A} \text { alternate } \\
\text { with } 1.5 \mathrm{D}\end{array}$ & 102 & 1 & 0 & 90 & 51 & 102 & $1 \mathrm{~A} / \mathrm{D}$ & 68 & 0 & 0 & 60 & 44 & 44 \\
\hline BUF & 1.5 A/1Dmax & 85 & 0 & 0 & 60 & 55 & 85 & $1 \mathrm{~A} / \mathrm{D}$ & 57 & 0 & 0 & 60 & 36 & 36 \\
\hline BUR & $1.25 \mathrm{~A} / \mathrm{D}$ & 71 & 0 & 0 & 75 & 52 & 71 & $1 \mathrm{~A} / \mathrm{D}$ & 60 & 0 & 0 & 60 & 38 & 38 \\
\hline BWI & $1.5 \mathrm{~A} / \mathrm{D}$ & 158 & 0 & 58 & 105 & 122 & 158 & $1.5 \mathrm{~A} / \mathrm{D}$ & 158 & 0 & 58 & 105 & 122 & 65 \\
\hline $\mathrm{CHS}$ & $\begin{array}{l}1.75^{*} \mathrm{~A} \text { alternate } \\
\text { with } 2 \mathrm{D} \\
\end{array}$ & 99 & 0 & 0 & 120 & 50 & 99 & $1 \mathrm{~A} / \mathrm{D}$ & 57 & 0 & 0 & 60 & 37 & 37 \\
\hline CLE & $2 A / D$ & 115 & 0 & 0 & 120 & 74 & 115 & $1.25 \mathrm{~A}+1 \mathrm{D}$ & 72 & 60 & 72 & 60 & 72 & 60 \\
\hline CLT & $2 A / D$ & 173 & 0 & 0 & 180 & 111 & 173 & $2 A / D$ & 173 & 0 & 0 & 180 & 111 & 111 \\
\hline $\mathrm{CMH}$ & $2 A / D$ & 113 & 60 & 56 & 120 & 93 & 113 & Dep A/D & 113 & 0 & 0 & 120 & 73 & 73 \\
\hline $\cos$ & $\begin{array}{l}1.5 \mathrm{~A} \text { alternate } \\
\text { with } 2 \mathrm{D} \text { with } \\
\text { Free } \mathrm{D} \\
\end{array}$ & 86 & 0 & 0 & 120 & 43 & 86 & $1 \mathrm{~A} / \mathrm{D}$ & 57 & 0 & 0 & 60 & 37 & 37 \\
\hline CRP & $\begin{array}{l}1.5 \mathrm{~A} \text { alternate } \\
\text { with } 1.5 \mathrm{Dmax} \\
\text { with } 0.75 \mathrm{Amin} \\
\end{array}$ & 85 & 0 & 42 & 90 & 63 & 85 & $1 \mathrm{~A} / \mathrm{D}$ & 56 & 0 & 0 & 60 & 36 & 36 \\
\hline CVG & $\begin{array}{l}2 \mathrm{~A} / \mathrm{D}+1 \mathrm{~A}+ \\
0.5 \mathrm{Dmax}\end{array}$ & 173 & 0 & 58 & 150 & 131 & 173 & $2 A / D+1 A$ & 173 & 0 & 58 & 120 & 131 & 74 \\
\hline DAB & $\begin{array}{l}1.75 \mathrm{~A} \text { alternate } \\
\text { with } 2 \mathrm{Dmax}\end{array}$ & 110 & 0 & 0 & 120 & 55 & 110 & $1 \mathrm{~A} / \mathrm{D}$ & 63 & 0 & 0 & 60 & 40 & 40 \\
\hline $\mathrm{DAL}$ & $1 A+1 D$ & 56 & 60 & 56 & 60 & 56 & 56 & $1 \mathrm{~A}+1 \mathrm{D}$ & 56 & 60 & 56 & 60 & 56 & 60 \\
\hline DAY & $1.75 \mathrm{~A} / \mathrm{D}$ & 112 & 0 & 0 & 120 & 72 & 112 & $1.75 \mathrm{~A} / \mathrm{D}$ & 112 & 0 & 0 & 120 & 72 & 72 \\
\hline $\mathrm{DCA}$ & $1.25 \mathrm{~A}+1 \mathrm{D}$ & 71 & 60 & 71 & 60 & 71 & 71 & 1.25A/1D & 71 & 0 & 0 & 60 & 46 & 46 \\
\hline DEN & $3 A+3 D$ & 236 & 120 & 118 & 240 & 193 & 236 & $3 A+3 D$ & 236 & 120 & 118 & 240 & 193 & 195 \\
\hline DFW & $3 A+2 D+1 A / D$ & 227 & 120 & 113 & 240 & 186 & 227 & $3 A+2 D$ & 227 & 120 & 113 & 240 & 186 & 193 \\
\hline DSM & $\begin{array}{l}\text { 1A/1D with } 0.75 \\
\text { Amin }\end{array}$ & 57 & 0 & 43 & 60 & 50 & 57 & $1 \mathrm{~A} / \mathrm{D}$ & 57 & 0 & 0 & 60 & 37 & 37 \\
\hline DTW & $2 A+1 D+1 A / D$ & 169 & 60 & 113 & 120 & 149 & 169 & $2 A+2 D$ & 113 & 120 & 113 & 120 & 113 & 120 \\
\hline ELP & $1 A+1 D$ & 56 & 60 & 56 & 60 & 56 & 56 & $1 \mathrm{~A} / \mathrm{D}$ & 56 & 0 & 0 & 60 & 36 & 36 \\
\hline EUG & $1 \mathrm{~A} / \mathrm{D}$ & 60 & 0 & 0 & 60 & 38 & 60 & $1 \mathrm{~A} / \mathrm{D}$ & 60 & 0 & 0 & 60 & 38 & 38 \\
\hline EWR & \begin{tabular}{|l}
$A+1 D+0.25$ \\
$A / D$ with Dmax \\
limit \\
\end{tabular} & 70 & 60 & 56 & 75 & 65 & 70 & $1 A+1 D$ & 56 & 0 & 56 & 60 & 56 & 60 \\
\hline FAT & $\begin{array}{l}\text { 1.5 A/D with } \\
\text { 1.5Dmax } \\
\end{array}$ & 91 & 0 & 0 & 90 & 58 & 91 & $1 \mathrm{~A} / \mathrm{D}$ & 61 & 0 & 0 & 60 & 39 & 39 \\
\hline FLL & 1.5A/D & 119 & 0 & 0 & 120 & 76 & 119 & $1 \mathrm{~A} / \mathrm{D}$ & 119 & 0 & 0 & 120 & 76 & 76 \\
\hline FNT & $1.25 \mathrm{~A} / \mathrm{D}$ & 75 & 0 & 0 & 75 & 47 & 75 & $1 \mathrm{~A} / 1.25 \mathrm{D}$ & 59 & 0 & 0 & 75 & 38 & 47 \\
\hline FXE & $1 \mathrm{~A} / 1.4 \mathrm{Dmax}$ & 68 & 1 & 0 & 84 & 44 & 68 & $1 \mathrm{~A} / \mathrm{D}$ & 68 & 0 & 0 & 60 & 44 & 44 \\
\hline GFK & $1.25 \mathrm{~A} / \mathrm{D}$ & 72 & 0 & 0 & 75 & 46 & 72 & $1 \mathrm{~A} / \mathrm{D}$ & 58 & 0 & 0 & 60 & 37 & 37 \\
\hline GRR & $1.75 \mathrm{~A} / \mathrm{D}$ & 101 & 0 & 0 & 105 & 65 & 101 & $1 \mathrm{~A} / \mathrm{D}$ & 58 & 0 & 0 & 60 & 37 & 37 \\
\hline
\end{tabular}


Table B-8. 110-Airport ROT-Limited Capacities for 2025 NextGen

\begin{tabular}{|c|c|c|c|c|c|c|c|c|c|c|c|c|c|c|}
\hline \multirow[b]{2}{*}{ Apt } & \multicolumn{7}{|c|}{ Visual meteorological conditions } & \multicolumn{7}{|c|}{ Instrument meteorological conditions } \\
\hline & Configuration & Amax & Aeq & Amin & Dmax & Deq & Free & Configuration & Amax & Amin & Aeq & Dmax & Deq & Free \\
\hline GSO & $\begin{array}{l}1.25 \mathrm{~A} / 0.5 \text { Free } \\
\text { alternate } \\
1.25 \mathrm{Dmax}\end{array}$ & 113 & 0 & 0 & 120 & 73 & 113 & $1 \mathrm{~A} / \mathrm{D}$ & 113 & 0 & 0 & 120 & 73 & 73 \\
\hline GYY & \begin{tabular}{|l|}
$1.5 \mathrm{~A} / 1.0$ Free \\
alternate \\
$1.5 \mathrm{Dmax}$ \\
\end{tabular} & 107 & 0 & 0 & 90 & 53 & 107 & $1 \mathrm{~A} / \mathrm{D}$ & 73 & 0 & 0 & 65 & 47 & 47 \\
\hline $\mathrm{HNL}$ & $0.5 \mathrm{~A} / 2 \mathrm{D}+1 \mathrm{~A}$ & 84 & 0 & 56 & 120 & 74 & 84 & $1 \mathrm{~A}+1 \mathrm{D}$ & 56 & 0 & 56 & 60 & 56 & 66 \\
\hline $\mathrm{HOU}$ & $1 \mathrm{~A} / 1.5 \mathrm{D}, \mathrm{E} \& \mathrm{~F}$ & 61 & 0 & 0 & 90 & 39 & 61 & $1 \mathrm{~A} / \mathrm{D}+0.5 \mathrm{Dmax}$ & 61 & 30 & 0 & 90 & 61 & 69 \\
\hline HPN & $\begin{array}{l}1.15 \mathrm{~A} / 1.25 \mathrm{D} 1 \mathrm{~F}, \\
1 \mathrm{E}\end{array}$ & 76 & 0 & 0 & 75 & 43 & 76 & $1 \mathrm{~A} / \mathrm{D}$ & 66 & 0 & 0 & 60 & 43 & 43 \\
\hline IAD & $2 A / D+1 A$ & 175 & 60 & 58 & 180 & 133 & 175 & $2 A / D$ & 116 & 60 & 0 & 180 & 75 & 135 \\
\hline $\mathrm{IAH}$ & $1 \mathrm{~A} / \mathrm{D}+2 \mathrm{~A}+1.5 \mathrm{D}$ & 170 & 60 & 57 & 180 & 129 & 170 & $2 A / D+1 A$ & 170 & 60 & 57 & 180 & 129 & 133 \\
\hline ICT & $\begin{array}{l}1 \mathrm{~A} / \mathrm{D}+0.5 \mathrm{~A} \\
\text { alternate with } \\
0.75 \mathrm{D}\end{array}$ & 85 & 45 & 28 & 105 & 56 & 85 & $0.25 A / 1.25 D+1 A$ & 71 & 0 & 56 & 75 & 63 & 38 \\
\hline IND & $2 \mathrm{~A} / \mathrm{D}+0.1 \mathrm{D}$ & 112 & 60 & 0 & 180 & 73 & 112 & $2 A / D$ & 112 & 60 & 0 & 180 & 73 & 133 \\
\hline ISP & $\begin{array}{l}\text { 1A/D + 1Amax + } \\
0.5 \mathrm{Dmax}\end{array}$ & 138 & 1 & 0 & 90 & 114 & 138 & $1 \mathrm{~A} / \mathrm{D}$ & 61 & 0 & 0 & 60 & 39 & 39 \\
\hline JAX & $\begin{array}{l}\text { 1A/D + 1D with } \\
\text { 1.25Amax }\end{array}$ & 113 & 0 & 0 & 120 & 73 & 113 & $1.25 \mathrm{~A} / \mathrm{D}+0.75 \mathrm{D}$ & 113 & 0 & 0 & 120 & 73 & 73 \\
\hline JFK & $2 A / D$ & 110 & 0 & 0 & 80 & 71 & 110 & $2 A / D$ & 110 & 0 & 0 & 80 & 71 & 71 \\
\hline JNU & $1 \mathrm{~A} / \mathrm{D}$ & 67 & 0 & 0 & 60 & 43 & 67 & $1 \mathrm{~A} / \mathrm{D}$ & 67 & 0 & 0 & 60 & 43 & 43 \\
\hline LAN & $\begin{array}{l}1 \mathrm{~A} / \mathrm{D}+1.25 \\
\mathrm{Dmax}\end{array}$ & 57 & 0 & 0 & 75 & 37 & 57 & $1 \mathrm{~A} / \mathrm{D}$ & 57 & 0 & 0 & 60 & 37 & 37 \\
\hline LAS & $1 \mathrm{~A} / \mathrm{D}+1 \mathrm{~A}$ & 115 & 0 & 58 & 60 & 95 & 115 & $1.1 \mathrm{~A}+1 \mathrm{D}$ & 63 & 60 & 63 & 60 & 63 & 60 \\
\hline LAX & $2 A+2 D$ & 114 & 120 & 114 & 120 & 114 & 114 & $2 A+2 D$ & 114 & 120 & 114 & 120 & 114 & 120 \\
\hline LGA & $1 A+1 D$ & 57 & 60 & 57 & 60 & 57 & 57 & $1 A+1 D$ & 57 & 60 & 57 & 60 & 57 & 60 \\
\hline LGB & $\begin{array}{l}1 \mathrm{~A} / \mathrm{D} \text { with } \\
\text { alternating } 1.5 \\
\text { Amax } 1.5 \mathrm{Dmax} \\
0.5 \text { Free } \\
\end{array}$ & 105 & 0 & 0 & 90 & 53 & 105 & $1 \mathrm{~A} / \mathrm{D}$ & 62 & 0 & 0 & 60 & 39 & 39 \\
\hline LIT & $\begin{array}{l}\text { 2A/D with Dmax } \\
\text { limit }\end{array}$ & 113 & 0 & 0 & 80 & 73 & 113 & $1 \mathrm{~A} / \mathrm{D}$ & 57 & 0 & 0 & 60 & 36 & 36 \\
\hline $\mathrm{MCl}$ & $2 \mathrm{~A} / \mathrm{D}$ & 113 & 0 & 0 & 120 & 73 & 113 & $2 A / D$ & 113 & 0 & 0 & 120 & 73 & 73 \\
\hline $\mathrm{MCO}$ & $3 \mathrm{~A} / \mathrm{D}+0.5 \mathrm{D}$ & 173 & 30 & 0 & 210 & 111 & 173 & 1CSP + 1Dep & 138 & 96 & 0 & 145 & 131 & 131 \\
\hline MDW & $1.25 \mathrm{~A} / \mathrm{D}$ & 74 & 0 & 0 & 75 & 47 & 74 & $1 \mathrm{~A} / \mathrm{D}$ & 59 & 0 & 0 & 60 & 38 & 38 \\
\hline MEM & $\begin{array}{l}1 \mathrm{~A} / \mathrm{D}+1.75 \mathrm{~A}+ \\
1 \mathrm{D}\end{array}$ & 156 & 60 & 99 & 120 & 136 & 156 & $1 A+1 D+1 A / D$ & 114 & 60 & 57 & 120 & 94 & 97 \\
\hline MHT & $1 A+1 D$ & 57 & 60 & 57 & 60 & 57 & 57 & $1 \mathrm{~A} / \mathrm{D}$ & 57 & 0 & 0 & 60 & 37 & 37 \\
\hline MIA & $2 A+2 D$ & 113 & 120 & 113 & 120 & 113 & 113 & $2 A / D$ & 113 & 0 & 0 & 120 & 73 & 73 \\
\hline MKE & $1.5 \mathrm{~A} / \mathrm{D}$ & 122 & 0 & 0 & 120 & 78 & 122 & $1 \mathrm{~A} / \mathrm{D}$ & 120 & 0 & 0 & 120 & 77 & 77 \\
\hline MLB & $1.5 \mathrm{~A} / \mathrm{D}+0.25 \mathrm{D}$ & 90 & 15 & 0 & 105 & 58 & 90 & $1 \mathrm{~A} / \mathrm{D}$ & 60 & 0 & 0 & 60 & 39 & 39 \\
\hline MSN & $1 \mathrm{~A} / \mathrm{D}$ & 58 & 0 & 0 & 60 & 37 & 58 & $1 \mathrm{~A} / \mathrm{D}$ & 58 & 0 & 0 & 60 & 37 & 37 \\
\hline MSP & $\begin{array}{l}2 \mathrm{~A} / \mathrm{D}+ \\
\text { alternating } 1 \\
\text { Amax and } 1 \\
\text { Dmax } \\
\end{array}$ & 170 & 0 & 0 & 180 & 85 & 170 & Dep Pair +1D & 80 & 95 & 0 & 150 & 73 & 133 \\
\hline MSY & 1.2 A/1Dmax & 112 & 0 & 0 & 120 & 72 & 112 & 1.2 A/1Dmax & 112 & 0 & 0 & 120 & 72 & 72 \\
\hline OAK & $1.5 \mathrm{~A} / \mathrm{D}$ & 94 & 0 & 0 & 90 & 60 & 94 & $1 \mathrm{~A} / \mathrm{D}$ & 58 & 0 & 0 & 60 & 37 & 37 \\
\hline $\mathrm{OKC}$ & $1.5 \mathrm{~A} / \mathrm{D}$ & 137 & 61 & 68 & 120 & 113 & 137 & $1 \mathrm{~A}+1 \mathrm{D}$ & 137 & 60 & 68 & 120 & 113 & 104 \\
\hline OMA & 2 A/1Dmax & 137 & 1 & 0 & 60 & 88 & 137 & 1.5 A/1Dmax & 102 & 0 & 0 & 60 & 66 & 44 \\
\hline ONT & $1.75 \mathrm{~A} / \mathrm{D}$ & 108 & 0 & 0 & 105 & 69 & 108 & $1 \mathrm{~A} / \mathrm{D}$ & 59 & 0 & 0 & 60 & 38 & 38 \\
\hline ORD & $1 A / D+2 A+2 D$ & 225 & 120 & 112 & 240 & 185 & 225 & $1 A / D+1 A+2 D$ & 225 & 120 & 112 & 240 & 185 & 193 \\
\hline ORF & $\begin{array}{l}1 \mathrm{~A} / \mathrm{D}+1.25 \\
\mathrm{Dmax} \\
\end{array}$ & 102 & 1 & 0 & 90 & 66 & 102 & $1 \mathrm{~A} / \mathrm{D}$ & 68 & 67 & 0 & 92 & 68 & 68 \\
\hline OXR & $1 \mathrm{~A} / \mathrm{D}$ & 72 & 1 & 0 & 60 & 48 & 72 & $1 \mathrm{~A} / \mathrm{D}$ & 72 & 0 & 0 & 60 & 48 & 48 \\
\hline PBI & $1 \mathrm{~A} / \mathrm{D}$ & 97 & 0 & 0 & 90 & 62 & 97 & $1 \mathrm{~A} / \mathrm{D}$ & 62 & 0 & 0 & 60 & 39 & 39 \\
\hline PDX & $2 A / D$ & 118 & 0 & 0 & 120 & 76 & 118 & Dep pair & 118 & 0 & 0 & 120 & 76 & 76 \\
\hline PHF & $\begin{array}{l}1 \mathrm{~A} / \mathrm{D} \text { alternate } \\
\text { with } 0.25 \mathrm{Dmax}\end{array}$ & 68 & 1 & 0 & 75 & 34 & 68 & $1 \mathrm{~A} / \mathrm{D}$ & 68 & 0 & 0 & 60 & 44 & 44 \\
\hline $\mathrm{PHL}$ & $\begin{array}{l}1 \mathrm{~A}+1 \mathrm{D} \\
+0.5^{\star} \mathrm{A} / \mathrm{D} \\
\end{array}$ & 115 & 0 & 0 & 120 & 74 & 115 & $1 A+1 D+0.5^{\star} A / D$ & 115 & 0 & 0 & 120 & 74 & 74 \\
\hline
\end{tabular}


Table B-8. 110-Airport ROT-Limited Capacities for 2025 NextGen

\begin{tabular}{|c|c|c|c|c|c|c|c|c|c|c|c|c|c|c|}
\hline \multirow[b]{2}{*}{ Apt } & \multicolumn{7}{|c|}{ Visual meteorological conditions } & \multicolumn{7}{|c|}{ Instrument meteorological conditions } \\
\hline & Configuration & Amax & Aeq & Amin & Dmax & Deq & Free & Configuration & Amax & Amin & Aeq & Dmax & Deq & Free \\
\hline $\mathrm{PHX}$ & $1 A+1 D+1 A / D$ & 114 & 60 & 57 & 120 & 94 & 114 & $2 A / D$ & 114 & 0 & 0 & 120 & 73 & 73 \\
\hline PIE & $\begin{array}{l}1 \mathrm{~A} / \mathrm{D}+0.75 \mathrm{~A}+ \\
0.5 \mathrm{D}\end{array}$ & 119 & 31 & 51 & 90 & 95 & 119 & $1 \mathrm{~A} / \mathrm{D}$ & 68 & 0 & 0 & 60 & 44 & 44 \\
\hline PIT & $1 A+1 D+1 A / D$ & 118 & 60 & 59 & 120 & 96 & 118 & $2 A / D$ & 118 & 0 & 0 & 120 & 75 & 75 \\
\hline PVD & $1 \mathrm{~A} / \mathrm{D}$ & 61 & 0 & 0 & 60 & 39 & 61 & $1 \mathrm{~A} / \mathrm{D}$ & 59 & 0 & 0 & 60 & 38 & 38 \\
\hline RDU & $2 A / D$ & 113 & 60 & 57 & 120 & 93 & 113 & $\begin{array}{l}\text { Dep pair - with } \\
1.5^{\star} \mathrm{Dmax} \text { Sgl }\end{array}$ & 113 & 60 & 57 & 120 & 93 & 96 \\
\hline RFD & $1.75 \mathrm{~A} / \mathrm{D}$ & 119 & 1 & 0 & 105 & 78 & 119 & $1.25 \mathrm{~A} / \mathrm{D}$ & 76 & 0 & 0 & 90 & 49 & 49 \\
\hline RIC & $1.25 \mathrm{~A} / \mathrm{D}$ & 85 & 1 & 0 & 75 & 55 & 85 & $1 \mathrm{~A} / \mathrm{D}$ & 68 & 0 & 0 & 60 & 44 & 44 \\
\hline RNO & $1.5 \mathrm{~A} / \mathrm{D}$ & 86 & 0 & 0 & 90 & 55 & 86 & $1 \mathrm{~A} / \mathrm{D}$ & 57 & 0 & 0 & 60 & 37 & 37 \\
\hline ROC & $1.25 \mathrm{~A} / \mathrm{D}$ & 71 & 0 & 0 & 75 & 46 & 71 & $1 \mathrm{~A} / \mathrm{D}$ & 57 & 0 & 0 & 60 & 37 & 37 \\
\hline RSW & $1 \mathrm{~A} / \mathrm{D}$ & 114 & 0 & 0 & 120 & 73 & 114 & $1 \mathrm{~A} / \mathrm{D}$ & 114 & 0 & 0 & 120 & 73 & 73 \\
\hline SAN & $1 \mathrm{~A} / \mathrm{D}$ & 58 & 0 & 0 & 60 & 37 & 58 & $1 \mathrm{~A} / \mathrm{D}$ & 58 & 0 & 0 & 60 & 37 & 37 \\
\hline SAT & $2 A / D$ & 127 & 1 & 0 & 120 & 81 & 127 & $1 \mathrm{~A} / \mathrm{D}$ & 60 & 60 & 0 & 120 & 39 & 99 \\
\hline SBA & $\begin{array}{l}1 \mathrm{~A} / \mathrm{D}+0.25 \mathrm{~A}+ \\
0.25 \mathrm{D}\end{array}$ & 77 & 15 & 15 & 75 & 55 & 77 & $1 A+1 D$ & 62 & 60 & 62 & 60 & 62 & 60 \\
\hline SDF & $2 \mathrm{~A} / \mathrm{D}$ & 112 & 0 & 0 & 120 & 72 & 112 & $2 A / D$ & 112 & 0 & 0 & 120 & 72 & 72 \\
\hline SEA & 2A/dep Dmax & 114 & 0 & 0 & 120 & 73 & 114 & $1 A+\operatorname{DepD}$ & 114 & 0 & 0 & 120 & 73 & 73 \\
\hline SFO & $2 \mathrm{~A} / \mathrm{D}$ & 98 & 19 & 0 & 120 & 68 & 98 & $1 \mathrm{~A} / \mathrm{D}+0.5 \mathrm{D}$ & 58 & 30 & 0 & 90 & 37 & 67 \\
\hline SJC & $\begin{array}{l}1 \mathrm{~A}+1 \mathrm{D} \\
+0.25 \mathrm{~A} / \mathrm{D}\end{array}$ & 76 & 60 & 61 & 75 & 70 & 76 & CSP w/Sgl Dmax & 58 & 56 & 0 & 60 & 58 & 58 \\
\hline SLC & $\begin{array}{l}\text { 2A/D with } \\
2.25 \mathrm{Amax}\end{array}$ & 134 & 0 & 0 & 120 & 91 & 134 & 2A/D with $2.25 \mathrm{Amax}$ & 134 & 0 & 0 & 120 & 91 & 76 \\
\hline SMF & $2 A / D$ & 113 & 0 & 0 & 120 & 73 & 113 & $2 A / D$ & 113 & 0 & 0 & 120 & 73 & 73 \\
\hline SNA & $1.5 \mathrm{~A} / \mathrm{D}$ & 100 & 1 & 0 & 90 & 64 & 100 & $1 \mathrm{~A} / \mathrm{D}$ & 60 & 0 & 0 & 60 & 39 & 39 \\
\hline STL & $1 A+1 D+1 A / D$ & 114 & 60 & 57 & 120 & 94 & 114 & $2 A / D$ & 114 & 0 & 0 & 120 & 73 & 73 \\
\hline SWF & $1 \mathrm{~A} / \mathrm{D}$ & 69 & 1 & 0 & 60 & 44 & 69 & $1 \mathrm{~A} / \mathrm{D}$ & 60 & 0 & 0 & 60 & 38 & 38 \\
\hline SYR & $1.3 \mathrm{~A} / \mathrm{D}$ & 89 & 1 & 0 & 78 & 57 & 89 & $1 \mathrm{~A} / \mathrm{D}$ & 68 & 0 & 0 & 60 & 44 & 44 \\
\hline TEB & $1 \mathrm{~A} / \mathrm{D}+0.5 \mathrm{D}$ & 68 & 31 & 0 & 90 & 44 & 68 & $1 \mathrm{~A} / \mathrm{D}$ & 68 & 0 & 0 & 60 & 44 & 44 \\
\hline TPA & $\begin{array}{l}\text { 2A/D with Dmax } \\
\text { limit }\end{array}$ & 118 & 0 & 59 & 80 & 97 & 118 & 2A/D with Dmax limit & 118 & 0 & 59 & 80 & 97 & 80 \\
\hline TUL & $1.6 \mathrm{~A} / \mathrm{D}$ & 205 & 2 & 0 & 180 & 133 & 205 & $1.5 \mathrm{~A} / \mathrm{D}$ & 137 & 0 & 0 & 120 & 88 & 88 \\
\hline TUS & $2 A / D$ & 133 & 1 & 0 & 120 & 85 & 133 & $1 \mathrm{~A} / \mathrm{D}+0.5 \mathrm{D}$ & 59 & 30 & 0 & 90 & 38 & 68 \\
\hline TVC & $\begin{array}{l}1.25 \mathrm{~A} / \mathrm{D} \text { with } \\
\text { interpolated } \mathrm{E}\end{array}$ & 85 & 1 & 0 & 90 & 44 & 85 & $1 \mathrm{~A} / \mathrm{D}$ & 68 & 0 & 0 & 60 & 44 & 44 \\
\hline TYS & $1.25 \mathrm{~A} / \mathrm{D}$ & 85 & 1 & 0 & 75 & 55 & 85 & $1 \mathrm{~A} / \mathrm{D}$ & 68 & 0 & 0 & 60 & 44 & 44 \\
\hline VNY & $1.25 \mathrm{~A} / \mathrm{D}$ & 85 & 1 & 0 & 75 & 55 & 85 & $1 \mathrm{~A} / \mathrm{D}+0.25 \mathrm{D}$ & 68 & 15 & 0 & 75 & 44 & 59 \\
\hline
\end{tabular}




\section{Appendix C IFR Delays at 310 LMINET Airports}

This appendix contains the detailed results of the analysis of arrival and departure delays under all-day IFR conditions. Table C-1 shows the results for the 110 major airports, and Table C-2 shows the results for the 200 smaller airports.

Table C-1. Average IFR Flight-Delay Minutes, Major 110 Airports

\begin{tabular}{|c|c|c|c|c|c|c|}
\hline \multirow[b]{2}{*}{ Airport } & \multicolumn{3}{|c|}{ Arrivals } & \multicolumn{3}{|c|}{ Departures } \\
\hline & 2007 & 2015 & 2025 & 2007 & 2015 & 2025 \\
\hline$A B Q$ & 1.3 & 3.1 & 2.8 & 2.0 & 2.9 & 3.3 \\
\hline ALB & 2.0 & 3.9 & 3.3 & 2.9 & 4.3 & 4.8 \\
\hline ANC & 68.5 & 70.2 & 27.2 & 62.8 & 49.4 & 27.3 \\
\hline ATL & 16.8 & 26.8 & 3.2 & 2.5 & 2.8 & 4.2 \\
\hline AUS & 1.7 & 3.4 & 4.4 & 2.9 & 4.4 & 6.0 \\
\hline BDL & 0.9 & 1.3 & 2.1 & 1.9 & 3.1 & 5.0 \\
\hline BFL & 0.2 & 0.3 & 0.3 & 0.7 & 1.0 & 0.8 \\
\hline $\mathrm{BHM}$ & 1.5 & 2.2 & 2.3 & 2.4 & 3.0 & 3.3 \\
\hline BNA & 0.3 & 0.5 & 0.6 & 0.6 & 0.9 & 1.0 \\
\hline $\mathrm{BOI}$ & 1.9 & 4.6 & 2.3 & 2.0 & 3.3 & 2.8 \\
\hline BOS & 136.8 & 9.6 & 4.2 & 3.3 & 8.4 & 7.2 \\
\hline BTR & 0.3 & 0.5 & 0.5 & 0.9 & 1.0 & 1.1 \\
\hline BUF & 0.8 & 1.2 & 0.7 & 1.0 & 1.3 & 1.3 \\
\hline BUR & 2.5 & 4.8 & 2.7 & 2.3 & 3.1 & 3.3 \\
\hline BWI & 1.7 & 3.4 & 0.5 & 2.6 & 4.2 & 1.1 \\
\hline $\mathrm{CHS}$ & 0.4 & 0.7 & 0.6 & 1.1 & 1.2 & 1.0 \\
\hline CLE & 6.6 & 12.5 & 7.2 & 3.1 & 5.7 & 10.1 \\
\hline CLT & 6.6 & 4.0 & 4.0 & 6.0 & 2.8 & 6.8 \\
\hline $\mathrm{CMH}$ & 0.5 & 0.8 & 0.3 & 0.7 & 1.2 & 0.6 \\
\hline $\cos$ & 0.5 & 0.7 & 0.6 & 0.8 & 0.8 & 0.8 \\
\hline CRP & 0.0 & 0.1 & 0.1 & 0.4 & 0.5 & 0.4 \\
\hline CVG & 3.5 & 5.6 & 5.8 & 4.3 & 5.7 & 8.8 \\
\hline DAB & 2.4 & 3.9 & 1.2 & 1.6 & 3.1 & 1.9 \\
\hline DAL & 2.8 & 10.9 & 3.1 & 1.7 & 2.5 & 4.0 \\
\hline DAY & 0.3 & 0.2 & 0.3 & 0.4 & 0.4 & 0.4 \\
\hline DCA & 6.5 & 5.7 & 3.1 & 8.6 & 6.7 & 4.2 \\
\hline DEN & 1.8 & 3.3 & 1.0 & 1.1 & 1.7 & 2.2 \\
\hline DFW & 1.5 & 2.8 & 1.6 & 1.0 & 1.9 & 1.9 \\
\hline DSM & 0.4 & 0.6 & 0.4 & 0.9 & 1.1 & 0.9 \\
\hline DTW & 6.9 & 10.4 & 4.3 & 3.6 & 5.1 & 5.5 \\
\hline ELP & 0.1 & 0.3 & 0.1 & 0.7 & 0.8 & 0.6 \\
\hline EUG & 0.4 & 1.0 & 0.7 & 1.1 & 1.2 & 1.1 \\
\hline EWR & 99.2 & 121.5 & 4.0 & 5.3 & 6.3 & 5.3 \\
\hline FAT & 2.0 & 4.1 & 1.4 & 2.3 & 3.2 & 2.4 \\
\hline FLL & 24.7 & 1.1 & 1.3 & 17.1 & 1.6 & 2.3 \\
\hline FNT & 0.2 & 0.3 & 0.4 & 0.5 & 0.5 & 0.6 \\
\hline
\end{tabular}


Table C-1. Average IFR Flight-Delay Minutes, Major 110 Airports

\begin{tabular}{|c|c|c|c|c|c|c|}
\hline \multirow[b]{2}{*}{ Airport } & \multicolumn{3}{|c|}{ Arrivals } & \multicolumn{3}{|c|}{ Departures } \\
\hline & 2007 & 2015 & 2025 & 2007 & 2015 & 2025 \\
\hline FXE & 4.6 & 9.5 & 2.9 & 2.9 & 6.5 & 4.5 \\
\hline GFK & 1.0 & 0.6 & 0.8 & 1.4 & 1.3 & 1.3 \\
\hline GRR & 0.2 & 0.3 & 0.5 & 0.7 & 0.9 & 1.0 \\
\hline GSO & 0.6 & 0.2 & 0.3 & 1.0 & 0.4 & 0.5 \\
\hline GYY & 0.1 & 0.2 & 0.2 & 0.4 & 0.4 & 0.5 \\
\hline $\mathrm{HNL}$ & 62.4 & 95.2 & 7.1 & 2.2 & 2.2 & 3.5 \\
\hline $\mathrm{HOU}$ & 5.2 & 16.1 & 3.5 & 2.2 & 2.8 & 4.9 \\
\hline HPN & 15.3 & 25.2 & 11.9 & 7.5 & 16.9 & 12.8 \\
\hline IAD & 7.6 & 11.4 & 6.3 & 6.4 & 5.3 & 8.0 \\
\hline $\mathrm{IAH}$ & 5.5 & 23.9 & 11.3 & 6.4 & 24.3 & 15.1 \\
\hline ICT & 0.3 & 0.4 & 0.2 & 0.6 & 0.6 & 0.5 \\
\hline IND & 0.3 & 0.4 & 0.2 & 0.9 & 1.5 & 0.6 \\
\hline ISP & 1.1 & 2.6 & 1.9 & 1.7 & 2.8 & 2.3 \\
\hline JAX & 0.3 & 0.5 & 0.5 & 0.8 & 1.0 & 0.9 \\
\hline JFK & 8.6 & 9.5 & 6.6 & 8.1 & 9.7 & 11.3 \\
\hline JNU & 6.6 & 11.0 & 8.9 & 5.1 & 6.0 & 9.5 \\
\hline LAN & 0.2 & 0.2 & 0.3 & 0.9 & 1.0 & 0.9 \\
\hline LAS & 122.0 & 131.7 & 4.4 & 2.8 & 2.9 & 3.3 \\
\hline LAX & 23.2 & 94.3 & 6.9 & 2.1 & 3.3 & 4.2 \\
\hline LGA & 75.1 & 80.1 & 3.0 & 4.1 & 4.5 & 3.5 \\
\hline LGB & 2.3 & 3.5 & 1.7 & 2.1 & 2.2 & 2.5 \\
\hline LIT & 0.7 & 1.0 & 0.7 & 1.2 & 1.5 & 1.4 \\
\hline $\mathrm{MCl}$ & 0.7 & 1.1 & 1.7 & 1.0 & 1.7 & 2.5 \\
\hline $\mathrm{MCO}$ & 0.2 & 0.4 & 0.4 & 0.4 & 0.6 & 0.7 \\
\hline MDW & 29.5 & 41.1 & 20.3 & 28.1 & 38.3 & 23.1 \\
\hline MEM & 8.1 & 9.1 & 1.7 & 4.0 & 4.4 & 4.1 \\
\hline MHT & 0.7 & 1.0 & 0.8 & 1.8 & 2.2 & 1.7 \\
\hline MIA & 1.1 & 1.9 & 1.6 & 1.6 & 2.2 & 2.3 \\
\hline MKE & 5.0 & 0.9 & 1.0 & 3.4 & 1.1 & 1.4 \\
\hline MLB & 0.2 & 0.1 & 0.2 & 0.4 & 0.5 & 0.4 \\
\hline MSN & 0.6 & 0.8 & 1.6 & 1.1 & 1.9 & 1.9 \\
\hline MSP & 8.0 & 21.0 & 7.2 & 2.7 & 4.5 & 6.5 \\
\hline MSY & 0.8 & 1.6 & 0.3 & 1.5 & 2.7 & 0.5 \\
\hline OAK & 6.2 & 28.2 & 19.2 & 4.6 & 22.1 & 19.8 \\
\hline OKC & 0.3 & 0.2 & 0.1 & 0.5 & 0.3 & 0.2 \\
\hline OMA & 0.4 & 0.7 & 1.0 & 1.1 & 1.3 & 1.7 \\
\hline ONT & 1.3 & 1.8 & 0.9 & 1.6 & 1.5 & 1.4 \\
\hline ORD & 156.0 & 24.4 & 7.2 & 5.3 & 10.2 & 8.8 \\
\hline ORF & 0.6 & 0.9 & 0.4 & 1.1 & 1.2 & 0.6 \\
\hline OXR & 0.3 & 0.4 & 0.4 & 0.5 & 0.7 & 0.7 \\
\hline PBI & 1.5 & 2.3 & 1.2 & 1.5 & 1.4 & 1.6 \\
\hline PDX & 1.4 & 1.7 & 2.0 & 1.7 & 2.2 & 3.7 \\
\hline PHF & 3.7 & 8.2 & 3.5 & 3.0 & 5.0 & 5.8 \\
\hline $\mathrm{PHL}$ & 13.0 & 18.5 & 18.8 & 4.3 & 19.8 & 29.9 \\
\hline $\mathrm{PHX}$ & 7.9 & 15.3 & 21.0 & 6.5 & 10.6 & 22.4 \\
\hline PIE & 0.2 & 0.2 & 0.2 & 0.4 & 0.4 & 0.3 \\
\hline PIT & 0.4 & 0.3 & 0.3 & 0.6 & 0.5 & 0.5 \\
\hline
\end{tabular}


Table C-1. Average IFR Flight-Delay Minutes, Major 110 Airports

\begin{tabular}{|c|r|r|r|r|r|r|}
\hline & \multicolumn{3}{|c|}{ Arrivals } & \multicolumn{3}{|c|}{ Departures } \\
\cline { 2 - 7 } Airport & 2007 & 2015 & 2025 & 2007 & 2015 & 2025 \\
\hline PVD & 1.4 & 2.1 & 2.0 & 1.7 & 2.2 & 2.2 \\
\hline RDU & 1.8 & 2.8 & 1.2 & 2.6 & 3.7 & 1.7 \\
\hline RFD & 0.1 & 0.2 & 0.3 & 0.5 & 0.4 & 0.6 \\
\hline RIC & 0.9 & 1.8 & 1.1 & 1.4 & 1.9 & 2.4 \\
\hline RNO & 1.1 & 3.3 & 2.1 & 1.6 & 3.4 & 3.0 \\
\hline ROC & 0.8 & 1.3 & 1.1 & 1.5 & 2.2 & 1.7 \\
\hline RSW & 1.4 & 3.0 & 0.5 & 1.7 & 2.4 & 0.6 \\
\hline SAN & 7.2 & 13.2 & 6.5 & 6.7 & 11.4 & 8.8 \\
\hline SAT & 6.6 & 20.0 & 2.4 & 5.6 & 16.0 & 2.1 \\
\hline SBA & 0.4 & 0.5 & 0.4 & 0.5 & 0.6 & 0.6 \\
\hline SDF & 1.1 & 2.9 & 3.7 & 1.9 & 2.7 & 4.8 \\
\hline SEA & 53.3 & 4.0 & 4.9 & 4.2 & 3.9 & 6.6 \\
\hline SFO & 105.3 & 203.6 & 85.7 & 5.4 & 12.0 & 78.8 \\
\hline SJC & 2.4 & 6.8 & 3.2 & 1.7 & 3.9 & 2.8 \\
\hline SLC & 5.4 & 9.4 & 4.9 & 4.4 & 5.2 & 5.9 \\
\hline SMF & 0.3 & 0.4 & 0.7 & 0.6 & 0.8 & 1.1 \\
\hline SNA & 13.8 & 43.6 & 14.5 & 10.9 & 35.0 & 16.0 \\
\hline STL & 0.4 & 0.6 & 0.6 & 0.8 & 1.2 & 1.0 \\
\hline SWF & 1.1 & 2.0 & 1.9 & 1.9 & 2.1 & 3.1 \\
\hline SYR & 0.6 & 0.6 & 0.5 & 0.8 & 0.8 & 0.8 \\
\hline TEB & 8.1 & 16.7 & 5.3 & 5.6 & 11.7 & 8.1 \\
\hline TPA & 1.0 & 1.4 & 0.6 & 1.4 & 2.0 & 0.9 \\
\hline TUL & 0.4 & 0.5 & 0.1 & 0.7 & 0.7 & 0.2 \\
\hline TUS & 1.0 & 1.2 & 0.3 & 0.5 & 0.6 & 0.6 \\
\hline TVC & 0.3 & 0.5 & 0.4 & 0.6 & 0.8 & 0.8 \\
\hline TYS & 1.0 & 1.6 & 1.0 & 1.2 & 1.5 & 1.5 \\
\hline VNY & 31.0 & 65.3 & 9.6 & 5.3 & 7.2 & 11.5 \\
\hline & & & & & & \\
\hline
\end{tabular}

Table C-2. Average IFR Flight-Delay Minutes, Smaller 200 Airports

\begin{tabular}{|c|r|r|r|r|r|r|}
\hline & \multicolumn{3}{|c|}{ Arrivals } & \multicolumn{3}{|c|}{ Departures } \\
\cline { 2 - 7 } Airport & 2007 & 2015 & 2025 & 2007 & 2015 & 2025 \\
\hline $40 \mathrm{~N}$ & 0.0 & 0.0 & 0.0 & 0.0 & 0.0 & 0.0 \\
\hline 55J & 9.8 & 10.4 & 10.4 & 9.0 & 8.7 & 8.7 \\
\hline ABE & 0.9 & 1.3 & 1.5 & 1.4 & 1.6 & 1.9 \\
\hline ABI & 0.1 & 0.1 & 0.1 & 0.2 & 0.3 & 0.3 \\
\hline ABR & 13.8 & 18.2 & 22.7 & 18.0 & 20.5 & 26.5 \\
\hline ABY & 0.2 & 0.2 & 0.2 & 0.4 & 0.4 & 0.5 \\
\hline ACK & 31.0 & 55.8 & 75.7 & 18.9 & 30.2 & 61.6 \\
\hline ACT & 0.0 & 0.0 & 0.1 & 0.3 & 0.3 & 0.3 \\
\hline ACV & 13.8 & 15.6 & 17.7 & 11.5 & 14.4 & 19.6 \\
\hline ACY & 0.1 & 0.1 & 0.3 & 0.4 & 0.5 & 0.5 \\
\hline ADQ & 0.1 & 0.1 & 0.1 & 0.3 & 0.4 & 0.4 \\
\hline ADS & 0.7 & 0.7 & 0.9 & 1.2 & 1.4 & 1.5 \\
\hline AEG & 7.1 & 20.6 & 20.1 & 7.9 & 17.9 & 16.6 \\
\hline
\end{tabular}


Table C-2. Average IFR Flight-Delay Minutes, Smaller 200 Airports

\begin{tabular}{|c|c|c|c|c|c|c|}
\hline \multirow[b]{2}{*}{ Airport } & \multicolumn{3}{|c|}{ Arrivals } & \multicolumn{3}{|c|}{ Departures } \\
\hline & 2007 & 2015 & 2025 & 2007 & 2015 & 2025 \\
\hline AEX & 0.1 & 0.2 & 0.3 & 0.3 & 0.4 & 0.5 \\
\hline AFW & 0.1 & 0.3 & 0.2 & 0.3 & 0.5 & 0.7 \\
\hline AGS & 0.1 & 0.2 & 0.2 & 0.3 & 0.4 & 0.4 \\
\hline $\mathrm{AHN}$ & 0.3 & 0.3 & 0.4 & 0.4 & 0.6 & 0.5 \\
\hline ALN & 0.2 & 0.1 & 0.1 & 0.3 & 0.3 & 0.4 \\
\hline ALO & 0.1 & 0.1 & 0.1 & 0.3 & 0.3 & 0.4 \\
\hline AMA & 0.3 & 0.3 & 0.3 & 0.6 & 0.5 & 0.6 \\
\hline APA & 3.3 & 7.0 & 12.6 & 1.6 & 2.2 & 5.0 \\
\hline APF & 0.7 & 1.3 & 1.5 & 1.0 & 1.4 & 2.1 \\
\hline ARR & 0.1 & 0.1 & 0.3 & 0.3 & 0.5 & 0.7 \\
\hline ASE & 0.9 & 1.8 & 2.8 & 1.9 & 2.1 & 3.6 \\
\hline ATW & 0.1 & 0.1 & 0.2 & 0.4 & 0.4 & 0.5 \\
\hline ATY & 11.5 & 11.3 & 12.5 & 6.6 & 7.4 & 7.4 \\
\hline AUG & 8.3 & 11.2 & 11.2 & 7.2 & 11.1 & 11.1 \\
\hline AVL & 0.5 & 1.0 & 1.4 & 1.0 & 1.2 & 1.7 \\
\hline AVP & 0.3 & 0.6 & 1.6 & 0.7 & 0.8 & 1.1 \\
\hline AWM & 11.0 & 12.9 & 12.9 & 10.0 & 12.0 & 12.0 \\
\hline AZO & 0.2 & 0.3 & 0.2 & 0.5 & 0.5 & 0.5 \\
\hline BCT & 0.3 & 0.4 & 0.9 & 0.6 & 0.8 & 0.9 \\
\hline BED & 1.0 & 2.1 & 3.9 & 1.3 & 1.6 & 2.3 \\
\hline BET & 1.4 & 2.1 & 3.3 & 1.8 & 2.0 & 3.5 \\
\hline BFF & 8.7 & 9.0 & 9.0 & 8.9 & 7.1 & 7.1 \\
\hline BFI & 32.1 & 36.7 & 61.4 & 14.5 & 23.4 & 37.4 \\
\hline BFM & 0.2 & 0.2 & 0.2 & 0.3 & 0.4 & 0.4 \\
\hline BGM & 0.2 & 0.2 & 0.2 & 0.5 & 0.5 & 0.5 \\
\hline BGR & 0.3 & 0.4 & 0.6 & 0.5 & 0.5 & 0.6 \\
\hline BIL & 0.5 & 0.9 & 1.7 & 0.8 & 0.8 & 1.2 \\
\hline BIS & 0.2 & 0.2 & 0.4 & 0.5 & 0.5 & 0.6 \\
\hline BJC & 0.0 & 0.0 & 0.0 & 0.3 & 0.3 & 0.3 \\
\hline BKL & 0.1 & 0.2 & 0.8 & 0.5 & 0.5 & 0.7 \\
\hline BLI & 0.7 & 0.8 & 1.2 & 1.4 & 1.4 & 1.6 \\
\hline BLV & 0.1 & 0.1 & 0.1 & 0.1 & 0.1 & 0.1 \\
\hline BMI & 0.1 & 0.2 & 0.1 & 0.3 & 0.4 & 0.3 \\
\hline BPT & 0.2 & 0.3 & 0.2 & 0.4 & 0.5 & 0.6 \\
\hline BTM & 8.7 & 7.7 & 7.7 & 6.1 & 5.2 & 5.2 \\
\hline BTV & 0.4 & 0.6 & 0.7 & 0.8 & 0.9 & 1.2 \\
\hline BVY & 0.1 & 0.2 & 0.2 & 0.4 & 0.5 & 0.6 \\
\hline BZN & 0.6 & 0.8 & 1.6 & 1.1 & 1.1 & 1.6 \\
\hline CAE & 0.5 & 1.1 & 1.7 & 0.7 & 1.0 & 1.3 \\
\hline CAK & 0.6 & 0.6 & 1.1 & 0.7 & 1.0 & 1.1 \\
\hline CCR & 0.3 & 0.3 & 0.4 & 0.8 & 0.8 & 0.9 \\
\hline CDC & 9.2 & 11.9 & 11.9 & 8.4 & 10.1 & 10.1 \\
\hline CGF & 0.2 & 0.4 & 0.4 & 0.5 & 0.6 & 0.8 \\
\hline CGI & 0.1 & 0.1 & 0.1 & 0.3 & 0.4 & 0.4 \\
\hline $\mathrm{CHA}$ & 0.3 & 0.7 & 0.7 & 0.8 & 1.1 & 1.2 \\
\hline $\mathrm{CHO}$ & 0.4 & 0.7 & 1.1 & 0.8 & 1.0 & 1.1 \\
\hline CID & 0.1 & 0.3 & 0.4 & 0.5 & 0.5 & 0.6 \\
\hline
\end{tabular}


Table C-2. Average IFR Flight-Delay Minutes, Smaller 200 Airports

\begin{tabular}{|c|r|r|r|r|r|r|}
\hline & \multicolumn{3}{|c|}{ Arrivals } & \multicolumn{3}{|c|}{ Departures } \\
\cline { 2 - 7 } Airport & 2007 & 2015 & 2025 & 2007 & 2015 & 2025 \\
\hline CKB & 0.2 & 0.3 & 0.4 & 0.6 & 0.6 & 0.6 \\
\hline CLL & 0.2 & 0.1 & 0.2 & 0.4 & 0.5 & 0.4 \\
\hline CMI & 0.0 & 0.0 & 0.0 & 0.2 & 0.2 & 0.2 \\
\hline COU & 0.0 & 0.0 & 0.0 & 0.2 & 0.2 & 0.2 \\
\hline CPR & 0.1 & 0.2 & 0.2 & 0.4 & 0.4 & 0.4 \\
\hline CPS & 0.1 & 0.1 & 0.4 & 0.6 & 0.5 & 0.6 \\
\hline CRW & 0.5 & 0.7 & 0.9 & 0.9 & 1.2 & 1.6 \\
\hline CSG & 0.2 & 0.4 & 0.4 & 0.5 & 0.6 & 0.7 \\
\hline CWA & 0.0 & 0.0 & 0.0 & 0.3 & 0.3 & 0.4 \\
\hline CYS & 0.2 & 0.2 & 0.2 & 0.3 & 0.3 & 0.3 \\
\hline DLH & 0.3 & 0.4 & 0.6 & 0.6 & 0.8 & 0.9 \\
\hline DPA & 0.3 & 1.0 & 2.0 & 0.7 & 1.0 & 1.4 \\
\hline DTO & 0.3 & 0.3 & 0.3 & 0.5 & 0.5 & 0.5 \\
\hline DVT & 1.6 & 3.0 & 4.4 & 1.7 & 2.5 & 2.6 \\
\hline EAU & 0.3 & 0.3 & 0.3 & 0.6 & 0.7 & 0.6 \\
\hline EFD & 0.0 & 0.0 & 0.0 & 0.2 & 0.2 & 0.2 \\
\hline EGE & 0.4 & 0.5 & 1.6 & 0.7 & 0.8 & 1.0 \\
\hline EQY & 11.4 & 10.6 & 10.6 & 11.8 & 11.9 & 11.9 \\
\hline ERI & 0.1 & 0.2 & 0.2 & 0.4 & 0.4 & 0.5 \\
\hline EVV & 0.4 & 0.3 & 0.6 & 0.7 & 0.7 & 0.7 \\
\hline FAI & 0.7 & 1.5 & 2.1 & 0.9 & 0.9 & 1.5 \\
\hline FAR & 0.2 & 0.2 & 0.2 & 0.5 & 0.5 & 0.5 \\
\hline FAY & 0.2 & 0.1 & 0.2 & 0.3 & 0.5 & 0.6 \\
\hline FDK & 9.3 & 11.4 & 13.0 & 10.4 & 13.3 & 13.6 \\
\hline FFZ & 2.1 & 5.1 & 12.5 & 1.2 & 2.8 & 5.6 \\
\hline FNL & 0.1 & 0.2 & 0.2 & 0.3 & 0.4 & 0.4 \\
\hline FOE & 0.0 & 0.0 & 0.0 & 0.3 & 0.3 & 0.2 \\
\hline FRG & 1.1 & 1.7 & 3.0 & 1.3 & 1.5 & 2.5 \\
\hline FSD & 0.3 & 1.0 & 1.2 & 1.1 & 1.6 & 2.0 \\
\hline HIOF & 0.1 & 0.3 & 0.3 & 0.4 & 0.5 & 0.7 \\
\hline FTG & 0.1 & 0.2 & 0.5 & 0.4 & 0.4 & 0.5 \\
\hline FTW & 0.3 & 0.4 & 1.5 & 0.6 & 0.7 & 1.4 \\
\hline FTY & 0.4 & 1.5 & 4.0 & 0.7 & 1.3 & 3.7 \\
\hline FWA & 0.1 & 0.1 & 0.2 & 0.7 & 1.3 & 1.6 \\
\hline GCN & 0.0 & 0.0 & 0.0 & 0.0 & 0.0 & 0.0 \\
\hline GEG & 0.4 & 1.2 & 0.3 & 0.6 & 0.8 & 0.4 \\
\hline GEU & 0.2 & 0.4 & 0.4 & 0.7 & 0.6 & 0.6 \\
\hline GJT & 0.2 & 0.2 & 0.4 & 0.5 & 0.6 & 0.8 \\
\hline GNV & 0.0 & 0.1 & 0.1 & 0.3 & 0.4 & 0.4 \\
\hline GPT & 0.0 & 0.2 & 0.4 & 0.3 & 0.3 & 0.4 \\
\hline GRB & 0.1 & 0.1 & 0.2 & 0.4 & 0.4 & 0.5 \\
\hline GRI & 0.1 & 0.1 & 0.1 & 0.2 & 0.3 & 0.4 \\
\hline GSP & 0.5 & 0.8 & 0.1 & 1.0 & 1.4 & 0.4 \\
\hline 0.1 & 0.2 & 0.5 & 0.4 & 0.4 & 0.6 \\
\hline HIO & 0.0 & 0.1 & 0.0 & 0.3 & 0.2 & 0.2 \\
\hline
\end{tabular}


Table C-2. Average IFR Flight-Delay Minutes, Smaller 200 Airports

\begin{tabular}{|c|c|c|c|c|c|c|}
\hline \multirow[b]{2}{*}{ Airport } & \multicolumn{3}{|c|}{ Arrivals } & \multicolumn{3}{|c|}{ Departures } \\
\hline & 2007 & 2015 & 2025 & 2007 & 2015 & 2025 \\
\hline $\mathrm{HQZ}$ & 9.6 & 7.7 & 10.0 & 9.1 & 6.2 & 12.8 \\
\hline HRL & 0.0 & 0.0 & 0.1 & 0.4 & 0.3 & 0.4 \\
\hline HSV & 0.1 & 0.2 & 0.2 & 0.3 & 0.3 & 0.3 \\
\hline HWD & 1.3 & 1.8 & 1.6 & 1.8 & 1.7 & 2.1 \\
\hline IAG & 0.0 & 0.1 & 0.0 & 0.3 & 0.3 & 0.3 \\
\hline IDA & 0.2 & 0.2 & 0.2 & 0.4 & 0.4 & 0.5 \\
\hline ILG & 0.2 & 0.2 & 0.5 & 0.5 & 0.6 & 0.7 \\
\hline ILM & 0.7 & 0.7 & 1.2 & 1.1 & 1.4 & 1.9 \\
\hline ISM & 0.6 & 1.0 & 1.6 & 0.7 & 1.1 & 1.3 \\
\hline ITO & 0.5 & 0.6 & 0.5 & 0.9 & 1.2 & 1.1 \\
\hline IWA & 0.7 & 0.9 & 1.5 & 0.6 & 0.7 & 1.2 \\
\hline JAC & 0.3 & 0.5 & 0.7 & 0.6 & 1.0 & 1.3 \\
\hline JAN & 0.1 & 0.1 & 0.2 & 0.2 & 0.3 & 0.3 \\
\hline JST & 0.1 & 0.2 & 0.1 & 0.4 & 0.3 & 0.3 \\
\hline KOA & 0.4 & 0.5 & 0.5 & 0.7 & 0.8 & 1.0 \\
\hline KTN & 0.1 & 0.1 & 0.1 & 0.3 & 0.4 & 0.4 \\
\hline LAL & 0.2 & 0.2 & 0.2 & 0.4 & 0.4 & 0.5 \\
\hline LBB & 0.1 & 0.1 & 0.2 & 0.4 & 0.4 & 0.4 \\
\hline LBE & 0.2 & 0.1 & 0.2 & 0.4 & 0.4 & 0.5 \\
\hline LBF & 11.1 & 13.1 & 13.1 & 9.1 & 9.1 & 9.1 \\
\hline LBL & 9.0 & 12.1 & 12.1 & 7.8 & 9.6 & 9.6 \\
\hline LCK & 2.2 & 1.0 & 1.9 & 5.0 & 4.8 & 5.7 \\
\hline LEX & 0.7 & 1.0 & 1.3 & 1.0 & 1.3 & 1.3 \\
\hline LFT & 0.1 & 0.2 & 0.5 & 0.4 & 0.5 & 0.7 \\
\hline LIH & 0.2 & 0.2 & 0.6 & 0.4 & 0.5 & 0.8 \\
\hline LNK & 0.1 & 0.1 & 0.1 & 0.3 & 0.4 & 0.3 \\
\hline LSE & 0.2 & 0.2 & 0.3 & 0.4 & 0.5 & 0.5 \\
\hline LWM & 0.3 & 0.3 & 0.4 & 0.6 & 0.9 & 0.9 \\
\hline MAF & 0.0 & 0.0 & 0.1 & 0.4 & 0.4 & 0.4 \\
\hline MBS & 0.2 & 0.3 & 0.2 & 0.5 & 0.6 & 0.7 \\
\hline MDT & 0.4 & 0.4 & 0.3 & 0.7 & 0.8 & 0.9 \\
\hline MFE & 0.4 & 0.5 & 0.8 & 0.8 & 1.0 & 1.2 \\
\hline MHK & 0.2 & 0.2 & 0.2 & 0.4 & 0.4 & 0.4 \\
\hline MLI & 0.1 & 0.2 & 0.2 & 0.4 & 0.4 & 0.5 \\
\hline MLU & 0.2 & 0.3 & 0.3 & 0.4 & 0.5 & 0.6 \\
\hline MMU & 2.6 & 3.7 & 4.7 & 2.7 & 4.0 & 3.7 \\
\hline MOB & 0.1 & 0.1 & 0.2 & 0.3 & 0.4 & 0.4 \\
\hline MOT & 0.2 & 0.2 & 0.3 & 0.5 & 0.5 & 0.5 \\
\hline MSO & 0.3 & 0.3 & 0.4 & 0.6 & 0.7 & 1.0 \\
\hline MTN & 0.5 & 0.5 & 0.8 & 1.0 & 1.1 & 1.3 \\
\hline MYR & 0.4 & 0.7 & 0.9 & 1.0 & 1.0 & 1.4 \\
\hline OGG & 2.6 & 3.8 & 11.3 & 2.5 & 3.1 & 6.3 \\
\hline OPF & 0.7 & 0.5 & 2.0 & 0.3 & 0.4 & 0.4 \\
\hline ORL & 0.2 & 0.3 & 0.6 & 0.8 & 0.9 & 1.2 \\
\hline PAE & 0.7 & 0.9 & 1.9 & 0.8 & 0.9 & 1.3 \\
\hline PDK & 5.5 & 14.0 & 39.5 & 3.6 & 6.3 & 18.1 \\
\hline PIA & 0.3 & 0.4 & 0.5 & 0.7 & 0.8 & 0.8 \\
\hline
\end{tabular}


Table C-2. Average IFR Flight-Delay Minutes, Smaller 200 Airports

\begin{tabular}{|c|r|r|r|r|r|r|}
\hline & \multicolumn{3}{|c|}{ Arrivals } & \multicolumn{3}{|c|}{ Departures } \\
\cline { 2 - 7 } Airport & 2007 & 2015 & 2025 & 2007 & 2015 & 2025 \\
\hline PIR & 12.5 & 17.3 & 17.1 & 13.2 & 14.3 & 14.2 \\
\hline PNE & 0.4 & 0.7 & 1.3 & 0.7 & 1.0 & 1.1 \\
\hline PNS & 0.6 & 0.7 & 1.4 & 1.2 & 1.3 & 1.7 \\
\hline PRC & 0.4 & 0.7 & 0.7 & 0.6 & 0.7 & 0.7 \\
\hline PSC & 0.0 & 0.1 & 0.1 & 0.3 & 0.4 & 0.4 \\
\hline PSM & 0.2 & 0.1 & 0.1 & 0.5 & 0.5 & 0.5 \\
\hline PSP & 0.1 & 0.1 & 0.2 & 0.4 & 0.4 & 0.5 \\
\hline PTK & 0.5 & 1.1 & 2.2 & 0.8 & 1.1 & 1.4 \\
\hline PUB & 0.1 & 0.1 & 0.2 & 0.4 & 0.4 & 0.5 \\
\hline PWK & 1.7 & 2.7 & 4.2 & 0.9 & 1.2 & 1.9 \\
\hline PWM & 0.4 & 0.4 & 0.9 & 0.7 & 0.7 & 1.0 \\
\hline RAP & 0.4 & 0.4 & 0.5 & 0.7 & 0.9 & 1.0 \\
\hline RBD & 0.3 & 0.5 & 0.5 & 0.7 & 0.9 & 0.9 \\
\hline RNT & 0.9 & 0.5 & 0.5 & 0.8 & 0.9 & 1.0 \\
\hline ROA & 0.2 & 0.2 & 0.2 & 0.5 & 0.5 & 0.6 \\
\hline RST & 0.1 & 0.1 & 0.2 & 0.3 & 0.5 & 0.6 \\
\hline RYY & 0.5 & 0.5 & 0.5 & 1.3 & 1.2 & 1.1 \\
\hline SAF & 0.3 & 0.3 & 0.7 & 0.4 & 0.5 & 0.8 \\
\hline SAV & 0.2 & 0.3 & 0.2 & 0.5 & 0.8 & 0.4 \\
\hline SBN & 0.0 & 0.1 & 0.1 & 0.3 & 0.4 & 0.5 \\
\hline SDL & 1.9 & 2.9 & 6.5 & 2.2 & 3.5 & 5.2 \\
\hline SDM & 0.2 & 0.6 & 0.3 & 0.6 & 1.2 & 0.8 \\
\hline SEE & 3.3 & 6.1 & 14.1 & 2.2 & 4.5 & 7.1 \\
\hline SFB & 0.4 & 0.6 & 1.4 & 0.7 & 0.9 & 1.2 \\
\hline SGF & 0.3 & 0.7 & 0.7 & 0.7 & 0.8 & 1.0 \\
\hline SGR & 0.3 & 0.5 & 0.5 & 0.7 & 1.1 & 1.5 \\
\hline SHV & 0.4 & 0.4 & 0.6 & 0.7 & 1.0 & 1.2 \\
\hline SJU & 0.2 & 0.1 & 0.2 & 0.2 & 0.2 & 0.3 \\
\hline SMX & 0.6 & 0.7 & 1.0 & 1.0 & 1.4 & 1.5 \\
\hline SRQ & 0.2 & 0.8 & 0.7 & 0.7 & 0.9 & 0.8 \\
\hline STP & 2.2 & 1.6 & 4.3 & 2.2 & 2.0 & 2.8 \\
\hline STT & 0.7 & 0.8 & 1.1 & 1.2 & 1.3 & 1.3 \\
\hline SUS & 1.0 & 2.5 & 2.3 & 0.7 & 0.9 & 1.3 \\
\hline SIP & 0.1 & 0.5 & 1.2 & 0.5 & 0.6 & 0.8 \\
\hline SUX & 0.2 & 0.1 & 0.2 & 0.3 & 0.3 & 0.3 \\
\hline TKI & 0.2 & 0.4 & 0.4 & 0.4 & 0.5 & 0.6 \\
\hline TLH & 0.1 & 0.1 & 0.2 & 0.4 & 0.5 & 0.6 \\
\hline TMB & 1.5 & 2.9 & 4.3 & 1.2 & 2.1 & 2.8 \\
\hline TOA & 0.7 & 0.7 & 0.8 & 0.8 & 0.9 & 1.4 \\
\hline TOL & 0.6 & 0.4 & 0.6 & 1.3 & 1.2 & 1.2 \\
\hline TRI & 0.3 & 0.6 & 1.4 & 0.6 & 0.8 & 1.0 \\
\hline TTD & 0.5 & 0.5 & 0.7 & 0.9 & 0.8 & 1.1 \\
\hline VGT & 0.3 & 0.5 & 0.9 & 0.6 & 0.8 & 1.1 \\
\hline & 0.9 & 4.6 & 30.5 & 1.1 & 2.1 & 23.9 \\
\hline YNA & 0.0 & 0.0 & 0.0 & 0.2 & 0.2 & 0.2 \\
\hline
\end{tabular}





\section{Appendix D \\ Abbreviations}

\begin{tabular}{|l|l|}
\hline AAR & Aircraft Arrival Rate \\
\hline AATT & Advanced Air Transportation Technologies \\
\hline AC & air carrier \\
\hline ACE & Aviation Capacity Enhancement \\
\hline ADR & Aircraft Departure Rate \\
\hline ADT & Airspace Design Tool \\
\hline AEM & Air Equivalency Method \\
\hline AFE & above field elevation \\
\hline AGL & above ground level \\
\hline APT & airport \\
\hline ASPM & Aviation System Performance Metrics \\
\hline ASQP & Airline Service Quality Performance \\
\hline AT & air taxi \\
\hline ATC & air traffic control \\
\hline ATM & air traffic management \\
\hline ATO-F & Air Traffic Organization-Finance \\
\hline ATO-P & Air Traffic Organization-Planning \\
\hline BADA & Base of Aircraft Data \\
\hline C/AFT & $\begin{array}{l}\text { Communication Navigation Surveillance/Air Traffic } \\
\text { Management Focused Team }\end{array}$ \\
\hline CAA & Clean Air Act \\
\hline CAEP & Committee on Aviation Environmental Protection \\
\hline CLEEN & Continuous Lower Energy, Emissions and Noise \\
\hline CO & Carbon monoxide \\
\hline CONUS & Continental United States \\
\hline dB & Decibel \\
\hline D/C & Demand-to-capacity \\
\hline DNL & day-night level \\
\hline EA & Environmental assessment \\
\hline EDMS & Emissions and Dispersion Modeling System \\
\hline & \\
\hline & \\
\hline
\end{tabular}




\begin{tabular}{|l|l|}
\hline EI & Emissions index \\
\hline EIS & Environment impact statement \\
\hline EPA & Environmental Protection Agency \\
\hline ETMS & Enhanced Traffic Management System \\
\hline FAA & Federal Aviation Administration \\
\hline FACT & Future Airport Capacity Task \\
\hline FIP & Federal implementation plan \\
\hline FSG & Flight Segment Generator \\
\hline ft & foot \\
\hline GA & general aviation \\
\hline GD & Gate Demand \\
\hline GDP & Ground Delay Programs \\
\hline GMT & Greenwich Mean Time \\
\hline HC & hydrocarbons \\
\hline ICAO & International Civil Aviation Organization \\
\hline IFR & instrument flight rules \\
\hline ILS & Instrument Landing System \\
\hline IMC & instrument meteorological conditions \\
\hline INM & Integrated Noise Model \\
\hline IPSA & Interagency Portfolio and Systems Analysis Division \\
\hline JPDO & Joint Planning and Development Office \\
\hline kg & kilogram \\
\hline kt & knot \\
\hline LTO & landing and takeoff operations \\
\hline MIT & miles-in-trail \\
\hline MITRE & MITRE Corporation \\
\hline MSL & mean sea level \\
\hline NAAQS & National Ambient Air Quality Standards \\
\hline NAS & National Airspace System \\
\hline NASEIM & NAS-wide Environmental Impact Model \\
\hline NAVAID & navigation aid \\
\hline NEPA & National Environmental Policy Act \\
\hline NextGen & Noise Integrated Routing System \\
\hline NIRS & \\
\hline nm & nautical mile \\
\hline & \\
\hline
\end{tabular}




\begin{tabular}{|l|l|}
\hline NO2 & Nitrogen dioxide \\
\hline NOx & Nitrogen oxide \\
\hline NPD & noise power distance \\
\hline NPIAS & National Plan for an Integrated Airport System \\
\hline OEP & operational evolution plan \\
\hline OMB & Office of Management and Budget \\
\hline OOOI & out-off-on-in \\
\hline Pb & lead \\
\hline PM & Particulate matter \\
\hline POET & Post-Operations Evaluation Tool \\
\hline R\&D & research and development \\
\hline RNAV & area navigation \\
\hline RNP & required navigation performance \\
\hline ROT & runway occupancy time \\
\hline RVR & runway visual range \\
\hline SIP & State implementation plan \\
\hline SO2 & Sulfur dioxide \\
\hline SOx & Sulfur oxide \\
\hline TAF & Terminal Area Forecast \\
\hline TMA & Traffic Management Advisor \\
\hline TRACON & Terminal Radar Approach Control \\
\hline VAMS & Virtual Airspace Modeling and Simulation \\
\hline VFR & visual flight rules \\
\hline VMC & visual meteorological conditions \\
\hline & \\
\hline
\end{tabular}


The public reporting burden for this collection of information is estimated to average 1 hour per response, including the time for reviewing instructions, searching existing data sources, gathering and maintaining the data needed, and completing and reviewing the collection of information. Send comments regarding this burden estimate or any other aspect of this collection of information, including suggestions for reducing this burden, to Department of Defense, Washington Headquarters Services, Directorate for Information Operations and
Reports (0704-0188), 1215 Jefferson Davis Highway, Suite 1204, Arlington, VA 22202-4302. Respondents should be aware that notwithstanding any other provision of law, no person

shall be subject to any penalty for failing to comply with a collection of information if it does not display a currently valid OMB control number
PLEASE DO NOT RETURN YOUR FORM TO THE ABOVE ADDRESS.
1. REPORT DATE (DD-MM-YYYY)
2. REPORT TYPE
3. DATES COVERED (From - To)
$01-01-2010$

4. TITLE AND SUBTITLE

Integrated Analysis of Airport Capacity and Environmental Constraints 5a. CONTRACT NUMBER

5b. GRANT NUMBER

5c. PROGRAM ELEMENT NUMBER

5d. PROJECT NUMBER

NNX08AD15A

5e. TASK NUMBER

5f. WORK UNIT NUMBER

305295.02.07.07.02

\section{PERFORMING ORGANIZATION NAME(S) AND ADDRESS(ES)}

NASA Langley Research Center

Hampton, VA 23681-2199

\section{SPONSORING/MONITORING AGENCY NAME(S) AND ADDRESS(ES)}

National Aeronautics and Space Administration

Washington, DC 20546-0001
10. SPONSOR/MONITOR'S ACRONYM(S)

NASA

11. SPONSOR/MONITOR'S REPORT NUMBER(S)

NASA/CR-2010-216188

\section{DISTRIBUTIONIAVAILABILITY STATEMENT}

Unclassified - Unlimited

Subject Category 03

Availability: NASA CASI (443) 757-5802

\section{SUPPLEMENTARY NOTES}

Langley Technical Monitor: Rosa M. Oseguera-Lohr

\section{ABSTRACT}

LMI conducted an integrated analysis of airport capacity and environmental constraints, identifying and ranking the key factors limiting achievement of NextGen capacity goals. The primary metric used was projected throughput, which was estimated for the years 2015 and 2025 based on the unconstrained demand forecast from the Federal Aviation Administration, and planned improvements including those proposed in the NextGen plan. A set of 310 critical airports was identified, collectively accounting for more than 99 percent of domestic air traffic volume; a one-off analytical approach was used to isolate the constraint being assessed. The study considered three capacity constraints (runway, taxiway, and gate) and three environmental constraints (fuel, NOx emissions, and noise). For the ten busiest airports, runway and noise are the primary and secondary constraints in both 2015 and 2025 . For the OEP 35 airports and overall for the remaining airports, the most binding constraint is noise. Six of the 10 busiest airports, will face runway constraints in 2025 , and 95 will face gate constraints. Nearly every airport will be subject to constraints due to emissions and NOx. Runway and taxi constraints are more concentrated in the large airports; environmental constraints are present at almost every airport regardless of size.

\section{SUBJECT TERMS}

Airport; Capacity; Constraints; Environmental; Throughput

\section{SECURITY CLASSIFICATION OF:} \begin{tabular}{l|l|l|}
\hline a. REPORT & b. ABSTRACT & c. THIS PAGE
\end{tabular} $\mathrm{U}$ $\mathrm{U}$ $\mathrm{U}$ 18. NUMBER PAGES 236 19a. NAME OF RESPONSIBLE PERSON

STI Help Desk (email: help@sti.nasa.gov) 19b. TELEPHONE NUMBER (Include area code)
(443) 757-5802 